\title{
Österreichischer Forschungs- und Technologiebericht 2009
}

Bericht der Bundesregierung an den Nationalrat gem. § 8 (2) FOG über die Lage und Bedürfnisse von Forschung, Technologie und Innovation in Österreich

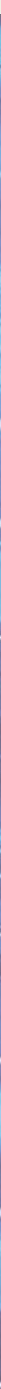


Der vorliegende Bericht ist im Auftrag des Bundesministeriums für Wissenschaft und Forschung (BMWF), Verkehr, Innovation und Technologie (BMVIT) und Wirtschaft, Familie und Jugend (BMWFJ) entstanden. Die Erstellung des Berichts erfolgte durch Joanneum Research (JR), das Österreichische Institut für Wirtschaftsforschung (WIFO), Austrian Research Centers (ARC) sowie unter Beteiligung der Statistik Austria.

AutorInnenteam: Andreas Schibany (Koordination, JR), Martin Berger (JR), Bernhard Dachs (ARC), Rahel Falk (WIFO), Helmut Gassler (JR), Peter Huber (WIFO), Karl-Heinz Leitner (ARC), Brigitte Nones (JR), Michael Peneder (WIFO), Andreas Reinstaller (WIFO), Doris Schartinger (ARC), Gerhard Streicher (JR), Fabian Unterlass (WIFO), Birgit Woitech (JR).

Impressum

Medieninhaber (Verleger):

Bundesministerium für Wissenschaft und Forschung gemeinsam mit Bundesministerium für Verkehr, Innovation und Technologie sowie Bundesministerium für Wirtschaft und Arbeit 1010 Wien

Alle Rechte vorbehalten

Auszugsweiser Nachdruck nur mit Quellenangabe gestattet

Gestaltung und Produktion:

Peter Sachartschenko \& Mag. Susanne Spreitzer OEG, Wien

Umschlagfotos: Ivan Petrov/Fotolia.com

Druck:

AV+Astoria Druckzentrum GmbH, 1030 Wien

Wien, 2009 


\section{Vorwort}

Wissenschaft, Forschung und Entwicklung zählen gerade in Zeiten der Krise und der globalen Veränderungen zu den Schwerpunkten der neuen Bundesregierung, denn Investitionen in Forschung und Entwicklung sind langfristig und nachhaltig wirksame Investitionen in den Wohlstand und die Zukunftsfähigkeit unseres Landes. Österreich hat in den letzten Jahren einen bemerkenswerten Aufholprozess geschafft, der uns in Bezug auf die Ausgaben für Forschung und Entwicklung an die Spitze der europäischen Staaten geführt hat. Dem Ziel, bis $20103 \%$ unseres BIP in Forschung und Entwicklung - und damit in unsere $\mathrm{Zu}$ kunft - zu investieren, werden wir uns trotz der Wirtschaftskrise ambitioniert annähern.

Um den Erfolg der letzten Jahre abzusichern und um uns im Wettbewerb mit den Wachstumsregionen dieser Welt zu behaupten, bedarf es der breiten Diskussion über die zukünftige strategische Ausrichtung unserer Forschungsund Innovationspolitik.

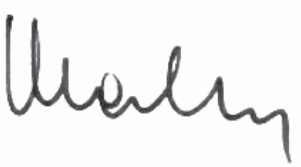

Dr. Johannes Hahn

Bundesminister für Wissenschaft und Forschung
Aufbauend auf dem Österreichischen Forschungsdialog und dem vom WIFO vorgelegten Evaluierungsbericht über das österreichische Forschungsförderungssystem wird die Bundesregierung eine langfristige Strategie für Österreichs Forschung erarbeiten.

Um über den künftigen Weg zu entscheiden, ist es wichtig, eine möglichst umfassende Sicht der gegenwärtigen Situation zu gewinnen. In diesem Sinne verstehen wir den Forschungs- und Technologiebericht 2009 auch als einen wichtigen Beitrag für den kommenden Strategieprozess. Darüber hinaus bietet der Forschungs- und Technologiebericht aber natürlich in gewohnter Weise eine hervorragende und breite Darstellung des österreichischen Forschungs- und Innovationssystems und kann damit zu einer Intensivierung einer faktenbasierten politischen Diskussion über die Bedeutung von Forschung und Entwicklung beitragen.

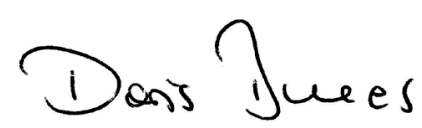

BM Doris Bures

Bundesministerin für Verkehr, Innovation und Technologie 



\section{Inhalt}

$\begin{array}{lr}\text { Executive Summary } & 9\end{array}$

1 Aktuelle Entwicklungen in Forschung und Technologie 16

1.1 Aktuelle Entwicklung der F\&E-Ausgaben in Österreich 16

1.1.1 Ergebnisse der Globalschätzung $2009 \quad 16$

1.1.2 Finanzierung und Durchführung von F\&E in Österreich 19

1.1.3 Der Zusammenhang zwischen der Konjunktur und F\&E-Ausgaben 22

1.1.4 Die Position Österreichs im European Innovation Scoreboard (EIS) 26

1.2 F\&E in Österreich $2002-2006$

$\begin{array}{ll}1.2 .1 \text { Allgemeines } & 30\end{array}$

1.2.2 Finanzierung und Ausgaben 30

1.2.3 Der Unternehmenssektor $\quad 34$

1.2.4 Der Hochschulbereich 36

1.2.5 F\&E-Beschäftigte $\quad 36$

1.2.6 Frauenanteil bei den F\&E-Beschäftigten 38

$\begin{array}{ll}1.2 .7 \text { Resümee } & 43\end{array}$

1.3 Förderung von F\&E - FFG, aws, FWF

1.3.1 Die Forschungsförderungsgesellschaft (FFG) 45

1.3.2 Die Austria Wirtschaftsservice (aws) 49

1.3.3 Exkurs: Die Förderung junger High-Tech-Unternehmen $\quad 51$

1.3.4 Der Wissenschaftsfonds (FWF)

2 Das österreichische Innovationssystem: Bestandsaufnahmen und Perspektiven 60

2.1 Systemevaluierung der Forschungsförderung und -finanzierung $\quad 61$

$\begin{array}{ll}2.1 .1 \text { Einleitung } & 61\end{array}$

2.1.2 Die Instrumente der Forschungsförderung und -finanzierung im Überblick 62

2.1.3 Die steuerliche Förderung der F\&E in Österreich 64

2.1.4 Die direkte Förderung der Forschung in Österreich $\quad 67$

2.1.5 Das Zusammenspiel der steuerlichen und der direkten Forschungsförderung 71

2.1.6 Fördereffekte $\quad 76$

2.1.7 Weitere wichtige Befunde der Systemevaluierung 78

$\begin{array}{ll}2.1 .8 \text { Zusammenfassung } & 79\end{array}$

2.2 Der österreichische Forschungsdialog $\quad \mathbf{8 0}$

2.2.1 Der Bericht der CREST Policy-Mix-ExpertInnengruppe 82 


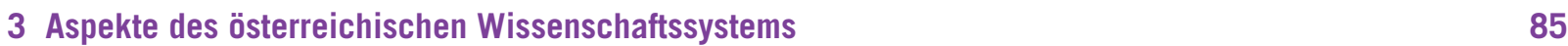

3.1 Struktur und Entwicklung der Grundlagenforschung in Österreich 85

3.1.1 Monetäre Aufwendungen für Grundlagenforschung im internationalen Vergleich $\quad 85$

3.1.2 Grundlagenforschung in Österreich $\quad 88$

3.1.3 Output - Ausgewählte bibliometrische Indikatoren im Überblick 93

3.2 Gibt es ein „europäisches Paradoxon“ (in Österreich)? 97

$\begin{array}{ll}3.2 .1 \text { Forschung } & 98\end{array}$

$\begin{array}{ll}3.2 .2 \text { Lehre } & 101\end{array}$

3.2.3 Resümee $\quad 106$

3.3 Qualifikationsstruktur der MigrantInnen in Österreich 107

3.3.1 Qualifikationsstruktur der im Ausland geborenen AkademikerInnen in Österreich 108

3.3.2 Der Einsatz der im Ausland geborenen Arbeitskräfte 112

3.3.3 Resümee $\quad 117$

4 Innovationen im österreichischen Unternehmenssektor $\quad 118$

4.1 Wachstum und Strukturwandel in der Wissensökonomie $\quad 118$

4.1.1 Aktuelle Befunde $\quad 119$

4.1.2 Resümee $\quad 125$

4.2 „Open Innovation“ in Österreich $\quad 125$

4.2.1 Das Konzept der Open Innovation 126

4.2.2 Empirische Befunde zu Open Innovation 127

4.2.3 Open Innovation in Österreich: Ergebnisse des Community Innovation Survey (CIS) 128

4.2.4 Resümee 134

4.3 Die Wirkung von Innovationen auf die Arbeitsproduktivität $\quad 135$

$\begin{array}{ll}\text { 4.3.1 Resümee } & 139\end{array}$

4.4 Zur Rolle der Kreativwirtschaft im österreichischen Innovationssystem 139

4.4.1 Abgrenzung und statistischer Überblick der Kreativwirtschaft 139

4.4.2 Kreativwirtschaft im österreichischen Innovationssystem 142

4.4.3 Humankapitalmobilität $\quad 146$

4.4.4 Wissenschaftskooperationen und Netzwerkbildung $\quad 149$

$\begin{array}{ll}\text { 4.4.5 Resümee } & 151\end{array}$

4.5 Technologiediffusion in der österreichischen Sachgüterproduktion $\quad 151$

4.6 Berufliche Selbstständigkeit und Unternehmensgründungen von Frauen 158

4.6.1 Die berufliche Selbstständigkeit von Frauen 159

$\begin{array}{ll}\text { 4.6.2 Frauen gründen } & 165\end{array}$

4.6.3 Gründungen von Frauen in Forschung und Technologie 168 
5 Evaluierung von Forschungs- und Technologiepolitik in Österreich

5.1 Kontext und Ratio

5.2 Rechtlicher Rahmen für Evaluation

5.3 Projekte, Programme, Institutionen und das System

5.4 Die Ergebnisse ausgewählter Evaluierungen

178

5.4.1 Zwischenevaluierung - AplusB Academia Business Spin-off Gründungen

5.4.2 Geistige Eigentumsrechte an den Hochschulen:

Evaluierung des Programms uni:invent (2004 - 2006)

6 Referenzen

183

Anhang

190

Statistischer Anhang 



\section{Executive Summary}

Der Forschungs- und Technologiebericht 2009 ist ein Bericht der Österreichischen Bundesregierung und gibt einen Überblick über jüngste Entwicklungen im Bereich Forschung, Technologie und Innovation (FTI). Auf der Basis aktueller Daten, Befunde und Einschätzungen werden zentrale Entwicklungslinien des österreichischen Innovationssystems nachgezeichnet und in ausgewählten Bereichen internationale Vergleiche angestellt. Der vorliegende Bericht ist im Auftrag des Bundesministeriums für Wissenschaft und Forschung (BMWF), Verkehr, Innovation und Technologie (BMVIT) und Wirtschaft, Familie und Jugend (BMWFJ) entstanden. Sämtliche Beiträge wurden in interministeriellen Arbeitgruppen, in denen alle Ressorts vertreten waren, diskutiert und akkordiert.

\section{Entwicklung der F\&E-Quote}

Gemäß der aktuellen Globalschätzung der Statistik Austria werden die gesamten Ausgaben für in Österreich durchgeführte Forschung und experimentelle Entwicklung im Jahr 2009 mehr als 7,652 Mrd. $€$ betragen. Gegenüber dem Jahr 2008 bedeutet dieses Ergebnis der Globalschätzung eine Steigerung der gesamten F\&E-Ausgaben in Österreich von 1,8 \%, der in den vergangenen Jahren überaus starke F\&EWachstumspfad Österreichs wäre somit im Jahr 2009 deutlich abgeschwächt. Aufgrund des zu erwartenden Rückgangs des BIP ist aber mit einer weiteren Steigerung der F\&EQuote und zwar von 2,66 \% (2008) auf 2,73\% (2009) zu rechnen. Nachdem jahrelang der pri- vate Unternehmenssektor mit seinen hohen Wachstumsraten bezüglich F\&E die treibende Kraft für die Finanzierung von Forschung und Entwicklung in Österreich darstellte, geht diese Dynamik nun im Zeichen der Finanz- und Wirtschaftskrise abrupt zu Ende, es ist sogar mit einem Rückgang der entsprechenden Finanzierungsbeiträge des Unternehmenssektors (sowie des Auslands) zu rechnen. Der Bund (und auf niedrigerem absoluten Niveau auch die Länder) übernimmt nunmehr die Vorreiterrolle, bezogen auf die Entwicklung der F\&E-Finanzierung in Österreich. Trotz - oder wegen - der Krise kann der öffentliche Sektor seine F\&E-Finanzierung weiter erhöhen und zwar mit ca. $9 \%$ in durchaus beachtlichem Ausmaß. Insgesamt gelingt es Österreich aber, die F\&E-Quote auch im Jahr 2009 weiter zu steigern. Eine Punktlandung für das $3 \%$-Ziel im Jahr 2010 ist aufgrund der nunmehr vorliegenden Zahlen unter den gegebenen Rahmenbedingungen nicht mehr zu erwarten.

\section{Der Zusammenhang zwischen der konjunkturellen Entwicklung und F\&E}

Angesichts der abrupten Verschlechterung der konjunkturellen Lage der Weltwirtschaft und der Wirtschaft in Österreich ab Herbst 2008 rückt die Frage nach den Auswirkungen der sich im Jahr 2009 weiter verschärfenden Rezession auf die F\&E-Ausgaben insgesamt und vor allem jene des Unternehmenssektors in neues Licht. Die durch zunehmende Unsicherheit und Investitionszurückhaltung geprägte wirtschaftliche Lage verschlechtert auch die 
Rahmenbedingungen für Investitionen in Forschung und Entwicklung. Die Ergebnisse einer ökonometrischen Panel-Regression über 14 Länder zeigen, dass die Forschungsausgaben insgesamt und die des Unternehmenssektors im Besonderen mit der Konjunktur „parallel" verlaufen (prozyklisch); es zeigen sich Hinweise, dass in konjunkturell schlechten Zeiten die F\&E-Ausgaben des Unternehmenssektors sogar stärker schrumpfen als das BIP. Daher ist unter den derzeitigen Konjunkturannahmen mit einem deutlichen Rückgang der F\&E-Ausgaben des Unternehmenssektors zu rechnen. Das Ausmaß dieses möglichen Rückgangs ist für Österreich schwer abzuschätzen, da die F\&E-Ausgaben (i) stark auf einige wenige Großunternehmen konzentriert sind und (ii) die Reaktion des hohen Auslandsanteils in der F\&E-Finanzierung nur ungenügend abschätzbar ist. Es ist wahrscheinlich, dass dieser Anteil überproportional auf BIP-Rückgänge reagieren wird. Bei einem BIP-Rückgang im EU-Raum von etwa $2 \%$ könnte auf Basis der hier angestellten Überlegungen der erwartete Rückgang der Finanzvolumina aus dem Ausland in Österreich bei durchaus 3-5\% liegen. Die Analyse der historischen Entwicklungen der 14 Vergleichsländer zeigt aber auch, dass in Rezessionszeiten die staatliche Finanzierung von Forschungsvorhaben - eventuell im Rahmen anzi-zyklischer Maßnahmen - gestiegen ist. Die Resultate zeigen in diesem Fall (d.h. bei einem Rückgang des BIP um etwa $2 \%$ ) eine Zunahme der staatlichen Ausgaben um etwa $1 / 2-1 \%$.

\section{Die österreichische Position im European Innovation Scoreboard}

In dem methodologisch verbesserten und unter Zugrundelegung aussagekräftigerer Indikatoren erstellten aktuellen European Innovation Scoreboard (EIS) nimmt Österreich 2008 den 6. Platz ein. Österreich führt damit die Gruppe der Innovation Followers an. Vor Österreich liegt die Gruppe der "Innovation Leaders", bestehend aus den Ländern Schweden, Finnland, Deutschland, Dänemark und dem Vereinigten Königreich. Österreich ist knapp daran, zu den Innovationsspitzenreitern aufzuschließen.

Auf der Ebene der Einzelindikatoren bestätigt der EIS 2008 die bereits bekannten Stärken/Schwächen im österreichischen Innovationssystem. Die Stärken liegen vor allem im Unternehmensbereich mit einem deutlich überdurchschnittlichen Abschneiden bei den meisten innovationsbezogenen Indikatoren. Schwächen liegen im unterdurchschnittlichen Abschneiden im Bereich der Humanressourcen, insbesondere bei den technisch- wissenschaftlichen Studienabgängern. Die Schwäche bei der Risikofinanzierung wird ebenfalls durch den EIS 2008 bestätigt.

\section{F\&E zwischen 2002 und 2006}

Seit Vorliegen der Ergebnisse der F\&E-Vollerhebung 2006 lassen sich intertemporale Vergleiche anstellen. Dabei zeigt sich, dass zwischen 2002 und 2006 die F\&E-Ausgaben insgesamt um $35 \%$ und die Zahl der F\&E durchführenden Einheiten um $17 \%$ gestiegen sind. Vor allem der Unternehmenssektor zeichnet sich bei beiden Indikatoren durch die höchsten Steigerungsraten aus $1+42 \%$ bei den F\&E-Ausgaben und $+24 \%$ bei der Anzahl forschender Unternehmen). Diese Steigerungsraten - vor allem, was die Erhöhung der Anzahl forschender Unternehmen betrifft - sind ein Indiz dafür, dass sich die Forschungsbasis der österreichischen Wirtschaft auch in Zukunft noch erweitern wird.

Die Finanzierungsstrukturen der gesamten F\&E-Ausgaben sind für den untersuchten Zeitraum relativ stabil. Bei einer inhaltlichinstitutionellen Interpretation finanziert der Unternehmenssektor (inländische und ausländische Unternehmen zusammen) 65 \% der 
gesamten Forschungsausgaben in Österreich. Dieser Anteil entspricht dem OECD- sowie dem EU-Durchschnitt.

Mit einem Anteil von $32 \%$ an den F\&EAusgaben des Unternehmenssektors sowie einer F\&E-Intensität (Anteil der F\&E-Ausgaben an der Bruttowertschöpfung) von $22 \%$ nimmt der High-Tech-Sektor in der Sachgüterindustrie die wichtigste Rolle ein. Den höchsten $\mathrm{Zu}-$ wachs verzeichnen hingegen die forschungsund technologieintensiven Dienstleistungen, deren F\&E-Anteil an der Bruttowertschöpfung von $11 \%$ auf knapp unter $17 \%$ gestiegen ist. $14 \%$ aller F\&E-Ausgaben sind 2006 in diesem Sektor getätigt worden.

Die F\&E-Ausgaben des Hochschulsektors stiegen in dem genannten Zeitraum um $+20 \%$. Das Fördervolumen durch den öffentlichen Sektor - als den wichtigsten Finanzierungssektor für die F\&E im Hochschulsektor - stieg um $+17 \%$. Mit $+55 \%$ fiel hingegen die Steigerung der Finanzierung der F\&E im Unternehmenssektor durch den öffentlichen Sektor deutlich höher aus. Unter Berücksichtigung der Forschungsprämie (welche im Jahr 2006 knapp 156 Mio. $€$ betrug) finanziert der öffentliche Sektor $10 \%$ der F\&E-Ausgaben des Unternehmenssektors. Das Verhältnis der Finanzierung der Unternehmens-F\&E (428 Mio. €) zur Finanzierung der Hochschul-F\&E (1.355 Mio. €) durch den öffentlichen Sektor betrug im Jahr 2006 somit ca. 1:3.

Die Beschäftigten im F\&E-Bereich haben zwischen 2002 und 2006 um $+28 \%$ auf 84 Tsd. (Kopfzahlen) zugenommen, was 49 Tsd. VZÄ entspricht. Der Frauenanteil bei den F\&E-Beschäftigten ist zwar zwischen 2002 und 2006 geringfügig gestiegen, ist aber mit 29 \% (Kopfzahlen) immer noch relativ gering. Allerdings zeigt die geschlechtsspezifische Analyse des wissenschaftlichen Personals an den Universitäten, dass sich der - verglichen mit 2002 - gestiegene Frauenanteil bei den jüngeren Altersgruppen auch im weiteren Karriereverlauf fortsetzt.

\section{Perspektiven und Einschätzungen des österreichischen Innovationssystems}

Das österreichische Innovationssystem war in den Jahren 2008/09 Gegenstand von Einschätzungen, Diskussionen sowie einer umfassenden Evaluierung. Versuchte der breit und umfassend angelegte Forschungsdialog möglichst viele Themen der FTI zu diskutieren und damit der gesamten Community ein Forum des Meinungsaustausches und Diskurses zu bieten, stellte die CREST Policy-Mix-ExpertInnengruppe die Sicht von außen dar. Angeführt werden die 24 Empfehlungen von der Feststellung, dass in Österreich eine gemeinsame und ganzheitliche Vision fehle und Österreich von der „Formulierung ganzheitlicher F\&E- und innovationsbezogener Strategien und der daraus folgenden Artikulierung eines kohärenten policy mix profitieren würde".

Der vorliegende Bericht enthält eine Darstellung wichtiger Ergebnisse, die im Rahmen der Systemevaluierung erbracht wurden. Diese beruhen auf Analysen, die im Rahmen der Systemevaluierung durchgeführt wurden. Schlussfolgerungen und Empfehlungen sind auf politischer Ebene noch zu diskutieren. Der Schwerpunkt der Darstellung sind die Hauptinstrumente der Forschungsförderung und -finanzierung (direkte und steuerliche F\&E Förderung). Hinsichtlich der steuerlichen Förderung zeigt sich, dass deren Ausmaß in den vergangenen Jahren überschätzt wurde. Entgegen den Schätzungen des Rechnungshofs und des Bundesministeriums für Finanzen liegen die Kosten der steuerlichen F\&E Förderung etwas über 250 Mio. $€$ zu konstanten Preisen. Was die Zielgruppen der steuerlichen Förderung anbelangt, so zeigen die Untersuchungen im Rahmen der Systemevaluierung, dass Unternehmen mit mehr als 100 Beschäftigten ca. $40 \%$ der Förderempfänger für den FFB-Alt und $30 \%$ für den FFB-Neu darstellen. Diese Unternehmen haben im Jahr 2005 ca. 90 \% der gesamten 
steuerlichen Förderung aus dem FFB-Alt und dem FFB-Neu in Anspruch genommen. Andererseits hat die Einführung der Forschungsprämie zu einer Ausweitung der Inanspruchnahme steuerlicher Förderung geführt. Viele der neu gewonnenen Nutzer sind Klein- und Mittelbetriebe (KMU), die auch bezüglich der Fördervolumen die Hauptnutznießer der Forschungsprämie sind.

Bezüglich der direkten Forschungsförderung zeigt sich, dass unterschiedliche Interventionsund Handlungslogiken zu einer Überlappung des Angebots bei den Zielgruppen führt. Die Ergebnisse der Analyse legen somit nahe, dass eine bessere und flexiblere Abstimmung der Förderangebote aufeinander die Steuerungswirkung der direkten Forschungsförderung erhöhen könnte. Hinsichtlich der Nutzerzufriedenheit deuten die Ergebnisse darauf hin, dass der oft ins Treffen geführte „Förderdschungel“ von den Nutzern nicht als solcher empfunden wird. Die Untersuchungen belegen vielmehr, dass sich die Nutzer sehr agil durch das System bewegen. Ergebnisse deuten darauf hin, dass diese sich mit dem Ziel im System bewegen, Förderwahrscheinlichkeit und Höhe der Förderzusagen zu maximieren. Damit entpuppt sich der "Förderdschungel" in einem positiven Sinn eher als "Fördersupermarkt". Die Untersuchungen der Evaluierung deuten auch darauf hin, dass Ziele und Aufgaben der Programme klarer definiert werden müssen. Sie sollen auch vermehrt auf Exzellenz und radikale Innovation hinwirken.

Die Analyse des Zusammenspiels der steuerlichen und der direkten Förderung zeigt, dass beide Förderungsformen notwendig sind. Das Ergebnis, dass sich das Zusammenspiel von direkter und indirekter Förderung positiv auf den Fördererfolg sehr dynamischer FTI-aktiver Unternehmen auswirkt, ist sehr robust und deckt sich weitgehend mit internationalen Befunden zu dem Zusammenspiel der direkten und indirekten Forschungsförderung. Weitergehen- de Ergebnisse der Systemevaluierung belegen, dass der Mangel an hochqualifizierten Humanressourcen auf allen Ebenen einen Flaschenhals und damit die große Herausforderung an die Politik darstellt. Generell zeigen die Analysen auf, dass die gesamte Forschungsförderungs und -finanzierungspolitik stärker mit anderen Politikbereichen verbunden werden muss, die einen Einfluss auf Innovation haben, so wie Bildung, Finanzierung udgl.

\section{Forschung und Ausbildung im Hochschulsektor}

Auf die Grundlagenforschung entfallen derzeit ca. 0,41 \% des BIP. Damit liegt Österreich im internationalen Vergleich im Mittelfeld. Berücksichtigt man allerdings die gute Position Österreichs was die allgemeine F\&E-Quote betrifft, so lässt sich eine etwas zu geringe Allokation von Ressourcen für Grundlagenforschung konstatieren. Auf der Grundlage der gesamten F\&E-Ausgaben entfallen derzeit nur $17 \%$ der gesamten Forschungsaufwendungen auf die Grundlagenforschung (35\% für angewandte Forschung und $47 \%$ für experimentelle Entwicklung), womit Österreich eher am unteren Ende jener OECD-Länder liegt, die entsprechende Daten bereitstellen. Die Grundlagenforschung geschieht zu $70 \%$ im Hochschulsektor, wodurch dem öffentlichen Sektor eine besondere Verantwortung bei der Finanzierung zukommt. Die Bereitstellung einer angemessenen Finanzierung und ihre Abschirmung vor Konjunkturschwankungen sind daher ein wichtiges Ziel der Bundesregierung.

Die öffentliche Finanzierung ist auch nötig, um die Forschungsausbildung von UniversitätsabsolventInnen zu unterstützen. Diesen kommt eine besondere Bedeutung $\mathrm{zu}$, tragen sie doch neues Wissen in den Unternehmenssektor und bilden die Basis für Innovationen und Wettbewerbsfähigkeit. Die im Bericht verwendeten Indikatoren geben jedoch Hinweise darauf, dass vor allem im technisch- 
naturwissenschaftlichen Bereich ein Mangel an AbsolventInnen herrscht und sich in naher Zukunft noch verschärfen wird. Schnell veraltetes Wissen und die Notwendigkeit einer hohen technologischen Kompetenz erfordern es, das Humankapital einer Gesellschaft ständig zu erweitern, anzupassen und zu erneuern. Versäumnisse in diesem Feld sind in kurzer und mittlerer Frist nicht zu kompensieren, da die Reaktionszeiten einer Umsteuerung im Qualifikationssystem sehr lang sind und die Ergebnisse erst nach vielen Jahren zur Verfügung stehen.

\section{Qualifikationsstruktur der MigrantInnen}

Bezüglich der Qualifikationsstruktur der im Ausland Geborenen weist Österreich spezifische Probleme auf. Vor allem in der Gruppe der Hochqualifizierten ist Österreich das OECDLand mit dem geringsten Anteil (11,3 \%) von AkademikerInnen unter den im Ausland geborenen Personen. Allerdings weisen internationale Vergleiche Österreich auch als ein Land aus, in dem überdurchschnittlich viele AusländerInnen studieren. Der Anteil der ausländischen Studierenden liegt bei 11,5\% und ist der vierthöchste unter den 23 OECD-Ländern, für die vergleichbare Daten vorliegen. Österreich kann als Land bezeichnet werden, in dem zwar viele AusländerInnen studieren, aber nur ein geringer Anteil hoch qualifizierter MigrantInnen lebt.

Die Daten der Arbeitskräfteerhebung zeigen, dass im Vergleich zur österreichischen Bevölkerung die im Ausland geborene Bevölkerung in Österreich nach wie vor stark an den beiden Extremen der Bildungsverteilung angesiedelt ist und unterdurchschnittliche Anteile im mittleren Qualifikationssegment aufweist.

Die Bemühungen der Migrationspolitik der letzten Jahre, verstärkt hoch qualifizierte Arbeitskräfte anzuziehen, dürften zu einer leich- ten Verbesserung der Bildungsstruktur der $\mathrm{Zu}$ gewanderten beigetragen haben. Im Ausland geborene Hochqualifizierte sind aber deutlich öfter als ihre im Inland geborenen KollegInnen beschäftigungslos und arbeiten auch deutlich häufiger als in Österreich Geborene unter ihrem Qualifikationsniveau: Rund die Hälfte $(47 \%)$ der im Ausland geborenen AkademikerInnen arbeitet in einem Beruf unter ihrem Qualifikationsniveau, bei den im Inland Geborenen liegt dieser Anteil bei 29 \%. AkademikerInnen, Zugewanderte aus traditionellen Gastarbeiterländern, Personen, die im Alter von mehr als 20 Jahren zuwanderten und $\mathrm{Zu}$ gewanderte der Jahre 1989 bis 1993 sind davon besonders stark betroffen.

Die Handlungsspielräume zur Veränderung dieser Situation liegen einerseits in der Attraktivierung des Standortes Österreich für hoch qualifizierte Arbeitskräfte, andererseits in migrationspolitischen Interventionen in Richtung einer stärkeren Selektion der Zuwanderung nach Ausbildungskriterien. Darüber hinaus bedarf es Maßnahmen zur leichteren Transferierbarkeit von Qualifikationen nach Österreich (d.h. Anerkennung von im Ausland erworbenen formalen Bildungsabschlüssen). Abgesehen von der sprachlichen Integration scheinen dabei einige institutionelle Besonderheiten des österreichischen Arbeitsmarktes eine wichtige Barriere darzustellen. Außerdem stellen Frauen und Personen, die im Alter zwischen 16 und 19 Jahren zuwandern, eine besondere Zielgruppe dar. Bei ersteren gilt es, die doppelte Diskriminierung der hoch qualifizierten im Ausland geborenen Frauen am Arbeitsmarkt zu verringern. Bei letzteren dürfte hingegen die migrationsbedingte Diskontinuität in der Bildungskarriere oftmals zu einem Abbruch der Schullaufbahn führen. Deshalb sollte auch das Schulwesen in eine umfassende Strategie zur Qualifizierung der im Ausland Geborenen einbezogen werden. 


\section{Wachstum und Strukturwandel}

Gesamtwirtschaftliches Wachstum wird durch viele Faktoren determiniert. Die vorhandene Branchenstruktur spielt dabei eine besondere Rolle und der signifikante Einfluss der Anteile sowohl von technologie- wie auch humankapitalintensiven Branchen auf das gesamtwirtschaftliche Wachstum wurde in einer Reihe von Untersuchungen bestätigt. Österreich wies diesbezüglich in den 1990er Jahren trotz hoher Wachstumsraten ein Strukturdefizit auf (Struktur-Performance Paradoxon). Neuere und umfassendere Analysen zeigen, dass Österreich im Zeitraum 1995-2005 eine markante Veränderung zugunsten wissensintensiver Produktionszweige zu verzeichnen hat, wodurch der Rückstand bei den innovationsintensiven Sektoren verringert werden konnte. Aber auch andere Länder verzeichnen einen Strukturwandel, wodurch trotz des beschleunigten Wandels in der Branchenspezialisierung in der Gruppe der ausbildungsintensiven Sektoren der Abstand Österreichs zur EU15 weiterhin besteht. Neben den laufenden Anstrengungen in der Innovationspolitik wird daher die Wirtschaftspolitik ihre Aufmerksamkeit in Zukunft verstärkt auf die Qualität und Effizienz der Ausbildung richten müssen.

\section{Innovation im Unternehmenssektor}

Innovationsprozesse von Unternehmen sind vielfältige und offene Prozesse. Der positive Einfluss von Innovation auf die Arbeitsproduktivität konnte in einer internationalen Studie empirisch nachgewiesen werden. Auch öffnen sich Unternehmen zunehmend für externe Impulse, um so die Effizienz und Effektivität der Investitionen in F\&E und Innovation zu erhöhen.

Nun stellt die derzeitige Krise am Finanzmarkt sowie die zu erwartende Rezession ernste Barrieren für Unternehmen dar: Mit der Ver- ringerung von Unternehmensgewinnen wird auch die Finanzierung von Innovationen deutlich schwieriger und eine sinkende Nachfrage erschwert zudem die Einführung neuer Produkte im Markt. Gleichzeitig können innovative Unternehmen veränderte Marktbedingungen auch als Chance nutzen, um durch Innovationen - wie die Neuorganisation interner Abläufe und die daraus resultierenden Produktivitätsgewinne für die Entwicklung neuer und leistungsfähigerer Produkte zu nutzen - neue Nachfrage zu generieren und sich im Wettbewerb neu zu positionieren. Die in Zeiten rückläufiger Kapazitätsauslastung freiwerdenden Ressourcen können für Zukunftsinvestitionen genutzt werden, wodurch vor allem hochqualifizierten Beschäftigten eine besondere Rolle zukommt. Denn es ist das unternehmensinterne Knowhow, welches in einer Phase der Konjunkturschwäche verstärkt Innovationsprojekte vorantreibt, um in Zeiten des Aufschwungs mit einer neuen Produktpalette sowie effizienten Prozessen in den Wettbewerb treten zu können. Dies löst zusätzliche Nachfrage aus und setzt neue gesamtwirtschaftliche Wachstumsimpulse.

\section{Die Kreativwirtschaft}

Insgesamt kann die Kreativwirtschaft als ein bedeutsamer Wachstumssektor betrachtet werden, dessen Gewicht mittlerweile auch gesamtwirtschaftlich nicht zu unterschätzen ist. Immerhin jedes zehnte Unternehmen und jede/r 20. Beschäftigte ist der Kreativwirtschaft zuzuordnen, der Anteil an der gesamten Wertschöpfung beträgt $5 \%$. Gleichzeitig lässt sich die Kreativwirtschaft als ein Querschnittssektor einstufen, dessen Produkte und Dienstleistungen vor allem auch in anderen Branchen zum Einsatz kommen und von dem daher wichtige Impulse auf die Gesamtwirtschaft ausgehen. Neben dem direkten Beitrag zum Innovationsgeschehen durch eigene Innovationen kommt der Kreativwirtschaft aber auch 
durch weitere Mechanismen eine wichtige Funktion im Innovationssystem Österreichs zu. So beschäftigte die Kreativwirtschaft überdurchschnittlich qualifiziertes Humankapital und weist eine überdurchschnittliche Personalmobilität auf. Beschäftigte der Kreativwirtschaft wechseln regelmäßig die Branche und stellen somit anderen Branchen ihre kreativen Fähigkeiten zur Verfügung.

\section{Unternehmensgründungen von Frauen}

Die berufliche Selbstständigkeit und Unternehmensgründungen von Frauen wiesen in den letzten Jahren eine sehr dynamische Entwicklung auf. So ist der Frauenanteil an den Neugründungen in den letzten Jahren um knapp 14 \%-Punkte gestiegen und im Jahr 2007 wurde bereits jedes dritte Unternehmen von einer Frau gegründet. Allerdings bestehen nach wie vor geschlechterspezifische Unterschiede in der Branchenwahl. So ist auch für Österreich zu vermuten, dass der Anteil der von Frauen gegründeten Unternehmen im High-Tech-Sektor niedrig ist (in Deutschland beträgt der An- teil knapp $10 \%$ ). Dies ist einerseits Ausdruck der spezifischen Studien- und Berufswahl von Frauen, andererseits aber auch Folge von gesellschaftsbedingten Barrieren.

\section{Evaluierung in Österreich}

Der CREST Review Bericht bestätigt, dass „Österreich nun eine der am besten entwickelten und eingebetteten Innovationsevaluierungskulturen in Europa" besitzt. Eine entwickelte Evaluierungskultur stellt einen zentralen Bestandteil einer lernenden und strategisch orientierten FTI-Politik dar und ist gleichsam Voraussetzung und Folge einer guten, also effizienten, transparenten und fairen Politik. Gleichzeitig kann die in Österreich herrschende Programmvielfalt und die daraus resultierende Menge an Evaluierungen auch zu einer „Paralyse durch Analyse“ führen. Evaluierungen erfüllen nur dann ihren Zweck, wenn ihre Ergebnisse in künftige Politikformulierung und -praxis einfließen und entsprechenden Mechanismen dies sicherstellen. 


\section{Aktuelle Entwicklungen in Forschung und Technologie}

\subsection{Aktuelle Entwicklung der F\&E-Ausgaben in Österreich}

\subsubsection{Ergebnisse der Globalschätzung 2009}

Gemäß der aktuellen Globalschätzung der Statistik Austria werden die gesamten Ausgaben für in Österreich durchgeführte Forschung und experimentelle Entwicklung im Jahr 2009 mehr als 7,652 Mrd. $€$ betragen. Zu beachten ist dabei (sowie bei allen folgenden Ausführungen), dass aufgrund der globalen Finanzund Wirtschaftskrise die diesjährige Prognose mit außergewöhnlich hohen Unsicherheiten behaftet ist. Diese Unsicherheiten betreffen sowohl die Abschätzung des BIP für das Jahr 2009 als auch die Abschätzungen für die F\&EAusgaben der jeweiligen Durchführungs- und Finanzierungssektoren (mit Ausnahme jener des Bundes, die ja im entsprechenden Budgetgesetz verankert sind).

Gegenüber dem Jahr 2008 bedeutet dieses Ergebnis der Globalschätzung eine Steigerung der gesamten F\&E-Ausgaben in Österreich um $1,8 \%$ - der in den vergangenen Jahren überaus starke F\&E-Wachstumspfad Österreichs wäre im Jahr 2009 somit deutlich abgeschwächt. Aufgrund des zu erwartenden Rückgangs des BIP ist aber mit einer weiteren Steigerung der F\&E-Quote und zwar von 2,66\% (2008) auf $2,73 \%$ (2009) zu rechnen (siehe Abbildung 1).

Am deutlichsten schlagen sich die zu erwartenden Folgen der globalen Finanz- und Wirtschaftskrise hinsichtlich der F\&E-Aufwendungen auf die Entwicklungen der einzelnen Finanzierungssektoren nieder. Während der Bund (und die Bundesländer) ihre F\&EFinanzierungsbeiträge weiter steigern, muss mit einem Rückgang der F\&E-Finanzierung des privaten Unternehmenssektors und des Auslands (hier ist wiederum die Finanzierung von F\&E-Aktivitäten österreichischer Töchter durch ihre ausländischen Mutterunternehmen ausschlaggebend) gerechnet werden. Bund und Länder werden 2009 voraussichtlich 2,947 Mrd. $€$ zur F\&E-Finanzierung aufwenden, davon entfallen 2,545 Mrd. $€(+8,9 \%)$ auf den Bund und 0,402 Mrd. $€(+9,5 \%)$ auf die Länder. Dem stehen sinkende Finanzierungsbeiträge des privaten Unternehmenssektors mit 3,440 Mrd. $€(-1,3 \%)$ und des Auslands mit 1,132 Mrd. $€(-6,2 \%)$ gegenüber. Für die Restkategorie "Sonstiges" (z.B. Gemeinden ohne Wien, Kammern und Sozialversicherungsträger sowie privater gemeinnütziger Sektor) wird ein F\&E-Finanzierungsbeitrag von 0,133 Mrd. € I+ 9,6\%) erwartet.

Die Ergebnisse der Globalschätzung zeigen somit, dass sich die Finanz- und Wirtschaftskrise auf folgende Weise auf die Entwicklung der F\&E-Finanzierung auswirkt:

- Nachdem jahrelang der private Unternehmenssektor mit seinen hohen Wachstumsraten bezüglich F\&E die treibende Kraft für die Finanzierung von Forschung und Entwicklung in Österreich darstellte, geht diese Dynamik nun abrupt zu Ende, es ist sogar mit einem weiteren Rückgang der entsprechenden Finanzierungsbeiträge zu rechnen.

- Der Finanzierungssektor Ausland hat sich seit 2002 auf hohem Niveau konsolidiert, die Wachstumsraten waren seither zwar geringer aber immer noch positiv. Für das Jahr 
2009 muss nun aber ebenfalls ein Rückgang der F\&E-Finanzierung des Auslands für Forschung in Österreich erwartet werden. Dieser Rückgang ist aufgrund der sinkenden Finanzierung multinationaler Konzernunternehmen für die F\&E-Aktivitäten ihrer österreichischen Töchter zurückzuführen.

- Der Bund (und auf niedrigerem absoluten Niveau auch die Länder) übernimmt nunmehr die Vorreiterrolle, was die Entwicklung der F\&E-Finanzierung in Österreich betrifft. Trotz - oder wegen - der Krise kann der öffentliche Sektor seine F\&E-Finanzierung weiter erhöhen und zwar mit ca. $9 \%$ in durchaus beachtlichem Ausmaß.

Insgesamt gelingt es Österreich - in Verein mit dem sinkenden BIP, das ja als Divisor in die Berechnung eingeht - die F\&E-Quote auch im Jahr 2009 weiter zu steigern. Eine Punktlandung für das 3-\%-Ziel im Jahr 2010 ist aber aufgrund der nunmehr vorliegenden Zahlen bzw. Abschätzungen unter den gegebenen Rahmenbedingungen nicht mehr zu erwarten.

\section{Abbildung 1: Forschung und Entwicklung in Österreich nach Finanzierungssektoren}

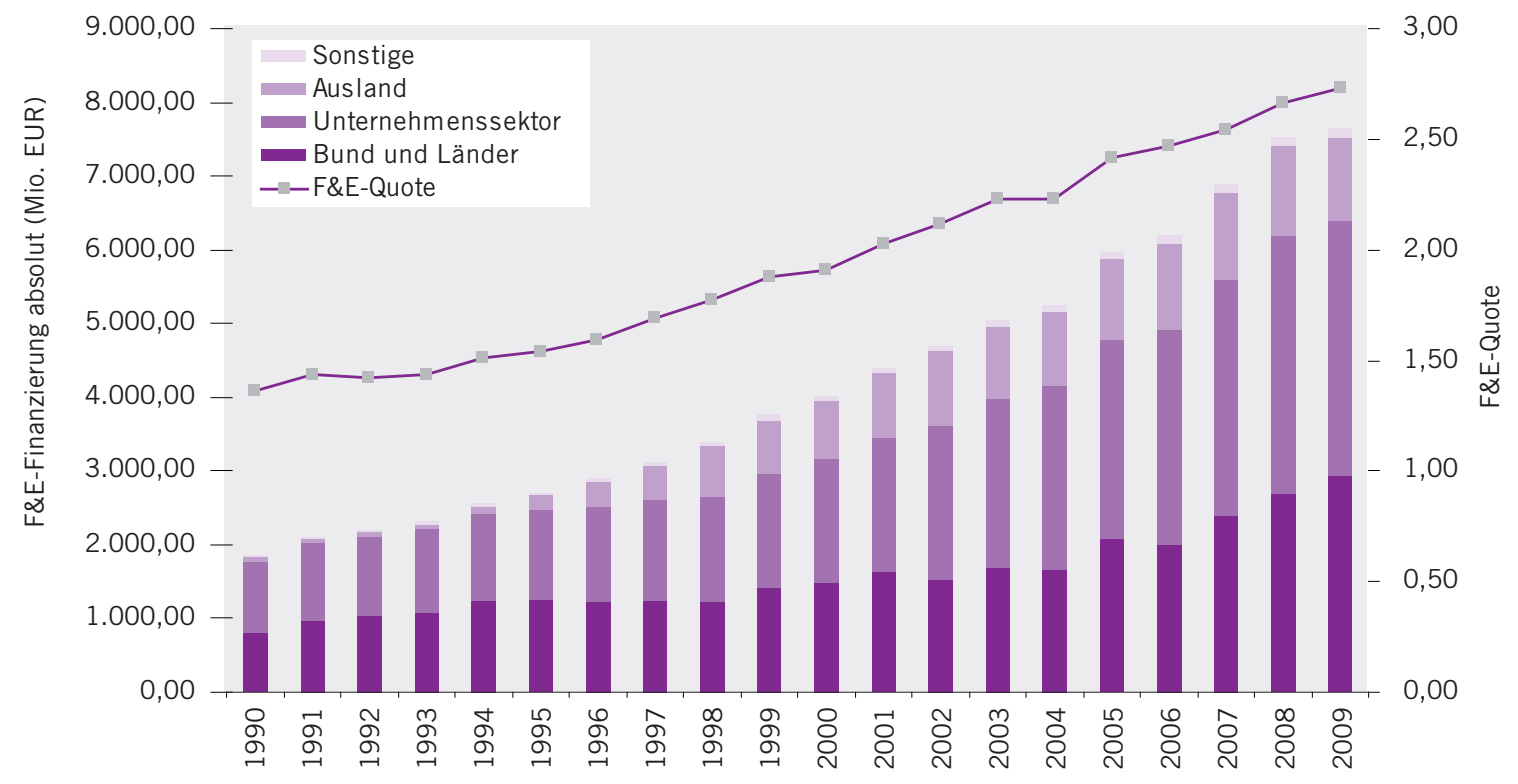

Quelle: Statistik Austria, Globalschätzung 2009 - Stand: 4. Mai 2009

\section{Internationaler Vergleich der F\&E-Quoten}

Für einen internationalen Vergleich der Entwicklung der F\&E-Quoten musste zwecks Datenverfügbarkeit und Vergleichbarkeit der Zeitraum 2000 bis 2006 zugrunde gelegt werden. Für Österreich bedeuteten die 6,3 Mrd. Forschungsausgaben im Jahre 2006 eine Forschungsquote von 2,45\% des BIP (Bruttoinlandsprodukt), nach 2,12\% im Jahr 2002 und
2,22 \% im Jahr 2004. Hinsichtlich der Zielsetzung, im Jahr 2010 eine Forschungsquote von $3 \%$ des BIP zu erreichen, sind die Fortschritte Österreichs über die letzten Jahre durchaus eindrucksvoll. Ausgehend von einer deutlich unterdurchschnittlichen Forschungsquote in den 1980er Jahren (1981 betrug sie 1,1 \% des BIP, gegenüber einem Schnitt der EU15 von 1,64 \%) konnte sie kontinuierlich - und speziell seit 1995 durchaus rasant - gesteigert wer- 
den. 1998 wurde der (nun bei 1,83 \% liegende) EU15-Schnitt übertroffen. Seit 2004 liegt Österreich nun auch über dem Schnitt der OECD-Staaten. Von den in Abbildung 2 angeführten EU- (und OECD) Staaten weist Österreich mit $+0,53$ Prozentpunkten die größte Veränderung auf. Auch lässt sich leicht erkennen, warum die F\&E-Quote auf europäischer Ebene stagniert. Da die großen Mitgliedslän- der wie Frankreich (-0,04 Prozentpunkte) und Großbritannien (-0,07 Prozentpunkte) jeweils einen Rückgang und Deutschland $1+0,08$ Prozentpunkte) und Italien $(+0,04$ Prozentpunkte) nur marginale Erhöhungen erreichten, veränderte sich die F\&E-Quote für Europa kaum. Die EU ist von dem ambitionierten Ziel einer F\&E-Quote von 3 \% für das Jahr 2010 weiter entfernt denn je.

\section{Abbildung 2: Entwicklung der F\&E-Ausgaben als Anteil am Bruttoinlandsprodukt im Ländervergleich}

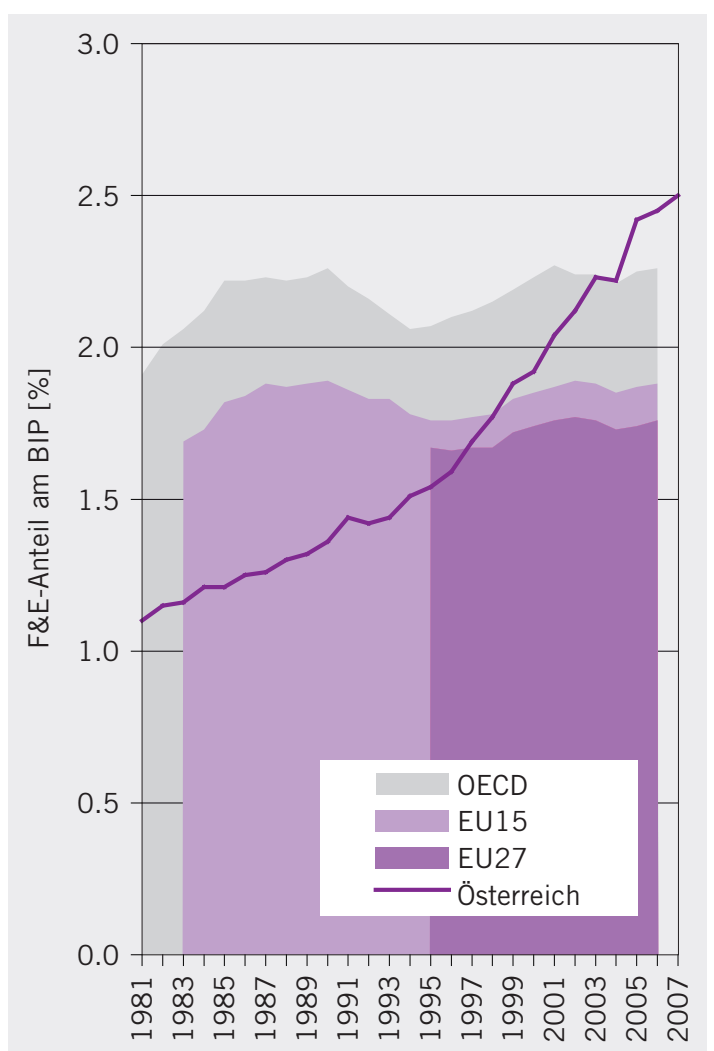

\begin{tabular}{|c|c|c|c|}
\hline & 2000 & 2006 & $5-2000)$ \\
\hline Belgien & 1.97 & 1.83 & -0.14 \\
\hline Dänemark & 2.18 (1999) & 2.43 & +0.25 \\
\hline Deutschland & 2.45 & 2.53 & +0.08 \\
\hline Finnland & 3.34 & 3.45 & +0.11 \\
\hline Frankreich & 2.15 & 2.11 & -0.04 \\
\hline Griechenland & 0.60 (1999) & 0.57 & -0.03 \\
\hline Großbritannien & 1.85 & 1.78 & -0.07 \\
\hline Irland & 1.12 & 1.32 & +0.20 \\
\hline Italien & 1.05 & $1.09(2005)$ & +0.04 \\
\hline Niederlande & 1.83 & 1.67 & -0.16 \\
\hline Norwegen & 1.64 (1999) & 1.52 & -0.12 \\
\hline Österreich & 1.92 & 2.45 & +0.53 \\
\hline Polen & 0.64 & 0.56 & -0.08 \\
\hline Portugal & 0.76 & 0.83 & +0.07 \\
\hline Schweden & 3.57 (1999) & 3.73 & +0.16 \\
\hline Slowakische Republik & 0.65 & 0.49 & -0.16 \\
\hline Spanien & 0.91 & 1.20 & +0.29 \\
\hline Tschechische Republik & 1.21 & 1.54 & +0.33 \\
\hline Ungarn & 0.78 & 1.00 & +0.22 \\
\hline EU27 & 1.74 & 1.76 & +0.02 \\
\hline EU15 & 1.85 & 1.88 & +0.03 \\
\hline Kanada & 1.92 & 1.94 & +0.02 \\
\hline Japan & 3.04 & 3.39 & +0.35 \\
\hline Schweiz & 2.53 & 2.90 (2003) & +0.37 \\
\hline USA & 2.74 & 2.62 & -0.12 \\
\hline OECD & 2.23 & 2.26 & +0.03 \\
\hline China & 0.90 & 1.42 & +0.52 \\
\hline
\end{tabular}

Quelle: OECD (MSTI), Berechnungen Joanneum Research 


\subsubsection{Finanzierung und Durchführung von F\&E in Österreich}

Für das Jahr 2006 wurde durch die Statistik Austria eine Vollerhebung bei den F\&E durchführenden Institutionen in allen volkswirtschaftlichen Sektoren durchgeführt. Das ermöglicht eine Analyse der Finanzierungsströme zwischen den wesentlichen Finanzierungs- bzw. Durchführungssektoren, sowie auch eine Einschätzung der Interdependenzen der einzelnen Sektoren.

In der Statistik wird zwischen vier Durchführungssektoren (Hochschulsektor, Sektor Staat, Privater gemeinnütziger Sektor und Unternehmenssektor) und vier Finanzierungssektoren (Öffentlicher Sektor, Unternehmenssektor, Privater gemeinnütziger Sektor und Ausland) unterschieden. Wobei zu bemerken ist, dass der Hochschulsektor kein Finanzierungssektor ist, wogegen das "Ausland" dem Inlandskonzept entsprechend kein Durchführungssektor sein kann. Für diese Analyse wurde der „Unternehmenssektor" weiter aufgesplittert: Zum einen in den firmeneigenen Bereich /umfasst im Wesentlichen die in der Absicht zur Erzielung eines Ertrags oder sonstigen wirtschaftlichen Vorteils für den Markt produzierenden Unternehmen des Produzierenden Bereichs und des Dienstleistungsbereichs) und zum anderen in den kooperativen Bereich (sind Dienstleistungseinrichtungen, die Forschung und experimentelle Entwicklung für Unternehmen betreiben; mehrheitlich nicht in der Absicht zur Erzielung eines Ertrags oder sonstigen wirtschaftlichen Vorteils, wie etwa die Mitglieder in der Vereinigung der kooperativen Forschungseinrichtungen der österreichischen Wirtschaft - ACR, die Austrian Research Centers - ARC, Joanneum Research oder die Kompetenzzentren).

Die folgende Tabelle 1 gibt einen Überblick über die Aufteilung der gesamten F\&E-Ausgaben für das Jahr 2006 nach Durchführungsund Finanzierungssektoren.

Tabelle 1: F\&E-Ausgaben nach Durchführung und Finanzierung (2006)

\begin{tabular}{|c|c|c|c|c|c|}
\hline Durchfẗhrungssektoren & in Mio. $€$ & Anteile in \% & Finanzierungssektoren & in Mio. $€$ & Anteile in \% \\
\hline Unternehmenssektor & 4.448 & 70,4 & Unternehmenssektor & 3.057 & 48,4 \\
\hline kooperative Bereich & 428 & 6,8 & Öffentlicher Sektor & 2.071 & 32,8 \\
\hline firmeneigener Bereich & 4.020 & 63,6 & Privater gemeinnütziger Sektor & 27 & 0,4 \\
\hline Hochschulsektor & 1.523 & 24,1 & Ausland & 1.163 & 18,4 \\
\hline Sektor Staat ${ }^{1}$ & 330 & 5,2 & ohne EU & 1.059 & 16,8 \\
\hline Privater gemeinnütziger Sektor ${ }^{2}$ & 17 & 0,3 & $E U$ & 104 & 1,6 \\
\hline Gesamt & 6.318 & 100,0 & Gesamt & 6.318 & 100,0 \\
\hline
\end{tabular}

1 Bundesinstitutionen (unter Ausklammerung der im Hochschulsektor zusammengefassten), Landes-, Gemeinde- und Kammerinstitutionen, F\&E-Einrichtungen der Sozialversicherungsträger, von der öffentlichen Hand finanzierte und/oder kontrollierte private gemeinnützige Institutionen sowie F\&E-Einrichtungen der Ludwig BoltzmannGesellschaft; einschließlich Landeskrankenanstalten. Die Landeskrankenanstalten wurden nicht mittels Fragebogenerhebung erfasst, sondern es erfolgte eine Schätzung der F\&E-Ausgaben durch Statistik Austria unter Heranziehung der Meldungen der Ämter der Landesregierungen.

2 Private gemeinnützige Institutionen, deren Status ein vorwiegend privater oder privatrechtlicher, konfessioneller oder sonstiger nicht öffentlicher ist.

Quelle: Statistik Austria, Berechnungen Joanneum Research 
Zur Darstellung der Interdependenzen in den Finanzierungsströmen („,wer finanziert was") zeigt Abbildung 3 eine entsprechende Matrix mit folgenden Informationen für das Jahr 2006:

- Die F\&E-Ausgaben der jeweiligen Durch- führungssektoren werden in den Kästchen angegeben.

- Die Angaben neben den Pfeilen stellen die Finanzierungsvolumina dar.

- Die Prozentangaben verdeutlichen die Veränderung zu 2004. ${ }^{1}$

\section{Abbildung 3: Durchführung und Finanzierung von F\&E in Österreich 2006 (versus 2004)²}

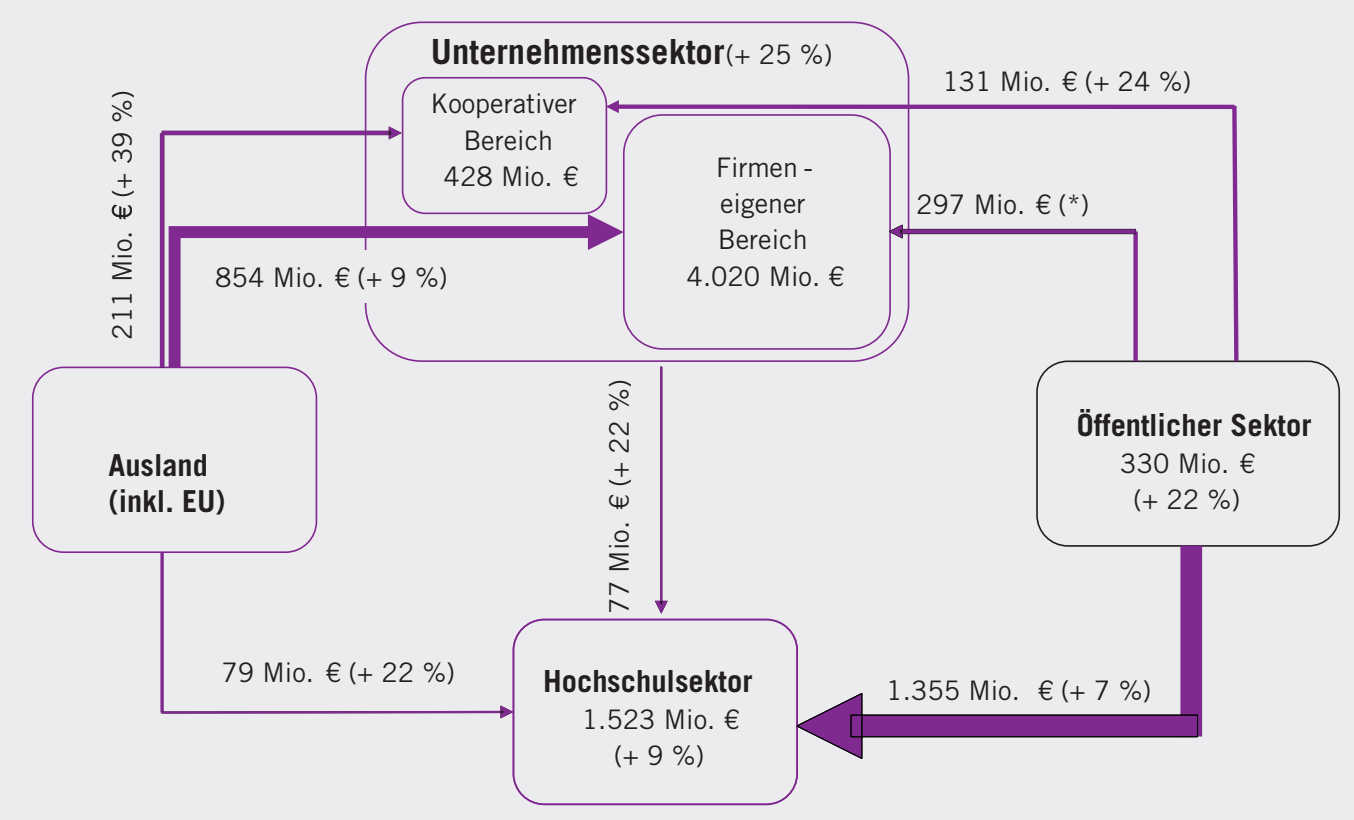

* Bei diesem Finanzierungsstrom ist die Vergleichbarkeit mit 2004 nicht möglich. Siehe dazu die Erläuterungen im Haupttext; gerundet auf Mio.

Quelle: Statistik Austria; Berechnungen Joanneum Research

Der Unternehmenssektor gab somit im Jahre 2006 insgesamt 4.448 Mio. $€$ für F\&E aus $(4.020+428)$, was eine Steigerung gegenüber 2004 von $+25 \%$ bedeutet. Der Hochschulsektor hingegen konnte seine F\&E-Ausgaben um $9 \%$ auf 1.523 Mio. $€$ steigern. Diese beiden Sektoren stellen somit mit fast $95 \%$ den größten Anteil an den gesamten nationalen F\&EAusgaben (Tabelle 1). Wie werden nun diese Ausgaben finanziert?
Es existieren drei wesentliche Finanzierungsströme. Der erste dieser Ströme ist die Eigenfinanzierung des Unternehmenssektors, der seine F\&E-Aktivitäten zum überwiegenden Teil selbst finanziert: denn der öffentliche Sektor finanziert insgesamt 428 Mio. $€$ $(297+131)$ und aus dem Ausland fließen 1.065 Mio. $€(211+854)$ in den Unternehmenssektor. Die restlichen zwei Drittel (2.955 Mio. €) finanziert der Unternehmenssektor selbst.

1 Für den Vergleich mit den Jahren 2004, 2002 und 1998 muss auf frühere Forschungs- und Technologieberichte zurückgegriffen werden

2 Der private gemeinnützige Sektor wurde aus Rücksicht auf die Übersichtlichkeit und auf Grund des geringen Anteils in der Darstellung nicht berücksichtigt. 
Bei der direkten öffentlichen Finanzierung von unternehmensinterner F\&E wurde bei der Erhebung 2006 erstmals die Forschungsprämie ${ }^{3}$ separat erfasst und ist somit - dem Konzept des Frascati-Handbuchs folgend ${ }^{4}-$ Teil der Finanzierung durch den öffentlichen Sektor (siehe dazu Schiefer 2008). Aus diesem Grund ist die Vergleichbarkeit der Finanzierung durch den öffentlichen Sektor mit den vorangegangenen Erhebungen nur eingeschränkt möglich. Das Volumen der Forschungsprämie betrug 156 Mio. $€$ und stellt somit die wichtigste Finanzierungsquelle dar. Unter Abzug der Forschungsprämie ergibt sich für 2006 somit ein öffentlicher Finanzierungsanteil von 272 Mio. $€(131+297-156)$, der mit dem Wert von 2004 (229 Mio. €) vergleichbar ist. Bei dieser Berechnungsart kommt es somit zu einer Steigerung der öffentlichen Finanzierung der Unternehmens-F\&E von $18,8 \%$. Inkludiert man jedoch legitimerweise die Forschungsprämie, so finanziert der öffentliche Sektor mit einem Volumen von 428 Mio. $€$ knapp $10 \%$ der F\&E des Unternehmenssektors. Eine detaillierte Darstellung der Finanzierung der Ausgaben für F\&E im Unternehmenssektor 2006 zeigt die folgende Tabelle:

Tabelle 2: Finanzierung der Ausgaben für F\&E im Unternehmenssektor 2006 (in Tsd. €)

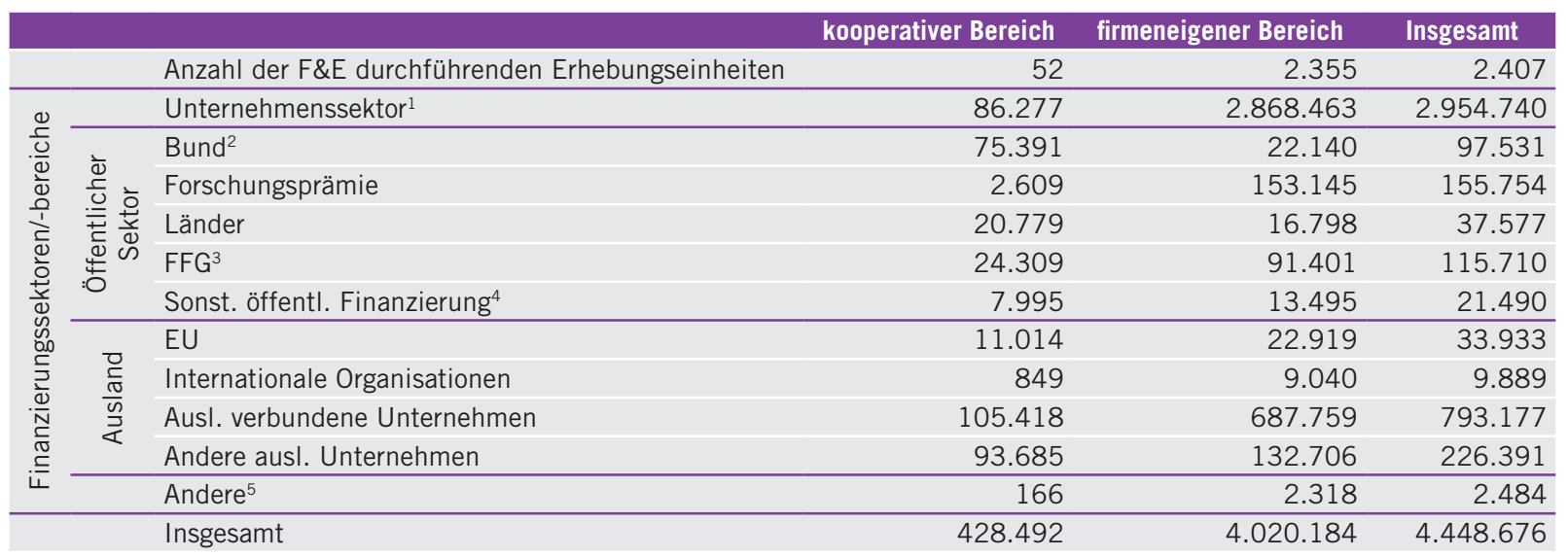

1 Umfasst eigene Mittel der Unternehmen, am Kapitalmarkt aufgenommene Mittel, Darlehen aus öffentlichen Fördermitteln und Mittel anderer inländischer Unternehmen;

2 Umfasst diejenigen Mittel, die direkt vom Bund (den Bundesdienststellen) finanziert werden, d.h. Fördermittel (Zuschüsse, Beihilfen) sowie auch Entgelte für im Auftrag des Bundes durchgeführte Forschungsprojekte. Beispiele für Förderprogramme des Bundes sind etwa die "Impulsprogramme“ des Bundesministeriums für Verkehr, Innovation und Technologie, wie „FIT-IT“ (Informationstechnologie) oder „Nachhaltig Wirtschaften“. Das Management der Förderprogramme und die Abwicklung der Förderungen werden dabei häufig von externen Einrichtungen und nicht direkt von den Bundesdienststellen durchgeführt. Unabhängig von der abwickelnden Stelle werden alle Fördermittel aus Förderprogrammen des Bundes unter "Bund“ angeführt.

3 Beinhaltet nur Zuschüsse (dazu zählen auch Kreditkostenzuschüsse), die die FFG zu Forschungsvorhaben von Unternehmen gewährt. Das sind vor allem Mittel aus der „Basisförderung“ bzw. aus den „Basisprogrammen“ der FFG oder Zuschüsse für Kooperationsprojekte im Rahmen des EUREKA-Programms. Angegeben werden die tatsächlich ausbezahlten Beträge und nicht die „Förderbarwerte“. So genannte „Anschlussförderungen“ an FFG-geförderte F\&E-Vorhaben aus Fördermitteln der Bundesländer oder ihrer ausgegliederten Fonds sind unter „Länder“ bzw. unter „Sonstige“ subsumiert. In Regionalfördergebieten besteht weiters die Möglichkeit einer Kofinanzierung von geförderten F\&E-Projekten aus Mitteln des „Europäischen Fonds für die regionale Entwicklung“ (EFRE). Diese Mittel werden zu „EU“ hinzugezählt. Geförderte Darlehen der FFG sind im „Unternehmenssektor" enthalten.

4 Umfasst Mittel von Gemeinden, Kammern, Sozialversicherungsträgern und sonstige öffentliche Finanzierung;

5 Inklusive Privater gemeinnütziger Sektor

Quelle: Statistik Austria, Schiefer (2008)

3 Die Forschungsprämie ist ein Instrument der indirekten Forschungsförderung. Seit dem Kalenderjahr 2002 ist es Unternehmen möglich (alternativ zum Forschungsfreibetrag), eine Forschungsprämie in maximaler Höhe von 8 \% der F\&E-Ausgaben zu beantragen. Da die Forschungsprämie - im Gegensatz zu den beiden Arten des Forschungsfreibetrages - einen direkten Transfer auf das Steuerkonto eines Unternehmens darstellt, ist laut Frascati-Handbuch diese Art der Finanzierung unter dem Finanzierungssektor "government sector" zu subsumieren.

4 The Measurement of Scientific and Technological Activities. Proposed Standard Practice for Surveys on Research and Experimental Development. Frascati Manual 2002, Paragr. 393, S. 114 f., OECD, Paris 2002. 
Zweiter wichtiger Financier von Forschung und Entwicklung in Österreich ist der öffentliche Sektor - Gebietskörperschaften (also Bund, Länder, Gemeinden, Kammern und Sozialversicherungsträger). Die Mittel des öffentlichen Sektors kommen vor allem den Hochschulen und eigener Forschung im öffentlichen Sektor zugute. Das Finanzierungsvolumen der F\&E im Hochschulsektor ist gegenüber 2004 um $7 \%$ auf 1.355 Mio. $€$ gestiegen. Das Verhältnis der Finanzierung der Unternehmens-F\&E (428 Mio. €) zur Finanzierung der Hochschul-F\&E (1.355 Mio. €) beträgt somit 1:3.

Dritter wichtiger Finanzierungssektor ist das Ausland. Dieser Sektor umfasst sowohl die Mittel ausländischer Unternehmen und internationaler Organisationen für $\mathrm{F} \& \mathrm{E}$ in Österreich als auch die Rückflüsse aus den Rahmenprogrammen der Europäischen Union. Will man ein differenziertes Bild erhalten, so entfallen vom Gesamtvolumen von 1,2 Mrd. $€$ aus dem Ausland 104 Mio. $€$ auf die EU. Der Hochschulsektor ist mit 52 Mio. $€$ der Hauptadressat der EU-Finanzmittel. Insgesamt finanziert die EU 1,6 \% der gesamten österreichischen F\&E-Ausgaben.

Folgende Entwicklungen sind besonders zu erwähnen:

- Von allen Durchführungssektoren konnte der Unternehmenssektor mit $+25 \%$ die F\&E-Ausgaben am deutlichsten steigern. Dies spiegelt sich auch in der Steigerung der öffentlichen Finanzierung der Unternehmens-F\&E wider, die mit $+18,8 \%$ gegenüber 2004 deutlich höher ausfiel als die staatliche Finanzierung der F\&E im Hochschulsektor $(+7 \%)$.

- Durch die Forschungsprämie werden in Summe 3,8 \% der firmeneigenen F\&E finanziert.
- Der kooperative Sektor wies 2006 ein Ausgabenvolumen von 428 Mio. $€$ aus. Finanziert wurde dieses Volumen überwiegend durch die öffentliche Hand (131 Mio. €) sowie das Ausland (211 Mio. €), was zusammen einen Anteil von $80 \%$ ergibt. Dies erzeugt ein verzerrtes Bild, da neben den Kompetenzzentren auch die beiden größten außeruniversitären Forschungseinrichtungen (Austrian Research Centers und Joanneum Research) diesem Sektor hinzugerechnet werden, deren Finanzierungsstrukturen jedoch nicht diesem Bild entsprechen. Der Grund für diese Verzerrung liegt in den Kriterien statistischer Konvention: Aufgrund ihrer außerordentlichen Mitgliedschaft bei der Austrian Cooperative Research (ACR) wird auch die AVL-List GmbH dem „kooperativen Bereich" zugerechnet ${ }^{5}$.

\subsubsection{Der Zusammenhang zwischen der Konjunktur und F\&E-Ausgaben}

Angesichts der abrupten Verschlechterung der konjunkturellen Lage der Weltwirtschaft und der Wirtschaft in Österreich ab Herbst 2008 rückt die Frage nach den Auswirkungen der sich im Jahr 2009 weiter verschärfenden Rezession auf die F\&E-Ausgaben insgesamt und vor allem jene des Unternehmenssektors in neues Licht. Die durch zunehmende Unsicherheit und Investitionszurückhaltung geprägte wirtschaftliche Lage verschlechtert auch die Rahmenbedingungen für Investitionen in Forschung und Entwicklung. Das nationale und europäische Credo von der positiven Wirkung von F\&E auf Wachstum und Beschäftigung muss nun auch von der anderen Seite diskutiert werden, d.h. welche Faktoren beeinflussen F\&E-Entscheidungen und sind gleichzeitig stark konjunk-

5 Siehe: http://www.acr.at/61.0.html 
turabhängig? In der Literatur werden dazu einige Übertragungsmechanismen diskutiert, ${ }^{6}$ welche in der Einschätzung der gegenwärtigen Situation sowie auch des Ziels einer Steigerung der Forschungsquote auf $3 \%$ des BIP (Ziel von Barcelona) berücksichtig werden sollten (siehe dazu Schibany et al. 2006):

- Die aktuelle konjunkturelle Situation beeinflusst die internen Finanzierungsbedingungen von Unternehmen. In Zeiten hoher und wachsender Nachfrage ist die Profitabilität der Unternehmen meist überdurchschnittlich gut und damit auch die Eigenfinanzierungsmöglichkeiten von unsicheren Investitionen aus dem Cashflow. F\&E-Investitionen in Unternehmen werden $\mathrm{zu}$ einem Großteil aus dem Cashflow finanziert. ${ }^{7}$

- Weiters beeinflusst die konjunkturelle Situation auch die Nachfrage- und Angebotsverhältnisse auf dem Kapitalmarkt. Unternehmen (insbesondere KMU) sind bei der Fremdfinanzierung von F\&E vor dem Hintergrund der aktuellen Entwicklung auf den Finanzmärkten mit besonderen Barrieren konfrontiert: Wegen der hohen und nur schwer bezifferbaren technischen und kommerziellen Risiken sind Fremdkapitalgeber, besonders Banken, bei der Finanzierung von F\&E-Projekten sehr zurückhaltend. F\&E unterliegt häufig einer starken Kreditrationierung, was auch den generell geringen Anteil von Bankkrediten in der F\&E-Finanzierung erklärt. Dass Fremdfinanzierung dennoch eine Rolle spielt, ist damit zu erklären, dass F\&E-Projekte i.d.R. nicht explizit nach Finanzierungsmöglichkeiten definiert werden, sondern diese Teile einer umfassenderen Investitionsstrategie sind.

- Besonders stark prozyklischer Natur sind alternative Finanzierungsformen, wie z.B.
Risikokapital. Privates Risikokapital ist in Hochkonjunkturphasen wegen des starken Zuflusses an Beteiligungskapital aufgrund der erwarteten prozyklischen Erhöhung von Unternehmenswerten und den günstigen Ausstiegsoptionen für Wagniskapitalinvestoren über die Aktienmärkte (hohes Kursniveau) in der Regel leichter erhältlich als in Rezessionsphasen.

- Im Konjunkturverlauf verändern sich auch die Preise für den Faktor Arbeit, wobei für F\&E-Entscheidungen vor allem die Löhne für höher Qualifizierte relevant sind. Da Personalaufwendungen ca. die Hälfte der F\&E-Ausgaben des Unternehmenssektors ausmachen, werden Preisänderungen in den Löhnen Rückwirkungen auch auf die Ausgabenentscheidungen der Unternehmen im F\&E-Bereich haben.

- Der wichtigste Einflussfaktor ist die aktuelle Entwicklung der Gesamtnachfrage auf den Absatzmärkten der Unternehmen. Entscheidungen über Investitionen in F\&E werden unter Unsicherheit getroffen, wobei der zukünftige Ertrag aus dieser Investition nicht nur vom „technologischen Erfolg" des Projektes, sondern vor allem von der zukünftigen Akzeptanz durch den Markt und der Nachfrage nach den aus dem F\&E-Projekt resultierenden neuen Produkten abhängt. Damit tragen wachsende Märkte zu einer Stimulierung der Investitionsentscheidungen bei, während eine aktuell schrumpfende Nachfrage die Bereitschaft, Zukunftsinvestitionen zu tätigen, einschränken kann.

- Darüber hinaus wirken diese konjunkturabhängigen Übertragungsmechanismen unterschiedlich auf Unternehmen unterschiedlicher Größe: Während KMU aufgrund von Unteilbarkeiten von F\&E-Aktivitäten (Min-

6 Guellec und Ioannidis (1999), Guellec und van Pottlesberghe de la Potterie (2001), Le Bas (2001), Geroski und Walters (1995), Geroski und Machin (1993), Rammer et al. (2004).

7 In Österreich werden im Schnitt nahezu zwei Drittel (64 \%) der gesamten F\&E-Aufwendungen in Unternehmen aus dem Cashflow finanziert (siehe Schibany und Jörg 2005, Schibany et al. 2004). 
destgröße von F\&E-Projekten) wenig Spielraum für partielle Anpassungen haben, steht dem eine höhere Flexibilität in der Umorientierung von unternehmensinternen Ressourcen gegenüber (z.B. zwischen $\mathrm{F} \& \mathrm{E}$ und Innovation oder anderen Unternehmensbereichen, wie Produktion und Vertrieb). Aufgrund der stärkeren Abhängigkeit von externen Finanzierungsquellen (Kredite) weisen KMU eine andere Finanzierungsstruktur auf als Großunternehmen und sind dementsprechend stärker von konjunkturbedingten Veränderungen des Realzinses betroffen. Gleichzeitig ist anzunehmen, dass KMU aufgrund des geringeren Internationalisierungsgrades eine stärkere Abhängigkeit von der Binnenkonjunktur aufweisen als Großunternehmen, was jedoch bei einer globalen Krise wenig Unterschied macht.

In den folgenden Ausführungen wird der Versuch unternommen, aktuelle Hinweise auf einen Zusammenhang zwischen Wirtschaftswachstum und F\&E-Ausgaben zu finden und diesen - falls möglich - quantitativ abzuschätzen. ${ }^{8}$ Dazu wurden F\&E-relevante Daten von 14 Ländern für den Zeitraum 1981-2007 verwendet, die von der OECD in harmonisierter Form zur Verfügung gestellt werden.

Auf der Basis dieser 14 Länder wird ein ökonometrisches Panel-Modell geschätzt, in dem der langfristige Zusammenhang zwischen den (realen) F\&E-Ausgaben und der (realen) Wirt- schaftsleistung (BIP) modelliert wird (beide Größen sind in Logarithmen geschätzt; der Schätzkoeffizient kann daher direkt als Elastizität interpretiert werden). Da alle Zeitreihen deutliches Trendverhalten aufweisen, wird ein Kointegrationsmodell geschätzt. ${ }^{10}$ Dabei werden die Veränderungen in F\&E-Ausgaben in Abhängigkeit von Veränderungen des BIP sowie den verzögerten (d.h. letztiährigen) Abweichungen von diesem langfristigen Zusammenhang modelliert und geschätzt.

Zusätzlich wird untersucht, ob sich Hinweise auf Asymmetrie der F\&E-Ausgaben zeigen, d.h. ob sich die Reaktion der Forschungsausgaben auf das Wirtschaftswachstum in Rezessionsjahren anders darstellt als in "normalen" Jahren. Dazu wird das Wirtschaftswachstum $\Delta \log (\mathrm{GDP})$ ein zweites Mal in die Regression inkludiert, allerdings multipliziert mit einer Dummy-Variablen DGDP, die den Wert 1 aufweist, wenn das Wirtschaftswachstum in einem Jahr negativ war, und sonst den Wert 0.

Das Modell lautet:

$\Delta \log \left(\mathrm{F} \&\right.$ E-Ausg $\left._{i, t}\right)=\alpha_{i}+\beta 1 \Delta \log \left(\mathrm{GDP}_{\mathrm{i}, \mathrm{t}}\right)+\beta 2$

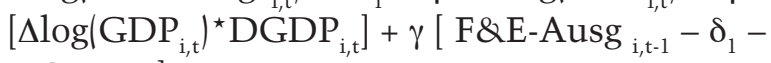
$\left.\delta_{2} \mathrm{GDP}_{\mathrm{i}, \mathrm{t}-\mathrm{l}}\right]$

\section{( $\mathrm{i}=$ Länderindex; $\mathrm{t}=$ Zeitindex $)$}

Für die F\&E-Ausgaben werden - in 4 verschiedenen Regressionen - GERD, BERD, HERD bzw. GOVERD verwendet. ${ }^{11}$ Wenn die An-

8 Die folgenden Ausführungen basieren auf einem von Gerhard Streicher (Joanneum Research) im Rahmen eines Forschungsauftrages des BMWF entwickelten ökonometrischen Modells.

9 AUT, BEL, CAN, DEU, DNK, ESP, FIN, FRA, GBR, IRL, ITA, JPN, NLD, USA.

10 Alle Zeitreihen, sowohl jene für das (reale) Bruttoinlandsprodukt als auch jene für die (realen) Forschungsausgaben, sind stark trendbehaftet; ein Unit Root Test liefert deutliche Hinweise, dass den Zeitreihen integrierte stochastische Prozesse zugrunde liegen. Dies ist insofern ein Problem, als die Verwendung integrierter Prozesse in ökonometrischen Schätzungen zu sogenannten "spurious regressions" führen kann - dies kann (muss aber nicht) dazu führen, dass ein nur scheinbarer Zusammenhang zwischen an sich völlig unabhängigen Variablen geschätzt wird. Um dies zu vermeiden, kann auf das Instrumentarium zurückgegriffen werden, das in der Theorie der "ko-integrierten Prozesse" entwickelt wurde. Vereinfacht gesprochen wird hier ein ko-integrativer Zusammenhang zwischen integrierten Variablen geschätzt (und getestet), der anschließend in ein Fehlerkorrekturmodell eingebaut wird; dieses Fehlerkorrekturmodell wird nicht für die ursprünglichen Variablen, sondern für deren erste Differenzen geschätzt (die Differenz zwischen dem Wert eines Jahres und dem Wert des Vorjahres). Damit sind die statistisch-mathematischen Voraussetzungen erfüllt, um das Problem der „spurious regression“ zu vermeiden.

11 GERD=Gross Expenditure on R\&D; GDP=Gross Domestic Product; BERD=Business Expenditure on R\&D; HERD=Higher Education Expenditure on R\&D; GOVERD=Government Expenditure on R\&D 
nahme eines kointegrativen Zusammenhanges stimmt, muss der Koeffizient der Abweichungsvariablen $\gamma$ signifikant negativ sein. $\beta_{1}$ misst den Zusammenhang zwischen Wirtschaftswachstum und Forschungsausgaben in "normalen" Jahren (d.h. jene mit positivem Wirtschaftswachstum); $\beta_{2}$ misst den Unter- schied zwischen diesem Zusammenhang in normalen und Rezessionsjahren (der Koeffizient, der für Rezessionsjahre gültig ist, beträgt daher $\beta_{1}+\beta_{2}$ ).

Die Ergebnisse sind in Tabelle 3 zusammengefasst:

\section{Tabelle 3: Schätzergebnisse des Kointegrationsmodells}

\begin{tabular}{|l|r|r|r|r|r|r|r|}
\hline & $\operatorname{dlog}($ GERD) & & dlog(BERD) & dlog(HERD) & dlog(GOVERD) \\
\hline$\alpha$ & $0.02(0.00)$ & $* * *$ & $0.02(0.00)$ & $* * *$ & $0.04(0.00) * * *$ & $-0.01(0.01)$ \\
\hline$\beta_{1}$ & $0.85(0.11)$ & $* * *$ & $0.97(0.16)$ & $* * *$ & $0.43(0.15)$ & $* * *$ & $0.84(0.15)$ \\
\hline$\beta_{2}$ & $-0.42(0.43)$ & & $0.71(0.58)$ & $-1.17(0.63)$ & $*$ & $-2.37(0.58)$ \\
\hline$\gamma$ & $-0.23(0.04)$ & $* * *$ & $-0.21(0.04)$ & $* * *$ & $-0.37(0.05)$ & $* * *$ & $-0.37(0.04)$ \\
\hline$\delta_{2}$ & $0.98(0.09)$ & $* * *$ & $1.33(0.13)$ & $* * *$ & $0.69(0.11) * * *$ & $0.44(0.10)$ \\
\hline $\mathrm{R}^{2} / \mathrm{DW}$ & $0.41 / 1.31$ & & $0.37 / 1.19$ & $0.34 / 1.42$ & $0.33 / 1.73$ \\
\hline
\end{tabular}

In Klammer die Standardabweichung des Schätzers; *** und * kennzeichnen statistische Signifikanz auf dem 1, 5 bzw. 10\%-Niveau

Quelle: OECD (MSTI), Berechnungen Joanneum Research

Folgende Schlüsse können gezogen werden:

- Langfristig liegt die Entwicklung von BERD über jener des BIP $\left(\delta_{2}=1,33\right.$ impliziert, dass pro $1 \%$-iger Steigerung des BIP die Ausgaben für BERD um 1,33\% steigen), GERD und BIP zeigen langfristig praktisch gleiche Wachstumsraten $\left(\delta_{2}=0,98\right)$; HERD (und noch stärker GOVERD) wachsen deutlich unter der BIP-Rate $\left(\delta_{2}=0,68\right.$ bzw. 0,44$)$.

- In der kurzen Frist implizieren die Schätzergebnisse, dass eine Erhöhung des realen BIP um 1\% zu einer Erhöhung von GERD um 0,85\% führt. Ein noch deutlicherer Zusammenhang mit dem BIP lässt sich für BERD schätzen: Die Erhöhung des BIP um $1 \%$ geht mit einer Erhöhung von BERD um 0,97 \% einher - BIP und BERD laufen kurzfristig praktisch „parallel“.

- Die Annahme eines kointegrativen Zusammenhangs zwischen Wirtschaftsleitung und Forschungsausgaben scheint durchaus gerechtfertigt: Der Koeffizient der verzögerten Abweichungen vom langfristigen Zusammenhang ist negativ und statistisch signifikant.
- Zur Frage der Asymmetrie: BERD scheint in "schlechten Zeiten" stärker zu schrumpfen als das BIP, die anderen Komponenten der Forschungsausgaben weniger stark: In Rezessionsjahren wird die Elastizität zwischen BIP und BERD auf 0,97+0,71=1,68 geschätzt (d.h. ein Rückgang des BIP um -1 $\%$ geht mit einem Rückgang von BERD um $-1 *(0,97+0,71)=-1,7 \%$ einher. Die Reagibilität von GERD geht zurück, bleibt aber positiv $(0,85-0,42=0,43)$. HERD und GOVERD werden in Rezessionsjahren sogar mit negativer Elastizität geschätzt (d.h. im Fall von HERD etwa, dass bei einem BIP-Rückgang um $-1 \%$ die HERD-Ausgaben um $-1^{\star}(0,43$ $1,17)=+0,74 \%$ steigen $;$ ähnlich im Fall von GOVERD). Dies kann darauf hindeuten, dass die staatlichen Ausgaben für F\&E (HERD und GOVERD werden zum Großteil staatlich finanziert) im Rahmen von anti-zyklischen Maßnahmen erhöht werden (bzw. in besseren Zeiten budgetiert wurden). Alle "asymmetrischen Koeffizienten" sind allerdings mit Vorsicht $\mathrm{zu}$ interpretieren: Sie sind statistisch kaum signifikant. 
Rezessionsjahre sind über den beobachteten Zeitraum recht seltene Ereignisse - es ist schwierig, für diese seltenen Ereignisse statistisch gesicherte Ergebnisse abzuleiten.

Die hier vorgestellten Schätzresultate sind das Ergebnis einer Panel-Regression und stellen ein "durchschnittliches" Ergebnis dar, gemittelt über 14 Länder und 26 Jahre. Massive Rezessionen stellen über den Beobachtungszeitraum eine deutliche Ausnahme dar, was die Übertragung auf die derzeitige Situation verkompliziert. Die Resultate reflektieren also nicht die spezifische österreichische Situation, sondern jene einer vergleichbaren Gruppe von Ländern. Die Ergebnisse zeigen, dass bei den derzeitigen Konjunkturannahmen mit einem deutlichen Rückgang der F\&E-Ausgaben des Unternehmenssektors zu rechnen ist. Das Ausmaß dieses möglichen Rückgangs ist für Österreich schwer abzuschätzen, da die F\&E-Ausgaben (i) stark auf einige wenige Großunternehmen konzentriert sind und (ii) die Reaktion des Auslandsanteils in der Finanzierung von F\&E auf Rezessionen auf Basis der Vergleichsgruppe nur ungenügend abbildbar ist. So ist anzunehmen, dass das vom Ausland finanzierte Forschungsvolumen (dessen Anteil in Österreich mit fast $20 \%$ sehr hoch ist) nicht nur von der Wirtschaftsentwicklung des Forschungslandes (also des Ziellandes der Auslandsfinanzierung), sondern auch von der Wirtschaftsentwicklung des Geberlandes abhängt. Da es sich bei auslandsfinanzierter F\&E in erster Linie um von ausländischen Unternehmen finanzierte F\&E handelt, kann argumentiert werden, dass diese ähnlich wie BERD reagieren dürfte - es ist daher zu erwarten, dass diese Forschungsausgaben überproportional auf BIP-Rückgänge in den Geberländern reagieren werden. Bei einem BIP-Rückgang im EU-Raum von etwa $2 \%$ könnte der auf der Basis der hier angestell- ten Überlegungen erwartete Rückgang der Finanzvolumina aus dem Ausland in Österreich durchaus bei $3-5 \%$ liegen. Die Analyse der Vergleichsländer zeigt aber auch, dass in Rezessionszeiten die staatliche Finanzierung von Forschungsvorhaben - eventuell im Rahmen anti-zyklischer Maßnahmen - gestiegen ist. Die Resultate weisen in diesem Fall auf eine Zunahme der staatlichen Ausgaben um etwa $1 / 2-1 \%$ hin.

\subsubsection{Die Position Österreichs im European Innovation Scoreboard (EIS)}

Der Europäische Innovationsanzeiger (European Innovation Scoreboard - EIS) ist ein Instrument des Lissabonprozesses, mit dem die Innovationsentwicklung in der EU sowie der EU gegenüber den anderen Märkten (v.a. USA und Japan) abgebildet werden soll. Diese (quantifizierbare) Performancedarstellung erfolgt auf Basis bestimmter Indikatoren, die im Laufe der Jahre mit dem Ziel weiterentwickelt wurden, ein realistisches Bild über die Innovationsentwicklung zu bekommen. ${ }^{12}$

Eine bessere Datenbasis sowie die konstante Weiterentwicklung der Analysemethoden ließen die Vergleichbarkeit zwischen den Ländern und damit die Aussagekraft des EIS mit der Zeit steigen. Trotz dieser Verbesserungen muss jedoch auch die Grenze einer indikatorenbasierten Abbildung eines Innovationssystems bedacht werden, zumal die im EIS verwendeten Einzelindikatoren zu einem Summary Innovation Index (SII) zusammengefasst werden, woraus sich die Notwendigkeit einer höchst vorsichtigen Interpretation dieser Zahl ergibt. Denn es liegt auf der Hand, dass sich nicht sämtliche Determinanten und Einflussgrößen mittels quantifizierbarer Indikatoren erfassen lassen. Aber diese Grenzen berücksichtigend hat sich der EIS als geeigne-

12 Eine ausführliche Diskussion des EIS findet sich im Forschungs- und Technologiebericht 2008 (S. 17ff.) 
tes Instrument erwiesen, um Entwicklungen nachzuzeichnen und Positionierungen in bestimmten Bereichen vorzunehmen. Für eine ausführliche Diskussion dieser Aspekte siehe Schibany und Streicher (2008).

Im Laufe des Jahres 2008 fand eine sehr intensive Diskussion über die methodologische Verbesserung des EIS sowie eine bessere Datengenerierung und damit Vergleichbarkeit statt. ${ }^{13}$ Dadurch wurde einer aufkommenden Kritik Rechnung getragen und die in einem Workshop diskutierten Ideen ${ }^{14}$ flossen in die Entwicklung eines neuen Indikatorensets sowie neuer Analysemethoden (siehe Hollanders und van Cruysen 2008). Der EIS 2008 basiert somit auf teilweise neuen Indikatoren, welche verstärkt auch die nicht-technologischen Aspekte von Innovation berücksichtigen. Seine Datenbasis ist nun stabiler, transparenter und nachvollziehbarer. Auch wurden die Trendentwicklungen im EIS 2008 aussagekräftiger, da sie nicht mehr auf den EU-Durchschnitt bezogen werden, sondern die 5-Jahresdurchschnitte der Absolutwerte berechnet werden.

\section{Die Ergebnisse des EIS 2008}

Die Ergebnisse des Summary Innovation Index (SII) zeigen nun eine Reihung der EU-Mitgliedstaaten, die im Prinzip seit der Einführung des Scoreboard gleichgeblieben ist. Die Führungsgruppe (Innovation Leaders) umfasst 5 Länder: Schweden, Finnland, Deutschland, Dänemark und Großbritannien. In der Gruppe der „Innovation Followers" finden sich mit Österreich, Irland, Luxemburg, Belgien, Frankreich und den Niederlanden jene 6 Länder, die noch über dem Durchschnitt der 27 EU-Mitgliedstaaten liegen (siehe Abbildung 4). Österreich nimmt im Gesamtranking unter den EU-Mitgliedsländern den 6. Platz ein und steht an der Spitze innerhalb der Gruppe der „Innovation Follower".

\section{Abbildung 4: Ländervergleich auf Basis des ElS 2008 (inklusive Vergleich mit ElS 2004)}

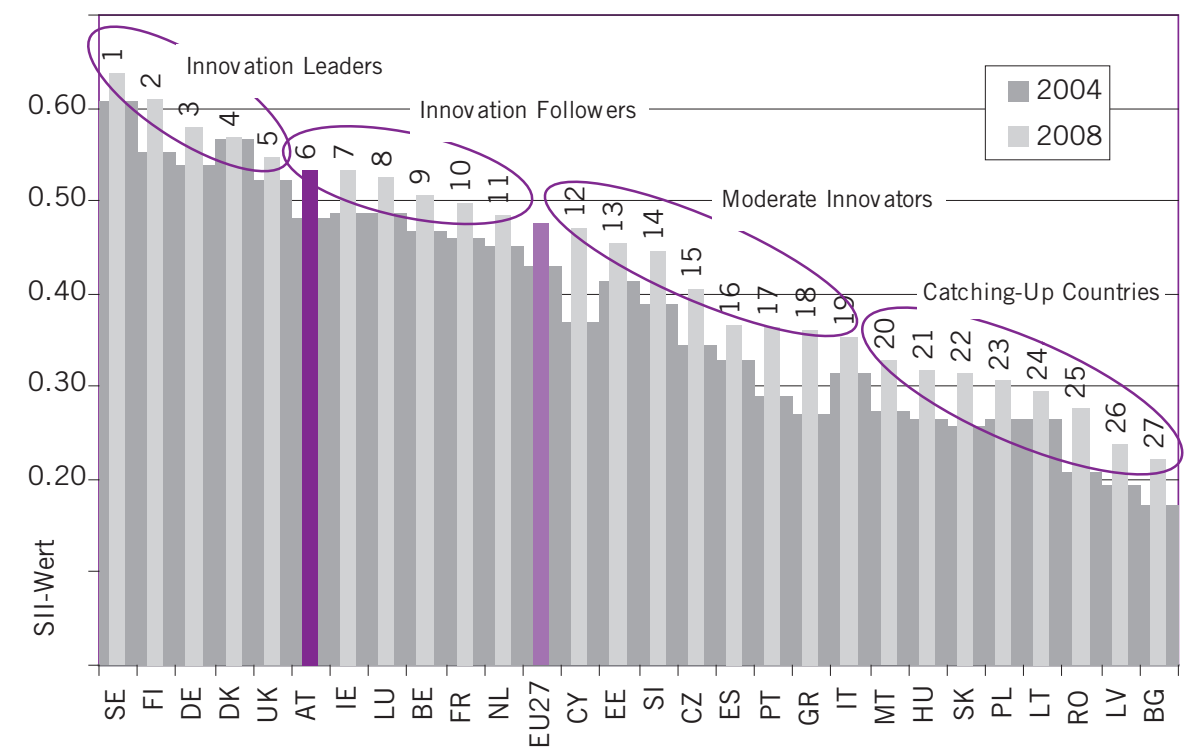

Quelle: InnoMetrics; Berechnungen Joanneum Research

13 Österreich hat sich an dieser Diskussion aktiv beteiligt. Auf der Basis einer vom BMWF, BMVIT und BMWFJ beauftragten Studie (Schibany, Streicher, Gassler 2007) übermittelte das BMWFJ im April 2008 eine akkordierte Stellungnahme bezüglich Änderungsvorschlägen zum European Innovation Scoreboard an die Europäische Kommission.

14 „Improving the European Innovation Scoreboard“; 16. Juni 2008, Brüssel. 
Die Gruppe der „Moderate Innovators“ umfasst die Länder Zypern, Estland, Slowenien, Tschechische Republik, Spanien, Portugal, Griechenland und Italien (Positionen 12-19); die Gruppe der "Catching Up-Countries" umfasst die übrigen „neuen“ Mitgliedstaaten.

Wie der Vergleich mit Ergebnissen früherer EIS zeigt, sind diese Gruppen recht stabil; Änderungen in der relativen Positionierung erfolgen in erster Linie innerhalb dieser Gruppen. Österreich konnte dabei seine Position innerhalb der Gruppe der "Innovation Followers" verbessern und hält nun innerhalb dieser Gruppe die Spitzenposition.

Ein Blick auf die 29 Einzelindikatoren (in der folgenden Abbildung sind die österreichischen Werte zusammen mit den Minima bzw. Maxima der EU27 dargestellt, jeweils bezogen auf den EU27-Durchschnitt) zeigt, dass Österreich nur bei weniger als einem Drittel der Einzelindikatoren, nämlich sieben, (deutlich) unter dem EU27-Schnitt liegt; zwei davon weisen auf die relativ niedrige Akademikerquote hin. Speziell der Indikator zu den neuen Bachelor- und Master-Abschlüssen in wissenschaftlichen Studien liegt dabei sehr nahe beim Minimum der EU27; interessanterweise liegt demgegenüber der Anteil der Doktoratsabsolventen deutlich über dem EU27-Schnitt. Rein rechnerisch bedeutet das, dass in Österreich auf nur 12 Master-Abschlüsse ein Doktoratsabschluss kommt - dies ist der beste Wert unter den EU27, deren Durchschnitt bei einem Doktorat pro 37 Master-Abschlüssen liegt. Weitere drei Indikatoren mit für Österreich unterdurchschnittlichen Werten weisen einen High-Tech-Bezug auf: die geringe Ausstattung mit Venture Capital, die schwache Handelsbilanz bei Technologie-Gütern sowie Schwächen beim Export wissensorientierter Dienstleistungen.

Stärken zeigen sich insbesondere bei den Unternehmensinnovationen und -kooperationen sowie bei den „Throughputs" - Patenten, Trademarks und Design (siehe Abb: 5).

Der EIS 2008 zeigt, dass Österreich neben Irland in der Gruppe der "Innovation Follower" die höchste Leistungssteigerung verzeichnen kann. Neben den bereits bekannten Stärken Österreichs in den Bereichen innovationsbezogener Indikatoren bestätigt der EIS 2008 aber auch die teilweisen Schwächen im Bereich der Humanressourcen, sowie in der Venture Capital-Finanzierung. 
Abbildung 5: detaillierte Ergebnisse des ElS 2008; Österreich vs. Minimum/Maximum der EU27 (Index EU27=1)

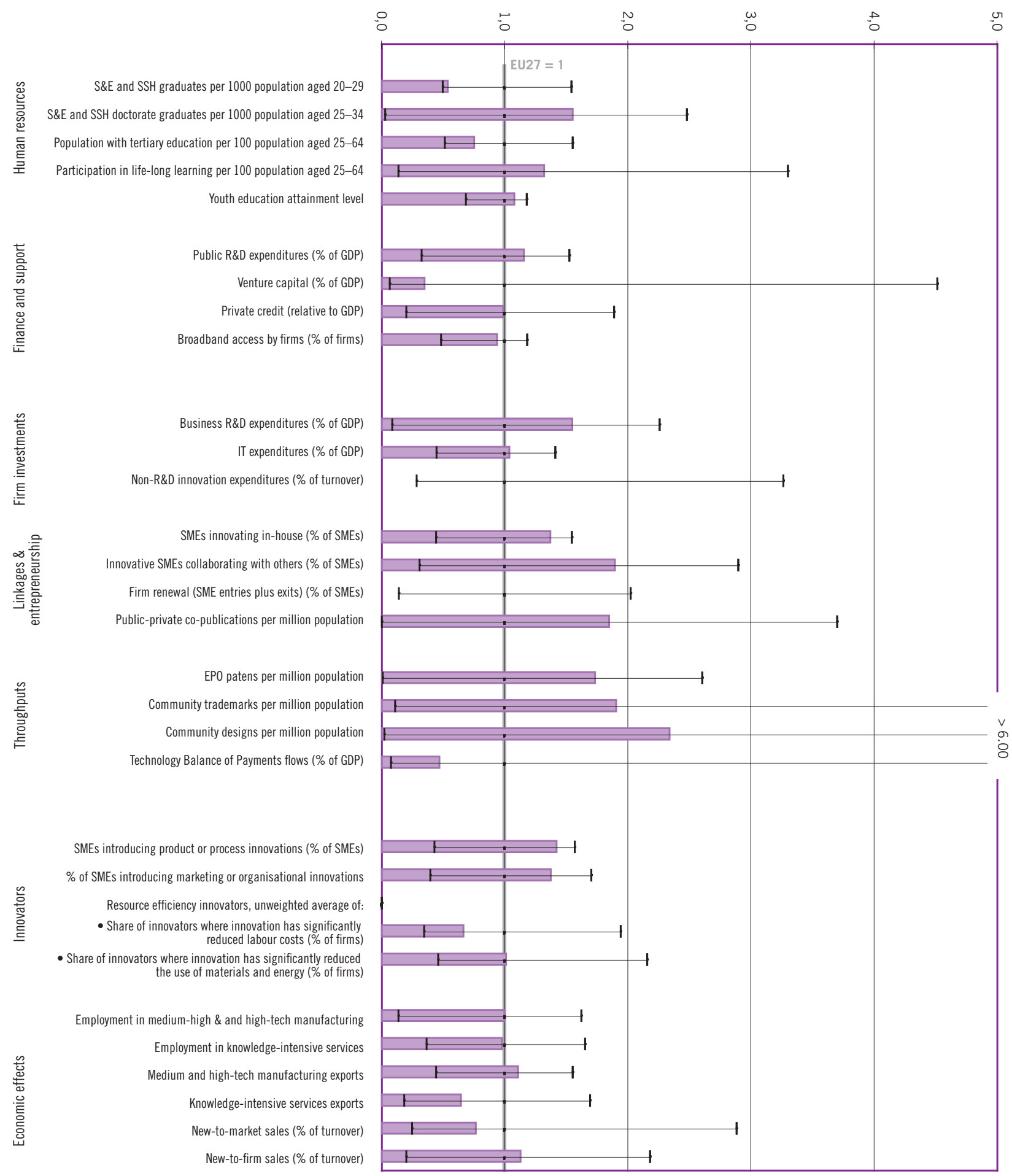

Quelle: InnoMetrics, Berechnungen Joanneum Research 


\subsection{F\&E in Österreich $2002-2006$}

\subsubsection{Allgemeines}

Im folgenden Kapitel sollen einige Ergebnisse der F\&E-Globalerhebungen, die von der Statistik Austria in den Jahren 2002, 2004 und 2006 durchgeführt wurden, präsentiert werden. ${ }^{15}$ Dieser intertemporale Vergleich wird durch internationale Querschnittsvergleiche ergänzt. ${ }^{16}$

Ein Vergleich der Erhebungsjahre 2002 bis 2006 zeigt eine deutliche Ausweitung, sowohl der forschenden Erhebungseinheiten wie auch der Ausgaben für F\&E:

Tabelle 4: Erhebungseinheiten und Ausgaben für F\&E in Österreich, 2002-2004-2006

\begin{tabular}{|c|c|c|c|c|c|c|c|c|}
\hline & \multicolumn{4}{|c|}{ F\&E duchftihrende Einheiten } & \multicolumn{4}{|c|}{ Ausgaben für F\&E [Mio. $€$ ] } \\
\hline Durchführungssektor & 2002 & 2004 & 2006 & $\begin{array}{r}\text { (Veränd. } \\
2002 \text { - 2006) }\end{array}$ & 2002 & 2004 & 2006 & $\begin{array}{r}\text { (Veränd. } \\
2002 \text { - 2006) }\end{array}$ \\
\hline Hochschulsektor & 969 & 1038 & 1162 & $+20 \%$ & 1266 & 1402 & 1523 & $+20 \%$ \\
\hline Sektor Staat & 308 & 226 & 254 & $-18 \%$ & 266 & 270 & 330 & $+24 \%$ \\
\hline Privater gemeinnütziger Sektor & 71 & 55 & 40 & $-44 \%$ & 21 & 22 & 17 & $-21 \%$ \\
\hline Unternehmenssektor & 1942 & 2123 & 2407 & $+24 \%$ & 3131 & 3556 & 4449 & $+42 \%$ \\
\hline gesamt & 3290 & 3442 & 3863 & $+17 \%$ & 4684 & 5250 & 6319 & $+35 \%$ \\
\hline
\end{tabular}

Quelle: Statistik Austria (F\&E-Erhebung), Berechnungen Joanneum Research

Die Zahl der forschenden Erhebungseinheiten stieg zwischen 2002 und 2006 um 17 \% (von 3290 auf 3863 Einheiten), die Ausgaben für F\&E um $35 \%$ (von 4,7 auf 6,3 Mrd. €). Speziell der Unternehmenssektor weitete seine Ausgaben mit $+42 \%$ (von 3,1 auf 4,5 Mrd. €) sehr deutlich aus; ein Rückgang muss nur beim privaten gemeinnützigen Sektor verzeichnet werden (um ein Fünftel, bei recht geringem Niveau).

\subsubsection{Finanzierung und Ausgaben}

Die Finanzierungsstruktur der Ausgaben für Forschung und Entwicklung in Österreich zeigt für den Zeitraum 2002 bis 2006 nur relative geringe Verschiebungen.

15 Siehe dazu auch: http://www.statistik.at/web_de/statistiken/forschung_und_innovation/index.html 16 Basierend auf den Main Science and Technology Indicators (MSTI) der OECD. 
Abbildung 6: F\&E-Ausgaben in Mio. €: 2002/04/06 nach Finanzierungsbereichen in Österreich

\begin{tabular}{|c|c|c|c|c|c|c|c|}
\hline Durchfïhrungssektor & & 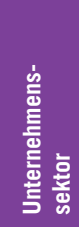 & 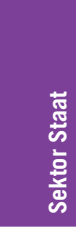 & 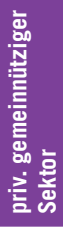 & 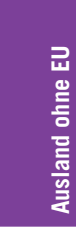 & 표 & 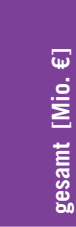 \\
\hline \multirow[t]{3}{*}{ Hochschulsektor } & 2002 & 51,3 & 1156,9 & 8,2 & 11,8 & 37,8 & 1266,1 \\
\hline & 2004 & 62,6 & 1262,4 & 11,4 & 19,1 & 46,1 & 1401,6 \\
\hline & 2006 & 76,8 & 1354,7 & 13,1 & 26,8 & 51,9 & 1523,2 \\
\hline \multirow[t]{3}{*}{ Sektor Staat } & 2002 & 16,0 & 236,8 & 2,0 & 3,9 & 7,8 & 266,4 \\
\hline & 2004 & 17,8 & 237,3 & 2,7 & 1,9 & 10,3 & 269,8 \\
\hline & 2006 & 22,5 & 287,3 & 1,8 & 1,9 & 16,8 & 330,2 \\
\hline \multirow{3}{*}{$\begin{array}{l}\text { Privater } \\
\text { gemeinnütziger } \\
\text { Sektor }\end{array}$} & 2002 & 5,2 & 5,0 & 6,3 & 1,9 & 2,6 & 20,9 \\
\hline & 2004 & 4,6 & 3,4 & 9,9 & 1,0 & 2,7 & 21,6 \\
\hline & 2006 & 3,0 & 1,3 & 10,8 & 0,1 & 1,3 & 16,5 \\
\hline \multirow{3}{*}{$\begin{array}{l}\text { Unternehmens- } \\
\text { sektor }\end{array}$} & 2002 & 2018,1 & 175,5 & 1,0 & 906,2 & 30,1 & 3130,9 \\
\hline & 2004 & 2390,6 & 229,2 & 1,2 & 907,6 & 27,9 & 3556,5 \\
\hline & 2006 & 2954,7 & 428,1 & 1,3 & 1030,7 & 33,9 & 4448,7 \\
\hline \multirow[t]{3}{*}{ gesamt } & 2002 & 2090,6 & 1574,2 & 17,5 & 923,7 & 78,3 & 4684,3 \\
\hline & 2004 & 2475,5 & 1732,2 & 25,2 & 929,6 & 87,0 & 5249,5 \\
\hline & 2006 & 3057,0 & 2071,3 & 26,9 & 1059,5 & 103,9 & 6318,6 \\
\hline
\end{tabular}

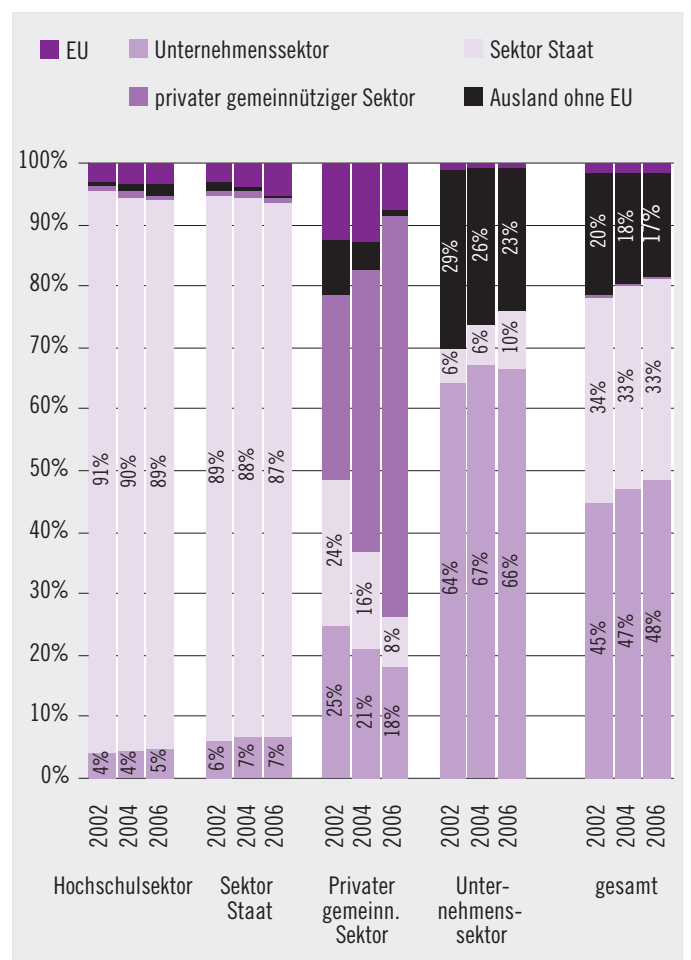

Quelle: Statistik Austria (F\&E-Erhebung), Berechnungen Joanneum Research

Vor dem Hintergrund dieser stabilen Finanzierungsstrukturen zwischen 2002 und 2006 zeigen sich allerdings einige interessante Entwicklungen: So stieg der Anteil des Sektors Staat an den Forschungsausgaben des Unternehmenssektors von $6 \%$ auf $10 \%$, der Auslandsanteil sank von $29 \%$ auf $23 \%$ (in absoluten Zahlen bedeutet dies allerdings keinen Rückgang: die Auslandsfinanzierung stieg von 924 auf 1060 Mio. $€$; dieser Zuwachs von $+15 \%$ bleibt allerdings deutlich hinter dem Gesamtzuwachs von +35\% zurück). Der Eigenfinanzierungsanteil des Unternehmenssektors blieb bei $64-66 \%$ praktisch stabil.

Hochschul- und Staatssektor sind überwiegend öffentlich finanziert, wenn auch die Staatsanteile leicht sinken; gleichzeitig konnten Unternehmens- und EU-Anteil leicht gesteigert werden, bleiben allerdings in einem Bereich von $3-7 \%$. Als einziger zeigt der pri- vate gemeinnützige Sektor deutlich Verschiebungen in seiner Finanzierungsstruktur.

Neben der Erreichung einer Forschungsquote von $3 \%$ des BIP im Jahre 2010 - welches in Barcelona 2002 für die gesamteuropäische Ebene definiert wurde - besteht das „Barcelona Ziel" auch noch aus einem zweiten Teil, nämlich der Erhöhung des Unternehmensanteils an der Finanzierung der gesamten Forschungsausgaben auf $67 \%$. Dieses Ziel wird bei wörtlicher Interpretation der Statistik weit verfehlt, ist bei inhaltlicher Interpretation allerdings bereits seit einiger Zeit (beinahe) erfüllt:

Der nominelle Unternehmensanteil an der Finanzierung der gesamten Forschungsausgaben beträgt $48 \%$ im Jahr 2006 und liegt damit etwas höher als im Jahr 2002 (45\%). Damit liegt auf den ersten Blick das $67 \%$-Ziel für den Unternehmensanteil in weiter Ferne. Al- 
lerdings hat Österreich einen mit 18,4\% im internationalen Vergleich sehr hohen Auslandsanteil - dieser wird aber zu ca. $90 \%$ von ausländischen Unternehmen, die F\&E-Aktivitäten ihrer österreichischen Töchter finanzieren gestellt /der Anteil der EU an den vom Ausland finanzierten F\&E-Mittel betrug im
Jahr 2006 knapp 9 \%). Zusammen bestreiten also in- und ausländische Unternehmen etwa 65 \% der gesamten Forschungsausgaben in Österreich - dies bedeutet eine Beinahe-Erfüllung des $67 \%$-Ziels ${ }^{17}$. Bei dieser Berechnungsart wird das Ziel auch auf Ebene der EU15 und EU27 bereits (fast) erfüllt:

\section{Abbildung 7: Finanzierungsstruktur der Forschungsausgaben 2005 im Ländervergleich}

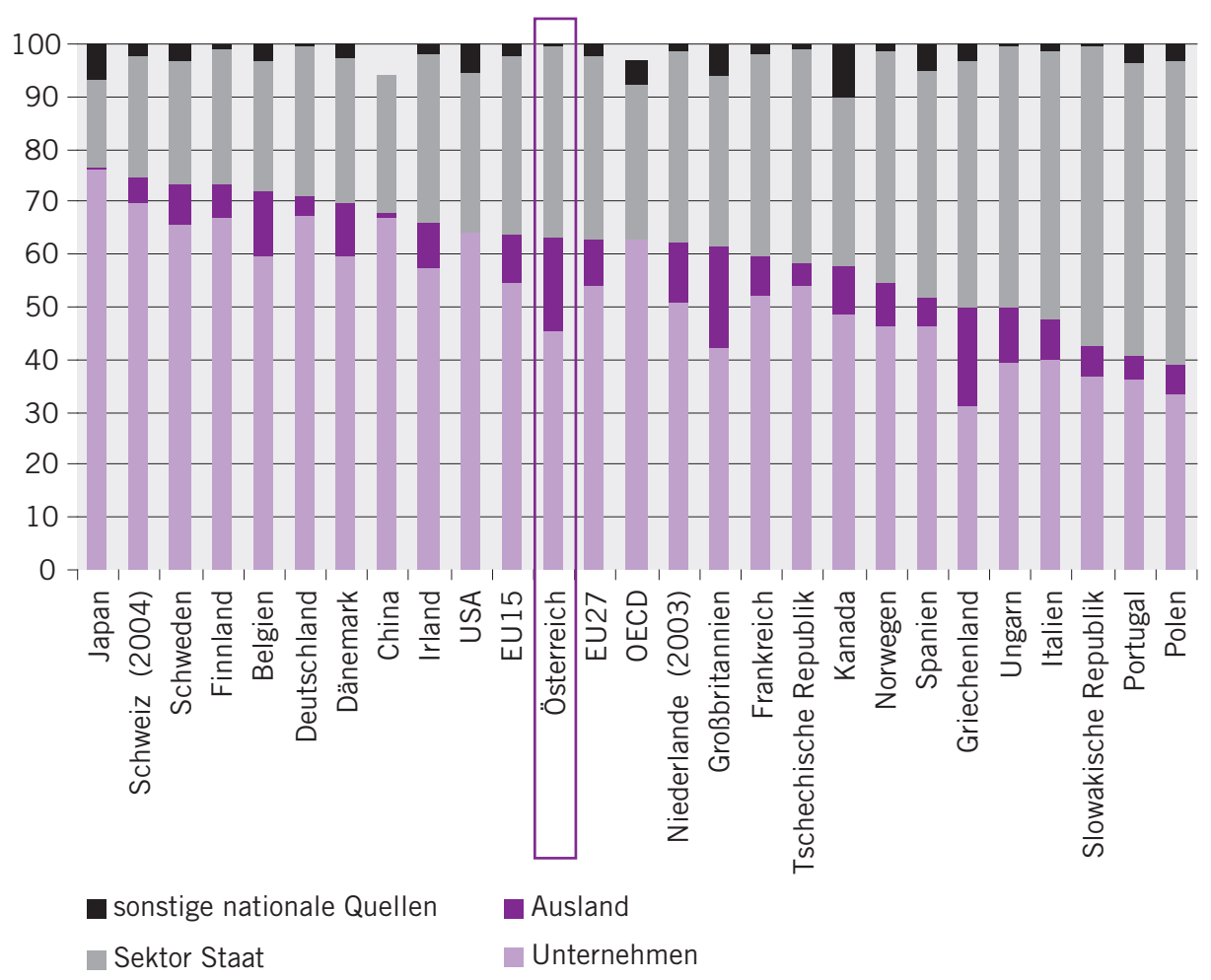

Quelle: OECD (MSTI), Berechnungen Joanneum Research

17 Der hohe Finanzierungsanteil durch ausländische Unternehmen hat natürlich gewisse Implikationen für die Forschungsstruktur in Österreich: Einerseits sind es eben nicht inländische Unternehmen, die die Forschungsausgaben bestreiten - sie haben daher geringere Forschungsausgaben (nämlich nur den „offiziellen“ Anteil von $48 \%$ ); das bedeutet auch, dass die Forschungsausgaben von österreichischen Institutionen (wenn von der möglichen, aber unbekannten Finanzierung ausländischer F\&E abgesehen wird) geringer sind als es die Gesamtquote von etwa 2,50 impliziert. Andererseits legt der hohe Auslandsanteil eine durchaus hohe Attraktivität des „Forschungsstandorts Österreich" nahe. 
Die höchsten Unternehmensanteile (bzw. kombinierte Unternehmens- und Auslandsanteile) weisen Japan und Schweiz mit jeweils knapp 75 $\%$ auf. Österreich liegt genau im Durchschnitt der EU15 bzw. EU27 (wenn auch mit einem deutlich höheren Auslandsanteil). Die Rangordnung der Länder zeigt auch, dass die Forschungsquote stark vom Unternehmenssektor bestimmt wird: Tendenziell weisen Länder mit hohem Unternehmensanteil auch hohe Forschungsquoten auf.

\section{Ausgabenarten}

Die größten Anteile an den Forschungsausgaben entfallen auf Personal und Sachausgaben; Bau- und Ausrüstungsinvestitionen sind zusammen für weniger als $10 \%$ der Ausgaben verantwortlich.

\section{Abbildung 8: F\&E-Ausgaben 2002/04/06 nach Ausgabenarten}

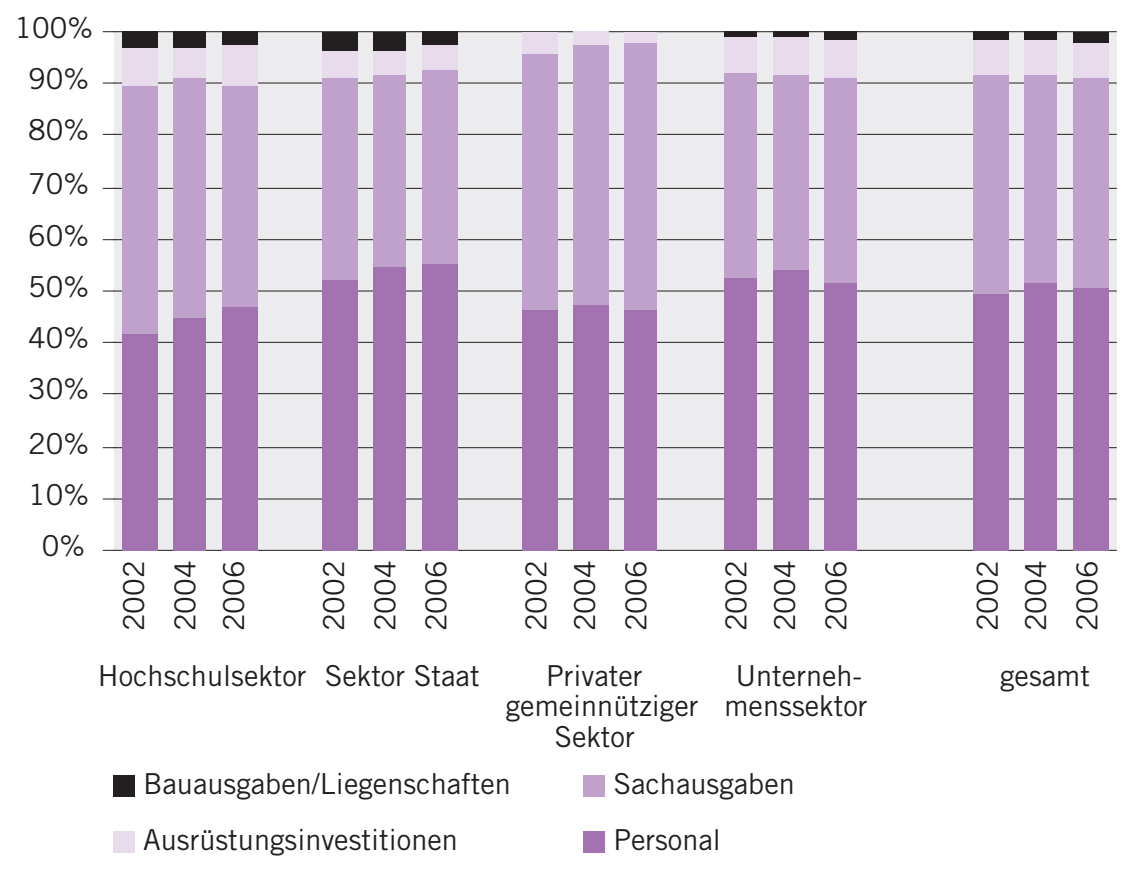

Quelle: Statistik Austria (F\&E-Erhebung), Berechnungen Joanneum Research 


\subsubsection{Der Unternehmenssektor}

Auf die beiden wichtigsten Träger der Forschung und Entwicklung, den Unternehmensund den Hochschulsektor, soll im Folgenden etwas detaillierter eingegangen werden. Beim Unternehmenssektor wird dabei nach Wirtschaftsbereichen und Technologieintensität ${ }^{18}$ unterschieden, im Hochschulbereich nach Wissenschaftsdisziplinen.

Tabelle 5: F\&E-Ausgaben und Wertschöpfung im Unternehmenssektor, 2002 und 2006

\begin{tabular}{|c|c|c|c|c|c|c|c|c|c|c|c|c|}
\hline \multirow{3}{*}{ Sektor } & \multicolumn{6}{|c|}{2006} & \multicolumn{6}{|c|}{2002} \\
\hline & 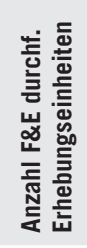 & 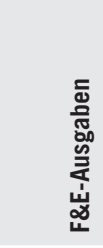 & 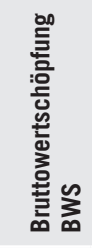 & 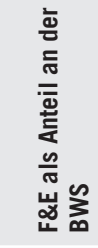 & 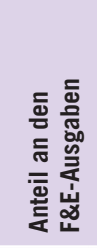 & 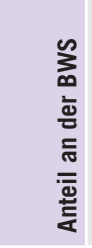 & 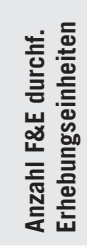 & 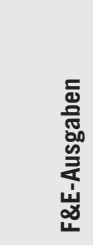 & 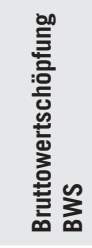 & 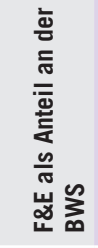 & 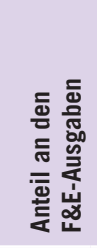 & 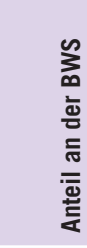 \\
\hline & & [Mio. €] & Mrd. €] & {$[\%]$} & {$[\%]$} & [\%] & & Mio. €] & Mrd. €] & [\%] & [\%] & [\%] \\
\hline Land- u.Forstwirtschaft, Fischerei & 3 & 1 & 3,9 & $0,0 \%$ & $0 \%$ & $2 \%$ & 4 & 2 & 4,0 & $0,1 \%$ & $0 \%$ & $2 \%$ \\
\hline Bergbau & 10 & 7 & 1,2 & $0,6 \%$ & $0 \%$ & $1 \%$ & 9 & 3 & 0,8 & $0,3 \%$ & $0 \%$ & $0 \%$ \\
\hline Sachgütererzeugung & 1324 & 3159 & 46,2 & $6,8 \%$ & $71 \%$ & $20 \%$ & 1169 & 2273 & 39,5 & $5,7 \%$ & $73 \%$ & $20 \%$ \\
\hline High-Tech & 275 & 1439 & 6,4 & $22,4 \%$ & $32 \%$ & $3 \%$ & 229 & 1029 & 5,5 & $18,6 \%$ & $33 \%$ & $3 \%$ \\
\hline Medium Tech & 767 & 1534 & 24,9 & $6,2 \%$ & $34 \%$ & $11 \%$ & 672 & 1114 & 19,4 & $5,7 \%$ & $36 \%$ & $10 \%$ \\
\hline Sonstige Sachgüter & 282 & 187 & 14,9 & $1,3 \%$ & $4 \%$ & $6 \%$ & 268 & 130 & 14,6 & $0,9 \%$ & $4 \%$ & $7 \%$ \\
\hline Energie- und Wasserversorgung & 25 & 9 & 6,0 & $0,2 \%$ & $0 \%$ & $3 \%$ & 17 & 14 & 4,5 & $0,3 \%$ & $0 \%$ & $2 \%$ \\
\hline Bauwesen & 82 & 26 & 17,9 & $0,1 \%$ & $1 \%$ & $8 \%$ & 53 & 12 & 14,8 & $0,1 \%$ & $0 \%$ & $7 \%$ \\
\hline Dienstleistungen & 963 & 1246 & 157,3 & $0,8 \%$ & $28 \%$ & $68 \%$ & 690 & 828 & 134,6 & $0,6 \%$ & $26 \%$ & $68 \%$ \\
\hline Hi-Tech Knowledge Intensive & 459 & 605 & 3,6 & $16,8 \%$ & $14 \%$ & $2 \%$ & 299 & 373 & 3,4 & $11,1 \%$ & $12 \%$ & $2 \%$ \\
\hline Sonstige Dienstleistungen & 504 & 641 & 153,7 & $0,4 \%$ & $14 \%$ & $66 \%$ & 391 & 455 & 131,2 & $0,3 \%$ & $15 \%$ & $66 \%$ \\
\hline Gesamt & 2407 & 4449 & 232,5 & $1,9 \%$ & $100 \%$ & $100 \%$ & 1942 & 3131 & 198,3 & $1,6 \%$ & $100 \%$ & $100 \%$ \\
\hline
\end{tabular}

Anm.: Auf Grund der Datenverfügbarkeit erfolgte die Klassifikation der Technologiezugehörigkeit auf NACE-2-Steller Ebene.

Quelle: Statistik Austria (F\&E-Erhebung, Volkswirtschaftliche Gesamtrechnung), Berechnungen Joanneum Research

Als Anteil an der Bruttowertschöpfung wurden die F\&E-Ausgaben zwischen 2002 und 2006 von insgesamt 1,6 auf 1,9\% gesteigert /die entsprechenden Anteile am Bruttoinlandsprodukt betragen 1,4 bzw. 1,7 \%); eine Steigerung des F\&E-Anteils kann in (fast) allen Sektoren beobachtet werden. Den höchste Zuwachs verzeichnen High-Tech-Knowledge-Intensive Dienstleistungen, deren F\&E-Anteil an der Bruttowertschöpfung von $11 \%$ auf knapp un-

18 Aus Gründen der Datenverfügbarkeit ist die Technologie-Definition etwas abweichend von der (üblicherweise verwendeten) Definition der OECD: Der High-Tech-Sachgüterbereich umfasst die Branchen NACE 24, 30, 32 und 33; der Medium-Tech-Sachgüterbereich die Branchen 25-29, 31, 34, 35. Zu den High-Tech-Knowledge-Intensive-Dienstleistungen gehören NACE 72 und 73. NACE 64, der auch zu den High-Tech- Sektoren zählt, kann auf der Basis der publizierten F\&E-Erhebung nicht aus der Gruppe NACE 60-64 (Verkehr und Nachrichtenübermittlung) herausgelöst werden und wird daher unter Sonstige Dienstleistungen subsumiert (für eine Liste der Branchen und ihrer Zuordnung vgl. den Anhang). 
ter $17 \%$ gestiegen ist. Damit entfielen im Jahr 2006 insgesamt $14 \%$ aller F\&E-Ausgaben auf diesen Sektor. Die höchsten F\&E-Intensitäten wie auch der größte Anteil an den F\&E-Ausgaben sind nach wie vor in der High-Tech-Sach- güterindustrie zu verzeichnen: Mit einer F\&EIntensität von fast $23 \%$ (nach $19 \%$ im Jahr 2002) stellen diese Branchen ein Drittel aller F\&E-Ausgaben.

Tabelle 6: Finanzierung der F\&E-Ausgaben im Unternehmenssektor, 2006

\begin{tabular}{|c|c|c|c|c|c|c|c|c|c|c|c|c|}
\hline \multirow{3}{*}{ Sektor } & \multicolumn{12}{|c|}{2006} \\
\hline & 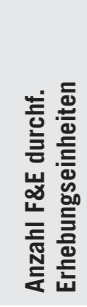 & 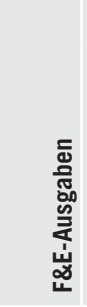 & 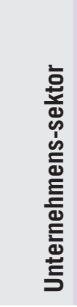 & 司 & 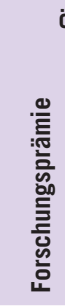 & 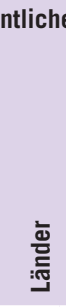 & Sektor & 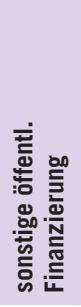 & 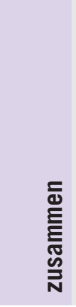 & 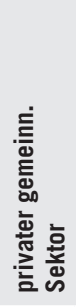 & 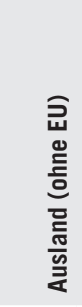 & 岀 \\
\hline & \multicolumn{2}{|c|}{ [Mio. €] } & [\%] & [\%] & [\%] & [\%] & [\%] & [\%] & [\%] & [\%] & [\%] & [\%] \\
\hline Land- u.Forstwirtschaft, Fischerei & 3 & 1 & $79 \%$ & $0 \%$ & $1 \%$ & $9 \%$ & $11 \%$ & $0 \%$ & $21 \%$ & $0 \%$ & $0 \%$ & $0 \%$ \\
\hline Bergbau & 10 & 7 & $57 \%$ & $0 \%$ & $0 \%$ & $0 \%$ & $1 \%$ & $0 \%$ & $1 \%$ & $0 \%$ & $42 \%$ & $0 \%$ \\
\hline Sachgütererzeugung & 1324 & 3159 & $72 \%$ & $0 \%$ & $4 \%$ & $0 \%$ & $2 \%$ & $0 \%$ & $7 \%$ & $0 \%$ & $21 \%$ & $0 \%$ \\
\hline High-Tech & 275 & 1439 & $56 \%$ & $1 \%$ & $5 \%$ & $0 \%$ & $2 \%$ & $0 \%$ & $8 \%$ & $0 \%$ & $35 \%$ & $0 \%$ \\
\hline Medium Tech & 767 & 1534 & $84 \%$ & $0 \%$ & $3 \%$ & $0 \%$ & $2 \%$ & $0 \%$ & $6 \%$ & $0 \%$ & $10 \%$ & $0 \%$ \\
\hline Sonstige Sachgüter & 282 & 187 & $95 \%$ & $0 \%$ & $2 \%$ & $0 \%$ & $2 \%$ & $0 \%$ & $5 \%$ & $0 \%$ & $0 \%$ & $0 \%$ \\
\hline Energie- und Wasserversorgung & 25 & 9 & $93 \%$ & $0 \%$ & $3 \%$ & $0 \%$ & $1 \%$ & $0 \%$ & $4 \%$ & $0 \%$ & $0 \%$ & $3 \%$ \\
\hline Bauwesen & 82 & 26 & $91 \%$ & $0 \%$ & $3 \%$ & $1 \%$ & $4 \%$ & $0 \%$ & $8 \%$ & $0 \%$ & $0 \%$ & $0 \%$ \\
\hline Dienstleistungen & 963 & 1246 & $52 \%$ & $7 \%$ & $2 \%$ & $2 \%$ & $4 \%$ & $1 \%$ & $16 \%$ & $0 \%$ & $30 \%$ & $2 \%$ \\
\hline Hi-Tech Knowledge Intensive & 459 & 605 & $54 \%$ & $12 \%$ & $3 \%$ & $5 \%$ & $5 \%$ & $2 \%$ & $26 \%$ & $0 \%$ & $17 \%$ & $3 \%$ \\
\hline Sonstige Dienstleistungen & 504 & 641 & $52 \%$ & $5 \%$ & $2 \%$ & $2 \%$ & $3 \%$ & $1 \%$ & $13 \%$ & $0 \%$ & $33 \%$ & $2 \%$ \\
\hline Gesamt & 2407 & 4449 & $66 \%$ & $2 \%$ & $4 \%$ & $1 \%$ & $3 \%$ & $0 \%$ & $10 \%$ & $0 \%$ & $23 \%$ & $1 \%$ \\
\hline
\end{tabular}

Anm.: Auf Grund der Datenverfügbarkeit erfolgte die Klassifikation der Technologiezugehörigkeit auf NACE-2-Steller Ebene.

Quelle: Statistik Austria (F\&E-Erhebung), Berechnungen Joanneum Research

Insgesamt werden zwei Drittel der F\&E-Ausgaben der Unternehmen vom Unternehmenssektor selbst finanziert, gefolgt vom Ausland (mit etwas unter einem Viertel) und dem öffentlichen Sektor mit 10 \%. Die EU spielt bei der Finanzierung der Unternehmens-F\&E nur eine marginale Rolle, der gemeinnützige Sektor praktisch keine. Überdurchschnittliche Auslandsanteile zeigen - abgesehen vom quantitativ unbedeutenden Bergbausektor - der High-Tech-Sachgüterbereich mit $35 \%$ und die Dienstleistungen mit $30 \%$. Der Dienstleistungssektor verzeich- net darüber hinaus relativ hohe Anteile an öffentlichen (16\%) und EU-Mitteln (2\%) und relativ geringe Anteile an Finanzierung durch den Unternehmenssektor (52\%). Innerhalb des Sachgüterbereichs ist eine nicht uninteressante Korrelation von Finanzierungsstruktur und Technologiegehalt beobachtbar: Je höher dieser ist, desto höher ist der Anteil des öffentlichen Sektors (speziell der Forschungsprämie) und des Auslands; der "Selbstfinanzierungsgrad" (Finanzierung durch den Unternehmensbereich) verhält sich umgekehrt dazu. 


\subsubsection{Der Hochschulbereich}

Im Hochschulbereich dominiert naturgemäß die Finanzierung der F\&E-Ausgaben durch den öffentlichen Sektor. Insgesamt finanziert die öffentliche Hand im Durchschnitt 89 \% der F\&E-Ausgaben des Hochschulsektors.

Tabelle 7: Finanzierung der F\&E-Ausgaben im Hochschulbereich, 2006

\begin{tabular}{|c|c|c|c|c|c|c|c|c|c|c|c|}
\hline \multirow[b]{2}{*}{ Wissenschaftsdisziplin } & \multicolumn{11}{|c|}{2006} \\
\hline & 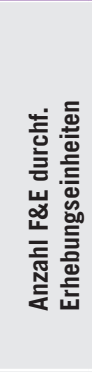 & 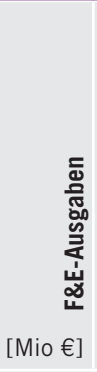 & 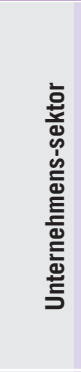 & 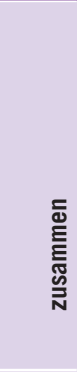 & 픔 & 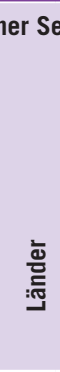 & 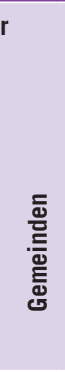 & 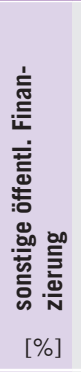 & 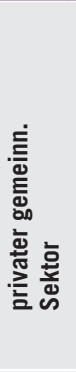 & 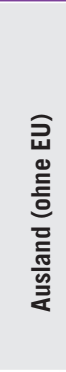 & $\mathrm{z}$ \\
\hline 1.0 bis 4.0 zusammen & 673 & 1.162 & $6 \%$ & $87 \%$ & $73 \%$ & $2 \%$ & $0 \%$ & $13 \%$ & $1 \%$ & $2 \%$ & $4 \%$ \\
\hline 1.0 Naturwissenschaften & 252 & 477 & $4 \%$ & $89 \%$ & $72 \%$ & $2 \%$ & $0 \%$ & $15 \%$ & $0 \%$ & $2 \%$ & $5 \%$ \\
\hline 2.0 Technische Wissenschaften & 192 & 218 & $13 \%$ & $79 \%$ & $66 \%$ & $4 \%$ & $0 \%$ & $8 \%$ & $1 \%$ & $2 \%$ & $5 \%$ \\
\hline 3.0 Humanmedizin & 172 & 397 & $5 \%$ & $88 \%$ & $74 \%$ & $1 \%$ & $0 \%$ & $13 \%$ & $1 \%$ & $3 \%$ & $3 \%$ \\
\hline 4.0 Land- und Forstwirtschaft, VetMed & 57 & 70 & $2 \%$ & $93 \%$ & $87 \%$ & $1 \%$ & $0 \%$ & $5 \%$ & $1 \%$ & $2 \%$ & $3 \%$ \\
\hline 5.0 und 6.0 zusammen & 489 & 361 & $2 \%$ & $94 \%$ & $84 \%$ & $2 \%$ & $0 \%$ & $8 \%$ & $1 \%$ & $0 \%$ & $2 \%$ \\
\hline 5.0 Sozialwissenschaften & 274 & 209 & $4 \%$ & $92 \%$ & $86 \%$ & $2 \%$ & $0 \%$ & $4 \%$ & $2 \%$ & $0 \%$ & $2 \%$ \\
\hline 6.0 Geisteswissenschaften & 215 & 152 & $1 \%$ & $98 \%$ & $82 \%$ & $3 \%$ & $0 \%$ & $12 \%$ & $0 \%$ & $0 \%$ & $1 \%$ \\
\hline 1.0 bis 6.0 Insgesamt & 1.162 & 1.523 & $5 \%$ & $89 \%$ & $75 \%$ & $2 \%$ & $0 \%$ & $11 \%$ & $1 \%$ & $2 \%$ & $3 \%$ \\
\hline
\end{tabular}

Quelle: Statistik Austria (F\&E-Erhebung), Berechnungen Joanneum Research

Der Anteil der öffentlichen Hand ist bei den Geisteswissenschaften mit 98 \% am höchsten, in den technischen Wissenschaften mit $79 \%$ am geringsten. Der Anteil des Unternehmenssektors verläuft umgekehrt dazu: Im Durchschnitt bei $5 \%$, liegt sein Anteil zwischen 1 (Geisteswissenschaften) und $13 \%$ (technische Wissenschaften); eine ähnliche Rangordnung zeigt sich für die EU-Mittel und das Ausland (Durchschnitt bei 3 bzw. $2 \%$ ). „Sonstige öffentliche Mittel", in welchen die Fördervolumina des Wissenschaftsfonds enthalten sind, tragen $11 \% \mathrm{zu}$ den Forschungsausgaben der Hochschulen bei. Auch diese Mittel haben aber deutlich unterschiedliches Gewicht: Am geringsten ist ihr Anteil in den Sozialwissen- schaften $(4 \%)$, am höchsten in Naturwissenschaften, Humanmedizin und Geisteswissenschaften $(15,13$ und $12 \%)$.

\subsubsection{F\&E-Beschäftigte}

Die Beschäftigung (als Kopfzahl) im F\&E-Bereich hat zwischen 2002 und 2006 um +28\% auf fast 84000 zugenommen; Träger dieser Ausweitung sind der Unternehmens- und Hochschulbereich mit +33 bzw. $+30 \%$. Der Sektor Staat und der private gemeinnützige Bereich weisen Rückgänge auf /die im Fall der Gemeinnützigen mit $-35 \%$ recht massiv sind). 
Tabelle 8: Beschäftigung in F\&E, 2002-2004-2006

\begin{tabular}{|c|c|c|c|c|c|c|c|c|c|c|c|}
\hline & \multicolumn{4}{|c|}{ Beschäftigte - Kopfzahlen } & \multicolumn{4}{|c|}{ Beschäftigte - Vollzeitäquivalente } & \multicolumn{3}{|c|}{ Verhältnis VZÄ/Köpfe } \\
\hline Durchführungssektor & 2002 & 2004 & 2006 & $\begin{array}{r}\text { (Veränd. } \\
2002-2006 \text { ) }\end{array}$ & 2002 & 2004 & 2006 & $\begin{array}{r}\text { (Veränd. } \\
2002-2006 \text { ) }\end{array}$ & 2002 & 2004 & 2006 \\
\hline Hochschulsektor & 25072 & 29358 & 32715 & $+30 \%$ & 9879 & 11502 & 12668 & $+28 \%$ & $39 \%$ & $39 \%$ & $39 \%$ \\
\hline Sektor Staat & 6010 & 5531 & 5511 & $-8 \%$ & 2060 & 2035 & 2423 & $+18 \%$ & $34 \%$ & $37 \%$ & $44 \%$ \\
\hline Priv.gemeinnütziger Sektor & 623 & 565 & 404 & $-35 \%$ & 227 & 212 & 161 & $-29 \%$ & $36 \%$ & $38 \%$ & $40 \%$ \\
\hline Unternehmenssektor & 34020 & 38737 & 45336 & $+33 \%$ & 26728 & 29143 & 34126 & $+28 \%$ & $79 \%$ & $75 \%$ & $75 \%$ \\
\hline gesamt & 65725 & 74191 & 83966 & $+28 \%$ & 38893 & 42891 & 49377 & $+27 \%$ & $59 \%$ & $58 \%$ & $59 \%$ \\
\hline
\end{tabular}

Quelle: Statistik Austria (F\&E-Erhebung), Berechnungen Joanneum Research

In Vollzeitäquivalenten (VZÄ) war die $\mathrm{Zu}$ nahme mit $+27 \%$ geringfügig kleiner (auf gut 49 000). Der "Auslastungsgrad“ (das Verhältnis von Vollzeitäquivalenten und Kopfzahlen) eines typischen F\&E-Beschäftigten ist bei etwa 59 \% konstant geblieben. Im Unternehmenssektor ist dabei der Auslastungsgrad gesun- ken (liegt dort mit $75 \%$ aber immer noch am höchsten). Ausdehnen konnten den Auslastungsgrad der private gemeinnützige Sektor und vor allem der Staatssektor.

Nach Beschäftigtenkategorien und Vollzeitäquivalenten zeigt sich folgendes Bild:

\section{Abbildung 9: F\&E-Beschäftigungsstruktur in VZÄ 2002/04/06 in Österreich}

\begin{tabular}{|c|c|c|c|c|c|}
\hline Durchführungssektor & 产 & 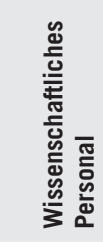 & 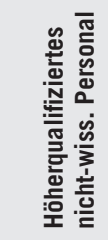 & 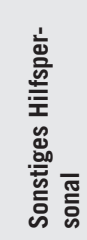 & $\begin{array}{l}: \\
\text { : } \\
\text { E. }\end{array}$ \\
\hline \multirow[t]{3}{*}{ Hochschulsektor } & 2002 & 6977 & 1475 & 1427 & 9879 \\
\hline & 2004 & 8281 & 1569 & 1652 & 11502 \\
\hline & 2006 & 9261 & 1856 & 1551 & 12668 \\
\hline \multirow[t]{3}{*}{ Sektor Staat } & 2002 & 999 & 342 & 719 & 2060 \\
\hline & 2004 & 1030 & 300 & 706 & 2035 \\
\hline & 2006 & 1349 & 357 & 718 & 2423 \\
\hline \multirow{3}{*}{$\begin{array}{l}\text { Privater } \\
\text { gemeinnütziger } \\
\text { Sektor }\end{array}$} & 2002 & 148 & 50 & 30 & 227 \\
\hline & 2004 & 137 & 49 & 27 & 212 \\
\hline & 2006 & 118 & 26 & 16 & 161 \\
\hline \multirow[t]{3}{*}{ Unternehmenssektor } & 2002 & 16001 & 8326 & 2400 & 26728 \\
\hline & 2004 & 16508 & 10150 & 2485 & 29143 \\
\hline & 2006 & 18471 & 12583 & 3072 & 34126 \\
\hline \multirow[t]{3}{*}{ gesamt } & 2002 & 24124 & 10194 & 4575 & 38893 \\
\hline & 2004 & 25955 & 12067 & 4869 & 42891 \\
\hline & 2006 & 29199 & 14822 & 5357 & 49377 \\
\hline
\end{tabular}

Quelle: Statistik Austria (F\&E-Erhebung), Berechnungen Joanneum Research

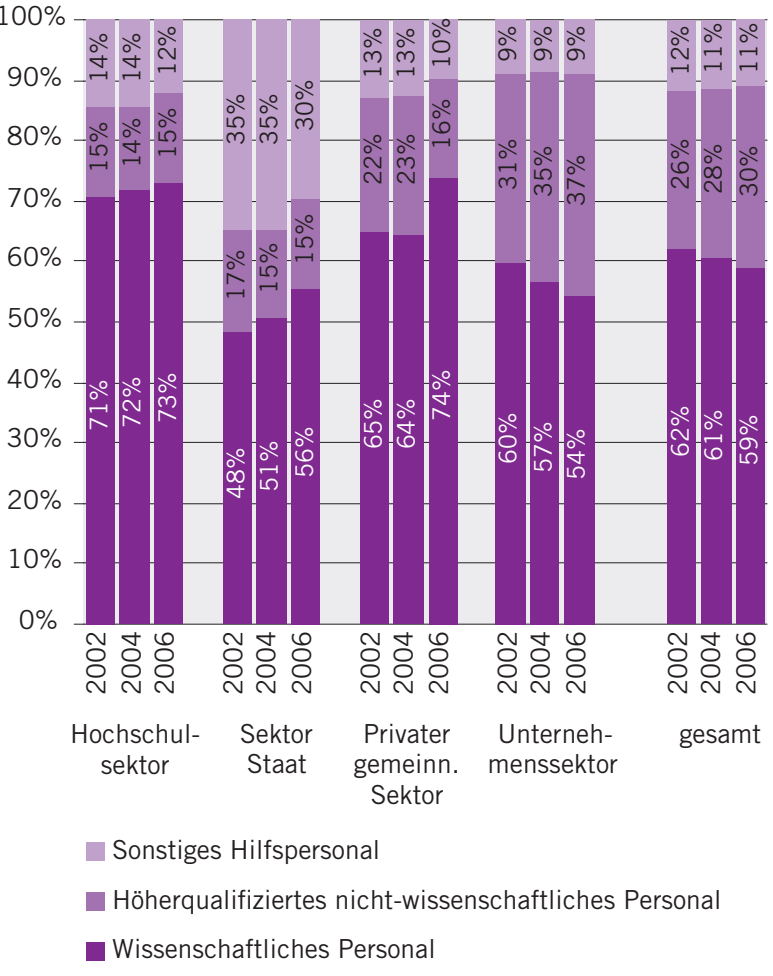


Getrieben vom Unternehmenssektor zeigt sich ein Rückgang des Anteils von wissenschaftlichem Personal (in Vollzeitäquivalenten) von 62 auf $59 \%$ zugunsten des höherqualifizierten nicht-wissenschaftlichen Personals (von 26 auf $30 \%$ ). Am höchsten qualifiziert sind der Hochschulsektor (mit einem wissenschaftlichen Anteil von $73 \%$ ) und der private, gemeinnützige Sektor, die ihre Qualifikationsstruktur im Vergleich zu den Jahren 2002 und
2004 deutlich verbessern konnten. Dies trifft auch auf den Staatssektor zu.

\subsubsection{Frauenanteil bei den F\&E-Beschäftigten}

Der Frauenanteil bei den F\&E-Beschäftigten ist zwar zwischen 2002 und 2006 geringfügig gestiegen, ist aber mit $29 \%$ Kopfanteil immer noch relativ gering:

Tabelle 9: Frauenanteil nach Durchführungssektoren und Beschäftigtenkategorien, 2002 und 2006

\begin{tabular}{|c|c|c|c|c|c|c|c|c|c|}
\hline \multirow{3}{*}{ Durchfïhrungssektor } & \multirow{3}{*}{ Jahr } & \multirow{2}{*}{\multicolumn{2}{|c|}{ Insgesamt }} & \multicolumn{6}{|c|}{ davon } \\
\hline & & & & \multicolumn{2}{|c|}{$\begin{array}{l}\text { Wissenschaft- } \\
\text { liches Personal }\end{array}$} & \multicolumn{2}{|c|}{$\begin{array}{l}\text { Höherqualifiziertes } \\
\text { nichtwiss. Personal }\end{array}$} & \multicolumn{2}{|c|}{$\begin{array}{c}\text { Sonstiges } \\
\text { Hilfspersonal }\end{array}$} \\
\hline & & Kopf & VZÄ & Kopf & VZÄ & Kopf & VZÄ & Kopf & VZÄ \\
\hline \multirow[t]{2}{*}{ Insgesamt } & 2002 & $28 \%$ & $22 \%$ & $21 \%$ & $16 \%$ & $32 \%$ & $26 \%$ & $53 \%$ & $45 \%$ \\
\hline & 2006 & $29 \%$ & $24 \%$ & $25 \%$ & $19 \%$ & $28 \%$ & $24 \%$ & $52 \%$ & $46 \%$ \\
\hline \multirow[t]{2}{*}{ 1. Hochschulsektor } & 2002 & $41 \%$ & $38 \%$ & $30 \%$ & $27 \%$ & $65 \%$ & $65 \%$ & $70 \%$ & $66 \%$ \\
\hline & 2006 & $44 \%$ & $41 \%$ & $35 \%$ & $31 \%$ & $65 \%$ & $65 \%$ & $71 \%$ & $68 \%$ \\
\hline \multirow[t]{2}{*}{ 2. Sektor Staat } & 2002 & $46 \%$ & $41 \%$ & $35 \%$ & $32 \%$ & $50 \%$ & $50 \%$ & $55 \%$ & $48 \%$ \\
\hline & 2006 & $45 \%$ & $41 \%$ & $39 \%$ & $34 \%$ & $48 \%$ & $48 \%$ & $53 \%$ & $49 \%$ \\
\hline \multirow[t]{2}{*}{ 3. Privater gemeinnütziger Sektor } & 2002 & $50 \%$ & $48 \%$ & $38 \%$ & $36 \%$ & $63 \%$ & $66 \%$ & $80 \%$ & $74 \%$ \\
\hline & 2006 & $57 \%$ & $59 \%$ & $52 \%$ & $53 \%$ & $65 \%$ & $74 \%$ & $79 \%$ & $82 \%$ \\
\hline \multirow[t]{2}{*}{ 4. Unternehmenssektor } & 2002 & $15 \%$ & $14 \%$ & $10 \%$ & $10 \%$ & $18 \%$ & $18 \%$ & $32 \%$ & $32 \%$ \\
\hline & 2006 & $17 \%$ & $16 \%$ & $14 \%$ & $12 \%$ & $17 \%$ & $17 \%$ & $34 \%$ & $34 \%$ \\
\hline
\end{tabular}

Quelle: Statistik Austria (F\&E-Erhebung), Berechnungen Joanneum Research

Die insgesamt niedrige Frauenquote ist in erster Linie auf den Unternehmenssektor zurückzuführen: Hier sind nur $17 \%$ der Beschäftigten Frauen (bzw. $16 \%$ der Vollzeitäquivalente). Nicht zuletzt ist das auf die Struktur der F\&E im Unternehmenssektor zurückzuführen: Hier handelt es sich in erster Linie um technische Forschung. Der Frauenanteil in den technischen Wissenschaften ist aber auch im Universitäts- bereich gering (mit $15 \%$ beim wissenschaftlichen Personal (vgl. Abbildung 12) im Vergleich zu $14 \%$ im Unternehmenssektor).

Im internationalen Vergleich weist Österreich eine sehr geringe Frauenquote im Bereich der Forschung und Entwicklung auf (der folgende Vergleich betrifft Kopfzahlen des wissenschaftliche Personals, also jenes mit akademischer bzw. gleichwertiger Ausbildung): 
Abbildung 10: Frauenanteil am wissenschaftlichen Personal (AkademikerInnen und gleichwertige Kräfte; Kopfzahlen) im internationalen Vergleich, 2004/05

\begin{tabular}{|c|c|c|c|c|c|}
\hline & 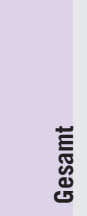 & 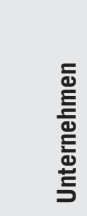 & 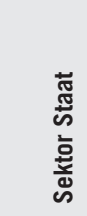 & 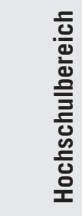 & 흥 \\
\hline Portugal & 44,4 & 26,4 & 56,6 & 46,9 & (2005) \\
\hline Slowakische Republik & 41,5 & 32,4 & 42,7 & 43 & (2005) \\
\hline Polen & 39,3 & 26,6 & 40,4 & 41 & (2005) \\
\hline Spanien & 36,7 & 26,8 & 46,6 & 38 & (2005) \\
\hline Griechenland & 36,4 & 28 & 40,8 & 38 & (2005) \\
\hline Schweden & 35,8 & 25,2 & 37,2 & 48,3 & (2005) \\
\hline Ungarn & 34,2 & 22,6 & 38,2 & 36,6 & (2005) \\
\hline Italien & 32,4 & 20,3 & 39,9 & 34,6 & (2005) \\
\hline Norwegen & 31,7 & 20,3 & 37,4 & 39,4 & (2005) \\
\hline Irland & 30,3 & 20,2 & 35,4 & 38,2 & (2005) \\
\hline Finnland & 30,2 & 17,7 & 41,9 & 43,7 & (2005) \\
\hline Dänemark & 29,7 & 24,9 & 36,4 & 35,7 & (2005) \\
\hline Belgien & 29,6 & 20,5 & 31,4 & 36,2 & (2005) \\
\hline Tschechische Republik & 28,8 & 17,8 & 35,5 & 33,6 & (2005) \\
\hline Frankreich & 27,8 & 20,1 & 32,9 & 34,2 & (2005) \\
\hline Schweiz & 26,7 & 21,1 & 25,5 & 29,6 & (2004) \\
\hline Österreich & 23,6 & 12,6 & 36,2 & 32,8 & (2004) \\
\hline Deutschland & 21,4 & 11,6 & 28,5 & 29,7 & (2005) \\
\hline Niederlande & 18 & 10,1 & 29,4 & 28,9 & (2005) \\
\hline Japan & 11,9 & 6,5 & 12,5 & 21,5 & (2005) \\
\hline ungew. Mittelwert & 30,5 & 20,6 & 36,3 & 36,5 & \\
\hline
\end{tabular}

Quelle: OECD (MSTI), Berechnungen Joanneum Research

Unter den 20 Ländern, für die vergleichbare Daten verfügbar waren, weist Österreich den viertkleinsten Frauenanteil auf, nur vor Deutschland, den Niederlanden und Japan. Im Unternehmensbereich ist der Frauenanteil zwar in allen Ländern geringer als in den Sektoren Staat und Hochschule (auch hier eine Folge der technischen Ausrichtung der Unternehmens-F\&E), allerdings weist Österreich auch hier einen sehr geringen Wert auf - wiederum den viertschlechtesten vor Deutschland, den Niederlanden und Japan. Im Staatssektor liegt

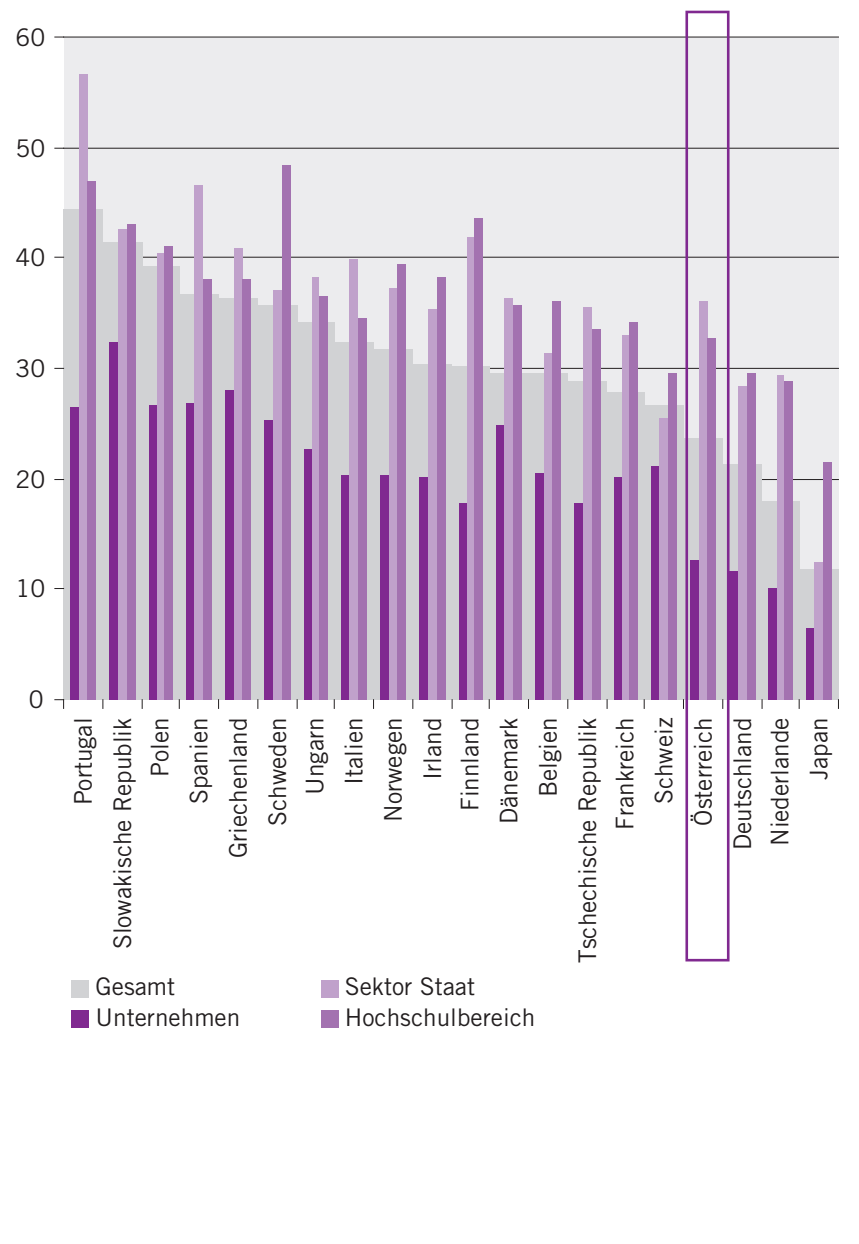

Österreichs Frauenanteil im Durchschnitt der 20 Länder, im Hochschulbereich etwas darunter.

Tabelle 9 zeigt noch zwei weitere Aspekte:

Mit sinkendem Qualifikationsniveau steigt der Frauenanteil. Auf der Basis aller F\&EBeschäftigten beträgt der Frauenanteil beim wissenschaftlichen Personal $25 \%$, beim höherqualifizierten nicht-wissenschaftlichen Personal $28 \%$ und $52 \%$ beim Hilfspersonal; ein ähnliches Muster ist auch bei allen Einzelsektoren $\mathrm{zu}$ beobachten. 
Ein weiterer Aspekt ist auch der geringere "Auslastungsgrad" der weiblichen Beschäftigten, definiert als das Verhältnis von Vollzeitäquivalenten zu Kopfzahlen. Dies zeigt sich in der Tabelle 9 durch einen höheren Frauenanteil bei den Köpfen als bei den Vollzeitäquivalenten. Deutlicher wird es aber in der folgenden Tabelle:

\section{Tabelle 10: Auslastungsgrad nach Durchführungssektoren und Geschlecht, 2002 und 2006}

\begin{tabular}{|c|c|c|c|c|c|c|c|c|c|}
\hline \multirow{3}{*}{ Durchfïhrungssektor } & \multirow{3}{*}{ Jahr } & \multirow{2}{*}{\multicolumn{2}{|c|}{ Insgesamt }} & \multicolumn{6}{|c|}{ davon } \\
\hline & & & & \multicolumn{2}{|c|}{$\begin{array}{l}\text { Wissenschaft- } \\
\text { liches Personal }\end{array}$} & \multicolumn{2}{|c|}{$\begin{array}{l}\text { Höherqualifiziertes } \\
\text { nichtwiss. Personal }\end{array}$} & \multicolumn{2}{|c|}{$\begin{array}{l}\text { Sonstiges } \\
\text { Hilfspersonal }\end{array}$} \\
\hline & & männl. & weibl. & männl. & weibl. & männl. & weibl. & männl. & weibl. \\
\hline \multirow[t]{2}{*}{ Insgesamt } & 2002 & $64 \%$ & $46 \%$ & $65 \%$ & $47 \%$ & $66 \%$ & $50 \%$ & $56 \%$ & $41 \%$ \\
\hline & 2006 & $64 \%$ & $47 \%$ & $63 \%$ & $45 \%$ & $66 \%$ & $51 \%$ & $57 \%$ & $45 \%$ \\
\hline \multirow[t]{2}{*}{ 1. Hochschulsektor } & 2002 & $42 \%$ & $36 \%$ & $42 \%$ & $36 \%$ & $38 \%$ & $37 \%$ & $44 \%$ & $36 \%$ \\
\hline & 2006 & $41 \%$ & $36 \%$ & $41 \%$ & $35 \%$ & $37 \%$ & $38 \%$ & $41 \%$ & $36 \%$ \\
\hline \multirow[t]{2}{*}{ 2. Sektor Staat } & 2002 & $38 \%$ & $30 \%$ & $44 \%$ & $39 \%$ & $26 \%$ & $26 \%$ & $35 \%$ & $27 \%$ \\
\hline & 2006 & $47 \%$ & $40 \%$ & $52 \%$ & $42 \%$ & $31 \%$ & $32 \%$ & $48 \%$ & $42 \%$ \\
\hline \multirow{2}{*}{$\begin{array}{l}\text { 3. Privater gemeinnütziger } \\
\text { Sektor }\end{array}$} & 2002 & $38 \%$ & $35 \%$ & $40 \%$ & $37 \%$ & $31 \%$ & $35 \%$ & $40 \%$ & $29 \%$ \\
\hline & 2006 & $38 \%$ & $41 \%$ & $41 \%$ & $43 \%$ & $28 \%$ & $41 \%$ & $29 \%$ & $34 \%$ \\
\hline \multirow[t]{2}{*}{ 4. Unternehmenssektor } & 2002 & $79 \%$ & $75 \%$ & $83 \%$ & $77 \%$ & $73 \%$ & $75 \%$ & $73 \%$ & $72 \%$ \\
\hline & 2006 & $76 \%$ & $70 \%$ & $82 \%$ & $73 \%$ & $71 \%$ & $70 \%$ & $66 \%$ & $65 \%$ \\
\hline
\end{tabular}

Quelle: Statistik Austria (F\&E-Erhebung), Berechnungen Joanneum Research

Im Durchschnitt beträgt der Auslastungsgrad eines männlichen Beschäftigten $64 \%$, einer weiblichen Beschäftigten aber nur $47 \%$ (beide praktisch unverändert gegenüber 2002). Dies kann durch zwei Effekte erklärt werden, deren relatives Gewicht aber auf Basis der vorliegenden Daten nicht bestimmt werden kann: erstens ein höherer Anteil von Teilzeitbeschäftigung, zweitens ein höherer Anteil nicht-forschender Tätigkeiten bei weiblichen Beschäftigten. Das Muster einer geringeren Auslastung weiblicher Beschäftigter zeigt sich in (fast) allen Sektoren und Beschäftigtenkategorien, wobei der Unterschied zwischen männlichen und weiblichen Auslastungsgraden in den Sektoren Staat und Hochschule am größten ist. Der Unternehmenssektor weist die weitaus höchsten Auslastungsgrade sowie (abgesehen von den Gemein- nützigen) den relativ geringsten Unterschied zwischen den Geschlechtern auf.

Insgesamt zeigt sich eine Entwicklung in Richtung eines höheren Frauenanteils an der F\&E-Beschäftigung, wenngleich diese auch nur schrittweise erfolgt. Dies ist allerdings bis zu einem gewissen Grad zu relativieren: Forschungskarrieren dauern mehrere Jahrzehnte, jeglicher "Strukturwandel" in diesem Bereich ist also notwendigerweise mit einer substantiellen zeitlichen Komponente verbunden, die in einem 4-Jahres-Vergleich (der F\&E-Erhebungen 2002 und 2006) natürlich keine sprunghaften Veränderungen erwarten lässt. Veränderungen in der Geschlechterstruktur sind allerdings durchaus zu beobachten, wie die folgende $\mathrm{Ab}$ bildung 11 für das wissenschaftliche Personal im Universitätsbereich zeigt. ${ }^{19}$

19 Der Universitätsbereich ist ein Teilbereich des Hochschulsektors; für die anderen Sektoren sind aus den veröffentlichten Daten der F\&E-Erhebungen keine vergleichbaren Analysen ableitbar. 


\section{Abbildung 11: Frauenanteil am wissenschaftlichen Personal an den Universitäten (VZÄ) nach Alters- gruppen, 2002 und 2006}

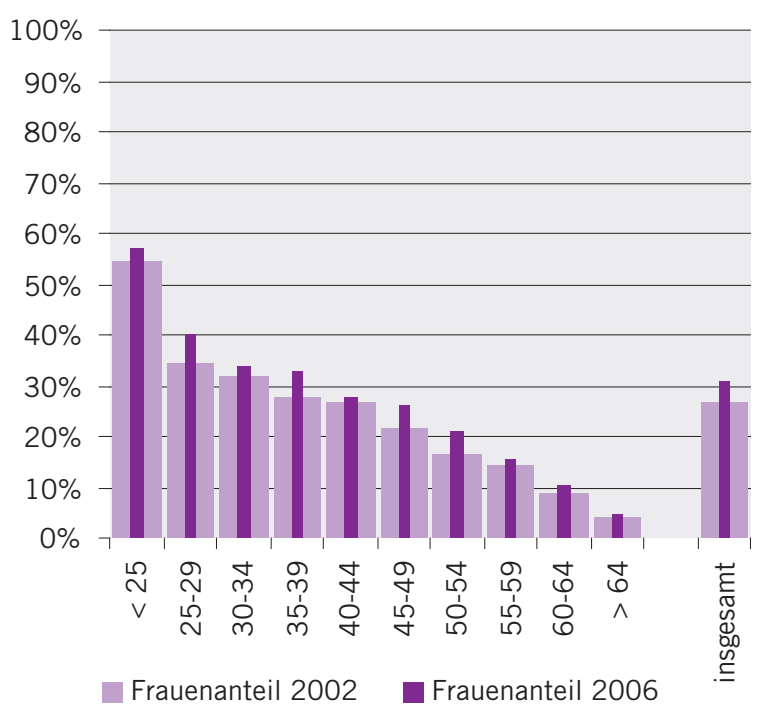

Quelle: Statistik Austria (F\&E-Erhebung), Berechnungen Joanneum Research

Die Abbildung zeigt zweierlei:

Erstens, dass die Zunahme des Frauenanteils alle Altersgruppen umfasst (am schwächsten bei den Über-64-Jährigen, wo der geringe Frauenanteil neben dem Aspekt der "historischen Geschlechterstruktur" auch das geringere Pensionsalter von Frauen reflektiert).

Zweitens, und das ist für die zukünftige Entwicklung vielleicht bedeutsamer, zeigt sich, dass sich der höhere Frauenanteil bei den jüngeren Altersgruppen im Karriereverlauf fortsetzt; Frauen scheinen also nicht in höherem Maß als Männer aus den Universitätskarrieren „herauszufallen". Sichtbar ist dies im Vergleich von zwei aufeinanderfolgenden Altersgruppen im Jahr 2002 und 2006 (wenn auch die Periodizität von Altersgruppen und F\&E-Erhebung nicht identisch ist - die Altersgruppen umfassen 5 Jahre, der Abstand der Erhebungen beträgt nur 4 Jahre): der Frauenanteil einer bestimmten Altersgruppe im Jahr 2002 entspricht recht gut dem Frauenanteil der nächst-älteren Gruppe im Jahr 2006; dies gilt für alle Altersgruppen mit Ausnahme der beiden ältesten (bei der das bereits erwähnte geringere Pensionsalter von Frauen für eine relative Zunahme des Männeranteils sorgt) sowie bei der jüngsten Altersgruppe, die allerdings nur sehr geringe Fallzahlen aufweist (Forscherkarrieren werden eher nur in Ausnahmefällen so früh begonnen). Es scheint also - zumindest auf den Universitäten - nicht der Fall zu sein, dass die Forscherkarrieren von Frauen deutlich öfter als jene von Männern frühzeitig abgebrochen werden ${ }^{20}$. Die Altersabhängigkeit des Frauenanteils ist aber nach wie vor sehr stark und wird es aufgrund des erwähnten Strukturwandels auch noch für die nächsten 20 loder mehr) Jahre bleiben.

Im Wesentlichen kann diese Zunahme des Frauenanteils in allen Altersstufen auch auf Ebene der einzelnen Wissenschaftsdisziplinen beobachtet werden: 


\section{Abbildung 12: Frauenanteil am wissenschaftlichen Personal an den Universitäten (VZÄ) nach Altersgruppen und Wissenschaftszweigen, 2002 und 2006}
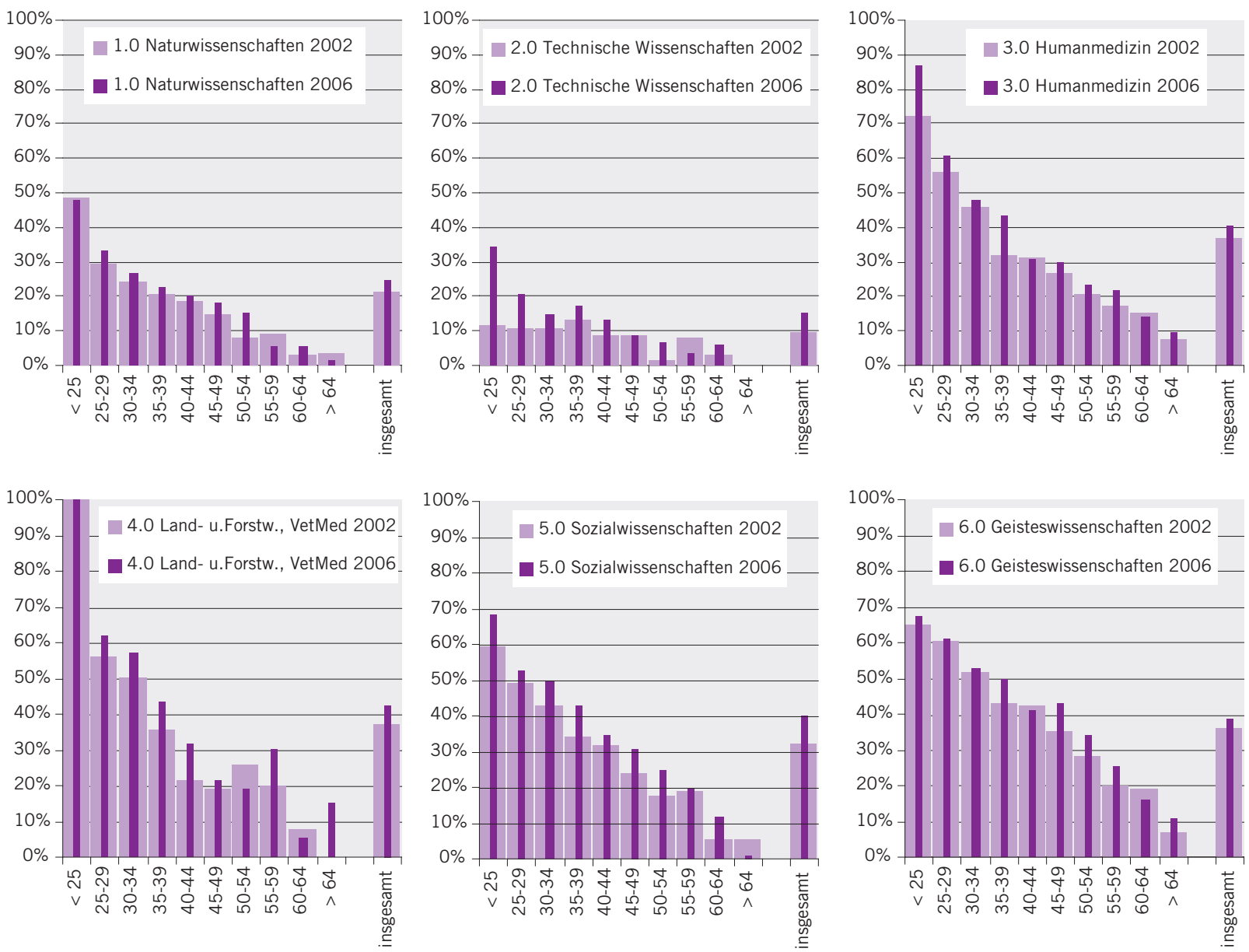

Quelle: Statistik Austria (F\&E-Erhebung), Berechnungen Joanneum Research

In den Disziplinen Humanmedizin, Land- und Forstwirtschaft, sowie den Sozial- und Geisteswissenschaften liegt der Frauenanteil bei den jüngeren Altersgruppen durchaus hoch: Bei den Unter-35-Jährigen ist er jeweils knapp unter oder sogar über $50 \%$; bei den Unter-30Jährigen liegt er - mit Ausnahme der Sozialwissenschaften - sogar über $60 \%$. Die relativ höchste Steigerung des Frauenanteils /wenn auch auf recht geringem Niveau) ist bei den technischen Wissenschaften $\mathrm{zu}$ beobachten: Innerhalb von 4 Jahren konnte der Frauenan- teil um mehr als $58 \%$ von unter $10 \%$ auf über $15 \%$ gesteigert werden. Wenn auch mit starker Zunahme seit 2002 ist der Frauenanteil bei den 25-29-Jährigen mit gut $20 \%$ immer noch relativ gering (und der mit Abstand geringste aller Wissenschaftsdisziplinen). Die Naturwissenschaften liegen in Entwicklung und Struktur zwischen den technischen und den anderen Wissenschaftszweigen.

Die Geschlechterstruktur des wissenschaftlichen Personals im Unternehmensbereich zeigt die folgende Tabelle 11: 
Tabelle 11: Qualifikations- und Geschlechterstruktur des wissenschaftlichen Personals im Unternehmenssektor, Vollzeitäquivalente, 2006

\begin{tabular}{|c|c|c|c|c|c|c|c|c|c|c|c|c|c|c|c|c|}
\hline & & & & & & & & & An & eile & & & & & & \\
\hline Sektor & 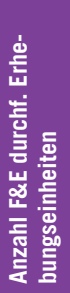 & 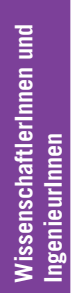 & & 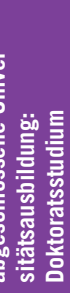 & & 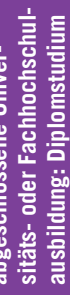 & : & 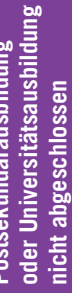 & & 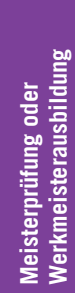 & & 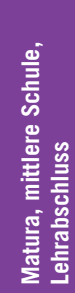 & & 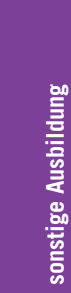 & & 㫷 \\
\hline & & [VZÄ] & $\begin{array}{r}\text { Anteil } \\
\text { an } \\
\text { VZÄ }\end{array}$ & $\begin{array}{l}\text { davon } \\
\text { Anteil } \\
\text { Frauen }\end{array}$ & $\begin{array}{r}\text { Anteil } \\
\text { an } \\
V Z \ddot{A}\end{array}$ & $\begin{array}{l}\text { davon } \\
\text { Anteil } \\
\text { Frauen }\end{array}$ & $\begin{array}{r}\text { Anteil } \\
\text { an } \\
V Z A ̈\end{array}$ & $\begin{array}{l}\text { davon } \\
\text { Anteil } \\
\text { Frauen }\end{array}$ & $\begin{array}{r}\text { Anteil } \\
\text { an } \\
V Z \ddot{A}\end{array}$ & $\begin{array}{l}\text { davon } \\
\text { Anteil } \\
\text { Frauen }\end{array}$ & $\begin{array}{r}\text { Anteil } \\
\text { an } \\
\text { VZÄ }\end{array}$ & $\begin{array}{l}\text { davon } \\
\text { Anteil } \\
\text { Frauen }\end{array}$ & $\begin{array}{r}\text { Anteil } \\
\text { an } \\
\text { VZÄ }\end{array}$ & $\begin{array}{l}\text { davon } \\
\text { Anteil } \\
\text { Frauen }\end{array}$ & $\begin{array}{r}\text { Anteil } \\
\text { an } \\
\text { VZÄ }\end{array}$ & $\begin{array}{l}\text { davon } \\
\text { Anteil } \\
\text { Frauen }\end{array}$ \\
\hline Land- und Forstwirtschaft, Fischerei & 3 & 5 & $12 \%$ & $83 \%$ & $88 \%$ & $23 \%$ & - & - & - & - & - & - & - & - & $100 \%$ & $31 \%$ \\
\hline Sachgütererzeugung & 1324 & 12480 & $14 \%$ & $15 \%$ & $39 \%$ & $8 \%$ & $3 \%$ & $17 \%$ & $3 \%$ & $5 \%$ & $36 \%$ & $8 \%$ & $4 \%$ & $23 \%$ & $100 \%$ & $10 \%$ \\
\hline High-Tech & 275 & 6050 & $18 \%$ & $19 \%$ & $40 \%$ & $10 \%$ & $3 \%$ & $23 \%$ & $1 \%$ & $21 \%$ & $34 \%$ & $13 \%$ & $3 \%$ & $27 \%$ & $100 \%$ & $14 \%$ \\
\hline Medium Tech & 767 & 5739 & $9 \%$ & $6 \%$ & $39 \%$ & $5 \%$ & $3 \%$ & $8 \%$ & $4 \%$ & $1 \%$ & $39 \%$ & $4 \%$ & $5 \%$ & $21 \%$ & $100 \%$ & $5 \%$ \\
\hline Low Tech & 282 & 691 & $11 \%$ & $19 \%$ & $34 \%$ & $22 \%$ & $8 \%$ & $29 \%$ & $10 \%$ & $5 \%$ & $31 \%$ & $14 \%$ & $5 \%$ & $22 \%$ & $100 \%$ & $18 \%$ \\
\hline Energie- und Wasserversorgung & 25 & 38 & $20 \%$ & $7 \%$ & $50 \%$ & $12 \%$ & $12 \%$ & $21 \%$ & $8 \%$ & - & $9 \%$ & $9 \%$ & - & - & $100 \%$ & $11 \%$ \\
\hline Bauwesen & 82 & 87 & $12 \%$ & $1 \%$ & $43 \%$ & $1 \%$ & $8 \%$ & - & $4 \%$ & - & $33 \%$ & $3 \%$ & $1 \%$ & $20 \%$ & $100 \%$ & $2 \%$ \\
\hline Dienstleistungen & 963 & 5853 & $25 \%$ & $20 \%$ & $49 \%$ & $15 \%$ & $5 \%$ & $14 \%$ & $1 \%$ & $5 \%$ & $16 \%$ & $14 \%$ & $4 \%$ & $46 \%$ & $100 \%$ & $17 \%$ \\
\hline Hi-Tech Knowledge Intensive & 459 & 3256 & $30 \%$ & $22 \%$ & $51 \%$ & $17 \%$ & $5 \%$ & $17 \%$ & $0 \%$ & $16 \%$ & $14 \%$ & $14 \%$ & $1 \%$ & $11 \%$ & $100 \%$ & $18 \%$ \\
\hline Other & 504 & 2598 & $18 \%$ & $14 \%$ & $47 \%$ & $12 \%$ & $5 \%$ & $10 \%$ & $2 \%$ & $2 \%$ & $20 \%$ & $13 \%$ & $8 \%$ & $51 \%$ & $100 \%$ & $16 \%$ \\
\hline Gesamt & 2407 & 18470 & $17 \%$ & $17 \%$ & $42 \%$ & $11 \%$ & $4 \%$ & $15 \%$ & $2 \%$ & $5 \%$ & $30 \%$ & $9 \%$ & $4 \%$ & $30 \%$ & $100 \%$ & $12 \%$ \\
\hline
\end{tabular}

Quelle: Statistik Austria (F\&E-Erhebung), Berechnungen Joanneum Research

Die höchste formale Qualifikationsstruktur weist das wissenschaftlicher Personal im wissensintensiven Dienstleistungssektor auf: Mehr als $80 \%$ verfügen hier über einen Abschluss eines Doktorats- (30\%) oder Diplomstudiums $(51 \%)$; relativ gering ist hier mit $20 \%$ der Anteil an sekundär oder geringer Ausgebildeten. Deren Anteil ist mit $43 \%$ naturgemäß in der Sachgütererzeugung relativ hoch (für Werkstätten- und Labortätigkeiten). Es scheint eine gewisse Korrelation zwischen Technologie- und Qualifikationsniveau beobachtbar.

Der Frauenanteil ist mit $12 \%$ (auch im internationalen Vergleich, s. oben) recht gering. Am relativ höchsten ist er bei den höchsten und niedrigsten Qualifikationsstufen: Mit $17 \%$ ist der Frauenanteil bei den Abschlüssen auf Doktoratsniveau leicht, beim Personal mit "sonstiger Ausbildung" deutlich (30 \%) überdurchschnittlich. Sehr gering ist der Frauenanteil bei den handwerklichen Qualifikationen (5\% beim Personal mit Meisterprüfung).

\subsubsection{Resümee}

Ein intertemporaler Vergleich der Forschungsausgaben zeigt, dass zwischen 2002 und 2006 die F\&E-Ausgaben insgesamt um $35 \%$ und die gesamten F\&E durchführenden Einheiten um $17 \%$ gestiegen sind. Dabei zeichnet sich vor allem der Unternehmenssektor bei beiden Indikatoren durch die höchsten Steigerungsraten aus $1+42 \%$ bei den F\&E-Ausgaben und $+24 \%$ bei der Anzahl forschender Unternehmen). 
Diese Steigerungsraten - vor allem was die Erhöhung der Anzahl forschender Unternehmen betrifft - zeigen, dass sich die Forschungsbasis der österreichischen Wirtschaft in den vergangenen Jahren erheblich ausgeweitet hat.

Die Finanzierungsstrukturen der gesamten F\&E-Ausgaben sind für den untersuchten Zeitraum relativ stabil. Auf dem Unternehmenssektor (inländische Unternehmen plus F\&E-Finanzierung durch ausländische Mutterunternehmen) entfallen in etwa $65 \%$ der gesamten Forschungsausgaben in Österreich. Dieser Anteil entspricht dem OECD- sowie dem EU-Durchschnitt.

Mit einem Anteil von $32 \%$ an den F\&EAusgaben des Unternehmenssektors sowie einer F\&E-Quote von $23 \%$ nimmt der HighTech-Sektor in der Sachgüterindustrie die wichtigste Rolle ein. Den höchsten Zuwachs verzeichnen hingegen die forschungs- und technologieintensiven Dienstleistungen, deren F\&E-Anteil an der Bruttowertschöpfung von 11 auf knapp unter $17 \%$ gestiegen ist. $14 \%$ aller F\&E-Ausgaben sind 2006 in diesem Sektor getätigt worden.

Die Beschäftigten im F\&E-Bereich haben zwischen 2002 und $2006 \mathrm{um}+28 \%$ auf 84 Tsd. (Kopfzahlen) zugenommen, was 49 Tsd. VZÄ entspricht. Der Frauenanteil bei den F\&E-Beschäftigten ist zwar zwischen 2002 und 2006 geringfügig gestiegen, ist aber mit 29 \% (Kopfzahlen) immer noch relativ gering. Allerdings zeigt die geschlechtsspezifische Analyse des wissenschaftlichen Personals an den Universitäten, dass sich der Frauenanteil bei den jüngeren Altersgruppen im Karriereverlauf fortsetzt; Frauen scheinen also nicht in höherem Maße als Männer aus den Universitätskarrieren „herauszufallen".

\subsection{Förderung von F\&E - FFG, aws, FWF}

In Österreich hat sich seit den 1990er Jahren - und verstärkt durch den EU-Beitritt - eine wachsende politische Bedeutung von Forschung, Technologie und Innovation (FTI) beobachten lassen. Neben deutlich erhöhten Mittelflüssen haben sich die Veränderungen auch auf der Ebene der Institutionen niedergeschlagen. Die schon in der Evaluierung des FFF und des FWF (Arnold 2004) enthaltenen Empfehlungen liefen in die Richtung einer stärkeren Konzentration vor allem der unternehmensbezogenen Forschungsförderung und damit stärker aufeinander abgestimmter Aktivitäten. Damit sollte die stark fragmentierte Bundesförderung reduziert und effektiver gestaltet werden, um damit Synergieeffekte lukrieren zu können. Mit der Gründung der aws (Austria Wirtschaftsservice) im Jahr 2002 und vor allem der FFG (Forschungsförderungsgesellschaft) 2004 wurden die institutionellen Voraussetzungen für ein effektiveres und effizienteres Fördersystem geschaffen. In diesem Kapitel werden daher neben den bereits erwähnten Agenturen auch der Wissenschaftsfonds (FWF) als die dritte große Fördereinrichtung des Bundes in ihren Förderaktivitäten kurz charakterisiert und mit Kennzahlen beschrieben, sodass deren Rolle und Bedeutung im österreichischen Innovationssystem nachvollziehbar wird. ${ }^{21}$ Die Ausführungen können mitnichten die Vielfalt und das Ausmaß der jeweiligen Förderagenturen umfassend beschreiben und analysieren. Dazu dienen die jeweiligen Jahresberichte. Aber das Hervorheben bestimmter Themen soll auf förderpolitisch relevante Aspekte verweisen. So enthält das folgende Kapitel u.a. eine Branchenklassifikation der durch die FFG geförderten Projekte und die Ausführungen zur aws

21 Siehe dazu auch den Forschungs- und Technologiebericht 2006, S. $23 \mathrm{ff}$. 
beinhalten einen Exkurs zur Förderung junger High-Tech- Unternehmen. Beim FWF wird auf die Bewilligungsraten näher eingegangen.

\subsubsection{Die Forschungsförderungsgesellschaft (FFG)}

Mit der Gründung der $\mathrm{FFG}^{22}$ wurden $2004^{23}$ die Förder- und Beratungseinrichtungen im
Bereich nationaler und internationaler anwendungsorientierter Forschung und Technologieförderung zusammengefasst. In fünf operativen Bereichen werden Basisförderungen im eigenen Wirkungsbereich sowie Aufgaben der beauftragten Programmabwicklung durchgeführt.

Tabelle 12: Gesamtübersicht der Förderzusagen der FFG (2008)

\begin{tabular}{|c|c|c|c|c|c|c|}
\hline Bereich & Projekte & Akteure $^{1}$ & Beteiligungen & $\begin{array}{r}\text { Gesamtkosten } \\
(\text { Mio. €) }\end{array}$ & $\begin{array}{l}\text { Gesamtförderung inkl. } \\
\text { Haftungen (Mio. } € \text { ) }\end{array}$ & $\begin{array}{l}\text { Barwert } \\
\text { (Mio. €) }\end{array}$ \\
\hline Basisprogramme (BP) & 1421 & 1498 & 2166 & 541 & 283 & 159 \\
\hline Strukturprogramme (SP) & 339 & 878 & 1219 & 424 & 144 & 144 \\
\hline Thematische Programme (TP) & 533 & 905 & 1413 & 195 & 109 & 109 \\
\hline Europäische und Internationale Programme (EIP) & 216 & 90 & 216 & 1,6 & 1,2 & 1,2 \\
\hline Agentur für Luft und Raumfahrt (ALR) & 36 & 48 & 74 & 11 & 7 & 7 \\
\hline FFG Gesamt & 2545 & 3419 & 5088 & 1173 & 544 & 420 \\
\hline
\end{tabular}

1 bereinigt ergeben sich 2863 Akteure

Quelle: FFG

Die FFG bewegt mit ihren Förderaktivitäten ein Gesamtprojektvolumen von über 1,1 Mrd. $€$ (=Gesamtkosten). Dieses Gesamtprojektvolumen wird mit 544 Mio. $€$ gefördert. Da neben der klassischen Förderung durch nichtrückzahlbare Zuschüsse in den Basisprogrammen auch andere Förderinstrumente wie Haftungen, Kreditkostenzuschüsse oder Darlehen zum Einsatz kommen, entspricht die Gesamtförderung von 544 Mio. $€$ einem Barwert von 420 Mio. $€$. Knapp über einem Drittel davon entfallen auf die Basisprogramme (BP).

Eine wesentliche Funktion öffentlicher Forschungsförderung besteht auch darin, Unternehmen bei der Aufnahme von Forschungsaktivitäten zu unterstützen. Die Anzahl for- schungsaktiver Unternehmen und solcher, die mit F\&E-Aktivitäten beginnen, ist ein wichtiger Indikator für die Ausweitung der Forschungsbasis und somit für das Forschungspotential einer Volkswirtschaft. Die FFG kann seit ihrem Bestehen auf eine kontinuierliche Ausweitung dieser Basis verweisen, indem jedes Jahr neue Antragsteller gewonnen werden können.

Aus der Gesamtzahl aller an FFG-Programmen beteiligten Unternehmen wiesen im Zeitraum 2003 bis 2007 im Schnitt 38 \% keine Erfahrung mit geförderten Projekten auf und sind somit erstmalig in ein von der FFG gefördertes Forschungsprojekt involviert. Die erstaunlich hohe Zahl an Newcomern im Jahre 2008 ist da- 
bei auf die Einführung des Innovationsschecks zurückzuführen, wodurch sich besonders viele KMU aus dem Dienstleistungssektor angesprochen fühlten. Dieses Förderprogramm vergibt auf einfache Weise kleine Beträge an viele kleine Unternehmen, wodurch sich die schiere Anzahl der Newcomer ein wenig verzerrt (Abbildung 13).

\section{Abbildung 13: Geförderte Unternehmen - Newcomer vs. Erfahrenen}

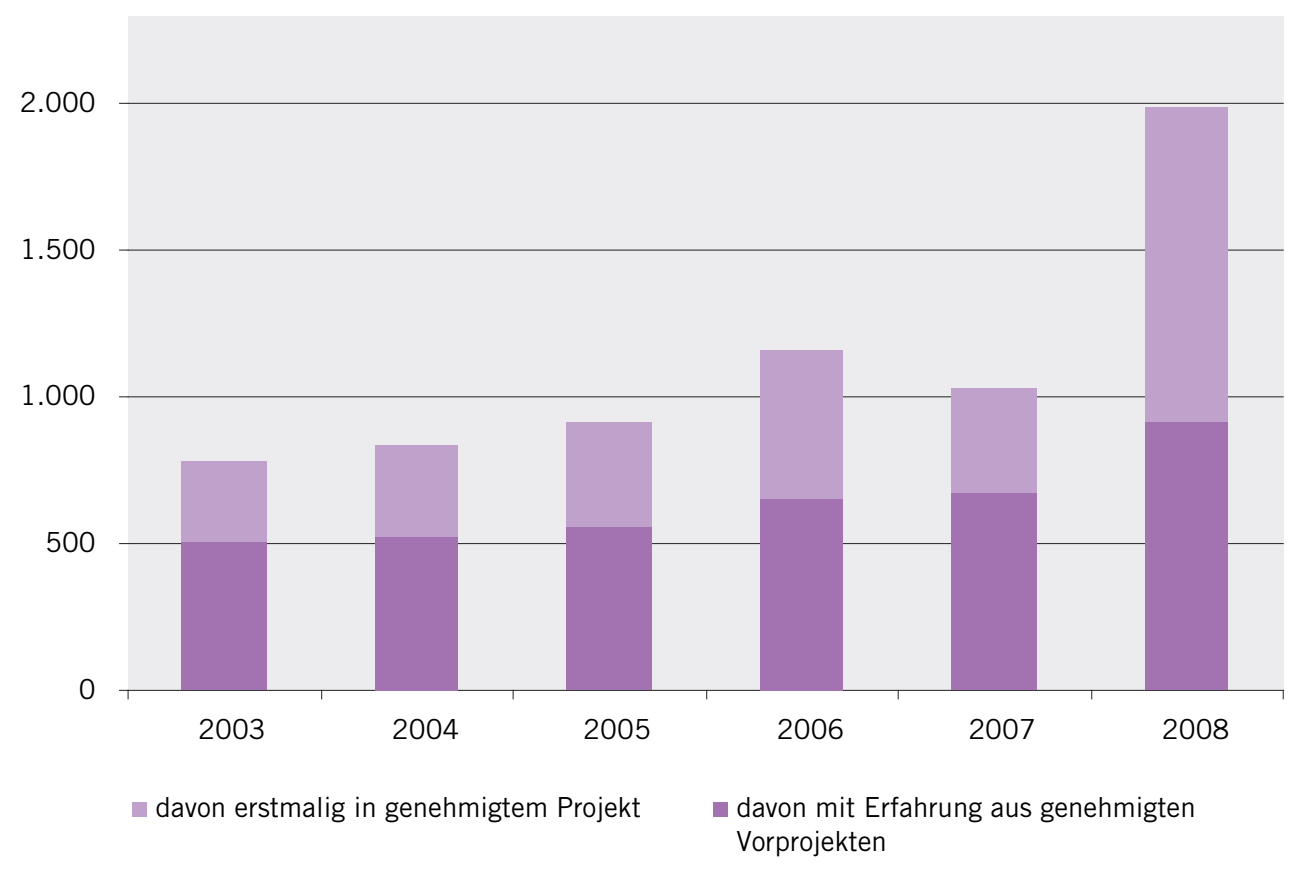

Quelle: FFG

Einer der Förderschwerpunkte der letzten Jahre, i.e. die Förderung der Kooperationen zwischen Wissenschaft und Wirtschaft, hat auch zu einer erhöhten Diversität der Akteure bei der FFG geführt. Durch die starke Präsenz von nicht-unternehmerischen Forschungsorganisationen in den Strukturprogrammen und thematischen Programmen, hat sich der Anteil von Forschungseinrichtungen und Universitäten seit der Gründung der FFG von $18 \%$ auf 39 \% erhöht. Parallel dazu sank der Unternehmensanteil von $79 \%$ auf $57 \%$. Ein Vergleich der Förderbarwerte zeigt jedoch, dass mit $51 \%$ noch knapp die Hälfte auf den Unternehmenssektor entfällt (siehe Abbildung 14).

Rechnet man innerhalb des Unternehmenssektors den Anteil der KMU heraus, so entfallen von den insgesamt 1783 Beteiligungen des Unternehmenssektors 70 \% (1 250 Beteiligungen) auf KMU. Das entspricht einem Anteil von $46 \%$ an der Gesamtbeteiligung. Auf der Ebene der Barwerte sinkt der KMU-Anteil innerhalb des Unternehmenssektors auf $53 \%$, was einem Anteil am Barwert der Gesamtförderung von $37 \%$ entspricht. 
Abbildung 14: Anteile der Organisationstypen an Beteiligungen und Barwerten in \% (2008)

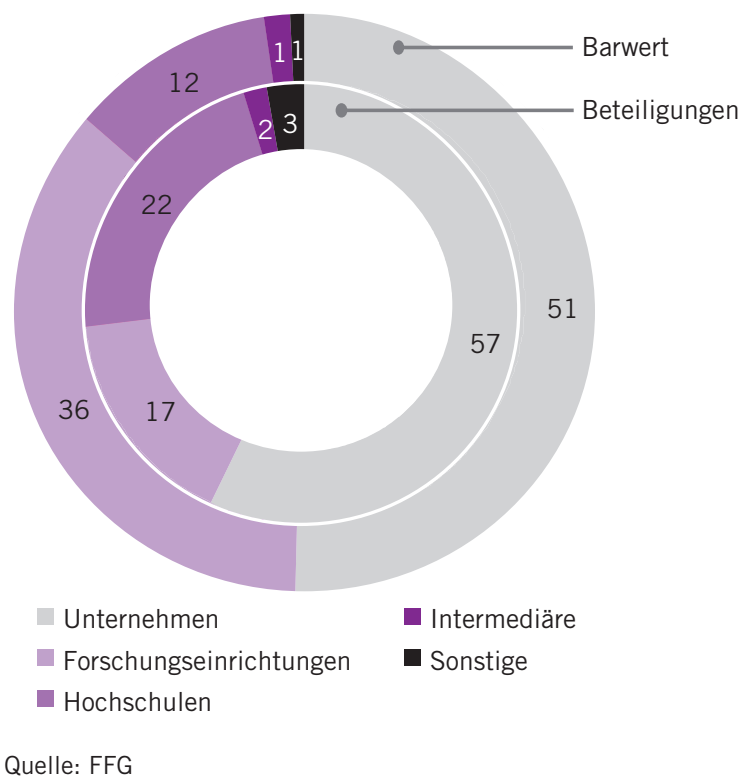

Neben der Ausweitung der Forschungsbasis hat öffentliche, wirtschaftsorientierte Forschungsförderung auch zum Strukturwandel beizutragen. Hier wurde oftmals die Kritik laut, die österreichische Technologie- und Forschungsförderung forciere zu wenig den Strukturwandel in Richtung Hightech-Industrien und wissensbasierte Dienstleistungen. Diese Kritik spiegelt zunächst ein sehr enges und mechanistisches Verständnis von Strukturwandel wider und verkennt auch das Ausmaß, welches die Forschungsförderung volkswirtschaftlich spielt: 2008 betrugen die Bruttoinlandsausgaben für $F \& E$ 7,5 Mrd. $€$. Die FFG finanziert mit einem Fördervolumen von 420 Mio. $€$ einen Anteil von 5,6 \%. Dies ist relativ gering und erklärt, warum der gesamtwirtschaftliche Strukturwandel - der ein eher langsamer und gradueller Prozess ist - wenig durch die öffentliche F\&E-Förderung beeinflusst werden kann. Die Branchenstruktur kann kein geeigneter Indikator für die Messung des Erfolgs einer Förderagentur sein. Dennoch scheint die Frage virulent, auf welche Branchen sich das Fördervolumen verteilt. Die Daten der FFG ordnen dabei nicht das Unternehmen, sondern die geförderten Projekte gemäß ihrer technologischen Ausrichtung der OECD-Branchenklassifikation (NACE) zu.

Die folgende Tabelle 13 ordnet sämtliche im Jahre 2008 geförderten FFG-Projekte (insgesamt 2545) den Branchen des Sachgüterbereichs bzw. den entsprechenden Dienstleistungsbranchen zu. Dabei wird für den Sachgüterbereich eine 4-stufige Skala und für den Dienstleistungssektor eine 6-stufige Skala der Technologieintensität zugrunde gelegt. Die Zuordnung erfolgt nach der gängigen OECDKlassifikation. 


\section{Tabelle 13: Geförderte Projekte nach Branchenklassifikation - FFG gesamt (2008)}

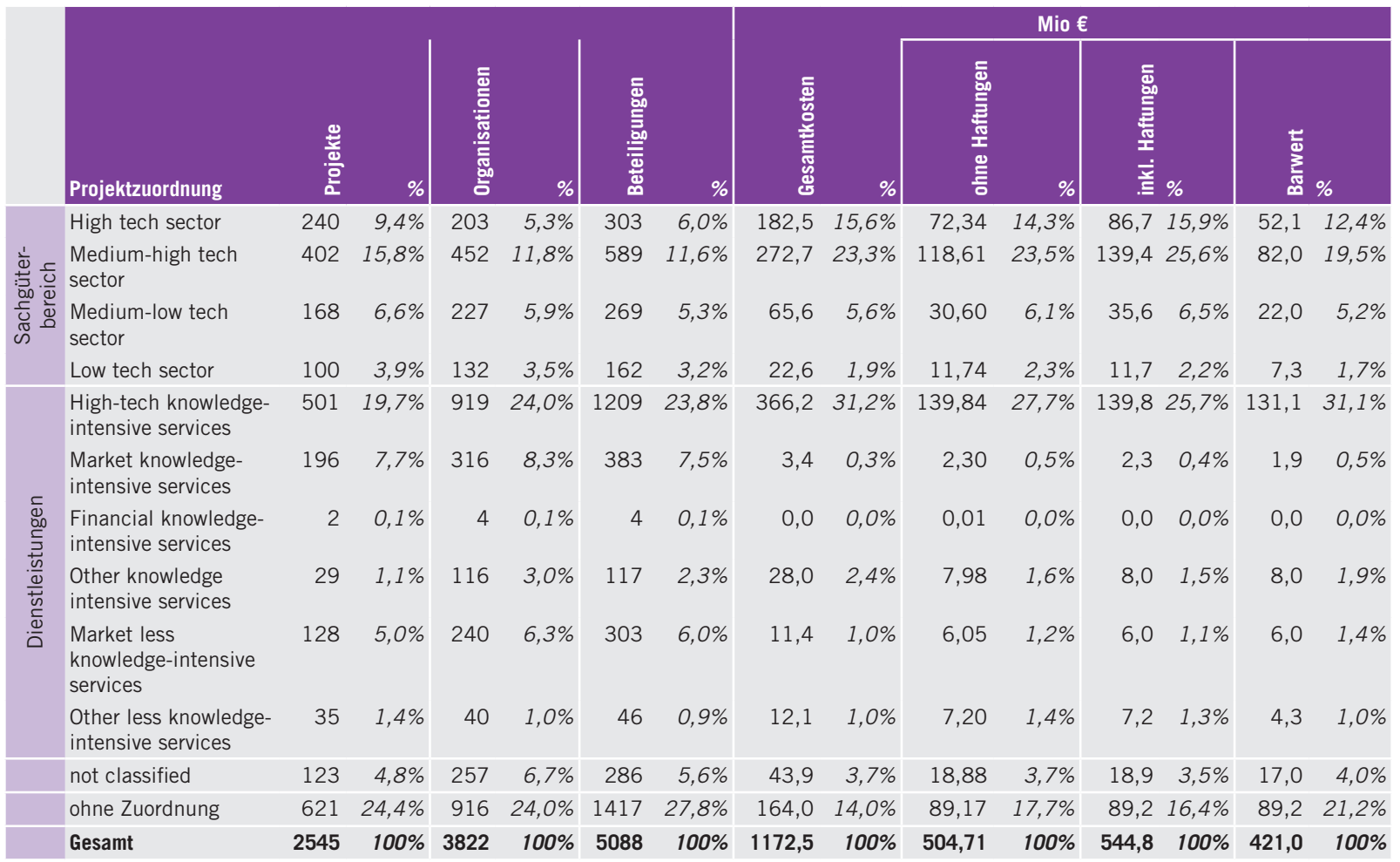

Quelle: FFG; Berechnungen Joanneum Research

Fasst man die jeweiligen obersten Segmente zusammen, so machen der "High-tech sector" sowie der Sektor der „High-tech knowledge intensive services" einen kombinierten Anteil von knapp unter $30 \%$ der geförderten Projekte aus. Der Anteil dieser beiden Sektoren beträgt jedoch $44 \%$ auf der Ebene der Fördervolumina. Der Medium-Bereich konzentriert sich im Wesentlichen auf den Sachgüterbereich mit einem Anteil von knapp über $22 \%$ und den wissensintensiven Dienstleistungen mit einem
Anteil von ca. 9 \% der geförderten Projekte. Fasst man den High-Tech und den MediumHigh-Tech Sektor sowie die technologie- und forschungsintensiven Dienstleistungen zusammen, so entfallen $54 \%$ der geförderten Projekte und $66 \%$ des Fördervolumens auf diese Branchen. ${ }^{24}$

Interessant in diesem Zusammenhang sind auch einige branchenspezifische Projektkennzahlen (Tabelle 14). 
Tabelle 14: Projektkennzahlen nach Branchenklassifikation

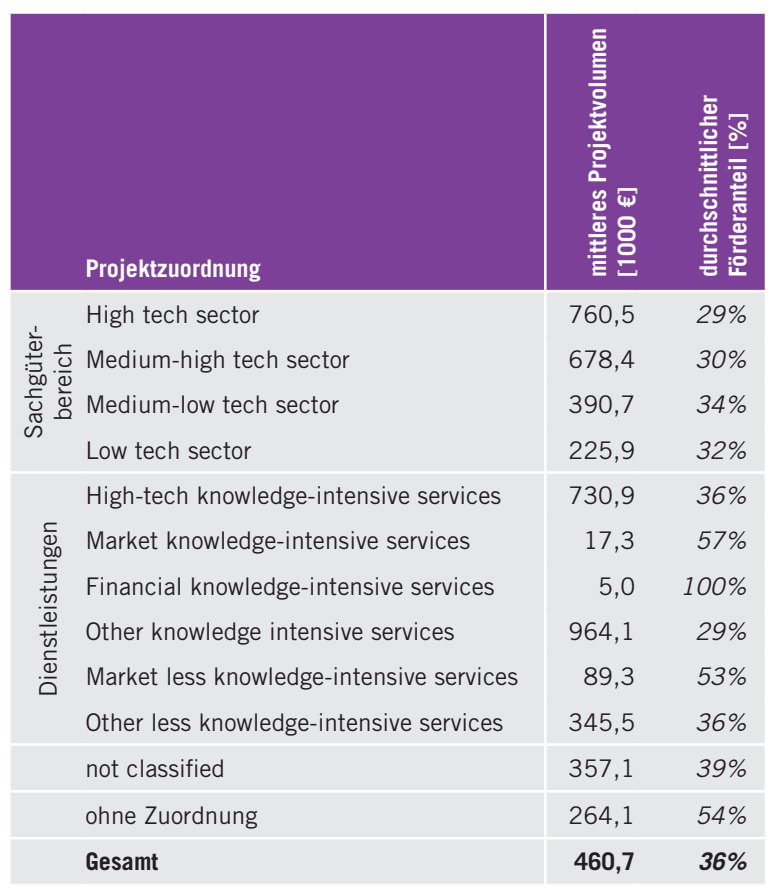

Quelle: FFG; Berechnungen Joanneum Research

Dabei wird das mittlere Projektvolumen als Gesamtkosten/Anzahl der Projekte und die durchschnittliche Förderrate als das Verhältnis des Förderbarwerts zu den Gesamtkosten (Barwert/ Gesamtkosten) berechnet. Über alle geförderten Projekte gerechnet beträgt das durchschnittliche Projektvolumen 460,7 Tsd. $€$ mit einer durchschnittlichen Förderrate von $36 \%$.

Die Projektzuordnung der FFG-Projekte auf der Basis der NACE-Klassifikation scheint auf den ersten Blick eine nachvollziehbare Zuordnung, da (i) der Schwerpunkt der FFG in der Projektförderung liegt, (ii) die Basisprogramme, welche keine branchenspezifischen Aus- schlusskriterien kennen, den größten Anteil an den Fördeaktivitäten haben, und (iii) die Branchenzugehörigkeit eines Unternehmens nicht deckungsgleich mit jener des Forschungsprojektes sein muss.

Dennoch muss diese Klassifikation mit Vorsicht interpretiert werden:

- Wie schon in der FFF-Evaluierung empfohlen, sollte neben der Projektklassifikation zusätzlich auch die Branchenklassifikation der geförderten Unternehmen erfolgen. Diese Ergänzung ist im Begriffe umgesetzt $z u$ werden.

- Es ist auch fraglich, ob eine NACE-Branchenklassifikation einen hohen Aussagewert über den Technologiegehalt von Forschungsprojekten besitzt, d.h. ob diese eine adäquate Klassifizierung darstellt.

- Denn auch innerhalb einer Klasse gibt es große Unterschiede im Technologiegehalt von Projekten. Branchen sind in sich mitnichten homogene Klassifizierungen.

\subsubsection{Die Austria Wirtschaftsservice (aws)}

Die aws ${ }^{25}$ wurde im Jahre 2002 als die zentrale Förderbank des Bundes zur Abwicklung der unternehmensbezogenen Wirtschaftsförderung gegründet. ${ }^{26}$ Resultierend aus der $\mathrm{Zu}$ sammenführung von Fördereinrichtungen, die in den Bereichen der allgemeinen Wirtschaftsförderung, der KMU-Förderung, der Innovationsförderung und der Regionalförderung tätig waren, ist das Förderspektrum der aws sehr breit und besteht aus einer Vielzahl von Programmlinien. Tabelle 15 gibt einen Leistungsüberblick auf der Basis der eingesetzten Förderinstrumente. 
Tabelle 15: Leistungsüberblick nach Förderinstrumenten der aws (2008)

\begin{tabular}{|lrrrr|} 
& Projekte & $\begin{array}{r}\text { Förderungsleistung } \\
\text { in Mio. } €\end{array}$ & $\begin{array}{r}\text { Gesamtprojektvolumen }{ }^{1} \\
\text { in Mio. } €\end{array}$ & $\begin{array}{r}\text { Förderungsbarwert }_{\text {in Mio. } €} \\
\hline \text { Zuschüsse }\end{array}$ \\
Haftungen & 3.009 & 167 & 1.695 & 167 \\
\hline Kredite, Darlehen & 681 & 370 & 8.051 & 10 \\
\hline Beratung/Service & 257 & 451 & 1.138 & 56 \\
\hline Summe & 1.235 & - & - & - \\
\hline
\end{tabular}

1 ohne Bereinigung von kombinierten Fördermaßnahmen

Quelle: aws

Insgesamt wurden von der aws im Jahre 2008 Mittel im Wert von 988 Mio. € genehmigt. Da dieser Wert auch Darlehen, Garantien und Haftungen enthält, ist der tatsächlich „wirksame" Betrag (Barwert) mit 233 Mio. € deutlich geringer. Die aws konnte 2008 insgesamt 5.182 Projekte fördern, dem stand ein Gesamtprojektvolumen von $10,8 \mathrm{Mrd}$. $€$ gegenüber.

Das Förderangebot der aws ist sehr umfassend und weist gleichzeitig, bedingt durch die hohe Anzahl an Förderlinien, einen hohen Grad an Differenziertheit auf. Das Förderspektrum reicht von der finanziellen Unterstützung von JungunternehmerInnen, über Tourismusförderung bis hin zur Schaffung von strukturellen Voraussetzungen für Verwertungs- und Vermittlungsprozesse. Dies ist sowohl das Ergebnis der wirtschaftspolitischen Zielsetzungen als auch der beihilfenrechtlichen Rahmenbedingungen.

Die Schwerpunkte des Technologieförderungsangebotes, die durch die aws betreut werden, liegen hierbei in der kommerziellen Umsetzung und wirtschaftlichen Nutzung von Forschungsergebnissen und werden in zwei Kernaufgaben erfüllt: Einerseits durch das
Schützen, Lizenzieren und Verwerten von Patenten und andererseits durch die Recherche, Beratung und finanzielle Unterstützung von/ bei Vorgründungs-, Gründungs- und Wachstumsvorhaben. Zusätzlich werden für einzelne Zukunftsfelder, beispielsweise Life Sciences und Creative Industries Schwerpunktprogramme mit branchenspezifischen Vernetzungs-, Beratungs- und Finanzierungsleistungen angeboten.

Dies gelingt durch die synergetische Nutzung monetärer Instrumente (in Form von Zuschüssen, zinsgünstigen ERP-Darlehen und Garantien), intensiven Management- und Beratungsaktivitäten sowie einem fein abgestimmten Instrumentarium von awarenessbildenden Maßnahmen. Dadurch kann der forschungs-, entwicklungs- und innovationsorientierte Sektor der österreichischen Wirtschaft in allen unternehmerischen Phasen - Gründung und Wachstum - nachhaltig und effizient unterstützt werden.

Der Anteil der F\&E-relevanten Instrumente beträgt knapp $10 \%$ an der gesamten Förderleistung und bewirkt ein Gesamtprojektvolumen von 284 Mio. $€$ (siehe Tabelle 16). 
Tabelle 16: Leistungsüberblick Forschung und Entwicklung (2008)

\begin{tabular}{|c|c|c|c|}
\hline & Projekte & $\begin{array}{r}\text { Förderungsleistung } \\
\text { in TEUR }\end{array}$ & $\begin{array}{r}\text { Gesamtprojektvolumen } \\
\text { in TEUR }\end{array}$ \\
\hline Zuschüsse & 88 & 16.711 & 128.415 \\
\hline Haftungen & 19 & 14.221 & 44.540 \\
\hline Kredite, Darlehen & 23 & 57.561 & 111.273 \\
\hline Beratung/Service & 1.209 & 5.421 & - \\
\hline Summe & 1.339 & 93.914 & 284.228 \\
\hline
\end{tabular}

Schwerpunkte im F\&E-relevanten Bereich sind neben dem Recherche- und Patentmanagement die Beratung, Betreuung und Vermittlung im Hochtechnologiesektor. Programme wie Life Science Austria, die Beteiligungsvermittlung Business Angels oder das Seedfinancing Programm für Hightech Gründungen ermöglichen die nachhaltige Unterstützung von Unternehmensgründungen im HightechSektor. In diesem Sektor sind Unternehmen in besonderem Maß von Momenten des Marktversagens betroffen und vor dem Hintergrund steigender Risikoaversion seitens des privaten Sektors sollte die öffentliche Hand in diesem Bereich verstärkt Akzente setzen.

\subsubsection{Exkurs: Die Förderung junger High-Tech-Unternehmen}

Technologieorientierte kleine und mittlere Unternehmen haben für die technologischen Entwicklungen und damit für die Innovationsfähigkeit einer Volkswirtschaft große Bedeutung (siehe dazu Jörg und Schibany 2006). Junge, technologieorientierte Unternehmen können

- Marktnischen abdecken, die von großen Unternehmen nicht wahrgenommen werden;

- flexibel auf Marktveränderungen reagieren;

- rasch neue technische Möglichkeiten aufgreifen und damit die Innovationskraft einer Volkswirtschaft stärken;
- in Strukturkrisen aufgrund ihrer Anpassungsfähigkeit stabilisierende Wirkung haben.

Nun ist für junge Unternehmen, die sich erst am Markt etablieren müssen, die Möglichkeit der Selbstfinanzierung aus dem eigenen Cashflow naturgemäß sehr beschränkt. Um neue Ideen verwirklichen und daraus marktfähige Produkte oder Dienstleistungen anbieten zu können, sind sie auf die Bereitschaft zur Finanzierung von Außenstehenden angewiesen. Die Beziehung zwischen Unternehmensgründern und externen Kapitalgebern ist dabei oft durch erhebliche Informationsdefizite belastet. Zum einen können neu gegründete Unternehmen nicht auf eine belegbare Entwicklung ihrer Projekte in der Vergangenheit hinweisen und die Information über die zu erwartende Entwicklung und die Erfolgsaussichten neuer Unternehmen lassen sich von außen nur schwer beurteilen. Zum anderen sind für kleine und mittelständische Unternehmen die Transaktionskosten einer eingehenden Bewertung und Überprüfung durch die Kapitalgeber vergleichsweise hoch.

Diese Finanzierungsprobleme sind bei technologieorientierten Unternehmen aus folgenden Gründen sogar noch stärker ausgeprägt (siehe dazu Carpenter und Petersen 2003):

- Das Chancen-Risikoprofil ist von besonders großer Unsicherheit geprägt und daher v. a. 
für Kreditgeber, die an dem höheren Ertragspotenzial nicht beteiligt sind, wenig attraktiv.

- Das Ertragspotenzial für neue, technologisch anspruchsvolle Produkte und Verfahren ist meist nur durch erfahrene Fachleute hinreichend gut abzuschätzen. Der Informationsvorsprung der UnternehmerInnen gegenüber dem Kapitalgeber und damit die Qualitätsunsicherheit in Bezug auf die Projekte sind deshalb besonders groß.

- Gleichzeitig steigt auch die Unsicherheit in Bezug auf das Risikoverhalten der UnternehmerInnen, die als EmpfängerInnen der Residualerträge einen Anreiz haben können, nach erhaltener Finanzierung ein größeres Risiko einzugehen.

- Schließlich ist zu berücksichtigen, dass stärker als in anderen Branchen der Firmenwert eines besonders innovationsorientierten Unternehmens von intangiblen Vermögenswerten abhängt, die sich kaum zur Besicherung von Krediten eignen.

Während große und etablierte TechnologieUnternehmen diesen Problemen durch Innenfinanzierung aus dem Cashflow bzw. die Aufnahme von Beteiligungskapital begegnen können, sind junge, innovationsorientierte Unternehmen am meisten von der Finanzierungslücke betroffen. Die fehlende Eigenkapitalbasis, die Informationsasymmetrie zwischen Kreditgeber und -nehmer über den tatsächlichen Risikograd resultiert in einer Kreditrationierung für junge High-Tech-Unternehmen

Um jedoch die vermuteten Vorteile einer sich stetig erneuernden Unternehmenspopulation und eines hohen Bestandes an flexiblen und hoch kompetenten kleinen Unternehmen für die technologische Leistungsfähigkeit der eigenen Volkswirtschaft, und damit für deren internationale Wettbewerbsfähigkeit, nutzen zu können - oder zumindest keine diesbezüglichen Nachteile hinnehmen zu müssen - kam in den letzten Jahren eine Vielzahl von Unter- stützungsmaßnahmen zum Einsatz. Aus den genannten Gründen unterstützen staatliche Förderprogramme junge High-Tech-Unternehmen in der Frühphase und schließen damit eine bestehende Finanzierungslücke. Gleichzeitig gehen die Fördermaßnahmen über die reine Finanzierung hinaus, indem das Management der Unternehmen mit spezifisch auf das Technologiefeld und das Unternehmen zugeschnittenen strategischen Betreuungsleistungen unterstützt wird. Dadurch sollen letztlich wichtige Signale an den privaten Venture Capital und Private Equity Markt gesendet und die weitere Finanzierung durch den Kapitalmarkt erleichtert werden.

Mit diesen Zielsetzungen sind unterschiedliche Maßnahmen implementiert:

- Staatliche Beteiligungsprogramme sollen die Eigenkapitaldecke der entsprechenden Unternehmen stabilisieren;

- Refinanzierungsmaßnahmen und Haftungsübernahmen für private Investoren sollen das Ausfallsrisiko für private Kapitalgeber verringern und ihr Finanzierungs-Engagement steigern;

- Maßnahmen zum Zusammenbringen von Investoren und Unternehmen, die Wagniskapital suchen, um die Suchprozesse effizienter und kostengünstiger zu gestalten;

- Förderung von Business Angels, die zu einem verstärktem Engagement von Privatpersonen bei der Finanzierung junger Unternehmen beitragen soll;

- Beratungsangeboten und Awarenessmaßnahmen vor allem im Hinblick auf Spin-offGründungen aus öffentlichen Forschungseinrichtungen, z.B. im Rahmen von thematischen Clustern oder regionalen Netzwerken.

In Österreich hat sich um die frühen Phasen der Unternehmensentwicklung ein generöses und umfassendes Förderangebot entwickelt (Abbildung 15), um damit eine Finanzierungslücke zu schließen. Insbesondere in der Sti- 
mulierung des Gründungsgeschehens aus Forschungseinrichtungen sah die Politik in den 90er Jahren großen Handlungsbedarf. So genannte akademische Spin-offs sind ein wichtiges Element zur kommerziellen Nutzung von wissenschaftlichen Ergebnissen und forcieren den Wissens- und Technologietransfer. Die
Bedeutung der Universitäten und Forschungsreinrichtungen für die Gründung von innovativen Hightech-Unternehmen mit hohem Wachstumspotential zeigt das Unternehmensportfolio des Seedfinancing-Programms: Bei der Mehrheit der Unternehmen handelt es sich um Spinoffs aus dem universitären Bereich.

\section{Abbildung 15: Finanzierungsphasen und Förderangebote in Österreich}

\begin{tabular}{|c|c|c|c|c|}
\hline & \multicolumn{3}{|c|}{ Early Stage } & \multirow{2}{*}{$\begin{array}{l}\text { Expansion } \\
\text { Expansion }\end{array}$} \\
\hline & Pre-Seed & Seed & Start up & \\
\hline \multirow{5}{*}{ 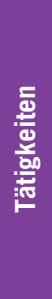 } & Produktkonzept & $\begin{array}{l}\text { Einbringen von Rechten und } \\
\text { Patenten }\end{array}$ & Unternehmensgründung & Erschließung neuer Märkte \\
\hline & Marktanalyse & Gesellschaftsstruktur & $\begin{array}{l}\text { Entwicklung bis zur } \\
\text { Produktionsreife }\end{array}$ & Erweiterung Produktportfolio \\
\hline & Businessplan & Finalisierung Businessplan & Markteintritt & Ausbau Vertrieb \\
\hline & Infrastruktur/Standort & Suche nach Finanzierung & Marketingkonzept & Wachstumsfinanzierung \\
\hline & $F \& E$ & $F \& E$ & F\&E & Produktionsbeginn \\
\hline \multirow{5}{*}{ 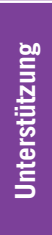 } & AplusB & FFG & Gründerzentren & VC \\
\hline & Pre-Seed & Seed & Bundesländer & aws/ERP \\
\hline & LISA & LISA & FFG & FFG \\
\hline & $\mathrm{BOB}$ & 12 & Double Equity & Bankkredite \\
\hline & Bundesländer & Double Equity & Jungunternehmerförderung & Mezzaninfinanzierung \\
\hline
\end{tabular}

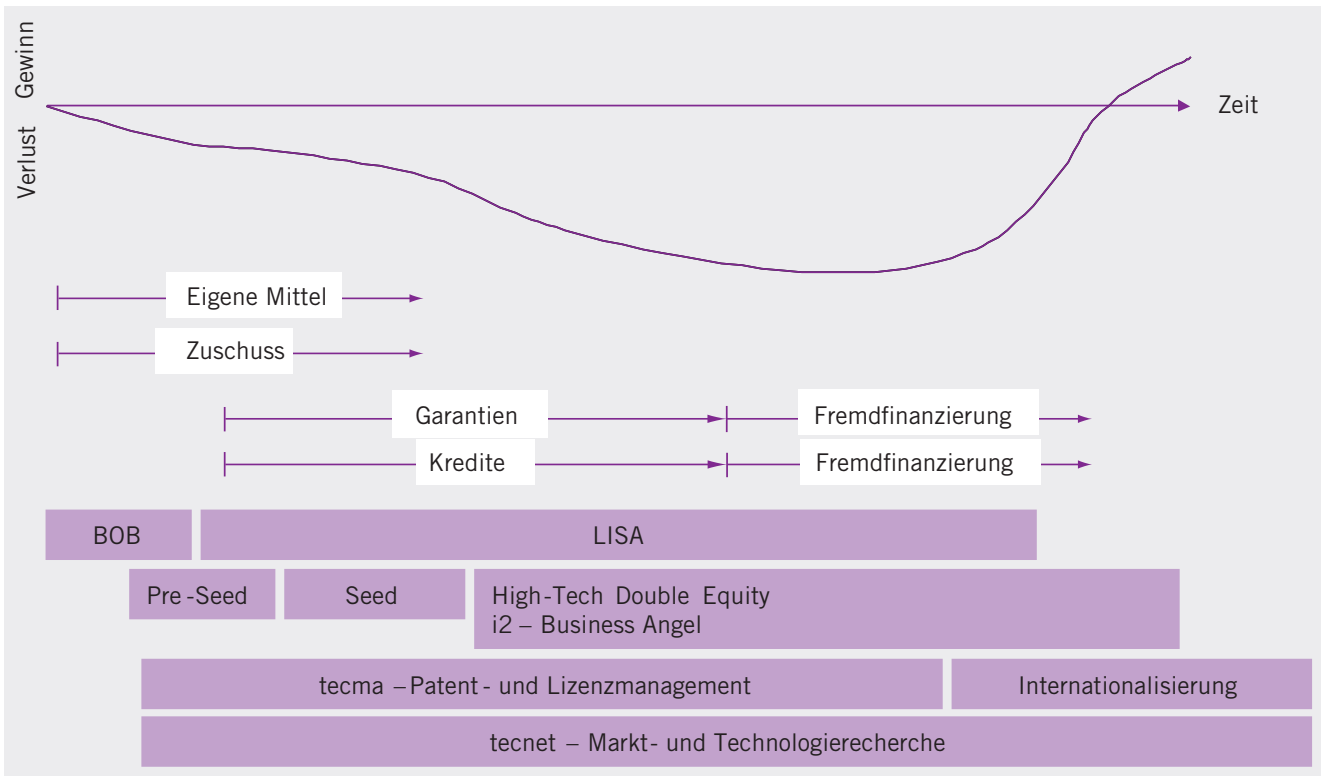

Quelle: Jörg und Schibany (2006) 
Die Unternehmensgründung durch WissenschafterInnen bzw. die Verwertung von Forschungsergebnissen über Unternehmensgründungen sind dabei von ganz spezifischen Hindernissen betroffen. Neben Awareness- und Stimulierungsmaßnahmen, welche das Terrain aufbereiten und das Interesse potentieller UnternehmensgründerInnen wecken, sind vor allem Management-Beratung und Qualifizierung wichtige Unterstützungsmaßnahmen. Hier leisten die AplusB Zentren wertvolle Arbeit. Weiters sehen sich potentielle GründerInnen massiven Finanzierungsproblemen gegenüber und stoßen auf besondere Hemmnisse bei der Fremdfinanzierung. Zum Zeitpunkt der Gründung existiert meist eine Produktidee oder bestenfalls ein Prototyp. Das technische Risiko ist daher sehr hoch. Für die zu entwickelnden Produkte gibt es häufig noch keinen Markt, an dem man sich hinsichtlich der Preissetzung und des Nachfragepotenzials orientieren kann. Zusätzlich gibt es keinen track record des Unternehmens, also keine Erfahrungswerte, anhand derer die externen Geldgeber ein quantitatives Rating vornehmen könnten, bzw. das als Indiz für die Qualität des Managements dienen kann. Der Finanzierungsbedarf beinhaltet typischerweise einen sehr hohen Anteil von „weichen Kosten“ (insbesondere Personalkosten), die nicht besicherbar sind. Gerade für wissensbasierte Gründungen mit hoher Unsicherheit in Bezug auf den technologischen und kommerziellen Erfolg kommt es daher zu einem Marktversagen. Daraus leitet sich die Legitimation für den staatlichen Eingriff ab, der in seiner Ausformung sehr differenziert sein kann, aber angemessen sein muss. Im Wesentlichen umfassen die staatlichen Fördermaßnahmen die Bereitstellung von Kapital und Frühphasenfinanzierungsinstrumenten (Preseed, Seed, Double Equity), von Beratungs-, Qualifikations- und Recherchediensten (LISA, tecnet, tecma, uni:invent) sowie die Vermittlung von Investoren durch Zugang zu Risikokapitalgebern (i2 - Business Angels).
Der Wert eines jungen High-Tech-Unternehmens besteht somit vor allem in seinem Wachstumspotential, einer möglichst patentierbaren Technologie und dem Humankapital. Neben der hohen Innovationskraft sind auch betriebsexterne Voraussetzungen, wie effiziente Vertriebskanäle und Partnerschaften mit anderen Unternehmen, maßgebliche Erfolgskriterien.

\subsubsection{Der Wissenschaftsfonds (FWF)}

Der Wissenschaftsfonds FWF (Fonds zur Förderung der wissenschaftlichen Forschung) ist Österreichs zentrale Einrichtung zur Förderung der Grundlagenforschung. Der FWF ist allen Wissenschaften gleichermaßen verpflichtet und ist - da in Österreich die wissenschaftliche Forschung fast ausschließlich an den Universitäten stattfindet - die wichtigste Förderagentur für die universitäre Forschung. Der Mix aus kompetitiver Forschungsfinanzierung im Rahmen der Einzelprojektförderung sowie der Förderung von Netzwerken (Spezialforschungsbereiche - SFB und Nationale Forschungsnetzwerke - NFN) kann als effektives Verfahren betrachtet werden, um Themen zu priorisieren und flexibel auf neue Themen zu setzen. Die Förderkriterien des FWF liegen einzig in der wissenschaftlichen Qualität der Förderanträge und deren Behandlung erfolgt unabhängig von einem festgelegten Verteilungsschlüssel - sämtliche Anträge an den FWF finden ein „level playing field“ vor.

Den höchsten Anteil in der Forschungsförderung nimmt der autonome Bereich ein, in dem zu 94 \% Einzelprojekte (neben anderen Programmen) für maximal 3 Jahre gefördert werden. Der wesentlich kleiner dimensionierte „beauftragte Bereich" enthält Förderlinien wie das START-Programm oder den Wittgenstein-Preis (Tabelle 17). 
Tabelle 17: Förderung des FWF im Überblick (2008)

\begin{tabular}{|c|c|c|c|c|c|}
\hline Förderlinie & $\begin{array}{l}\text { Förderungen } \\
\qquad(\text { Mio. €) }\end{array}$ & Anträge & $\begin{array}{c}\text { Anträge } \\
\text { entschieden }\end{array}$ & $\begin{array}{l}\text { Anträge } \\
\text { bewilligt }\end{array}$ & $\begin{array}{r}\text { Bewilligungsrate } \\
\text { der eingereichten } \\
\text { Fördersummen }\end{array}$ \\
\hline \multicolumn{6}{|l|}{ autonomer Bereich } \\
\hline Einzelprojekte & 89,9 & 869 & 833 & 357 & $39 \%$ \\
\hline Spezialforschungsbereiche (SFB) ${ }^{1}$ & 11 & 47 & 48 & 23 & $33 \%$ \\
\hline Nationale Forschungsnetzwerke (NFN) ${ }^{2}$ & 12 & 32 & 32 & 12 & $25 \%$ \\
\hline Internationale Programme ${ }^{3}$ & 9,7 & 187 & 160 & 67 & $28 \%$ \\
\hline Doktoratskollegs-plus (DKs) ${ }^{4}$ & 16,1 & 7 & 7 & 3 & $29 \%$ \\
\hline Erwin-Schrödinger-Stipendien & 4,1 & 127 & 125 & 75 & $61 \%$ \\
\hline Lise-Meitner-Programm & 4,2 & 84 & 75 & 26 & $37 \%$ \\
\hline Translational Brainpower ${ }^{5}$ & 1,5 & 12 & 12 & 5 & $38 \%$ \\
\hline Elise-Richter-Programm & 3 & 31 & 35 & 13 & $30 \%$ \\
\hline Translational Research (TRP) & 12,4 & 135 & 139 & 51 & $34 \%$ \\
\hline Publikationsförderung ${ }^{6}$ & 0,5 & 108 & 106 & 71 & $60 \%$ \\
\hline Gesamt & 164,4 & 1639 & 1572 & 703 & $38 \%$ \\
\hline \multicolumn{6}{|l|}{ beauftragter Bereich } \\
\hline START-Programm & 6,1 & 46 & 46 & 8 & $10 \%$ \\
\hline Wittgenstein-Preis & 1,5 & 13 & 13 & 1 & $8 \%$ \\
\hline Hertha-Firnberg-Programm & 2,6 & 50 & 41 & 13 & $32 \%$ \\
\hline Impulsprojekte & 0 & 0 & 0 & 0 & - \\
\hline Provison & 1,54 & 7 & 26 & 10 & $24 \%$ \\
\hline Gesamt & 11,74 & 116 & 126 & 32 & $12 \%$ \\
\hline
\end{tabular}

1 2-stufiges Verfahren; die hier ausgewiesenen Zahlen entsprechend Teilprojekten von Vollanträgen (2. Stufe)

2 2-stufiges Verfahren; die hier ausgewiesenen Zahlen entsprechend Teilprojekten von Vollanträgen (2. Stufe)

3 Anbahnung, internationale Kooperationen etc.

4 Ist eine 2008 implementierte Weiterentwicklung der 2004 etablierten DK (Doktoratskollegs).

5 Ist ein 2007 gestartetes Programm zur Finanzierung von GastwissenschafterInnen im Rahmen von TRP-Projekten.

6 Selbstständige Publikationen, Übersetzungskosten, Zeitschriftenpublikationen

Quelle: FWF

Im Jahre 2008 wurden insgesamt 1639 Anträge (mit einem Antragsvolumen von 389 Mio. $€)$ auf Förderung von wissenschaftlichen Projekten im autonomen Bereich eingereicht. Davon wurden 703 Projekte gefördert. Das durchschnittliche Volumen eines bewilligten
Projektes betrug rund $233.000 €$. Bei Einzelprojekten betrug die Bewilligungsrate $39 \%$ der beantragten Fördersumme. Das gesamte Bewilligungsvolumen des FWF betrug insgesamt knapp über 164 Mio. $€$ im autonomen Bereich (siehe auch Abbildung 16). 
Abbildung 16: FWF Fördervolumina im autonomen und beauftragten Bereich (1998-2008)

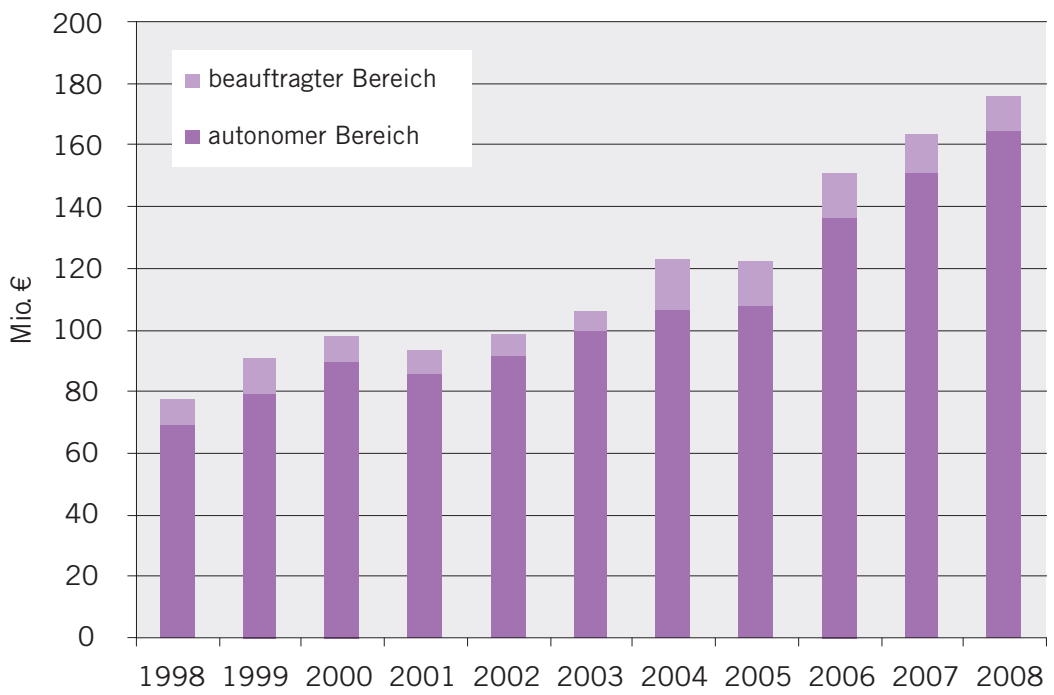

Quelle: FWF

Damit erhöhte sich das Bewilligungsvolumen im autonomen Bereich von 70 Mio. $€$ im Jahre 1998 auf 164 Mio. im Jahre 2008, was einer Steigerungsrate von $137 \%$ entspricht.

Forschung - insbesondere wissenschaftliche Forschung - ist vor allem personenbezogen. Mehr als $80 \%$ der bewilligten Mittel werden für die Abdeckung der Personalkosten - also die Anstellung junger WissenschafterInnen eingesetzt. Damit hatte der FWF 2008 mehr als 2700 wissenschaftlich tätige Personen gleichsam auf seiner "Payroll" stehen (Tabelle 18).

Tabelle 18: Durch den FWF finanziertes Forschungspersonal

\begin{tabular}{lrrr} 
& $\mathbf{2 0 0 6}$ & $\mathbf{2 0 0 7}$ & $\mathbf{2 0 0 8}$ \\
\hline Postdocs & 670 & 860 & 830 \\
DoktorandInnen & 1280 & 1359 & 1526 \\
Erwin-Schrödinger-Stipendien & 93 & 111 & 102 \\
Lise-Meitner-Stellen & 33 & 45 & 45 \\
Hertha-Firnberg-Stellen & 41 & 46 & 40 \\
Elise-Richter-Stellen & 8 & 24 & 29 \\
Charlotte-Bühler-Stellen & 10 & 3 & 0 \\
Impulsprojekte & 13 & 13 & 7 \\
Technisches Personal & 102 & 118 & 123 \\
Gesamt & 2250 & 2579 & 2702
\end{tabular}

Eine Analyse der Mittelverteilung nach Wissenschaftsdisziplinen (Tabelle 19) zeigt, dass die Naturwissenschaften deutlich voran liegen. $62 \%$ der bewilligten Mittel entfallen auf diesen Wissenschaftsbereich, was eine deutliche Steigerung von fast 10 Prozentpunkten gegenüber dem Vorjahr bedeutet. Diese Steigerung ging hauptsächlich zulasten der Humanmedizin, welche von $20 \%$ auf $11 \%$ im letzten Jahr zurückfiel. Auf die Geistes- und Sozialwissenschaften entfiel ein Anteil $21 \%$.

Tabelle 19: Bewilligungen nach Wissenschaftsdisziplinen 2008, autonomer Bereich

\begin{tabular}{lrr} 
& in Mio. $€$ & $\%$ \\
\hline Naturwissenschaften & 102,2 & 62 \\
Technische Wissenschaften & 4,6 & 3 \\
Humanmedizin & 18,5 & 11 \\
Land- und Forstwirtschaft, Veterinärmedizin & 3,8 & 2 \\
Sozialwissenschaften & 13,8 & 8 \\
Geisteswissenschaften & 21,5 & 13 \\
Gesamt & 164,4 & 100 \\
Quelle: FWF & & \\
& & \\
& &
\end{tabular}

Quelle: FWF 
Ein vielfach diskutiertes und auch zuletzt von der CREST-ExpertInnengruppe angesprochenes Charakteristikum des FWF liegt in der Bewilligungsrate von Forschungsanträgen (CREST 2008). ${ }^{27}$ Diese scheint - so der CREST-Bericht - ein wenig hoch zu sein, „,...und verdient näher untersucht zu werden". Die Entwicklung der letzten Jahre zeigt, dass die Bewilligungsrate der Einzelprojektanträge (Anzahl) bis in das Jahr 2004 tendenziell rückläufig und auf $36 \%$ gesunken ist. Bis 2008 ist die Rate wieder auf $43 \%$ gestiegen. Die Entwicklung der Bewilligungssummen zeigt eine ähnliche Entwicklung und liegt knapp unter $40 \%$ (vgl. Abbildung 17).

\section{Abbildung 17: Bewilligungsrate für Einzelprojekte (1995 - 2008)}

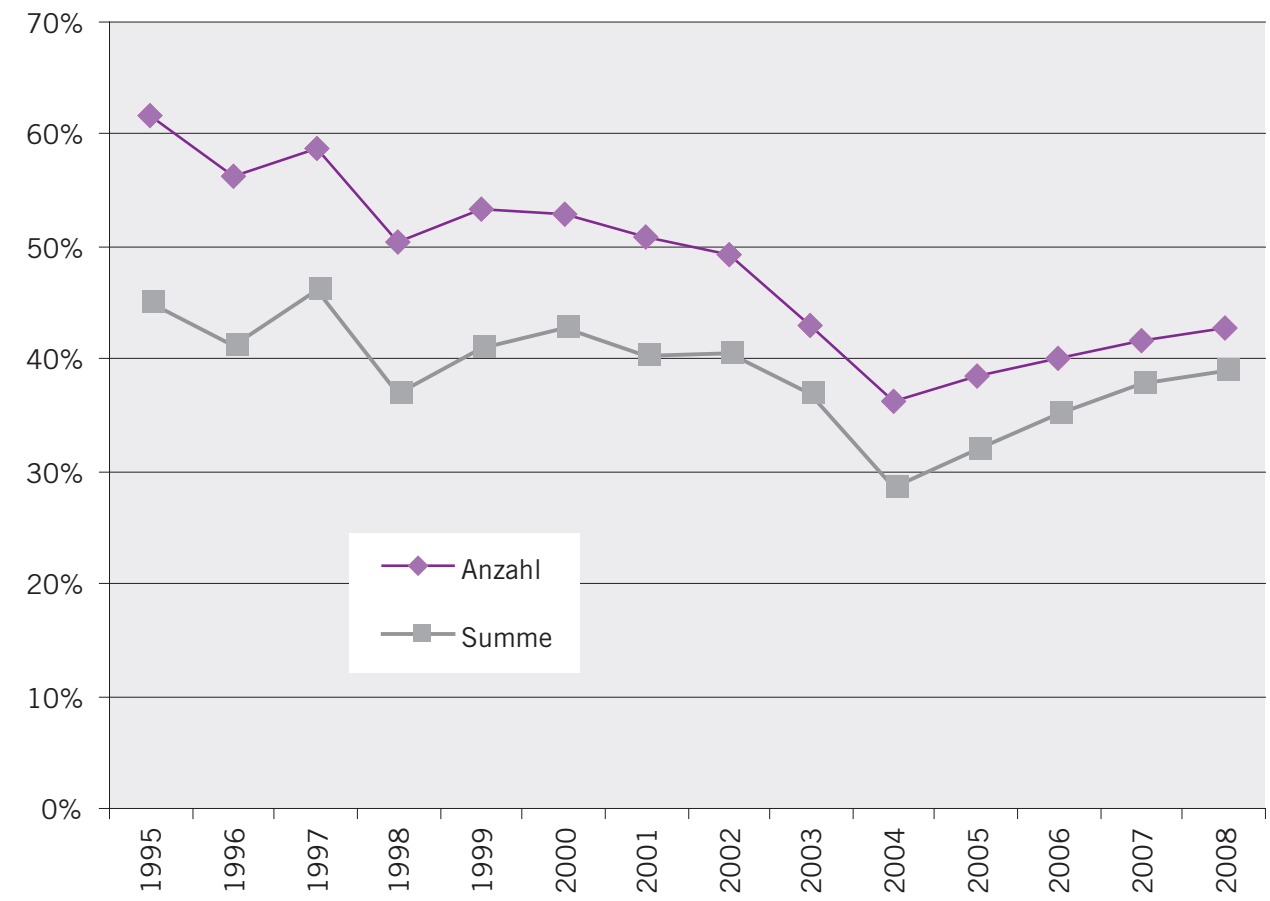

Quelle: FWF

Die Bemerkungen durch CREST verwundern ein wenig, wendet doch der FWF in der Beurteilung der Anträge das international üblich „Peer Review" Verfahren an, d.h. ein mehr oder weniger standardisiertes Verfahren zur Qualitätssicherung zur Bewertung wissenschaftlicher Projektanträge oder wissenschaftlicher Publi- kationen durch FachkollegInnen. Peer-Review Verfahren tragen dabei dem Umstand Rechnung, dass es für Forschungsvorhaben keine anderen formalen Qualitätsstandards gibt, die es erlauben würden, Qualität, Innovativität und Chancen der erfolgreichen Durchführung zu bestimmen. Weiters stellte auch die 2004

27 „Die hohe Erfolgsquote von Projektanträgen an den FWF im Vergleich zur Erfolgsquote in anderen Ländern wurde mit Interesse zur Kenntnis genommen. Sie könnte natürlich einfach mit der hohen Qualität der österreichischen Wissenschaft und Forschungsgemeinschaft zu tun haben. Andererseits könnte sie auch bedeuten, dass die Qualitätsvorschriften niedriger sind als anderswo. Diese Situation verdient es daher, näher untersucht zu werden." (CREST 2008, S. 23) 
durchgeführte Evaluierung des FWF (Arnold et al. 2004) dem Begutachtungssystem ein sehr positives Zeugnis aus. ${ }^{28}$

Die Analyse der Bewilligungsraten bei anderen europäischen - und mit dem FWF vergleichbaren - Wissenschaftsfonds zeigt hingegen, dass die jeweiligen Raten sehr unter- schiedlich sind. So liegen die Bewilligungsraten von Einzelprojektanträgen bei der Deutschen Forschungsgemeinschaft DFG bei knapp über $50 \%$, beim Schweizer Nationalfonds SNF seit Jahren zwischen 60 und $70 \%$ und bei der Finnischen Akademie der Wissenschaften bei 27 \% im Jahre 2007 (siehe Abbildung 18).

\section{Abbildung 18: Bewilligungsraten von Einzelprojektanträgen im Vergleich (in \%)}
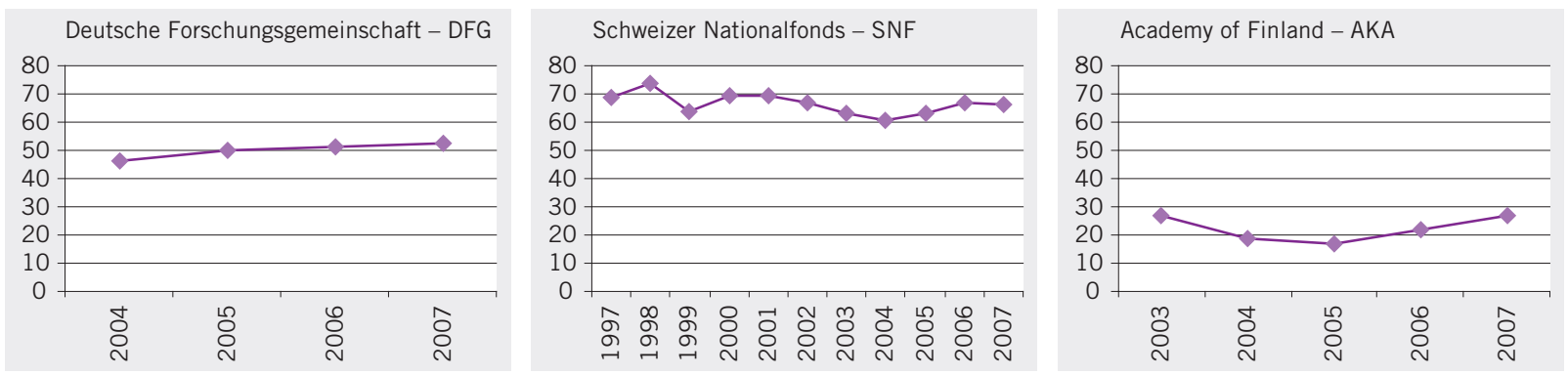

Quelle: DFG, SNF, AKA; Berechnungen Joanneum Research

Auffallend ist, dass es große Differenzen zwischen den einzelnen Förderagenturen gibt und dass es schlichtweg unmöglich ist, ex cathedra die optimale Höhe der Bewilligungsquote zu bestimmen. Förderagenturen sind Teil des Innovationssystems und deren Förderaktivitäten sind wesentlich geprägt von Kontextbedingungen wie zusätzlich existierenden Fördermöglichkeiten, den Finanzierungsstrukturen der Hochschulen, den herrschenden Anreizstrukturen für WissenschafterInnen und anderen Determinanten. In Österreich finanziert der FWF ca. $11 \%$ der F\&E-Ausgaben des Hoch- schulsektors und $75 \%$ der Bund, was darin resultiert, dass von der potentiell antragsberechtigten Community nur das oberste Segment (ca. $20-25 \%$ ) Anträge beim FWF stellt. ${ }^{29}$

Völlig anders sieht die Situation in Ländern wie UK aus, wo die Mittel für die Hochschulforschung prinzipiell nur über die Councils eingeworben werden können und damit der Anreiz, Forschungsanträge zu stellen, auf einen viel umfassenderen Personenkreis zutrifft. Die unterschiedlichen Strukturen haben somit Einfluss auf die Bewilligungsquoten.

28 "No changes in the peer review system seem to be necessary within the current regime. It is open to newcomers in term of disciplines etc, while at the same time the portfolio of funding schemes allows for some accumulation of critical resources. There are no barriers set up in the decision procedures that would give some researchers better access than others. Even individual researchers with prestigious prizes have a moderate level of projects from the council" (Arnold et al. 2004, S. 85)

29 Um dieses Segment zu verbreitern, wurde vom BMWF als Teil einer Exzellenzinitiative die Vergütung von Overheadkosten der Universitäten für FWF-Projekte [2008, rückwirkend mit Dezember 2007] eingeführt, die eine selbstverstärkende positive Dynamik in den Forschungsstätten in Gang bringen soll. Weiterer wichtiger Teil dieser Initiative ist eine verbesserte Doktoratsausbildung (siehe dazu auch Universitätsbericht S.132f.). 
Generell lässt sich auch anführen, dass die Antragsqualität gestiegen ist. Zwar existieren noch keine harten Fakten und Untersuchungen dazu, aber auf der Basis der Beobachtungen der SachbearbeiterInnen in den Fachabteilungen lassen sich folgende Schlüsse ziehen:

- Der gesteigerte Wettbewerbsdruck und die damit verbundenen hohen Ablehnungsquoten von rund $70 \%$ und mehr in den Jahren 2004 und 2005 haben zur gesteigerten Qualitätsorientierung beigetragen.

- Weiters führt die kumulative Erfahrung in der Einreichung von Projektanträgen vor allem unter jüngeren WissenschafterInnen zu einer „Internalisierung" bestehender Qualitätsstandards.

- Die den AntragstellerInnen seit 2003 voll inhaltlich zur Verfügung gestellten Gutachten haben in vielen Fällen zur Überarbeitung und neuerlichen Einreichung von Anträgen geführt. Damit einher ging oft eine deutliche Qualitätssteigerung und höhere Bewilligungschance.

- Eine kontinuierliche Verbesserung der Antragsrichtlinien und Vorgaben im Zusam- menhang mit der seit 2005 verlangten Projekteinreichung auch auf elektronischem Datenträger hat offenbar besser strukturierte und präzisere Anträge begünstigt.

- Mit den seit September 2006 vor allem für junge WissenschafterInnen angebotenen "Coaching Workshops" hat der FWF seine Beratungstätigkeit auf ein neues Feld ausgedehnt. Dieses Coaching erhöht nachweislich die Bewilligungschancen.

Es mag vielleicht ein Fehlschluss sein, von der Bewilligungsquote auf die Qualität der österreichischen Wissenschaft zu schließen - wie die CREST-ExpertInnen es fragend taten - hingegen scheint es allemal legitim zu sein, auf die Qualität der Forschungsanträge zu schließen. Aber will man den Schluss von der Bewilligungsquote auf die Qualität der Wissenschaft gelten lassen, so könnte sich Österreich an der Schweiz ein Beispiel nehmen. Denn diese schneidet im internationalen Vergleich der Forschungsleistung am weitaus besten ab (siehe Tabelle 29), und das trotz (oder vielleicht sogar wegen) der hohen Bewilligungsquote des Schweizer Nationalfonds. 


\section{Das österreichische Innovationssystem: Bestandsaufnahmen und Perspektiven}

Das österreichische Innovationssystem war in den Jahren 2008/09 Gegenstand von Einschätzungen und Diskussionen. Mit unterschiedlichen Zielsetzungen wurden Initiativen gestartet, welche durch eine möglichst umfassende Gesamteinschätzung die Grundlagen für eine nationale FTI-Strategie legen sollen. ${ }^{30}$

Im Frühjahr 2008 hat das Bundesministerium für Verkehr, Innovation und Technologie (BMVIT) gemeinsam mit dem Bundesministerium für Wirtschaft und Arbeit (BMWA - jetzt BMWFJ) die Systemevaluierung der Forschungsförderung und -finanzierung in Auftrag gegeben. Ziel der Evaluierung ist es, die Forschungsförderung und -finanzierung in Hinblick auf die Leistungsfähigkeit des österreichischen Innovationssystems $\mathrm{zu}$ analysieren und allfälligen Handlungsbedarf $\mathrm{zu}$ seiner Verbesserung $\mathrm{zu}$ identifizieren. Im besonderen Blickfeld steht dabei die Wirkungsweise der Instrumente der Forschungsförderung. Durchgeführt wird die Evaluierung von einer Bietergemeinschaft, bestehend aus dem Österreichischen Institut für Wirtschaftsforschung WIFO als Projektleiter, der KMU Forschung Austria, Prognos AG sowie dem Subunternehmer convelop. ${ }^{31}$

Anders war die Zielsetzung des im Sommer 2007 begonnenen und in Alpbach 2008 präsentierten Forschungsdialoges. Hier wurde durch die Einbeziehung der gesamten Community ein Forum geschaffen, in dem nahezu sämtliche Themen der Wissenschaft und der Forschung diskutiert und dokumentiert wurden. Unter Federführung des BMWF und mit Unterstützung der gesamten Bundesregierung war der Forschungsdialog möglichst breit angelegt, um den Beteiligten die Möglichkeit zu bieten, Standpunkte und Sichtweisen darzulegen und zu diskutieren. Gesammelt und zugänglich gemacht wurden die Diskussionen in einer 265-seitigen Ergebnisdokumentation. ${ }^{32}$ Die Schlussfolgerungen des Forschungsministers aus dem Forschungsdialog wurden in den sog. Zukunftsbotschaften zusammengefasst. ${ }^{33}$

Um eine Sicht von außen zu erhalten, wurden europäische Experten und Expertinnen Mitglieder des Ausschusses für wissenschaftlich-technische Forschung (CREST) der EU ${ }^{34}$ - eingeladen, einen Länderbericht über Österreich zu erstellen. Der Policy-Mix-Peer-Review Länderbericht ${ }^{35}$ spiegelt die kollektive Einschätzung der österreichischen Forschungs-, Technologie- und Innovationslandschaft durch ein Review Team wider und ist das Ergebnis eines Konsultationsprozesses mit VertreterInnen politischer Entscheidungsebenen, der Förderagenturen, der Beratungsorgane sowie nationaler Experten und Expertinnen. Die Einschät-

\footnotetext{
30 Siehe Regierungsprogramm für die XXIV. Gesetzgebungsperiode (2008-2013), S. 45.

31 Siehe dazu: http://www.bmvit.gv.at/innovation/forschungspolitik/systemevaluierung/index.html

32 Siehe dazu: http://www.bmwf.gv.at/forschung/oesterr forschungsdialog/

33 http://www.bmwf.gv.at/forschung/oesterr forschungsdialog/

34 CREST ist der Ausschuss für wissenschaftliche und technische Forschung der Europäischen Union. Als Teil der Methode der Offenen Koordinierung - zyklische Aktivitäten zur Unterstützung der Bemühungen der EU, die F\&E-Ausgaben innerhalb der EU auf $3 \%$ des BIP zu erhöhen - hat CREST während des zweiten Zyklus der Aktivität eine Reihe von Policy-Mix-Peer-Reviews begonnen und sie im dritten und vierten Zyklus weitergeführt.

35 http://www.bmwf.gv.at/forschung/oesterr forschungsdialog/
} 
zungen des Review-Teams bezogen sich auf die Bereiche Wissenschaftssystem, industrielle $\mathrm{F} \& \mathrm{E}$ und Innovation, Humanressourcen und die für Österreich besonders wichtigen Empfehlungen bezüglich Strukturen und Prozessen der Politikformulierung.

In den folgenden Kapiteln werden die wichtigsten Einschätzungen und Empfehlungen dieser drei Aktivitäten zusammengefasst, womit auch dokumentiert werden soll, dass die für eine nationale FTI-Strategie notwendigen Grundlagen vorhanden sind.

\subsection{Systemevaluierung der Forschungsförderung und -finanzierung}

\subsubsection{Einleitung}

Österreich hat in den letzten 15 Jahren seine technologische Position kontinuierlich verbessert. Anfang der neunziger Jahre lag es bei den meisten Technologie- und Innovationsindikatoren unterhalb oder im Schnitt der Europäischen Union. Mittlerweile zeigen die Indikatoren, dass Österreich zum Spitzenfeld aufgeschlossen hat. ${ }^{36}$ Besonders augenfällig ist die positive Entwicklung bei der F\&E-Quote, die sich von 1,44\% im Jahr 1993 auf 2,66 \% im Jahr 2008 (bzw. auf prognostizierte 2,73\% im Jahr 2009) verbesserte. Österreich ist damit eines der wenigen europäischen Länder, das sich auf einem Pfad in Richtung der anvisierten 3-\%-Quote befindet (gleichwohl aus heutiger Sicht unter den neuen Rahmenbedingungen eine exakte Zielerreichung im Jahr 2010 unrealistisch erscheint). Im selben Zeitraum wurde die öffentliche Forschungsförderung und -finanzierung maßgeblich aus- und umgebaut. Als wichtigste Ereignisse sind hier auf der Seite der direkten For- schungsförderung die Gründung des aws (Austria Wirtschaftsservice) im Jahre 2002 und jene der FFG (Forschungsförderungsgesellschaft) im Jahre 2004 zu vermerken. Auf der Seite der steuerlichen Forschungsförderung wurden im Jahr 2002 die Weichen für den Forschungsfreibetrag und für die Forschungsprämie auf der Grundlage der Definition von Forschungsausgaben nach dem Frascati Handbuch der OECD gestellt. Die stetige Erhöhung der Mittelflüsse und die zunehmende Ausdifferenzierung des Förderportfolios haben auf der politischen Ebene und der Ebene der Institutionen die Notwendigkeit verspüren lassen, eine Evaluierung des österreichischen Systems der Forschungsförderung und finanzierung durchzuführen.

Im Herbst des Jahres 2007 erfolgte unter Federführung des Bundesministeriums für Verkehr, Innovation und Technologie (BMVIT) und des Bundesministeriums für Wirtschaft, Familie und Jugend (BMWFJ, damals noch Bundesministerium für Wirtschaft und Arbeit, BMWA) die Ausschreibung für eine Systemevaluierung der Forschungsförderung und -finanzierung. Unter mehreren Bewerbern wurde der Bietergemeinschaft um das Österreichische Institut für Wirtschaftsforschung (WIFO) bestehend aus der KMU Forschung Austria, der Prognos AG Deutschland und convelop im Frühjahr 2008 der Zuschlag für dieses Vorhaben erteilt. Der Auftrag wurde im April 2009 abgeschlossen. ${ }^{37}$ Der Evaluierungsauftrag umfasste drei Ebenen. ${ }^{38}$

1. Rahmenbedingungen: Hierzu zählen einerseits jene Umfeldbedingungen, die nicht direkt der FTI-Politik zuzuordnen sind, aber dennoch die Funktionsfähigkeit des Forschungssystems in positiver oder negativer Weise beeinflussen (z.B. Marktregulierungen, Steuergesetze, Universitätsgesetz). ${ }^{39}$ Anderer-

36 Siehe dazu etwa Kapitel 1.1.4 in diesem Forschungs- und Technologiebericht.

37 Aiginger et al. (2009), Endbericht der Systemevaluierung.

38 Das Leistungsbild der Ausschreibung ist im Anhang abgedruckt.

39 Janger et al. (2009), Rahmenbedingungen - Teilbericht 1 der Systemevaluierung. 
seits werden die strategischen GovernanceStrukturen des FTI-Systems im engeren Sinne analysiert ${ }^{40}$, sowie die Interaktionen zwischen strategischer und operativer Ebene. ${ }^{41}$

2. Instrumente: Maßnahmen der indirekten, direkten und - als Teil davon - der institutionellen Förderung werden in drei zunächst getrennten Portfolioanalysen erfasst und hinsichtlich ihrer Ausgestaltung, Angemessenheit und Wirkungsweise bewertet. Hier stehen vor allem Aspekte der Zielgruppenabdeckung und -zufriedenheit sowie des Zielgruppenverhaltens im Vordergrund. ${ }^{42}$

3. Systemische Interaktion: Zunächst werden die systemischen Anforderungen an ein kohärentes und anreizkompatibles Gesamtsystem der Forschungsförderung erarbeitet. Weiters geht es um das operative Zusammenspiel zwischen den Institutionen und Akteuren sowie drittens um die Kohärenz des Instrumentenmix: das Zusammenspiel der direkten und indirekten Förderinstrumente. ${ }^{43}$

Erste Ergebnisse mit Schwerpunkt auf den Instrumenten der direkten und der steuerlichen Forschungsförderung und deren Zusammenspiel werden in diesem Abschnitt des Forschungs- und Technologieberichtes präsentiert. Schlussfolgerungen aus diesen Ergebnissen sind auf politischer Ebene noch zu bewerten.

\subsubsection{Die Instrumente der Forschungsförderung und -finanzierung im Überblick}

Die direkte Förderung zeichnet sich dadurch aus, dass Einnahmen als Förderungen an An- tragsteller auf der Grundlage eines Begutachtungsverfahrens vergeben werden. Direkte Förderung besteht also aus Transfers von der öffentlichen Hand zu begünstigten Antragstellern. Bei den indirekten oder steuerlichen Instrumenten erfolgt die Förderung über steuerliche Entlastungen. In Österreich setzen die steuerlichen Forschungsförderungsinstrumente an der Steuerbemessungsgrundlage bzw. an der Steuerschuld an. Forschungsfreibeträge reduzieren die Steuerbemessungsgrundlage; bei der Forschungsprämie sinkt die Steuerschuld um einen bestimmten Anteil der Forschungsausgaben. ${ }^{44}$ Besteht mangels entsprechender Gewinne keine Steuerschuld, wird die Prämie von derzeit $8 \%$ der anrechenbaren Aufwendungen dem Unternehmen direkt gutgeschrieben. Drei Viertel der über steuerliche Forschungsförderinstrumente induzierten Steuereinnahmeausfälle für das Steuerjahr 2005 entfielen auf die Forschungsprämie. Im Jahr 2007 hat der Österreichische Rechnungshof die durch steuerliche Forschungsförderinstrumente induzierten Steuereinnahmeausfälle für 2005 auf insgesamt 418 Mio. $€$ geschätzt.

Bei der steuerlichen Förderung wird generell von einem hohen Maß an Neutralität ausgegangen, da die steuerliche Förderung im Prinzip weder im Hinblick auf Art und Inhalt der begünstigten Projekte, noch hinsichtlich der sektoralen Zugehörigkeit der Begünstigten diskriminiert. Sie fördert forschende KMUs in gleichem Maße wie forschende Großunternehmen. Wer den Fördertatbestand erfüllt, kann einen Rechtsanspruch auf Förderung geltend

40 Gerhardter et al. (2009), Strategische Governance - Teilbericht 2 der Systemevaluierun

41 Ruhland et al. (2009), Governance in der FTI-Politik im Wechselspiel zwischen Ministerien und Agenturen - Teilbericht 3 der Systemevaluierung.

42 Falk (2009), Tax Incentives for R\&D - Teilbericht 4 der Systemevaluierung; Mayer et al. (2009), Das Angebot der direkten FTI-Förderung in Österreich - Teilbericht 5 der Systemevaluierung; Klose et al. (2009), Grundlagenfinanzierte Forschungs-und Wissenschaftseinrichtungen - Teilbericht 6 der Systemevaluierung; Streicher et al. (2009), Public RTDI Funding in Austria - the Target Groups' Perspectives - Teilbericht 7 der Systemevaluierung.

43 Falk (2009), Kohärenz des Instrumentenmix - Teilbericht 8 der Systemevaluierung; Astor et al. (2009), Systemische Analyse des Innovations- und Interventionssystems - Teilbericht 9 der Systemevaluierung.

44 Zur Struktur der steuerlichen Förderung in Österreich s. BMVIT und BMWF (2007), etwas ausführlicher in BMF/BMWA (2008). 
machen, ohne dass die öffentliche Hand den Anspruch erhebt, auf unternehmerische Entscheidungen steuernd einzugreifen. Insofern ist die steuerliche Förderung als niedrigschwelliges, nicht-selektives Instrument mit potentieller Breitenwirksamkeit zu klassifizieren. Die steuerliche Förderung wurde in der Literatur zuweilen kritisch gesehen (David et al. 2000), da sie aufgrund der nicht vorgegebenen inhaltlich-thematischen Schwerpunktsetzung von ihrer Konstruktion her eher Forschungsaktivitäten mit hohen privaten Erträgen und nicht notwendigerweise solche mit hohen sozialen Erträgen fördert. Da ferner der Barwert der Förderung in der Regel sehr niedrig angesetzt ist - jedenfalls niedriger als im Vergleich zur direkten Förderung - sind aus unternehmerischer Perspektive wenig Anreize gegeben, das Ausmaß und die Art der Forschungsaktivitäten substantiell zu erhöhen, bzw. abzuändern.

Die direkte Forschungsförderung setzt demgegenüber vom Ansatz her bewusst inhaltlichthemenbezogene und/oder akteursbezogene Schwerpunkte. So können beispielsweise als wichtig erachtete Forschungsvorhaben sehr zielgenau angestoßen, oder mittels spezieller Programme FTI-Kooperationen zwischen grundlagenorientierter Forschung in wissenschaftlichen Einrichtungen und anwendungsorientierter Forschung in Unternehmen forciert werden. Über eine entsprechende Auswahl der
Fördergegenstände, -kriterien und -begünstigten ist es im Prinzip leichter möglich, Mitnahmeeffekte auszuschließen, also nur solche Aktivitäten mit öffentlichen Mitteln zu bezuschussen, die nicht ohnehin privat finanziert worden wären. Aus der stärken Selektivität können jedoch nicht nur eine bessere Steuerbarkeit und potentiell höhere Anreizwirkungen erwachsen, sondern ebenso hohe Anforderungen an den Auswahl-, Bewilligungs- und Abwicklungsprozess. Bei thematischen Vorgaben besteht häufig auch das Problem, dass nur unzulänglich Information über die Zukunftspotentiale einzelner Technologien oder Branchen vorliegt und es dadurch zu Fehlallokationen knapper Fördermittel kommt. Zudem kann eine stärkere Selektivität im Förderangebot leicht zu einer wachsenden Zersplitterung und Unübersichtlichkeit der Förderungslandschaft und deren Anforderungen führen. Der Informationsbedarf seitens der Nachfrager steigt damit. Ebenso können Förderkriterien und -entscheidungen an Transparenz einbüßen und die Planungssicherheit auf Seiten der Unternehmen kann sinken. Demgegenüber steht wiederum die hohe Planungssicherheit der öffentlichen Hand, da Programme in der Regel mit fixen Mitteln dotiert werden, während auf der anderen Seite steuerliche Anreize die Budgetkontrolle unterlaufen und zukünftige Einnahmeausfälle nur prognostiziert werden können.

Tabelle 20: Gegenüberstellung der steuerlichen und direkten Forschungsförderung

\begin{tabular}{|lcc|}
\hline & Steuerliche Förderung & Direkte Förderung \\
\hline Zutrittsschwelle für Förderberechtigte & Niedrig & Hoch \\
\hline Steuerungswirkung & Gering & Hoch \\
\hline Administrativer Aufwand & Gering & Hoch \\
\hline $\begin{array}{l}\text { Planungssicherheit } \\
\text { Seitens der Unternehmen } \\
\text { Seitens der öffentlichen Hand }\end{array}$ & Hoch & Gering \\
\hline Anreizwirkung & Mittelmäßig & Hoch \\
\hline
\end{tabular}

Quelle: WIFO Darstellung 
Vor- und Nachteile der unterschiedlichen Förderansätze stehen spiegelbildlich zueinander (Tabelle 20). Insofern sind beide Instrumente vom konzeptionellen Ansatz her komplementär ausgestaltet.

\subsubsection{Die steuerliche Förderung der F\&E in Österreich}

Die Instrumente der steuerlichen F\&E Förderung in Österreich

Im österreichischen Steuerrecht sind steuerliche oder indirekte F\&E Förderungen vorgesehen. Es kennt drei Forschungsfreibeträge gemäß $§ 4$ Abs.4 Z 4 bis Z 4b EStG, sowie die Forschungsprämie gemäß $\S 108 \mathrm{c}$ EStG. Die Bemessungsgrundlage der Forschungsfreibeträge gem. § 4 Abs. 4 Z 4 und Z 4b EStG (,FFB$\mathrm{Neu}^{\prime \prime}$ ) sowie die Forschungsprämie sind Aufwendungen für Forschung und Entwicklung entsprechend dem OECD-Definitionsgerüst des Frascati-Handbuches. Aufwendungen für Auftragsforschung werden in Z 4b EStG geregelt. Ab dem Veranlagungsjahr 2004 wurde im
Zuge des Wachstums- und Standortgesetztes 2003 ein Freibetrag von 25 \% festgelegt. Die Forschungsprämie beträgt hingegen $8 \%$ der Aufwendungen nach der Frascati Definition gem. $\S 4$ Abs. 4 Z 4. Für den Forschungsfreibetrag gemäß $\S 4$ Abs. 4 Z 4a EStG (,FFB-Alt") gehen Aufwendungen zur Entwicklung oder Verbesserung so genannter volkswirtschaftlich wertvoller Erfindungen in die Bemessungsgrundlage ein. Seit dem Steuerreformgesetz 2000 wurde ab dem Wirtschaftsjahr 2000 ein Freibetragssatz von 25 \% für Aufwendungen, welche sich nicht vom F\&E-Aufwandsniveau des Vorjahres unterscheiden, festgesetzt. Für darüber hinausgehende Leistungen kann ein sog. inkrementeller Freibetragssatz von bis zu $35 \%$ geltend gemacht werden. Voraussetzung für die Inanspruchnahme ist das Vorliegen eines Bundeszertifikats in Form einer Bescheinigung des BMWFJ oder eines Patentes. Tabelle 21 gibt einen Überblick über die Förderung bezüglich der veranlagten F\&E Kosten der Forschungsfreibeträge und der Forschungsprämie. So hat im Jahr 2005 die Förderung durch den „FFB-Neu“ 6,25 Cent je $1 €$ F\&E Aufwendungen von Körperschaften betragen.

\section{Tabelle 21: Steuerliche Förderung der F\&E im Überblick}

\begin{tabular}{|c|c|c|c|c|c|c|}
\hline & \multicolumn{2}{|c|}{ FFB-Alt ${ }^{1)}$} & FFB-Neu ${ }^{1)}$ & $\begin{array}{c}\text { FFB-Neu }{ }^{1)} \\
\text { für Auftragsforschung }\end{array}$ & Forschungsprämie & $\begin{array}{l}\text { Forschungsprämie für } \\
\text { Auftragsforschung }\end{array}$ \\
\hline & Aufwendung & inkrementell & & & & \\
\hline 2002 & $8,50 \%$ & $11,90 \%$ & $3,40 \%$ & & $3 \%$ & \\
\hline 2003 & $8,50 \%$ & $11,90 \%$ & $5,10 \%$ & & $5 \%$ & \\
\hline 2004 & $8,50 \%$ & $11,90 \%$ & $8,50 \%$ & & $8 \%$ & \\
\hline seit 2005 & $6,25 \%$ & $8,75 \%$ & $6,25 \%$ & $6,25 \%$ & $8 \%$ & $8 \%$ \\
\hline
\end{tabular}

Bemerkung: ${ }^{1)}$ Die Förderung der Freibeträge wird durch die Multiplikation des Freibetragssatzes mit dem jeweils geltenden Körperschaftssteuersatz ermittelt. Bei Einkommenssteuerpflichtigen ergibt sich die Förderung aus der Multiplikation des Freibetragssatzes mit dem jeweiligen Grenzsteuersatz.

Quelle: Einkommensteuerstatistik, WIFO Berechnungen

Die oben beschriebenen Änderungen der steuerlichen Förderung von F\&E haben $z u$ einer Steigerung des Fördervolumens geführt (Abbildung 19). Im Veranlagungsjahr 2005 betrugen die Kosten der steuerlichen F\&E Förderung etwas über 250 Mio. $€$ zu konstanten Preisen. Dies entspricht 276,6 Mio. $€$ zu laufenden Preisen und liegt damit unter den Einschätzungen des Rechnungshofes von 418 Mio. $€$. 


\section{Abbildung 19: Kosten der steuerlichen F\&E Förderung}

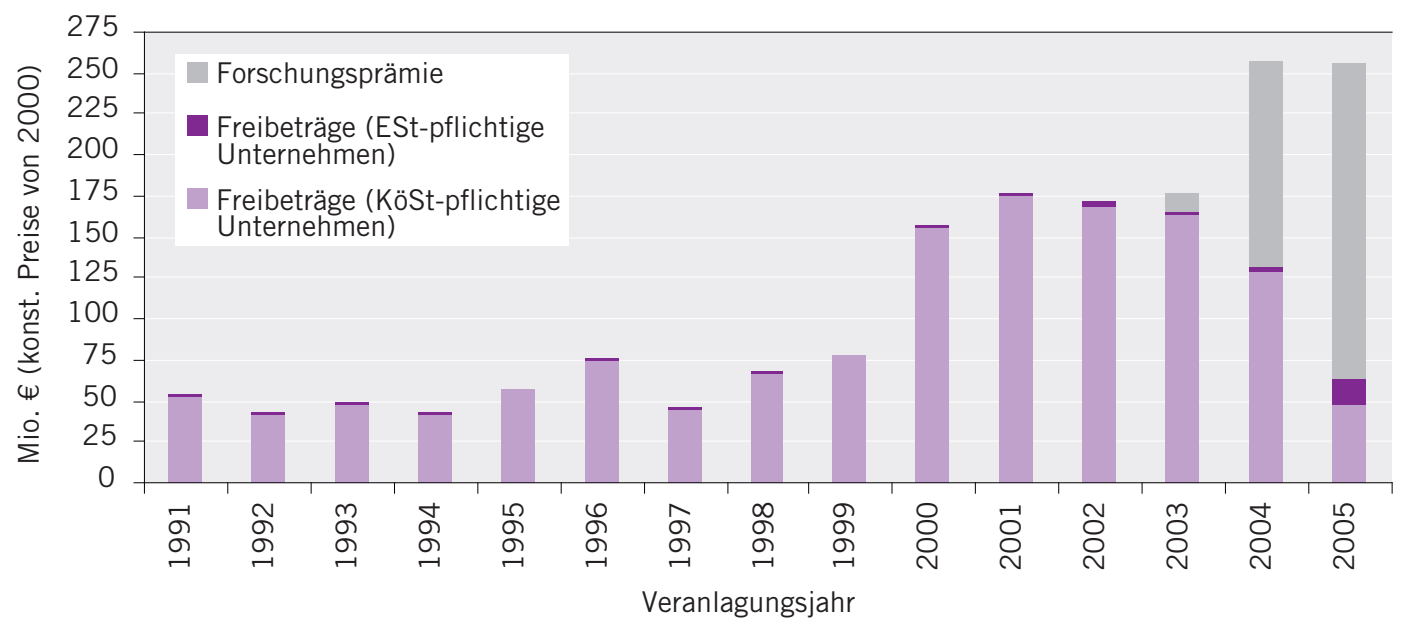

Anmerkung: Zum Zeitpunkt der Erhebung waren Daten bis zu dem Veranlagungsjahr 2005 verfügbar.

Quelle: Körperschaftssteuerstatistik, Einkommenssteuerstatistik, Mitteilungen des Bundesministerium für Finanzen, WIFO Berechungen

\section{Nutzung der Instrumente der steuerlichen Förderung}

Abbildung 20 zeigt die Verteilung der steuerlichen Förderung im Jahr 2005 auf eine Branchenklassifikation der Unternehmen, die die Technologie- bzw. Wissensintensität abbildet. Es zeigt sich, dass alle Formen der steuerlichen Förderung vor allem von technologiein- tensiven Unternehmen in Anspruch genommen werden. Am stärksten trifft dies auf den FFB-Neu zu; 68,8 \% der gesamten steuerlichen Förderung, die über den FFB-Neu geltend gemacht wurde, flossen technologieintensiven Unternehmen zu. Wissensintensive Dienstleistungsunternehmen nehmen vor allem die Forschungsprämie in Anspruch (22\%).

\section{Abbildung 20: Verteilung der steuerlichen Förderung nach Branchen im Jahre 2005}

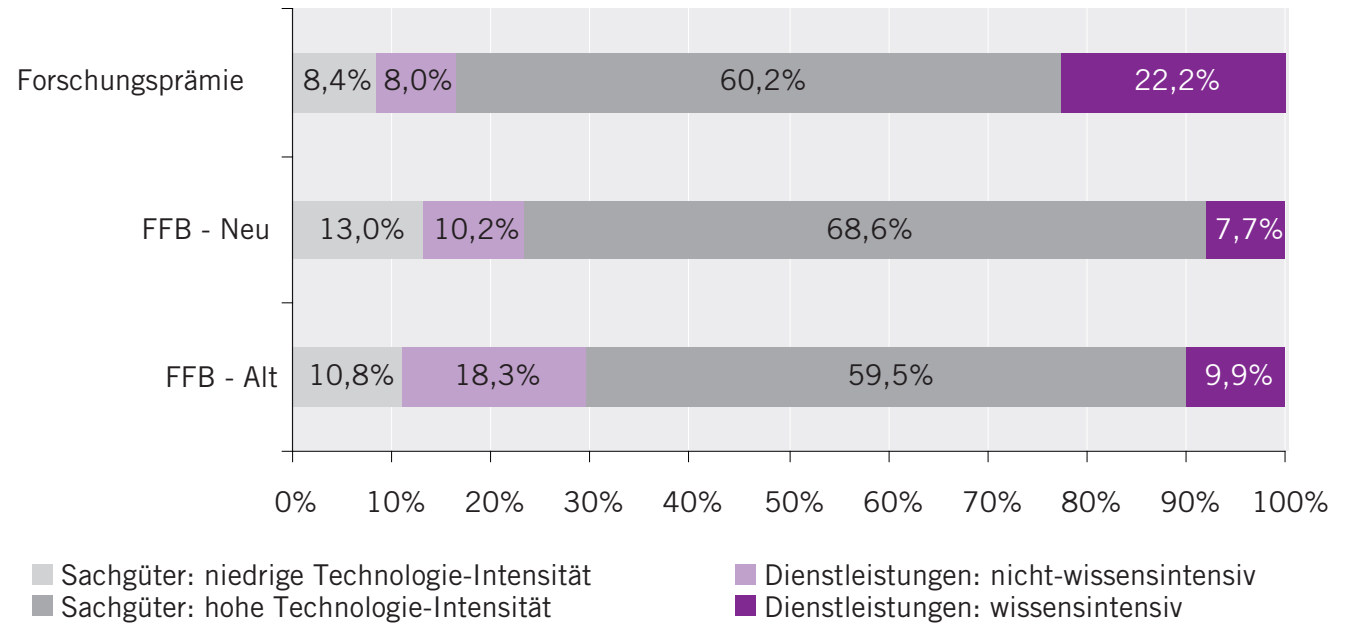

Quelle: Körperschaftssteuerstatistik, Einkommenssteuerstatistik, Mitteilungen des Bundesministerium für Finanzen, WIFO Berechungen 
Betrachtet man die Verteilung der steuerlichen F\&E Förderung nach Größenklassen der Unternehmen, so zeigen Analysen, dass Unternehmen mit mehr als 100 Beschäftigten ca. $40 \%$ der Förderempfänger für den FFB-Alt und $30 \%$ für den FFB-Neu darstellen. Diese Unternehmen haben im Jahr 2005 ca. 90 \% der gesamten steuerlichen Förderung aus dem FFBAlt und dem FFB-Neu in Anspruch genommen. Innerhalb der Unternehmen mit mehr als 100 Mitarbeitern zählen vor allem Großunternehmen mit mehr als 500 Beschäftigten zu den stärksten Nutznießern der steuerlichen Förderung auf der Grundlage von Freibeträgen. Damit stellen gemessen an der Anzahl der Empfänger steuerlicher Förderung v.a. KMUs den größten Anteil dar, während gemessen an den Fördervolumen Großunternehmen den größten Anteil der Förderungen erhalten. Bei der Forschungsprämie verhält es sich bei der Anzahl der Förderempfänger ähnlich wie bei den Freibeträgen. Auch stellen Unternehmen mit mehr als 100 Beschäftigten etwa $30 \%$ aller Förderempfänger dar. Anders als bei den Freibeträgen kommen aber KMUs bis 100 Beschäftigten über $70 \%$ der gesamten über die Forschungsprämie verteilten Fördervolumen zugute. Damit sind bezogen auf die Fördervolumen Unternehmen mit mehr als $100 \mathrm{Be}-$ schäftigten die Zielgruppe der Freibeträge und KMUs jene der Forschungsprämie.

Betrachtet man die Förderintensitäten der steuerlichen Förderung, d.h. das Verhältnis zwischen Förderungen und den getätigten Forschungsausgaben, so zeigen die Ergebnisse der diesbezüglichen Untersuchungen, dass in den Größenklassen ab einer Größe von zehn Mitarbeitern die Förderintensitäten zwischen $4,5 \%$ und 7,7 \% schwanken. Für Kleinstunternehmen sind die verfügbaren Daten aufgrund einer statistischen Untererfassung nicht aussagekräftig. Die durchschnittliche Förderintensität über alle Größenklassen hinweg beträgt $6,9 \%$. Insgesamt ist die steuerliche Förderung neutral bezüglich der Unternehmensgröße. Bei einer branchenspezifischen Aufschlüsselung zeigen sich größere Schwankungen als bei der Betrachtung nach Größenklassen. Im Bereich des produzierenden Gewerbes wurden die niedrigsten Förderintensitäten bei Unternehmen mit niedrig-mittlerer Technologieintensität, die höchsten bei Unternehmen mit mittel-hoher Technologieintensität beobachtet. Im Hochtechnologiesegment liegt die Förderintensität knapp über 5,6 \%. Im Bereich der Dienstleistungen wurde in Branchen mit hoher Wissensintensität eine Förderintensität von knapp unter $5 \%$ beobachtet. Die über alle Unternehmen hinweg beobachteten geringen Förderintensitäten in Hochtechnologiebranchen sowie im wissensintensiven Segment der Dienstleistungsbranchen spiegeln die Spezialisierung der österreichischen Wirtschaft wider.

\section{Fördereffekte der steuerlichen Förderung}

Das Anheben der Forschungsprämie auf $8 \%$ im Jahr 2004 und die Senkung des Körperschaftssteuersatzes von $34 \%$ auf $25 \%$ im Jahr 2005 hat zu einer stärkeren Inanspruchnahme der Forschungsprämie zulasten der Forschungsfreibeträge geführt. Die Einführung der Forschungsprämie hat die Anzahl der indirekt geförderten Unternehmen stark ansteigen lassen. Wurden im Jahr 2001 noch 853 Unternehmen durch den „FFB-Alt" gefördert, so ist die Anzahl der Unternehmen, die einen steuerlichen Freibetrag oder eine Prämie in Anspruch genommen haben im Jahr 2005 auf mindestens 2374 bis maximal 3144 angestiegen. Die Schwankungsbreite erklärt sich durch die Möglichkeit der multiplen Inanspruchnahme der einzelnen Instrumente. Der Prämie kommt dabei der größte Anteil zu. Knapp über 1800 Unternehmen wurden durch die Forschungsprämie steuerlich begünstigt. Viele der neu gewonnenen Nutzer sind Kleinund Mittelbetriebe (KMU). 


\subsubsection{Die direkte Förderung der Forschung in Österreich}

Im Rahmen der Systemevaluierung der Forschungsförderung und -finanzierung wurde das Angebot der direkten Forschungs-, Technologie-, und Innovationsförderung auf Bundesebene untersucht. Untersuchungsgegenstand waren 77 verschiedene Fördermaßnahmen. Die Programme teilen sich dabei folgendermaßen auf die Agenturen auf: Forschungsförderungsgesellschaft (FFG) 39, Fond für die Wissenschaftliche Forschung (FWF) 19, Christian Doppler Gesellschaft (CDG) 1 und Austria Wirtschaftsservice (aws) 18.

Ausgangspunkt der Analyse waren die verfügbaren Dokumente der für die Evaluierung relevanten Förderungsmaßnahmen. Zusätzliche Information wurde durch qualitative Inter- views mit ExpertInnen aus den Fachressorts, aus dem Bundesministerium für Finanzen, aus den Agenturen, sowie mit ExpertInnen aus dem Kreis der Zielgruppen gewonnen. Zuletzt wurde eine Befragung der Zielgruppen durchgeführt und ausgewertet.

\section{Ressourcenausstattung und Zielgruppen ${ }^{45}$}

Wie aus Tabelle 22 ersichtlich wurden die im untersuchten Zeitraum (2002-2007) genehmigten Mittel zur Forschungsförderung zu den größten Teilen durch die FFG $(62,5 \%)$ und den FWF $(31,8 \%)$ vergeben. Die aws und die CDG haben an der gesamten Forschungsförderung relativ geringe Anteile. Bei aws und CDG konnten keine beantragten Summen festgestellt werden.

Tabelle 22: Entwicklung der beantragten und genehmigten Förderungsmittel (Förderbarwerte) von FFG, AWS, FWF, und CDG, 2002-2007, in Mio. €

\begin{tabular}{|l|c|c|c|c|c|c|c|c|}
\hline & \multicolumn{2}{c|}{ CDG } & \multicolumn{2}{c|}{ FFG } & \multicolumn{3}{c|}{ AWS } & \multicolumn{3}{c|}{ FWF } \\
\hline & beantr. & genehmigt & beantr. & genehmigt & beantr. & genehmigt & beantr. & genehmigt \\
\hline 2002 & - & 3,8 & 602,2 & 214,2 & - & 7,7 & 227,2 & 100,8 \\
2003 & - & 5,5 & 490,8 & 169,8 & - & 11,8 & 324,6 & 105,8 \\
2004 & - & 5,8 & 472,9 & 183,2 & - & 17,5 & 355,2 & 123,5 \\
2005 & - & 6,3 & 535,1 & 257,7 & - & 26,6 & 384,6 & 122,1 \\
2006 & - & 6,9 & 656,5 & 281,8 & - & 27,0 & 424,1 & 151,0 \\
2007 & - & 8,4 & 1045,8 & 398,5 & - & 9,4 & 417,1 & 163,3 \\
\hline Gesamt & - & 36,8 & 3803,3 & 1505,1 & - & 100,0 & 2132,8 & 766,5 \\
\%-Verteilung & - & $1,5 \%$ & - & $62,5 \%$ & - & $4,2 \%$ & - & $31,8 \%$ \\
\hline
\end{tabular}

Anmerkungen:

FFG (bzw. Vorläuferorganisationen): ohne Biomed (2002), SELP (2005), IEA (2003), NAWI-Beauftragungen (2007)

AWS inkludiert folgende Programme: Double Equity, Eigenkapitalgarantien, i2, Produktfindung, ERP Technologieprogramme, F\&E Garantien (Förderbarwerte)

Quelle: Angaben der Förderagenturen. Berechnung und Darstellung KMFA.

45 Vgl. auch Abschnitt 1.3 
Die CDG zeigt eine kontinuierliche Steigerung der vergebenen Fördersummen. Die aws hatte bis 2007 auch ein steigendes Fördervolumen. Im Jahr 2007 war dann ein Rückgang der genehmigten Fördersummen zu beobachten, der auf das Auslaufen einiger Technologieprogramme (Seedfinancing inkl. LISA, protec-INNO, protec-TRANS) zurückzuführen ist. Die vergebenen Förderungen der FFG bzw. deren Vorläuferorganisationen sind im Zeitraum 2002-2004 gesunken und haben erst wieder 2005 das Niveau von 2002 erreicht. Seitdem ist das Fördervolumen des FFG kontinuierlich gestiegen. In der FFG wird der größte Teil der Förderungen durch die Basisprogramme vergeben (s.a. Abschnitt 1.3.1 dieses Berichtes), für die auch die meisten Anträge eingehen. In den Strukturprogrammen wie auch den thematischen Programmen sind 2007 sowohl die Anzahl der Anträge als auch die genehmigten Fördermittel gestiegen. Dies ist einerseits auf die erste COMET Ausschreibung, andererseits auf eine Reihe neuer Ausschreibungen im Bereich der thematischen Programme zurückzuführen. Die genehmigten Förderungen des FWF sind von 100,8 Mio. € im Jahr 2002 auf 163,3 Mio. $€$ im Jahr 2007 gestiegen. Hier ist bemerkenswert, dass während die beantragten Fördermittel im Beobachtungszeitraum um $83 \%$ gestiegen sind, die genehmigten Fördermittel lediglich um $62 \%$ gewachsen sind. Damit ist der Wettbewerb um Fördermittel in der vom FWF angesprochenen Zielgruppe über die Zeit gestiegen.

Was die operative Umsetzung der Maßnahmen der Förderagenturen anbelangt, so zeigt die Analyse, dass nicht rückzahlbare Zuschüsse das dominante Instrument sind, dessen Bedeutung über die Zeit sogar zugenommen hat. Bei rund $86 \%$ der in der Systemevaluierung untersuchten Programme werden nicht rückzahlbare Zuschüsse vergeben. An zweiter Stel- le rangiert die geförderte Beratung mit $9 \%$. Es folgen mit jeweils zwischen einem bis fünf Prozent Haftungen, gewinnabhängige Rückzahlungen und Darlehen. Risikokapital und Preise für besondere Leistungen rangieren mit jeweils 1\% am unteren Ende der Skala und haben über die Zeit an Bedeutung verloren.

Eine Zielgruppen-Analyse der Programmdokumente der insgesamt 77 Programme der direkten Forschungsförderung in Österreich zeigt, dass etwa $65 \%$ davon für Unternehmen zugänglich sind. Von diesen sind rund $41 \%$ ohne weitere Einschränkung nach Größe oder Alter des Unternehmens zugänglich. $13 \%$ sind auf KMUs beschränkt und jeweils ca. $5 \%$ legen einen Schwerpunkt auf KMUs bzw. auf die Gründungsfinanzierung. In einigen wenigen Programmen liegt der Fokus auf dem technologischen Profil der Unternehmen. Für WissenschafterInnen/ForscherInnen/ ErfinderInnen sind etwa 285 der Programme der direkten Forschungsförderung zugänglich. Hier sind die Förderungen des FWF dominant, wenn auch Förderungsprogramme der FFG ebenso für ForscherInnen als Personen erreichbar sind. Für Universitäten und andere Forschungseinrichtungen sind ca. $36 \%$ der Förderungen zugänglich. Intermediäre werden in $7 \%$ der Programme als Zielgruppe angesprochen. Andere Einrichtungen (z.B. Museen, Schulen, potentielle Anwender) werden in $11 \%$ als Zielgruppe definiert und lediglich drei Programme wenden sich an außeruniversitäre Forschungseinrichtungen. Es zeigt sich, dass die FFG gleichermaßen Unternehmen, Hochschulen und Forschungseinrichtungen anspricht. Der FWF zielt primär auf EinzelforscherInnen und in deutlich geringerem Ausmaß Unternehmen, Hochschulen und andere Forschungseinrichtungen ab. Die aws legt den Schwerpunkt auf Unternehmen und hier v.a. auf KMUs und Gründungen. 
Abbildung 21: Verteilung der genehmigte Fördersummen nach Förderagenturen und Organisationstypen (2002 - 2007)

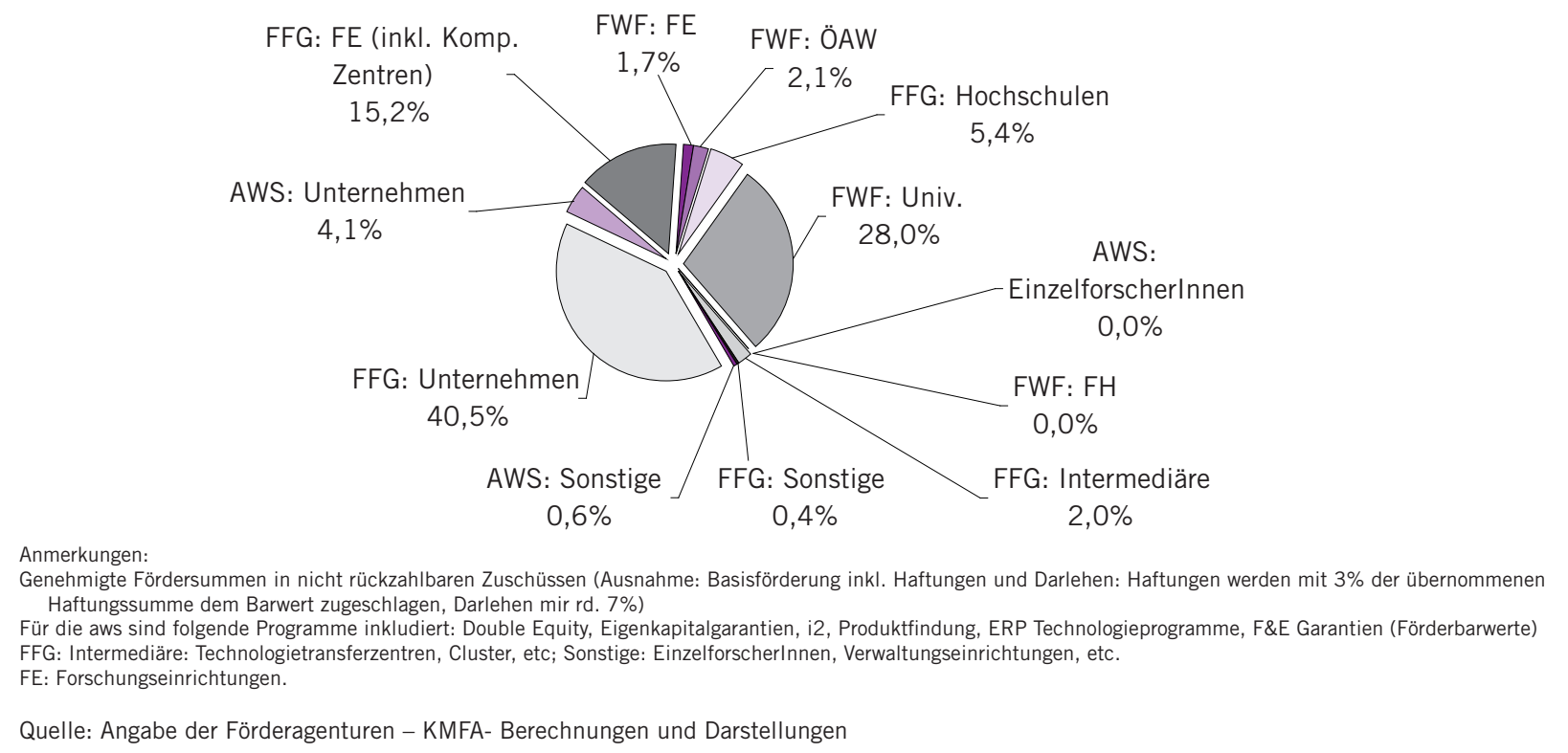

Wie Abbildung 21 zeigt, entfällt fast die Hälfte der genehmigten Förderungen auf Unternehmen (vor allem durch die FFG), ca. ein Drittel auf Universitäten bzw. ForscherInnen an Universitäten (primär durch den FWF), und knapp $20 \%$ entfallen auf Forschungseinrichtungen inklusive der Kompetenzzentren. Weiterführende Analysen haben auch gezeigt, dass für Innovationstätigkeiten im Dienstleistungssektor vergleichsweise geringe Fördervolumen vergeben werden. Andererseits zeigt sich auch, dass die Forschungsförderung durchaus imstande ist neue Akteure ins System zu bringen, wiewohl es schwierig ist festzulegen, wie viele davon Unternehmen sind, die keine oder nur wenig Erfahrung im F\&E Bereich haben.

\section{Zielgruppenüberlappungen der Programme und das Verhältnis thematisch orientierter Programme und themenfreier Förderung zueinander}

Die Analysen zeigen, dass es einer Menge verschiedener Instrumentarien bedarf, um die unterschiedlichen Anforderungen und Zielgrup- pen in geeigneter Weise ansprechen zu können. Zur Förderung von Unternehmen mit keiner Erfahrung in F\&E sind andere Instrumente notwendig, als für Unternehmen, die kontinuierlich $F \& E$ betreiben und bei denen das vorrangige Ziel ist, das Anspruchsniveau ihrer F\&E Aktivitäten zu heben. Hier hat sich in Österreich ein sehr ausdifferenziertes Fördersystem entwickelt. Wurden in den 1990er Jahren noch Förderlücken bemängelt, so wird heute eher kritisiert, dass neue Förderprogramme zumeist das Mittel der Wahl sind, sobald ein Handlungsbedarf identifiziert wird. Dies hat zu einer Vielzahl von Programmen geführt.

Programme der direkten Forschungsförderung können danach unterschieden werden, ob sie thematische Vorgaben machen, oder ob die Themenwahl frei erfolgt. Andererseits können Programme das Ziel haben strukturelle Defizite zu beseitigen, oder aber bewährte Strukturen an neue Herausforderungen anzupassen. Programme können auch nach unterschiedlichen Zielgruppen differenziert werden. Dementsprechend ergeben sich unterschiedliche 
Förderperspektiven und Interventionslogiken. Die Analyse der einzelnen Fördermaßnahmen zeigt aber, dass es aufgrund dieser Differenzen zu Überlappungen zwischen einzelnen Programmen kommt.

Im Zusammenspiel zwischen themenfreier Förderung und thematisch orientierter Förderung der FFG hat sich z.B. gezeigt, dass zu Beginn einer neuen thematisch orientierten Förderung Erstantragsteller angezogen werden. Mit zunehmender Laufzeit bildet sich jedoch eine fixe Klientel heraus und die Anzahl der Erstantragsteller nimmt ab. Es gibt Technologiebereiche, die aus der themenfreien Förderung mehr Förderungsmittel beziehen, als aus den thematischen Programmen. In manchen Fällen sind Indizien für opportunistisches Verhalten der Antragsteller hin zu Programmen mit höheren Bewilligungs- und Förderquoten gegeben. So ist im Zeitverlauf festzustellen, dass themenorientierte Ausschreibungen bei annähernd konstantem Gesamttrend zu einer
Migration der Zielgruppe aus der themenfreien Förderung führen. Dieses Bild ist jedenfalls nicht für alle Technologiebereiche gleich und eine differenzierte Betrachtung ist nötig. Die Ergebnisse der Analyse legen aber nahe, dass eine bessere und flexiblere Abstimmung der Förderangebote aufeinander die Steuerungswirkung erhöhen könnte.

\section{Zufriedenheit der Systemnutzer}

Studien in der Vergangenheit haben das stark ausdifferenzierte System der direkten Forschungsförderung in Österreich als „Förderdschungel" bezeichnet. Damit sollte veranschaulicht werden, dass die hohe Anzahl der Programme verwirrend auf Antragsteller wirkt und damit sowohl auf der Nutzer- als auch auf der Angebotsseite zu Ineffizienzen führt. Es wurde daher im Rahmen der Systemevaluierung die Nutzerzufriedenheit erhoben.

\section{Abbildung 22: Unternehmensbefragung - Anzahl der Programme bei FWF, aws, oder FFG, bei denen Unternehmen im Zeitraum 2005-2007 zumindest einen Antrag eingereicht haben, in Prozent}

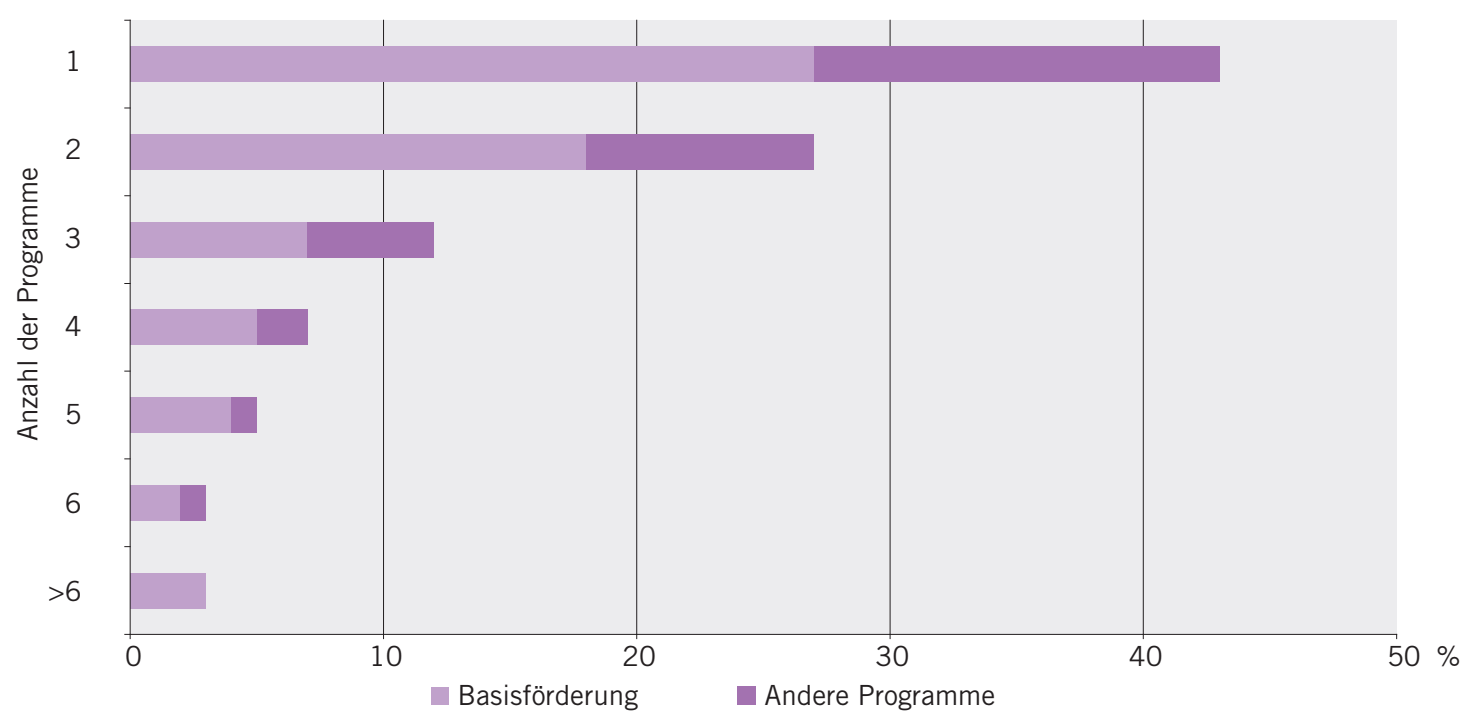

Anmerkungen: $\mathrm{n}=850$

Quelle: WIFO/KMFA Erhebung im Rahmen der Systemevaluierung der Forschungsförderung und -finanzierung - KMFA Berechnungen und Darstellung 
Die Analysen zeigen, dass sowohl Unternehmen als auch Forschungsinstitutionen systematisch Mehrfachanträge bei unterschiedlichen Programmlinien und auch bei unterschiedlichen Agenturen stellen. Abbildung 22 zeigt, dass ungefähr $40 \%$ der befragten Unternehmen angegeben haben, mindestens einen Antrag bei zwei oder drei spezifischen Programmen bei FWF, aws oder FFG eingereicht zu haben. Rund ein Fünftel hat Anträge bei vier oder mehr Programmen eingereicht. Einfachanträge betreffen in der Regel die Basisförderung der FFG. Diese wird aber auch am häufigsten mit anderen Programmen kombiniert.

Betrachtet man die Häufigkeit der Einreichungen, so zeigt sich, dass rund $50 \%$ der befragten Unternehmen im Erhebungszeitraum ein bis zwei Anträge bei FWF, aws oder FFG eingereicht haben. Etwa $1 / 3$ hat zwischen drei und sechs Anträge gestellt und rund $12 \%$ der befragten Unternehmen haben mehr als sechs Anträge bei einer der Agenturen eingereicht. Die Antragshäufigkeit steht in einem klaren Zusammenhang mit der Unternehmensgröße. $43 \%$ der Unternehmen, die lediglich ein bis zwei Anträge gestellt haben, waren Kleinstunternehmen mit weniger als neun Beschäftigten. Am anderen Ende der Skala waren von den Unternehmen, die mehr als sechs Förderanträge gestellt haben, rund $33 \%$ Großunternehmen mit mehr als 500 Beschäftigten. Bei Forschungsinstitutionen werden ähnliche Muster beobachtet, doch ist aufgrund der Bedeutung der Drittmittel für Forschungsinstitutionen eine höhere Anzahl von Anträgen zu beobachten.

Die Nutzerbefragung im Rahmen der Systemevaluierung hat ergeben, dass ihre $\mathrm{Zu}-$ friedenheit hinsichtlich der Klarheit des Förderangebots, des Zugangs zu relevanter Information und hinsichtlich der Qualität der Beratung hoch ist. Bemängelt wird vor allem der administrative Aufwand und die mangelnde Transparenz und Nachvollziehbarkeit von Förderentscheidungen. Bei Unternehmen hängt die Entscheidung für einen Antrag bei einem spezifischen Programm primär von der Wahrscheinlichkeit, dass dieser auch angenommen wird, und der Höhe der Förderungen ab. Forschungsinstitutionen orientieren sich neben diesen ersten beiden Gesichtspunkten auch an der thematischen Übereinstimmung des Programms mit ihrem Antrag.

Damit zeigt die Erhebung zur Zufriedenheit der Systemnutzer im Rahmen der Systemevaluierung, dass diese das bestehende Förderangebot nicht als "Förderdschungel" empfinden. Es zeigt sich vielmehr, dass sich die Nutzer sehr agil durch das System bewegen und sich teilweise auch dahingehend opportunistisch verhalten, dass die Möglichkeiten des komplexen Systems bestmöglich zum eigenen Vorteil ausgenutzt werden. Dies unterstreicht den Befund, dass durch eine bessere und flexiblere Abstimmung der Förderangebote aufeinander die Steuerungswirkung der direkten Forschungsförderung erhöht werden könnte.

\subsubsection{Das Zusammenspiel der steuerlichen und der direkten Forschungsförderung}

Für Unternehmen sind konzeptionelle Unterschiede in der Ausgestaltung der steuerlichen und direkten Instrumente von Bedeutung. Es ist weiters wichtig, (i) welcher Unternehmenstyp über einen direkten Förderansatz erreicht wird und welcher über einen indirekten, (ii) wie stark das Ausmaß der Mehrfachförderung ist (also gleichzeitige Nutzung beider Instrumente) und (iii) auf welche Programme sich die Mehrfachförderung konzentriert. Schließlich sind die Fördereffekte von Bedeutung und die Frage, ob es im Hinblick auf bestimmte FTI-politische Zielsetzungen eine optimale Arbeitsteilung zwischen der steuerlichen und der direkten Forschungsförderung gibt. 


\section{Nutzung der Instrumente}

Die fiskalischen Kosten der steuerlichen Forschungsförderung in Form entgangener Steuereinnahmen betrugen für das letzte verfügbare Veranlagungsjahr (2005) rund 277 Mio. $€$ (nominell). Demgegenüber stehen 272 Mio. $€$, die im Jahr 2006 über antragsinduzierte direkte Förderungen ausgezahlt wurden. ${ }^{46}$ In etwa 46-47 \% der im Jahr 2006 ausgezahlten direkten öffentlichen Fördermittel flossen in den kooperativen Bereich - auf den nicht ganz $10 \%$ der gesamten F\&E-Ausgaben entfielen. Die im kooperativen Bereich zusammengefassten (Dienstleistungs-)Einrichtungen sind mehrheitlich nicht gewinnorientiert, sondern forschen für, bzw. in Kooperation mit gewinnorientierten Unternehmen des firmeneigenen Bereichs. ${ }^{47}$

\section{Abbildung 23: Verteilung der Fördermittel nach Branchen (2005)}

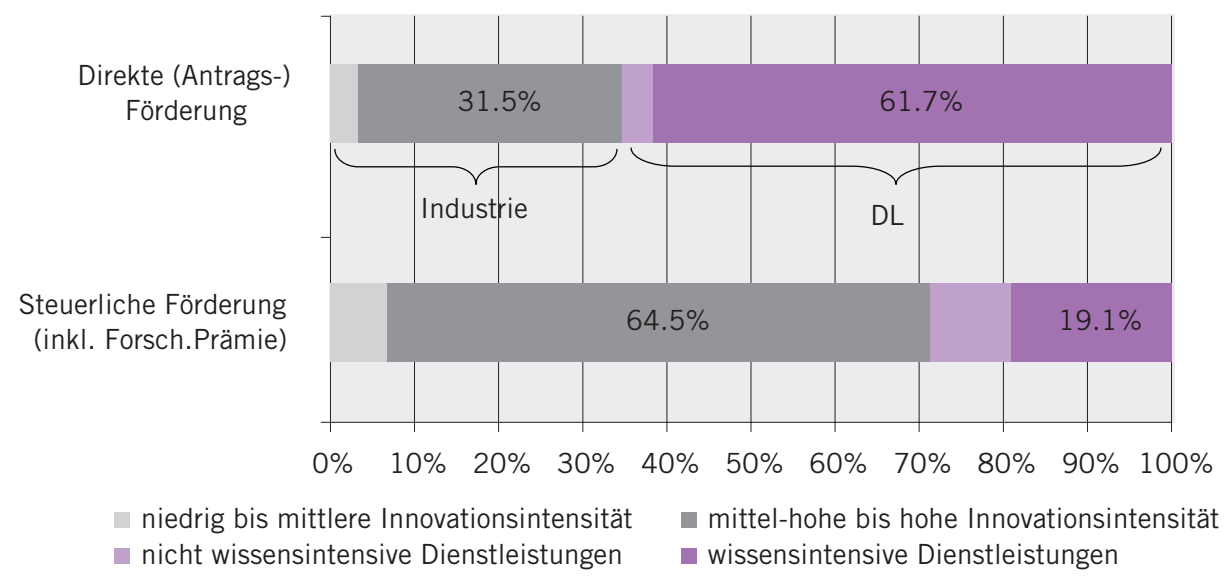

Quelle:F\&E-Erhebung 2004, 2006; BMF - Wifo Berechnungen. - Die Daten zur steuerlichen Förderung sind ausführlich in Falk (2009), Anhang 2 dokumentiert; die letzten verfügbaren Informationen betreffen das Veranlagungsjahr 2005. Angaben zu direkten Mittelflüssen in 2005 stellen interpolierte Werte für die Jahre 2004 und 2006 dar.

Die Verteilung der Mittel auf unterschiedliche Branchenaggregate erfolgt weitgehend komplementär. Abbildung 23 weist den Anteil der Mittel aus, der auf Dienstleistungsunternehmen und auf Unternehmen der Sachgüterindustrie entfällt. Gut $2 / 3$ der steuerlichen Förderung kommen der Sachgüterindustrie zugute, knapp 2/3 der direkten Förderung gehen an Dienstleistungsunternehmen. Der Dienstleis- tungssektor wird im Folgenden in wissensintensive und traditionelle Segmente unterteilt, wobei in die letztgenannten Unternehmen aus der Energie- und Wasserversorgung fallen, aus dem Bauwesen, dem Handel und dem Beherbergungs- und Gaststättenwesen. Die Werte des wissensintensiven Dienstleistungssektors sind, wie schon zuvor erwähnt, von dem kooperativen Sektor getragen.

46 Insgesamt schüttete die öffentliche Hand 2006 Forschungsmittel in Höhe von rund 428 Mio. $€$ an Unternehmen aus (s. F\&E-Erhebung 2006, Tabelle 13). Hierin inkludiert sind jedoch 156 Mio. $€$, die über die Forschungsprämie ausbezahlt wurden; der antragsinduzierte Teil beträgt somit 272 Mio. $€$.

47 Der kooperative Sektor umfasst neben außeruniversitären Forschungsinstituten wie den ARC auch öffentlich geförderte Kompetenzzentren, die ein von Unternehmen und wissenschaftlichen Institutionen gemeinsam definiertes Forschungsprogramm bearbeiten und die durch entsprechende Förderprogramme mitfinanziert werden, statistisch aber dem Unternehmenssektor zugeordnet werden. Die hohe beobachtete F\&E-Intensität wie auch die hohen Förderintensitäten der wissensintensiven Dienstleistungsbranchen ergeben sich damit aus der Konstruktion dieser Förderprogramme. 


\section{Abbildung 24: Sektorale Förderintensitäten (2005) - steuerliche Förderung vs. direkte (antragsinduzierte) Förderung}

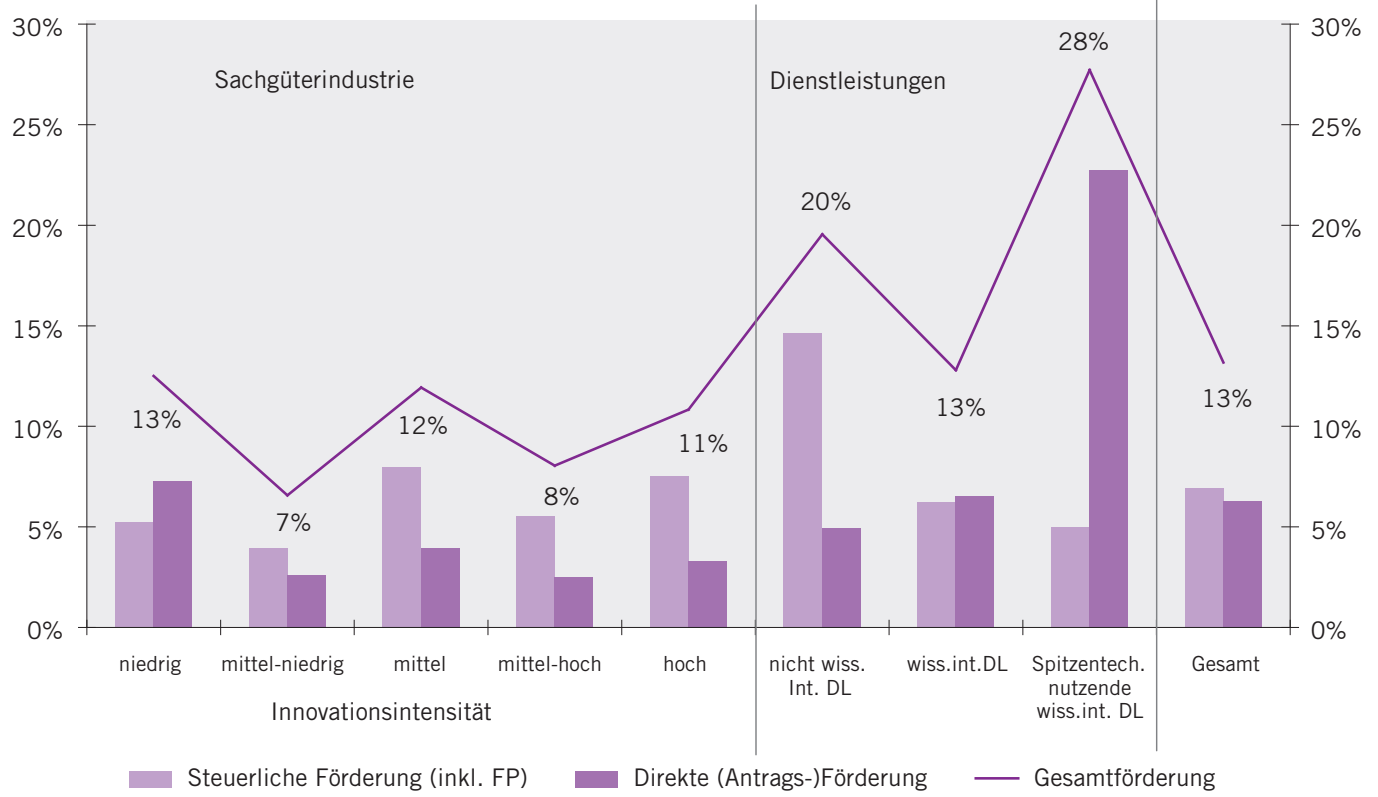

Quelle:F\&E-Erhebung 2004, 2006; BMF - Wifo Berechnungen. - Die Daten zur steuerlichen Förderung sind ausführlich in Falk (2009), Anhang 2 dokumentiert; die letzten verfügbaren Informationen betreffen das Veranlagungsjahr 2005. Angaben zu direkten Mittelflüssen in 2005 stellen interpolierte Werte für die Jahre 2004 und 2006 dar.

In Abbildung 24 werden die Wirtschaftszweige der Sachgüterindustrie nach einer neuen Taxonomie von Peneder klassifiziert, die die vielfach als unzureichend kritisierte OECDTerminologie auf Grundlage der F\&E-Intensitäten durch sog. Innovationsintensitäten ersetzt. ${ }^{48,49}$ Mit einem Anteil von 93 \% konzentriert sich die direkte Förderung etwas stärker auf Branchen mit mittel-hoher bis hoher Innovationsintensität, bzw. auf wissensintensive Dienstleistungen. Der Vergleichswert für die steuerliche Förderung beträgt $84 \%$. Beide Instrumente unterstützen damit den vielfach eingeforderten Strukturwandel hin zu einer stärkeren Wissens- und Technologieorientierung, wobei der direkten Förderung hierbei vordergründig betrachtet eine etwas stärkere Rolle zufällt. An dieser Stelle ist jedoch zum einen anzumerken, dass gemäß der gegenwärtigen Struktur des steuerlichen Förderinstrumentariums in Österreich nur diejenigen einen Nutzen aus dem indirekten Förderansatz ziehen können, die grundsätzlich gewinnsteuerpflichtig sind. Für den kooperativen Bereich gilt dies nur eingeschränkt - der kooperative Bereich ist ausnahmslos den wissensintensiven Dienstleistungen zuzuordnen. Zum anderen gehören Unternehmen der Sachgüterindustrie natürlich auch zu den Nutznießern der kooperativen Einrichtungen. Diese Auswertungen zeigen, dass der indirekte Förderansatz forschungsintensive Branchen unmit-

48 Peneder (2008)

49 Siehe dazu BMWF und BMVIT (2007). Da steuerliche Förderungen nur auf technisch-definierte Innovationen gewährt werden (Ansatz an Frascati-F\&E), bleibt das herkömmliche OECD-Konzept insgesamt gut anwendbar. Anders stellt sich die Lage für direkte Förderungen dar, die auch nicht-technisch definierte Innovationen adressiert. 
telbar begünstigt. Fast die Hälfte der direkten Fördermittel fließt in den kooperativen Sektor und erreicht forschende Unternehmen somit vornehmlich über geförderte Kooperationsprojekte in Kompetenzzentren und andere auf Forschungskooperationen ausgerichtete Förderprogramme.

Abbildung 24 setzt die Förderungen in Beziehung zu den getätigten Forschungsausgaben („Förderintensitäten“). Die Balken weisen steuerliche und direkte Förderintensitäten aus, deren Summe als Linie dargestellt ist. Da 2005 annähernd gleich viel Mittel über beide Instrumente wirksam wurden, sind folglich auch die Förderintensitäten annähernd gleich hoch $16,9 \%$ für die steuerliche gegenüber $6,3 \%$ für die direkte Förderung). Gemäß der EurostatKlassifizierung wird der Dienstleistungssektor weiter unterteilt in wissensintensive und Spitzentechnologie nutzende, wissensintensive Segmente, dem Unternehmen aus den ÖNACE-2005er Branchen 72 (Datenverarbeitung und Datenbanken) und 73 (Forschung und Entwicklung) zugerechnet werden. ${ }^{50,51}$ Der Branchenvergleich zeigt erwartungsgemäß eine starke Variabilität. Der hohe Anteil der wissensintensiven Dienstleistungen an den direkten Förderungen ergibt sich wieder durch den kooperativen Sektor, der direkte geförderte Kompetenzzentren einschließt. Die Förderintensitäten der nicht-wissensintensiven Dienstleistungen sind andererseits mit der statistischen Zurechnung einiger forschungs- intensiver Unternehmen zur Handelsbranche zu erklären. Die aggregierten Forschungsausgaben der Unternehmen mit einer geringen Innovationsintensität und die der Spitzentechnologie nutzenden wissensintensiven Dienstleister werden (sehr viel) stärker über direkte antragsinduzierte öffentliche Mittel gefördert. Ein ausgeglichenes Bild ergibt sich für wissensintensive Dienstleister. Alle anderen Branchen profitieren sehr viel stärker von der steuerlichen Förderung, die forschende Unternehmen branchenneutraler stützt, als das bei der direkten Förderung der Fall ist.

Abbildung 25 zeigt, dass sich die steuerliche Förderung als relativ größenneutral erweist. Mit anderen Worten, das relative Ausmaß der Förderung ist über alle Größenklassen annähernd gleich hoch. Die direkte Förderung begünstigt selektiver. Für Unternehmen mit mehr als 100 Beschäftigten erweist sich die steuerliche Förderung in der aggregierten Perspektive als attraktiver. Für Kleinstunternehmen (weniger als 9 Beschäftigte) ist die relative Bedeutung der direkten und indirekten Forschungsförderung aufgrund statistischer Erhebungsprobleme der Gesamtforschungsausgaben in diesem Unternehmenssegment kaum zu bewerten. Aus diesem Grund werden hier entsprechende Daten nicht ausgewiesen. Unternehmensdaten zeigen aber, dass Kleinstunternehmen stärker über steuerliche Instrumente gefördert werden, sofern sie forschen.

51 Eurostat klassifiziert auch die Nachrichtenübermittlung (ÖNACE-2005 64) als Spitzentechnologie nutzende wissensintensive Dienstleistungsbranche (WID). Diese Branche wird in der F\&E-Erhebung von StAt jedoch nicht einzeln ausgewiesen, sondern geht in das Aggregat „Verkehr und Nachrichtenübermittlung" ein (ÖNACE-2005 60-64). Eine konsistente Förderintensität erlangt man folglich nur dann, wenn Verkehr- und Nachrichtenübermittlung" als Ganzes entweder den wissensintensiven DL zugerechnet werden, oder den Spitzentechnologie nutzenden WID. 


\section{Abbildung 25: Förderintensitäten nach Unternehmensgröße (2005)}

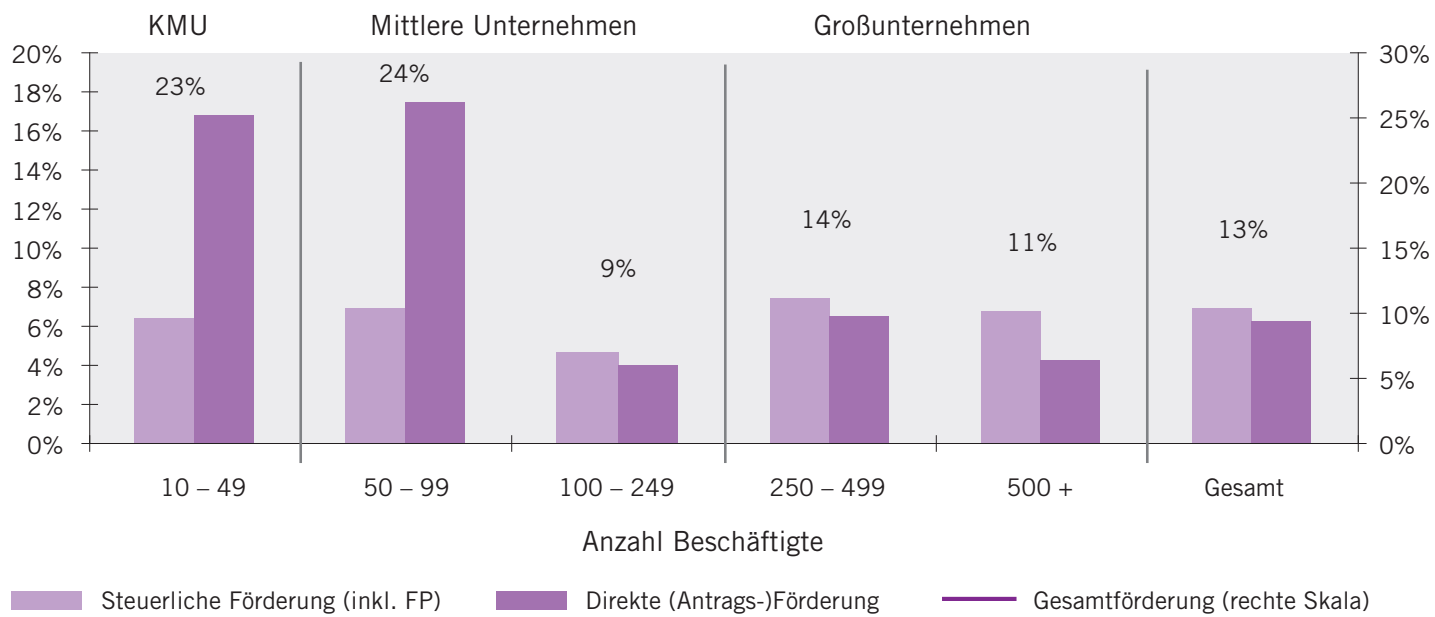

Quelle: F\&E-Erhebung 2004, 2006; BMF - Wifo Berechnungen. - Die Daten zur steuerlichen Förderung sind ausführlich in Falk (2009), Anhang 2 dokumentiert; die letzten verfügbaren Informationen betreffen das Veranlagungsjahr 2005. Angaben zu direkten Mittelflüssen in 2005 stellen interpolierte Werte für die Jahre 2004 und 2006 dar.

Um ein genaueres Bild davon zu erhalten, wie die steuerliche Förderung mit der direkten Förderung auf der Ebene der einzelnen Unternehmung zusammenspielt, wurde im Rahmen der Systemevaluierung eine Unternehmensbefragung durchgeführt. ${ }^{52}$ Von den rund 1200 FTIaktiven Unternehmen wurden - im Zeitraum 2005-2007 - $15 \%$ gar nicht gefördert. $13 \%$ der Unternehmen nutzten nur steuerliche Förderungen, $24 \%$ nur Maßnahmen der Förderagenturen. Fast jedes zweite FTI-aktive Unternehmen, nämlich $48 \%$, nutzte im besagten Zeitraum sowohl die steuerliche Förderung und wurde gleichzeitig mit mindestens einem Projekt über antragsinduzierte Förderungen erreicht. Die Mehrfachförderung konzentriert sich dabei auf sehr wenige Programme. Dies sind - in absteigender Reihenfolge - erstens die FFG Basisförderung, über die jedes zweite auch steuerlich geförderte Unternehmen im Zeitraum 2005-2007 mit mindestens einem Projekt gefördert wurde. In fast gleichem Ausmaß werden zweitens Programme der Bundesländer genutzt. Hier schlägt sich die gelebte Praxis der Bundesländerförderung durch, Basisförderprojekte regelmäßig kozufinanzieren. An dritter und vierter Stelle rangieren EU-Förderprogramme und die K-Zentren/COMETProgramme, an denen $23 \%$, respektive $22 \%$ der steuerlich geförderten Unternehmen beteiligt waren. Weitere sieben Programme wurden von wenigstens fünf Prozent der steuerlich geförderten Unternehmen genutzt - BRIDGE, Innovationscheck, Christian-Doppler Labore, FIT-IT, Double Equity und Energiesysteme der Zukunft (mit den Programmlinien „Energie/ Haus und Fabrik der Zukunft" |, sowie das erpProgramm Technologie. Alle übrigen Program-

52 Die Stichprobe konzentrierte sich zum einen auf forschungsintensive Branchen - Unternehmen aus dem High- und Medium-High-Tech Segment der Sachgüterindustrie, sowie aus den forschungsintensiven unternehmensnahen Dienstleistungen. Aus diesem Kreis wurden Unternehmen mit 10 und mehr Beschäftigten angeschrieben („Herold-Stichprobe“). Dieser Datensatz wurde angereichert mit Unternehmen, die innerhalb der letzten 5 Jahre bei Förderagenturen des Bundes (FFG und aws) um Forschungsförderung angesucht haben (aber nicht notwendigerweise tatsächlich gefördert wurden). Während diese „Agentur-Stichprobe" sicherstellt, dass der Datensatz eine hinreichende Anzahl FTI-aktiver Kleinstunternehmen und Dienstleister enthält, die in sekundärstatistischem Material erheblich und systematisch unterrepräsentiert sind, wirkt die „Herold-Stichprobe“ der Verzerrung in Richtung direkt (antrags-) geförderter Unternehmen entgegen. 
me spielen im Hinblick auf etwaige Mehrfachförderungen eine geringe Rolle.

\subsubsection{Fördereffekte}

Der letzte Abschnitt beleuchtet die Arbeitsteilung der beiden Fördermaßnahmen im Hinblick auf ihren Beitrag zu den zwei FTIpolitischen Grundzielen, nämlich einerseits die Anzahl der F\&E treibenden Unternehmen $\mathrm{zu}$ erhöhen und andererseits die Forschungstiefe der Unternehmen zu erhöhen, die bereits Forschung treiben. Abbildung 26 zeigt zunächst anhand der sektoralen Daten der F\&E-Erhebung, wie sich die Forschungsbasis im Zeitraum 1998 - 2006 entwickelt hat. Dem sind die durchschnittlichen gesamten Förder- intensitäten gegenübergestellt (i.e. Förderintensität der jeweiligen Jahre - in den jeweiligen Branchenaggregaten - gemittelt über die Zeit). Über alle Unternehmen hinweg zeigen die Daten, dass eine stärkere Förderintensität mit höheren Wachstumsraten der Anzahl forschender Unternehmen übereinstimmt. Der spezielle Befund für die Dienstleistungssektoren (nicht wissensintensive Dienstleistungen, Spitzentechnologie nutzende wissensintensive Dienstleistungssektoren) spiegelt die bereits beschriebenen Eigenheiten der statistischen Zurechnung wider und sollte daher nicht dahingehend interpretiert werden, dass das Förderwesen dem Tertiarisierungsprozess in besonderem Maße Rechnung getragen hat.

\section{Abbildung 26: Forschungsbasis vs. Förderintensität}

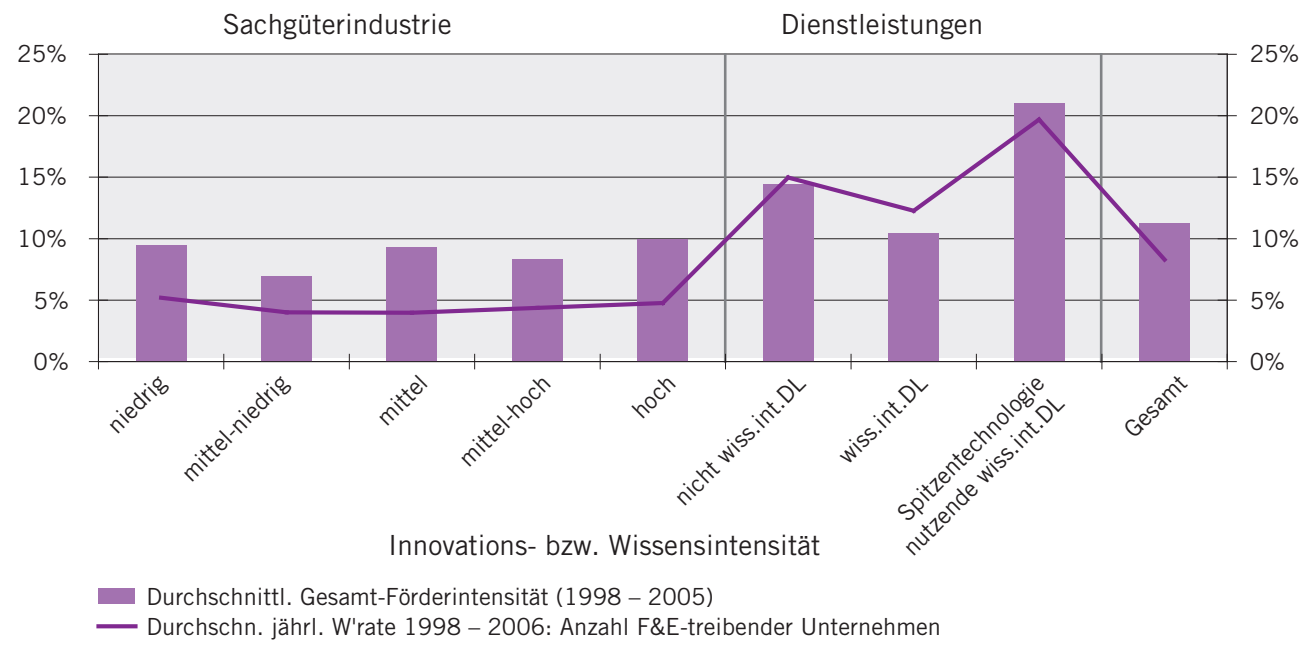

Quelle: F\&E-Erhebung 1998, 2002, 2004, 2006; BMF - Wifo Berechnungen. - Die Daten zur steuerlichen Förderung sind ausführlich in Falk (2009), Anhang 2 dokumentiert; die letzten verfügbaren Informationen betreffen das Veranlagungsjahr 2005. Angaben zu direkten Mittelflüssen in 2005 stellen interpolierte Werte für die Jahre 2004 und 2006 dar.

Auf der Grundlage der erhobenen Befragungsdaten wurde anhand ökonometrischer Analysen der Fördereffekt der direkten und indirekten Förderung auf die Innovationsleistung geförderter Unternehmen im Vergleich $z u$ nicht geförderten Unternehmen untersucht. Abbildung 27 zeigt, wie sich die Wahrscheinlichkeiten für die erfolgreiche Einführung be- stimmter Innovationstypen ändern, wenn ein Unternehmen in der einen oder anderen Art (bzw. sowohl in der einen, als auch in der anderen Art) gefördert wird. Als Vergleich dienen jeweils FTI-aktive Unternehmen, die im Zeitraum 2005-2007 nicht gefördert wurden.

Abbildung 27 zeigt zunächst, dass die Realisierung niedrigschwelliger Innovationen (An- 
passungsinnovationen, Verbesserungen bestehender Produkte, Verfahren oder Dienstleistungen, Imitationsinnovationen) in geringem Ausmaß über Förderinstrumente stimuliert wird. Bei mehrfach geförderten Unternehmen ist die Wahrscheinlichkeit, dass diese in dieser Weise innovationsaktiv sind, gegenüber der Vergleichsgruppe nicht geförderter FTI-aktiver Unternehmen über alle Innovationskategorien hinweg signifikant höher. Positive Fördereffekte zeigen sich in erster Linie im Hinblick auf die erfolgreiche Einführung wirklicher Marktneuheiten. Unternehmen, die nur steuerlich gefördert werden, weisen eine um 14 Prozent- punkte höhere Wahrscheinlichkeit für solche "radikalen" Innovationen aus im Vergleich zur Referenzgruppe der nicht geförderten FTIaktiven Unternehmen. Wird ein Unternehmen nur über direkte (Antrags-)Förderung erreicht, steigt die Erfolgswahrscheinlichkeit für Radikalinnovationen um 17 Prozentpunkte. ${ }^{53}$ Wird es sowohl steuerlich gefördert, als auch über Programme der direkten Forschungsförderung, steigt die Erfolgswahrscheinlichkeit um 24 Prozentpunkte. Dies würde darauf hindeuten, dass sich in der von den Daten abgedeckten Dreijahresperspektive nur für sehr dynamische FTI-Akteure Fördererfolge einstellen. ${ }^{54}$

\section{Abbildung 27: Wahrscheinlichkeit für erfolgte Innovationen im Zeitraum 2005 - 2007 - nach Innovations- $\operatorname{grad}^{1)}$. Jeweils Änderungen gegenüber nicht geförderten, FTI-aktiven Unternehmen ${ }^{2)}$}

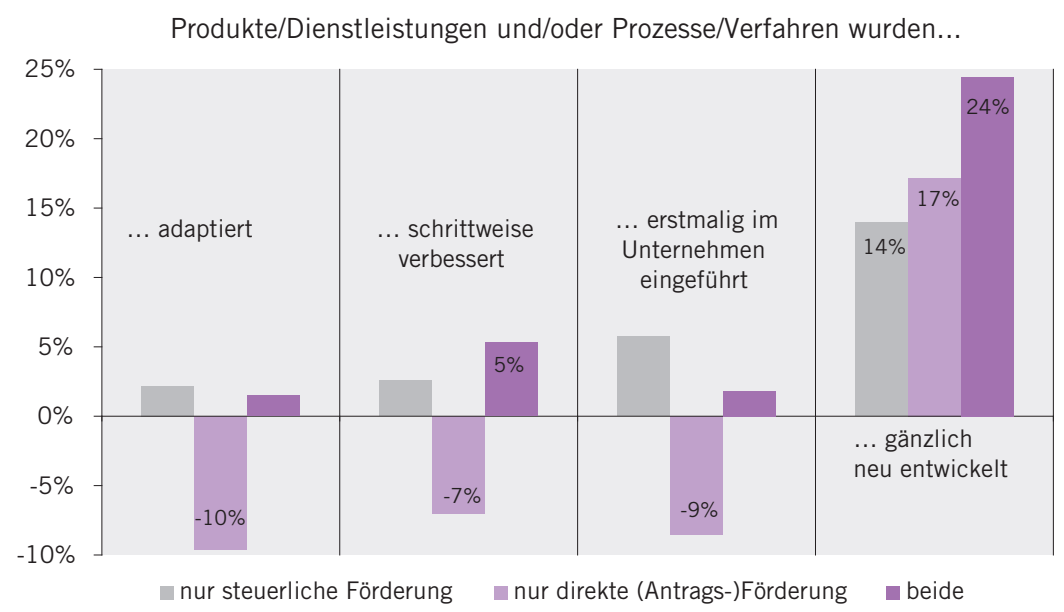

Quelle: WIFO/KMFA (2008): Forschungsförderung für Unternehmen. Fragebogen zur Beurteilung des österreichischen Forschungsfördersystems WIFO Berechnungen. - 1) Adaptierende Innovationen: N = 1132; Modifizierende/inkrementelle Innovationen (,Schrittweise Verbesserung “): N = 1136; „Smart-Follow“ Innovationen (neu für das Unternehmen, aber nicht neu für den Markt): N = 1103; Marktneuheiten/“radikale“ Innovationen: $\mathrm{N}=1146 .-2)$ statistisch signifikante Änderungen sind mit einer Größenangabe markiert. Das Befragungsdesign war so angelegt, dass jeder der Innovationstypen einzeln aufgelistet war, jeweils mit den Antwortoptionen ,ja“ oder „,nein“.

53 Die in Abbildung 27 ausgewiesenen Erfolgswahrscheinlichkeiten sind rein deskriptiver Natur. Faktoren, die die Förderentscheidung nachhaltig beeinflussen (sog. „Förderdeterminanten“), bleiben unberücksichtigt. Diese wurden anhand einfacher Probitmodelle zunächst identifiziert. Anhand der Befragungsdaten lässt sich der Förderstatus als solcher beobachten, nämlich ob ein Unternehmen über ein bestimmtes Programm (z.B. „Basisförderung") oder eine bestimmte Programmgruppe (z.B. „Thematische Programme“) gefördert wird oder nicht. Der hier verwendete Probitansatz modelliert die nicht direkt beobachtbare stochastische Variable "Förderwahrscheinlichkeit" in Abhängigkeit von Branchenzugehörigkeit, Unternehmensgröße, Alter, Organisationsform, FTI-Erfahrung, -Routine und -Intensität. Daran anschließend wurde die Wahrscheinlichkeit für „radikale“ Innovationserfolge in Abhängigkeit dieser Förderdeterminanten und des Förderstatus selbst geschätzt. Dieser ökonometrische Ansatz endogenisiert die Förderentscheidung und ",kontrolliert" somit den Umstand, dass sich bestimmte Faktoren nicht nur positiv auf den Innovationserfolg auswirken, sondern gleichzeitig die Förderwahrscheinlichkeit positiv beeinflussen. Die Koeffizienten für die entscheidenden Fördervariablen weisen quasi den Netto-Fördereffekt aus, also Innovationserfolge, die allein der Intervention zuzuschreiben sind.

54 Ein Fördererfolg wird als solcher gewertet, wenn die Innovations- oder Outputperformance nicht geförderter innovationsaktiver Unternehmen signifikant niedriger ausfällt. 
Dieses Ergebnis muss mit Vorsicht interpretiert werden. Der Förderstatus gibt noch keine Information über das Ausmaß der Förderung. Eine Detailanalyse zeigt, dass steuerlich geförderte Unternehmen mindestens ein antragsinduziertes Förderprojekt pro Jahr benötigen, um signifikant häufiger Marktneuheiten einführen zu können als die Vergleichsgruppe. Der Fördereffekt exzellenzorientierter Programme ist hierbei höher im Vergleich zu den Programmen der angewandten Forschung. Es stehen hinter diesen Ergebnissen auch keine Fördervolumina. Da Förderungen mit Exzellenzanspruch ein zunehmend größeres Gewicht an den gesamten vergebenen Mitteln einnehmen, kann das Ergebnis darauf hindeuten, dass der Förderstatus für radikale Innovationen eine bedeutendere Rolle spielt als für Innovationserfolge auf niedrigeren Stufen.

Diese Aspekte konnten im Rahmen der Evaluierung auf Grundlage der erhobenen Daten und weitergehender Befragungen nicht restlos geklärt werden. Das Ergebnis, dass sich das Zusammenspiel von direkter und indirekter Förderung positiv auf den Fördererfolg sehr dynamischer FTI-aktiver Unternehmen auswirkt, ist sehr robust und deckt sich weitgehend mit internationalen Befunden zu dem Zusammenspiel der direkten und indirekten Forschungsförderung (z.B. De Jong et al. 2007). Für diese Unternehmen wirken die steuerlichen und die direkten Förderungen komplementär. Unternehmen zeigen sich nur dann als deutlich erfolgreicher im Hinblick auf ihre F\&E-Ausgabenperformance, ihre Wachstumsperformance und bei der Einführung von Marktneuheiten, wenn sie über beide Fördermechanismen erreicht werden.

\subsubsection{Weitere wichtige Befunde der Systemevaluierung}

Neben den hier schwerpunktmäßig behandelten Aspekten der direkten und indirekten (steuerlichen) Förderung der Forschung wurden in der Systemevaluierung auch die Rahmenbedingungen für Forschung und Innovation, die Steuerung des Systems der Forschungsföderung und -finanzierung, die Wirkung der Grundfinanzierung auf Forschungsinstitutionen, sowie das Stärken-Schwächenprofil des Systems untersucht. Die Befunde hinsichtlich der Systemsteuerung zeigen, dass sich die Steuerungsmechanismen kaum an die sich wandelnden Rahmenbedingungen anpassen. Dies ist einerseits auf das Fehlen einer normativen Orientierung des Systems der Forschungsförderung und -finanzierung zurückzuführen, andererseits aber auch auf eine fortschreitende Fragmentierung von Kompetenzen und eine enge Vernetzung der maßgeblichen Entscheidungsträger im System. Dies führt, so die Analysen, einerseits zu engen, selbstbezogenen, wenig offenen Kommunikations- und Organisationsstrukturen, die die Anpassungsfähigkeit des Systems an neue langfristig wirkende Herausforderungen und reflexives Lernen innerhalb des Systems erschweren. Andererseits ermöglichen die beobachteten Kommunikations- und Interaktionsstrukturen aber im Rahmen einer kurzfristigen Perspektive das System graduell zu verbessern.

Die Analyse der Rahmenbedingungen zeigt, dass neben dem Mangel an privatem Beteiligungskapital für innovative Unternehmen vor allem der Mangel an hochqualifizierten Humanressourcen eine zentrale Herausforderung für die weitere Entwicklung der Innovationsfähigkeit der österreichischen Wirtschaft darstellt. Die Stärken-Schwächen Analyse des Systems zeigt auch, dass Humanressourcen und Bildungsmaßnahmen die stärkste Wirkung auf das Innovationssystem entfalten können. Die Ergebnisse der Analysen der Systemevaluierung weisen daher einer als Querschnittsmaterie begriffenen FTI-Politik abseits der Förderagenturen ein sehr viel stärkeres Mandat zu. 


\subsubsection{Zusammenfassung}

Die Ergebnisse der Systemevaluierung zeigen, dass das System der Forschungsförderung und -finanzierung aus einer Vielzahl von Instrumenten besteht, die eine breite Zielgruppenabdeckung, Zielgruppenerreichung und Zielgruppenzufriedenheit zur Folge haben. Das System hat erfolgreich Impulse zur Verbesserung der Forschungs- und Innovationstätigkeit in Österreich gesetzt. Auf der Seite der Evaluatoren bestehen aber Zweifel, inwieweit Organisation, Schwerpunktsetzung und verfügbare Instrumente des Systems imstande sein werden, den aus ihrer Sicht notwendigen Wandel $\mathrm{zu}$ einem von Wissenschaft, Technologie und Innovation getragenen Wachstumsparadigma zu vollziehen. So zeigen die Analysen, dass der Mangel an hochqualifizierten Humanressourcen auf allen Ebenen einen Flaschenhals und damit die große Herausforderung an die Politik darstellt. Generell zeigen die Analysen auf, dass die gesamte Forschungsförderungs- und Forschungsfinanzierungspolitik stärker mit anderen Politikbereichen verbunden werden muss, die einen Einfluss auf Innovation haben, so wie Bildung, Finanzierung udgl.

Auf der Ebene der Detailbefunde, die das Konsortium der Systemevaluierung auf Basis von eigenen Untersuchungen erstellt hat, zeigt sich, dass das Ausmaß der steuerlichen Förderung in den vergangenen Jahren überschätzt wurde. Entgegen den Schätzungen des Rechnungshofs und des Bundesministeriums für Finanzen liegen die Kosten der steuerlichen F\&E Förderung etwas über 250 Mio. $€$ zu konstanten Preisen. Was die Zielgruppen der steuerlichen Förderung anbelangt, so zeigen die Untersuchungen im Rahmen der Systemevaluierung, dass Unternehmen mit mehr als 100 Beschäftigten ca. $40 \%$ der Förderempfänger für den FFB-Alt und 30 \% für den FFB-Neu darstellen. Diese Unternehmen haben im Jahr 2005 ca. $90 \%$ der gesamten steuerlichen Förderung aus dem FFB-Alt und dem FFB-Neu in Anspruch genommen. Andererseits hat die Einführung der Forschungsprämie zu einer Ausweitung der Inanspruchnahme steuerlicher Förderung geführt. Viele der neu gewonnenen Nutzer sind Klein- und Mittelbetriebe (KMU), die auch bezüglich der Fördervolumen die Hauptnutznießer der Forschungsprämie sind.

Bezüglich der direkten Förderung der Forschung zeigt sich, dass unterschiedliche Interventions- und Handlungslogiken zu einer Überlappung des Angebots bei den Zielgruppen führen. Die Ergebnisse der Analyse legen somit nahe, dass eine bessere und flexiblere $\mathrm{Ab}$ stimmung der Förderangebote aufeinander die Steuerungswirkung der direkten Forschungsförderung erhöhen könnte. Hinsichtlich der Nutzerzufriedenheit deuten die Ergebnisse darauf hin, dass der oft ins Treffen geführte „Förderdschungel" von den Nutzern nicht als solcher empfunden wird. Es zeigt sich vielmehr, dass sich die Nutzer sehr agil durch das System bewegen und sich auch teilweise dahingehend opportunistisch verhalten, die Möglichkeiten des komplexen Systems bestmöglich zum eigenen Vorteil zu nutzen. Ergebnisse deuten in der Tat darauf hin, dass Nutzer sich mit dem Ziel im System bewegen, Förderwahrscheinlichkeit und Höhe der Förderzusagen zu maximieren. Damit entpuppt sich der "Förderdschungel" in einem positiven Sinn eher als "Fördersupermarkt". Die Untersuchungen der Evaluierung deuten auch darauf hin, dass Ziele und Aufgaben der Programme klarer definiert werden müssen. Sie sollen auch vermehrt auf Exzellenz und radikale Innovation hinwirken.

Die Analyse des Zusammenspiels der steuerlichen und der direkten Förderung zeigt, dass beide Förderungsformen notwendig sind. Das Ergebnis, dass sich das Zusammenspiel von direkter und indirekter Förderung positiv auf den Fördererfolg sehr dynamischer FTI-aktiver Unternehmen auswirkt, ist sehr robust und deckt sich weitgehend mit internationalen Be- 
funden zu dem Zusammenspiel der direkten und indirekten Forschungsförderung

\subsection{Der österreichische Forschungsdialog}

In Alpbach 2007 wurde unter dem Titel „Österreichischer Forschungsdialog" ein Prozess initiiert, der zum Ziel hatte, "Ideen und Inputs zu sammeln, um Österreich als attraktiven Forschungs- und Wissenschaftsstandort bis $2020 \mathrm{zu}$ positionieren" (BMWF 2008, S. 4). Durch die Einbindung möglichst vieler Akteure und Meinungsbildner sollte damit auch der Grundstein für eine FTI-Strategie gelegt werden. Die neue Bundesregierung bezieht sich im Regierungsprogramm ausdrücklich auf die Ergebnisse des Forschungsdialogs als Input zur Erstellung einer nationalen FTI-Strategie.

Unter der Federführung des BMWF wurde in enger Kooperation mit dem BMVIT sowie BMWA ein Dialog mit wichtigen Stakeholdern und Politikinteressierten gestartet, welcher mittels Dialogforen und Kamingesprächen quer durch Österreich reiste. Insgesamt haben so in den Jahren 2007/08 über 2.200 Personen an Live-Veranstaltungen teilgenommen. Darüber hinaus wurde dieser Prozess zusätzlich durch Onlinedialoge und Joint Ventures, welche durch Dritte veranstaltet wurden, unterstützt.

Mit insgesamt 21 für die FTI-Politik relevanten Themen wurde der Diskurs bewusst sehr breit angelegt, was sich als unabdingbar für die Akzeptanz, die Teilnahmebereitschaft sowie die Diskussionen herausgestellt hat. Einige der zentralen Befunde, Wahrnehmungen und Positionen sollen nun kurz dargestellt werden:

Österreich hat in den vergangenen Jahren einen eindrucksvollen Aufhol- und Überholprozess im Bereich Forschung und Entwicklung absolviert. Die F\&E-Ausgaben sind sowohl absolut als auch relativ gesehen maßgeblich gestiegen, die Qualifikationsstruktur der Arbeitskräfte hat sich in den Unternehmen erhöht und ein genereller Trend zu höherer Bildung hat sich manifestiert. Österreich darf sich somit zu jenen Ländern zählen, welche sich dem Barcelona-Ziel von $3 \%$ erfolgreich genähert haben. Um diesen Wandel nun auch in Zukunft fortzusetzen, gilt es als essentiell, neben der Weiterentwicklung und Verbesserung der Rahmenbedingungen zur Förderung der anwendungsorientierten Forschung, auch die Förderung der Grundlagenforschung weiter auszubauen. Bislang rangiert Österreich 2006 mit etwa 1064 Mio. Euro (oder 0,41\% des BIP) für die Grundlagenforschung international gesehen im Mittelfeld; ein aus dem Forschungsdialog heraus formuliertes Ziel ist, die Investitionen in die Grundlagenforschung bis 2020 auf das Niveau von führenden Nationen (wie bspw. die Schweiz), nämlich auf etwa $1 \%$ des BIP, zu steigern.

Einen wesentlichen Erfolgsfaktor stellen dabei die österreichischen Hochschulen dar. Sind diese per Implementierung des UG 2002 aufgefordert, sich dezidiert mit einem Forschungsprofil auszuweisen und sich am Wissensmarkt zu positionieren, so gilt es hier insbesondere die Exzellenz zu fördern. Ziel ist, Exzellenz vor allem im Wettbewerb entstehen zu lassen, wobei bestehende Kompetenzen zu nutzen sowie die Berufung von jungen WissenschafterInnen zu fördern sind. Atypische Karriereverläufe und Mobilitätserfahrung sind dabei ebenso anzuerkennen wie die Gewährung der Option auf durchgängige Laufbahnsysteme. Was Letztere betrifft, so geht es vor allem darum, ,"early stage researchers" auf DoktorandInnen- wie auch Postdoc-Ebene besser (finanziell) abzusichern.

Besonderen Augenmerks bedarf in diesem Kontext auch die Förderung von Frauen in Wissenschaft, Forschung und Technologie. Österreich stellt mit einem Professorinnenanteil von 11,3\% im Jahr 2006 das Schlusslicht unter den Universitäten im europäischen Vergleich dar, was dringenden Handlungsbedarf widerspiegelt. Dabei soll es nicht nur Ziel 
sein, den Frauenanteil in Führungspositionen zu erhöhen, sondern auch weiterhin GenderDimensionen in der Forschung und deren Förderung zu setzen sowie Karrieremodelle zu entwickeln, welche eine bessere Vereinbarkeit von Beruf und Familie erlauben.

„Nachhaltigkeit" spielt eine immer größere Rolle in modernen Industrienationen. Problemlösungen sowie die Befriedigung bestimmter gegenwärtiger oder antizipierter gesellschaftlicher Bedürfnisse stehen dabei im Mittelpunkt und veranlassen die FTI-Politik, sich vermehrt mit gesellschaftsrelevanten Fragestellungen und somit mit einer neuen Missionsorientierung auseinanderzusetzen. Diese charakterisiert sich vor allem durch ihre thematische Breite und die Verschränkung mit anderen Politikbereichen (wie Umwelt, Klima, Energie, Gesundheit, Verkehr etc.) und hält damit seit Jahren Einzug in verschiedene nationale Entwicklungs- und Umweltpläne, Infrastrukturrahmenpläne etc. In diesem Zusammenhang kommt auch den Geistes-, Sozial- und Kulturwissenschaften (GSK) verstärkt Bedeutung zu. Ihre Aufgabe ist es, die jeweiligen gesellschaftlichen Anforderungen und Bedürfnisse bzw. auch die Problemwahrnehmung kritisch zu reflektieren. Entsprechende institutionelle, infrastrukturelle und monetäre Rahmenbedingungen sind daher in Zukunft zu schaffen, um nicht nur eine Qualitätssteigerung, eine weitere Internationalisierung sowie eine disziplinenübergreifende Vernetzung voranzutreiben, sondern auch um die Ergebnisse der Gesellschaft - mit der Gewährleistung, dass die Erkenntnisse unter Einhaltung ethnischer Grundregeln gewonnen wurden zu kommunizieren.

Österreich verfügt mittlerweile über eine - auch international anerkannte - hohe „Kooperationskultur". Wesentlicher Bestandteil dieser Kultur ist es, auf eine breite Palette von Fördermaßnahmen zurückgreifen zu können, die allesamt die Förderung der „industry-sci- ence linkages" zum Ziel haben und somit die rasche Verwertung neuer wissenschaftlicher Erkenntnisse sicherstellen. Gerade die Ausweitung von Technologie- und Strukturprogrammen hat diesen Prozess seit den 90er Jahren unterstützt, sodass zur Förderung des Wissensund Technologietransfers heute prominente Förderprogramme wie die Kompetenzzentren und -netzwerke, deren Nachfolge COMET, die Christian Doppler Gesellschaft (CDG), FHplus, AplusB und Bridge zur Verfügung stehen. Auch sind die jüngsten Maßnahmen zwecks Regelung und Verwertung des geistigen Eigentums an den Universitäten zu nennen.

Der Unternehmenssektor stellt aber nicht nur im Wissens- und Technologietransfer eine tragende Säule dar, auch ist die deutliche und nachhaltige Steigerung der F\&E-Quote Österreichs auf die Ausweitung von F\&E-Aktivitäten in Unternehmen zurückzuführen. Dies spiegelt sich sowohl in der zunehmenden Anzahl forschender Unternehmen als auch in deren Forschungsintensität wider. Dennoch aber kann der Anteil jener Unternehmen, insbesondere der KMU, die aktiv F\&E betreiben, in Zukunft durchaus noch gesteigert werden. So stellen neben den bestehenden Unternehmen gerade die jungen, innovativen bzw. die Unternehmensgründungen eine wichtige Zielgruppe der künftigen Förderpolitik dar. Aufgabe ist es, insbesondere die jungen technologieintensiven Unternehmen zu unterstützen, indem deren Hauptproblem, nämlich die Finanzierungsfrage, Unterstützung erfährt. Dabei ist zum einen eine adäquate Verfügbarkeit von Risikokapital sowie zum anderen ein funktionierender Private Equity Markt zu nennen. Zusätzlich gilt es, auch den akademischen Spin-off Gründungen, welche insbesondere zur Durchlässigkeit zwischen Wissenschaft und Wirtschaft beitragen, verstärkt Aufmerksamkeit zukommen zu lassen.

Eine weitere treibende Kraft zeigt sich in der Dynamik der Internationalisierung, welche in 
den letzten Jahren zunehmend auch den Bereich der F\&E erfasst hat. So lässt sich beobachten, dass Österreichs Unternehmen heute im Bereich der F\&E international viel aktiver agieren als jene Deutschlands. Ein Hauptgrund dafür mag - aus österreichischer Sicht - die Enge des heimischen Marktes sein und somit das Bestreben, Produkte und Prozesse an regionale Kundenwünsche anzupassen. Letzteres ist auch ausschlaggebend dafür, dass Unternehmen zunehmend in emerging economies wie China tätig sind. Dies hat zur Folge, dass China heute für Österreich nach den USA nicht nur der zweitwichtigste außereuropäische Handelspartner ist, sondern bereits rund $1 \%$ aller österreichischen Exporte und Direktinvestitionen nach China fließt. Mit der Internationalisierung geht somit auch eine Verschärfung des Standortwettbewerbs einher. Österreich steht angesichts dessen vor der Herausforderung, seine Attraktivität für Headquarters und F\&E treibende Unternehmen gemeinsam mit leistungsfähigen Forschungsinstitutionen weiterzuentwickeln. Im Rahmen dessen sind weiterhin F\&E-Beziehungen nicht nur mit führenden Industrienationen, sondern auch mit emerging economies auszubauen sowie Plattformen, Programme und Initiativen auf europäischer Ebene zur Entwicklung von Zukunftstechnologien zu nutzen.

Österreichs Weg zum 3\%-Ziel scheint erfolgreich zu sein und wird somit von einem bemerkenswerten Ausbau von F\&E-Aktivitäten - sowohl im Unternehmensbereich als auch im Hochschulsektor - unterstützt. Darüber hinaus wird dieser Prozess durch die steuerliche F\&E-Förderung, wie auch durch die projektbezogene F\&E-Förderung forciert, welche zudem als klassische Politikinstrumente von innovationsstimulierenden Aktivitäten im
Bereich des öffentlichen Beschaffungswesen in Zukunft begleitet werden sollen. Alles in allem ist es aber gerade die Governance und somit mitunter die Effizienz des Policy-Mix, welche für eine erfolgreiche FTI-Politik in den nächsten Jahren ausschlaggebend sein wird.

\subsubsection{Der Bericht der CREST Policy-Mix- ExpertInnengruppe}

Der Österreichische Forschungsdialog wurde auch um einen Policy-Mix-Peer-Review aus externer Sicht ergänzt (CREST 2008). ${ }^{55}$ Dieser Review fand im Mai 2008 statt und ist Teil des von CREST, dem Ausschuss für wissenschaftliche und technische Forschung der Europäischen Union, initiierten und entwickelten Policy-Mix-Peer-Review-Prozesses, dem sich bereits mehrere Länder unterzogen haben. ${ }^{56}$ Das Policy-Mix-Peer-Review-Team bestand dabei aus VertreterInnen von Mitgliedstaaten der EU und einer Beobachterin von der Europäischen Kommission und wurde von einem unabhängigen Berater geleitet. Ziel war es, F\&E-politische Optionen für Österreich zu diskutieren. Im Folgenden werden einige der Empfehlungen und Einschätzungen (bzw. Korrektur derselben) durch CREST kurz vorgestellt:

Zentraler Punkt dieses Reviews ist, dass Österreich derzeit eine gemeinsame, ganzheitliche Vision sowie kohärente politische Maßnahmen zur Stimulierung der Entwicklung des gesamten F\&E- und Innovationssystems fehlen. Die mangelnde Gesamtstrategie sowie Defizite bzgl. der Koordinations- und Kommunikationsmechanismen würden laut Einschätzung der CREST-ExpertInnen zu Ineffizienzen in der österreichischen Governance-Struktur führen, welche sich in manchen Bereichen punktuell in Kleinteiligkeit, Überschneidun-

55 http://www.bmwf.gv.at/euinternationales/euforschung/lissabon_prozess/crest/

56 Peer-Review-Berichte auf: http://www.era.gv.at/space/11442/directory/11661/doc/11662.html 
gen, unklaren Zuständigkeitsverteilungen in Bezug auf die Förderagenturen sowie einem „Programmdschungel" manifestierten. Beispielgebend führen die CREST-ExpertInnen Länder wie Finnland, Dänemark oder die Niederlande an, wo die obersten politischen EntscheidungsträgerInnen in viel stärkerem Ausmaß in strategischen Koordinationseinrichtungen präsent sind. Demnach würde „,[...] Österreich von einer Neubewertung seiner existierenden Strukturen und Prozesse zur Formulierung ganzheitlicher F\&E- und innovationsbezogener Strategien und der daraus folgenden Artikulierung eines kohärenten policy mix profitieren" (S. 32).

Bezüglich der Rolle der Beratungsgremien greift der CREST-Review eine bereits durch die OECD in ihrer Länderstudie über Österreich formulierte Empfehlung auf (OECD 2007), i.e. eine stärkere (personelle) Verschränkung des Rates für Forschung und Technologieentwicklung (RFT) sowie des Wissenschaftsrates, um dadurch die Rolle, die Bedeutung und das Gewicht eines derartigen Gremiums zu stärken. Dies hängt von der Politik, die einem Beratungsgremium qua Inanspruchnahme und Reaktion auf Empfehlungen eine bestimmte Bedeutung zuerkennt, wie auch vom Beratungsgremium selbst ab. Hier empfiehlt das PeerReview-Panel eine Überprüfung der Rolle, der Zusammensetzung und des modus operandi des RFT, um dessen Beitrag für die politische Entscheidungsfindung zu stärken.

Eine in Österreich bereits seit langem diskutierte und von den CREST-ExpertInnen aufgegriffene Frage ist jene, inwieweit das Förderniveau über ein extrem breites Spektrum von Technologiebereichen und Industriesektoren aufrechtzuerhalten ist, oder ob es doch mehr Ressourcen in Bereiche mit strategischer Schlüsselrelevanz für Österreich umzulenken gilt. „Viele wirtschaftlich größere Länder als Österreich haben die Gefahr erkannt, die darin liegt, Ressourcen dünn zu verteilen ..." (S. 13).
Allerdings verkennen die CREST-ExpertInnen, dass (i) die technologischen Schlüsselbereiche der wirtschaftlich großen Länder auch jene wirtschaftlich kleiner Länder sind und (ii) es für den Fall, dass sich ein kleines Land für eine riskante Nischenstrategie entscheiden sollte, schwierig ist, durch wen und wie ein für Österreich strategisch relevanter Bereich definiert werden soll. Denn dies hängt ganz wesentlich vom Standpunkt des- oder derjenigen $a b$, der oder die diesen Bereich definiert.

Auch ist zu überdenken, inwieweit die hohe Vielfalt an Politik- und vor allem Förderinstrumenten eine gewisse Abhängigkeit der Unternehmen von öffentlichen Förderungen und damit eine "Förderkultur" hervorruft, welche sich negativ auf das eigentliche Innovationsverhalten von Unternehmen auswirkt. Allerdings ist diesbezüglich den Einschätzungen von CREST entgegenzuhalten, dass die internationalen Statistiken (wie der European Innovation Scoreboard) hier mit Vorsicht $\mathrm{zu}$ interpretieren sind. Der Innovationscheck beispielsweise wird den Anteil geförderter Unternehmen wesentlich erhöhen, ohne dass dieser aufgrund seines Fördervolumens (bis zu 5.000 $€$ pro Unternehmen) marktinduziertes Innovationsverhalten ersetzen könnte.

Weiterhin gilt es die Möglichkeit für Forschende, mit internationalen Partnern zu arbeiten, zu maximieren; Probleme der Fragmentierung und der Mangel an kritischer Masse müssen auf allen Ebenen mittels Teilnahme an Initiativen wie ERANETs oder Technologieplattformen überwunden werden. Eine diesbezügliche Strategie sollte daher auch die Wechselwirkung zwischen nationalen Programmen und EU-Rahmenprogrammen betonen, um sicherzustellen, dass die Möglichkeiten für österreichische Forschende, mit internationalen Partnern zu arbeiten, maximiert werden. Die weiteren Beteiligungen von Forschungsprogrammen an ERANETs, Technologieplattformen sollten im Rahmen einer Gesamtstrate- 
gie unter Berücksichtigung auch der Initiative für die österreichische Beteiligung an JointProgramming-Aktivitäten geschehen. Ziel soll es auch sein, den Handlungsspielraum für die zunehmende internationale Kooperation mit im Forschungsbereich führenden Ländern zu erhöhen.

Ausführlich werden der Wissenschaftssektor sowie der Sektor industrieller F\&E in dem CREST-Review behandelt, wobei hier anfangs eine Einschätzung gemacht wird, die zu hinterfragen ist. Wenn von einem „substantiellen Anstieg der öffentlichen Förderbeträge für Forschung an den Universitäten" (S. 21) und von einem „geringen Anstieg der öffentlichen Unterstützung für industrielle F\&E" (S. 25) die Rede ist, so ist diese Einschätzung auf der Basis der F\&E-Erhebungen 2002 bis 2006 nicht nachvollziehbar (siehe Abbildung 6). Für diesen Zeitraum stieg nämlich die öffentliche F\&EFörderung des Hochschulsektors lediglich um $17 \%$, die der industriellen F\&E hingegen um $55 \%$ (exklusive der Forschungsprämie). Unter Berücksichtigung der Forschungsprämie stieg sie um $144 \%$.

Was die Universitäten betrifft, so stellen für die CREST-ExpertInnen die Leistungsvereinbarungen ein gutes Instrument dar, die Zielsetzungen in Zukunft ambitionierter zu gestalten. Die Autonomie ermöglicht es den
Universitäten maßgeschneiderte Strategien dafür zu entwickeln.

Bezüglich der Förderung industrieller F\&E war das Review-Team überrascht über das hohe Ausmaß der Unterstützung von Einzelfirmen und den geringen Förderanteil kollaborativer F\&E.

Im Bereich der Humanressourcen verdeutlicht der CREST-Review den Handlungsbedarf in Österreich. Das Thema Humanressourcen im Bereich F\&E und Innovation wird von drei Hauptthemen dominiert: die Anzahl, die Qualität sowie die Mobilität des qualifizierten Personals. Hier gilt es vor allem entsprechende Anreize zu setzen, um die Anzahl der Personen mit Tertiärbildung zu erhöhen.

Insgesamt weist der CREST-Review 24 Empfehlungen auf ${ }^{57}$, welche allesamt zusätzlich zu den Erkenntnissen, die aus dem Österreichischen Forschungsdialog gewonnen wurden, sowie den Ergebnissen der Systemevaluierung in die strategische Ausrichtung der österreichischen FTI-Politik einfließen sollen. Dabei besteht laut CREST-Review die zentrale Herausforderung in Zukunft vor allem darin, begonnene institutionelle Reformen bei forschenden und fördernden Einrichtungen weiterzuführen sowie eine Optimierung der Governance in der FTI-Politik anzustreben.

57 Anregungen der CREST-Review bezüglich der Bewilligungsquote des FWF siehe Kapitel 1.3.4. Die Empfehlungen bezüglich Evaluierung finden in Kapitel 5 Erwähnung. 


\section{Aspekte des österreichischen Wissenschaftssystems}

\subsection{Struktur und Entwicklung der Grundlagenforschung in Österreich}

Die Thematik Grundlagenforschung ist jüngst in Österreich zunehmend in den Vordergrund der forschungspolitischen Diskussion geraten. Dies ist vor dem Hintergrund zu sehen, dass der beachtliche Aufholprozess Österreichs hinsichtlich der aggregierten F\&E-Quote mittlerweile als weitgehend „abgeschlossen" betrachtet werden kann und Österreich nunmehr eine Position über dem jeweiligen Durchschnitt der EU-15 (sowie der EU-27) als auch der OECD einnimmt. Die noch in den 1990er Jahren zentral diskutierten Defizite im Innovationssystem Österreichs, nämlich der mangelnde Beitrag des Unternehmenssektors sowie das geringe Ausmaß an Interaktionen zwischen Wissenschaft und Wirtschaft, konnten in den vergangenen Jahren - nicht zuletzt aufgrund erfolgreicher programmatischer Maßnahmen von Seiten der öffentlichen Hand - deutlich abgebaut werden. Auch das lange Zeit als sehr ambitioniert eingestufte $3 \%$-Ziel hinsichtlich der F\&E-Quote kommt in die Nähe der Realisierung. Somit verlagert sich die Zieldiskussion auf die Ebene einzelner Forschungsarten. Vor dem Hintergrund dieser Entwicklung der F\&E-Landschaft in Österreich werden also auch Rolle und Aufgabe der Grundlagenforschung neu diskutiert. Im Rahmen dieses Ka- pitels soll zunächst das quantitative Gewicht der Grundlagenforschung in Österreich in Relation zum BIP sowie zu den gesamten F\&EAusgaben beleuchtet und im internationalen Vergleich dargestellt werden. Anschließend werden einige zentrale bibliometrische Outputindikatoren der Grundlagenforschung diskutiert.

\subsubsection{Monetäre Aufwendungen für Grundlagenforschung im internationalen Vergleich}

Mit 1,06 Mrd. $€$ beträgt der Anteil der Grundlagenforschung am BIP in Österreich derzeit (2006) 0,41\%. Damit befindet sich Österreich in einem „mittleren" Bereich der OECD-Länder (vgl. Abbildung 28). Beachtet werden muss dabei, dass einige Länder seit geraumer Zeit (darunter auch z.B. Deutschland, Schweden, Finnland oder auch Großbritannien) der OECD keine genaue Aufteilung nach Forschungsarten melden und somit hier leider nicht berücksichtigt werden können (Guellec 2001). Länder mit einem besonders hohen Grundlagenforschungsanteil sind die Schweiz mit 0,83\% (2004) und Israel mit 0,76 \% (2004). Die USA weisen eine Grundlagenforschungsquote von 0,48 \% (2006) auf. Japan liegt mit 0,40 \% (2006) knapp unter dem österreichischen Niveau. 


\section{Abbildung 28: F\&E und Grundlagenforschung im internationalen Vergleich (in \% des BIP, 2006 bzw. jeweils aktuellste verfügbare Daten)}

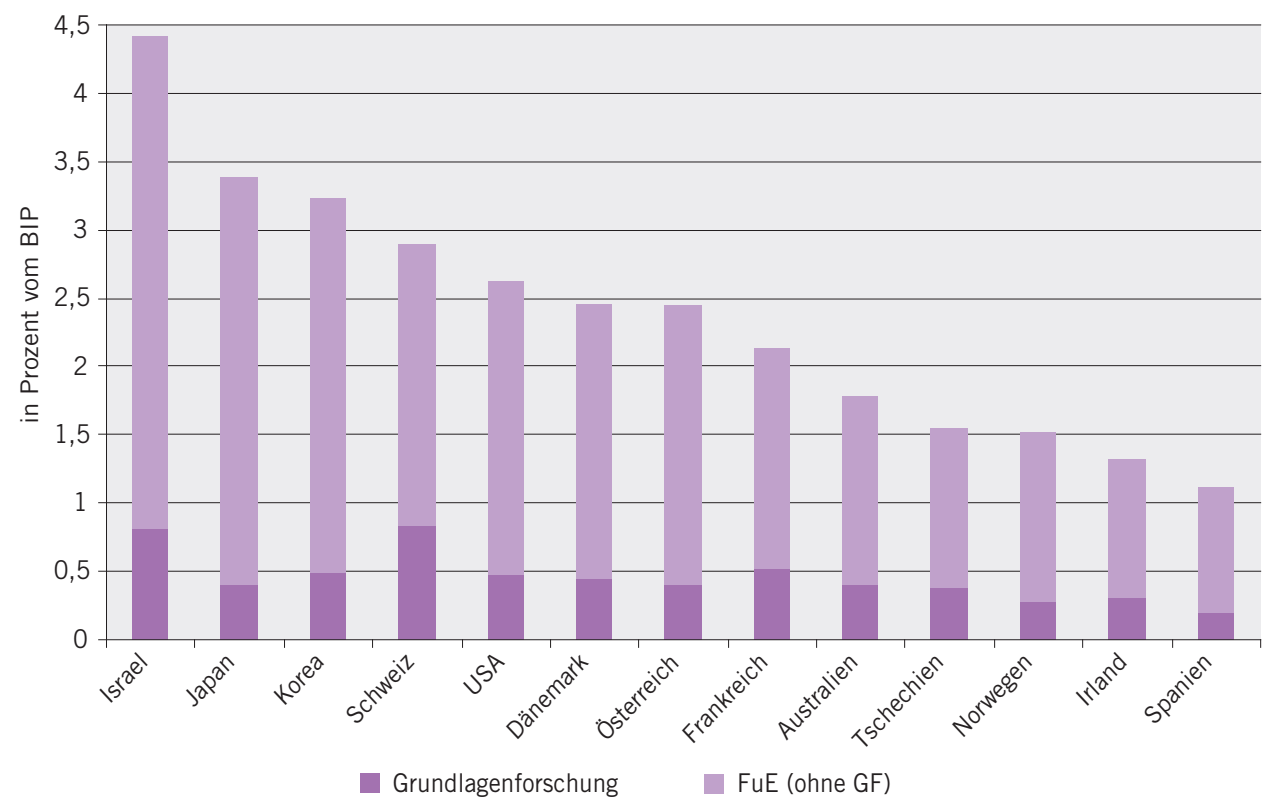

Quelle: OECD (MSTI 2008/1); Berechnungen Joanneum Research

In Abbildung 29 ist die relative Grundlagenforschungsorientierung der F\&E-Aktivitäten in ausgewählten OECD-Ländern dargestellt. Die Abbildung zeigt, wie hoch der Anteil der Grundlagenforschung an den gesamten F\&EAusgaben eines Landes ist. Wiederum liegt die Schweiz an der Spitze, und zwar mit einem Anteil der Grundlagenforschung von knapp $29 \%$. In Österreich beträgt dieser Anteil ca. $17 \%$, womit sich Österreich im unteren Mittelfeld der hier betrachteten Länder befindet. Länder mit ähnlich hoher "relativer" Grundlagenforschungsorientierung sind z.B. Dänemark, Norwegen, Israel, die USA und Spanien. Damit zeigt sich also, dass das Ausmaß der Orientie- rung auf Grundlagenforschung ein nationales Spezifikum des jeweiligen Wissenschaftssystems ist, d.h. es ist nicht systematisch von der generellen Forschungsintensität abhängig, wodurch sich auch keine Korrelation zwischen Forschungsquote und Grundlagenforschungsorientierung feststellen lässt. Es finden sich Länder mit einer hohen Forschungsquote und einer relativ geringen Orientierung auf Grundlagenforschung (z.B. Korea, Japan) sowie auch umgekehrt Länder mit niedriger Forschungsquote, deren Forschung allerdings relativ stark auf die Grundlagenforschung hin orientiert ist (z.B. Tschechien, Irland). 


\section{Abbildung 29: Anteil der Grundlagenforschung an den gesamten Forschungsausgaben (2006 bzw. jeweils aktuell verfügbare Daten)}

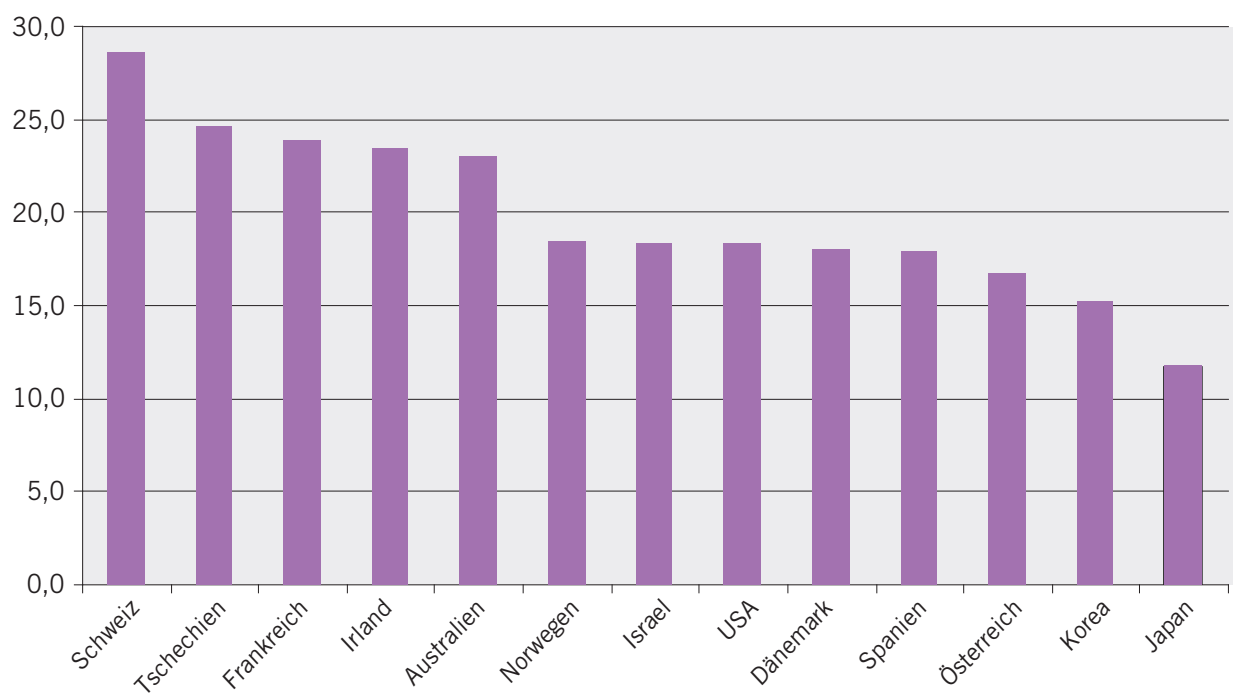

Quelle: OECD (MSTI/2008/1); Berechnungen Joanneum Research

In Abbildung 30 wird der Zusammenhang zwischen der allgemeinen F\&E-Quote und dem Anteil der Grundlagenforschung am BIP im internationalen Vergleich dargestellt. Der positive Zusammenhang ist natürlich zumindest teilweise "tautologisch", da ja die Grundlagenforschungsquote ein Teil der allgemeinen Forschungsquote ist und somit ceteris paribus ein Zusammenhang gegeben sein muss. Nichtsdestotrotz zeigen gerade die Abweichungen von der Trendgeraden einige interessante Details. So zeigt z.B. der große Abstand der Schweiz von der Trendgerade die spezielle Position dieses Landes mit seinem spezifischen Wissenschafts- und Forschungssystem, das in besonderem Maß auf die Grundlagenforschung hin orientiert ist. Das erklärt nicht zuletzt auch die herausragende Stellung, die die Schweiz bei vielen der bibliometrischen Outputindikatoren einnimmt (vgl. dazu die Diskussion am Ende dieses Kapitels). Umgekehrt lässt sich an der Position Koreas und Japans unterhalb der Trendgerade erkennen, dass die akademische Forschung (bzw. die Grundlagenforschung im speziellen) in diesen Ländern im Vergleich zur ihrer doch recht hohen Forschungsquote unterrepräsentiert ist. Österreich findet sich ebenfalls unterhalb der Trendgeraden, wenn auch in deutlich geringerem Ausmaß. Trotzdem ist das ein Hinweis, dass Österreich derzeit - bei gegebener allgemeiner Forschungsquote - „zu wenig" an Mitteln für die Grundlagenforschung alloziert. 
Abbildung 30: F\&E-Quote und Grundlagenforschungsquote im internationalen Vergleich

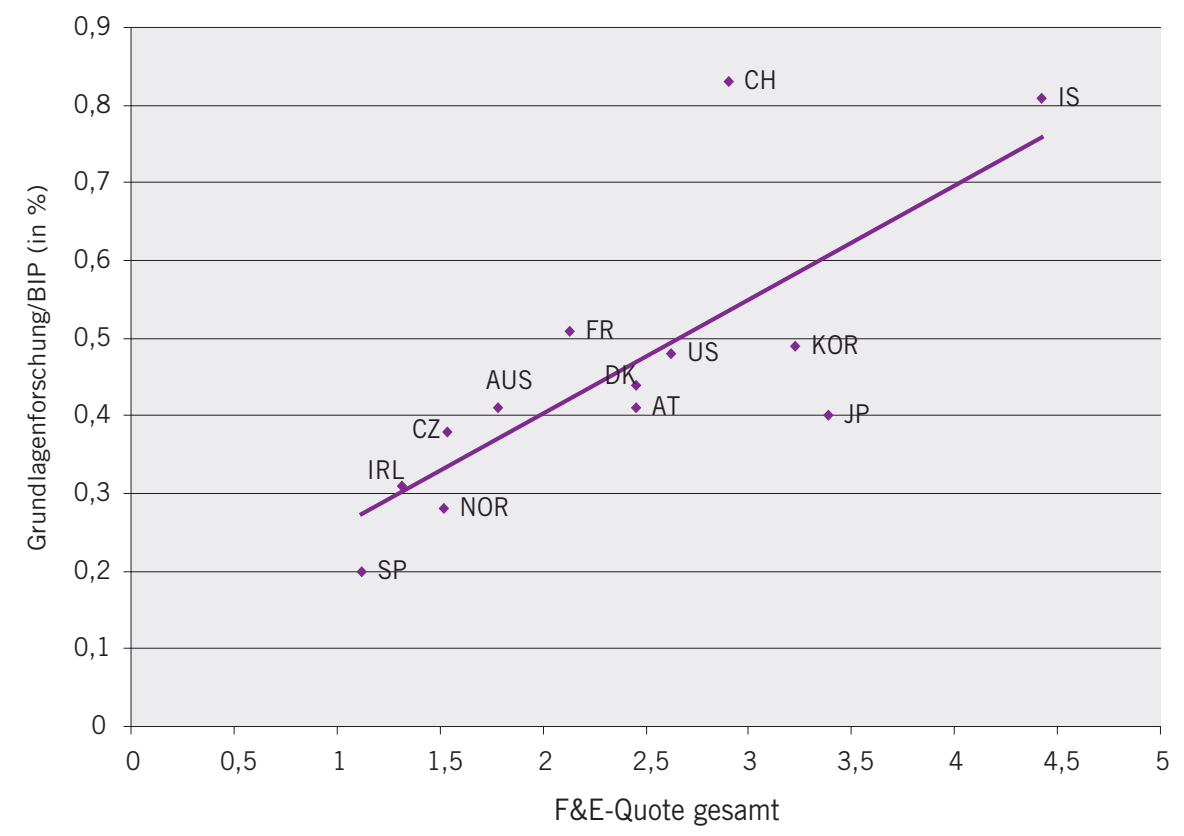

Quelle: OECD, Berechnungen Joanneum Research

\subsubsection{Grundlagenforschung in Österreich}

Auf die Grundlagenforschung entfallen im Jahr 2006 17,2 \% der gesamten F\&E-Aufwendungen Österreichs $(35,4 \%$ entfallen auf die angewandte Forschung, 47,4\% auf experimentelle Entwicklung). Gegenüber den Jahren 2002 und 2004 haben sich diese Anteile nur leicht verändert (vgl. Abbildung 31). Insgesamt hat der Anteil der experimentellen Entwicklung an den Forschungsausgaben etwas zugenommen (von 45 auf $47 \%$ ), in erster Linie auf Kosten der angewandten Forschung (von 38 auf $35 \%$ ); die Grundlagenforschung blieb praktisch konstant bei $17-18 \%$.

Innerhalb der einzelnen Sektoren ist die Orientierung auf die Grundlagenforschung (und spiegelbildlich dazu auf die beiden anderen Forschungsarten) sehr unterschiedlich ausgeprägt. Wenig überraschend ist im Hochschulsektor der Anteil der Grundlagenforschung am höchsten. Von den gesamten Forschungsausgaben im Hochschulsektor entfällt knapp die Hälfte $(49 \%)$ auf die Grundlagenforschung, knapp $42 \%$ sind hier angewandte Forschung und $9 \%$ experimentelle Entwicklung. Im Sektor Staat beträgt der Anteil der Grundlagenforschung knapp ein Drittel $(32,2 \%)$. Im Unternehmenssektor macht die Grundlagenforschung nur knapp über $5 \%$ der Forschungsausgaben aus (in den eigentlichen privaten Unternehmen - also exklusive kooperativer Bereich 3,4 \%). 


\section{Abbildung 31: F\&E-Ausgaben 2002/04/06 nach Forschungsarten}

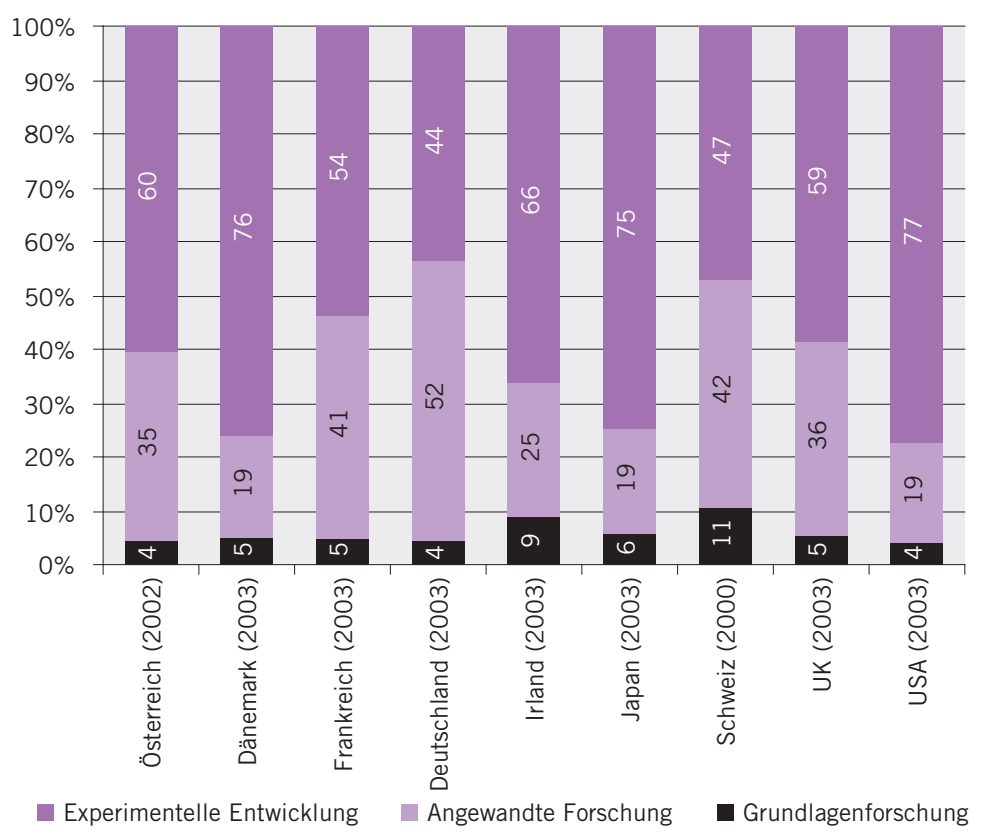

Quelle: Statistik Austria (F\&E-Erhebung), Berechnungen Joanneum Research

Eine in diesem Zusammenhang besonders interessante Frage ist, wie der relativ geringe Anteil der Grundlagenforschung im Unternehmenssektor zu bewerten ist. Schließlich wurde in der Grundlagenforschung des Unternehmenssektors das Potential für radikale Innovationen gesehen. Mit der vorhandenen Struktur der F\&E-Tätigkeiten im Unternehmenssektor, „... lassen sich zwar Aufholprozesse gewin- nen, aber keine „Front Runner" Positionen erreichen" (Aiginger et al. 2006, S. 131f.). Nun zeigt aber ein internationaler Vergleich der Forschungsarten im Unternehmenssektor, dass der Anteil der Grundlagenforschung wenig mit der Entwicklung neuer Technologien bzw. mit wirtschaftlichem Erfolg zu tun hat (Abbildung 32). 


\section{Abbildung 32: F\&E-Ausgaben nach Forschungsarten im Unternehmenssektor}

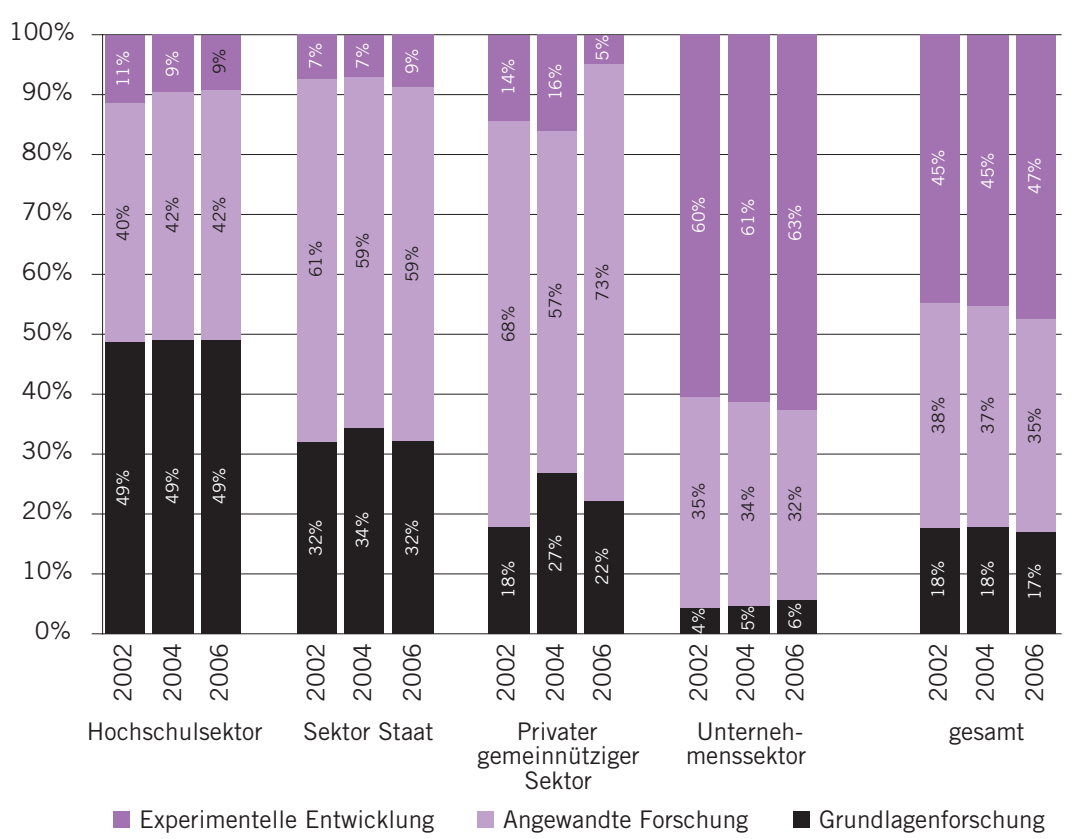

Quelle: OECD, Berechnungen Joanneum Research

Der Anteil der Grundlagenforschung ist relativ gering im Unternehmenssektor und gerade in jenem Land, welches als Vorzeigeland technologischer Durchbrüche gilt (USA), am geringsten. Der wirtschaftliche Erfolg technologischer Entwicklungen passiert im Bereich der „Experimentellen Entwicklung“. Grundlagenforschung wird außerhalb des Unternehmenssektors betrieben, was angesichts immer kürzer werdender Produktlebenszyklen und großen Wettbewerbsdrucks auch nachvollziehbar ist.

Die Grundlagenforschung wird in Österreich hauptsächlich im Hochschulsektor (Universitäten einschließlich Uni-Kliniken; Universitäten der Künste, Akademie der Wissenschaften, Fachhochschulen, Privatuniversitäten, Donauuniversität Krems) durchgeführt, auf den 70,1 \% der österreichischen Grund- lagenforschung entfallen. Es folgt der Unternehmenssektor mit $23 \%$, wobei hier aber ein nicht unbeträchtlicher Teil auf den kooperativen Bereich (also z.B. Austrian Research Centers, Kplus- und COMET-Zentren, Joanneum Research) entfällt. Rechnet man den kooperativen Bereich heraus und betrachtet nur die eigentlichen Unternehmen (firmeneigener Bereich), entfallen im Jahr 2006 12,9 \% der Ausgaben für Grundlagenforschung auf diesen Sektor. Der Sektor Staat (Institutionen des Bundes und der Länder sowie der Gemeinden) spielt mit 6,5\% nur eine geringe Rolle. Ein sehr geringes Gewicht hat in Österreich der private gemeinnützige Sektor $(0,3 \%)$. Der genaue Überblick über die jeweiligen absoluten Aufwendungen bzw. deren Anteile, gegliedert nach den drei Forschungsarten, findet sich in Tabelle 23. 
Tabelle 23: F\&E-Ausgaben nach Forschungsarten und Durchführungssektoren

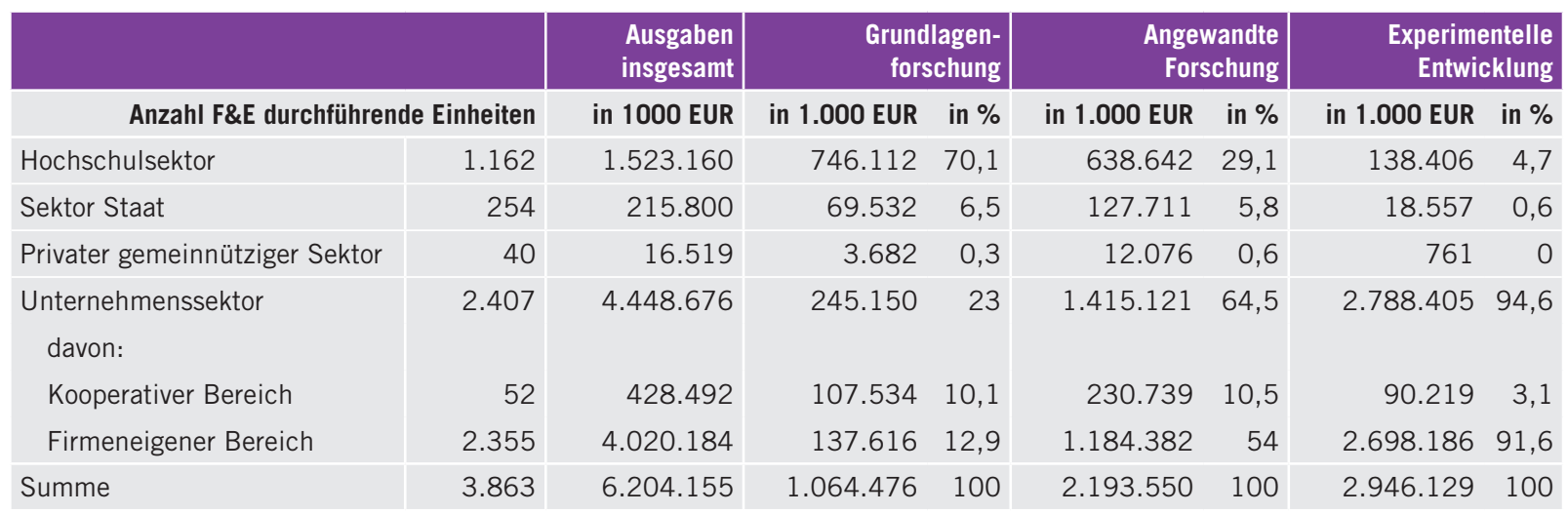

Quelle: Statistik Austria; Berechnungen Joanneum Research

Tabelle 24 gibt einen Überblick über die Struktur der Forschungsausgaben im Unternehmenssektor, wobei dieser nach Technologieklassen gegliedert ist. Zudem wird noch zwischen dem firmeneigenen Bereich im eigentlichen Sinn und dem sogenannten kooperativen Bereich (hier handelt es sich v.a. um als Unternehmen geführte öffentliche Forschungseinrichtungen wie ARC oder Joanneum Research sowie die Kompetenzzentren, die rechtlich ebenfalls als Unternehmen geführt werden) getrennt. Auf Ebene der Technologieklassen zeigt sich das überraschende Ergebnis, dass gerade der HighTech-Sektor die geringste Orientierung auf Grundlagenforschung aufweist (lediglich $1 \%$ der gesamten Forschungsausgaben entfallen hier auf Grundlagenforschung, während die experimentelle Entwicklung mit 78 \% eindeutig dominiert). Aus Tabelle 24 wird offensichtlich, dass gerade in der Sachgüterproduktion in ers- ter Linie die experimentelle Entwicklung und - in bereits etwas geringerem Ausmaß - die angewandte Forschung dominieren. Grundlagenforschung ist hier lediglich eine Restgröße.

Bemerkenswert ist weiters das vergleichsweise große Aufkommen an Grundlagenforschung im Dienstleistungssektor. Dies lässt sich vor allem auf die Wirtschaftszweige in der Branchengruppe „Technologie- und wissensintensive Dienstleistungen" zurückführen (zu denen auch der Wirtschaftszweig „Forschung und Entwicklung" gehört, wo wiederum der erwähnte kooperative Bereich zu finden ist), aber auch im Bereich der unternehmensnahen Dienstleistungen findet sich mit knapp 49 Mio. $€$ eine bemerkenswert hohe Aktivität in der Grundlagenforschung (immerhin werden hier auch knapp 14 \% der gesamten F\&E-Ausgaben der Grundlagenforschung zugerechnet). 
Tabelle 24: F\&E-Ausgaben nach Forschungsarten im Unternehmenssektor

\begin{tabular}{|c|c|c|c|c|c|c|c|c|}
\hline \multirow[t]{2}{*}{ Technologietyp } & \multirow{2}{*}{$\begin{array}{l}\text { Anzahl der F\&E } \\
\text { durchftihrenden } \\
\text { Einheiten }\end{array}$} & \multirow{2}{*}{$\begin{array}{c}\text { interne } \\
\text { F\&E-Ausgaben } \\
\text { insgesamt }\end{array}$} & \multicolumn{2}{|c|}{ Grundlagenforschung } & \multicolumn{2}{|c|}{ Angewandte Forschung } & \multicolumn{2}{|c|}{ Experimentelle Entwicklung } \\
\hline & & & in 1.000 EUR & in $\%$ & in 1.000 EUR & in $\%$ & in 1.000 EUR & in $\%$ \\
\hline Primärer Sektor & 13 & 7.966 & 657 & 8 & 4.031 & 51 & 3.278 & 41 \\
\hline High Tech & 202 & 1.314 .044 & 14.187 & 1 & 270.625 & 21 & 1.029 .232 & 78 \\
\hline Medium High Tech & 508 & 1.284 .780 & 57.176 & 4 & 433.053 & 34 & 794.553 & 62 \\
\hline Medium Low Tech & 333 & 373.931 & 20.613 & 6 & 105.899 & 28 & 247.419 & 66 \\
\hline Low Tech & 277 & 170.678 & 6.694 & 4 & 44.460 & 26 & 119.524 & 70 \\
\hline Sonstige & 107 & 34.923 & 1.880 & 5 & 14.402 & 41 & 18.641 & 53 \\
\hline Unt.nahe Dienstl. & 243 & 350.902 & 48.978 & 14 & 163.445 & 47 & 138.479 & 39 \\
\hline High Tech Dienstl. & 459 & 605.371 & 88.086 & 15 & 276.947 & 46 & 240.338 & 40 \\
\hline Sonstige Dienstl. & 261 & 290.122 & 6.379 & 2 & 97.921 & 34 & 185.822 & 64 \\
\hline \multicolumn{9}{|l|}{ Unt.Sektor insgesamt: } \\
\hline Firmeneigener Bereich & 2.355 & 4.020 .184 & 137.616 & 3 & 1.184 .382 & 30 & 2.698 .186 & 67 \\
\hline Kooperativer Bereich & 52 & 428.492 & 107.534 & 25 & 230.739 & 54 & 90.219 & 21 \\
\hline Unt. Sektor insgesamt & 2.407 & 4.448 .676 & 245.150 & 6 & 1.415 .121 & 32 & 2.788 .405 & 63 \\
\hline
\end{tabular}

Anm.: Die Klassifikation der Technologiezugehörigkeit erfolgte auf NACE-3-Steller Ebene.

Quelle: Statistik Austria; Berechnungen Joanneum Research

In Tabelle 25 wird die Forschung im Hochschulsektor nach den verschiedenen Wissenschaftszweigen differenziert. Der Wissenschaftszweig „Naturwissenschaften" weist mit 295,3 Mio. $€$ von der absoluten Zahl her die höchsten Mittel im Bereich der Grundlagenforschung auf. Es folgen Humanmedizin $(160,8$ Mio. $€)$ und die Geisteswissenschaften (106,3 Mio. €). Dahinter liegen die Sozialwissenschaften $(97,2$ Mio. $€)$, die Technischen Wissenschaften $(58,8$ Mio. €) sowie die Land- und Forstwirtschaft/ Veterinärmedizin (27,7 Mio. €). Tabelle 25 gibt weiters auch einen Überblick über den Stel- lenwert der Grundlagenforschung (d.h. den Anteil der Grundlagenforschung an den jeweiligen gesamten F\&E-Ausgaben) nach Wissenschaftszweigen bzw. Disziplingruppen. Die Orientierung auf Grundlagenforschung ist am höchsten in den Geisteswissenschaften $170 \%$ der Forschung ist hier Grundlagenforschung). An zweiter und dritter Stelle liegen Naturwissenschaften $(62 \%)$ und Sozialwissenschaften (46 \%). Es folgen Humanmedizin (41 \%), Landund Forstwirtschaft/Veterinär $(40 \%)$ und Technische Wissenschaften (27\%).

\section{Tabelle 25: Struktur der Forschungsausgaben im Hochschulsektor nach Wissenschaftszweigen}

\begin{tabular}{|c|c|c|c|c|c|c|c|}
\hline \multicolumn{2}{|c|}{ Ausgaben für F\&E insgesamt } & \multicolumn{2}{|c|}{ Grundlagenforschung } & \multicolumn{2}{|c|}{ Angewandte Forschung } & \multicolumn{2}{|c|}{ Experimentelle Entwicklung } \\
\hline Wissenschaftszweige & in 1000 EUR & in 1000 EUR & in $\%$ & in 1000 EUR & in $\%$ & in 1000 EUR & in $\%$ \\
\hline Naturwissenschaften/Technik/Medizin & 1.162 .243 & 542.563 & 46,7 & 496.761 & 42,7 & 122.919 & 10,6 \\
\hline Naturwissenschaften & 477.341 & 295.271 & 61,9 & 143.332 & 30 & 38.738 & 8,1 \\
\hline Technische Wissenschaften & 218.368 & 58.806 & 26,9 & 129.597 & 59,4 & 29.965 & 13,7 \\
\hline Humanmedizin & 396.862 & 160.783 & 40,5 & 186.774 & 47,1 & 49.305 & 12,4 \\
\hline Land- und Forstwirtschaft, Veterinärmedizin & 69.672 & 27.703 & 39,8 & 37.058 & 53,2 & 4.911 & 7 \\
\hline Sozial- und Geisteswissenschaften & 360.917 & 203.549 & 56,4 & 141.881 & 39,3 & 15.487 & 4,3 \\
\hline Sozialwissenschaften & 209.340 & 97.227 & 46,4 & 102.673 & 49,1 & 9.440 & 4,5 \\
\hline Geisteswissenschaften & 151.577 & 106.322 & 70,1 & 39.208 & 25,9 & 6.047 & 4 \\
\hline Summe & 1.523 .160 & 746.112 & 49 & 638.642 & 41,9 & 138.406 & 9,1 \\
\hline
\end{tabular}

Quelle: Statistik Austria; Berechnungen Joanneum Research 
Betrachtet man das "Gewicht" der einzelnen Wissenschaftszweige in Bezug auf die Gesamtsummen der Grundlagenforschung im Hochschulsektor, so zeigt sich, dass auf die "harten" Wissenschaften (Natur- und Ingenieurswissenschaften, Medizin, Land- und Forstwirtschaft/ Veterinärmedizin) 72,7 \% der Grundlagenforschung im österreichischen Hochschulsektor entfallen (vgl. Abbildung 33). Auf Ebene der Wissenschaftszweige liegen mit einem Anteil von knapp $40 \%$ die Naturwissenschaften an erster Stelle, gefolgt von der Humanmedizin mit 21,5\%. Auf die Technischen Wissenschaften entfallen hingegen lediglich knapp $8 \%$ der gesamten Ausgaben für Grundlagenforschung. Spiegelbildlich entfallen auf die Sozial- und Geisteswissenschaften die verbleibenden 27,3 \% der gesamten Mittel für Grundlagenforschung. Diese sind relativ gleich auf die Sozialwissenschaften mit $13 \%$ bzw. Geisteswissenschaften mit $14,3 \%$ verteilt.

\section{Abbildung 33: Anteile der Wissenschaftszweige an den gesamten Ausgaben für Grundlagenforschung im Hochschulsektor}
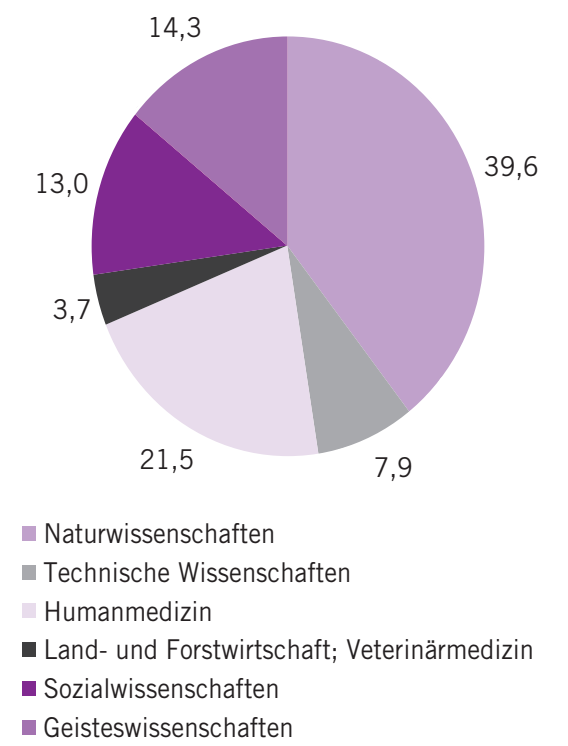

Quelle: Statistik Austria; Berechnungen Joanneum Research

\subsubsection{Output - Ausgewählte bibliometrische Indikatoren im Überblick}

Bislang wurden lediglich monetäre Inputgrößen der Grundlagenforschung diskutiert. Im Folgenden soll daher für die weitere Analyse die Outputseite betrachtet werden, wobei hierfür übliche bibliometrische Indikatoren herangezogen werden. Trotz aller damit verbundenen Probleme (unterschiedliche Wertigkeit von Publikationen, überproportionale Berücksichtigung englischsprachiger Publikationen etc.) lässt sich mit Hilfe bibliometrischer Indikatoren ein adäquates Bild hinsichtlich der Quantität und der thematischen Spezialisierung der Grundlagenforschung im internationalen Kontext zeichnen. Tabelle 26 stellt die Anzahl der wissenschaftlichen Publikationen in internationalen Fachjournalen sowie der entsprechenden Zitationen je WissenschafterIn dar. Im Unterschied zur Anzahl der Publikationen geben Zitationen Aufschluss über die Relevanz einer wissenschaftlichen Arbeit für andere WissenschafterInnen und sind somit im weitesten Sinn ein Indikator für die Qualität bzw. für die Exzellenz wissenschaftlicher Forschung. 
Tabelle 26: Publikationen und Zitationen im internationalen Vergleich (Durchschnitt 1997 - 2006)

\begin{tabular}{|c|c|c|}
\hline & $\begin{array}{l}\text { Publikationen je } \\
\text { Wissenschafter }\end{array}$ & $\begin{array}{c}\text { Zitationen je } \\
\text { Wissenschafter }\end{array}$ \\
\hline Schweiz & 14,27 & 196,38 \\
\hline Großbritannien & 12,17 & 140,69 \\
\hline USA & 11,19 & 146,04 \\
\hline Niederlande & 10,6 & 130,25 \\
\hline Dänemark & 9,07 & 112,02 \\
\hline Irland & 9,02 & 82,58 \\
\hline Schweden & 8,81 & 102,48 \\
\hline Italien & 8,2 & 76,12 \\
\hline Belgien & 7,95 & 83,15 \\
\hline EU-15 & 6,94 & 73,62 \\
\hline Norwegen & 5,94 & 58,45 \\
\hline Deutschland & 5,84 & 68,04 \\
\hline Frankreich & 5,68 & 55,54 \\
\hline Tschechische Rep. & 5,3 & 30,34 \\
\hline Finnland & 5,28 & 58,43 \\
\hline Griechenland & 5,06 & 30,01 \\
\hline Österreich & 4,48 & 44,18 \\
\hline Spanien & 4,42 & 35,15 \\
\hline Ungarn & 4,41 & 31,53 \\
\hline Island & 3,79 & 45,24 \\
\hline Japan & 3,33 & 27,04 \\
\hline Portugal & 2,77 & 17,88 \\
\hline
\end{tabular}

Quelle: Reckling (2007), UNESCO Datenbank. WIFO Berechnungen auf der Basis von Dosi-Llerena-Sylos-Labini (2006) vorgelegten Ergebnissen. Für die Anzahl der WissenschafterInnen wurde die Anzahl der im öffentlichen Sektor und in privaten Non-Profit Organisation tätigen WissenschafterInnen in Vollzeitäquivalenten herangezogen

Tabelle 26 zeigt, dass U.S.-WissenschafterInnen im Schnitt produktiver sind $(11,19$ gegen 6,95 Publikationen je Forscher über den Zeitraum 1997-2006) und auch mehr Zitationen auf ihre Forschungsergebnisse ziehen $(146,04$ Zitationen gegen 73,62 für die EU15) als im europäischen Durchschnitt. Dies deutet darauf hin, dass deren wissenschaftliche Arbeiten für andere WissenschafterInnen im Schnitt relevanter sind, als jene ihrer europäischen KollegInnen. Innerhalb Europas gibt es erhebliche
Unterschiede, wobei die Schweiz bei beiden Indikatoren an der Spitze liegt. Großbritannien führt die Liste der EU-Staaten - gefolgt von den Niederlanden - an. Diese beiden Länder (und mit einigen Abstrichen auch Schweden und Dänemark) sind die einzigen EU-Staaten, die zumindest annähernd das Niveau des US-amerikanischen Benchmark erreichen. Österreich liegt mit 4,48 Publikationen/WissenschafterIn sowie 44,18 Zitationen/WissenschafterIn in Hinblick auf diese beiden Indikatoren deutlich hinter den USA und den oben genannten europäischen Spitzenländern. Auch gegenüber dem EU-15-Schnitt fällt Österreich zurück.

Tabelle 27 stellt die Anzahl vielzitierter WissenschafterInnen - als Indikator für die „Exzellenz" eines Forschungssystems - in den forschungsstärksten Ländern gegenüber. Österreich rangiert diesbezüglich unter den zwanzig Spitzennationen, was diesen Indikator anbelangt. Mit zwölf vielzitierten WissenschafterInnen fällt Österreich aber von der Spitze ab. Vor allem der Vergleich mit der Schweiz fällt ungünstig aus. In der Schweiz gibt es achtmal mehr vielzitierte ForscherInnen. In Schweden und den Niederlanden ist die Zahl mehr als viermal höher und in Dänemark arbeiten immer noch mehr als doppelt so viele vielzitierte WissenschafterInnen wie in Österreich. Dieser Abstand Österreichs von den führenden Ländern zeigt sich auch, wenn man die absoluten Zahlen in Bezug zu den jeweiligen Einwohnerzahlen (z.B. Österreich 1,59 vielzitierte WissenschafterInnen pro 1 Mio. Einwohner gegenüber 16,28 in der Schweiz) oder zur Gesamtzahl der WissenschafterInnen $(0,68$ pro 1000 WissenschafterInnen gegenüber 9,69 in der Schweiz) setzt. 
Tabelle 27: Anzahl vielzitierter WissenschafterInnen auf Bevölkerung und WissenschafterInnen

\begin{tabular}{|c|c|c|c|c|c|}
\hline & $\begin{array}{r}\text { Anzahl vielzitierter } \\
\text { Wissenschafter }\end{array}$ & $\begin{array}{r}\text { Vielzitierte Wissenschafter } \\
\text { je } 1 \text { Mio. Ew. }\end{array}$ & Rang & $\begin{array}{r}\text { Vielzitierte Wissenschafter } \\
\text { je } 1000 \text { Wissenschafter }\end{array}$ & Rang \\
\hline USA & 3829 & 16,82 & 1 & 15,67 & 1 \\
\hline Schweiz & 103 & 16,28 & 2 & 9,69 & 2 \\
\hline Israel & 47 & 12,49 & 3 & k.A. & k.A. \\
\hline Großbritannien & 439 & 7,79 & 4 & 6,95 & 3 \\
\hline Australien & 105 & 7,13 & 5 & 2,06 & 10 \\
\hline Sweden & 59 & 7,09 & 6 & 3,23 & 6 \\
\hline Kanada & 172 & 7,03 & 7 & 4,01 & 5 \\
\hline Niederlande & 92 & 6,5 & 8 & 4,64 & 4 \\
\hline Dänemark & 28 & 5,47 & 9 & 3,05 & 7 \\
\hline Neuseeland & 17 & 5,46 & 10 & 1,73 & 13 \\
\hline Belgien & 35 & 3,55 & 11 & 2,48 & 8 \\
\hline Finnland & 15 & 3,14 & 12 & 1,01 & 17 \\
\hline Deutschland & 240 & 3,12 & 13 & 2,25 & 9 \\
\hline Norwegen & 12 & 2,93 & 14 & 1,29 & 15 \\
\hline Frankreich & 155 & 2,88 & 15 & 1,74 & 12 \\
\hline Japan & 247 & 2,12 & 16 & 1,1 & 16 \\
\hline Irland & 7 & 2,06 & 17 & 1,99 & 11 \\
\hline Singapur & 4 & 1,66 & 18 & 0,56 & 19 \\
\hline Österreich & 12 & 1,59 & 19 & 0,68 & 18 \\
\hline Italien & 72 & 1,28 & 20 & 1,67 & 14 \\
\hline
\end{tabular}

Quelle: Bauwens - Mion - Thisse (2008); UNESCO Datenbank. Anzahl der im öffentlichen Sektor und in privaten Non-Profit Organisationen tätigen Forscher in Vollzeitäquivalenten; WIFO Berechnungen.

In Tabelle 28 wird versucht, ein dissaggregiertes Bild des wissenschaftlichen Profils darzustellen, indem ein Spezialisierungsprofil auf der Basis der wissenschaftlichen Produktivität (P) und der wissenschaftlichen Wirkungskraft (W) abgebildet wird. Werte, die größer als Eins sind, bilden einen komparativen Vorteil ab (d.h. ein Land hat in diesem Wissenschaftsgebiet "bessere" Werte als im Durchschnitt). Formal ist der RCA-Wert in diesem Zusammenhang folgenderweise definiert:

$$
R C A I_{i, j}=\frac{x_{j}}{\bar{x}_{j}} / \frac{-}{\frac{x_{i}}{\bar{x}}}
$$

Wobei

$x_{i j} \quad \ldots$ Durchschnittliche Anzahl an Zitationen pro wissenschaftlichem Papier im _ $\quad$ Land $i$ und Disziplin $j$

$x_{i} \quad \ldots$ Durchschnittliche Anzahl an Zitationen pro wissenschaftlichem Papier im Land $i$ in allen Disziplinen

$\bar{x}_{j} \quad \ldots$ Durchschnittliche Anzahl an Zitationen pro wissenschaftlichem Papier in

_ Disziplin $j$ in allen Ländern

$\bar{x} \quad$... Durchschnittliche Anzahl an Zitationen pro wissenschaftlichem Papier in allen Ländern und Disziplinen

RCA-Werte messen also relative (!) (Spezialisierungs-|Vorteile, d.h. ein Wert über 1 in einem bestimmten Wissenschaftsgebiet $j$ bedeutet für das betreffende Land, das hier im Vergleich zur Gesamtposition bez. Zitationshäufigkeit 
des Landes (gemessen durch $\bar{x}_{i} / \bar{x}$ ) ein Vorteil besteht (obwohl u.U. die "absolute" Zitationshäufigkeit im Gebiet j trotzdem unterhalb jener der Spitzenländer liegt).

Es ist dabei zu beachten, dass der Indikator der „Produktivität" wegen der impliziten Annahme einer Gleichverteilung der WissenschafterInnen eines Landes auf alle Wissenschaftszweige verzerrt ist. Der Indikator für die Wirkungskraft, der abbildet, wie viele andere WissenschafterInnen auf die Ergebnisse einer
Publikation zurückgreifen, ist hingegen zuverlässiger. Die Analyse der Wirkungskraft zeigt, dass Österreichs Wissenschaft in den Bereichen Molekularbiologie und Genetik, Physik und Mathematik einen komparativen Vorteil genießt. In den Bereichen Neurowissenschaften und Mikrobiologie besteht auch noch ein schwacher komparativer Vorteil. Österreichs wissenschaftliche Forschung hat damit eine Spezialisierung im natur- und formalwissenschaftlichen Bereich.

Tabelle 28: Wissenschaftliches Spezialisierungsmuster in wissenschaftlicher Produktivität (P: Publikation/ WissenschafterIn) und wissenschaftlicher Wirkungskraft (W: Zitationen/Publikation)

\begin{tabular}{|c|c|c|c|c|c|c|c|c|c|c|}
\hline & \multicolumn{2}{|c|}{ Österreich } & \multicolumn{2}{|c|}{ Schweiz } & \multicolumn{2}{|c|}{ Dänemark } & \multicolumn{2}{|c|}{ Schweden } & \multicolumn{2}{|c|}{ USA } \\
\hline & $\mathbf{P}$ & W & $\mathbf{P}$ & W & $\mathbf{P}$ & W & $\mathbf{P}$ & W & $\mathbf{P}$ & $\mathbf{W}$ \\
\hline Agrarwissenschaften & 0.55 & 0.73 & 0.55 & 0.75 & 0.79 & 1.11 & 1.32 & 0.98 & 0.86 & 0.93 \\
\hline Biologie und Biochemie & 0.98 & 0.96 & 0.71 & 0.92 & 0.88 & 1.06 & 1.71 & 0.72 & 1.92 & 1.04 \\
\hline Chemie & 0.89 & 0.95 & 0.53 & 0.89 & 1.06 & 0.87 & 0.89 & 1.14 & 0.96 & 1.06 \\
\hline Computerwissenschaften & 1.05 & 0.77 & 0.75 & 0.62 & 0.61 & 1.09 & 0.88 & 0.95 & 1.51 & 1.38 \\
\hline Geowissenschaften & 0.86 & 0.86 & 0.86 & 0.97 & 0.63 & 0.99 & 1.09 & 1.06 & 0.92 & 0.93 \\
\hline Humanmedizin & 1.37 & 0.89 & 0.75 & 0.9 & 1.24 & 0.94 & 1.09 & 0.82 & 1.13 & 0.96 \\
\hline Immunologie & 1.21 & 0.98 & 1.41 & 0.94 & 1.44 & 0.9 & 0.88 & 0.86 & 1.43 & 1 \\
\hline Ingenieurswissenschaften & 0.71 & 0.97 & 1.11 & 0.93 & 0.97 & 0.8 & 0.75 & 0.95 & 2.33 & 1.03 \\
\hline Materialwissenschaften & 0.92 & 0.98 & 1.55 & 1.14 & 0.99 & 0.83 & 0.81 & 0.98 & 0.62 & 0.80 \\
\hline Mathematik & 0.98 & 1.12 & 0.77 & 0.97 & 0.46 & 0.98 & 0.78 & 0.9 & 0.72 & 0.98 \\
\hline Mikrobiologie & 0.90 & 1.04 & 0.96 & 0.8 & 0.76 & 1.05 & 0.62 & 1.36 & 0.87 & 0.84 \\
\hline Molekularbiologie und Genetik & 1.00 & 1.25 & 1.05 & 0.98 & 1.41 & 0.70 & 1.33 & 1.08 & 0.72 & 1.06 \\
\hline Neurowissenschaften & 1.07 & 1.09 & 0.78 & 0.96 & 1.42 & 1.03 & 0.95 & 0.95 & 1.15 & 1.11 \\
\hline Pharmakologie \& Toxikologie & 0.91 & 0.94 & 0.59 & 0.84 & 0.66 & 0.87 & 1.07 & 1.01 & 1.34 & 1.06 \\
\hline Physik & 1.07 & 1.17 & 1.04 & 1 & 1.1 & 0.84 & 1.23 & 0.97 & 1.19 & 1.08 \\
\hline Botanik \& Zoologie & 0.85 & 0.86 & 1.34 & 1.03 & 1.34 & 0.96 & 0.73 & 0.85 & 0.89 & 0.89 \\
\hline Psychiatrie/Psychologie & 0.71 & 0.91 & 1.26 & 0.93 & 1.05 & 1.02 & 0.54 & 0.99 & 0.86 & 0.97 \\
\hline Raumfahrtechnik & 0.91 & 0.62 & 0.67 & 0.93 & 1.31 & 0.99 & 0.98 & 0.84 & 1.09 & 0.92 \\
\hline Sozialwissenschaften & 0.55 & 0.69 & 0.98 & 1.05 & 0.75 & 0.99 & 0.60 & 0.91 & 0.80 & 1.23 \\
\hline Umweltwissenschaft und Ökologie & 0.68 & 0.90 & 1.09 & 0.84 & 1.17 & 0.98 & 1. 4 & 0.97 & 0.79 & 0.86 \\
\hline Wirtschaftswissenschaften & 0.76 & 0.82 & 1.08 & 1.07 & 1.03 & 0.94 & 0.84 & 1.05 & 0.64 & 1.06 \\
\hline
\end{tabular}

Q: Reckling (2007), OECD-MSTI, WIFO-Berechnung. Die hier verwendeten Verfahren sowie deren methodische Einschränkungen sind in Lattimore und Ravesz (1996) beschrieben.

Insgesamt deuten die hier präsentierten Statistiken darauf hin, dass Österreich zwar in der (erweiterten) internationalen Spitzengruppe angesiedelt ist, der Abstand zur Weltspitze (Benchmark USA oder europäischer Benchmark
Schweiz) aber aggregiert gesehen doch groß ist. Die wissenschaftliche Leistung in einzelnen Disziplinen weicht aber naturgemäß von den allgemeinen Indikatoren ab. So heben sich in Österreich vor allem einige natur- und formal- 
wissenschaftliche Disziplinen deutlich positiv von dem gesamtösterreichischen Bild ab.

Zusammenfassend lassen sich in Bezug auf die österreichische Situation in punkto Grundlagenforschung folgende Schlüsse ziehen:

- Auf die Grundlagenforschung entfallen derzeit ca. 0,41 \% des BIP. Damit liegt Österreich im internationalen Vergleich im Mittelfeld. Berücksichtigt man allerdings die gute Position Österreichs was die allgemeine F\&E-Quote betrifft (wo Österreich mittlerweile über dem OECD wie auch dem EUDurchschnitt liegt), so lässt sich eine etwas zu geringe Allokation von Ressourcen für Grundlagenforschung konstatieren. Derzeit entfallen nur $17 \%$ der gesamten Forschungsaufwendungen Österreichs auf die Grundlagenforschung (gegenüber $35 \%$ für angewandte Forschung und $47 \%$ für experimentelle Entwicklung), damit liegt Österreich eher am unteren Ende jener OECD-Länder, die entsprechende Daten bereitstellen.

- Die Grundlagenforschung geschieht zu $70 \%$ im „Hochschulsektor' (Universitäten, Akademie der Wissenschaften, Fachhochschulen etc.), auf die Universitäten allein entfallen dabei knapp 60 \% der Grundlagenforschung.

- Rund $13 \%$ der gesamten Ausgaben für Grundlagenforschung kommen aus dem Unternehmenssektor im engeren Sinn (= firmeneigener Bereich), machen dort aber nur ca. $3 \%$ der gesamten F\&E-Ausgaben des Sektors aus.

- Mit ca. 10 \% an den gesamten Ausgaben für Grundlagenforschung entfällt ebenfalls ein nennenswerter Beitrag auf den (zum Unternehmenssektor im weiteren Sinn gezählten) sogenannten ,kooperativen Bereich', also privatwirtschaftlich organisierte Forschungseinrichtungen mit starker öffentlicher Beteiligung/Finanzierung (ARC, ACR, JR, Kompetenzzentren). Innerhalb der F\&EAktivitäten dieses Subsektors macht die Grundlagenforschung ca. $25 \%$ aus.
- Im internationalen Vergleich zeigt sich insgesamt, dass Österreich - sowohl was den monetären Input als auch was den (bibliometrischen) Output betrifft - eine Position im (unteren) Mittelfeld einnimmt, jedoch deutlich hinter den entsprechenden globalen (USA) und europäischen (Schweiz) Benchmarks liegt.

\subsection{Gibt es ein „europäisches Paradoxon“ (in Österreich)?}

Der Begriff des „europäischen Paradoxons“ wurde erstmalig in einer Publikation der Europäischen Kommission im Jahr 1994 (Europäische Kommission 1994) geprägt. Das Konzept ist von Bedeutung, da es seit einigen Jahren die Argumentation wichtiger Empfehlungen der Europäischen Kommission zur Rolle der Universitäten im Lissabon-Prozess prägt (z.B. Europäische Kommission 2003a, b; 2005; 2006). Es besagt, dass viele europäische Universitäten zwar Forschung auf einem Spitzenniveau betreiben, ihr wissenschaftlicher Output aber aufgrund fehlender bzw. unzulänglicher Transmissionsmechanismen zu wenig in marktfähige Innovationen umgesetzt wird. Diese These wurde seit ihrer Formulierung viel diskutiert und ist mittlerweile sehr umstritten (vgl. Pavitt 2001; Dosi et al. 2005). Aus diesem Grund wird hier untersucht, inwieweit die Prämissen des Europäischen Paradoxons zutreffen. Auf Grundlage verfügbarer Daten wird die Leistung des europäischen und insbesondere des österreichischen Hochschulsektors in der Forschung im Vergleich zur USA abgebildet. Die zweite Komponente des Paradoxons wird in Form der Lehre, welche der bei weitem bedeutendste Transmissionsmechanismus von Wissen aus dem Hochschulsektor in den Unternehmenssektor hinein ist, diskutiert. Hier wird überprüft, ob die österreichischen Hochschulen die Nachfrage des österreichischen Unternehmenssektors nach den von ihm be- 
nötigten AbsolventInnen decken können. Der innereuropäische Vergleich (wie auch der Vergleich mit den USA) ist mangels vergleichbarer Daten nur äußerst eingeschränkt möglich.

\subsubsection{Forschung}

Wissenschaftliche Exzellenz ist der Angelpunkt der sich widersprechenden Befunde zur Bedeutung des Hochschulsektors für nationale Innovationssysteme. Während im Zusammenhang mit dem „europäischen Paradoxon" Europas Universitäten Exzellenz in der Forschung, aber mangelnder Wissenstransfer und damit ein unzulänglicher Beitrag zur nationalen Innovationsleistung unterstellt wird, wird in anderen Publikationen Exzellenz als Vorbedingung für Wissenstransfer gesehen (vgl. z.B. Mansfield 1995; Narin et al. 1997; Van Pottelsberghe 2007; Azoulay et al.; 2006, Breschi et al., 2007).
Obwohl wissenschaftliche Exzellenz generell schwer zu quantifizieren ist, liefert die Analyse wissenschaftlicher Publikationen und Zitationen in internationalen Fachorganen dennoch nützliche Hinweise, inwieweit die heimischen WissenschafterInnen in das internationale wissenschaftliche Geschehen eingebunden sind. Tabelle 29 stellt die Anzahl der wissenschaftlichen Publikation in internationalen Fachjournalen je Einwohner eines Landes, sowie die Zerlegung dieser Ziffer in Publikationen je ForscherIn und das Verhältnis zwischen der Einwohner- und der ForscherInnenzahl dar. In der unteren Hälfte der Tabelle wird die Darstellung für wissenschaftliche Zitationen von Publikationen, die an inländischen Forschungsinstitutionen verfasst wurden, wiederholt. Im Unterschied zur Anzahl der Publikationen geben Zitationen Aufschluss über deren Relevanz einer wissenschaftlichen Arbeit für andere WissenschafterInnen.

Tabelle 29: Die Forschungsleistung im internationalen Vergleich (1997 - 2006)

\begin{tabular}{|c|c|c|c|c|c|c|}
\hline & $\begin{array}{l}\text { Gesamtzahl der } \\
\text { Publikationen }\end{array}$ & $\begin{array}{l}\text { Publikationen je } 1000 \mathrm{EW} \\
\emptyset 1997-2006\end{array}$ & $=$ & $\begin{array}{l}\text { Publikationen je Wissenschafterln* } \\
\emptyset 1997-2006\end{array}$ & $x$ & $\begin{array}{l}\text { Wissenschafterln* je } 1000 \mathrm{EW} \\
\emptyset 1997-2006\end{array}$ \\
\hline USA & 2732816 & 9,65 & & 11,19 & & 0,86 \\
\hline Japan & 746020 & 5,87 & & 3,33 & & 1,76 \\
\hline EU15 & 3361168 & 8,90 & & 6,95 & & 1,28 \\
\hline Schweiz & 151694 & 20,99 & & 14,27 & & 1,47 \\
\hline Dänemark & 83265 & 15,58 & & 9,07 & & 1,72 \\
\hline Schweden & 160831 & 18,06 & & 8,81 & & 2,05 \\
\hline \multirow[t]{2}{*}{ Österreich } & 79071 & 9,74 & & 4,48 & & 2,18 \\
\hline & $\begin{array}{r}\text { Gesamtzahl der } \\
\text { Zitationen }\end{array}$ & $\begin{array}{l}\text { Zitationen je } 1000 \mathrm{EW} \\
\emptyset 1997-2006\end{array}$ & $=$ & $\begin{array}{l}\text { Zitationen je Wissenschafterln* } \\
\emptyset 1997-2006\end{array}$ & $\mathrm{x}$ & $\begin{array}{l}\text { Wissenschafterln* je } 1000 \text { EW } \\
\emptyset 1997-2006\end{array}$ \\
\hline USA & 35678385 & 125,93 & & 146,04 & & 0,86 \\
\hline Japan & 6051531 & 47,65 & & 27,04 & & 1,76 \\
\hline EU15 & 35603583 & 107,46 & & 73,62 & & 1,28 \\
\hline Schweiz & 2087710 & 288,84 & & 196,38 & & 1,47 \\
\hline Dänemark & 1027981 & 192,32 & & 112,02 & & 1,72 \\
\hline Schweden & 1871884 & 210,16 & & 102,48 & & 2,05 \\
\hline Österreich & 780243 & 96,14 & & 44,18 & & 2,18 \\
\hline
\end{tabular}

Quelle: Reckling (2007), UNESCO Datenbank. WIFO Berechnungen auf der Basis von Dosi-Llerena-Sylos-Labini (2006) vorgelegten Ergebnissen. * WissenschafterInnen: Anzahl der im öffentlichen Sektor (Hochschulen und andere öffentliche Einrichtungen) und in privaten Non-Profit Organisation tätigen WissenschafterInnen in Vollzeitäquivalenten. 
Die absolute Anzahl der Publikationen wie auch die Anzahl der Publikationen je Einwohner (erste und zweite Datenspalte) liefern zunächst ein Argument für die Existenz eines europäischen Paradoxons. Im EU 15 Raum erscheinen mehr Publikationen als in den USA. Umgelegt auf die Bevölkerungszahl relativiert sich dieser erste Eindruck, doch insgesamt zeigen die Zahlen, dass im EU 15 Raum nur geringfügig weniger wissenschaftliche Publikationen je Einwohner in internationalen wissenschaftlichen Fachzeitschriften erscheinen als in den USA. Die Zahl für Österreich liegt mit einem Wert von 9,74 sogar geringfügig über dem Wert der USA. Dies würde die erste Prämisse des „europäischen Paradoxons" bestätigen, da diese Zahlen nahelegen, dass die Dominanz der U.S. Forschung rein auf die Größe der USA zurückzuführen ist. Die Relevanz von Publikationen wird aber vor allem durch die Anzahl der Zitationen, die diese auf sich ziehen, angenähert. Bei dieser Kennzahl verschiebt sich das Bild zugunsten der USA (untere Hälfte von Tabelle 29). Hier fallen die Länder des EU 15 Raumes hinter die USA zurück. Einzelne Länder wie die Schweiz, Schweden oder Dänemark schneiden aber immer noch besser als die USA ab.

Aussagekräftiger als die Normierung der Publikationen und Zitationen mit der Bevölkerungszahl ist aber die Normierung mit der Anzahl der Forscher, die nicht im Unterneh- menssektor arbeiten ${ }^{58}$. Diese Kennziffer reflektiert stärker die Produktivität und Qualität der Arbeit der nationalen Forscherpopulationen. Die dritte Datenspalte in Tabelle 29 zeigt, dass U.S.-WissenschafterInnen im Schnitt produktiver sind (11,19 gegen 6,95 Publikationen je ForscherIn über den Zeitraum 1997-2006) und auch mehr Zitationen auf ihre Forschungsergebnisse ziehen (146 Zitationen gegen 73,6 für die EU 15). Dies deutet darauf hin, dass deren wissenschaftliche Arbeiten für andere WissenschafterInnen im Schnitt relevanter sind, als jene ihrer europäischen KollegInnen. Eine Ausnahme bildet hier die Schweiz, die bei beiden Indikatoren besser als die USA abschneidet. Der Grund für die Diskrepanz zwischen der mit der Bevölkerung und der mit der Anzahl der WissenschafterInnen normierten Kennziffern ist, dass es in den USA weniger ForscherInnen je Einwohner gibt. Dadurch ist der Vergleich bei einer Gegenüberstellung der auf die Einwohner umgelegten Zahlen zu ungunsten der USA nach unten verzerrt. Die Forscher in den USA sind aber wesentlich produktiver und produzieren - gemessen an den Zitationen - relevantere Arbeiten, als ihre europäischen Kolleginnen und Kollegen. Die Leistung der europäischen WissenschafterInnen fällt nun hinter jene der U.S.-ForscherInnen zurück, wobei Österreich unter dem EU Durchschnitt liegt.

58 Es wird in diesem Kapitel zur Normierung die Anzahl der im öffentlichen Sektor (Hochschulen und andere öffentliche Einrichtungen) und in privaten Non-Profit Organisationen tätigen ForscherInnen in Vollzeitäquivalenten verwendet. Dies ist aus zweierlei Gründen notwendig: 1) der überwiegende Teil der ForscherInnen, die im Unternehmenssektor tätig sind, veröffentlichen nicht oder nur sehr wenig in internationalen Fachzeitschriften, da Unternehmen aufgrund der strategischen Bedeutung der Erkenntnisse ihrer Forscher kaum Interesse an einer Verbreitung von Erkenntnissen haben, die im Rahmen von firmeneigenen Forschungstätigkeiten entstanden sind. 2) Würde auch die Zahl der im Unternehmenssektor tätigen ForscherInnen herangezogen, so wären die Ergebnisse zugunsten von Ländern mit einer wenig technikintensiven Industriestruktur verzerrt, da dort weniger ForscherInnen im Unternehmenssektor beschäftigt werden. 


\section{Abbildung 34: Komparative Vorteile nach Wissenschaftsdisziplinen - Zitationen/Publikation}

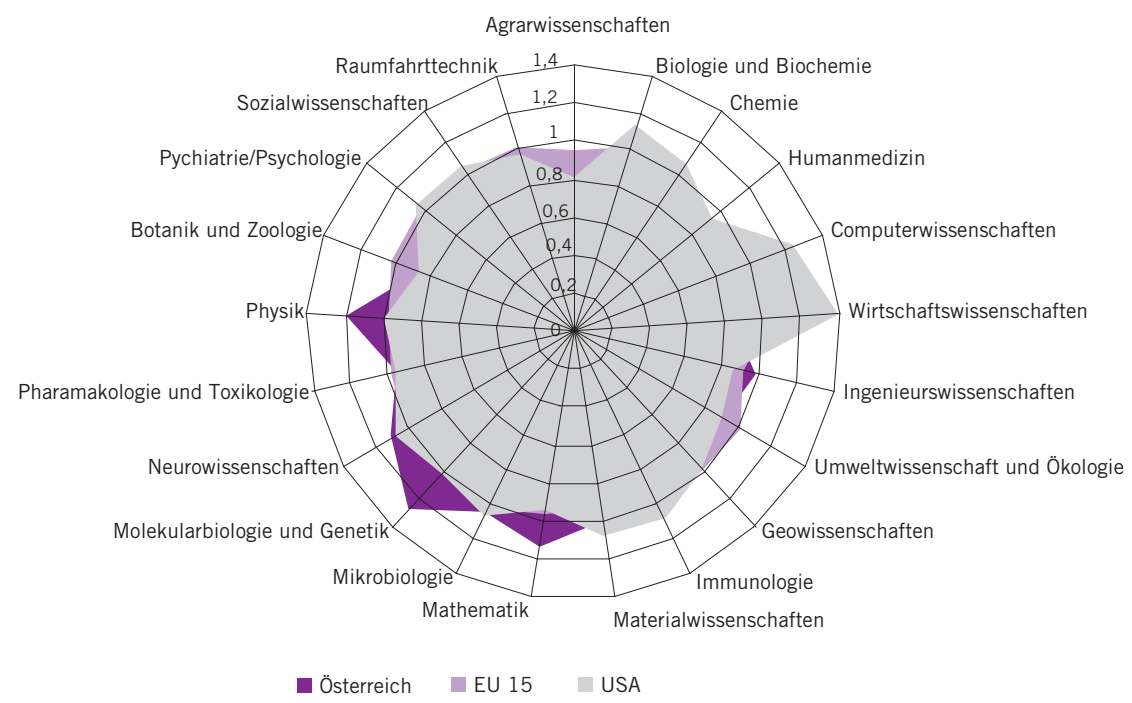

Quelle: Reckling (2007), UNESCO Datenbank; Darstellung und Berechnung durch WIFO

Zur weiteren Differenzierung dieser Ergebnisse sollte auch die Frage beantwortet werden, ob die Dominanz der U.S.-WissenschafterInnen sich über alle Forschungsdisziplinen hin erstreckt, oder ob der offensichtliche Vorteil in der Anzahl der Publikationen und Zitationen einzig auf das wissenschaftliche Spezialisierungsmuster der USA zurückzuführen ist. Ist nämlich ein Land in einigen wenigen Disziplinen stark, in denen in der Regel viele aber kürzere Aufsätze publiziert werden und ist in diesen Aufsätzen auch die durchschnittliche Anzahl der Zitationen höher als in anderen Disziplinen, so könnte der Befund der wissenschaftlichen Dominanz der USA verzerrt sein. Damit ein solcher Struktureffekt ausgeschlossen werden kann, stellt Abbildung 34 den relativen Vorteil eines Landes gemessen an den Zitationen je Publikation nach Disziplinen berechnet dar (siehe auch die Präsentation des Indikators in Kapitel 3.1.3). Damit wird der Spezialisierung der verglichenen Länder Rechnung getragen. Wie aus Abbildung 34 ersichtlich, erstreckt sich die Dominanz der U.S. Forschung auf viele Disziplinen. Damit kann nicht von einer Verzerrung des Gesamtbefundes aufgrund einer vorteilhaften wissenschaftlichen Spezialisierung der USA ausgegangen werden. Besondere stark ausgeprägt sind die Vorteile der USA in den Bereichen Wirtschaftswissenschaften und Computerwissenschaften. Für Österreich zeigen die Ergebnisse komparative Vorteile in den Bereichen Mathematik, Molekularbiologie und Genetik sowie Physik. 
Tabelle 30: Das europäische Paradoxon - vielzitierte WissenschafterInnen

\begin{tabular}{lrrr} 
& $\begin{array}{r}\text { Anzahl vielzitierter } \\
\text { Wissenschaftler }\end{array}$ & $\begin{array}{r}\text { Vielzitierte Wissenschaftler } \\
\text { je Mio. EW }\end{array}$ & $\begin{array}{r}\text { Vielzitierte Wissenschaftler } \\
\text { je 1000 Wissenschaftler* }\end{array}$ \\
\hline USA & 3829 & 16,82 & 15,67 \\
Japan & 247 & 2,12 & 1,1 \\
EU 15 & 1184 & 3,14 & 2,45 \\
\hline Schweiz & 103 & 16,28 & 9,69 \\
Dänemark & 28 & 5,47 & 3,05 \\
Sweden & 59 & 7,09 & 3,23 \\
Österreich & 12 & 1,59 & 0,68
\end{tabular}

* Anzahl der im öffentlichen Sektor und in privaten Non-Profit Organisationen tätigen ForscherInnen in Vollzeitäquivalenten; WIFO Berechnungen.

Quelle: Bauwens et al. (2008); UNESCO Datenbank.

Die wissenschaftliche Dominanz der USA wird bei Betrachtung der geographischen Verteilung vielzitierter WissenschafterInnen noch unterstrichen. Diese Kennziffer ist auch deshalb besonders wichtig, da vielzitierte WissenschafterInnen wichtige Akteure im Wissenstransfer zwischen Forschungs- und Unternehmenssektor sind (Zucker et al. 1998; Mathieu et al. 2008; van Looy et al., 2007). Tabelle 30 stellt die Anzahl vielzitierter WissenschafterInnen einiger Ländern gegenüber. Die USA liegen hier sowohl in der absoluten, als auch in der in Relation zur Bevölkerung und der Gesamtpopulation der WissenschafterInnen gesetzten Zahl an vielzitierten WissenschafterInnen weit vor dem EU-Durchschnitt. Österreich rangiert in diesem Indikator unter den zwanzig Spitzennationen weltweit. Mit zwölf vielzitierten WissenschafterInnen fällt Österreich aber von der Spitze ab. In der Schweiz arbeiten neunmal und in Schweden fünfmal mehr vielzitierte WissenschafterInnen. Aussagekräftiger sind diese Zahlen aber wiederum, wenn sie mit der Anzahl der WissenschafterInnen in einem Land normiert werden. Der neue Indikator drückt dann die Dichte vielzitierter WissenschafterInnen in der gesamten WissenschafterInnenpopulation aus. Der Vergleich mit anderen kleinen offenen Volkswirtschaften fällt hier für Österreich ungünstig aus. In der Schweiz gibt es vierzehnmal mehr vielzitierte WissenschafterInnen je tausend WissenschafterInnen, was aber immer noch weit hinter dem Wert der USA liegt. In Schweden und Dänemark ist deren Dichte immer noch fast fünfmal höher als in Österreich.

Die Zahlen untermauern die Existenz eines „europäischen Paradoxons" nicht. Die verwendeten Indikatoren deuten darauf hin, dass die Leistungsfähigkeit der wissenschaftlichen Forschung in den meisten europäischen Ländern und so auch in Österreich zwar in der internationalen Spitzengruppe angesiedelt ist, dass der Abstand zur Weltspitze aber fallweise groß ist. WissenschafterInnen an U.S.-Hochschulen sind produktiver und ihre Ergebnisse sind über alle Disziplinen hinweg relevanter als jene ihrer europäischen Kollegen.

\subsubsection{Lehre}

Die zweite Kernfunktion der Universitäten ist die Lehre und damit auch die Ausbildung hoch qualifizierter Arbeitskräfte. Dies ist gleichzeitig der wichtigste Mechanismus, wie Wissen, das an den Universitäten geschaffen wird, sich in der Gesellschaft im Allgemeinen und im Unternehmenssektor im Besonderen verbreitet. Dadurch kommt dieser Funktion eine besondere Bedeutung für das Innovationssystem 
zu. Die Studie von Peneder (2008) zeigt, dass sich im vergangen Jahrzehnt der Strukturwandel in der österreichischen Volkswirtschaft hin $\mathrm{zu}$ innovations- und wissensintensiveren Branchen beschleunigt hat (s.a. Kapitel 4.1). Dies impliziert, dass sich der Bedarf an hoch qualifizierten Arbeitskräften erhöht hat. Eine Beschäftigungsprognose (Fritz et al. 2008) zeigt, dass sich dieser Trend auch in naher Zukunft fortsetzen wird ${ }^{59}$ (siehe Tabelle 31).

Der stärkste Anstieg der Nachfrage nach Arbeitskräften wird in hoch qualifizierten Tätigkeiten erwartet, also in Berufen, deren Ausübung üblicherweise einen Hochschulabschluss voraussetzt (ISCO $88^{60}$, Code 2). Die Beschäftigung wird bis 2012 im Schnitt um 2,2 \% pro Jahr wachsen. Maßgeblich getrieben wird diese Entwicklung laut Modellrechnungen durch einen Anstieg der Nachfrage nach naturwissenschaftlich-technischen Berufen (4,8\% pro Jahr), im biowissenschaftlich-medizinischen Bereich $(3,1 \%$ pro Jahr) sowie in sozialwissenschaftlichen Berufen $13,2 \%$ pro Jahr). Starkes Beschäftigungswachstum wird auch für einige Berufsgruppen innerhalb der technischen und nicht-technischen Fachkräfte erwartet, die formell den Berufen mit Matura zugeordnet werden (ISCO 88, Code 3). Das gilt insbesondere für die Datenverarbeitungsfachkräfte mit einem jährlichen Beschäftigungsanstieg von $5,1 \%$ pro Jahr bis 2012 .

Inwieweit dieser Anstieg in der Nachfrage nach hoch qualifizierten Arbeitskräften und damit nach dem Wissen, das ihnen an den Hochschulen vermittelt wurde, gedeckt werden kann, ist auf Grundlage der verfügbaren Daten nur sehr schwer zu bewerten. Hier sind zwei Aspekte von Bedeutung. Einerseits stellt sich die Frage, ob eine ausreichende Anzahl von Personen in einem Land durch das Ablegen eines Studiums Teil dieses Diffusionsprozesses werden kann, oder ob ein Mangel an AbsolventInnen insgesamt den Wissenstransfer unterbindet. Andererseits ist für den Wissenstransfer auch wichtig, inwieweit bei einer gegebenen AbsolventInnenzahl die Verteilung der abgeschlossenen Studien auch zur bestmöglichen Verwendung dieser AbsolventInnen in Wirtschaft und Gesellschaft führt oder ob Fehlallokationen zu AbsolventInnenmangel in einigen Bereichen und AbsolventInnenüberschuss in anderen Bereichen führen.

59 Mögliche Auswirkungen der Wirtschaftskrise im letzten Quartal 2008 und 2009 sind dabei nicht berücksichtigt. 60 Internationale Standardklassifikation der Berufe, Ausgabe 1988 


\section{Tabelle 31: Prognose der Beschäftigungsveränderung in qualifizierten Tätigkeiten (2006 bis 2012)}

\begin{tabular}{|c|c|c|}
\hline Beschäftigungsentwicklung & Absolut & In \% pro Jahr \\
\hline ISC0 88, Code 2 - WissenschafterInnen / AkademikerInnen ${ }^{1}$ & 42.100 & 2,2 \\
\hline PhysikerInnen. ChemikerInnen. MathematikerInnen. Statistiker- und InformatikerInnen & 11.600 & 4,8 \\
\hline ArchitektInnen. IngenieurInnen & 3.000 & 2,2 \\
\hline Biowissenschafter-. MedizinerInnen & 6.300 & 3,1 \\
\hline Universitäts- und HochschullehrerInnen & 1.700 & 1,8 \\
\hline LehrerInnen im Sekundarbereich & 3.900 & 0,8 \\
\hline Wissensch. LehrerInnen des Primar-Norschulbereiches. SonderschullehrerInnen & 2.000 & 0,9 \\
\hline Unternehmensberatungs- und Organisationsfachkräfte & 4.100 & 3,1 \\
\hline JuristenInnen. wissenschaftliche Verwaltungsfachkräfte im öffentlichen Dienst & 3.300 & 2,4 \\
\hline Archiv-. Bibliotheks-. InformationswissenschafterInnen. SozialwissenschafterInnen & 4.100 & 3,2 \\
\hline SchriftstellerInnen. KünstlerInnen. Geistliche. Seelsorger & 2.100 & 2,0 \\
\hline ISCO 88, Code 3 - Technische und nicht-technische Fachkräfte ${ }^{2}$ & 58.500 & 1,4 \\
\hline Material- u. Ingenieurtechnische Fachkräfte & 8.900 & 1,2 \\
\hline Datenverarbeitungsfachkräfte & 8.700 & 5,1 \\
\hline BedienerInnen optischer/elektronischer Anlagen. Schiffs-/FlugzeugführerInnen. Sicherheits-/Qualitätskontrolle & 500 & 0,3 \\
\hline Medizinische Fachberufe (ohne Krankenpflege). BiotechnikerInnen. verwandte Berufe & 3.900 & 2,1 \\
\hline Krankenpflege- und Geburtshilfefachkräfte & 18.700 & 4,4 \\
\hline Nicht-wissenschaftliche Lehrkräfte & 7.600 & 3,3 \\
\hline Finanz- und Verkaufsfachkräfte & 2.400 & 0,2 \\
\hline HandelsmaklerInnen. Vermittler gewerblicher Dienstleistungen & -1.100 & $-1,6$ \\
\hline Verwaltungs-. Zoll-. Steuerfachkräfe. PolizeikommissarInnen. DetektivInnen & 3.100 & 0,4 \\
\hline Sozialpflegerische Berufe & 5.300 & 4,0 \\
\hline Künstl. Berufe. Unterhaltungs-/Sportberufe. Ordensbrüder/-schwestern & 700 & 0,8 \\
\hline
\end{tabular}

Quelle: WIFO-Branchen- und Berufsmodelle (auf Basis PROMETEUS und MultiREG). Werte auf 100 gerundet, Differenzen aufgrund von Rundungsfehlern möglich. Prognostiziert wird die unselbständige, voll versicherungspflichtige Beschäftigung (ohne Karenzbezieherlnnen und Präsenz-/ Zivildiener).

Bezüglich des ersten Aspektes geht aus den Daten das bekannte Bild hervor, dass mit $18 \%$ die österreichische AkademikerInnenquote weit unter dem OECD Mittel von $27 \%$ liegt (OECD 2008b, S. 44). Weiters studieren in Österreich auch weniger Personen als im Durchschnitt der EU 27 Länder. Abbildung 35 zeigt, dass in Österreich die Anzahl der Studierenden stagniert, während sie in anderen Ländern, wie Dänemark, den Niederlanden, Finnland oder Schweden und auch im EU 27-Schnitt zwischen 1998 und 2006 gestiegen ist. Verringert hat sich die Anzahl der StudienabbrecherInnen. Österreich liegt bei diesem Indikator nahe dem OECDMittel von ca. 30 \% (OECD 2008b, S. 98) und konnte gegenüber den Vorjahren diesen Aspekt der tertiären Ausbildung verbessern ${ }^{61}$. Insgesamt jedoch deuten diese Indikatoren darauf hin, dass zu wenige Personen eine tertiäre Ausbildung anstreben, bzw. in Bezug auf die bestehende Nachfrage eine solche auch erfolgreich abschließen. 


\section{Abbildung 35: Anteil der Personen, die eine Einrichtung des Tertiärbereichs (Hochschule oder sonstige Einrichtung des Tertiärbereichs) im Rahmen des regulären Bildungssystems des betreffenden Landes besuchen, an der Einwohnerzahl}

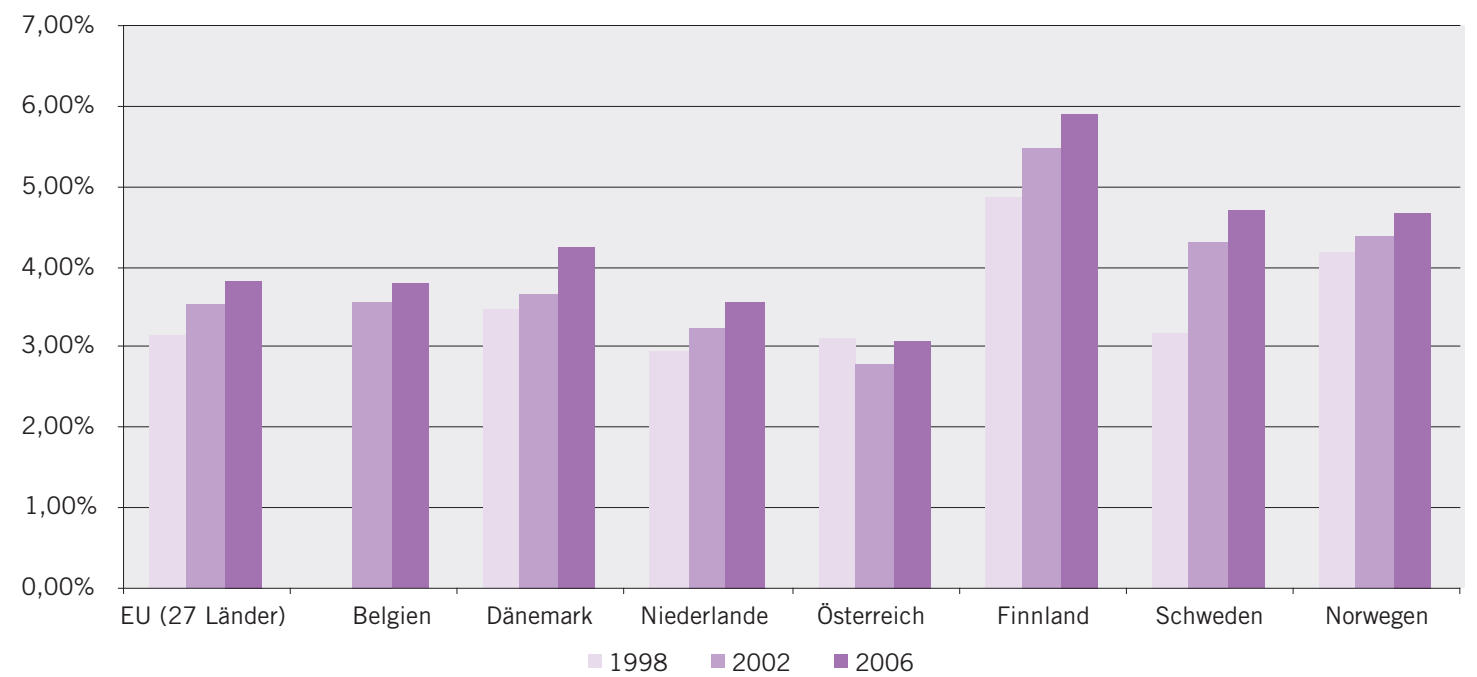

Quelle: Eurostat, WIFO-Darstellung.

Der zweite Aspekt des Wissenstransfers zwischen Universitäten und dem Unternehmenssektor ist mit der Frage verbunden, inwieweit der Wissenstransfer durch eine den Bedürfnissen entsprechende Verteilung der AbsolventInnenzahlen erfolgreich ist. Sie kann am Besten durch die Analyse der Beschäftigungschancen von HochschulabsolventInnen beantwortet werden. Ein mögliches Ungleichgewicht zwischen AbsolventInnenangebot und AbsolventInnennachfrage ist jedoch schwer abzubilden. Nur die Volkszählungsdaten des Jahres 2001 lassen verlässliche Aussagen $\mathrm{zu}$ Beschäftigungs- und Arbeitslosenquoten nach Studienrichtung $\mathrm{zu}^{62}$. Dieser Indikator kann jedoch nicht hochgerechnet werden, da hierzu auf Registerdaten der Arbeitsämter zurückgegriffen werden muss. Diese Daten wiederum erfassen aber nur jene Personen, die sich arbeitslos mel- den, bzw. bilden nachfrageseitig nur jene offenen Stellen ab, die von den Unternehmen an die Arbeitsämter gemeldet werden. Da jedoch Angebote für Stellen mit hohen Qualifikationsanforderungen nur in geringem Maße über Arbeitsämter besetzt werden, sind diese in diesen Daten unterrepräsentiert. Aus diesem Grund stellt Abbildung 36 die Arbeitslosenquote der fünfundzwanzig- bis fünfunddreißigjährigen AkademikerInnen ${ }^{63}$ für ausgewählte Studienfächer und Studienrichtungen im Jahr 2001 und die Veränderung der AMS Meldungen zwischen 2003 und 2007 getrennt dar.

Abbildung 36 zeigt, dass die AkademikerInnenarbeitslosenquoten 2001 am niedrigsten im Bereich der Ingenieurswissenschaften waren. Für AbsolventInnen einzelner natursowie wirtschafts- und sozialwissenschaftlichen Fächer konnten ähnlich niedrige Werte

62 Der Grund hierfür liegt darin, dass die Erfassung und Gliederung der österreichischen Arbeitsmarktdaten in Anlehnung an die internationale Standardklassifikation (ISCO 88, COM) nur Rückschlüsse auf das üblicherweise vorausgesetzte Ausbildungsniveau (Pflicht-, Sekundar- oder Tertiärabschluss), nicht aber über die tatsächliche Ausbildung der Beschäftigten erlaubt. In der Studie von Reinstaller - Unterlass - Prean (2008) werden diese Probleme ausführlich diskutiert.

63 Die Alterskohorte, in die die meisten StudienabgängerInnen fallen. 
beobachtet werden. Am höchsten war die Arbeitslosigkeit im Bereich der AbsolventInnen geisteswissenschaftlicher Studien. Die Veränderungen der AMS Meldungen zwischen 2003 und 2007 je 100 AbsolventInnen zeigen, dass sie für die abgebildeten Ingenieursfächer, in einigen natur- sowie wirtschafts- und sozial- wissenschaftlichen Studienrichtungen und im Bereich der Rechtswissenschaften zurückgegangen sind. Am stärksten war der Anstieg der AMS-Meldungen dagegen für Studienfächer im geisteswissenschaftlichen Bereich sowie für AbsolventInnen der Soziologie.

Abbildung 36: Arbeitslosenquote 2001 nach Studienrichtungen, Veränderung der AMS-Meldungen 2003-07, Unikat.at Karriere-Index für das Jahr 2007.

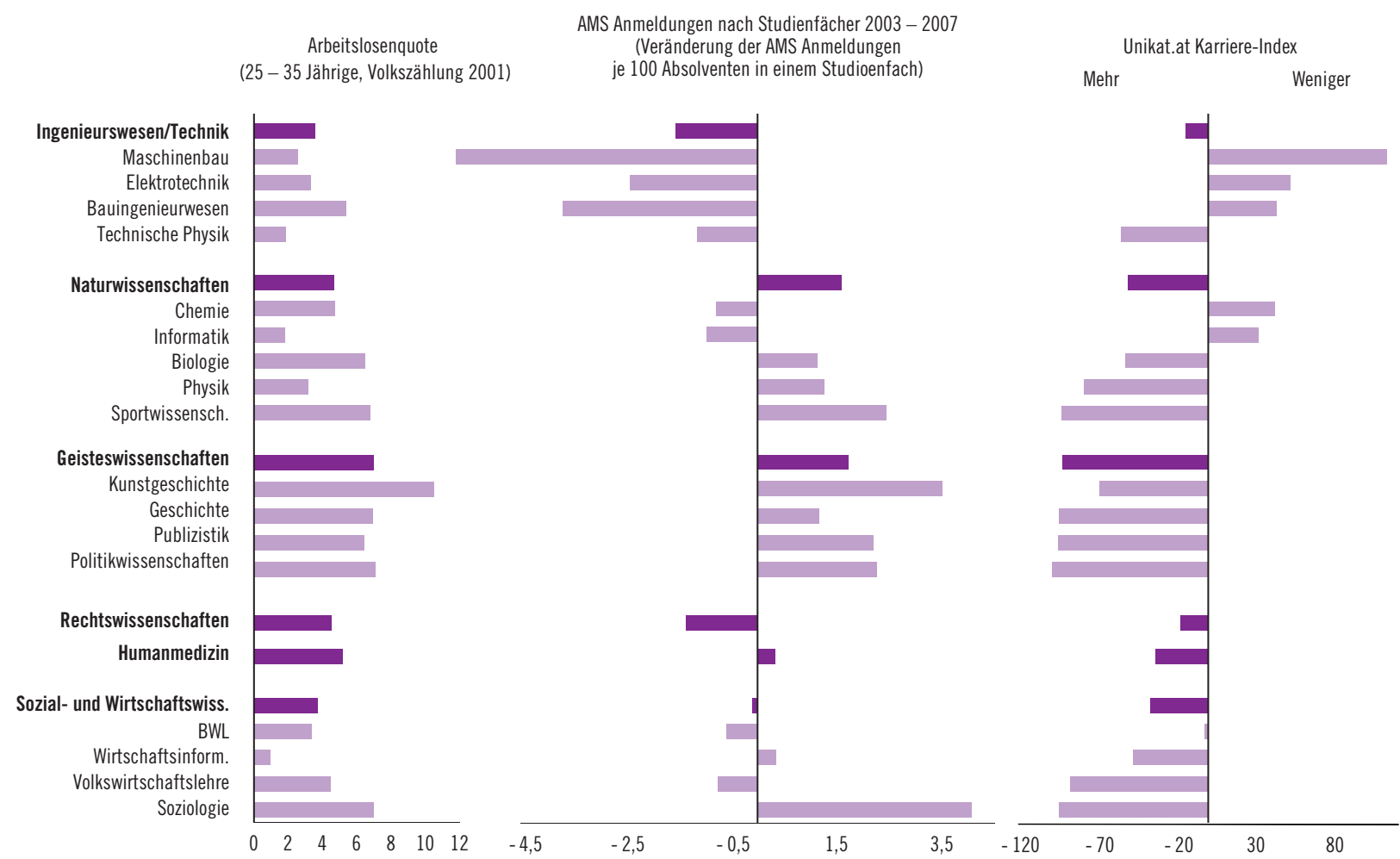

Quelle: Statistik Austria, Volkszählung 2001, AMS Meldestatisik, UNIKAT Hochschul- Informationssysteme GmbH. WIFO Darstellung. Arbeitslosenquote nach Lebensunterhalt I: Arbeitslose inkl. erstmals arbeitssuchend und Karenzgeldbezieherlnnen (wenn vorher arbeitslos); Erwerbstätige: unselbständig Beschäftigte inkl. geringfügig Beschäftigte, KarenzgeldbezieherInnen (wenn vorher erwerbstätig).

Der dritte Indikator in Abbildung 36, der Karriere-Index der Unikat.at Hochschulinformationsges.m.b.H. für das Jahr 2007, wird aus dem Verhältnis zwischen veröffentlichten Stellenangeboten im Privatsektor für AkademikerInnen ohne Berufserfahrung und der ge- schätzten Anzahl der HochschulabsolventInnen $^{64}$, die erstmalig eine Beschäftigung in der Privatwirtschaft suchen, gebildet. Dabei wird der Mitbewerb durch AbsolventInnen anderer Studienfächer (Universität, Fachhochschule, Höhere Technische Schule, Handelsakademi-

64 AbsolventInnen von Fachhochschulen sind in dieser Statistik aufgrund von Zuordnungsproblemen nicht erfasst. Eine analoge Auswertung wäre aber prinzipiell möglich. 
en) mitberücksichtigt. Bei einem Wert von 100 entsprechen einander die Zahl der AbsolventInnen und die Anzahl der ausgeschriebenen Stellen. Die Abbildung zeigt Abweichungen von diesem Referenzwert. Obwohl diese Daten eine starke Selektionsverzerrung aufweisen und nicht repräsentativ sind, bilden sie derzeit besser als andere Daten den Umstand ab, ob die erworbenen Studienqualifikationen fachspezifisch vom Unternehmenssektor nachgefragt werden ${ }^{65}$. Das Bild ist weitgehend spiegelbildlich zu jenem der Veränderungen der AMS Meldungen. Es werden mehr Stellen für AbsolventInnen in den technisch-naturwissenschaftlichen Fächern angeboten, als durch die Zahl der AbsolventInnen gedeckt werden kann. Das deutet auf einen Mangel an AbsolventInnen in diesen Fachgebieten hin.

Trotz aller datenspezifischen Einschränkungen und der mangelnden Vergleichbarkeit deuten diese Indikatoren darauf hin, dass hinsichtlich der eingangs erwähnten Beschäftigungsprognose und des Befundes eines verstärkten Strukturwandels hin zu wissensintensiven Branchen von einem verschärften Mangel an akademisch ausgebildeten Personen im Unternehmenssektor auszugehen ist. Dies wird vor allem Stellen betreffen, für die ein Universitätsstudium im naturwissenschaftlich-technischen Bereich notwendig ist und $\mathrm{zu}$ deren Aufgaben F\&E-nahe Tätigkeiten des Unternehmenssektors gehören. Damit wird auch der Wissenstransfer zwischen dem Hochschulbereich und dem Unternehmenssektor eingeschränkt, was wiederum Wettbewerbsnachteile nach sich ziehen kann. Die derzeitige Datenlage erfordert jedoch eine weitergehende und umfassende Erhebung der Karriereverläu- fe von HochschulabsolventInnen in den ersten Jahren nach ihrem Studienabschluss, damit die Lage genauer bewertet und entsprechende Maßnahmen getroffen werden können.

\subsubsection{Resümee}

Die Analyse zeigt, dass in Österreich sowie den meisten anderen EU Mitgliedsländern (und somit auf EU-Ebene insgesamt) die erste und entscheidende Bedingung für ein europäisches Paradoxon nicht erfüllt ist. Die Daten deuten darauf hin, dass die Leistungsfähigkeit der wissenschaftlichen Forschung in Österreich zwar in der internationalen Spitzengruppe angesiedelt ist, dass der Abstand zur absoluten Weltspitze in vielen Fällen jedoch groß ist. Die Literatur argumentiert aber, dass wissenschaftliche Exzellenz eine notwendige Bedingung für erfolgreichen Wissenstransfer zwischen Universitäten und Unternehmen in zweierlei Hinsicht ist. Einerseits fördert wissenschaftliche Exzellenz die Qualität der Lehre, andererseits fördert sie auch die Kooperation zwischen dem Hochschul- und dem Unternehmenssektor. Die Förderung wissenschaftlicher Exzellenz ist daher ein wichtiger Bestandteil einer Wachstumsstrategie, die sich vornehmlich auf wissenschaftliche Leistungsfähigkeit und ein hohes Innovationspotential des Unternehmenssektors stützt. Statistische Analysen zeigen, dass sich ein hohes allgemeines Bildungsniveau in einem Land, die Höhe der finanziellen Ressourcen je WissenschafterIn und die Autonomie der Universitäten in Budget-, Personal-, und Gehaltsfragen, aber auch der Wettbewerb (nicht zuletzt um Forschungsmittel) zwischen den Universitäten

65 Die Verzerrungen entstehen neben den Problemen, die auch für die anderen Indikatoren gelten, u.a. auch dadurch, dass StudienabsolventInnen oft Stellen direkt annehmen bzw. ihnen Stellen auch direkt angeboten werden. Andererseits ist auch die inhaltliche Bewertung des Problems schwierig da nicht alle Studien auf spezifische Berufsprofile vorbereiten aber trotzdem intellektuelle Fertigkeiten vermitteln, die in vielen akademisch anspruchsvollen Tätigkeitsfeldern sinnvoll eingesetzt werden können. So kann nicht von einer Fehlbesetzung gesprochen werden, wenn z. B. ein Mathematiker als EDV Analytiker arbeitet, oder aber ein Politikwissenschafter als Journalist. 
positiv auf die wissenschaftliche Leistung auswirken (Aghion et al. 2007, Bauwen et al. 2008, Reinstaller et al. 2008). Für Österreich wurden durch das UG 2002 diesbezüglich wichtige Impulse gesetzt, die nun weiterentwickelt werden müssen.

Besondere Bedeutung kommt in einer auf Wissenschaft und Innovation gegründeten Wachstumsstrategie auch den AbsolventInnen der Universitäten und anderer Hochschulen $z u$, da sie neues Wissen in die Unternehmen hineintragen und zur Anwendung bringen. Sie sind in vielen Fällen auch durch ihre Kontakte zu ihrer Alma Mater wichtige Knotenpunkte im Aufbau von Kooperationen zwischen Universitäten und Unternehmen. Trotz einer ungünstigen Datenlage liefern die in diesem Kapitel präsentierten Indikatoren Hinweise darauf, dass vor allem im technisch-naturwissenschaftlichen Bereich ein Mangel an AbsolventInnen herrscht und sich in naher Zukunft noch verschärfen wird. Insgesamt ist in Österreich die AkademikerInnenquote $\mathrm{zu}$ niedrig und die Verteilung der Studierenden zwischen unterschiedlichen Studienrichtungen ist im Hinblick auf bestehende Knappheiten ungünstig. Wie die Daten in diesem Kapitel zeigen, besteht in diesem Zusammenhang nicht nur in der Forschung, sondern zusätzlich auch in der Lehre noch ein Nachholbedarf in Österreich.

Hochschulen erfüllen ihre Schlüsselrolle in einem nationalen Innovationssystem dann am besten, wenn sie ihre traditionellen Kernaufgaben, Forschung und Lehre, auf höchstmöglichem Niveau wahrnehmen. Die Forschung ist aber nur in wenigen Wissenschaftsbereichen im absoluten globalen Spitzenfeld, wodurch die Hauptprämisse des „Europäischen Paradoxons" für Österreich (und die EU) nicht zutrifft. Der Wissenstransfer - festgemacht am Verhältnis zwischen Angebot und Nachfrage nach UniversitätsabsolventInnen - ist ebenfalls verbesserungswürdig. Damit ist das Potential der Hochschulen für das österreichi- sche Innovationssystem noch unzureichend ausgeschöpft.

\subsection{Qualifikationsstruktur der MigrantInnen in Österreich}

Die Anwerbung von GastarbeiterInnen in den sechziger und siebziger Jahren, die Polenkrise in den frühen achtziger Jahren, die kriegerischen Auseinandersetzungen im ehemaligen Jugoslawien am Beginn der neunziger Jahre, der Fall des Eisernen Vorhangs, der Beitritt Österreichs zur EU, die EU-Osterweiterung, die Neuregelung des Zugangs ausländischer Arbeitskräfte aus Drittstaaten auf den Arbeitsmarkt, der Familiennachzug niedergelassener ausländischer Arbeitskräfte, das demographische Ungleichgewicht zu weniger entwickelten Staaten, Lohnunterschiede und die zunehmende Globalisierung wirtschaftlicher Aktivitäten lassen die Größenordnung und Herkunft ausländischer Arbeitskräfte am österreichischen Arbeitsmarkt zunehmend komplexer werden. $\mathrm{Zu}$ den ausländischen Arbeitskräften aus den traditionellen Gastarbeiterregionen in Südeuropa und der Türkei gesellen sich Arbeitskräfte aus den alten und neuen EU-Staaten, besonders aus Deutschland, aus weiter entfernten Regionen Asiens und Afrikas, Flüchtlinge und AsylwerberInnen. Als Folge davon stieg der AusländerInnenanteil an der Bevölkerung und Beschäftigung und bewirkte einen Wandel in der Struktur und Zusammensetzung der ausländischen Wohnbevölkerung in Österreich nach Herkunftsregionen und Ausbildungsebenen.

Gleichzeitig hat sich die österreichische Wirtschaftsstruktur seit den neunziger Jahren deutlich gewandelt (siehe dazu Kapitel 4.1). Durch den technologischen Wandel und die zunehmende Globalisierung verschiebt sie sich zunehmend in Richtung höher qualifizierter Tätigkeiten mit den damit verbundenen Anforderungen an die Qualifikationen der Ar- 
beitskräfte. Vor diesem Hintergrund stellt sich die Frage, ob es Österreich im internationalen Vergleich gelingt, ausreichend hoch qualifizierte Arbeitskräfte zu erhalten, die entsprechend ihren Qualifikationen eingesetzt werden, zumal die AusländerInnenbeschäftigung in Österreich bislang hauptsächlich auf gering qualifizierte Arbeitskräfte aus den beiden traditionellen Gastarbeiterregionen des ehemaligen Jugoslawien und der Türkei ausgerichtet war. Aus technologiepolitischer Sicht ist hier vor allem die Situation der im Ausland geborenen AkademikerInnen in Österreich von besonderem Interesse, da diese am ehesten die heimischen Humanressourcen im Bereich Forschung und Entwicklung stärken könnten.

Dementsprechend widmet sich dieses Kapitel zwei Fragestellungen. Erstens soll untersucht werden, welche Qualifikationsstruktur die im Ausland geborenen Arbeitskräfte in Österreich aufweisen. Zweitens soll festgestellt werden, ob die in Österreich arbeitenden aber im Ausland geborenen AkademikerInnen auch ihren Qualifikationen entsprechend eingesetzt werden.

Die Datengrundlage für dieses Kapitel ist dabei die österreichische Arbeitskräfteerhebung (AKE). Dies ist eine repräsentative Stichprobenerhebung, im Zuge derer jedes Quartal etwa 22.500 zufällig ausgewählte Haushalte zu den Themen „Erwerbstätigkeit" und „Wohnen" befragt werden. Zum Thema Erwerbstätigkeit werden vor allem Fragen bezüglich des Erwerbsstatus, der geleisteten Arbeitszeit, Bildung und Beruf gestellt. Sie dient als Datengrundlage für europäische Vergleiche der Arbeitsmarktlage und ist damit der einzige jährlich erhobene Datensatz, welcher umfassende Informationen zum Bildungsstand der im Ausland geborenen Bevölkerung in Österreich beinhaltet.

Ein Nachteil der Arbeitskräfteerhebung ist allerdings, dass sie keine Vollerhebung darstellt. Dementsprechend ist sie mit einem
Stichprobenfehler behaftet. Zufällige Schwankungen können nicht ausgeschlossen werden. Diese sind vor allem dann zu erwarten, wenn kleine Gruppen betrachtet werden. Um die Probleme mit Stichprobenfehlern zu minimieren, werden in diesem Kapitel daher Durchschnitte für den Vierjahreszeitraum 2004 bis 2007 betrachtet. Obwohl diese Vorgehensweise die zufälligen Schwankungen verringert, verbleiben bei einigen Gruppen (insbesondere in der Landwirtschaft und im Bauwesen) weiterhin relativ große Stichprobenfehler. Wir übernehmen daher die in den Publikationen von Statistik Austria (z.B. Statistik Austria, 2007) vorgeschlagene Konvention, nach der Werte mit einem 95\% Konfidenzintervall von $+/-30 \%$ oder mehr in Klammern ausgewiesen werden.

\subsubsection{Qualifikationsstruktur der im Ausland geborenen AkademikerInnen in Österreich}

Entsprechend einer neueren OECD-Studie (OECD 2008a) zum internationalen Vergleich der $\mathrm{Zu}$ - und Abwanderung von so genannten "foreign-born" zwischen 28 OECD-Staaten, der allerdings auf das Jahr 2000 abstellt, ist Österreich dabei das OECD-Land mit dem geringsten Anteil an AkademikerInnen unter den im Ausland geborenen Personen. Der AkademikerInnenanteil unter den im Ausland Geborenen liegt nach diesem Vergleich in Österreich mit 11,3\% hinter Polen an letzter Stelle unter den OECD-Ländern. Damit bestätigt diese Datensammlung eine Reihe von neueren vergleichenden internationalen Studien, die zeigen, dass Österreich hinsichtlich der Qualifikationsstruktur der MigrantInnen hinter den meisten OECD-Ländern liegt. So weist Österreich auch nach den Ergebnissen von Belot und Hatton (2008) sowie Biffl (2006) den geringsten Anteil an hoch qualifizierten MigrantInnen mit einem tertiären Bildungsabschluss unter den OECD-Ländern auf. 
Tabelle 32: Kennzahlen zur Qualifikationsstruktur der Zu- und Abwanderung in OECD-Ländern (in \%)

\begin{tabular}{|c|c|c|c|}
\hline & $\begin{array}{l}\text { Mit einem } \\
\text { Universitäts- } \\
\text { abschluss }\end{array}$ & $\begin{array}{l}\text { Student- } \\
\text { Innen'1) }\end{array}$ & $\begin{array}{l}\text { Professio- } \\
\text { nalisten }^{2)}\end{array}$ \\
\hline & \multicolumn{3}{|c|}{$\ln \%$} \\
\hline Österreich & 11,3 & 11,5 & 13,3 \\
\hline Polen & 11,9 & 0,5 & 32,7 \\
\hline Italien & 12,2 & 1,2 & 17,5 \\
\hline Tschechien & 12,8 & 1,9 & 18,6 \\
\hline Deutschland & 14,9 & 8,1 & 10,2 \\
\hline Türkei & 15,2 & 1,3 & \\
\hline Slowakei & 15,7 & & 23,8 \\
\hline Griechenland & 15,9 & & 11,2 \\
\hline Frankreich & 18,1 & 7,3 & 22,1 \\
\hline Finnland & 18,9 & 1,7 & 21,6 \\
\hline Niederlande & 19,2 & & 25,3 \\
\hline Portugal & 19,3 & & 21,3 \\
\hline Ungarn & 19,8 & 2,6 & 31,8 \\
\hline Spanien & 21,1 & 1,7 & 15,5 \\
\hline Luxemburg & 21,7 & 30,5 & 23,3 \\
\hline Belgien & 23 & 4 & 31,6 \\
\hline Schweiz & 23,7 & 16 & 23,1 \\
\hline Dänemark & 23,9 & 6 & 16,9 \\
\hline Schweden & 24,3 & 4,5 & 19 \\
\hline Australien & 25,8 & 12,6 & 31,2 \\
\hline Vereinigte Staaten & 26,1 & 3,2 & \\
\hline Japan & 30 & 1,4 & \\
\hline Norwegen & 30,5 & 3,2 & 20,9 \\
\hline Neuseeland & 31 & 3,7 & 33,4 \\
\hline Mexiko & 34,8 & & 36,1 \\
\hline Großbritannien & 34,8 & 10,8 & 34,2 \\
\hline Kanada & 38 & 2,8 & 28,8 \\
\hline Irland & 41,1 & 4,8 & 38,1 \\
\hline
\end{tabular}

Quelle: OECD (2008a). ${ }^{1}$ ) Anteil an der Gesamtzahl der StudentInnen. ${ }^{2}$ ) Beschäftigte in den ISCO-Berufsgruppen 1 (Berufe mit einer leitenden Funktion) und 2 (WissenschafterInnen).

Allerdings weisen diese Vergleiche Österreich auch als ein Land aus, in dem überdurchschnittlich viele AusländerInnen studieren.
Der Anteil der ausländischen Studierenden liegt nach den Daten der OECD (2008a) bei $11,5 \%$ und ist (hinter Luxemburg, der Schweiz und Australien) der vierthöchste unter den 23 OECD-Ländern, für die hier Daten vorliegen. Auch dies bestätigen Ergebnisse früherer Studien. So gehörte Österreich auch nach einer vergleichenden Studie von Tremblay (2001) zu den Ländern mit einem der höchsten Anteile ausländischer Studierender. ${ }^{66}$

Laut Daten der Arbeitskräfteerhebung hat sich die Qualifikationsstruktur der Zuwanderung in Österreich allerdings seit dem Jahr 2000 etwas verbessert (Tabelle 33). Rund 36\% der in den späten achtziger Jahren zugewanderten Bevölkerung hatte höchstens die Pflichtschule abgeschlossen, nur 10\% der ausländischen Bevölkerung hatte einen Universitätsabschluss oder eine äquivalente Ausbildung. Im Zeitraum 2003 bis 2007 lag der Anteil der PflichtschulabsolventInnen zwar immer noch bei $31 \%$ (und damit deutlich höher als $21 \%$ in der einheimischen Bevölkerung), der Anteil der AkademikerInnen betrug allerdings bereits $19 \%$ und war damit ebenfalls höher als unter der in Österreich geborenen Bevölkerung (bei der der AkademikerInnenanteil bei 10\% lag). Im Vergleich zur österreichischen Bevölkerung ist die im Ausland geborene Bevölkerung in Österreich somit zunehmend stark an den beiden Extremen der Bildungsverteilung angesiedelt. Überdurchschnittlich hohen Anteilen an gering und hoch qualifizierten MigrantInnen stehen unterdurchschnittlich hohe Anteile im mittleren Qualifikationssegment gegenüber.

66 Ausländische Studierende in Österreich studieren dabei im internationalen Vergleich häufiger geisteswissenschaftliche bzw. künstlerische Fächer als in anderen Ländern; ingenieurswissenschaftliche Fächer werden eher seltener studiert. 
Tabelle 33: Bildungsstruktur der im In- und Ausland geborenen Bevölkerung im erwerbsfähigen Alter zwischen 15 und 64 Jahren nach Herkunftsregion, Zuwanderungsjahr und Alter bei der Zuwanderung (in \%)

\begin{tabular}{|c|c|c|c|c|}
\hline & Pflichtschule & Lehre, BMS & AHS, BHS, Meister & Universität, FH \\
\hline \multicolumn{5}{|l|}{ Herkunftsregion, Geburtsland } \\
\hline Österreich gesamt & 21 & 49 & 19 & 10 \\
\hline Ausland & 36 & 32 & 18 & 14 \\
\hline EU 15 (ohne Österreich) & 13 & 35 & 22 & 29 \\
\hline EU 12 (neue Mitgliedstaaten) & 16 & 37 & 32 & 16 \\
\hline Ehemaliges Jugoslawien (ohne SLO) & 46 & 39 & 11 & 4 \\
\hline Türkei & 73 & 18 & 7 & 2 \\
\hline Sonstige Länder & 30 & 21 & 22 & 26 \\
\hline \multicolumn{5}{|l|}{ Zuwanderungsjahr } \\
\hline $1942-1959$ & 20 & 47 & 20 & 12 \\
\hline $1960-1979$ & 47 & 29 & 13 & 11 \\
\hline $1980-1988$ & 35 & 30 & 19 & 15 \\
\hline $1989-1993$ & 36 & 37 & 17 & 10 \\
\hline $1994-2002$ & 35 & 26 & 21 & 19 \\
\hline 2003-2007 & 31 & 28 & 22 & 19 \\
\hline \multicolumn{5}{|l|}{ Alter bei der Zuwanderung } \\
\hline 0 bis 15 & 43 & 36 & 15 & 6 \\
\hline 16 bis 19 & 55 & 25 & 13 & 7 \\
\hline 20 bis 29 & 33 & 32 & 21 & 14 \\
\hline 30 bis 39 & 30 & 31 & 18 & 22 \\
\hline 40 und mehr & 33 & 29 & 15 & 23 \\
\hline
\end{tabular}

Quelle: Statistik Austria., Mikrozensus (gepoolter Datensatz 2004-2007).

Nach den Ergebnissen neuerer Analysen (siehe Huber et al. 2008) sind dabei (in der Reihenfolge ihrer Wichtigkeit) die Sendelandstruktur, die Zuwanderungspolitik und das Alter, in dem zugewandert wird, die wesentlichsten Einflussgrößen auf die Bildungsstruktur der MigrantInnen. So hängt die Bildungsstruktur der Zuwanderung auch nach Berücksichtigung anderer Faktoren ganz wesentlich von dem Sendeland ab. Die Wahrscheinlichkeit, dass (bei sonst gleichen persönlichen Charakteristika) eine im ehemaligen Jugoslawien oder der Türkei geborene Person höchstens einen Pflichtschulabschluss besitzt, ist markant höher als unter Personen aus den alten EU-Staaten (ehemaliges Jugoslawien: 46\%, Türkei: 73\%, EU15: 13\%) und die Wahrscheinlichkeit, dass Personen aus diesen Ländern einen tertiären Bildungsabschluss haben, ist deutlich niedriger. Staatsangehörige aus den 12 neuen EU-Staaten weisen im Vergleich zu den alten EU-Staaten dagegen lediglich eine um 8 Prozentpunkte höhere Wahrscheinlichkeit auf, höchstens die Pflichtschule abgeschlossen zu haben. Der Unterschied hinsichtlich der tertiären Ausbildung fällt mit 2 Prozentpunkten noch geringer aus (siehe Tabelle 33).

Insgesamt sind somit unter den zahlenmäßig relevantesten Gruppen am österreichischen Arbeitsmarkt Personen aus den 14 alten EU-Staaten, gefolgt von Staatsangehörigen aus den 12 neuen EU-Staaten, die am besten qualifizierten Arbeitskräfte, aus denen auch die meisten AkademikerInnen stammen. Die am schlechtesten qualifizierten Arbeitskräfte sind hingegen in der Türkei und Jugoslawien geboren. Außerdem haben das Zuwanderungsjahr und das Alter bei der Zuwanderung einen signifikanten Ein- 
fluss auf die Qualifikation der zugewanderten Personen. Zuwandernde, die in der Phase der Gastarbeiterzuwanderung in den sechziger und siebziger Jahren nach Österreich gekommen sind, haben im Vergleich zu den Personen, die vor 1960 nach Österreich gekommen sind, eine rund 27 Prozentpunkte höhere Wahrscheinlichkeit, höchstens die Pflichtschule abgeschlossen zu haben. Im Gegensatz dazu sind Personen, die seit der Einführung der Fremdengesetzesnovelle 2002 mit Jahresanfang 2003 nach Österreich gekommen sind, besser qualifiziert. Dies kann als Indiz dafür gewertet werden, dass die Bemühungen der Migrationspolitik der letzten Jahre, verstärkt hoch qualifizierte Arbeitskräfte anzuziehen, zu einer Verbesserung der Bildungsstruktur der Zuwandernden in Österreich geführt haben, die allerdings - wie bereits dargestellt - nicht ausreichte, um die relativ schlechte Position Österreichs im internationalen Vergleich zu verbessern.

Schlussendlich sind auch Jugendliche, die im Alter zwischen 16 und 19 Jahren nach Ös- terreich zugewandert sind, im Vergleich zu Jugendlichen, die bis zu einem Alter von 15 Jahren nach Österreich zugewandert sind, deutlich schlechter ausgebildet. Sie haben eine um 22 Prozentpunkte höhere Wahrscheinlichkeit, höchstens die Pflichtschule abgeschlossen zu haben als gebürtige ÖsterreicherInnen und auch eine geringere Wahrscheinlichkeit eines tertiären Abschlusses. Besser ausgebildet als Personen, die mit unter 15 Jahren nach Österreich gekommen sind, sind Personen, die im Alter von über 20 Jahren nach Österreich migrierten. Die Gruppe der Personen, die im Alter zwischen 16 und 19 Jahren zuwandert, stellt somit eine besondere Zielgruppe für die Bildungs- und Arbeitsmarktpolitik dar. Bei ihnen dürfte die migrationsbedingte Diskontinuität in der Bildungskarriere dazu beitragen, die Schullaufbahn abzubrechen. Dementsprechend wichtig ist es, das Schulwesen in eine umfassende Strategie zur höheren Qualifizierung der im Ausland Geborenen einzubeziehen.

\section{Tabelle 34: Fachstruktur der höchsten abgeschlossenen Ausbildung von erwerbsfähigen HochschulabsolventInnen im Alter von 15 bis 64 Jahren nach Herkunftsländern}

\begin{tabular}{|c|c|c|c|c|}
\hline & \multicolumn{4}{|c|}{ Fachbereich der höchsten abgeschlossenen Ausbildung } \\
\hline Herkunftsregion, Geburtsland & $\begin{array}{l}\text { Natur- und Ingenieurs- } \\
\text { wissenschaften }\end{array}$ & $\begin{array}{l}\text { Geistes- und Sozial- } \\
\text { wissenschaften }\end{array}$ & $\begin{array}{r}\text { Erziehungswissenschaft } \\
\text { und Gesundheit }\end{array}$ & $\begin{array}{r}\text { Andere } \\
\text { Ausbildungsfelder }\end{array}$ \\
\hline Österreich gesamt & 89.095 & 174.856 & 184.194 & 13.945 \\
\hline in \% & 19 & 38 & 40 & 3 \\
\hline Ausland & 38.589 & 55.384 & 29.562 & 5.935 \\
\hline in \% & 30 & 43 & 23 & 5 \\
\hline EU 15 (ohne Österreich) & 12.251 & 19.247 & 9.179 & 2.081 \\
\hline in $\%$ & 29 & 45 & 21 & 5 \\
\hline EU 12 (neue Mitgliedsstaaten) & 8.180 & 11.321 & 6.381 & $(1.095)$ \\
\hline in \% & 30 & 42 & 24 & 4 \\
\hline Ehemaliges Jugoslawien (ohne SLO) & 5.019 & 4.194 & 2.437 & (978) \\
\hline in $\%$ & 40 & 33 & 19 & 8 \\
\hline Türkei & $(871)$ & $(1.265)$ & $(1.097)$ & (86) \\
\hline in $\%$ & 26 & 38 & 33 & 3 \\
\hline Sonstige Länder & 12.145 & 19.200 & 10.371 & $(1.677)$ \\
\hline in $\%$ & 28 & 44 & 24 & 4 \\
\hline
\end{tabular}

Quelle: Statistik Austria, Mikrozensus 2004 - 2007 - Basis = Personen im erwerbsfähigen Alter von 15 bis 64 Jahren, Werte in Klammern= geringe Stichprobengröße. 
Die im Ausland geborenen AkademikerInnen in Österreich haben aber noch öfter als die in Österreich geborenen AkademikerInnen einen Geistes- oder Sozialwissenschaftlichen Abschluss (Tabelle 34), wobei dies vor allem auf die aus den EU15 Ländern zugewanderten AkademikerInnen zutrifft. Allerdings liegt auch der Anteil der im Ausland geborenen AkademikerInnen mit natur- und ingenieurswissenschaftlichen Abschlüssen, aufgrund des hohen Anteils der im ehemaligen Jugoslawien geborenen AkademikerInnen mit einem solchen Abschluss, höher als bei im Inland geborenen. Geringer als unter den in Österreich geborenen AkademikerInnen ist hingegen der Anteil der im Ausland geborenen AkademikerInnen mit Abschlüssen im Bereich Erziehungswissenschaft und Gesundheit.

\subsubsection{Der Einsatz der im Ausland geborenen Arbeitskräfte}

Im Ausland geborene Arbeitskräfte werden am österreichischen Arbeitsmarkt - selbst bei gleicher höchster abgeschlossener Ausbildung - auch anders eingesetzt als im Inland Geborene. So lag die Beschäftigungsquote bei den hoch qualifizierten ausländischen Arbeitskräften (AkademikerInnen) im Durchschnitt der Jahre 2004 bis 2007 mit 76\% (Männer 85\%, Frauen $67 \%$ ), obwohl am höchsten unter allen Bildungsgruppen, um rund 12 Prozentpunkte niedriger als bei den im Inland Geborenen. Diese Unterschiede zwischen im In- und im Ausland Geborenen sind dabei bei Frauen (mit annähernd 19 Prozentpunkten) erheblich größer als bei den Männern (ca. 5 Prozentpunkte) (Tabelle 35). Ähnlich sind die Arbeitslosenquoten unter den im Ausland geborenen AkademikerInnen mit rund 6,3\% (Männer 5,2\%, Frauen 7,6\%) bei ähnlichen Geschlechterunterschieden deutlich höher als bei den im Inland Geborenen. Selbst hoch qualifizierte im Ausland geborene Arbeitskräfte in Österreich sind daher mit einem deutlich höheren Arbeitslosigkeitsrisiko und geringeren Beschäftigungschancen konfrontiert als ihre im Inland geborenen KollegInnen. Unter den im Ausland geborenen Hochqualifizierten sind dabei Frauen besonders stark benachteiligt. Wie auch bei allen anderen Bildungsgruppen leiden im Ausland geborene Frauen dabei wohl unter einer doppelten Diskriminierung, aufgrund des Geschlechts und aufgrund der Herkunft. ${ }^{67}$

67 Eine detailliertere Analyse dieser doppelten Diskriminierung der im Ausland geborenen Frauen (nach z.B. Herkunftsregion, Alter oder Migrationszeitpunkt) scheitert leider an der geringen Stichprobengröße, die keine gesicherten Aussagen über Teilaggregate mehr zulässt. 
Tabelle 35: Beschäftigungs- und Erwerbsquoten der in Österreich lebenden Erwerbstätigen im Alter von 15 bis 64 Jahren nach Herkunft und Bildungsniveau

\begin{tabular}{|c|c|c|c|c|c|c|}
\hline & \multicolumn{2}{|c|}{$\begin{array}{l}\text { Bevölkerung im } \\
\text { erwerbsfähigen Alter }\end{array}$} & \multicolumn{2}{|c|}{$\begin{array}{l}\text { Beschäftigungsquote } \\
\text { in \% }\end{array}$} & \multicolumn{2}{|c|}{$\begin{array}{l}\text { Arbeitslosenquote } \\
\text { in } \%\end{array}$} \\
\hline & Österreich & Zuwanderer & Österreich & Zuwanderer & Österreich & Zuwanderer \\
\hline & \multicolumn{6}{|c|}{ Insgesamt } \\
\hline Max Pflichtschule & 992.963 & 335.825 & 47 & 51,2 & 8 & 14,2 \\
\hline Lehre, BMS & 2.280 .679 & 291.123 & 75,4 & 71 & 3,5 & 8,4 \\
\hline AHS, BHS, Meister & 888.786 & 165.998 & 74,3 & 63,4 & 2,9 & 8,9 \\
\hline Universität, FH & 462.090 & 129.072 & 88 & 75,8 & 2,1 & 6,3 \\
\hline \multirow[t]{2}{*}{ Insgesamt } & 4.624 .518 & 922.018 & 70,4 & 63,1 & 3,9 & 10 \\
\hline & \multicolumn{6}{|c|}{ Männer } \\
\hline Max Pflichtschule & 413.933 & 141.823 & 53,1 & 62,7 & 8 & 14,3 \\
\hline Lehre, BMS & 1.187 .223 & 156.442 & 81 & 77,3 & 3,2 & 8,5 \\
\hline AHS, BHS, Meister & 505.255 & 76.414 & 78 & 70,9 & 2,3 & 8,4 \\
\hline Universität, FH & 228.730 & 63.195 & 90,3 & 84,7 & 1,8 & 5,2 \\
\hline \multirow[t]{2}{*}{ Insgesamt } & 2.335 .142 & 437.874 & 76,3 & 72,5 & 3,5 & 9,7 \\
\hline & \multicolumn{6}{|c|}{ Frauen } \\
\hline Max Pflichtschule & 579.029 & 194.003 & 42,7 & 42,7 & 7,9 & 14,1 \\
\hline Lehre, BMS & 1.093 .456 & 134.681 & 69,3 & 63,8 & 3,9 & 8,3 \\
\hline AHS, BHS, Meister & 383.531 & 89.584 & 69,6 & 57 & 3,7 & 9,5 \\
\hline Universität, FH & 233.360 & 65.876 & 85,7 & 67,3 & 2,3 & 7,6 \\
\hline Insgesamt & 2.289 .376 & 484.144 & 64,3 & 54,6 & 4,4 & 10,3 \\
\hline
\end{tabular}

Quelle: Statistik Austria, Mikrozensus 2004-2007 - Basis = Personen im erwerbsfähigen Alter von 15 bis 64 Jahren, Werte in Klammern= geringe Stichprobengröße

Im Ausland geborene Arbeitskräfte in Österreich werden auch deutlich häufiger als in Österreich Geborene in Berufen eingesetzt, die unter ihrem Qualifikationsniveau liegen. Sie sind also oftmals für ihren Beruf überqualifiziert (siehe dazu auch Gächter 2006, Biffl et al. 2008). ${ }^{68}$ Insgesamt arbeitetet laut österreichischer Arbeitskräfteerhebung im Durchschnitt der Jahre 2004 bis 2007 rund die Hälfte (47\%) der im Ausland geborenen AkademikerInnen in einem Beruf unter ihrem Qualifikationsniveau, bei den im Inland Geborenen lag dieser Anteil bei 29\% (Tabelle 36). Bei MaturantInnen sind $67 \%$ der im Ausland Geborenen überqualifiziert, aber nur $48 \%$ der InländerInnen. Bei LehrabsolventInnen liegt dieses Verhältnis bei $22 \%$ (für im Ausland Geborene) zu 9\% (für im Inland Geborene).

68 Um die beruflichen Einsatzmuster der im Ausland geborenen Arbeitskräfte im Vergleich zu ihrem Bildungsniveau eingehender zu analysieren, wird der internationalen Literatur zur Über- bzw. Unterqualifizierung von Arbeitskräften (siehe Chiswick - Miller, 2007 für einen Überblick) folgend ein Indikator der Über- bzw. Unterqualifizierung gebildet. Dabei wurde folgendermaßen vorgegangen (siehe Gächter (2006), Biffl et al. (2008), Lassnigg - Vogtenhuber (2007) für ähnliche Vorgehensweisen in Österreich): Zunächst wurde anhand einer Kreuztabellierung der internationalen Berufsgruppensystematik (ISCO) mit dem Bildungstand jeder Berufsgruppe ein Qualifikationsniveau zugeordnet. Anschließend wurde für jede Person, die in der entsprechenden Berufsgruppe arbeitete, das tatsächliche Qualifikationsniveau festgestellt. Eine Person wurde als überqualifiziert eingestuft, wenn ihr tatsächliches Qualifikationsniveau höher war als das für den Beruf "normale". Als Indikator dafür, ob es im Ausland geborenen AkademikerInnen gelingt, ihr Humankapital in Österreich zu verwerten, kann dabei der Anteil der Überqualifizierten im Ausland geborenen AkademikerInnen im Vergleich zu den im Inland geborenen herangezogen werden. 
Tabelle 36: Unter- bzw. Überqualifizierung der in Österreich lebenden Erwerbstätigen im Alter von 15 bis 64 Jahren nach Herkunft und Bildungsniveau

\begin{tabular}{|c|c|c|c|c|c|c|}
\hline & \multicolumn{2}{|c|}{ Männer } & \multicolumn{2}{|c|}{ Frauen } & \multicolumn{2}{|c|}{ Insgesamt } \\
\hline & Österreich & Zuwanderer & Österreich & Zuwanderer & Österreich & Zuwanderer \\
\hline \multicolumn{7}{|c|}{ Max. Pflichtschule } \\
\hline Unterqualifiziert & 180.405 & 55.271 & 171.572 & 33.613 & 351.976 & 88.883 \\
\hline Entsprechend & 30.290 & 30.330 & 68.162 & 47.356 & 98.452 & 77.686 \\
\hline Insgesamt & 210.695 & 85.601 & 239.734 & 80.968 & 450.428 & 166.569 \\
\hline \multicolumn{7}{|c|}{ Lehre } \\
\hline Unterqualifiziert & 175.704 & 10.140 & 187.915 & 15.926 & 363.619 & 26.066 \\
\hline Entsprechend & 629.730 & 86.375 & 472.851 & 42.786 & 1.102 .581 & 129.161 \\
\hline Überqualifiziert & 79.955 & 18.587 & 67.884 & 24.966 & 147.839 & 43.554 \\
\hline Insgesamt & 885.389 & 115.102 & 728.649 & 83.679 & 1.614 .039 & 198.781 \\
\hline \multicolumn{7}{|c|}{ AHS, BHS } \\
\hline Unterqualifiziert & 28.698 & 2.986 & 13.591 & 1.484 & 42.288 & 4.471 \\
\hline Entsprechend & 154.532 & 12.629 & 106.969 & 14.310 & 261.501 & 26.939 \\
\hline Überqualifiziert & 145.899 & 32.134 & 131.088 & 32.188 & 276.988 & 64.322 \\
\hline Insgesamt & 329.128 & 47.750 & 251.648 & 47.982 & 580.777 & 95.732 \\
\hline \multicolumn{7}{|c|}{ AkademikerInnen } \\
\hline Entsprechend & 130.975 & 27.812 & 125.455 & 18.623 & 256.430 & 46.435 \\
\hline Überqualifiziert & 42.247 & 18.546 & 61.863 & 23.379 & 104.109 & 41.925 \\
\hline Insgesamt & 173.222 & 46.358 & 187.317 & 42.002 & 360.539 & 88.360 \\
\hline \multicolumn{7}{|c|}{ Anteile in \% } \\
\hline \multicolumn{7}{|c|}{ Max. Pflichtschule } \\
\hline Unterqualifiziert & 86 & 65 & 72 & 42 & 78 & 53 \\
\hline Entsprechend & 14 & 35 & 28 & 58 & 22 & 47 \\
\hline Insgesamt & 100 & 100 & 100 & 100 & 100 & 100 \\
\hline \multicolumn{7}{|c|}{ Lehre } \\
\hline Unterqualifiziert & 20 & 9 & 26 & 19 & 23 & 13 \\
\hline Entsprechend & 71 & 75 & 65 & 51 & 68 & 65 \\
\hline Überqualifiziert & 9 & 16 & 9 & 30 & 9 & 22 \\
\hline Insgesamt & 100 & 100 & 100 & 100 & 100 & 100 \\
\hline \multicolumn{7}{|c|}{ AHS, BHS } \\
\hline Unterqualifiziert & 9 & 6 & 5 & 3 & 7 & 5 \\
\hline Entsprechend & 47 & 26 & 43 & 30 & 45 & 28 \\
\hline Überqualifiziert & 44 & 67 & 52 & 67 & 48 & 67 \\
\hline Insgesamt & 100 & 100 & 100 & 100 & 100 & 100 \\
\hline \multicolumn{7}{|c|}{ AkademikerInnen } \\
\hline Entsprechend & 76 & 60 & 67 & 44 & 71 & 53 \\
\hline Überqualifiziert & 24 & 40 & 33 & 56 & 29 & 47 \\
\hline Insgesamt & 100 & 100 & 100 & 100 & 100 & 100 \\
\hline
\end{tabular}

Quelle: Statistik Austria, Mikrozensus 2004-2007 - Basis = Erwerbstätige im Alter von 15 bis 64 Jahren. 
Tabelle 37: Überqualifizierung der in Österreich erwerbstätigen HochschulabsolventInnen nach Herkunftsregion, Zuwanderungsjahr, Alter bei Zuwanderung und Fachbereich der höchsten abgeschlossenen Ausbildung

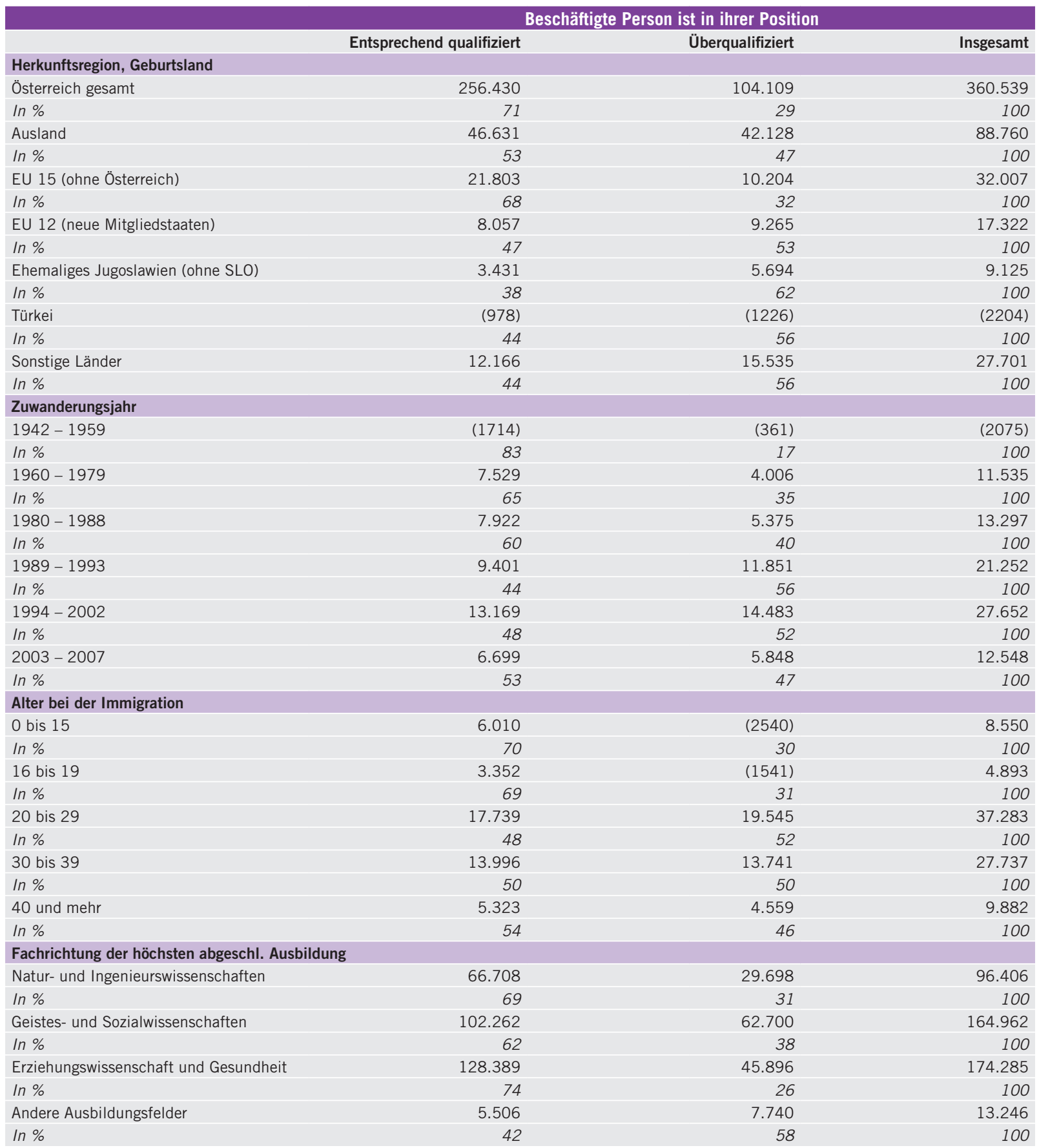

Quelle: Statistik Austria, Mikrozensus 2004-2007 - Basis = Erwerbstätige HochschulabsolventInnen im Alter von 15 bis 64 Jahre, Werte in Klammer weisen aufgrund der geringen Fallzahlen einen großen Stichprobenfehler auf. 
Wie auch schon bei Beschäftigungschancen und Arbeitslosigkeitsrisiko zeigt sich auch bei der überqualifizierten Beschäftigung eine deutliche Benachteiligung der im Ausland geborenen Frauen. Bei den Akademikerinnen arbeiten mehr als die Hälfte der Frauen in Berufen, die ein niedrigeres Qualifikationsniveau erfordern als sie haben und nur bei den AHS/BHS Absolventinnen ergeben sich keine Unterschiede zu den im Ausland geborenen Männern.

MigrantInnen können ihr im Ausland erworbenes Humankapital daher nur sehr bedingt auf dem österreichischen Arbeitsmarkt verwerten. Obwohl im Rahmen dieser Untersuchung nichts darüber gesagt werden kann, ob die Ursache für diese Probleme in unterschiedlichen Bildungssystemen, mangelnder Anrechenbarkeit von Abschlüssen, fehlenden Fremdsprachenkenntnissen, den institutionellen Bedingungen des österreichischen Arbeitsmarktes oder auch nur schlichtweg in einer Diskriminierung gegen im Ausland Geborene liegen, zeigt sich doch, dass dieser Befund für Zuwandernde aus fast allen Herkunftsländern gilt. Die einzige Ausnahme sind hier Personen, die in Deutschland oder einem anderen Land der (alten) EU 15 geboren sind. Bei ihnen ist das Ausmaß der Überqualifizierung in keinem der Ausbildungssegmente signifikant höher als bei in Österreich geborenen Arbeitskräften.

Unter den im Ausland geborenen AkademikerInnen haben Personen, die im ehemaligen Jugoslawien geboren wurden, besondere Probleme ihr im Ausland erworbenes Humankapital zu verwerten. Die Wahrscheinlichkeit, dass eine Person aus dieser Herkunftsregion, die AkademikerIn ist, in einem Beruf arbeitet, der unter ihrem Qualifikationsniveau liegt, ist um 33 Prozentpunkte höher als bei einer vergleichbaren Person, die in Österreich geboren wurde.

Neben diesen Unterschieden steigt das Risiko von AkademikerInnen, überqualifiziert beschäftigt zu sein, auch mit dem Zuwande- rungsalter der MigrantInnen. Insbesondere unterscheidet sich die Wahrscheinlichkeit, überqualifiziert beschäftigt zu sein, zwischen Personen, die im Alter von unter 15 Jahren zuwanderten und daher den Großteil ihrer Ausbildung in Österreich erhielten, kaum mehr von jener der in Österreich Geborenen.

Insgesamt deuten diese Ergebnisse darauf hin, dass die Wahrscheinlichkeit eines überqualifizierten Einsatzes von im Ausland geborenen AkademikerInnen stark von dem Zuwanderungsalter und dem Sendeland abhängen. Auffällig ist dabei, dass diese Probleme der ausländischen Bevölkerung in Österreich dort am geringsten sind, wo Sprachprobleme nur eine untergeordnete Rolle spielen (z. B. bei MigrantInnen aus Deutschland) oder ein Großteil der Ausbildung im Inland erworben wurde. Sprachliche Integration und verstärkte Bemühungen um eine formale Anerkennung von im Ausland erworbenen Qualifikationen gehören daher zu den wohl wirksamsten Maßnahmen zur Verhinderung von Überqualifikation. Neben solchen Maßnahmen scheinen aber auch einige institutionelle Besonderheiten des österreichischen Arbeitsmarktes (wie z. B. der hohe Insideranteil und die starke Betonung des Senioritätsprinzips) eine wichtige Barriere darzustellen, sodass auch hier Maßnahmen für eine bessere Integration überlegt werden könnten.

Einschränkend ist allerdings festzuhalten, dass die Probleme des Humankapitaltransfers auch ein Integrationsproblem darstellen. Es wäre daher zu vermuten, dass diese Probleme vor allem in den ersten Jahren nach der Migration von großer Bedeutung sind. Dies wird zum Teil durch die Ergebnisse in Tabelle 37 bestätigt. So unterscheidet sich nach unseren deskriptiven Auswertungen die Wahrscheinlichkeit einer überqualifizierten Beschäftigung zwischen im In- und Ausland geborenen AkademikerInnen vor allem für jene im Ausland Geborenen, die nach 1980 zuwanderten. Bei im Ausland gebo- 
renen AkademikerInnen, die in den sechziger oder siebziger Jahren nach Österreich wanderten, unterscheiden sich diese Wahrscheinlichkeiten nicht mehr so deutlich von im Inland Geborenen. Dies scheint darauf hinzudeuten, dass es den im Ausland Geborenen mit der Zeit gelingt, einen ihren Qualifikationen entsprechenden Beruf zu finden.

\subsubsection{Resümee}

Das Kapitel bietet einen Überblick über das Ausmaß und die Qualifikationsstruktur ausländischer Arbeitskräfte am Arbeitsmarkt. Österreich kann als ein Land bezeichnet werden, in dem zwar viele AusländerInnen studieren, aber nur ein geringer Anteil an hoch qualifizierten MigrantInnen lebt und aus dem eine starke Emigration hoch qualifizierter Einheimischer besteht. Jugendliche, die im Alter zwischen 15 und 19 Jahren zuwandern, haben eine auffällig schlechtere Bildungsstruktur als vergleichbare Zuwanderer, die jünger oder auch älter waren. Die Bemühungen der Migrationspolitik der letzten Jahre, verstärkt hoch qualifizierte Arbeitskräfte anzuziehen, dürften $\mathrm{zu}$ einer leichten Verbesserung der Bildungsstruktur der Zuwanderer beigetragen haben. Im Ausland geborene Hochqualifizierte sind aber deutlich öfter als ihre im Inland geborenen KollegInnen beschäftigungslos und arbeiten auch deutlich häufiger als in Österreich Geborene unter ihrem Qualifikationsniveau. AkademikerInnen, ZuwandererInnen aus tra- ditionellen Gastarbeiterländern, Personen die im Alter von mehr als 20 Jahren zuwanderten und ZuwandererInnen der Jahre 1989 bis 1993 sind davon besonders stark betroffen.

Insgesamt zeigen die vorliegenden Ergebnisse somit, dass die Qualifikationsstruktur der im Ausland Geborenen in Österreich durch einige spezifische Probleme geprägt ist. Die Handlungsspielräume zur Veränderung dieser Situation liegen einerseits in der Attraktivierung des Standortes Österreich für hoch qualifizierte Arbeitskräfte, andererseits in migrationspolitischen Interventionen in Richtung einer stärkeren Selektion der Zuwanderung nach Ausbildungskriterien. Darüber hinaus bedarf es Maßnahmen zur leichteren Transferierbarkeit von Qualifikationen nach Österreich. Abgesehen von der sprachlichen Integration scheinen dabei einige institutionelle Besonderheiten des österreichischen Arbeitsmarktes eine wichtige Barriere darzustellen. Außerdem stellen Frauen und Personen, die im Alter zwischen 16 und 19 Jahren zuwandern, eine besondere Zielgruppe dar. Bei ersteren gilt es, die doppelte Diskriminierung der hoch qualifizierten im Ausland geborenen Frauen am Arbeitsmarkt $z u$ verringern. Bei letzteren dürfte hingegen die migrationsbedingte Diskontinuität in der Bildungskarriere oftmals zu einem Abbruch der Schullaufbahn führen. Deshalb sollte auch das Schulwesen in eine umfassende Strategie zur Qualifizierung der im Ausland Geborenen einbezogen werden. 


\section{Innovationen im österreichischen Unternehmenssektor}

\subsection{Wachstum und Strukturwandel in der Wissensökonomie}

Dieser Abschnitt überprüft die These, dass in Österreich die Spezialisierung auf traditionelle, vornehmlich im mittleren Technologiesegment angesiedelte Branchen im Widerspruch $\mathrm{zu}$ dem insgesamt hohen Wohlstandsniveau sowie der positiven makroökonomischen Entwicklung steht. Es knüpft damit an die Debatte über das Österreichische Struktur-Performance Paradoxon (Peneder 1999; Tichy 2000; Janger 2007; Kattinger 2008) sowie die in jüngerer Zeit geführte Auseinandersetzung über die Bedeutung von sog. High-Tech vs. LowTech Branchen an (Falk und Unterlass, 2006; Leo et al., 2006; Schibany et al. 2007a).

Das Österreichische Struktur-Performance Paradoxon bezeichnet den Widerspruch zwischen zwei für die innovations- und standortpolitische Diskussion prägenden empirischen Befunden zur technologischen Leistungsfähigkeit der österreichischen Wirtschaft. Defizite in der Struktur der Sachgüterbranchen wurden bereits Ende der 80er Jahre aufgezeigt (Aiginger, 1987) und Mitte der 90er Jahre bestätigt (Hutschenreiter und Peneder 1997). Diesem pessimistischen Bild wurden wiederum die gute gesamtwirtschaftliche Entwicklung und das hohe Wohlstandsniveau Österreichs entgegengehalten. In der wirtschaftspolitischen Diskussion standen einander meist zwei konträre Wertungen gegenüber. Von den „Wachstumsoptimisten" wurde entweder ein kausaler Zusammenhang zwischen Branchenstruktur und Wachstum und/oder die Messgenauigkeit der bestehenden Klassifizierungssysteme, insbe- sondere die Abgrenzung von High-Tech versus Low-Tech Sektoren, in Zweifel gezogen. Im Gegenzug verweisen die "Strukturpessimisten" auf das erschöpfte historische Aufholpotenzial und interpretieren die Strukturdefizite v.a. als Indikatoren für die zukünftige Gefährdung des erreichten Wohlstands.

Vor diesem Hintergrund versuchte Peneder (1999) das Paradoxon unter Berücksichtigung der empirischen Evidenz auf beiden Seiten der Debatte aufzuarbeiten. Kurz zusammengefasst bestätigte die Untersuchung für die Gesamtwirtschaft einen Wachstumsvorsprung gegenüber vergleichbaren OECD-Ländern bei gleichzeitigen Defiziten in der Branchenstruktur. Diese Defizite betreffen v.a. den Anteil besonders wissensintensiver Produktionszweige. Weiters wurde anhand eines panelökonometrischen Schätzmodells für die 90er Jahre ein statistisch signifikanter Einfluss der Anteile sowohl von technologie- wie auch humankapitalintensiver Branchen auf das gesamtwirtschaftliche Wachstum nachgewiesen. Der positive Einfluss bestimmter Branchentypen auf das Wachstum wurde seither in einer Reihe empirischer Untersuchungen bestätigt (Peneder 2003A; Crespo-Cuaresma und Wörz, 2005; Falk 2007; Yoo 2008). Ceteris paribus, d.h. unter der Annahme alle anderen Faktoren wären gleich, folgt daraus, dass die bestehenden Strukturdefizite das Wachstum in Österreich bremsen.

Die Antwort auf das Paradoxon beginnt daher bei der Frage, welche Faktoren die ceteris paribus Bedingung verletzen. Mit anderen Worten, welche besonderen Standort- und Wettbewerbsvorteile können die bestehenden 
Strukturdefizite (mehr als) kompensieren? Eine Reihe österreichischer Besonderheiten bieten sich dafür als mögliche Erklärungen an. Ein erster Faktor ist die günstige wirtschaftsgeografische Lage inmitten zweier Hocheinkommensregionen (Süddeutschland und Norditalien) sowie in unmittelbarer Nachbarschaft zu den neuen Wachstumsregionen in Ost- und Südostmitteleuropa. Während die Nachbarschaft zu Süddeutschland und Norditalien v.a. den erfolgreichen wirtschaftlichen Aufholprozess (catching-up) begünstigt hat, war die Ostöffnung ein historischer Glücksfall, der gerade in jener Periode einsetzte, als die Aufholpotenziale weitgehend erschöpft waren. Diese Wachstumsimpulse wurden durch den Prozess der Europäischen Integration weiter verstärkt. Ein zweiter möglicher erklärender Faktor ist das über lange Zeiträume hinweg erstaunlich kohärente System der makroökonomischen Steuerung in Verbindung mit dem österreichischen Modell der sozialpartnerschaftlichen Arbeitsbeziehungen. Stichworte sind die antizyklische Fiskalpolitik in Verbindung mit einer erfolgreichen Inflationskontrolle durch die Hartwährungspolitik und der relativ hohen Reallohnflexibilität (Tichy 1984; Guger 1998). Drittens wurde auf spezifische unternehmerische Qualitäten verwiesen, die man vereinfachend mit Begriffen wie Anpassungsfähigkeit, flexibler Spezialisierung und erfolgreiche Nischenstrategien zusammenfassen kann (Tichy 2000; Peneder 2003B).

Das österreichische „Wachstumspuzzle" setzt sich somit aus vielen Faktoren zusammen und die Strukturbefunde zeigen davon nur einen, allerdings realen und ernst zu nehmenden Aspekt. Trotz guter gesamtwirtschaftlicher Leistung sind die relativ traditionellen Branchenstrukturen im Bereich der Sachgütererzeugung einerseits ein Indiz für in der Vergangenheit nicht realisierte Wachstumspotenziale sowie andererseits ein Anzeichen für in Zukunft bestehende Wachstumsrisiken, v.a. dann, wenn die Transformation vom Technologienehmer zum partiellen Technologiegeber nicht gelingt (Aiginger, Tichy, Walterskirchen, 2006).

Dieser Abschnitt untersucht daher die Frage, ob das bestehende Spannungsverhältnis zwischen traditioneller Branchenspezialisierung und gesamtwirtschaftlicher Leistung weiter besteht, oder ob sich langfristig eine Lösung des Paradoxons abzeichnet - entweder in Form geringeren Wachstums oder einem stärkeren Strukturwandel in Richtung wissensintensiver Wirtschaftszweige. Im Ergebnis ist eine Kombination aus beiden Tendenzen zu beobachten. Einerseits bestätigt sich der Einfluss des Struktur-Performance Paradoxons der 90er Jahre auf die aktuelle Wirtschaftsentwicklung, während sich andererseits eine Entspannung des Paradoxons, i.S. der Konvergenz zu europäischen Durchschnittswerten, abzeichnet.

\subsubsection{Aktuelle Befunde}

\section{Die Makroökonomie}

Wenn wir das Struktur-Performance Paradoxon im Licht der neueren Daten überprüfen, dann zeigt sich zunächst, dass Österreich den seit den 70er Jahren beständig schrumpfenden Wachstumsvorsprung (Marterbauer 2001) nach dem Jahr 2000 vollständig verloren hat. Hatte Österreich im Vergleich der Wachstumsraten in den 70er Jahren mit durchschnittlich 3,5\% p.a. noch den dritten Rang innerhalb der EU15 Länder belegt, so reichten sowohl in den $80 \mathrm{er}$ als auch in den 90er Jahren die erzielten 2,1\% p.a. nur mehr für den 8. Rang (Tabelle 38). Während zumindest in den 90er Jahren das österreichische Wachstum noch geringfügig über dem der EU15 lag, entsprechen die Wachstumsraten der Jahre 2001 bis 2007 mit 1,4\% genau dem Durchschnitt. Dieser Mittelwert wird allerdings von wenigen großen Ländern wie Italien, Frankreich und Deutschland stark 
gedrückt, während Österreich nur mehr den 10. Rang innerhalb der EU15 belegt. Der Ende der 90er Jahre im Zuge der Diskussion über das Paradoxon erwartete weitere Rückgang des gesamtwirtschaftlichen Wachstums ist somit eingetreten und der in der Aufholphase erzielte historische Wachstumsvorsprung im Vergleich langfristiger Mittelwerte verschwunden.

Tabelle 38: Langfristige Entwicklung des BIP pro Kopf (zu Preisen 2000)

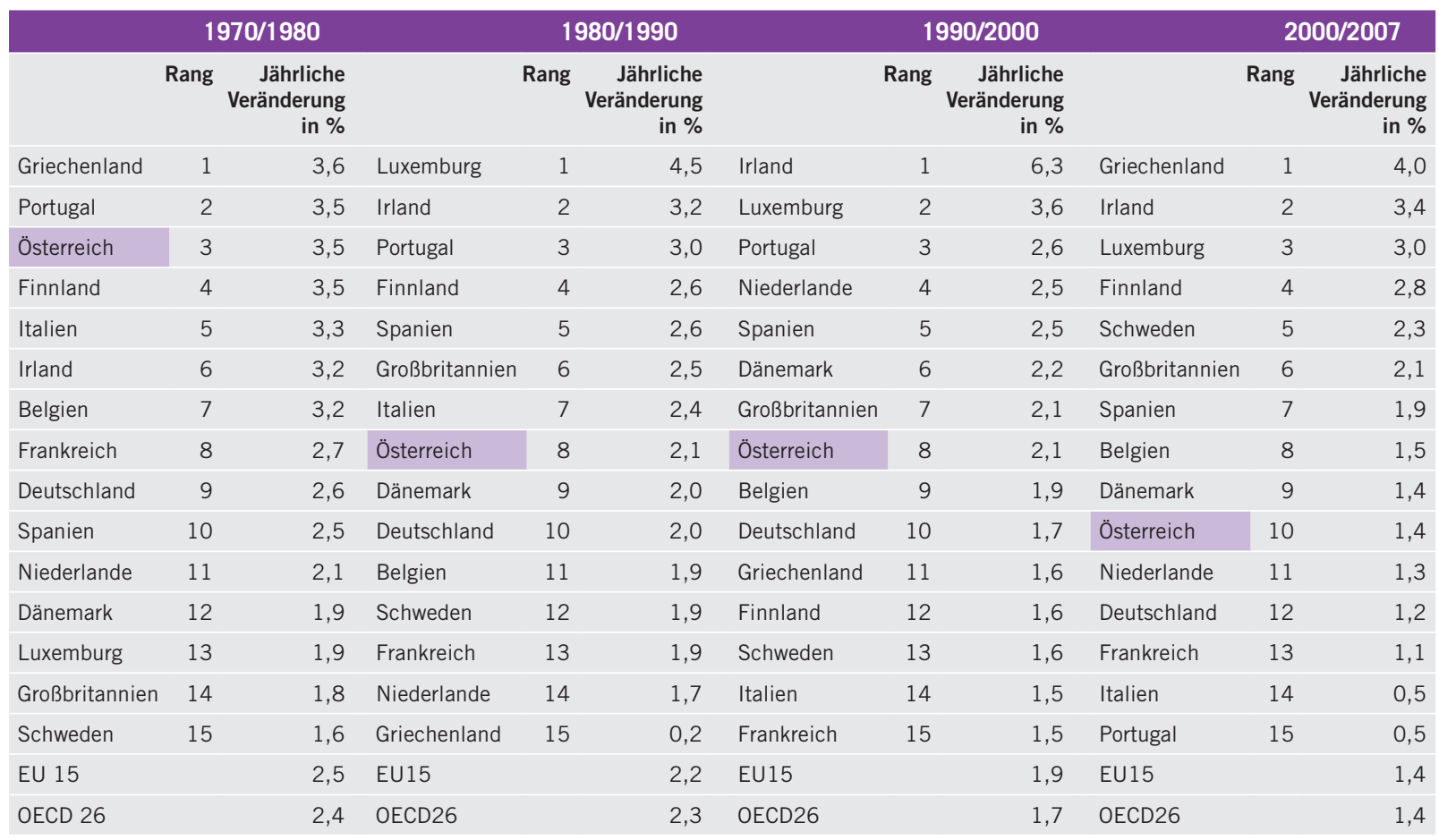

Quelle: Eurostat, Ameco, WIFO Berechnungen.

Einschränkend muss man betonen, dass die schlechte Wachstumsleistung in den Jahren nach 2000 durch die für eine Abschwungphase der Konjunktur ungewöhnlich ambitionierten Zielen der Budgetkonsolidierung verstärkt wurde, während die Kombination aus boomender Weltkonjunktur und hoher Exportorientierung in den Jahren 2006 und 2007 in Österreich eine Rückkehr zu hohen Wachstumsraten von 3,4 \% bzw. 3,1 \% brachte, die jeweils deutlich über jenen der EU15 (2,8 \% bzw. 2,7\%) lagen (Abbildung 37). Mit dem Konjunktureinbruch im Jahr 2008 sind die Wachstumsraten allgemein gesunken und für 2009 sind die Aussichten besonders schlecht. Allerdings zeigt Abbildung 37, dass Österreich in Zeiten schwacher Konjunktur meist besser abschneidet als die EU15, was auch für die nächsten Jahre einen positiven Wachstumsvorsprung wahrscheinlich macht. 
Abbildung 37: Reales Bruttoinlandsprodukt. Veränderung gegenüber dem Vorjahr in \% (Dreijahresdurchschnitte)

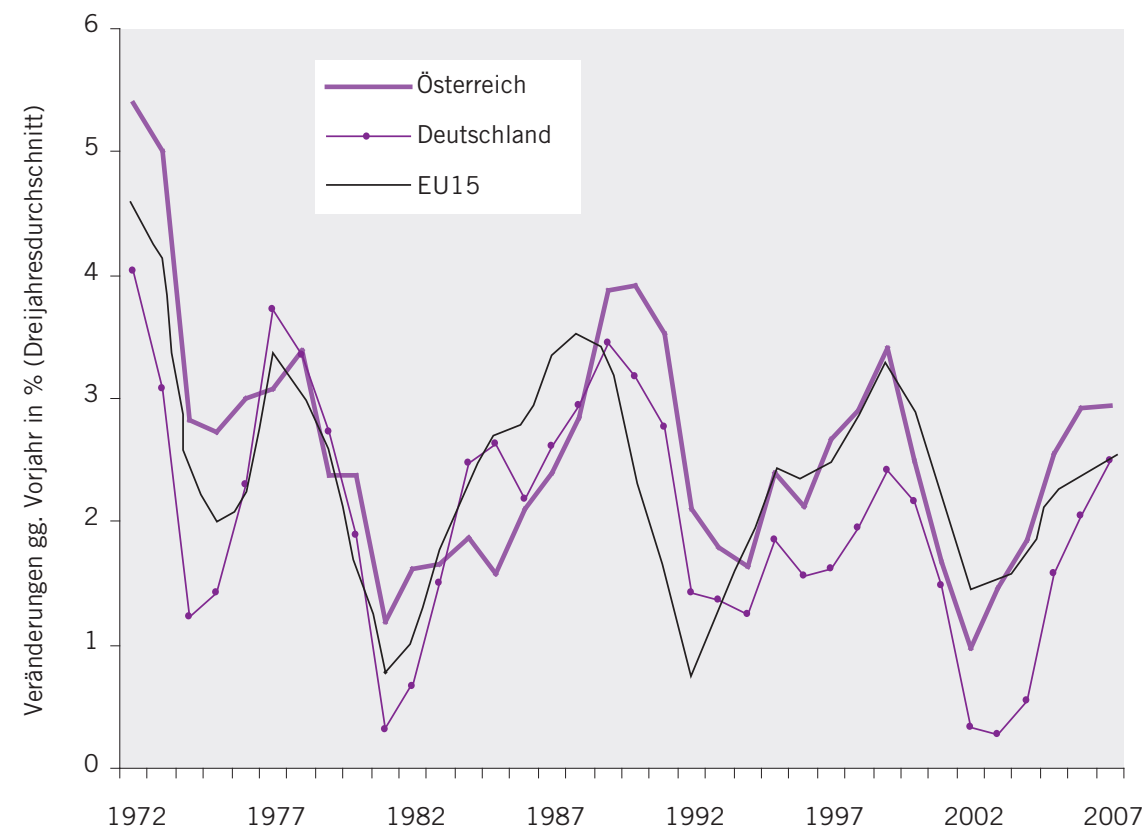

Quelle: Eurostat, Ameco.

\section{Strukturwandel}

Die meisten Befunde zur Wettbewerbsfähigkeit und Spezialisierung berücksichtigen nur die Sachgüterproduktion. Diese war zuletzt aber nur für rund $20 \%$ der gesamten Wertschöpfung und $17 \%$ der gesamten Beschäftigung in Österreich verantwortlich. Im Folgenden wird daher die traditionelle Strukturanalyse durch die Einbeziehung der Dienstleistungen erweitern. Die Instrumente dafür sind zwei neue Taxonomien zur Ausbildungs- sowie Innovationsintensität nach Sektoren, die in der folgenden Box kurz zusammengefasst sind. Im Anhang findet sich eine vollständige Auflistung.

Die beiden Panele in Abbildung 38 zeigen für die Länder der Europäischen Union sowie die USA und Japan die gemeinsame Entwicklung des BIP pro Kopf zu Kaufkraftparitäten sowie die Veränderung der Wertschöpfungsanteile für jene beiden Sektortypen, die am meisten mit der zunehmenden Bedeutung der „Wissensgesellschaft" assoziiert werden. Lediglich für die Gruppe der ausbildungsintensiven Branchen finden wir einen ausgeprägten positiven Anstieg der Wertschöpfungsanteile über die Zeit (Panel A). Während ihr Anteil in fast allen Ländern sehr konstant ansteigt, ist das Bild für die Gruppe der innovationsintensiven Branchen uneinheitlich. Wir beobachten einen ausgeprägten Strukturwandel zugunsten dieser Branchen in nur wenigen Ländern wie z.B. Finnland, Schweden oder Ungarn, aber relativ geringe Veränderungen in den meisten anderen Staaten (Panel B). ${ }^{69}$

69 Für die Gruppe der High-Tech-Sektoren nach der Definition der OECD sind in den meisten Ländern der EU, aber auch in den USA, die Wertschöpfungsanteile sogar rückläufig. Allerdings sind diese in der Branchengliederung der EU KLEMS-Datenbank nur relativ unscharf abbildbar. 


\section{Box 1: Die neuen WIFO Branchentaxonomien (Peneder 2007, 2008a)}

Branchentaxonomien sind Instrumente der Strukturanalyse, die darauf abzielt, Wachstum nicht nur als rein quantitatives Phänomen zu betrachten, sondern auch qualitative Veränderungen einbezieht. Sie dient der selektiven Abbildung von Heterogenität, mit der Absicht aus der i.d.R. unüberschaubaren Vielfalt der Einzelbeobachtungen jene Aspekte und Tendenzen herauszufiltern, denen im Hinblick auf den Untersuchungsgegenstand besondere Bedeutung zukommt. Die neuen WIFO Taxonomien zielen beide auf unterschiedliche Dimensionen der Wissensintensität der Produktion. Gegenüber den bisher zur Verfügung stehenden Klassifikationen weisen sie folgende Verbesserungen auf:

- In beiden Taxonomien werden Sachgüter und Dienstleistungen nach einheitlichen Kriterien gemeinsam klassifiziert. Einerseits wird damit der zunehmenden Unschärfe in der Grenzziehung zwischen Sachgüter- und Dienstleistungsbranchen Rechnung getragen, andererseits werden die bestehenden Unterschiede in der Ausbildungs- und Innovationsintensität entlang der neuen Kategorien der Klassifikationen konkreter abgebildet.

- Beide Klassifikationen sind mikrofundiert. Die Taxonomie zur Ausbildungsintensität beruht auf Individualdaten aus den europäischen Labour Force Surveys, jene zur Innovationsintensität auf Unternehmensdaten des Community Innovation Survey (CIS3). In beiden Fällen basiert die Berechnung auf der Verteilung unterschiedlicher Individual-/ Unternehmenstypen innerhalb eines Wirtschaftszweiges (anstelle von einfachen Branchenmittelwerten, z.B. der FせE-Intensität). Diese Vorgangsweise berücksichtigt die Heterogenität im Ausbildungsniveau der Beschäftigten bzw. im Innovationsverhalten einzelner Unternehmen und identifiziert darauf aufbauend Unterschiede in deren Verteilung auf Sektorebene. ${ }^{70}$

- Beide Klassifikationen berücksichtigen Daten für Österreich. Jene zur Ausbildungsintensität beruht zusätzlich auf vergleichbaren Daten für die USA, Großbritannien, Frankreich und Deutschland. Die Taxonomie zur Innovationsintensität wurde mithilfe der im Eurostat-Datenschutzraum zur Verfügung gestellten CIS-Mikrodaten von 22 europäischen Ländern (ink1. Österreich) identifiziert.

- Beide Taxonomien wurden mithilfe statistischer Clustermethoden bestimmt und im Rahmen einer systematischen Validierung auf ihre Robustheit hinsichtlich der Variation zwischen den Ländern getestet. Daten, Methode und Ergebnisse der Validierung sind für beide Klassifikationen ausführlich dokumentiert:

70 Die Beobachtung von sehr innovativen Unternehmen in Sektoren mit geringer durchschnittlicher Innovationsintensität und umgekehrt von wenig innovativen Unternehmen in sog. High-Tech-Sektoren ist daher kein Widerspruch, sondern ein selbstverständlicher Bestandteil der den Taxonomien zugrundeliegenden Verteilung. 
Im Gegensatz zur internationalen Entwicklung weist Österreich in beiden Dimensionen einen positiven Strukturwandel in Richtung mehr wissensintensiver Produktionszweige auf. Der Wertschöpfungsanteil der Sektoren mit besonders hoher Ausbildungsintensität ist von $10,5 \%(1985)$ auf $13,1 \%(1995)$ und $15,6 \%$ (2005) gestiegen; jener der Sektoren mit besonders hoher Innovationsintensität von 7,3 \% (1985) auf 8,0 \% (1995) und 9,2 \% im Jahr 2005. Die Strukturlücke relativ zu den EU15 ist trotz der großen Veränderungen in der Gruppe der Sektoren mit hoher Ausbildungsintensität von 3,6 Prozentpunkten (1985) auf 4,1 Prozentpunkte (2005) weiter angewachsen. Im Gegensatz dazu ging sie für die Sektoren mit hoher Innovationsintensität von 4 Prozentpunkten (1985) auf 1,8 Prozentpunkte zurück.
In Abbildung 39 wird der Strukturwandel nicht als absolute Differenz der Wertschöpfungsanteile in Prozentpunkten gemessen, sondern relativ zum Ausgangsniveau (d.h. als Quotient der Wertschöpfungsanteile von 1995) und die unterschiedlichen Sektortypen werden nach diesem Maß sortiert. Dabei zeigt sich ein überraschend klares Bild. Am meisten vom Strukturwandel profitiert haben die Sektoren mit hoher Ausbildungsintensität und jene mit hoher Innovationsintensität. In der ersten Gruppe war der Zuwachs in Österreich gleich hoch wie in der EU15, in der zweiten Gruppe sogar höher. Die Strukturanalyse zeigt Österreich somit in einem Aufholprozess mit markanten Veränderungen zugunsten wissensintensiver Produktionszweige.

\section{Abbildung 38: Wachstum und Strukturwandel $(1985,1995,2005)$}

\section{A. Branchen mit hoher Ausbildungsintensität}

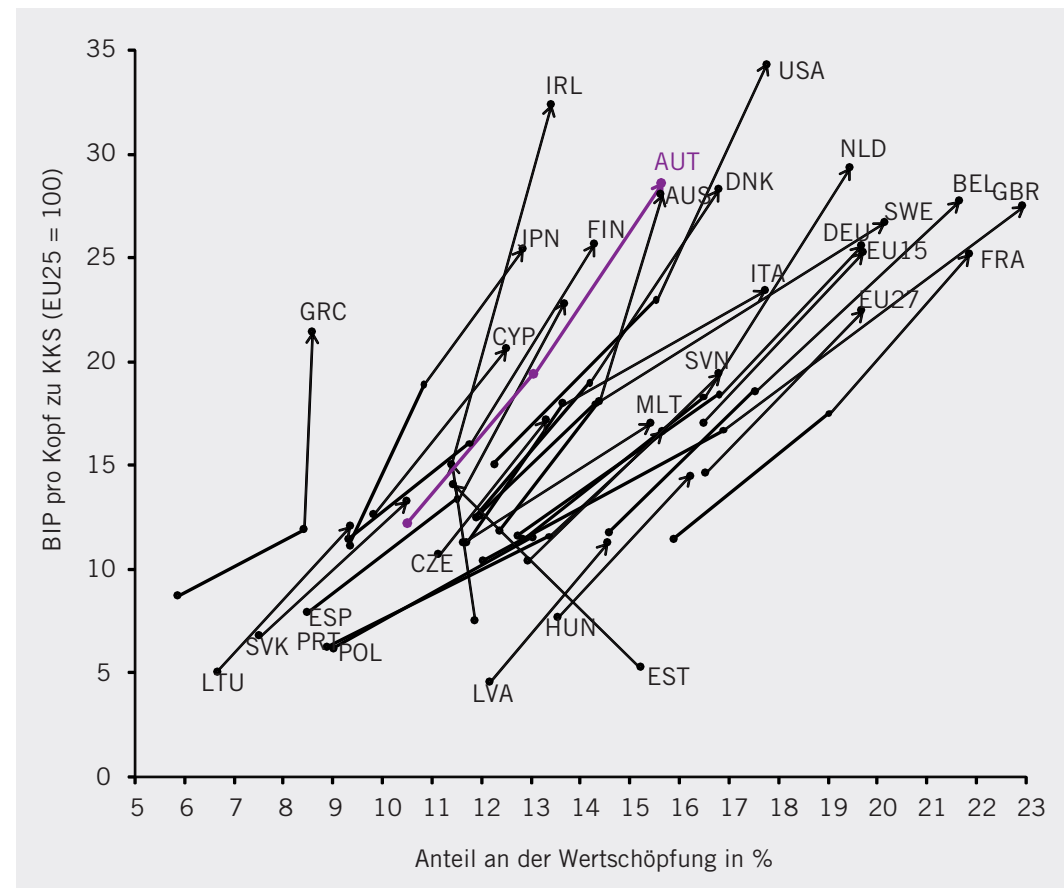

NB: Alle Pfeile beginnen im Jahr 1985 (1990) und enden im Jahr 2005.

Quelle: Eurostat, Ameco; EU KLEMS; WIFO Berechnungen. 


\section{B. Branchen mit hoher Innovationsintensität}

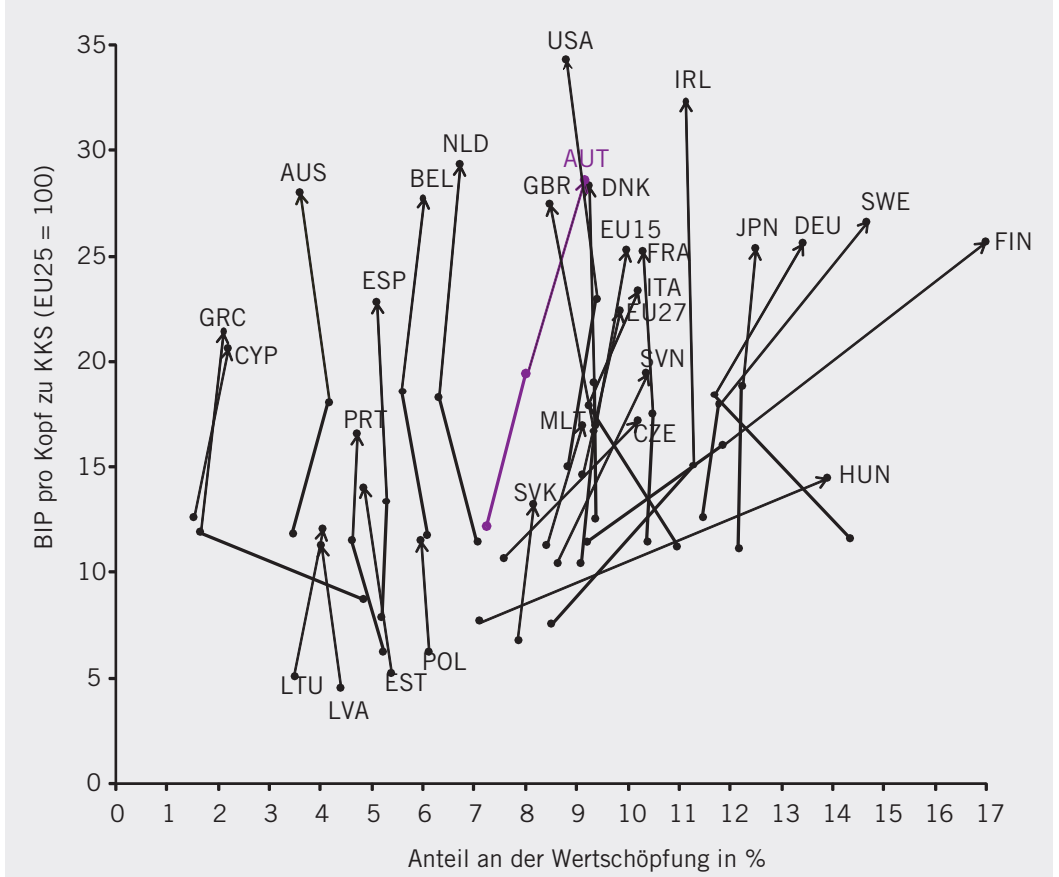

NB: Alle Pfeile beginnen im Jahr 1985 (1990) und enden im Jahr 2005.

Quelle: Eurostat, Ameco; EU KLEMS; WIFO Berechnungen.

\section{Abbildung 39: Relative Veränderung der Wertschöpfungsanteile 1995/2005 in \%}

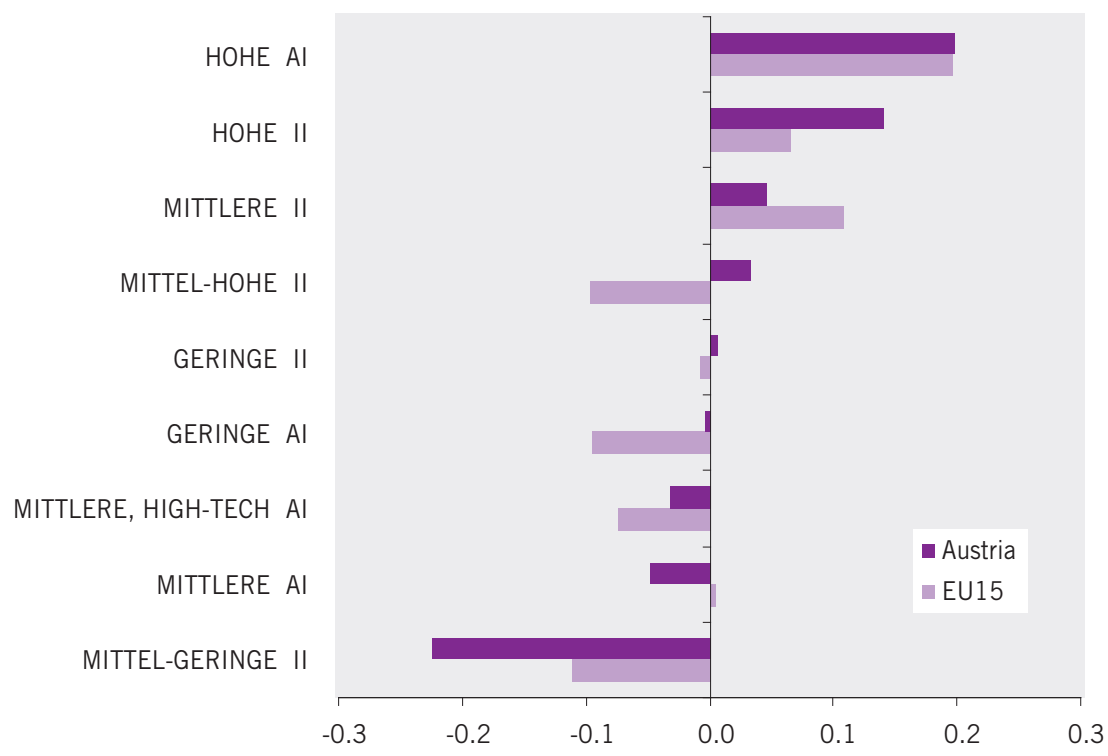

Legende: AI: Ausbildungsintensität; II: Innovationsintensität.

Quelle: Eurostat, Ameco; EU KLEMS; WIFO Berechnungen. 


\subsubsection{Resümee}

Das österreichische Struktur-Performance Paradoxon bezeichnet den in den 90er Jahren besonders auffallenden Widerspruch zwischen dem hohen Pro-Kopf Einkommen einerseits, sowie dem weitgehenden Verharren in traditionellen und wenig technologieintensiven Wirtschaftszweigen andererseits. Die aktuellen Befunde zeigen, dass der Widerspruch zwar noch besteht, sich aber von beiden Seiten einer Lösung nähert. In den Jahren nach 2000 ging der traditionelle Wachstumsvorsprung weitgehend verloren und markiert damit den endgültigen Abschluss der wirtschaftlichen Aufholphase, während zuletzt die traditionelle Strukturlücke durch den beschleunigten Wandel der Branchenspezialisierung verringert wurde. Allerdings trifft dieser Befund nicht für beide Formen der Wissensintensität in gleicher Weise zu. Während der Rückstand bei den innovationsintensiven Sektoren geringer wurde, bleibt in der Gruppe der ausbildungsintensiven Sektoren der deutliche Abstand zur EU15 trotz der hohen Zuwächse weiter bestehen. Neben den bestehenden Anstrengungen in der Innovationspolitik wird daher die Wirtschaftspolitik ihre Aufmerksamkeit in Zukunft vermehrt auf die Qualität und Effizienz der Ausbildung richten müssen.

Die Kombination aus boomender Weltkonjunktur und hoher Exportorientierung brachte in den Jahren 2006 und 2007 eine Rückkehr zu hohen gesamtwirtschaftlichen Wachstumsraten, gefolgt vom gegenwärtigen Wirtschaftsabschwung. Dem langfristigen Muster folgend und durch die Konjunkturpakete verstärkt, kann man für die nächsten Jahre wieder Wachstumsraten über dem EU Durchschnitt erwarten. Daraus lässt sich noch keine erfolgreiche Trendwende ablesen. Falls sich diese Tendenz über einen vollständigen Konjunkturzyklus hinweg bestätigen sollte, wird sich aber die Frage aufdrängen, ob die Rückkehr eines positiven Wachstumsvorsprungs als Anzeichen für den Erfolg struktureller Reformen und damit für ein verstärktes Voranschreiten mit der technologischen Grenze anstelle der traditionellen Aufholbewegung zu dieser gewertet werden kann.

\section{2 „Open Innovation“ in Österreich}

Unternehmen sind im Innovationsprozess vor drei wesentliche Herausforderungen gestellt (Pavitt 2005, S. 88): neues Wissen zu schaffen, aus dem Wissen funktionsfähige Produkte zu schaffen und ihre Produkte schließlich auf die wechselnden Marktbedürfnisse abzustimmen. Dieser Prozess erfordert die Kombination interner und externer Wissensbasen durch Kooperationen oder andere Formen des Lernens aus externen Quellen. Hier orten verschiedene Studien eine Schwäche des österreichischen Innovationssystems; der oft gehörte Befund ist, dass österreichische Unternehmen zu selten kooperieren und vor allem Wissenschaft und Wirtschaft zu wenig voneinander lernen. Der vorliegende Beitrag geht empirisch der Frage nach, ob sich an diesem Befund im letzten Jahrzehnt etwas geändert hat. Es gibt im Wesentlichen zwei Gründe, die vermuten lassen, dass Lernen aus externen Quellen in den Innovationsstrategien österreichischer Unternehmen heute tatsächlich einen höheren Stellenwert hat:

Zum einen ist die Steigerung der Kooperationsneigung seit Mitte der 1990er Jahre ein wesentliches Ziel der österreichischen Forschungs- und Technologiepolitik /vgl. Österreichischer Forschungs- und Technologiebericht 2006, S. 40 und 41). Innovationskooperationen werden mit einer Reihe von Instrumenten gefördert, so z.B. den früheren Kompetenzzentrenprogramm Kplus und K-ind/-net, nunmehr COMET, von AplusB, FHplus, bridge u.v.m.

Zum anderen argumentieren verschiedene Autoren, dass Unternehmen ihre Innovati- 
onsprozesse zunehmend öffnen, um Ideen für neue Technologien und Produkte aus verschiedenen externen Quellen zu nutzen. In jüngster Vergangenheit hat Henry Chesbrough (2003) für diese Strategie den Begriff „Open Innovation" geprägt, der seitdem in der Innovationsforschung intensiv diskutiert wird, wenngleich auch schon früher einige Autoren auf die Bedeutung externer Quellen hingewiesen haben (Bsp. Rosenberg 1982, Lundvall 1988).

Unabhängig von der Frage, was an Open Innovation tatsächlich fundamental neu ist, mobilisieren derartige Begriffe unternehmensinterne Entwicklungsprozesse und provozieren innovationspolitische Debatten. Im Folgenden soll untersucht werden, ob sich in der österreichischen Wirtschaft tatsächlich Hinweise auf eine verstärkte Öffnung der Innovationsprozesse finden lassen. Dabei wird zunächst auf die grundlegenden Ideen des Open Innovation-Paradigmas und ähnlicher Ansätze eingegangen. Danach wird untersucht, ob österreichische Unternehmen im Innovationsprozess tatsächlich vermehrt auf externes Wissen und Informationen zurückgreifen. Basis für diese Analyse sind Daten des Community Innovation Survey (CIS) aus vier Erhebungsdurchgängen, die einen Zeitraum von 12 Jahren abdecken.

\subsubsection{Das Konzept der Open Innovation}

Innovation ist ein Prozess, in dem unterschiedliche, intern generierte Wissensbasen mit externem Wissen kombiniert werden: Ergebnisse sind neue marktfähige Produkte und neuartige oder verbesserte Produktionsprozesse. Chesbrough (2003) hat diesen Entwicklungstrend im Begriff der "Open Innovation" pointiert zusammengefasst. Er definiert: „Open Innovation is a paradigm that assumes that firms can and should use external ideas as well as internal ideas, and internal and external paths to market, as the firms look to advance their technology" (Chesbrough 2003, xxiv).
Empirische Befunde für diesen Entwicklungstrend werden von diesem Autor am Beispiel einiger multinationaler Unternehmen angeführt. Bereits kurz nach Publikation seines Werks „Open Innovation" bekannten sich weltweit große Unternehmen ausdrücklich $z u$ dieser Strategie. So präsentierten etwa auf einer OECD-Konferenz in Ferrara im Herbst 2005 die Unternehmen IBM und Hitachi ihre F\&E-Strategien, welche dem Open InnovationParadigma folgen (OECD 2005).

Die Idee der offenen, vernetzten Innovation ist jedoch nicht neu: Bereits Rosenberg (1982), von Hippel (1986) oder Lundvall (1988) illustrierten die Bedeutung der Integration von und Kooperation mit Kunden, Lieferanten, Universitäten und Wettbewerbern für erfolgreiche F\&E- und Innovationstätigkeiten. Chesbrough (2003) betont mit seinem Modell jedoch besonders das Wechselspiel zwischen eigener Entwicklung und der internen Nutzung externen Wissens, zwischen eigener Kommerzialisierung und alternativen Verwertungsstrategien. Im Zeitalter von Open Innovation sind Unternehmen seiner Meinung nach dazu angehalten, so früh wie möglich auf externe Ressourcen zuzugreifen und F\&E in ständiger Interaktion mit dem Umfeld voranzutreiben. Durch die ausgeweitete Suchstrategie können neue Impulse für Entwicklungstätigkeiten gewonnen werden, indem Suchaktivitäten von der relativ engen Gruppe des eigenen Personals auf unterschiedlichste externe Akteure ausgeweitet werden, die allesamt ihre Ideen und Kompetenzen einbringen.

Chesbrough stellt das Modell der Open Innovation dem Modell der "Closed Innovation" gegenüber. Er versteht darunter die in der Praxis nach wie vor dominierende Vorgehensweise, neue Produkte und Prozesse in einer relativ isolierten F\&E-Abteilung zu entwickeln. Das Modell der Closed Innovation folgt der sequentiellen Vorstellung von betrieblicher Innovation, bei dem Unternehmen in F\&E-Abteilungen 
technologisches Wissen generieren, welches sodann in Form von verbesserten Produktionsabläufen und neuen Produkten am Markt durch das Unternehmen kommerzialisiert wird. Dabei ist die Marktorientierung und Absorption von Informationen über Kundenbedürfnisse vergleichsweise schwach ausgeprägt. Ganz anders im Open Innovation-Paradigma: Hier wird postuliert, dass Unternehmen gewinnbringende Ideen intern generieren oder extern erwerben und die darauf aufbauenden Produkte selbst, in Kooperation mit Dritten oder durch Lizenzierung am Markt kommerzialisieren.

Der Kunde ist eine wichtige Ideenquelle oder Kooperationspartner im Open Innovation-Modell. Von Hippel (1986) führte etwa das Konzept der "Lead User" (Pionieranwender) ein und weist auf die besondere Relevanz einer kleinen Gruppen von Kunden hin, die Marktbedürfnisse vorwegnehmen und wichtige Impulse für Innovationen liefern. Die Analyse der Beziehung von Produzenten und Kunden im Innovationsprozess gewann bei Lundvall (1988) eine umfassende Interpretation und war Ausgangspunkt für seine Entwicklung des Konzeptes der Nationalen Innovationssysteme. Forscher haben auch darauf hingewiesen, dass besonders neue Informations- und Fertigungstechnologien die Einbindung der Kunden in den Innovationsprozess ermöglichen (Rothwell 1994). Durch interaktive Softwaretools können so Kunden in den Innovationsprozess intensiv eingebunden werden oder es wird ihnen die Möglichkeit gegeben, neue Produkte und Produktvariationen selbst zu schaffen. Der Kunden selbst wird damit immer stärker zum Innovator, wofür auch der Begriff der User Innovation propagiert wurde (von Hippel 2005).

\subsubsection{Empirische Befunde zu Open Innovation}

Trotz der weiten Verbreitung von Open Innovation und ähnlichen Konzepten besteht in der Literatur Uneinigkeit darüber, für welche Unternehmenstypen, Branchen und Technologien diese Konzepte besonderes Potenzial bieten und welche Rahmenbedingungen die Umsetzung derartiger Strategien ermöglichen. Handelt es sich bei Open Innovation um ein universelles Paradigma, wie von Chesbrough (2003) postuliert? Oder ist die offene, vernetzte Entwicklung und Umsetzung von Innovationen auf bestimmte Branchen, Unternehmenstypen oder Produkte beschränkt?

Wenngleich sich einige große internationale Unternehmen Open Innovation-Strategien verschrieben haben und Open Innovation als Schlagwort firmiert, ist die empirische Literatur zu diesem Thema noch spärlich. Am besten untersucht ist dabei die Interaktion mit dem Kunden und eine Reihe von empirischen Studien hat die unterschiedlichen Rollen von Kunden im Innovationsprozess in verschiedenen Industrien untersucht. Lüthje und Herstatt (2004) konstatieren, dass im Sportartikelbereich und im Softwarebereich rund 10 bis 20\% der Anwender eigene Lösungen entwickelten. In einigen Industrien kommt sogar der Großteil neuer Produktideen von Nutzern, wie etwa im Medizintechnikbereich, oder bei der Entwicklung von Werkzeugen in der Halbleiterindustrie. Untersuchungen bei Unternehmen, die computergestützte Planungs- und Fertigungsinstrumente (CAD/CAM) anbieten, haben ebenfalls gezeigt, dass Prozessinnovationen häufig von Nutzern realisiert wurden. Aber auch in Low-Tech-Industrien wie der Baubranche wurde auf die Bedeutung von Kunden als Ideenlieferant oder Innovator hingewiesen (Slaughter 1993). Gewiss hängt die Bedeutung von Kunden und Anwendern von bestimmten Spezifika der Branche ab: So sind in der Biotechnologie Universitäten die wichtigste Quelle für Innovationen (Zucker et al. 1998), während bei der Entwicklung von wissenschaftlichen Messinstrumenten die Anwender dominieren (Riggs und von Hippel 1996). 
Die Studie von Herstadt et al. (2008) hat die Offenheit des Innovationsprozesses und die Verbreitung von Open Innovation-Strategien in Österreich, Belgien, Dänemark und Norwegen untersucht. Die Autoren kommen zum Schluss, dass sowohl Breite (Zahl der Partner) als auch Intensität der Innovationskooperationen österreichischer Unternehmen im internationalen Vergleich niedrig ist. Zusätzlich ist die Wertschätzung für Kunden als Quelle für innovationsrelevantes Wissen in Österreich geringer als in anderen Ländern. Beide Ergebnisse sind wichtig, da die AutorInnen weiters zeigen, dass Open Innovation-Strategien einen messbaren Einfluss auf den Unternehmenserfolg haben. Unternehmen, die diese Strategien anwenden, entwickeln mit einer höheren Wahrscheinlichkeit Marktneuheiten und erreichen einen höheren Umsatzanteil aus ihren Marktneuheiten. Auch Laursen und Salter (2006) finden, dass sich die Nutzung von Kunden als Quelle für Innovation positiv auf den Innovationsoutput auswirkt. Die Autoren finden aber keinen Konnex mit der Innovationshöhe: Kunden sind sowohl für radikale wie auch inkrementelle Innovationen gleichermaßen von Bedeutung.

Weiters liefert in Österreich die Untersuchung von Leitner (2003) zu den 50 besten industriellen Innovationen zwischen 1975 und 2000 Hinweise auf die Bedeutung unterschiedlicher externer Quellen im Innovationsprozess. Dabei zeigt sich, dass Kunden, Lieferanten und Wettbewerber wichtige Ideenlieferanten waren und insgesamt bei rund 30\% der untersuchten Innovationen mit hohem Innovationsgrad ihren Ausgang extern gehabt haben. Innovative Pilotkunden, mit denen gemeinsam erste Prototypen und Versuchsanlagen entwickelt und getestet wurden, spielten in rund einem Dutzend der Erfolgsgeschichten eine entscheidende Rolle.

\subsubsection{Open Innovation in Österreich: Ergebnisse des Community Innovation Survey (CIS)}

Die oben beschriebenen Untersuchungen zeigen, dass Open Innovation in bestimmten Sektoren große Bedeutung erlangt hat. Die Ergebnisse geben jedoch nicht Aufschluss darüber, ob es sich dabei um einen allgemeinen Trend oder eine Sondersituation einiger ausgewählter Branchen handelt. Wir werden deshalb im Folgenden auf die Ergebnisse einer repräsentativen Umfrage, des Community Innovation Surveys (CIS), zurückgreifen ${ }^{71}$. Der CIS erhebt das Innovationsverhalten von Unternehmen in der Europäischen Union und wird von EUROSTAT gemeinsam mit den nationalen statistischen Ämtern organisiert. Für Österreich stehen Ergebnisse des CIS für vier verschiedene Zeiträume zur Verfügung: 1994-1996 (CIS2), 1998-2000 (CIS3), 2002-2004 (CIS4) sowie 2004-2006 (CIS2006). Die Einzelerhebungen basieren auf gemeinsamen Definitionen und sind daher prinzipiell miteinander vergleichbar. CIS ist eine Stichprobenerhebung; die Ergebnisse einzelner Branchen und Beschäftigtengrößenklassen werden gemäß ihres Anteils an der Gesamtwirtschaft hochgerechnet.

Unterschiede zwischen den einzelnen Durchgängen bestehen erstens im Fragebogen selbst. Verschiedene Fragen wurden verändert oder in einzelnen Jahren nicht in den Fragebogen aufgenommen. Zweitens änderte sich die Stichprobe im Laufe der Zeit. So war CIS2 (1994/96) wesentlich stärker auf die Sachgüterproduktion fokussiert als spätere Erhebungen, was mit ein Grund für das starke Absinken der Innovatorenquote zwischen CIS2 auf CIS3 (1998/00) war (Falk und Leo 2004, S. 12). Drittens sollte bei einer Interpretation der Ergebnisse nicht vergessen werden, dass zwi-

71 Wir danken Andreas Schiefer (STATISTIK AUSTRIA) und Gerhard Schwarz (WIFO), die die Daten für den Beitrag zur Verfügung gestellt haben. 
schen erster und letzter Erhebung mehr als 10 Jahre liegen; während dieser Zeit hat sich das Bewusstsein für Innovation durch die Präsenz des Themas in der öffentlichen Diskussion erhöht, was möglicherweise zu einer Änderung im Antwortverhalten geführt hat. Auch gibt es große Unterschiede zwischen den einzelnen Erhebungen was das wirtschaftliche Umfeld betrifft: Die durchschnittliche Wachstumsrate des realen BIP betrug 1998/2000 3,5 \%, während der Vergleichswert für 2002/04 nur 1,1 \% ausmacht. Unternehmen ändern im Konjunkturzyklus mit sich ändernden Zukunftsaussichten auch ihre Innovationspläne.

\section{Die Bedeutung externer Informationsquellen im Innovationsprozess}

Der CIS beinhaltet zwei Fragen zum Lernen aus externen Quellen. Zum einen werden innovierende Unternehmen über ihre Einschätzung der Bedeutung verschiedener interner und externer Informationsquellen befragt. Diese
Quellen können etwa Gespräche mit Kunden, die Beobachtung von Konkurrenten oder veröffentlichte Forschungsergebnisse von Universitäten sein. Ein solcher Informationstransfer ist meist informell und setzt nicht zwingend das Einverständnis des Senders voraus.

Antworten auf die Frage nach externen Informationsquellen stehen für die Zeiträume 1994/96, 1998/00 und 2004/06 zur Verfügung. Für die Jahre 2002/04 fehlt die Frage nach den Informationsquellen im Fragebogen. Einschränkend muss außerdem angemerkt werden, dass nicht alle Informationsquellen in allen Perioden abgefragt wurden. Auch weichen die vorgegebenen Antwortkategorien für die Periode 1994/06 vom Fragebogen, der in den folgenden Erhebungen verwendet wur$\mathrm{de}, \mathrm{ab}^{72}$. Trotz dieser Änderungen zeigen die Daten deutlich, dass die Bedeutung externer Information für die Innovationsaktivitäten österreichischer Unternehmen über die Zeit zugenommen hat (Abbildung 40).

\section{Abbildung 40: Anteil der Innovatoren, die die Bedeutung verschiedener Informationsquellen als „groß“ einstufen}

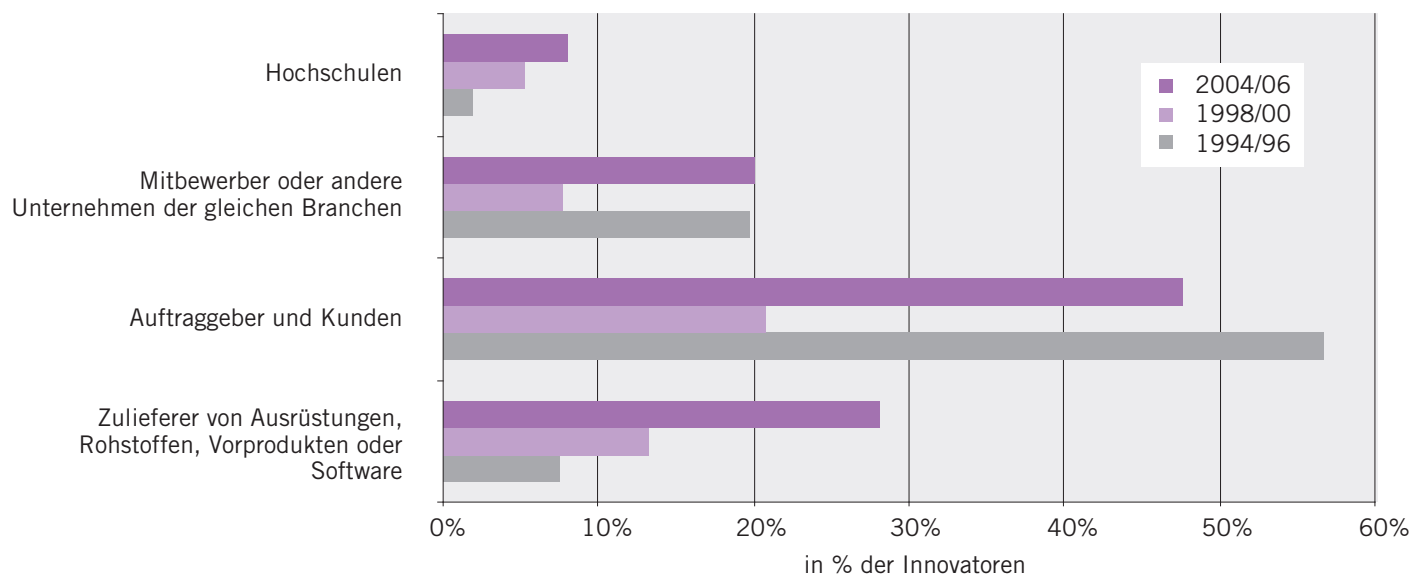

Anm.:1994/06: Anteil der Unternehmen, die eine bestimmte Informationsquelle als „sehr wichtig“ einstufen.

Quelle: WIFO, STATISTIK AUSTRIA

72 Gegenüber den späteren Perioden gibt der Fragebogen für den Zeitraum 1994/06 andere Antwortkategorien vor. Die Kategorien 1994/06 sind: nicht genutzt - wenig wichtig - wichtig - sehr wichtig. In den folgenden Erhebungen wird die Skala: keine Bedeutung - geringe Bedeutung - mittlere Bedeutung - große Bedeutung verwendet. Alle Erhebungen verwenden allerdings eine vierstufige Skala, sodass ein Vergleich möglich ist. Weiters beinhaltet der Hochschulsektor auch HTLs. 
Die Häufigkeit, in der eine bestimmte Quelle als wichtig eingeschätzt wurde, ist für den Zeitraum 2004/06 im Vergleich zu 1998/00 und 1994/06 durchwegs gestiegen. Im Vergleich der einzelnen Quellen zeigt sich - unabhängig von Branche oder Größe des Unternehmens - eine klare Rangordnung:

Auftraggeber oder Kunden stehen als bedeutendste externe Informationsquelle an erster Stelle. Allerdings lassen sich hier im Zeitablauf starke Schwankungen (besonders von 1994/96 auf 1998/00) beobachten. Unabhängig von der Branche sind Kunden die wichtigste Informationsquelle, sowohl in F\&E-intensiven Branchen wie der Elektro- und Elektronikindustrie als auch bei Konsumgütererzeugern oder verschiedenen Dienstleistungsbranchen. Die Uniformität dieses Ergebnisses ist erstaunlich, wenn man bedenkt, dass Kundenbeziehungen sowie die Konzentration und Marktmacht von Kunden zwischen Branchen äußerst unterschiedlich sind. Maschinenbauer haben typischerweise einen relativ überschaubaren Kundenkreis, die erzeugten Produkte sind oft Einzelanfertigungen. Nahrungsmittelhersteller produzieren hingegen standardisierte Produkte für eine große Zahl von Konsumenten, mit denen die Firmen nur indirekt über den Handel oder die Marktforschung kommunizieren. Verschiedene Branchen wie etwa die Automobilindustrie können ihre Lieferanten auf die Einhaltung bestimmter Qualitätsstandards verpflichten. In anderen Branchen haben Kunden diese Macht nicht.

Zweitwichtigste Informationsquelle sind die Zulieferer von Ausrüstungen, Rohstoffen oder Vorprodukten. Erwartungsgemäß werden Lieferanten vor allem in kapitalintensiven Branchen mit geringer F\&E-Intensität wie der Nahrungsmittelindustrie, Textilindustrie, der Holzbearbeitung und der Erzeugung von Papier und Pappe als wichtige Innovationsquelle geschätzt. Pavitt (1984) und andere Autoren haben Innovationsaktivitäten in diesen Branchen deshalb treffend als "supplier domi- nated industries" charakterisiert. Die Bedeutung von Lieferanten als Informationsquelle erreicht hier jene der Kunden. Obwohl heute auch verschiedene Dienstleistungsbranchen wie etwa der Bankensektor oder die Telekommunikation aufgrund ihrer umfangreichen Investitionen in Informations- und Kommunikationstechnologien (IKT) als „supplier dominated" bezeichnet werden können, erreichen Lieferanten in keiner Dienstleistungsbranche ähnliche Bedeutung wie in der Sachgüterproduktion. Zwischen großen, mittleren und kleinen Unternehmen gibt es keine Unterschiede in der Einschätzung der Bedeutung von Lieferanten als Quelle für Innovationen.

Drittwichtigste Quelle sind Konkurrenten. Die Funktionsweise neuer Produkte und Produktionsprozesse lässt sich oft nur unzureichend vor Nachahmung schützen (Cohen et al. 2000). Besonders schwierig ist der Schutz von Innovationen vor Nachahmung im Dienstleistungssektor. Als Folge werden Konkurrenten - oft unfreiwillig - zu wichtigen Informationsquellen im Innovationsprozess. Etwa jedes fünfte innovative Unternehmen in Österreich schätzt die Bedeutung von Mitbewerbern als Informationsquelle als wichtig ein. Im Unterschied zu Lieferanten werden Konkurrenten als Quelle für innovationsrelevante Informationen in Mittel- und Hochtechnologiebranchen wie dem Maschinenbau oder der Elektroindustrie tendenziell höher eingeschätzt als in Branchen mit geringer F\&E-Intensität. Ebenfalls höher ist ihre Wertschätzung im Dienstleistungssektor und hier vor allem bei Banken und im Handel.

Auch die Einschätzung der Bedeutung von Universitäten, Fachhochschulen sowie anderer gemeinnütziger Forschungseinrichtungen nimmt im Lauf der Zeit beträchtlich zu. Insgesamt bleibt die Bedeutung dieser wissenschaftlichen Quellen jedoch deutlich hinter jener der marktbezogenen Quellen zurück. Erwartungsgemäß ist die Einschätzung der Bedeutung in Mittel- und Hochtechnologiebranchen über- 
und in Niedrigtechnologiebranchen sowie im Dienstleistungssektor unterdurchschnittlich. Die Einschätzung von Universitäten als Informationsquelle steigt mit der Unternehmensgröße an. Die Erklärung dafür ist einfach: Für die Beobachtung, Aufnahme und Verarbeitung der Forschungsergebnisse von Universitäten braucht es bestimmte Kapazitäten an Forschungspersonal und permanente F\&E-Aktivitäten. Diese Ressourcen finden sich tendenziell häufiger in großen Unternehmen. Insgesamt bleibt die Einschätzung von Universitäten und anderen Forschungseinrichtungen aber auch bei Großunternehmen wesentlich hinter jener von Kunden oder Lieferanten zurück.

\section{Innovationskooperationen im österreichischen Unternehmenssektor}

Ein zweiter Indikator für das Lernen aus externen Quellen - neben der Einschätzung verschiedener Informationsquellen - ist das Ausmaß, in dem Unternehmen Innovationskooperationen eingehen. Im Unterschied zu Informationsquellen sind Kooperationen ein deutlich formaleres Instrument des Informations- und Wissensaustausches (Hagedoorn 2002; Caloghirou et al. 2003): Sie werden durch Übereinkommen geschlossen und haben deshalb einen höheren Grad an Verbindlichkeit. Kooperationen erlauben Unternehmen nicht nur Wissen und Informationen auszutauschen. Kooperation ermöglicht auch, im Innovationsprozess durch Zusammenarbeit Skaleneffekte zu nutzen, Entwicklungskosten zu teilen und so Risiko und Unsicherheit zu reduzieren. Darüber hinaus regeln Kooperationsvereinbarungen oft auch die Verwertung der Ergebnisse der Zusammenarbeit. Üblicherweise wird angenommen, dass in Kooperationen multilateraler Austausch herrscht. Die Auftragsvergabe an Dritte ohne aktive Zusammenarbeit wird deshalb nicht als Kooperation betrachtet.

Trotz der großen Aufmerksamkeit von Seiten der Politik hat sich die Kooperationsneigung über einen langen Zeitraum nur wenig verändert (vgl. Tabelle 39). Der Anteil kooperierender Unternehmen beträgt zwischen 1994/96 und 2002/04 relativ konstant ein Fünftel der Innovatoren. Der niedrigste Wert findet sich im Zeitraum 2002/04; möglicherweise haben das schwache Wirtschaftswachstum und unsichere Zukunftserwartungen in diesen Jahren die Bereitschaft zu Kooperation verringert. Eine konstante Zunahme der Innovationskooperationen über den gesamten Zeitraum lässt sich nur für Unternehmen mit mehr als 250 Beschäftigten beobachten. Tatsächlich werden die Maßnahmen zur Förderung von Innovationskooperationen wie Kplus vor allem von Großunternehmen in Anspruch genommen (Hutschenreiter 2004, S. 22).

\section{Tabelle 39: Anteil von Unternehmen mit Innovationskooperation an allen Innovatoren}

\begin{tabular}{lcccc} 
& $\mathbf{1 9 9 4 / 9 6}$ & $\mathbf{1 9 9 8 / 0 0}$ & $\mathbf{2 0 0 2 / 0 4}$ & $\mathbf{2 0 0 4 / 0 6}$ \\
\hline Insgesamt & $18,7 \%$ & $21,2 \%$ & $17,4 \%$ & $38,9 \%$ \\
Sachgütererzeugung & $19,7 \%$ & $18,5 \%$ & $18,8 \%$ & $37,9 \%$ \\
Dienstleistungen & $17,2 \%$ & $23,3 \%$ & $15,8 \%$ & $39,5 \%$ \\
$10-49$ Beschäftigte & $14,6 \%$ & $17,9 \%$ & $13,7 \%$ & $34,4 \%$ \\
$50-249$ Beschäftigte & $25,4 \%$ & $21,6 \%$ & $19,7 \%$ & $42,7 \%$ \\
250 und mehr Beschäftigte & $41,0 \%$ & $38,9 \%$ & $49,1 \%$ & $66,6 \%$
\end{tabular}

Quelle: WIFO, STATISTIK AUSTRIA 
Erst im Zeitraum 2004/06 ist ein deutlicher Anstieg der Kooperationsneigung zu beobachten. Es lässt sich allerdings nicht eindeutig sagen, ob diese Zunahme nicht durch Änderungen im Fragebogen mit erklärt werden $\mathrm{kann}^{73}$. Nach den letzten verfügbaren Zahlen der Erhebung 2004/06 gehen etwa 40 \% der österreichischen Innovatoren Innovationskooperationen ein. Die Kooperationsneigung ist im Dienstleistungssektor und der Sachgütererzeugung etwa gleich hoch. Sie steigt mit der Unternehmensgröße, was die relativ größeren Kapazitäten von Großunternehmen für Partnersuche, Überwachung von Kooperationen und die höheren absorptiven Kapazitäten großer Unternehmen reflektiert.

Österreichische Unternehmen sind in den letzten 15 Jahren zunehmend international tätig geworden. Gleichzeitig haben Tochtergesellschaften ausländischer Unternehmen ihre Innovationsaktivitäten in Österreich wesentlich ausgeweitet. Beide Entwicklungen sollten auch die Vernetzung des österreichischen Unternehmenssektors mit dem Ausland gestärkt haben. Die Daten zeigen allerdings keinen derartigen Effekt. Der Anteil der Unternehmen mit Kooperationspartnern im Ausland ist im Zeitablauf nahezu konstant geblieben /der Anteil von kooperierenden Unternehmen mit Partnern in Europa ist von 58,6 \% (1998/00) auf 60,2 \% (2004/06) gestiegen). Ebenso wie Kooperationen mit europäischen Partnern stagnieren die Anteile jener kooperierenden Unternehmen, die Partner in den USA oder anderen Ländern außerhalb Europas haben. Es lassen sich hier keine wesentlichen Unterschiede zwischen der Sachgüterproduktion und dem
Dienstleistungssektor erkennen. Erwartungsgemäß steigen internationale Kooperationen mit der Unternehmensgröße an.

Eine wesentliche Annahme der Literatur zu Open Innovation ist der Gedanke, dass Unternehmen zunehmend unterschiedliche Typen von Partnern als Wissensquellen für die Lösung verschiedener Probleme nutzen. Wurde früher ausschließlich mit Kunden kooperiert, schließt das Unternehmen heute für ein spezifisches Problem eine Kooperation mit einer Universität, während in einem anderen Segment die Zusammenarbeit mit einem Konkurrenten gesucht wird. Die Verbreitung von Open Innovation-Strategien in der Wirtschaft sollte also dazu führen, dass Unternehmen vermehrt mit unterschiedlichen Typen von Partnern kooperieren.

Die Ergebnisse des CIS zeigen, dass österreichische Unternehmen im Zeitablauf den Kreis ihrer Kooperationspartner tatsächlich erweitert haben (Abbildung 41). Besonders bemerkenswert im Sinne eines offenen Suchprozesses ist die Zunahme von Unternehmen, die mit Kunden und Lieferanten kooperieren. Waren es 1998/00 noch 54,8 \%, die mit Zulieferer bzw. $43,5 \%$, die mit Kunden kooperierten, arbeiten 2004/06 jeweils etwa $60 \%$ aller kooperierenden Unternehmen mit diesen beiden Gruppen von Partnern zusammen. Hier zeigen sich deutliche Parallelen mit der Einschätzung von Kunden und Lieferanten als Informationsquellen im Innovationsprozess. Die Wertschätzung für diese beiden Gruppen ist im Zeitablauf ebenfalls deutlich angestiegen. Vertikale Kooperationen mit Kunden oder Lieferanten sind die am meisten verbreitete Form der Zusammenarbeit.

73 Der Fragebogen des CIS4 definiert Innovationskooperation als "aktive Teilnahme Ihres Unternehmens zusammen mit anderen Unternehmen oder nicht-kommerziellen Einrichtungen an gemeinsamen Forschungsprojekten oder anderen Innovationsaktivitäten", während CIS2006 von „aktive Teilnahme Ihres Unternehmens zusammen mit anderen Unternehmen oder nicht-kommerziellen Einrichtungen an gemeinsamen Innovationsaktivitäten" spricht. Möglicherweise haben die Befragten des CIS4 nur F\&E-Kooperationen gemeldet. 


\section{Abbildung 41: Anteile verschiedener Typen von Kooperationspartnern sowie verschiedener Regionen an Innovationskooperationen in Österreich}

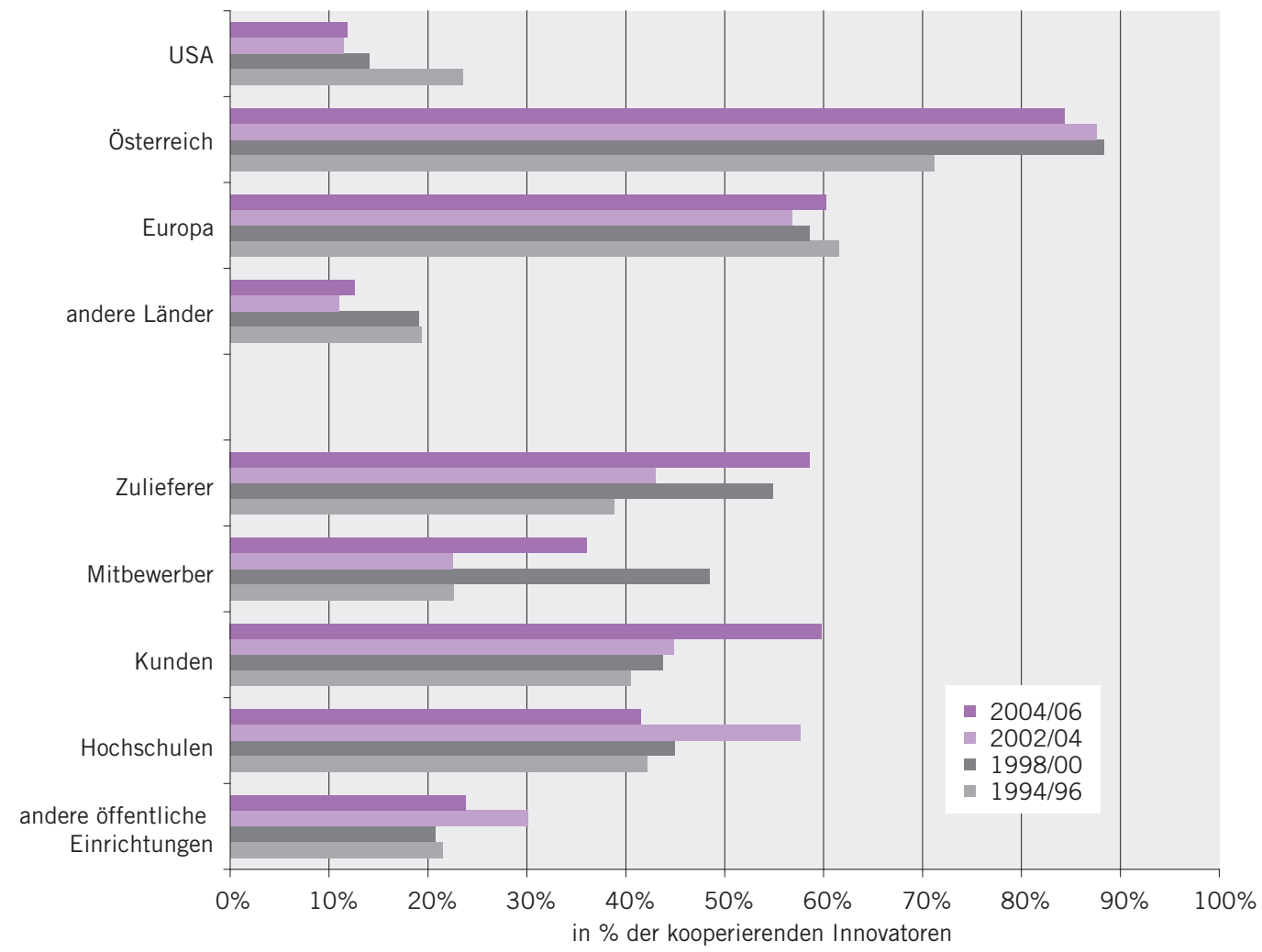

Quelle: WIFO, STATISTIK AUSTRIA

Hingegen stagniert der Anteil von kooperierenden Unternehmen, die mit Universitäten, Fachhochschulen oder anderen Forschungsreinrichtungen zusammenarbeiten. 1994/96 gaben $42 \%$ der kooperierenden Unternehmen an, mit einer Universität zusammenzuarbeiten, 2004/06 sind es 41,5 \%. Eine ähnliche Entwicklung ist bei Kooperationen mit anderen Forschungseinrichtungen zu beobachten. Dies ist überraschend, wenn man bedenkt, dass der Schwerpunkt vieler einschlägiger Förderprogramme in der Stimulierung der Kooperation zwischen Wissenschaft und Wirtschaft liegt und die Wertschätzung von Universitäten als Informationsquelle zugenommen hat.

Dieser Rückgang sollte allerdings mit Vorsicht interpretiert werden; es ist zu berück- sichtigen, dass sich dieser Anteil auf alle Unternehmen mit Innovationskooperationen bezieht. Wenn sich die generelle Kooperationsneigung erhöht, kann trotz sinkendem Anteil die absolute Zahl der Unternehmen, die mit Hochschulen kooperieren, zugenommen haben. Der Rückgang kommt in diesem Fall zustande, weil andere Kooperationsformen stärker gewachsen sind. Deshalb sollte der Befund mit anderen Daten abgeglichen werden, z.B. Ko-Patentierungen oder gemeinsamen Publikationen zwischen Unternehmen und Universitäten.

Im Gegensatz zur geografischen Verteilung zeigen sich bei der Verteilung nach Partnertypen beträchtliche Unterschiede zwischen Unternehmen der Sachgüterproduktion und 
Dienstleistern. Letztere kooperieren deutlich seltener mit Hochschulen oder Forschungsorganisationen, jedoch häufiger mit Mitbewerbern. Dienstleistungsinnovationen sind in einem geringeren Ausmaß technologiebasiert und häufiger nicht-technische Organisations- oder Marketinginnovationen (vgl. Miles 2005). Innovationen von Dienstleistungsunternehmen sind in einem höheren Ausmaß kundenspezifische Entwicklungen, was sich in den Daten allerdings nicht widerspiegelt. Möglicherweise ist dies eine Folge der Samplezusammensetzung, in der bestimmte Beratungsdienstleistungen, Einzelhandel sowie der Tourismus nicht enthalten sind und deshalb informations- und güterzentrierte Dienste ein Übergewicht haben.

\subsubsection{Resümee}

Der Beitrag untersucht die Frage, ob sich die Innovationsstrategien österreichischer Unternehmen im letzten Jahrzehnt hin zu mehr externer Kooperation und mehr Lernen aus externen Quellen entwickelt haben. Verschiedene Ansätze in der Innovationsliteratur wie etwa Open Innovation gehen davon aus, dass Unternehmen ihre Innovationsprozesse zunehmend für externe Impulse öffnen, um so die Effizienz und Effektivität der Investitionen in F\&E und Innovation zu erhöhen.

Ein Vergleich der Ergebnisse des Community Innovation Survey über 12 Jahre zeigt, dass österreichische Unternehmen im Innovationsprozess tatsächlich vermehrt auf externes Wissen und externe Informationen zurückgreifen. Die Daten zeigen eine zunehmende Wertschätzung für verschiedene externe Innovationsquellen, wobei die Bedeutung von Kunden, Lieferanten und Wettbewerbern im Vergleich zu anderen Quellen wie Universitäten und Forschungseinrichtungen überproportional gestiegen ist. Der Anteil kooperierender Innovatoren blieb lange Zeit unverändert und steigt erst seit 2002/04 deutlich an. Innerhalb der Gruppe der kooperierenden Unternehmen können wir vor allem eine Zunahme der Kooperationen mit Kunden und Auftraggebern sowie Zulieferern sehen. Hingegen stagniert bzw. sinkt der Anteil der kooperierenden Unternehmen, die mit Universitäten und anderen Forschungseinrichtungen zusammenarbeiten.

Zusätzlich steigern österreichische Unternehmen auch die Breite ihrer Suchprozesse: Die Zunahme der Kooperationen mit einzelnen Partnern geht nicht zulasten der Zusammenarbeit mit anderen Partnern, sodass das Partnerportfolio der Unternehmen insgesamt breiter wird. Ähnliches gilt für die Wertschätzung verschiedener Informationsquellen. Diese Erweiterung beschränkt sich allerdings auf den Typ des Partners; der Anteil von Unternehmen mit Partnern im Ausland hat sich über den gesamten Zeitraum kaum verändert.

Die Orientierung hin zu offenen Innovationsprozessen ist vom Standpunkt der Politik begrüßenswert, da Unternehmen, die diese Strategien verfolgen, eine höhere Erfolgsrate bei Produktentwicklungen aufweisen, mehr Marktneuheiten entwickeln und sich ein messbarer positiver Einfluss auf den Unternehmenserfolg nachweisen lässt (von Hippel 2005, Laursen und Salter 2006). Open Innovation schafft jedoch auch neue Herausforderungen für die Politik (Leitner 2009). Das Konzept der Open Innovation hinterfragt die Rolle von intellektuellen Eigentumsrechten als Anreiz für Innovationsaktivitäten. Wenngleich das Patentsystem seit seinem Bestehen immer wieder kritisch diskutiert wurde, gibt es letztlich Konsens, dass die Vorteile die Nachteile aufwiegen. In mehr und mehr Bereichen stellen sich intellektuelle Eigentumsrechte jedoch als Innovationshindernis dar und gerade die Open-Source-Bewegung ist eine Reaktion auf durch Patente geschaffene Monopole (Henkel und von Hippel 2005). Hier ist die Forschungsagenda derzeit umfangreich; Ziel ist, besser zu 
verstehen, in welchen Bereichen Patente sinnvoll sind und in welchen Bereichen sie negative wohlfahrtsökonomische Auswirkungen haben.

\subsection{Die Wirkung von Innovationen auf die Arbeitsproduktivität}

Seit Mitte der 1950er Jahre wird der technologische Wandel in der ökonomischen Theorie betrachtet und in neoklassischen Wachstumsmodellen berücksichtigt (Solow 1957; Arrow 1962; Romer 1990; Grossman und Helpman 1991). Dabei wird unter anderem ein Zusammenhang zwischen F\&E-Aktivitäten und Produktivitätswachstum prognostiziert (Romer 1990). Zur empirischen Überprüfung wurde in Folge neben Kapital und Arbeit auch der F\&EKapitalstock, als Variable für den Wissensbestand, in Produktionsfunktionen aufgenommen. Dabei war für die Analyse förderlich, dass seit Ende der 1990er Jahre zunehmend Daten aus Unternehmensbefragungen zum Innovationsverhalten (in Europa der Community Innovation Survey; CIS) zur Verfügung stehen. Zahlreiche Studien kommen dabei zu dem Ergebnis, dass der Innovationsoutput einen signifikant positiven Einfluss auf den Unternehmenserfolg hat - unabhängig davon, mit welchen Indikatoren dieser gemessen wird. Allerdings sind sowohl zwischen Indikatoren als auch zwischen Ländern deutliche Unterschiede zu beobachten ${ }^{74}$.

Ein kürzlich abgeschlossenes Projekt im Rahmen der OECD (vgl. OECD 2008d, Berger 2008) hat erstmalig gleichzeitig für Österreich und 17 weitere europäische und außereuropä- ische Länder ${ }^{75}$ vergleichbare Ergebnisse unter Verwendung von Mikrodaten aus Innovationsbefragungen und auf Basis einheitlicher ökonometrischer Modelle ${ }^{76}$ vorgelegt.

Dabei werden folgende Fragen beantworten:

1. Welche Unternehmensmerkmale beeinflussen die Entscheidung eines Unternehmens, Innovationsaktivitäten durchzuführen? Und welche Merkmale wirken sich auf die Höhe des Innovationsinputs (Innovationsaufwendungen pro Beschäftigtem / Beschäftigter) aus?

2. Welche Korrelation besteht zwischen dem Innovationsinput und dem Innovationsoutput (gemessen als Umsatz mit innovativen Produkten)?

3. Welcher Zusammenhang existiert zwischen dem Innovationsoutput und der Arbeitsproduktivität (gemessen in Umsatz pro Beschäftigtem / Beschäftigter)?

\section{Einflussfaktoren}

Hinsichtlich der ersten Frage sind die Ergebnisse eindeutig: In Österreich steigt die Wahrscheinlichkeit eines Unternehmens innovativ zu sein mit der Unternehmensgröße, sowie der Tatsache, dass ein Unternehmen auf ausländischen Märkten aktiv ist oder zu einer Unternehmensgruppe gehört. Dieses Ergebnis war zu erwarten, da international agierende Unternehmen einem größeren Wettbewerbsdruck unterliegen und größere Unternehmen mehr Ressourcen für Innovationen zur Verfügung haben. Überraschend ist hingegen, wie eindeutig dieser Zusammenhang in allen Ländern festgestellt werden kann.

\footnotetext{
74 Vgl. Crepon/ Duguet/ Mairesse 1998, Criscuolo/Haskel, 2002; Hagen et al. 2007, Hall/Mairesse 2006, Janz et al. 2004, Klomp/van Leeuwen 2001, Lööf/Heshmati 2002, 2006, Lööf, et al. 2003, Mohnen et al. 2006, Mohnen/ Therrien 2003, Raymond et al. 2006, van Leeuwen/Klomp 2006, Verspagen 2005

75 Australien, Österreich, Belgien, Brasilien, Kanada, Dänemark, Finnland, Frankreich, Deutschland, Italien, Südkorea, Luxemburg, Niederlande, Neuseeland, Norwegen, Schweden, Schweiz, Großbritannien.

76 in Anlehnung an Crepon / Duguet / Mairesse 1998
} 
Zudem lässt sich für Österreich zeigen, dass die Höhe der Innovationsausgaben mit der Tatsache, dass Unternehmen auf ausländischen Märkten aktiv sind, zusammenhängt. Zusätzlich spielen die staatliche Innovationsförderung und Innovationskooperationen eine positive Rolle.

\section{Die Rolle der öffentlichen Förderung}

Bei sonst gleichartigen Unternehmen erhöht die Tatsache, dass sie öffentliche Innovationsförderung erhalten, die Innovationsausgaben um $75 \%$, die Teilnahme an Innovationskooperationen um $41 \%$. Dabei ist allerdings darauf hinzuweisen, dass bei der Analyse nur der Tatbestand der Förderung und nicht deren Höhe berücksichtigt wird und überdies die Förderung in Österreich vielfach an die Existenz von Kooperationen gekoppelt ist. Diese Zusammenhänge lassen sich ebenfalls in der Mehrheit der Vergleichsländer finden, wobei im internationalen Vergleich der hohe Effekt der öffentlichen Förderung in Österreich auffällt.

Dieses Ergebnis ist technologiepolitisch höchst interessant: Ein gefördertes Unternehmen verzeichnet also um rund Dreiviertel höhere Innovationsausgaben als ein ansonsten identisches, aber nicht gefördertes Unternehmen. Die Ergebnisse stimmen mit vorhergehenden Untersuchungen österreichischer CIS3-Daten überein, wonach die nationale Innovationsförderung sowohl zu einer Erhöhung der F\&E- Intensität als auch des Umsatzes mit innovativen Produkten führt, während die EUFörderung keine signifikanten Auswirkungen hat (Mohnen und Garcia 2004). Auch eine Analyse der Inputadditionalität unternehmerischer F\&E-Förderung durch die österreichische Forschungsförderungsgesellschaft (FFG) weist auf eine signifikante und deutliche Additionalität hin (Streicher 2007). Da jedoch bei der vorliegenden Analyse keine Informationen über die Höhe der Förderung und deren Verwendung bekannt sind, lässt sich nur wenig über die Additionalitätswirkung der Förderung aussagen: Geförderte Unternehmen weisen zwar keinen vollständigen Mitnahmeneffekt auf (dann würden sich die Innovationsausgaben im Vergleich zu den nicht geförderten Unternehmen nicht unterscheiden), inwieweit jedoch eigene Ausgaben evtl. durch die Förderung substituiert werden, kann mangels Daten nicht untersucht werden.

Der im internationalen Vergleich hohe Effekt lässt sich teilweise mit der hohen Quote an geförderten Unternehmen in Österreich erklären, die zu den höchsten in Europa zählt (vgl. Abbildung 42). Allerdings ist bei der Interpretation des internationalen Vergleichs einschränkend hinzuzufügen, dass die Analyse in Österreich auf CIS3-Daten beruht, während die übrigen europäischen Länder CIS4-Daten verwenden, die zusätzlich zur direkten auch die indirekte Förderung erfassen. Dies schränkt die Vergleichbarkeit deutlich ein (vgl. Arundel et al. 2008). 


\section{Abbildung 42: Anteil der geförderten Unternehmen an a) allen Unternehmen und b) Unternehmen mit Innovationsaktivitäten in ausgewählten europäischen Ländern}

a) Alle Unternehmen*

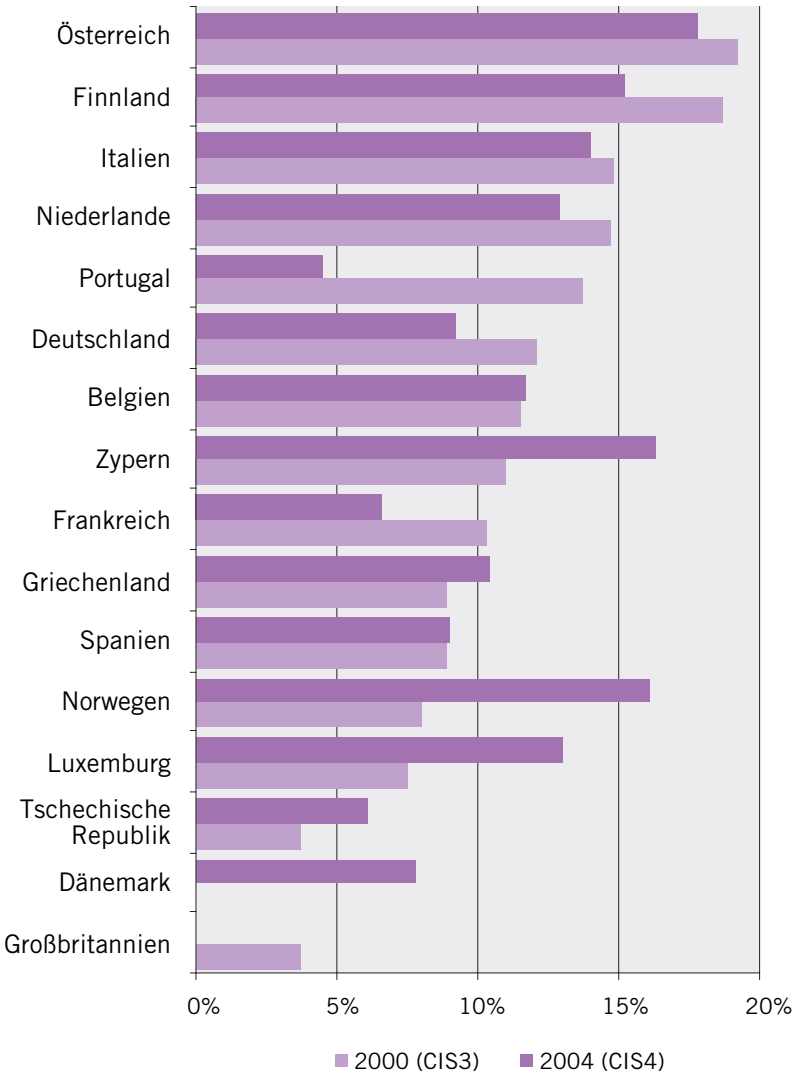

Quelle: Eurostat 2008, *eigene Berechnungen

\section{Innovationsinput und -output}

Hinsichtlich der zweiten Fragestellung zeigt sich, dass Unternehmen mit hohen Innovationsausgaben auch einen höheren Umsatz mit innovativen Produkten erzielen. Auch besteht ein positiver Zusammenhang zwischen dem Anteil von Hochschulabsolventen und -absolventinnen am Personal und dem Umsatzanteil mit neuen Produkten. Hingegen wirken sich die Größe eines Unternehmens, seine Zugehörigkeit zu einer Unternehmensgruppe, neu eingeführte Prozessinnovationen und Innovationskooperationen mit Kunden oder öffentlichen Forschungseinrichtungen nicht auf den Umsatz b) Unternehmen mit Innovationsaktivitäten

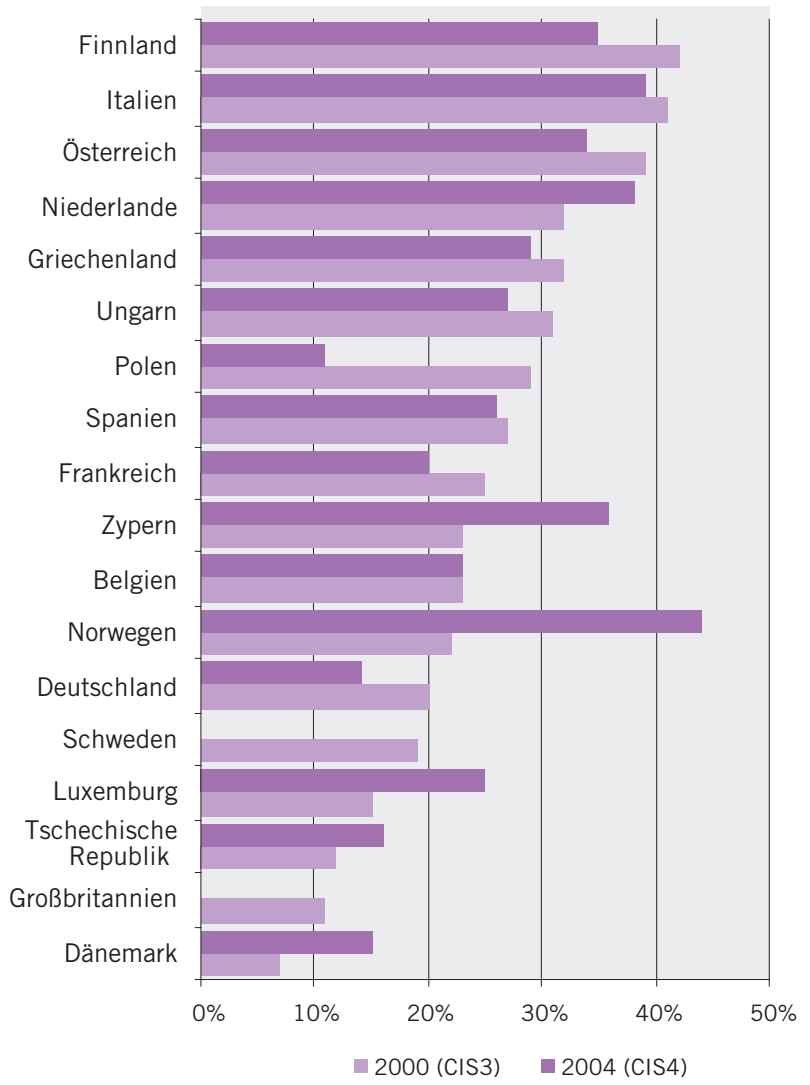

mit neuen Produkten aus. Erneut gelten die für Österreich gefundenen Zusammenhänge überwiegend auch in den anderen Ländern - einzig beim Einfluss der Unternehmensgröße gibt es international ein sehr widersprüchliches Bild.

\section{Die Wirkung auf die Arbeitsproduktivität}

Bei der dritten und zentralen Frage nach der Beziehung zwischen Innovationen und Arbeitsproduktivität zeigt sich, dass der Umsatz mit innovativen Produkten positiv mit dem Umsatz je Beschäftigtem / Beschäftigter zusammenhängt. Dies gilt auch in nahezu allen anderen untersuchten Ländern. Selbstverständlich 
haben aber nicht nur Produktneuerungen einen positiven Effekt: Auch die Zugehörigkeit zu einer Unternehmensgruppe und der Kapitalstock (gemessen als Bruttoinvestitionen in Sachanlagen je Beschäftigtem / Beschäftigter) wirken sich statistisch signifikant auf die Produktivität aus. Hingegen weisen größere Unternehmen und solche mit Prozessinnovationen erstaunlicherweise keine höhere Produktivität auf - ein im internationalen Vergleich nicht untypisches Ergebnis. Allerdings entspricht vor allem der fehlende Zusammenhang zu Prozessinnovationen nicht den Erwartungen. Die Einführung neuer oder verbesserter Prozesse (z.B. Herstellungsverfahren) sollte die Arbeitsproduktivität erhöhen. Dass diese Wirkung in den Daten - über alle Länder - nicht zu finden ist, ist schwer zu erklären. Mögliche Ursachen sind, dass auf Basis der CIS-Daten nur eine suboptimale Definition der Arbeitsproduktivität (Umsatz pro Beschäftigtem / Beschäftigter statt Wertschöpfung pro Beschäftigtem / Beschäftig- ter) möglich ist, oder dass Prozessinnovationen häufiger in wirtschaftlich schwierigen Zeiten, also bei geringen Umsätzen, durchgeführt werden, was mittels Querschnittsdaten nicht identifiziert werden kann - weitere Analysen scheinen diesbezüglich notwendig.

Zusammenfassend veranschaulicht Abbildung 43 die in der Untersuchung für Österreich gefundenen Zusammenhänge. Die Höhe der Innovationsausgaben hängt positiv mit dem Erhalt öffentlicher Innovationsförderungen, der Existenz von Innovationskooperationen und Exportaktivitäten zusammen. Je höher dabei die Innovationsausgaben sind und je größer der Anteil der HochschulabsolventInnen an den Beschäftigten ${ }^{77}$, desto höher ist auch der Umsatz mit innovativen Produkten. Dieser weist zudem eine positive Korrelation mit der Arbeitsproduktivität auf, auf die sich auch die Zugehörigkeit zu einer Unternehmensgruppe sowie Investitionen in Sachanlagen ${ }^{78}$ verstärkend auswirken.

\section{Abbildung 43: Zusammenhang zwischen Innovationen und Arbeitsproduktivität}

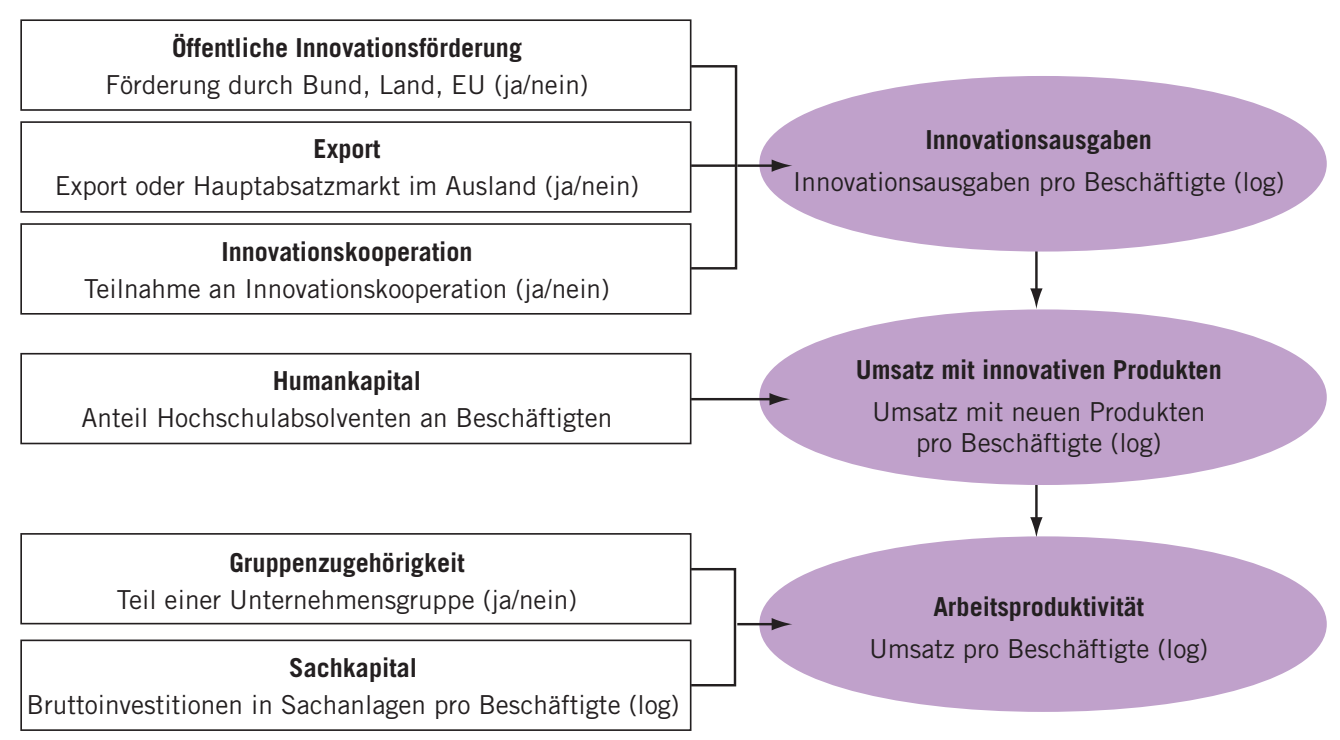

Darstellung: Joanneum Research

77 Das Basismodell der OECD sieht keine Berücksichtigung der Variablen Human- und Sachkapital vor. 78 siehe vorherige Fußnote. 


\subsubsection{Resümee}

Die große Leistung des OECD-Projektes ist es, erstmalig Innovationserhebungen einer Vielzahl von Ländern mit identischen ökonometrischen Modellen vergleichend ausgewertet zu haben. Die dabei erzielten Ergebnisse sind zwar nicht überraschend, aber doch über alle Modellvarianten und alle Länder bemerkenswert robust und klar: International tätige, große und verbundene Unternehmen haben eine größere Wahrscheinlichkeit innovativ zu sein und investieren mehr in Innovationsaktivitäten. Des Weiteren lässt sich ein positiver $\mathrm{Zu}$ sammenhang zwischen öffentlicher Förderung und der Höhe des Innovationsinputs finden. Ebenfalls korrelieren Innovationskooperationen positiv mit den Innovationsausgaben. Die Ausgaben ihrerseits weisen einen positiven Zusammenhang mit dem Umsatz mit innovativen Produkten auf, der sich seinerseits positiv auf die Arbeitsproduktivität auswirkt. Erfolgreiche (Produkt-) Innovationen und hohe Arbeitsproduktivität gehen also Hand in Hand. Wider Erwarten trifft dies nicht auf Prozessinnovationen $\mathrm{zu}$.

Während methodisch das verwendete Modell dem aktuellen Stand der Wissenschaft entspricht, leidet die Modellierung darunter, dass der CIS- Fragebogen nur wenige Variablen zu Unternehmensmerkmalen, insbesondere für nicht innovative Unternehmen, enthält. Dies schränkt die Gestaltungsmöglichkeiten bei der Analyse deutlich ein. Dieses Problem wird durch den internationalen Vergleich insofern verschärft, als dass das Modell auf Basis des ,kleinsten gemeinsamen Nenners' zu erfolgen hat und einige Variablen, die nicht für alle Länder verfügbar waren, außer Acht gelassen werden mussten.

\subsection{Zur Rolle der Kreativwirtschaft im österreichischen Innovationssystem}

\subsubsection{Abgrenzung und statistischer Überblick der Kreativwirtschaft}

Die sogenannte Kreativwirtschaft (,Creative Industries') wird heute allgemein als ein Sektor mit einer hohen Wachstumsdynamik und hohen Beschäftigungs- und Innovationspotentialen wahrgenommen. Darüber hinaus gelten die Creative Industries (CI) auch als wichtige Impulsgeber für Innovationen in anderen Branchen. Es sind im Wesentlichen drei Merkmale, die diese besondere "Zukunftsfähigkeit" der Kreativwirtschaft ausmachen:

Erstens fußen die Creative Industries definitionsgemäß auf dem „Produktionsfaktor Kreativität" und damit auf einem spezifischen Ausstattungsvorteil hoch entwickelter Volkswirtschaften. Seine Bedeutung wird im Vergleich zu traditionellen Produktionsfaktoren weiter zunehmen, sodass erwartet werden kann, dass das Gewicht der Creative Industries an der Gesamtwirtschaft weiter steigen wird.

Zweitens bieten die Creative Industries gerade solche Leistungen an, die in Volkswirtschaften mit hohem Pro-Kopf-Einkommen innerhalb der Gesamtnachfrage sukzessive an Gewicht gewinnen, nämlich immaterielle Güter, die auf die individuellen Bedürfnisse und Präferenzen der Kunden zugeschnitten sind. Creative Industries profitieren ganz besonders von dieser - empirisch zu beobachtenden - wachsenden Nachfrage nach hochwertigen Dienstleistungen.

Drittens sind die Creative Industries eine typische Querschnittsbranche, die mit ihren Leistungen für eine Vielzahl anderer Branchen Impulse geben kann, sei es bei der Entwicklung neuer Produkte und Dienstleistungen, bei deren Herstellung oder bei der Vermarktung und Distribution. Querschnittsbranchen haben meistens höhere langfristige Wachstums- 
perspektiven, da sie nicht von branchenspezifischen Entwicklungspfaden und/oder Schocks sowie einzelnen Verschiebungen in der Wettbewerbsfähigkeit zwischen Ländern abhängig sind.

Das Zentrum für Europäische Wirtschaftsforschung (ZEW) in Mannheim hat jüngst gemeinsam mit dem Fraunhofer Institut für System- und Innovationsforschung (ISI) in Karlsruhe und unter Mitarbeit der Joanneum Research Forschungsgesellschaft - eine groß angelegte, repräsentative Befragung bei CI-Unternehmen in Österreich durchgeführt, wobei die Erfassung des Innovationspotentials der CI und deren Ausstrahlung auf andere Branchen im Zentrum der Erhebung war (siehe Georgieff et al. 2008). Die Ergebnisse dieser Studie flossen in den Dritten Kreativwirtschaftsbericht (herausgegeben von der arge creativ wirtschaft austria in der Wirtschaftskammer Österreich) ein. Im Folgenden werden einige zentrale Fakten daraus dargestellt.

Aufgrund der Vielfalt kreativer Dienstleis- tungen ist eine genaue Definition, was im Folgenden als Kreativwirtschaft verstanden wird, notwendig. Konstitutiv für die übliche Abgrenzung der CI ist, dass Tätigkeiten im Fokus stehen, die inputseitig durch eine Dominanz von „Kreativität" als Produktionsfaktor definiert sind und die sich outputseitig durch eine privatwirtschaftliche Erbringung von Marktangeboten auszeichnen. Die CI umfassen demnach all jene „kreativen“ Unternehmen, die Güter im Markt anbieten und auf die Erzielung von Erlösen und - zumindest mittelfristig - Gewinnen ausgerichtet sind ${ }^{79}$.

Tabelle 40 gibt einen Überblick über ausgewählte Kennzahlen der österreichischen Kreativwirtschaft und deren Entwicklung in den letzten zehn Jahren. Die in Summe ca. 30.000 Unternehmen (das sind ca. $10 \%$ aller Unternehmen Österreichs!) dieser Branchengruppe beschäftigten insgesamt mehr als 100.000 Personen (bzw. 3,3\% aller Beschäftigten) und erwirtschaften eine Bruttowertschöpfung (zu Faktorkosten) von etwa 7,7 Mrd. $€$.

Tabelle 40: Die österreichische Kreativwirtschaft im Überblick

\begin{tabular}{lrrrrr} 
& $\mathbf{2 0 0 2}$ & $\mathbf{2 0 0 3}$ & $\mathbf{2 0 0 4}$ & $\mathbf{2 0 0 5}$ & $\mathbf{2 0 0 6}$ \\
\hline Unternehmen & 27183 & 28820 & 28681 & 29300 & 30299 \\
Unselbst. Beschäftigte & 103680 & 100886 & 101644 & 102250 & 104211 \\
Durchschnittl. Unternehmensgröße & 3,8 & 3,4 & 3,5 & 3,5 & 3,4 \\
Bruttowertschöpfung (Mio. €) & 6890 & 6889 & 7152 & 7376 & 7710 \\
Anteil Unternehmen & 9,5 & 9,7 & 9,6 & 10,4 & 10,5 \\
Anteil Beschäftigte & 3,7 & 3,6 & 3,5 & 3,4 & 3,3 \\
Anteil Bruttowertschöpfung & 5,3 & 5,2 & 5,1 & 5,2 & 5,1
\end{tabular}

Quelle: Dritter Kreativwirtschaftsbericht, 2008

79 Gemeinnützige und öffentliche Einrichtungen, die ebenfalls - teilweise im erheblichen Umfang - kreative Dienstleistungen anbieten, werden im Rahmen dieses Überblicks nicht berücksichtigt. 
Für eine detailliertere Darstellung ist es sinnvoll, die durchaus heterogene Branchengruppe der Kreativwirtschaft in einzelne Subbereiche zu gliedern, wobei folgende Aufteilung gewählt wurde (siehe auch Abbildung 44):

1. Design (Grafik, Web-Design, Industriedesign, Textil-, Schmuck-, Möbeldesign etc.)

2. Content (Film, Literatur, Journalismus, Komposition, Schauspielerei, Texterstellung, Übersetzung)

3. Architektur (inkl. Landschaftsplanung, Bauplanung)

4. Werbung (inkl. PR-Beratung und Marktforschung)

5. Software (Programmierung, ohne Web-Design, inkl. Datenbankdienste etc.)

6. Verlag/Druck (inkl. Vervielfältigung von Ton-, Bild- und Datenträgern)

7. Technische Büros (Planung für Nichtbaubereiche, inkl. F\&E-Dienstleistungen)

8. Beratung/Training (Unternehmensberatung, Coaching, Erwachsenenbildung)

Abbildung 44 zeigt die sektorinterne Struktur bezüglich Unternehmen und Beschäftigung der
Kreativwirtschaft nach diesen acht Subbereichen. Bezogen auf die Zahl der Unternehmen führen die Architekturbüros (knapp $20 \%$ ), gefolgt von Software $(16 \%)$ und den technischen Büros (15\%). Beratung/Training sowie Werbung halten sich mit einem Anteil von jeweils ca. $13 \%$ ungefähr die Waage. Design und Content weisen jeweils ca. $8 \%$ auf. An letzter Stelle liegt der Bereich Verlage/Druck, der einen Anteil von $5 \%$ aller Unternehmen der Kreativwirtschaft verbucht. Aufgrund unterschiedlicher durchschnittlicher Unternehmensgrößen ist das Bild bezüglich der Beschäftigung teilweise deutlich davon abweichend. Hier liegen mit einem Anteil von knapp $23 \%$ der Beschäftigten die technischen Büros deutlich an erster Stelle. Im Bereich Werbung sind ca. $17 \%$ aller Beschäftigten zu finden, gefolgt von Beratung/Training (15\%), Software (13\%) und Architektur (13\%). Mit Anteilen von $5 \%$ (Design) bzw. $6 \%$ (Content) bis knapp $8 \%$ (Verlag/Druck) weisen diese Bereiche ein quantitativ deutlich geringeres Gewicht auf.

\section{Abbildung 44: Struktur der Kreativwirtschaft nach Subbranchen (Anteile in Prozent)}

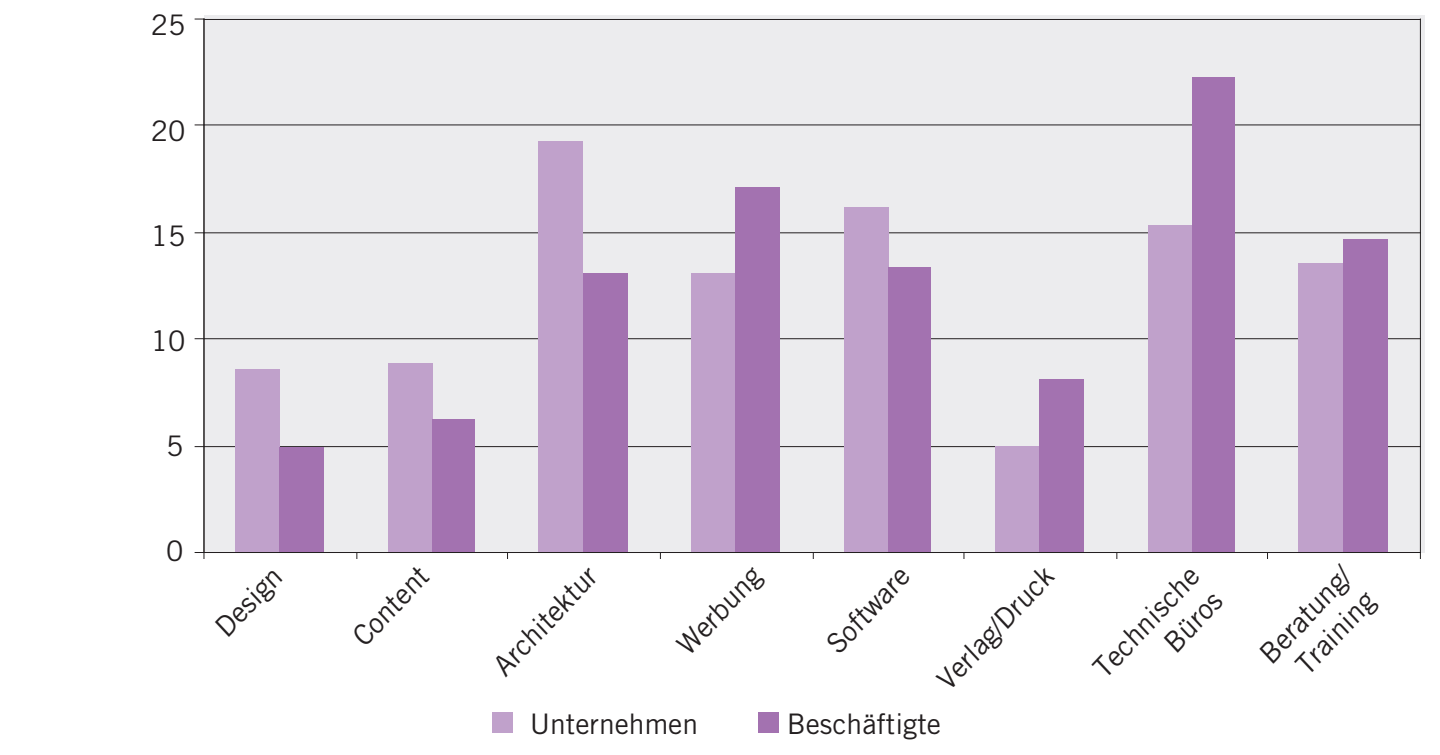

Quelle: Dritter Kreativwirtschaftsbericht, 2008 


\subsubsection{Kreativwirtschaft im österreichischen Innovationssystem}

Die Kreativwirtschaft ist nicht ausschließlich als dynamische Wachstumsbranche mit entsprechend positiven Effekten für Beschäftigung und Wertschöpfung von großem Interesse für die Wirtschaftspolitik, sondern vor allem auch aufgrund der erwarteten spezifischen Wirkungen für das Innovationssystem als Ganzes. Der Kreativwirtschaft kommt dabei aufgrund folgender Mechanismen eine besondere Rolle im Innovationssystem zu:

- Zunächst trägt sie mit eigenen F\&E-Anstrengungen und entsprechenden Innovationen sui generis zum Innovationsgeschehen in Österreich bei.

- Als Querschnittsbranche können die Innovationen der Kreativwirtschaft wiederum für eine Reihe von anderen Branchen wichtige Inputs liefern und somit weisen die CI eine Funktion als Innovationstreiber für die gesamte Wirtschaft auf.

- Als humankapitalintensiver Bereich trägt die Kreativwirtschaft qua Humankapitalmobilität (Wechsel von MitarbeiterInnen in andere Branchen) zum Wissenstransfer innerhalb des Innovationssystems bei.

- In der Kreativwirtschaft finden sich oft enge Verknüpfungen zwischen den Unternehmen und wissenschaftlichen Einrichtungen. Diese Netzwerkbildung stimuliert den Transfer zwischen Wissenschaft und Wirtschaft innerhalb eines Innovationssystems.

Diese Mechanismen sollen für Österreich im Folgenden mit empirischem Material dargestellt werden, wobei als Datenbasis die Ergebnisse einer umfangreichen CATI-gestützten Erhebung $^{80}$ bei ca. 5.000 CI-Unternehmen Österreichs des ZEW (Zentrum für Europäische
Wirtschaftsforschung, Mannheim) aus dem Frühjahr 2008 zur Verfügung stehen.

\section{F\&E und Innovation}

Über alle Unternehmen der Creative Industries hinweg betreiben $31 \%$ eigene Forschungsund Entwicklungsarbeit. In Summe sind dies hochgerechnet etwa 6.000 Unternehmen. Bei der Interpretation dieser Zahlen ist darauf $\mathrm{zu}$ achten, dass die hier verwendete Definition von $\mathrm{F} \& \mathrm{E}$ über den engeren Ansatz des Frascati-Manuals der OECD deutlich hinausgeht, der für internationale F\&E-Statistiken maßgebend ist. Auf Basis der Frascati-Definition betrieben in Österreich laut der jüngsten F\&E-Erhebung (2006) der Statistik Austria 2.356 Unternehmen F\&E Die deutlich höheren Zahlen in der CI-Befragung ergeben sich einerseits daraus, dass hier auf einen dreijährigen Referenzzeitraum abgestellt wird. Andererseits wurde F\&E wesentlich breiter definiert, nämlich als jede „systematische schöpferische Arbeit zur Erweiterung des vorhandenen Wissens im Unternehmen “. Es ist davon auszugehen, dass auch viele konzeptionelle Arbeiten und die kreative Auseinandersetzung mit Problemstellungen von den Unternehmen subjektiv als eigene F\&E-Tätigkeit eingestuft werden. Viele der CI-Unternehmen mit F\&E dürften auch nur unregelmäßig F\&E durchführen. Gleichwohl ist diese breitere Definition eher geeignet, das Ausmaß von systematischen Aktivitäten zur Erzielung von neuem, kommerziell relevantem Wissen in der Kreativwirtschaft zu erfassen als die enge, stark auf technische F\&EArbeiten abzielende Definition der offiziellen F\&E-Statistik. Denn viele „F\&E-Prozesse in den Creative Industries basieren nicht auf technisch-naturwissenschaftlichen Entwick- 
lungen, sondern haben überwiegend immateriellen Charakter und zielen darauf ab, kreative Ideen in für Kunden interessante Produkte und Dienstleistungen umzusetzen.

Differenziert nach den einzelnen CI-Bereichen (siehe Abbildung 45) finden sich die höchsten Anteile F\&E betreibender Unternehmen unter den technischen Büros (47 \%) sowie in der Software (46 \%). Über dem Durchschnitt liegt noch der Bereich Beratung/Training, in dem rund $34 \%$ aller CI-Unternehmen angeben, eigene Forschungs- und Entwicklungsarbeit durchzuführen. Die drei Kernbereiche der Creative Industries (Design, Content und Architektur) und die Werbung weisen dagegen eine unterdurchschnittliche F\&E-Tätigkeit auf. Mit Abstand am geringsten ist mit lediglich $14 \%$ der Anteil der F\&E betreibenden Unternehmen im Verlags- und Druckgewerbe.

\section{Abbildung 45: F\&E durchführende Unternehmen in den Creative Industries in Österreich nach Cl-Bereichen (2005 - 2007) (Anteil an allen Unternehmen in \%)}

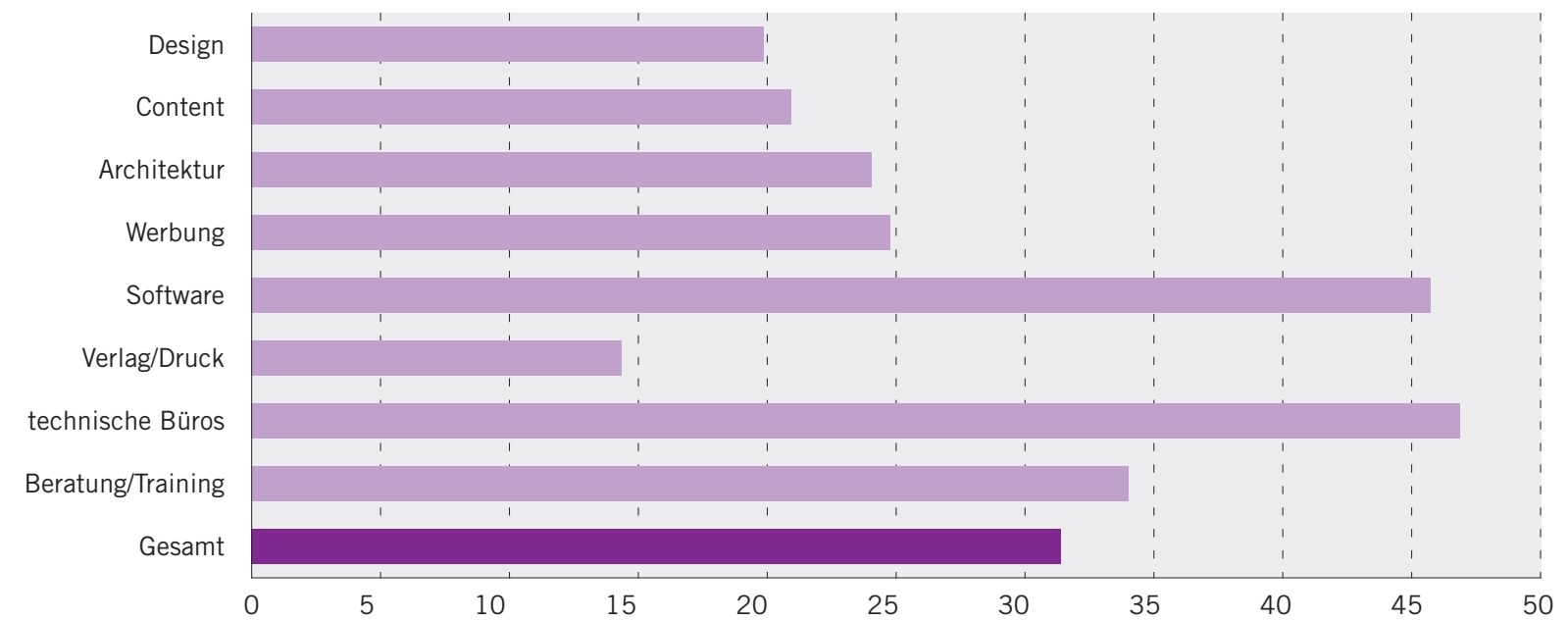

Quelle: ZEW: Befragung Creative Industries Österreich 2008 - Berechnungen des ZEW. Alle Angaben hochgerechnet auf die Gesamtzahl der Unternehmen in den Creative Industries in Österreich Anfang 2008.

Abbildung 46 zeigt die Innovationsaktivitäten der Kreativwirtschaft. Als Innovation wird hier die Einführung eines neuen Leistungsangebots im Markt durch ein Unternehmen („Produktinnovation“) oder die Einführung eines neuen Verfahrens zur Leistungserbringung im Unternehmen („Prozessinnovation“) bezeichnet. $\mathrm{Zu}$ berücksichtigen ist, dass hier ein subjektiver Innovationsbegriff (wie bei Innovationserhebungen in Form von Befragungen üblich) unterstellt wird, d.h. eine Innovation muss neu für das betreffende Unternehmen sein, sie kann aber durchaus von anderen
Unternehmen bereits zuvor angeboten bzw. eingesetzt worden sein. Innovationen sind in den CI-Unternehmen Österreichs sehr weit verbreitet. $71 \%$ der Unternehmen geben an, dass sie innerhalb eines Dreijahreszeitraums (2005-2007) eine Innovation eingeführt haben. Produktinnovationen sind dabei häufiger anzutreffen als Prozessinnovationen: 59 \% der CIUnternehmen führten neue Leistungsangebote im Markt ein, während 49 \% neue Verfahren im Unternehmen implementiert haben. $37 \%$ aller CI-Unternehmen haben sowohl Produktals auch Prozessinnovationen eingeführt. 


\section{Abbildung 46: Anteil der Unternehmen mit Produkt- und Prozessinnovationen 2005 - 2007 nach Cl-Bereichen (in \% aller Unternehmen)}

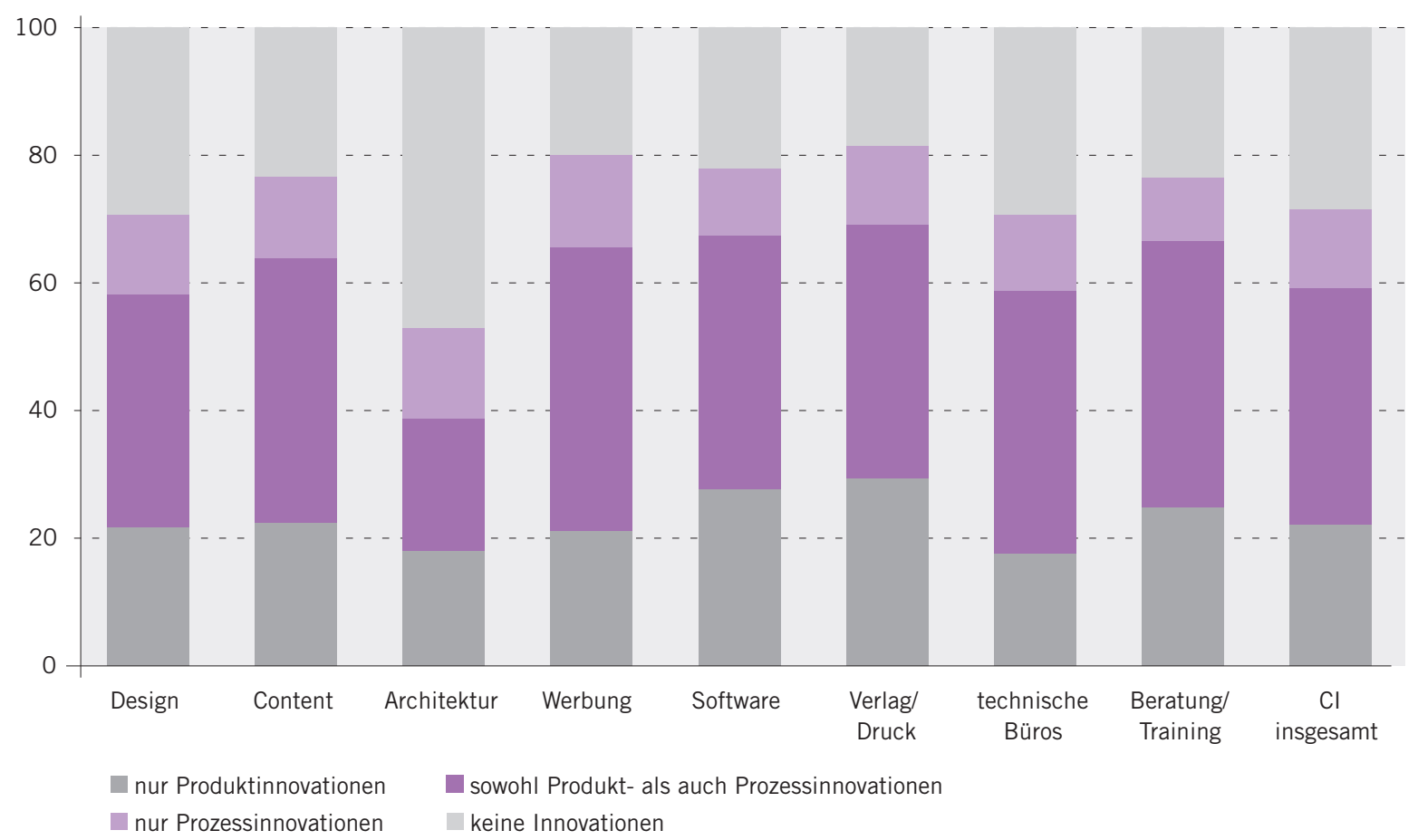

Quelle: ZEW: Befragung Creative Industries Österreich 2008 - Berechnungen des ZEW. Alle Angaben hochgerechnet auf die Gesamtzahl der Unternehmen in den Creative Industries in Österreich Anfang 2008.

Die höchsten Innovatorenquoten weisen die CI-Bereiche Verlag/Druck, Werbung, Software, Content und Beratung/Training auf, hier sind jeweils über drei Viertel der Unternehmen mit Innovationen erfolgreich gewesen. In all diesen CI-Bereichen sind Produktinnovationen deutlich weiter verbreitet als Prozessinnovationen und die meisten Prozessinnovatoren haben gleichzeitig auch neue Produkte in den Markt gebracht. In etwa durchschnittlich hoch ist die Innovatorenquote im Content-Bereich und in den technischen Büros. Der einzige CIBereich, der eine deutlich geringere Quote von erfolgreich innovierenden Unternehmen aufweist, ist der Bereich Architektur. Hier zählen nur $52 \%$ der Unternehmen zu den Innovatoren und nur $39 \%$ haben innerhalb eines Dreijahreszeitraums neue Leistungsangebote eingeführt. Dies zeigt an, dass sich der größe- re Teil der Unternehmen auf ein bestimmtes Leistungsangebot spezialisiert hat (z.B. Innenarchitektur für Büroräume, ökologisches Bauen, Entwurf/Planung für Einfamilienhäuser) und dieses über einen längeren Zeitraum beibehält. Gleichwohl werden innerhalb dieses konstanten Angebots für jeden einzelnen Auftrag kreative Leistungen erbracht.

\section{Innovationstreiber Kreativwirtschaft - Wirkungen auf andere Branchen}

Wie bereits erwähnt spielt die Kreativwirtschaft als Querschnittsbranche eine wichtige Funktion als Innovationstreiber für die gesamte Wirtschaft. Erstens fragen Unternehmen der Kreativwirtschaft innovative Produkte und Technologien von anderen Unternehmen nach und können so als Kunden Innovations- 
impulse an ihre Technologielieferanten geben. Die Funktion der Creative Industries als Innovationen fordernde Nachfrager ergibt sich aus dem nicht unerheblichen Umfang des Sektors - er stellt etwa $5 \%$ der Wertschöpfung des Unternehmenssektors - und vor allem aus der äußerst starken eigenen Innovationstätigkeit. Denn es sind gerade die innovativen Unternehmen, die an ihre Zulieferer und die in der Wertschöpfungskette vorgelagerten Unternehmen besonders hohe Ansprüche an Innovation und Technologie stellen. Zweitens bieten die Creative Industries ein vielfältiges Bündel an kreativen Leistungen an, das von anderen Unternehmen in ihre eigenen Innovationsprozesse integriert werden kann. Diese Innovationen unterstützenden oder begleitenden Leistungen können von Ideen für neue Produkte, die etwa CI-Unternehmen im Content- oder Beratungsbereich entwickeln, über F\&E-Arbeiten, das Design von neuen Produkten, die Entwicklung spezifischer Softwareanwendungen für neue Produkte und Prozesse, die Entwicklung neuer Vermarktungsstrategien oder Konstruktionsleistungen für effizientere Produktionsverfahren bis hin zu Konzepten für eine bessere Nutzung des innovativen Potenzials in Unternehmen, über neue Beratungs- und Trainingsansätze bis hin zu einer kommunikationsfreundlicheren und kreativitätsfördernden Gestaltung von Arbeitsplätzen durch neue architektonische Konzepte reichen.

In Abbildung 47 wird dieser letztere Aspekt, nämlich die Unterstützung von Kunden bei ihren Innovationsprozessen (i.w.S.), empirisch beleuchtet. Tatsächlich konnte fast die Hälfte aller CI-Unternehmen (46\%) ihre Unternehmenskunden dabei unterstützen, Innovationen einzuführen. Besonders stark auf die Rolle als Innovationsunterstützer ausgerichtet ist der Bereich Werbung, dort gaben $71 \%$ der Unternehmen an, Kunden bei der Einführung neuer Produkte in den Markt bzw. bei der Implementation von neuen Verfahren unterstützt zu haben. Ebenfalls über dem Durchschnitt liegen die CI-Bereiche Software, technische Büros und Beratung/Training. Recht niedrig ist der Anteil der CI-Unternehmen mit innovationsunterstützenden Aktivitäten in der Architektur (19\%) und im Bereich Content (24\%). Dies liegt einerseits am hier vergleichsweise niedrigen Anteil von CI-Unternehmen, unter deren Kunden sich auch Unternehmen befinden (69 bzw. 73 \%). Andererseits ist auch das Leistungsangebot dieser beiden Bereiche per se weniger dazu geeignet, Innovationsprozesse in der gewerblichen Wirtschaft direkt $z u$ unterstützen. Während Architektur in erster Linie auf die kreative Gestaltung baulicher Infrastruktur abzielt und dadurch bestenfalls indirekt in betriebliche Innovationsprozesse eingreifen kann, entwickeln Unternehmen im Content-Bereich typischerweise Ideen für Produktinhalte, die häufig von anderen CI-Bereichen aufgegriffen und umgesetzt werden, so etwa in Bezug auf künstlerische, fotografische oder texterstellende Leistungen. 
Abbildung 47: Anteil der Cl-Unternehmen, die Unternehmenskunden dabei unterstützt haben, Innovationen einzuführen, nach Cl-Bereichen (in \%)

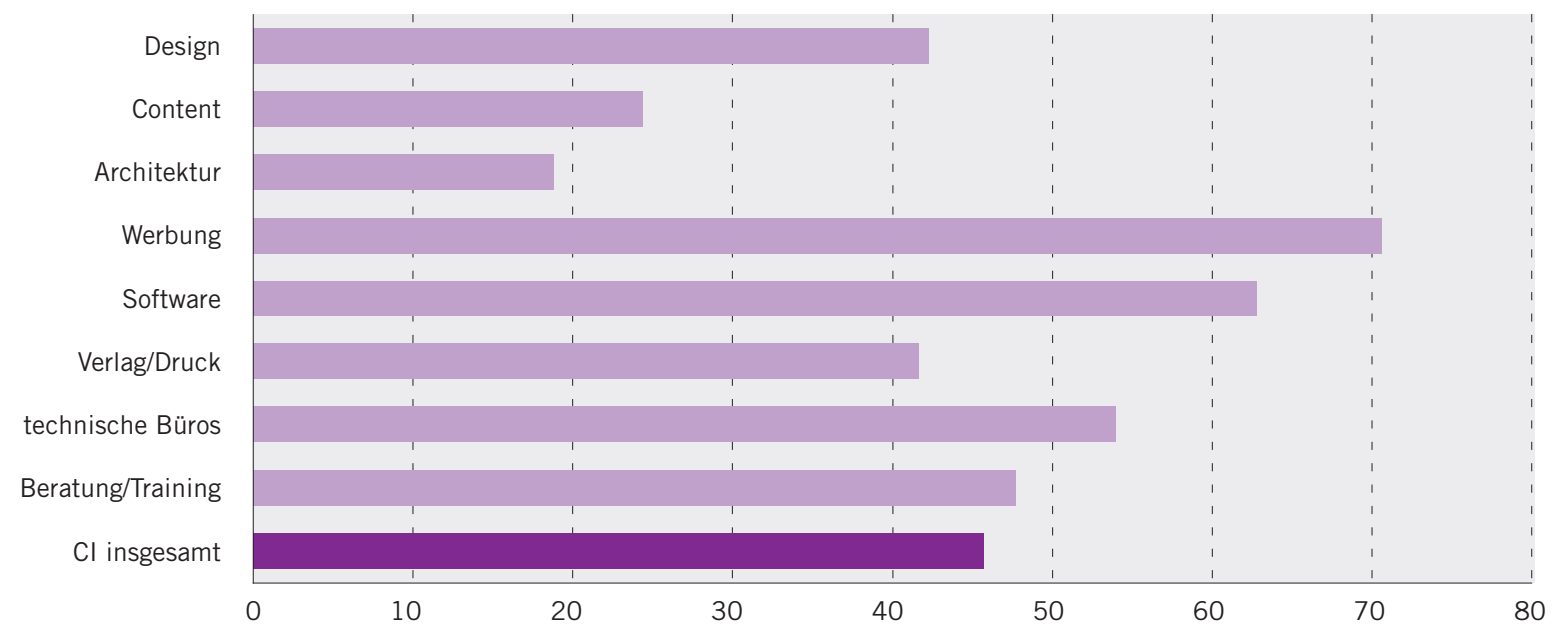

Quelle: ZEW: Befragung Creative Industries Österreich 2008 - Berechnungen des ZEW. Alle Angaben hochgerechnet auf die Gesamtzahl der Unternehmen in den Creative Industries in Österreich Anfang 2008.

\subsubsection{Humankapitalmobilität}

Eine wichtige Form des Wissenstransfers zwischen Unternehmen und Branchen ist der Wechsel von MitarbeiterInnen. Dies gilt ganz besonders für die Creative Industries. Humankapital, also die Fähigkeiten und Fertigkeiten der MitarbeiterInnen, ist der wichtigste Produktionsfaktor in dieser Branche. Mit der Personalfluktuation wandern auch Knowhow, berufliche Erfahrung und kreative Ideen und Ansätze. Um die Bedeutung der Creative Industries als Innovationsgeber für andere Wirtschaftszweige beurteilen zu können, ist es somit notwendig, auch diese Form von „Innovationsimpulsen" zu untersuchen.

Die Creative Industries zeichnen sich durch einen sehr hohen Anteil von freien MitarbeiterInnen aus. Anfang 2008 waren $17 \%$ aller Beschäftigten in den Creative Industries in Österreich freie MitarbeiterInnen. In der österreichischen Wirtschaft insgesamt waren es - auf Basis der Ergebnisse des Mikrozensus
- im Jahr 2007 lediglich 1,6 \%. Allerdings ist ein direkter Vergleich nur eingeschränkt möglich, da ein und dieselbe Person in mehreren Unternehmen als freie/r MitarbeiterIn tätig sein kann und somit mehrfach gezählt wird, während im Mikrozensus jede/r freie DienstnehmerIn nur einmal erfasst wird. Jedenfalls ist die Bedeutung von freien MitarbeiterInnen in den Creative Industries um ein Vielfaches höher als in der Gesamtwirtschaft.

Mit dieser hohen Zahl an freien MitarbeiterInnen geht auch eine hohe Personaldynamik in den Creative Industries einher. Dabei ist zunächst einmal festzuhalten, dass die Creative Industries im Zeitraum 2004-2007 ein enorm hohes Beschäftigungswachstum aufwiesen. In den Unternehmen, die sowohl im Jahr 2004 als auch Anfang 2008 wirtschaftlich aktiv waren (d.h. ohne Unternehmen, die in den Jahren 2005 bis 2007 entweder neu gegründet oder geschlossen wurden), stieg die Beschäftigtenzahl um $5 \%$ pro Jahr an. ${ }^{81}$ Ein deutlich überdurchschnittliches Wachstum zeigt sich in

81 Zum Vergleich: Die Zahl der unselbstständig Erwerbstätigen insgesamt wies in Österreich in den Jahren von 2005 bis 2008 Wachstumsraten zwischen 1 und $2 \%$ auf. 
den CI-Bereichen Software, Content und Beratung/Training, während Verlag/Druck und Architektur relativ niedrige Zuwachsraten der Beschäftigung und der Design-Bereich sogar eine Stagnation der Beschäftigtenzahlen zeigt (Tabelle 41).

Hinter diesem Nettowachstum verbirgt sich eine noch viel höhere Personaldynamik, wenn man die $\mathrm{Zu}$ - und Abgänge von MitarbeiterInnen betrachtet. In CI-Unternehmen, die sowohl 2004 als auch Anfang 2008 wirtschaftsaktiv waren, schieden pro Jahr 6,6\% der MitarbeiterInnen aus, gleichzeitig wurden pro Jahr 13,1 \% der MitarbeiterInnen neu eingestellt.
Dies bedeutet, dass am Jahresende nur mehr etwa $80 \%$ der MitarbeiterInnen von CI-Unternehmen auch zu Jahresbeginn in demselben Unternehmen tätig waren. Die Personalfluktuation ist in den Bereichen Content, Werbung und Software besonders hoch. Während die hohe Personalfluktuation in den Bereichen Content und Werbung mit einem hohen Anteil freier MitarbeiterInnen einhergeht, ist der Software-Bereich durch einen niedrigen Anteil von freien DienstnehmerInnen gekennzeichnet. Trotz des hohen Anteils freier MitarbeiterInnen ist im Bereich Beratung/Training die Personalfluktuation vergleichsweise niedrig.

Tabelle 41: Personalfluktuation in den Creative Industries in Österreich 2004 - 2007 nach Cl-Bereichen*

\begin{tabular}{|c|c|c|c|c|c|c|}
\hline & $\begin{array}{r}\text { durchschnittliches } \\
\text { jährliches Wachstum der } \\
\text { MitarbeiterInnenzahl } \\
\text { (in \%) }\end{array}$ & $\begin{array}{l}\text { Abgangsquote/ } \\
\text { Jahr (in \%) }\end{array}$ & $\begin{array}{r}\text { Einstellungs- } \\
\text { quote/Jahr } \\
\text { (in \%) }\end{array}$ & $\begin{array}{r}\text { Nettozuwachs an } \\
\text { MitarbeiterInnen } \\
\text { je Unternehmen } \\
\text { und Jahr }\end{array}$ & $\begin{array}{r}\text { Zahl der ausscheiden- } \\
\text { den Mitarbeiterinnen } \\
\text { pro Jahr und Unter- } \\
\text { nehmen }\end{array}$ & $\begin{array}{r}\text { Zahl der neu } \\
\text { eintretenden } \\
\text { Mitarbeiterinnen pro Jahr } \\
\text { und Unternehmen }\end{array}$ \\
\hline Design & 0.4 & 7.2 & 7.8 & 0.03 & 0.36 & 0.39 \\
\hline Content & 7.6 & 8.8 & 18.5 & 0.44 & 0.40 & 0.85 \\
\hline Architektur & 1.9 & 6.8 & 9.3 & 0.14 & 0.38 & 0.52 \\
\hline Werbung & 5.5 & 8.5 & 15.6 & 0.74 & 0.89 & 1.63 \\
\hline Software & 7.6 & 7.5 & 17.2 & 0.57 & 0.44 & 1.01 \\
\hline Verlag/Druck & 3.4 & 5.0 & 9.4 & 0.57 & 0.64 & 1.21 \\
\hline techn. Büros & 4.9 & 5.6 & 12.0 & 0.76 & 0.66 & 1.42 \\
\hline Beratung/Tr. & 7.1 & 5.0 & 14.1 & 0.75 & 0.42 & 1.17 \\
\hline $\mathrm{Cl}$ insgesamt & 5.0 & 6.6 & 13.1 & 0.50 & 0.52 & 1.02 \\
\hline
\end{tabular}

* In Unternehmen, die sowohl im Jahr 2004 als auch Anfang 2008 wirtschaftlich aktiv waren (d.h. ohne Gründungen der Jahre 2005 bis 2007 ).

Quelle: ZEW: Befragung Creative Industries Österreich 2008 - Berechnungen des ZEW. Alle Angaben hochgerechnet auf die Gesamtzahl der Unternehmen in den Creative Industries in Österreich Anfang 2008.

Rechnet man die Personaldynamik auf die Zahl der MitarbeiterInnen um, die im Durchschnitt pro Jahr ein CI-Unternehmen verlassen bzw. in ein CI-Unternehmen eintreten, zeigen sich angesichts der Kleinheit der CI-Unternehmen erstaunlich hohe Werte. So erhöht im Mittel jedes CI-Unternehmen im Zeitraum von zwei Jahren seine MitarbeiterInnenzahl um eine Person. Während pro Jahr und Unternehmen im Mittel eine Person neu eingestellt wird, verlässt innerhalb von zwei Jahren im Mittel eine Person ein CI-Unternehmen. Die so ge- messene Personaldynamik ist in der Werbung, im Bereich Verlag/Druck und in den technischen Büros besonders hoch und in den Bereichen Design und Architektur dagegen besonders niedrig.

Zwischen Anfang des Jahres 2005 und Ende des Jahres 2007 sind insgesamt knapp 30.000 MitarbeiterInnen aus Unternehmen der Creative Industries ausgeschieden (inklusive ausscheidende MitarbeiterInnen in Unternehmen, die in diesem Zeitraum neu gegründet wurden). Der größte Teil dieser MitarbeiterInnen 
wechselte in andere Unternehmen $(74 \%)(\mathrm{Ab}-$ bildung 48). Besonders häufig wechseln ausscheidende MitarbeiterInnen der CI-Bereiche technische Büros in Unternehmen, während in den Bereichen Design und Beratung/Werbung einen Wechsel in andere Unternehmen relativ selten ist. $8,7 \%$ haben sich selbständig gemacht. In absoluten Zahlen waren dies in den drei betrachteten Kalenderjahren 2005 - 2007 über 2.500 Personen.

\section{Abbildung 48: Verteilung der aus Cl-Unternehmen 2004 - 2007 ausgeschiedenen Mitarbeiterlnnen nach Zielsektoren, differenziert nach Cl-Bereichen}

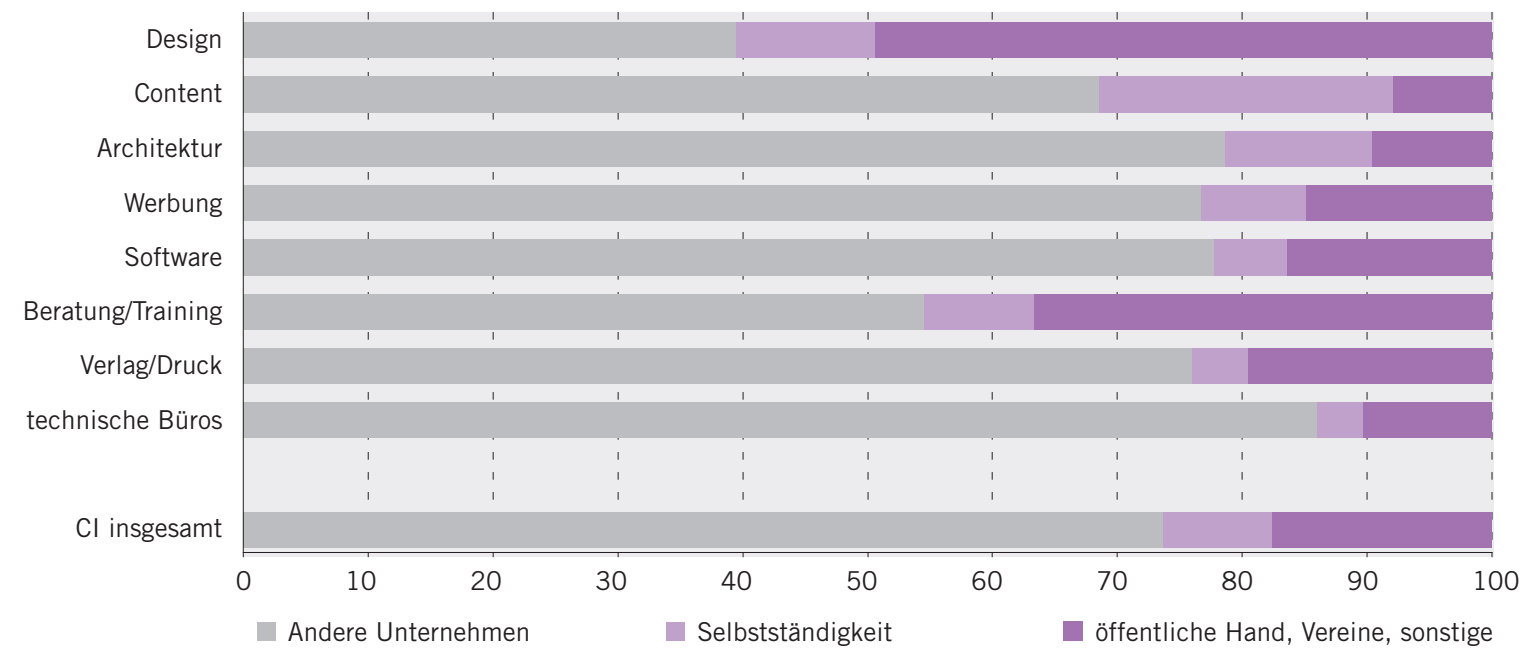

Quelle: ZEW: Befragung Creative Industries Österreich 2008 - Berechnungen des ZEW. Alle Angaben hochgerechnet auf die Gesamtzahl der Unternehmen in den Creative Industries in Österreich Anfang 2008, gerundete Hochrechnung.

Der Anteil der in die Selbstständigkeit wechselnden MitarbeiterInnen von CI-Unternehmen liegt geringfügig unter der durchschnittlichen Selbstständigenquote in der österreichischen Wirtschaft (ohne Land- und Forstwirtschaft), die im Mittel der Jahre 2004-2006 - nach Angaben aus dem Mikrozensus - 9,3 \% betrug. Besonders hoch ist der Anteil der in die Selbstständigkeit wechselnden MitarbeiterInnen im Content-Bereich (24\%), hohe Werte zeigen auch die Bereich Architektur (12\%) und Design (11\%). Im Bereich der technischen Büros und Verlag/Druck machen sich nur wenige der ausscheidenden MitarbeiterInnen selbstständig - ein Indiz der höheren Eintrittsbarrieren in diesem Bereich. Ein weiterer wichtiger Zielsektor sind öffentliche und gemeinnützige Einrichtungen. $18 \%$ aller aus CI-Unternehmen ausscheidenden MitarbeiterInnen gehen in diesen Bereich /der auch den Wechsel in die Nicht-Erwerbstätigkeit umfasst, der allerdings von sehr geringer Bedeutung ist).

Weiterhin ist ein Zusammenhang zwischen dem Bildungsgrad der in einem Unternehmen beschäftigten MitarbeiterInnen und der Abgangsquote sowie der Wahrscheinlichkeit, dass sich ausscheidende MitarbeiterInnen selbständig machen, zu erkennen (Tabelle 42). Klassifiziert man CI-Unternehmen danach, ob der Anteil der MitarbeiterInnen mit Hochschulstudium über oder unter dem Mittelwert im jeweiligen CI-Bereich des Unternehmens liegt, so zeigen Unternehmen mit überdurchschnittlich vielen AkademikerInnen eine höhere Abgangsquote, vor allem aber einen mehr als doppelt so hohen Anteil von ausscheidenden MitarbeiterInnen, die sich selbstständig machen (13\% gegenüber $6 \%$ ). Dies deutet da- 
rauf hin, dass innerhalb der Creative Industries eher die besser qualifizierten MitarbeiterInnen den Arbeitgeber wechseln, und dass höher qua- lifizierte MitarbeiterInnen eher den Schritt in die Selbstständigkeit wagen.

Tabelle 42: Abgänge von MitarbeiterInnen aus CI-Unternehmen differenziert nach dem Humankapital der MitarbeiterInnen (in \%)

\begin{tabular}{lcccc}
$\begin{array}{l}\text { Anteil der Beschäftigten mit } \\
\text { Hochschulstudium: }\end{array}$ & Abgangsquote & $\begin{array}{c}\text { Anteil der Abgänge in } \\
\text { andere Unternehmen }\end{array}$ & $\begin{array}{c}\text { Anteil der Abgänge in die } \\
\text { Selbständigkeit }\end{array}$ & $\begin{array}{c}\text { Anteil der Abgänge in öffentliche/ } \\
\text { gemeinnïtzige Einrichtungen, sonstige }\end{array}$ \\
\hline überdurchschnittlich & 7,7 & 75 & 13 & 12 \\
\hline unterdurchschnittlich & 6,3 & 73 & 6 & 21 \\
$\mathrm{Cl}$ insgesamt & 6,8 & 74 & 9 & 18
\end{tabular}

Quelle: ZEW: Befragung Creative Industries Österreich 2008 - Berechnungen des ZEW. Alle Angaben hochgerechnet auf die Gesamtzahl der Unternehmen in den Creative Industries in Österreich Anfang 2008.

\subsubsection{Wissenschaftskooperationen und Netzwerkbildung}

Einen ebenfalls wichtigen Beitrag zum Innovationssystem leistet die Rolle, die Unternehmen der Creative Industries im Rahmen des Wissenstransfers aus der Wissenschaft bzw. der generellen Netzwerkbildung Wissenschaft-Wirtschaft spielen. Eine Nähe zum Wissenschaftsbetrieb ist bei der Kreativwirtschaft schon von daher gegeben, dass UnternehmerInnen wie unselbstständig Beschäftigte und freie MitarbeiterInnen in den Creative Industries oftmals eine Hochschule besucht haben. Insgesamt haben $28 \%$ der Beschäftigten in den Creative Industries einen Hochschulabschluss ${ }^{82}$ und über
$60 \%$ der CI-Unternehmen haben zumindest eine/n MitarbeiterIn, die/der an einer Hochschule studiert hat. Ein Transfer von neuen Forschungsergebnissen in Marktangebote findet jedoch nicht nur durch die Rekrutierung von HochschulabsolventInnen statt, sondern insbesondere auch durch eine direkte Zusammenarbeit mit der Wissenschaft. Diese Interaktionen zwischen Wissenschaft und Wirtschaft können dabei sehr unterschiedliche Formen annehmen (vgl. Schartinger et al., 2002). Zwei wichtige Kooperationsformen sind gemeinsamen Forschungsprojekte sowie eine Zusammenarbeit bei der Betreuung von Diplom- oder Doktorarbeiten (siehe Abbildung 49). 
Abbildung 49: Kooperationen mit der Wissenschaft nach Cl-Bereichen (Anteil an allen Unternehmen in \%)

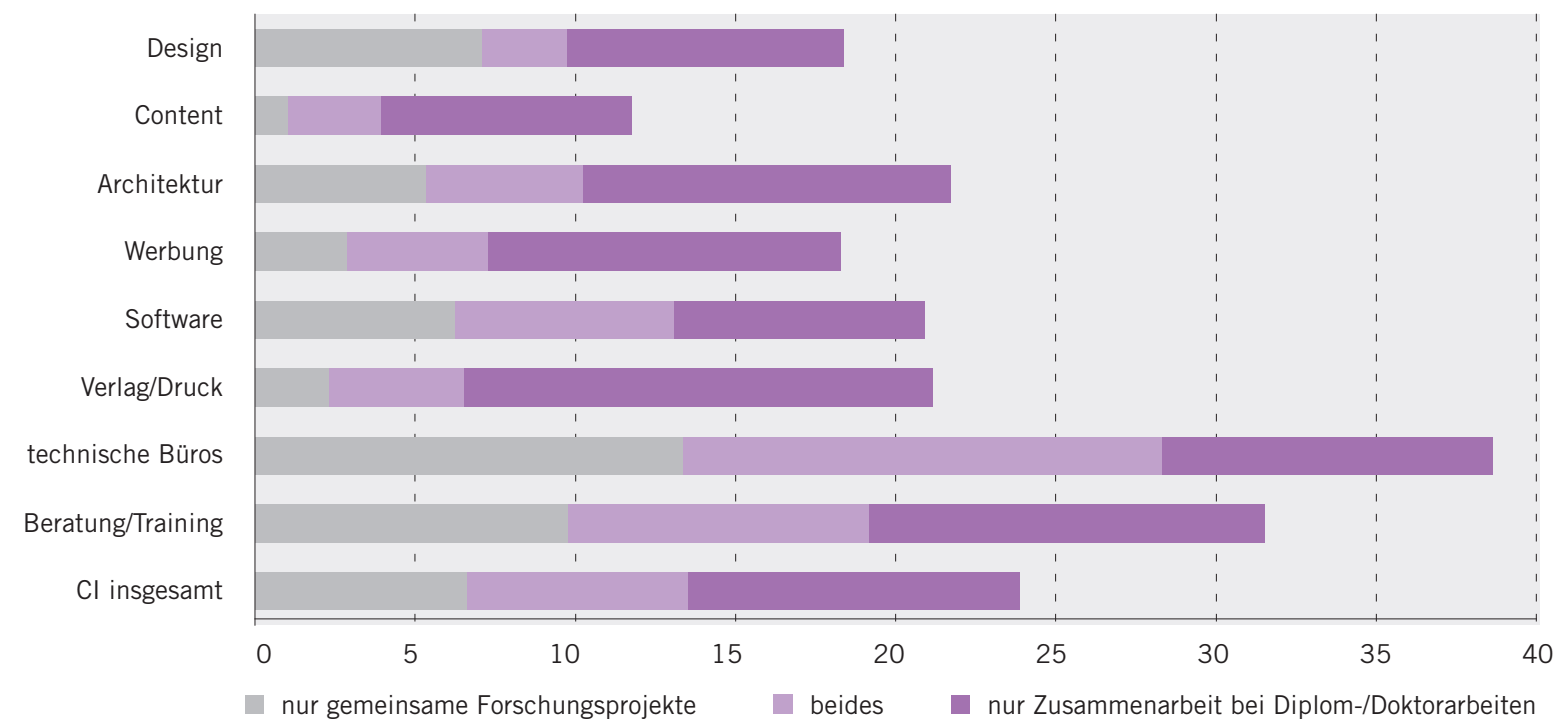

Quelle: ZEW: Befragung Creative Industries Österreich 2008 - Berechnungen des ZEW. Alle Angaben hochgerechnet auf die Gesamtzahl der Unternehmen in den Creative Industries in Österreich Anfang 2008.

Immerhin $24 \%$ aller CI-Unternehmen nutzen zumindest eine dieser beiden Formen der $\mathrm{Zu}$ sammenarbeit mit der Wissenschaft. $14 \%$ aller CI-Unternehmen führen gemeinsame Forschungsprojekte mit der Wissenschaft durch, $17 \%$ betreuen DiplomandInnen oder DoktorandInnen im Rahmen ihrer jeweiligen Abschlussarbeiten. Bei letzterer Form der Zusammenarbeit ist davon auszugehen, dass typischerweise auch eine Mitarbeit der StudentInnen in dem CI-Unternehmen stattfindet, z.B. als freie $/ \mathrm{r}$ MitarbeiterIn. $7 \%$ aller CI-Unternehmen haben sowohl gemeinsame Forschungsprojekte als Kooperationen bei Diplom- und Doktorarbeiten. Wissenschaftskooperationen sind vor allem bei technischen Büros stark verbreitet. $28 \%$ haben gemeinsame Forschungsprojekte mit Wissenschaftseinrichtungen und $24 \%$ betreuen DiplomandInnen oder DoktorandInnen. Aber auch Unternehmen des Bereichs Beratung/Training weisen überdurchschnittlich häufig Wissenschaftskooperationen auf. Auffällig ist, dass Wissenschaftskooperationen im Content-Bereich deutlich seltener als in anderen CI-Bereichen sind. Nur $12 \%$ der Content-Unternehmen weisen Wissenschaftskooperationen in den beiden hier betrachteten Formen auf, wobei lediglich $4 \%$ gemeinsame Forschungsprojekte durchführen.

Im Vergleich $\mathrm{zu}$ anderen technologie- und wissensintensiven Branchen zeigt sich, dass die Kooperation des CI-Sektors mit dem Wissenschaftsbereich im Branchenvergleich ausgesprochen hoch ist. Wie die Ergebnisse der Innovationserhebung 2005 durch die Statistik Austria (2006) zeigen, kommen selbst die forschungsintensiven Industriebranchen nicht auf einen so hohen Anteil an mit der Wissenschaft zusammenarbeitenden Unternehmen. Bezogen auf innovationsaktive Unternehmen ab 10 Beschäftigten - denn nur für diese Gruppe liegen Vergleichszahlen für andere Branchen vor - unterhalten $26 \%$ der CI-Unternehmen F\&EKooperationen mit der Wissenschaft (d.h. ohne Kooperationen im Bereich der Ausbildung von StudentInnen). In anderen forschungs- und wissensintensiven Branchen liegt diese Quote zwischen 10 und $20 \%$. 


\subsubsection{Resümee}

Insgesamt kann die Kreativwirtschaft als ein bedeutsamer Wachstumssektor betrachtet werden, dessen Gewicht mittlerweile auch gesamtwirtschaftlich nicht $\mathrm{zu}$ unterschätzen ist. Immerhin jedes zehnte Unternehmen und jeder 20. Beschäftigte ist der Kreativwirtschaft zuzuordnen, der Anteil an der gesamten Wertschöpfung beträgt $5 \%$. Gleichzeitig lässt sich die Kreativwirtschaft als ein Querschnittssektor einstufen, dessen Produkte und Dienstleistungen vor allem auch in anderen Branchen zum Einsatz kommen und von daher wichtige Impulse auf die Gesamtwirtschaft ausgehen. Dabei ist die Kreativwirtschaft sui generis ein hoch innovativer Sektor mit beträchtlichen F\&E-Aufwendungen und entsprechenden Innovationsanstrengungen. Neben diesem direkten Beitrag zum Innovationsgeschehen kommt der Kreativwirtschaft aber auch durch weitere Mechanismen eine wichtige Funktion im Innovationssystem Österreichs zu. So beschäftigte die Kreativwirtschaft überdurchschnittlich qualifiziertes Humankapital und weist eine überdurchschnittliche Personalmobilität auf. Beschäftigte der Kreativwirtschaft wechseln regelmäßig die Branche und stellen somit anderen Branchen ihre kreativen Fähigkeiten zur Verfügung. Die Ergebnisse der Befragung zeigen weiters, dass Unternehmen der Kreativwirtschaft in ihren Geschäftsbeziehungen zu Unternehmen anderer Branchen regelmäßig als Impulsgeber für Innovation dienen und/oder in die Innovationsprozesse ihrer Kunden mit einbezogen werden. Darüber hinaus weisen Unternehmen der Kreativwirtschaft oft enge Beziehungen zum Wissenschaftssystem auf (z.B. über gemeinsame Kooperationsprojekte oder über Ausbildungspartnerschaften bezüglich
Diplomarbeiten/Dissertationen) und spielen somit eine wichtige Rolle für den Technologieund Wissenstransfer zwischen Wissenschaft und Wirtschaft.

Die hier empirisch untermauerte große Bedeutung der kreativwirtschaftlichen Branchen für Österreichs Innovationssystem wird von Seiten der Wirtschafts- und Technologiepolitik anerkannt und im Regierungsprogramm ${ }^{83}$ ausdrücklich erwähnt. Mit dem Programm evolve wurden entsprechende Initiativen und Programme zur Stimulierung einschlägiger Aktivitäten in die Wege geleitet. ${ }^{84}$

\subsection{Technologiediffusion in der österreichischen Sachgüterproduktion}

Innovation ist nicht immer das Ergebnis von Forschung und Entwicklung. Auch die Anwendung existierender Technologien, etwa der Einsatz eines neuen Fertigungsverfahrens in einem Betrieb, kann zu Steigerungen der Produktivität und in weiterer Folge zu neuen Produkten führen. Technologiediffusion ist daher eine wesentliche Quelle für Wachstum und besonders kleine Länder wie Österreich profitieren in großem Umfang vom weltweit existierenden Pool an Technologien. Technologiediffusion hat in den letzten Jahrzehnten wesentlich zum Wachstum der österreichischen Wirtschaft beigetragen. Zerlegt man Wirtschaftswachstum in seine Hauptkomponenten, nämlich in die Beiträge der Produktionsfaktoren Arbeit und Kapital zur Veränderung der jährlichen Wirtschaftsleistung, so zeigt sich, dass Veränderungen des Kapitalstocks - etwa der Einsatz besserer Maschinen - im Zeitraum 1990 bis 2004 für mehr als die Hälfte des Wachstums der Wertschöpfung verantwortlich waren (Peneder et al. 2006).

83 Siehe Regierungsprogramm 2008-2013 für die XXIV. Gesetzgebungsperiode S. $12 f$. 84 Einen Überblick über die Aktivitäten bietet: http://www.evolve.or.at. 
Technologisches Wissen ist charakterisiert durch unterschiedliche Grade an Aneigenbarkeit (appropriability), unterschiedliche Möglichkeiten der Anwendung (technological opportunity), Kumulativität im Erlernen von technischem Know-how und tacit knowledge. Technologien entwickeln sich entlang unterschiedlicher Trajektorien, die sich aus den spezifischen technischen Eigenschaften und der absorptiven Kapazität der Firmen ergeben. Als Folge all dieser Eigenschaften ist eine unterschiedliche Verbreitung von Technologien eine fundamentale und permanente Charakteristik der Industrielandschaft (Silverberg et al. 1988). Einen Einblick in die umfassende Literatur zu Technologiediffusion geben u.a. Geroski (2000) und Hollenstein und Woerter (2008).

\section{European Manufacturing Survey (EMS)}

Im Rahmen des European Manufacturing Survey (EMS) befragte ARC systems research in den Jahren 2003 und 2006 österreichische Unternehmen zu Innovationen in der Produktion. Diese Umfrage war ein Gemeinschaftsprojekt mit dem deutschen Fraunhofer-Institut für Systemtechnik und Innovationsforschung. Insgesamt beteiligten sich ca. 280 Unternehmen an der Erhebung. Untersuchungsgegenstand waren die verfolgten Produktionsstrategien, der Einsatz innovativer Organisations- und Technikkonzepte in der Produktion, Fragen des Personaleinsatzes und der Qualifikation sowie Fragen zu Auslagerungen sowie zur Leistungstiefe und zum Management der Produktionsmodernisierung. Daneben wurden Leistungsindikatoren wie Produktivität, Flexibilität, erreichte Qualität und nicht zuletzt die Rendite erhoben. Mit diesen Informationen erlaubt die Umfrage Aussagen zur Modernität und Leistungskraft der österreichischen Industrie und internationale Vergleiche auf Branchenebene mit Deutschland, der Schweiz und einigen östlichen Nachbarstaaten, in denen diese Umfrage ebenfalls durchgeführt wurde.

Mehr Informationen auf der Homepage des Fraunhofer-Institut für Systemtechnik und Innovationsforschung (http://www.isi.fhg.de/pi/projekte/erhebung pi.htm)
Der Einsatz neuer Produktionstechnologien ist jedoch nur eine von mehreren Strategien, mit denen Leistungssteigerungen im Produktionssektor erreicht werden können. Über die letzten 40 Jahre haben verschiedene Entwicklungen stattgefunden. Rückblickend lassen sich vier Phasen im Wandel von Leitbildern der Produktionsmodernisierung unterscheiden (adaptiert nach Lay und Wengel 1998):

- Bis in die 1970er Jahre war der Automatisierungstrend dominant. Technische Lösungen und Maschinen wurden für immer mehr Produktionsaufgaben entwickelt und ersetzten die menschliche Arbeitskraft in der Produktion.

- Von den 1970er Jahren bis in die 1980er Jahre blieben die Verbesserung der Produktionstechnik und die verstärkte Automatisierung im Vordergrund, jedoch änderte sich der Fokus in Richtung flexible Automatisierung.

- Ende der 1980er Jahre wurde die Dominanz technologischer Innovationen als Instrument der Produktionsmodernisierung abgelöst: Verbesserungen in der Organisation der Produktion und die Fähigkeiten der Mitarbeiter und Mitarbeiterinnen rückten mehr in den Vordergrund.

- Seit Ende der 1990er Jahre rückt der Kunde verstärkt ins Blickfeld. Ergebnis ist eine stärkere Fokussierung auf die Anpassung der Produkte an seine Bedürfnisse und die Ergänzung des Produktangebotes um Dienstleistungen als Ausrichtung der Produktionsmodernisierung.

Dieser Wandel von Leitbildern über die Zeit ist damit auch eine Erklärung für die unterschiedliche Geschwindigkeit in der Verbreitung neuer Technologien: Wenn Unternehmen sich nicht allein auf neue technologische Lösungen als Innovationsstrategie konzentrieren, sondern ihr Gewicht zu einem erheblichen Teil auf nicht-technische Maßnahmen der Produktionsmodernisierung legen, so ist das auch ei- 
ne Ursache für die geringere Verbreitungsdynamik mancher Technologien.

Abbildung 50 verdeutlicht die unterschiedliche Gewichtung verschiedener Strategien zur Produktionsmodernisierung auf Branchenebene. Viele österreichische Unternehmen räumen technischen Innovationen, also dem Einsatz von neuen Maschinen, in der Produktionsmodernisierung einen sehr hohen Stellenwert, aber selten den ersten Platz ein. Insgesamt legen $41 \%$ aller Unternehmen den Schwerpunkt auf die Entwicklung neuer Produkte, gefolgt von $33 \%$ auf technische Innovationen im Produktionsprozess, $14 \%$ auf organisatorische Maßnahmen und $13 \%$ auf die
Ergänzung des Produktangebotes um Dienstleistungen. Es zeigen sich deutliche Unterschiede zwischen den Wirtschaftszweigen in der Bedeutung verschiedener Maßnahmen zur Steigerung ihrer Leistung. Vor allem in der Metallerzeugung und im Maschinenbau liegt der Schwerpunkt auf technischen Innovationen im Produktionsprozess. In den meisten anderen Branchen sind technische Innovationen im Produktionsprozess zweitwichtigste Maßnahme nach der Entwicklung neuer Produkte. Der Fahrzeugbau bildet eine Ausnahme; hier wird massiv auf organisatorische Maßnahmen gesetzt.

\section{Abbildung 50: Stellenwert der Technik in der Produktionsmodernisierung}

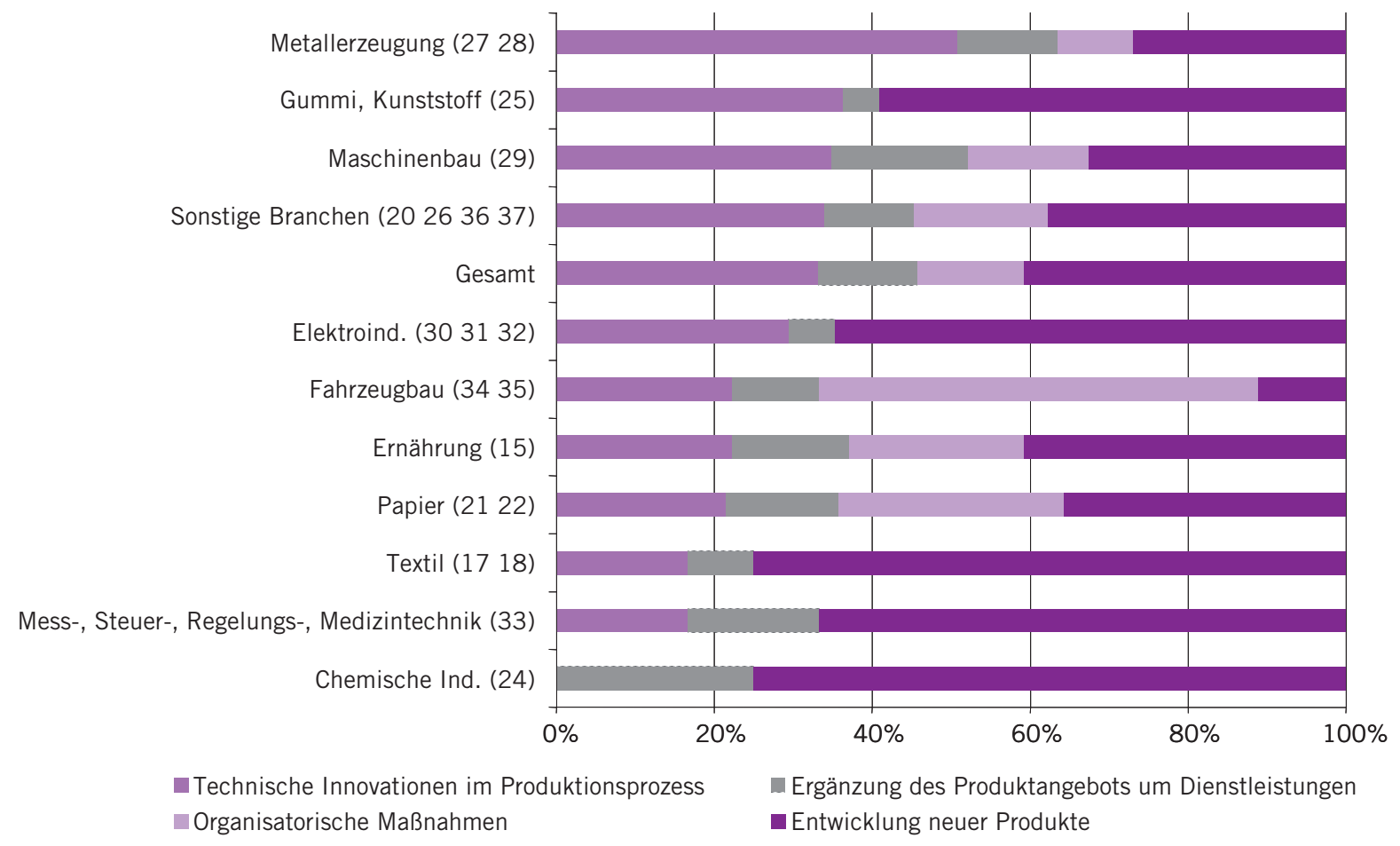

Anteil der Betriebe, bei denen die Maßnahme die Priorität 1 hat.

Quelle: ARC systems research, Erhebung Innovation in der Produktion 2006 (EMS). 
Betrachtet man die Betriebsgröße, so unterscheiden sich Modernisierungsstrategien im Produktionsprozess zwischen den einzelnen Größenklassen kaum. Ausnahme ist, dass große Unternehmen verstärkt auf die Entwicklung neuer Produkte setzen und Unternehmen unter 100 Beschäftigten tendenziell häufiger ihr Produktangebot um Dienstleistungen ergänzen.

Die unterschiedliche Bedeutung von technischen Innovationen in verschiedenen Branchen ist einerseits das Ergebnis der Anwendungsreife verschiedener Technologien, andererseits Resultat der potenziellen Anwendungsmöglichkeiten in einer Branche. Wie diese beiden Faktoren zusammenspielen, lässt sich am Beispiel von CAD/CAM sowie bio- und gentechnologischen Verfahren sehen. Abbildung 51 gibt einen Überblick über den Verbreitungsgrad und den geplanten Einsatz verschiedener Technologien. CAD und CAM sind dabei die am weitesten verbreiteten Technologien. Etwa $80 \%$ der befragten Firmen haben diese Technologien bereits vor dem Jahr 2000 eingeführt, nur noch ein geringer Anteil von Firmen hat Pläne für ihre Einführung. Die meisten Unternehmen, die CAD und CAM bis jetzt nicht eingeführt haben, sehen keine betrieblichen Anwendungsmöglichkeiten. CAD und CAM sind Technologien, die in eine Sättigungsphase eingetreten zu sein scheinen.

Am anderen Ende des Spektrums befinden sich der Einsatz von bio- und gentechnologischen Verfahren oder Katalysatoren und die Produktion unter Reinraumbedingungen, wie sie etwa bei Elektronikkomponenten, Nahrungsmitteln etc. notwendig ist: Über zwei Drittel der Unternehmen sehen hier keine betrieblichen Anwendungsmöglichkeiten. Die geringe Zahl von Firmen, die diese Technologien bereits eingeführt haben (4 bzw. $10 \%$ ), hat dies zu 80 bzw. $74 \%$ schon vor dem Jahr 2000 getan. Beide Technologien sind Nischenanwendungen und werden es vermutlich auch bleiben, weil das technische Potenzial für Anwendungen außerhalb ihrer ursprünglichen Branchen fehlt bzw. ihre Anwendung nicht wirtschaftlich erscheint. 
Abbildung 51: Die Verwendung und der geplante Einsatz verschiedener Technologien im österreichischen Produktionssektor im Jahr 2006

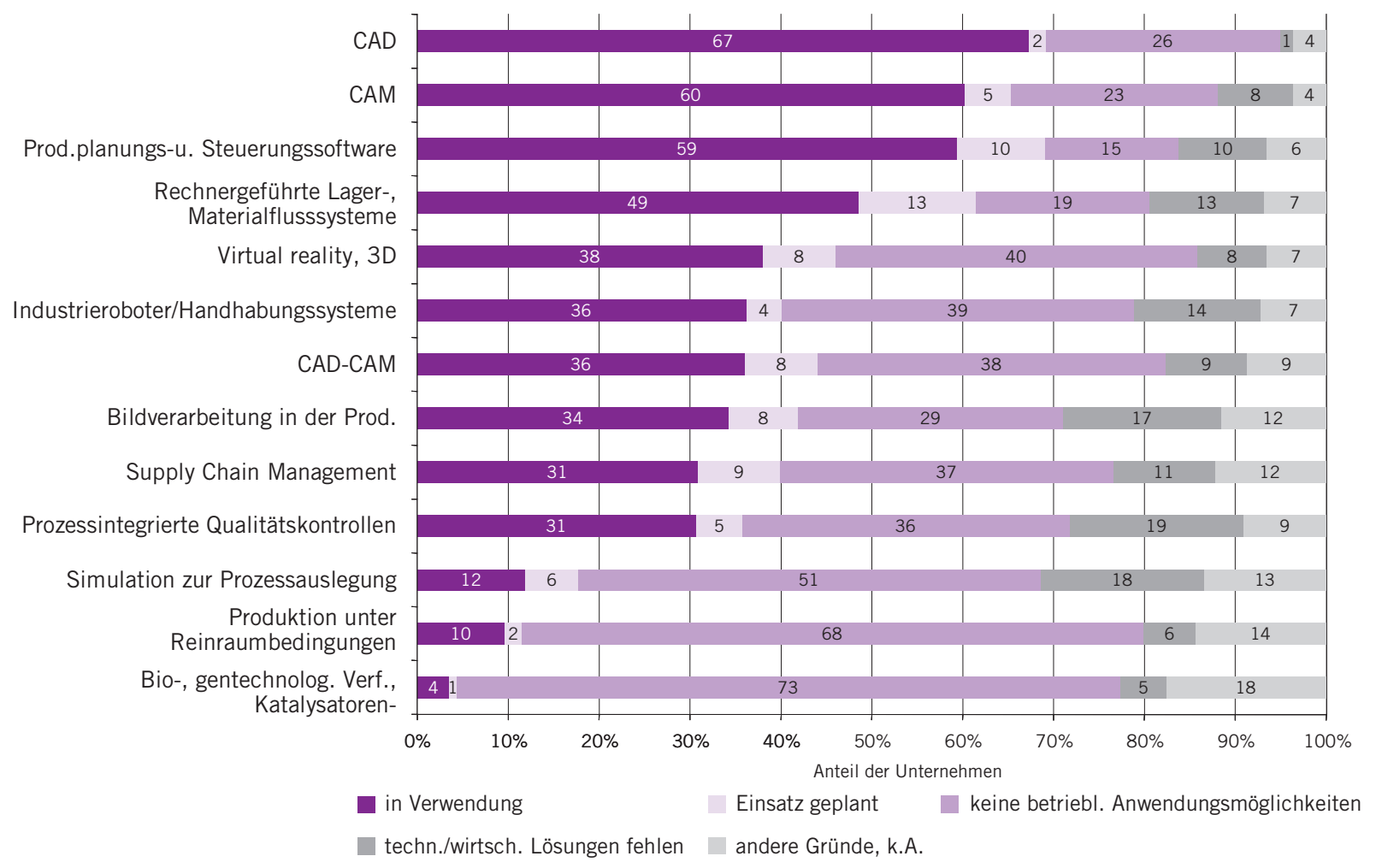

Quelle: ARC systems research, Erhebung Innovation in der Produktion 2006 (EMS).

Hinter den in Abbildung 51 erfassten Technologien steht eine sehr unterschiedliche Verbreitungsdynamik. Abbildung 52 zeigt, dass sich etwa Virtual Reality (Simulation zur Produktauslegung) und Supply Chain Management (Austausch von Dispositionsdaten mit anderen Unternehmen) durch eine hohe Verbreitungsdynamik seit dem Jahr 2000 auszeichnen. Es handelt sich dabei um relativ junge Technologien, die bereits eine hohe Anwendungsreife besitzen und die sich zurzeit in einer Wachstumsphase befinden. Mehr als die Hälfte bis zwei Drittel aller befragten Unternehmen, die die Technologien eingeführt haben, haben dies seit dem Jahr 2000 getan.

Am anderen Ende der Skala befinden sich CAD und CAM, die von rund $80 \%$ der befragten Unternehmen bereits vor dem Jahr 2000 eingeführt wurden. 


\section{Abbildung 52: Unterschiedliche Technologien und die Dynamik ihrer Diffusion}

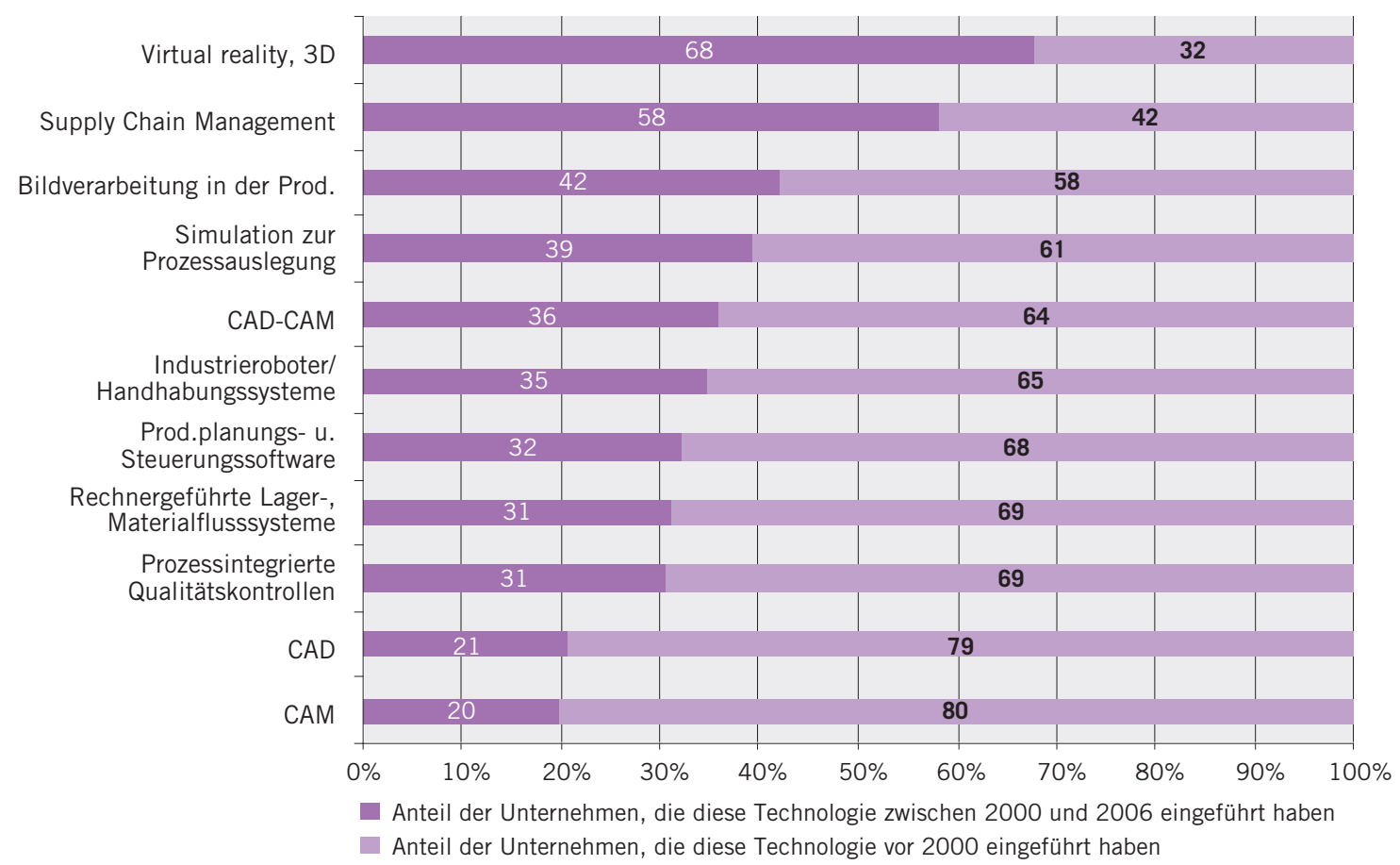

Quelle: ARC systems research, Erhebung Innovation in der Produktion 2006 (EMS).

Die Technologien im mittleren Bereich von Abbildung 52 sind mit Anwendungsbarrieren konfrontiert (siehe dazu auch Abbildung 53). Es handelt sich dabei um Technologien wie die prozessintegrierte Qualitätskontrolle (z. B. durch Laser, Ultraschall, Sensor), die Simulation zur Prozessauslegung, die Bildverarbeitung in der Produktion (z.B. Qualitätskontrolle, Prozessführung) und Industrieroboter bzw. Handhabungssysteme für Werkzeug- und Werkstückguthandhabung. Bei diesen Technologien wird sich in den nächsten Jahren herausstellen, ob sie sich durchsetzen oder ob sie scheitern, denn Scheitern ist in technologischen Neuerungsprozessen nichts Ungewöhnliches (Geroski, 2000). Diese Technologien werden nur zögerlich eingesetzt, weil vielfach die techni- schen oder wirtschaftlichen Lösungen fehlen.

Unterschiedliche Analysen von Hemmnisfaktoren bei technisch-organisatorischen Innovationsprojekten weisen darauf hin, dass es oft nicht die Reife der Technologie selbst ist, die ihre Einführung verhindert. Vielmehr deuten Inkompatibilitäten mit der im Betrieb vorhandenen Technik und Pfadabhängigkeiten durch vergangene Technologieinvestitionen auf einen Bedarf an Anpassentwicklungen hin: Bedarf an Software-Entwicklung, Schnittstellenprobleme, Inkompatibilitäten mit vorhandenen Maschinen oder mit der Organisation des Unternehmens. Es gibt hier wohl den größten Bedarf and Forschungs- und Entwicklungsanstrengungen, um $\mathrm{zu}$ anwendungsreifen Lösungen zu gelangen. 


\section{Abbildung 53: Technologische Unreife: eine Reihung unterschiedlicher Technologien}

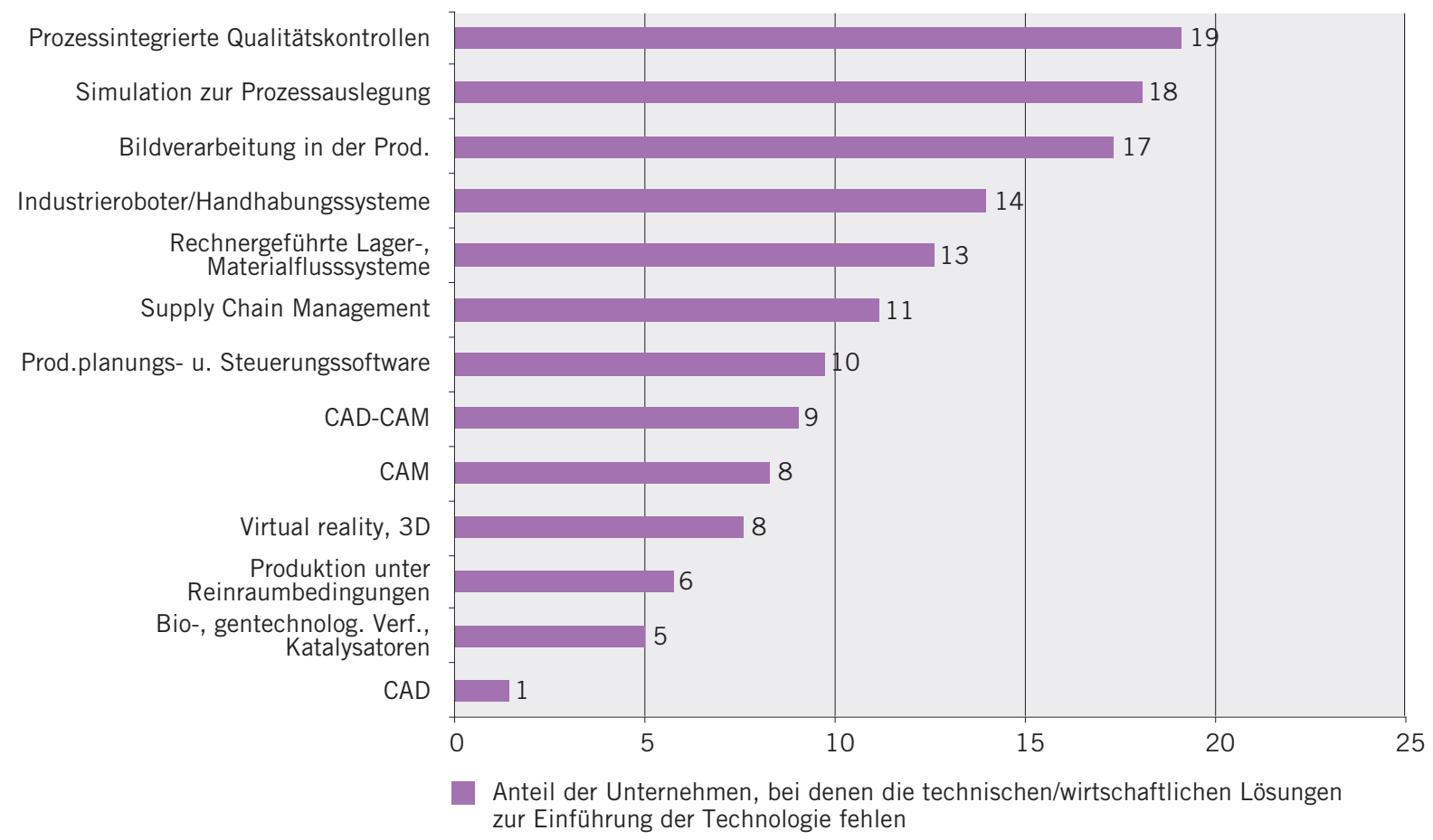

Quelle: ARC systems research, Erhebung Innovation in der Produktion 2006 (EMS).

Prozesstechnologien haben einen wesentlichen Einfluss auf die Produktivität eines Unternehmens. Neue Technologien nehmen in der Produktionsmodernisierung einen sehr hohen Stellenwert ein, österreichische Unternehmer legen ihr Gewicht aber auch zu einem erheblichen Teil auf Produkt- und organisatorische Innovationen und auch die Ergänzung des Produktangebotes um Dienstleistungen nimmt an Bedeutung zu. Es zeigen sich dabei deutliche Unterschiede zwischen den Wirtschaftszweigen in der Bedeutung verschiedener Maßnahmen zur Steigerung ihrer Leistung.

Daraus ergibt sich allerdings noch nicht automatisch ein Handlungsbedarf für die Politik. Intervention ist nicht notwendig, wenn es sich um Technologien handelt, deren Durchdringung bereits sehr hoch ist oder die derzeit ohnehin bereitwillig von den Unternehmen angenommen werden. Vielmehr sollte sich die Politik auf Bereiche mit technologischem Potenzial konzentrieren, deren Verbreitung stockt. Wenn sich herausstellt, dass dies das Resultat von Anwendungsbarrieren wie technologischer Unsicherheit, dem Fehlen von Pilotanwendungen oder zu hohen Kosten in der Eingangsphase ist, wäre dies ein Grund, Maßnahmen zur Überwindung dieser Hindernisse zu überlegen. 


\subsection{Berufliche Selbstständigkeit und Unternehmensgründungen von Frauen}

Unternehmensgründungen ${ }^{85}$ sind in den letzten Jahren immer stärker in das Interesse der Wirtschaftspolitik gerückt. So ist die Steigerung der Gründungsrate mittlerweile wesentlicher Bestandteil wirtschaftspolitischer Zielsetzungen, sowohl auf nationaler wie europäischer Ebene. Hervorgehoben wird vor allem die volkswirtschaftliche Bedeutung von Unternehmensgründungen für Wettbewerbsfähigkeit, Beschäftigung, Innovation und technologischen Wandel: Im Aktionsplan der EU wurde UnternehmerInnentum als wichtiger Faktor für Innovation, Wettbewerbsfähigkeit und Wachstum bezeichnet, verbunden mit der Feststellung, dass das unternehmerische Potenzial (noch) nicht ausgeschöpft wird (Europäische Kommission 2004). Zur Förderung der unternehmerischen Dynamik wurden strategische Politikbereiche festgelegt, die unter anderem die verstärkte Ermutigung zur Unternehmensgründung zum Ziel haben. Neben einem klaren Fokus auf kleine und mittlere Unternehmen wurden auch Frauen explizit als Zielgruppe von Maßnahmen genannt. Einen Ausgangspunkt bildete dabei die Feststellung, dass Frauen als Unternehmerinnen wie auch als Gründerinnen unterrepräsentiert sind, die unternehmerische Tätigkeit von Frauen aber „zunehmend als ein wichtiger Motor für die Beschäftigung und das Wachstumspotenzial angesehen wird" (OECD 2004, S. 10). Da es aber vielfach an entsprechenden Daten- und Analysegrundlagen zu weiblicher Unternehmenstätigkeit, über das Ausmaß des weiblichen Gründungspotenzials oder die Effekte von relevanten (Förder-)Maßnahmen fehlt, haben in den letzten Jahren geschlechterspe- zifische Aspekte zunehmend Eingang in die Gründungs- und Entrepreneurship-Forschung gefunden.

Diese empirischen Untersuchungen haben nicht nur einen Gender-Gap bei Unternehmensgründungen in Österreich, sondern auch Unterschiede im Gründungsverhalten von Frauen und Männern aufgezeigt: Unternehmensgründungen von Frauen sind durch Einzelgründungen, geringere Unternehmensgröße, traditionelle Branchen (z.B. Handel, Tourismus, personenbezogene Dienstleistungen), geringes Startkapital sowie schwächeres Wachstum gekennzeichnet. Die Gründungen von "rein technisch" dominierten Unternehmen stellt für Frauen nach wie vor die Ausnahme dar. Eine Analyse des Zentrums für Europäische Wirtschaftsforschung (ZEW) hat für das Jahr 2007 einen Anteil von Frauengründungen in den High-Tech-Sektoren von $8 \%$ in Deutschland ergeben - ein Wert, der sogar zwei Prozentpunkte unter jenem von 1995 liegt (Metzger et al. 2008). Entsprechend systematische Daten liegen für Österreich nicht vor. Anhaltspunkte können bspw. aus der exemplarischen Betrachtung des AplusB-Förderprogramms getroffen werden. Im November 2008 gab es in ganz Österreich neun AplusBZentren, in denen GründerInnen betreut werden. $7 \%$ der Gründenden bzw. Co-Gründenden waren Frauen.

Da es in der Entrepreneurship-Forschung jedoch keine einheitliche Definition von Gründungen gibt; der Begriff in verschiedenen Forschungsarbeiten also unterschiedlich verwendet wird, variieren auch die Angaben über Anzahl oder Intensität von Frauengründungen. Je nach Erfassungsmethode oder Definition liegt bspw. der Anteil von Frauen an den österreichischen Gründungen zwischen $25 \%$ und $40 \%$. 
Als Abgrenzungskriterien werden meist die Art der Gründung (Neugründung, Übernahme, neue Gewerbeberechtigung), die Verortung im Gründungsprozess (wie lange vor bzw. wie lange nach dem tatsächlichen Gründungszeitpunkt) oder eine Kombination beider Kriterien herangezogen. So unterscheidet der Global Entrepreneurship Monitor (GEM-Monitor ${ }^{86}$ ) Gründungen nach ihrer „Lebensdauer" (bei einer Dauer von weniger als 3 Monaten wird von Neugründungen, bei einer Dauer von 3-42 Monaten wird von neuen/jungen UnternehmerInnen gesprochen). Die Neugründungsstatistik der Wirtschaftskammer erfasst von den Gewerbebehörden übermittelte Daten über die Vergabe neuer Gewerbeberechtigungen. Aus Gründungsgesichtspunkten ist das nicht eindeutig, da es sich dabei bspw. auch um eine Erweiterung oder Übernahme bestehender Unternehmen handeln kann. Hinzu kommt, dass diese Statistiken nur Mitglieder der Wirtschaftskammer umfassen. Der größte Teil der österreichischen Gründungen findet zwar im Bereich der gewerblichen Wirtschaft statt, die Ausübung einer selbstständigen Tätigkeit beruht jedoch nicht zwangsläufig auf dem Besitz einer Gewerbeberechtigung. Es gibt ebenso GründerInnen, die über keinen Gewerbeschein verfügen müssen, auf Werkvertragsbasis arbeiten und als „Neue Selbstständige" bezeichnet werden. Die zunehmende De-Regulierung der Erwerbsformen bringt somit noch eine weitere
Heterogenität hinsichtlich der (sozial-)rechtlichen Form möglicher Gründungen mit sich.

Trotz dieser Einschränkungen stellen die Daten der Wirtschaftskammer die umfangreichsten und detailliertesten derzeit in Österreich vorliegenden zur Beschreibung des Gründungsgeschehens dar. Der folgende Überblick umfasst sowohl berufliche Selbstständigkeit wie auch Unternehmensgründungen von Frauen. Als Datenbasis wird neben Mikrozensus und Labour Force Survey auch die Mitgliederstatistik der Wirtschaftskammer verwendet. Daran schließt sich eine kurze Darstellung der Analyse von High-Tech-Gündungen von Frauen (exemplarisch für Deutschland) sowie der spezifischen Charakteristika und Rahmenbedingungen für Frauen in diesem Feld an.

\subsubsection{Die berufliche Selbstständigkeit von Frauen}

Nach Untersuchungen auf Basis des Mikrozensus gab es im Jahr 2007 insgesamt 481.500 selbstständig Erwerbstätige, davon waren 311.500 Männer $(64,7 \%)$ und 170.000 Frauen $(35,3 \%)$. Im Vergleich zum Vorjahr bedeutet das einen Anstieg um knapp $2 \%$, wobei die Zahl selbstständig erwerbstätiger Frauen stärker als die der Männer zugenommen hat. Im Vergleich zur EU - mit einem Frauenanteil unter den Selbstständigen von 29,9\% - liegt Österreich damit im europäischen Spitzenfeld.

86 Der GEM-Monitor untersucht unternehmerische Aktivität in 42 Ländern auf allen fünf Kontinenten. Im Rahmen einer repräsentativen Befragung der Bevölkerung im Alter zwischen 18 und 64 Jahren, ergänzt um eine Erhebung unter ausgewählten nationalen ExpertInnen, erfolgt zum einen ein Monitoring der unternehmerischen Aktivitäten. Zum anderen werden die Rahmenbedingungen für UnternehmerInnentum analysiert. Österreich hat sich im Jahr 2007 zum zweiten Mal beteiligt. 
Abbildung 54: Frauenanteil selbstständig Erwerbstätiger in der Europäischen Union, 2007

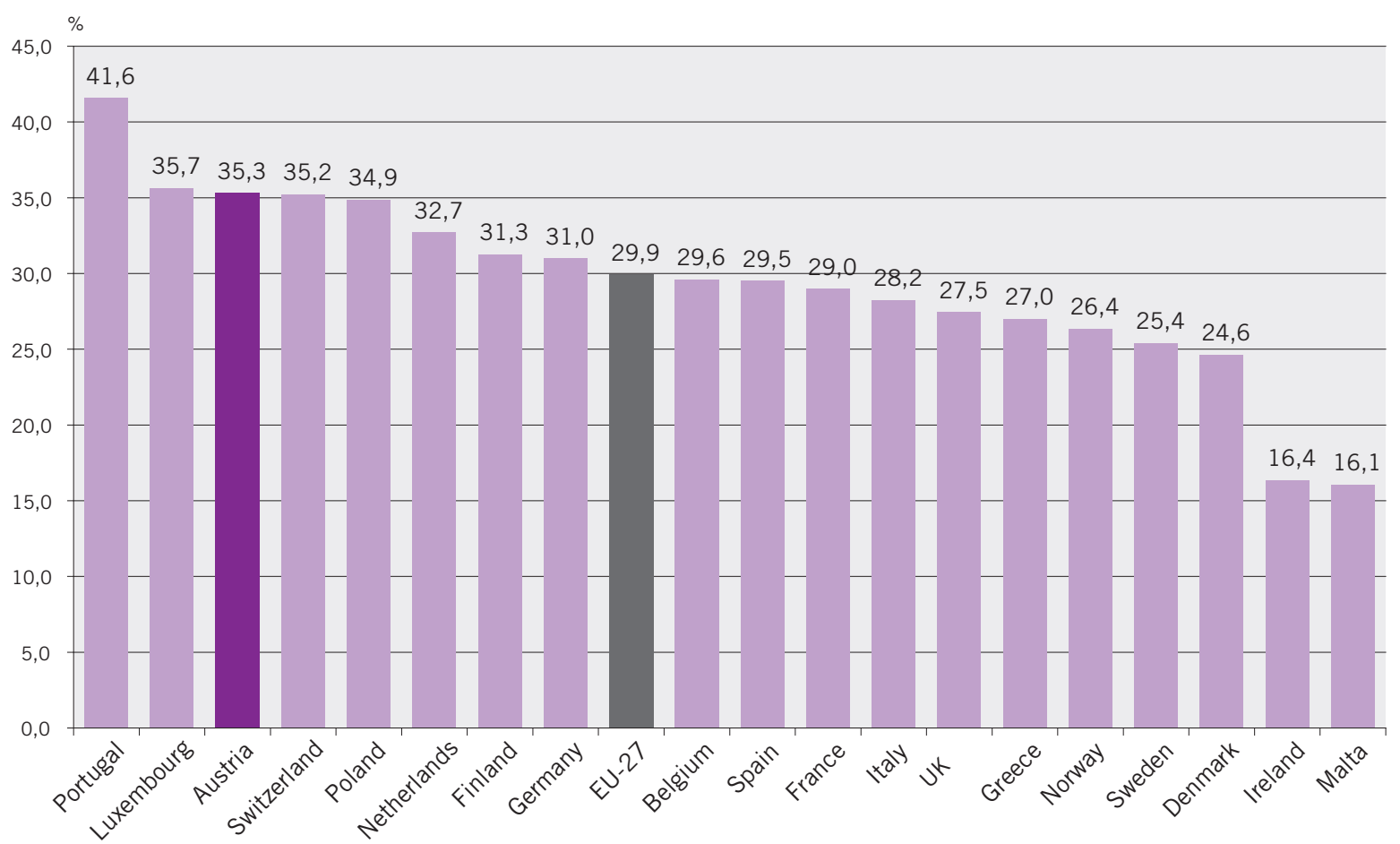

Quelle: EUROSTAT Labour Force Survey 2007

Die Entwicklung der letzten zehn Jahre zeigt ebenfalls eine stetige Zunahme der Selbstständigkeit: Von 1996 bis 2007 insgesamt um mehr als 20 Prozent, bei Frauen mit 23,4 \% etwas stärker als bei Männern $(18,1 \%)$. Im selben Zeitraum ist allerdings auch die Frauenerwerbsquote gestiegen. Die Selbstständigenquote von Frauen, also der Anteil selbstständi- ger Frauen an den erwerbstätigen Frauen, hat daher nur moderat zugenommen: von 8,6 \% im Jahr 1996 auf 9,1 \% im Jahr 2007. Im Vergleich dazu stieg die Selbstständigenquote von Männern im gleichen Zeitraum von 12,4 \% auf $14,1 \%$. Insgesamt lag die Selbstständigenquote 2007 bei $12 \%$. 
Abbildung 55: Erwerbs- und Selbstständigenquoten 1995 - 2007, nach Geschlecht, in \%
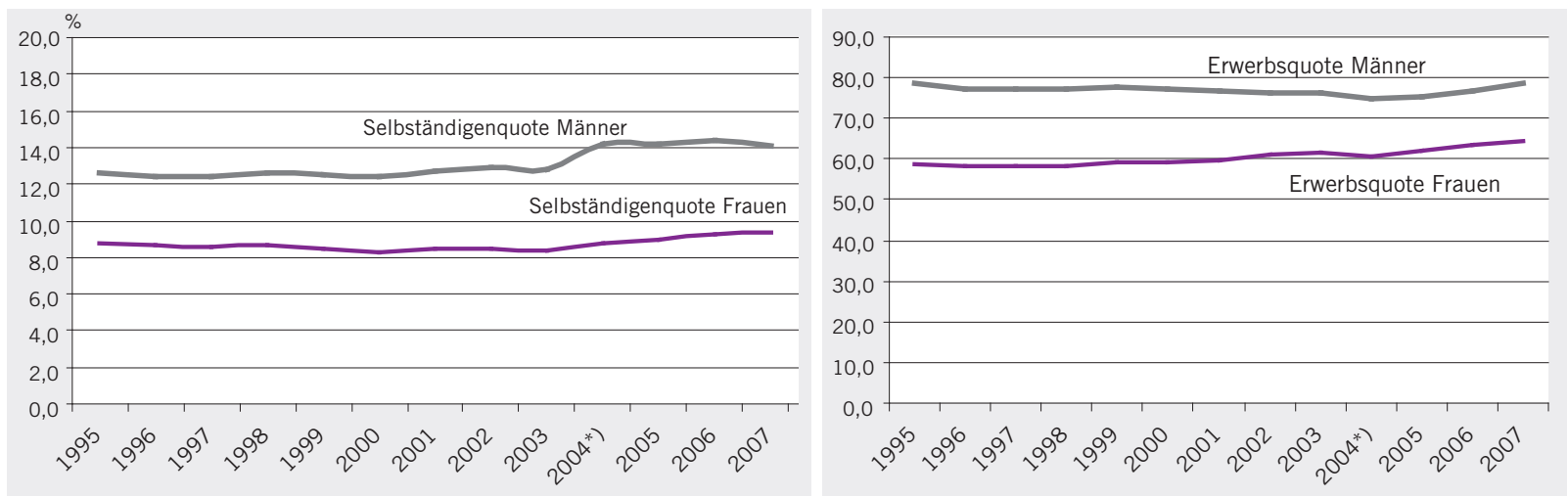

Quelle: Statistik Austria, Mikrozensus

Im Hinblick auf die Selbstständigenquote rangiert Österreich im europäischen Vergleich im Mittelfeld. Die durchschnittliche weibli- che Selbstständigenquote in der EU war mit $9,7 \%$ knapp halb so hoch wie jene der Männer $(18,2 \%)$.

\section{Abbildung 56: Selbstständigenquote von Frauen in der Europäischen Union, 2007}

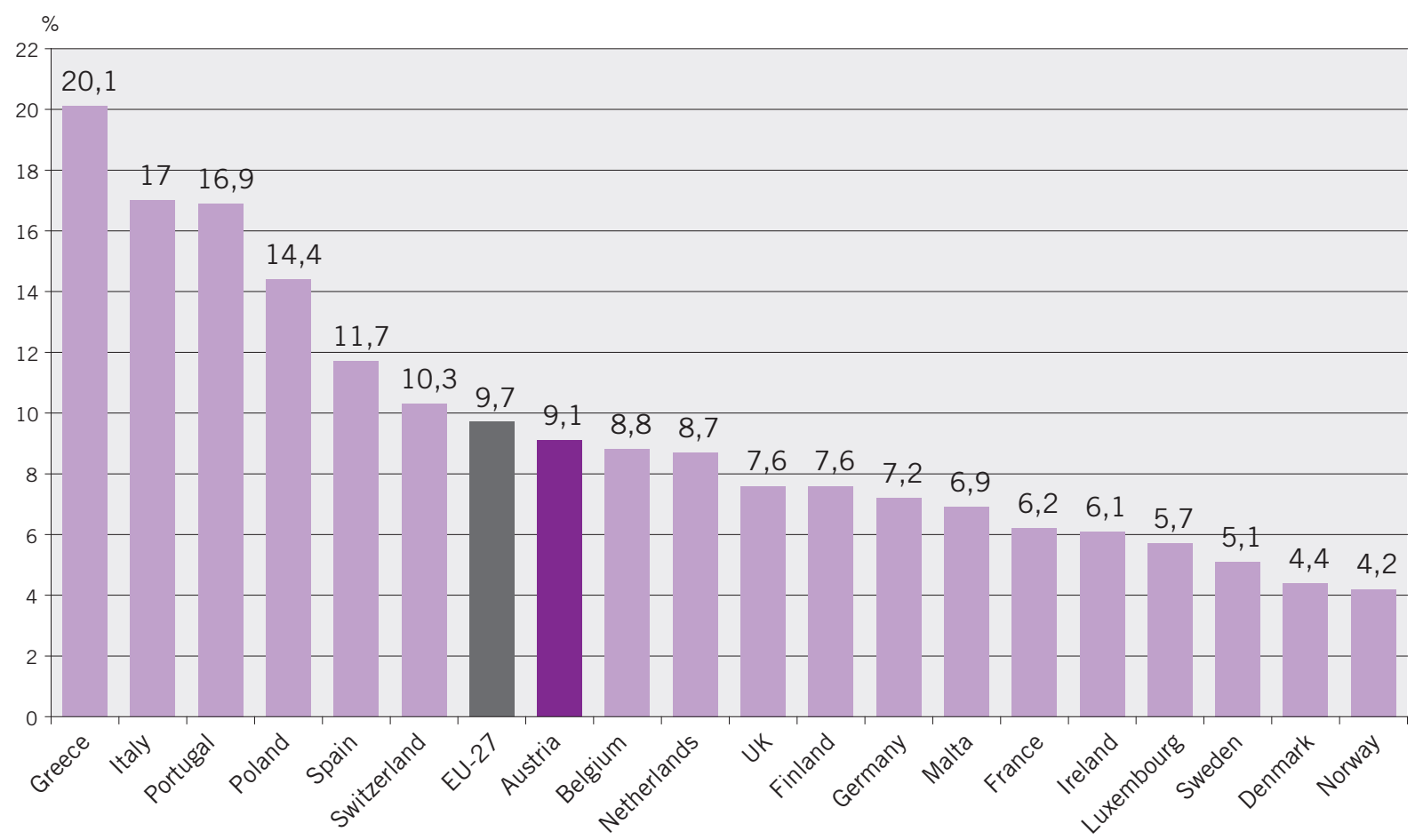

Quelle: Eurostat, http://epp.eurostat.ec.europa.eu/cache/ITY OFFPUB/KS-SF-08-099/EN/KS-SF-08-099-EN.PDF 
Dieser Gender Gap hat sich auf EU-Ebene im Zeitraum von 2000 bis 2007 kaum verändert (2000: 8,7 \%; 2007: 8,6\%). Große Variationen gibt es hingegen in einzelnen Mitgliedsstaaten.
Österreich zählt mit einem Gender Gap von 4,7 \% im Jahr $2007 \mathrm{zu}$ den Ländern mit den niedrigsten Differenzen, allerdings ist er in diesem Zeitraum um 0,5 \%-Punkte angestiegen.

\section{Abbildung 57: Gender Gap i.d. Selbstständigenquote von Frauen und Männern, 2000 und 2007}

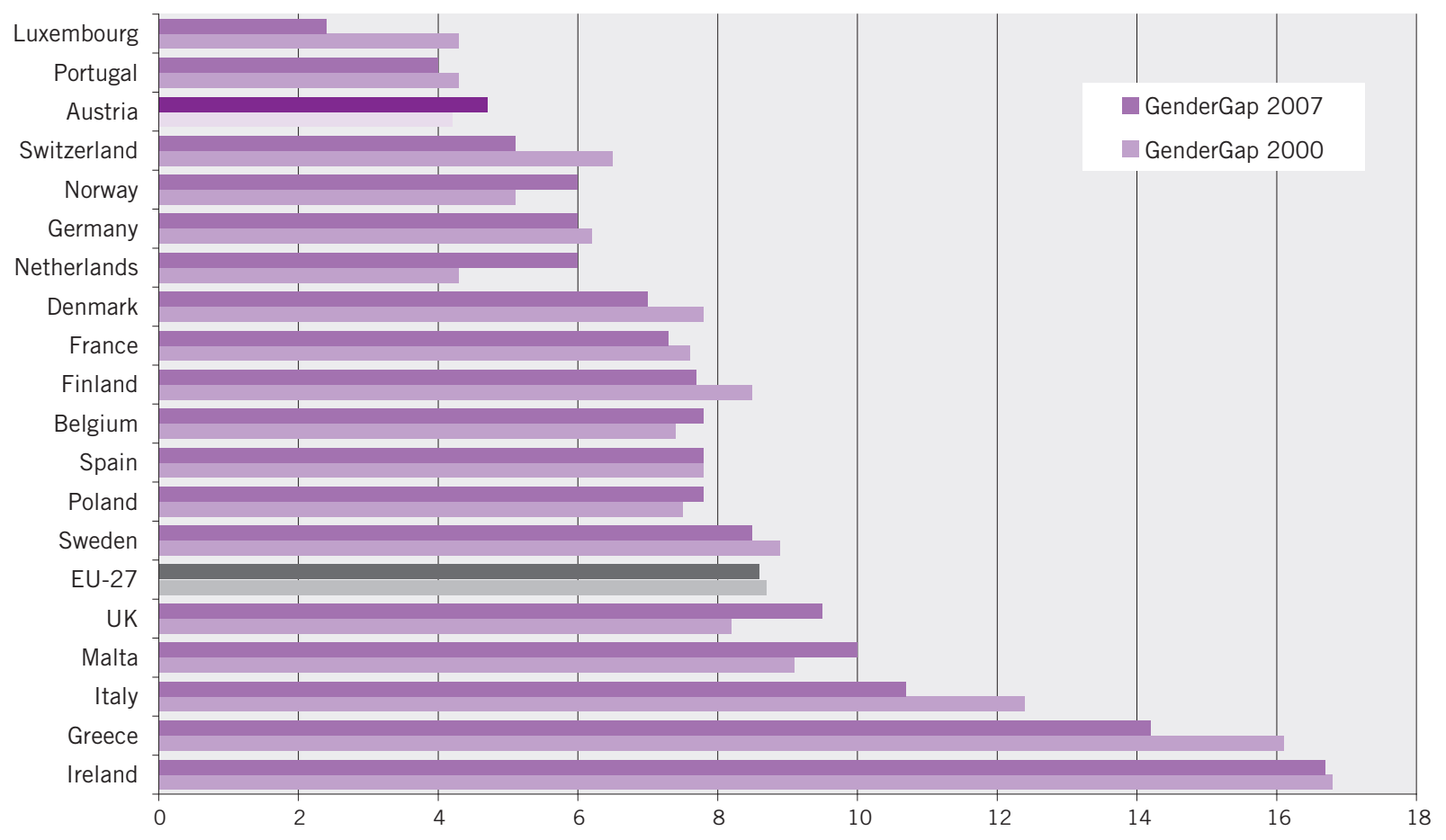

Quelle: Eurostat, http://epp.eurostat.ec.europa.eu/cache/ITY OFFPUB/KS-SF-08-099/EN/KS-SF-08-099-EN.PDF

Im Hinblick auf die berufliche Stellung selbstständiger Frauen zeigt sich ein Rückgang des Frauenanteils bei den Mithelfenden, der vor allem im landwirtschaftlichen Bereich sehr deutlich ausfällt. Zugenommen hat der Anteil selbstständiger Frauen in Handel, Gewerbe und Industrie, insbesondere bei mittleren und größeren Betrieben. 
Tabelle 43: Anteil selbstständiger Frauen, nach beruflicher Stellung, 1990, 2000 und 2006

\begin{tabular}{|l|r|r|c|}
\hline Berufliche Stellung & $\mathbf{1 9 9 0}$ & $\mathbf{2 0 0 0}$ & $\mathbf{2 0 0 6}$ \\
\hline Selbstständige und Mithelfende gesamt & Frauen in \%* & Frauen in \% & Frauen in \% \\
\hline Selbstständige in Landwirtschaft & 43,2 & 40,6 & 38,0 \\
\hline Mithelfende in der Landwirtschaft & 36,2 & 42,1 & 44,5 \\
\hline Selbstständige in Handel, Gewerbe und Industrie & 77,9 & 64,4 & 61,9 \\
\hline Kleinbetrieb ohne ArbeitnehmerInnen & 28,5 & 28,3 & 31,4 \\
\hline Kleinerer Betrieb & 42,0 & 33,1 & 37,6 \\
\hline Mittlerer Betrieb & 28,1 & 29,8 & 29,9 \\
\hline Größerer Betrieb & 18,1 & 20,5 & 24,9 \\
\hline Mithelfende in Handel, Gewerbe und Industrie & 10,9 & 13,5 & 14,7 \\
\hline
\end{tabular}

* Anteil an selbstständig Erwerbstätigen gesamt

Quelle: Statistik Austria, Mikrozensus

Die Land- und Forstwirtschaft ist traditionell die Branche mit den höchsten absoluten Selbstständigenzahlen (2007: 120.670), sowohl bei Frauen wie auch bei Männern, gefolgt von wirtschaftsbezogenen Dienstleistungen (72.764), Handel und Reparatur (61.230) sowie dem Beherbergungs- und Gaststättenwesen (47.363). Ingesamt arbeiten $64 \%$ aller selbstständig erwerbstätigen Frauen und $57 \%$ aller selbstständig erwerbstätigen Männer in diesen Branchen.

Abbildung 58: Selbstständige Personen nach Branchen u. Geschlecht, Frauenanteil in \%, 2007

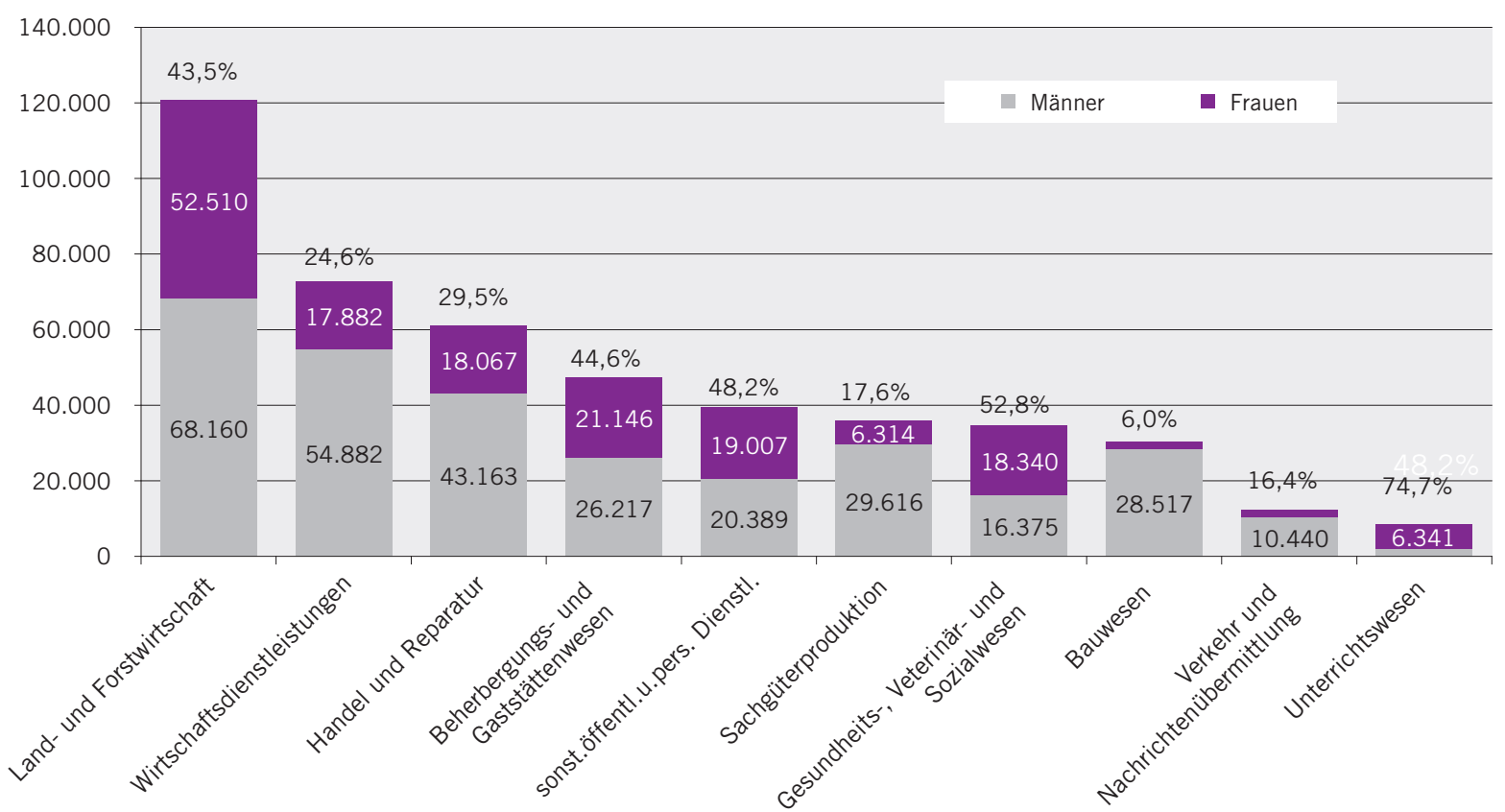

Quelle: Statistik Austria, Mikrozensus 
Werden Frauen- und Männeranteile innerhalb der einzelnen Branchen betrachtet, so zeigt sich, dass selbstständige Frauen ihren unternehmerischen Schwerpunkt häufiger im Unterrichts-, Gesundheits- und Sozialwesen, im Gastgewebe und in den sonstigen Dienstleistungen setzen. In all diesen Wirtschaftsbereichen liegen die Frauenanteile jeweils deutlich über dem Durchschnitt $(33,8$ \%). In der Produktion ebenso wie im Bauwesen sind dagegen vergleichsweise wenige Frauen selbstständig tätig. Gemeinsam mit dem Verkehrsbereich sowie den wirtschaftsbezogenen Dienstleistungen stellen sie Branchen mit einem überdurchschnittlichen Anteil selbstständig erwerbstätiger Männer dar.
Ein ähnliches Bild zeigt auch die Mitgliederstatistik der Wirtschaftskammer Österreich. Im Jahr 2007 waren von rd. 423.324 Spartenmitgliedern (natürliche Personen) rd. 130.538 bzw. 30,8 \% weiblich. Somit ist fast jedes dritte Kammermitglied und damit jede/r dritte UnternehmerIn der gewerblichen Wirtschaft eine Frau. Auch in den zwei größten Sparten (Gewerbe/Handwerk und Handel) sind jeweils rund $30 \%$ der Selbstständigen Frauen. Den höchsten Frauenanteil - mit über 40 \% - weist die Sparte Tourismus und Freizeitwirtschaft auf. Die drittgrößte Sparte Information und Consulting wird hingegen zu fast $80 \%$ von Männern dominiert.

\section{Abbildung 59: Sparten-Mitglieder WKÖ*, nach Geschlecht, Frauenanteil in \%, 2001 und 2007}

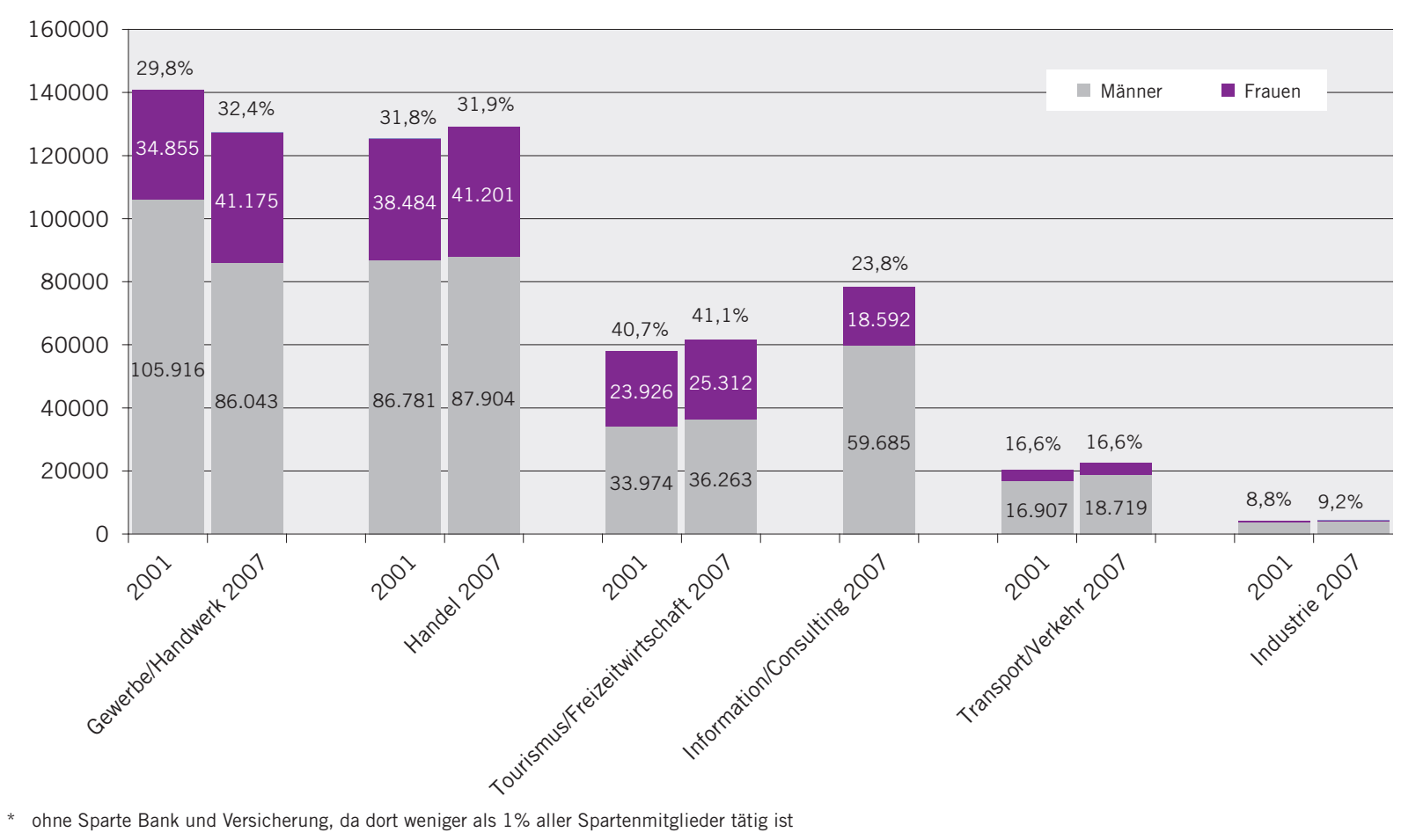

Quelle: Wirtschaftskammer Österreich, Mitgliederstatistik 
Diese Branchenverteilung spiegelt sich in den beruflichen Tätigkeiten Selbstständiger wider. So ist der Frauenanteil unter den Selbstständigen in handwerklichen und technischen Berufen deutlich niedriger als in kaufmännischen oder dienstleistungsorientierten Berufen. Für einzelne technische Berufe in Wien haben Reidl et al. (2006) die im Vergleich zu Männern geringe Selbstständigenquote von Frauen aufgezeigt: So machen Frauen mehr als 30 \% der unselbstständig Beschäftigten im IT-Bereich und der Architektur, aber nur knapp über $10 \%$ der Betriebsinhabenden aus.

\subsubsection{Frauen gründen}

Betrachtet man die Entwicklung der österreichischen Neugründungen in der gewerblichen
Wirtschaft über die letzte Dekade, so ist eine positive Gründungsdynamik sowohl allgemein als auch bei von Frauen neugegründeten Unternehmen festzustellen. Der Frauenanteil an den Gründungen ist seit 1993 ebenso deutlich gestiegen (+13\%-Punkte zwischen 1993 und 2007) wie die absolute Anzahl der gegründeten Unternehmen $\mid+15.000$ Neugründungen). Im Jahr 2007 lag der Frauenanteil unter neugegründeten Einzelunternehmen bei fast 40 \% und ist damit höher als der Frauenanteil unter den WKÖ-Spartenmitgliedern: Knapp 10.000 Unternehmen wurden 2007 von Frauen gegründet, im Vergleich zu 1994 hat sich die Zahl der Neugründungen durch Frauen mehr als verdreifacht. Ein Anstieg, der stärker ausfällt als die Zunahme von Neugründungen durch WKÖ-Mitglieder insgesamt.

\section{Abbildung 60: Entwicklung der österreichischen Unternehmensneugründungen*, nach Geschlecht, 1993 - 2005, absolut und Frauenanteil in \%}

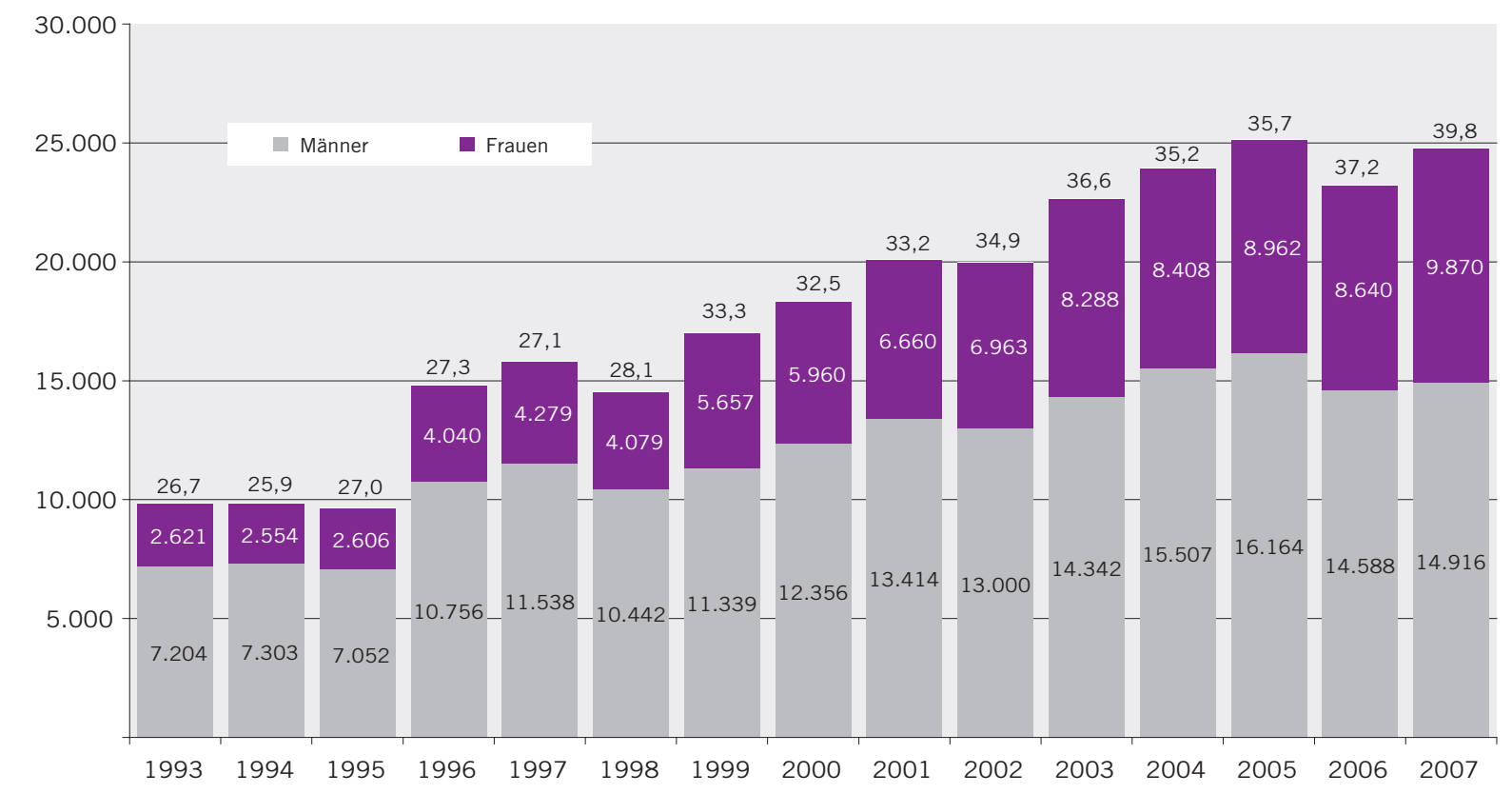

* Es werden die Neugründungen der Einzelunternehmen dargestellt, da nur für diese Unternehmen geschlechtsspezifische Aussagen getätigt werden können. Die Einzelunternehmen stellen rd. 80\% aller Unternehmensneugründungen der gewerblichen Wirtschaft dar.

Quelle: Wirtschaftskammer Österreich, Neugründungsstatistik 2007 
Die höchsten Frauenanteile unter den Gründungspersonen der gewerblichen Wirtschaft finden sich in den traditionell von Frauen dominierten Branchen. Die Betrachtung der zwanzig am stärksten besetzten Branchen zeigt die höchsten Frauenanteile im Bereich öffentliche und private Dienstleistungen (in den Fachgruppen FriseurInnen, FußpflegerInnen, KosmetikerInnen und MasseurInnen, 85,9 \%), im Direktvertrieb (66,2 \%) und dem allgemeinen Fachverband des Gewerbes (54,0 \%). Viele Gründungen von Frauen gibt es zudem im Druckgewerbe sowie im Textil- und Schuhhandel. Rd. ein Viertel aller neugegründeten Unternehmen von Frauen konzentriert sich in diesen Fachgruppen. Diese ausgeprägten geschlechterspezifischen Unterschiede in der Branchenwahl wurden in einer aktuellen Studie von Lueger et al. (2007) auch als erschwerende Faktoren der Unternehmensgründung, v. a. für die Ressourcenausstattung, identifiziert. Wie zu Beginn ausgeführt, sind die Daten der Wirtschaftskammer die ausführlichsten in Österreich verfügbaren, auf Basis derer auch ein Vergleich über die Zeit möglich ist. Sie erlauben aber keine exakte Bestimmung der Zahl der Unternehmensneugründungen oder der von Frauen gegründeten Unternehmen in Österreich. In den letzten Jahren haben sich verschiedene empirische Studien mit dem österreichischen Gründungsgeschehen sowie dem Frauenanteil unter den Gründerpersonen beschäftigt: Die Geschlechterverteilung hinsichtlich Ausmaß und Art der Unternehmensgründungen zeigt dabei ein sehr traditionelles Bild: Der Frauenanteil in der von Schwarz und Grieshuber (2003) untersuchten Gründungskohorte des Jahres 1999 lag bei rund einem Viertel $(25,1 \%)$. Eine Längsschnittanalyse der Teilnahmen am Unternehmens-Gründungsprogramm (UGP) des AMS errechnete einen durchschnittlichen Frauenanteil von $31 \%$ für den Zeitraum 1998-2005 (Dornmayer und Lenger, 2006). Eine aktuelle Erhebung von
Statistik Austria (2007) untersucht Unternehmen, die 2002 gegründet und 2005 noch am Markt aktiv waren. Der Frauenanteil unter den Gründungspersonen beträgt hier 27,2 \%. Der aktuelle GEM-Länderbericht (Apfelthaler et al., 2008) für das Jahr 2007 weist einen Frauenanteil an Unternehmensgründungen von sogar 38,1 \% aus. Die Schwankungsbreite in Frauen- (und Männer-)Anteilen beträgt somit knapp 15\%-Punkte, was eine präzise Aussage zur Gründungsintensität von Frauen und Männern erschwert und nicht zuletzt ein Hinweis auf das Fehlen einer genuinen Gründungsstatistik in Österreich (vgl. Schwarz et al., 2005) ist.

Im Hinblick auf die Altersverteilung zeigen sich ebenfalls Unterschiede zwischen Frauen und Männern: In den Studien von Schwarz und Grieshuber (2003) und Schwarz (2006) wie auch im GEM-Länderbericht 2007 für Österreich sind weibliche Gründerinnen tendenziell etwas älter als männliche Gründer: Frauen sind in der Altersgruppe der 45- bis 54-Jährigen am häufigsten vertreten (38\%), in der Gruppe der 35- bis 44-Jährigen ist der Frauenanteil (21\%) im Vergleich zu dem der Männer (32\%) deutlich niedriger.

Tabelle 44: Altersverteilung UnternehmensgründerInnen nach Geschlecht, Anteile in \%, 2007

\begin{tabular}{|l|r|r|}
\hline & Frauen & Männer \\
\hline 18-24 Jahre & $16 \%$ & $7 \%$ \\
\hline 25-34 Jahre & $19 \%$ & $14 \%$ \\
\hline 35-44 Jahre & $21 \%$ & $32 \%$ \\
\hline 45-54 Jahre & $38 \%$ & $33 \%$ \\
\hline 55-64 Jahre & $6 \%$ & $13 \%$ \\
\hline
\end{tabular}

Quelle: Apfelthaler G. et al. (2008), Global Entrepreneurship Monitor: Bericht 2007, S. 18

Schwarz (2006) weist darauf hin, dass sich dieser Altersunterschied vor allem auf karenzund betreuungsbedingte Unterbrechungen von Frauen zurückführen lässt, die die Unternehmensgründung von Frauen zeitlich etwas 
nach hinten verschieben. Anders als der GEMLänderbericht kommt Schwarz (2006) in ihrer Online-Befragung von UnternehmensgründerInnen zu dem Ergebnis, dass Unternehmer jünger als die Unternehmerinnen sind: 12,2 \% der Unternehmer waren zum Zeitpunkt der Befragung unter 30 Jahren während sich dieser Anteil bei Frauen nur auf 7,7 \% beläuft.

Gründerinnen unterscheiden sich nicht nur im Alter, sondern auch hinsichtlich ihres Bildungsniveaus von ihren männlichen Kollegen. Grundsätzlich zeigen Analysen des Grün- dungsgeschehens in der gewerblichen Wirtschaft eine deutliche Dominanz von Unternehmensgründungen aus Lehrberufen. Werden die Verhältniszahlen weiblicher zu männlichen Gründerpersonen in Bezug auf den höchsten Bildungsabschluss betrachtet, verfügen Gründerinnen tendenziell über ein niedrigeres Bildungsniveau als Gründer (Schwarz 2006, S. 26). Im mittleren Ausbildungsbereich sind Frauen etwas häufiger, im Bereich Lehre/Meisterprüfung, Matura und Universitätsstudium hingegen seltener vertreten als Männer.

\section{Abbildung 61: Höchste abgeschlossene Ausbildung von UnternehmensgründerInnen* nach Geschlecht, Anteile in \%, 2005}

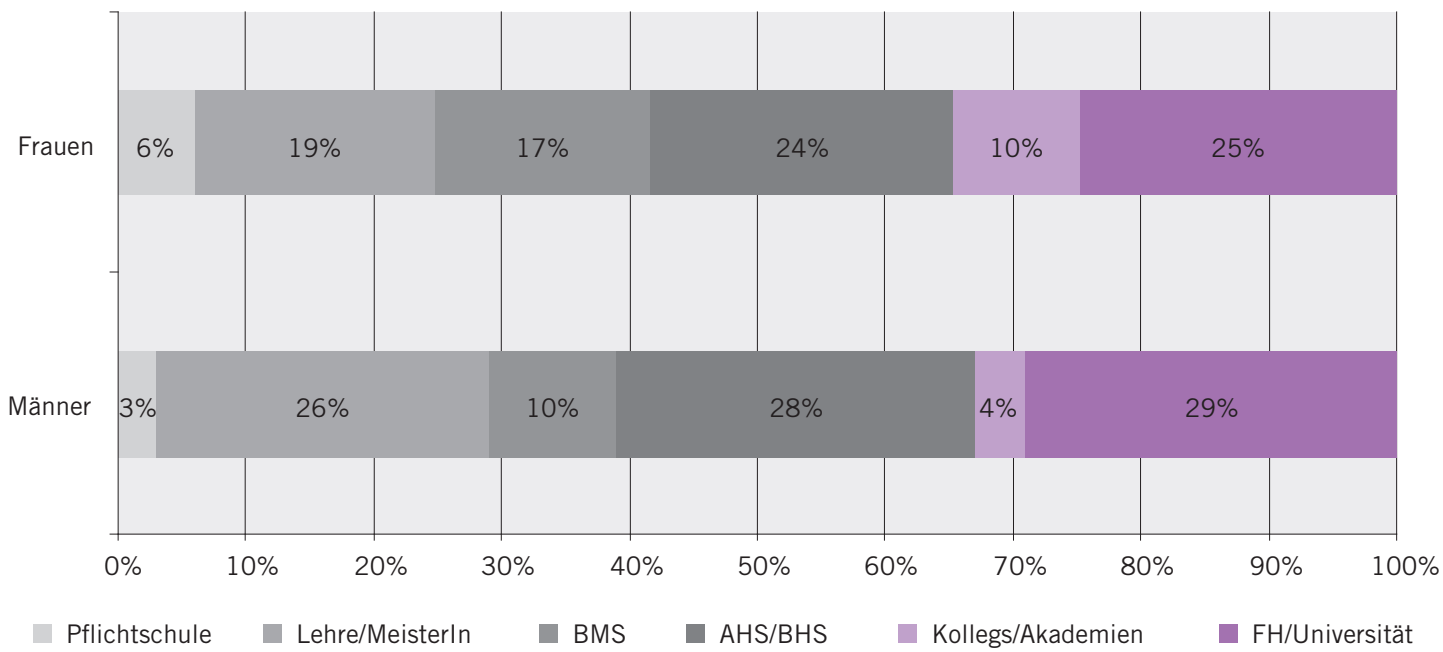

* österreichweite Online-Befragung von UnternehmensgründerInnen im November 2005, Stichprobenumfang 1.276

Anmerkung: Unterschiede in der höchsten Ausbildungskategorie sind signifikant

Quelle: Schwarz 2006, S. 26-27

Im Vergleich zu allen weiblichen Erwerbstätigen weisen Gründerinnen jedoch höhere Bildungsabschlüsse auf (vgl. Heckl et al. 2005). Aufgrund des gestiegenen Bildungsniveaus haben sich die geschlechterspezifischen Unterschiede insgesamt verringert, die bestehenden Unterschiede in fachlicher Spezialisierung oder der Wahl des Ausbildungsberufes haben aber nach wie vor Auswirkungen auf die Gründungsentscheidung.
Im Durchschnitt gründen Frauen kleinere Betriebe als Männer und starten ihr Unternehmen großteils ohne VollzeitmitarbeiterInnen, als Einzelunternehmen mit einem deutlich niedrigeren Startkapitel bzw. auch Finanzressourcenbedarf als Männer. Dies in Kombination mit einer geschlechterspezifisch segregierten Branchenwahl schlägt sich auch in den unterschiedlichen Wachstumsraten nieder. Lueger et al. (2007) kommen in ihrer Studie zum 
Ergebnis, dass das durchschnittliche Wachstum von Unternehmen, die von Männern gegründet wurden, in den Beobachtungszeiträumen signifikant vom Wachstum von durch Frauen gegründeten Unternehmen abweicht. Während sich bei von Männern gegründeten Unternehmen ein durchschnittliches Gesamtwachstum von 2,8 MitarbeiterInnen ergibt, liegt dieser Wert bei von Frauen gegründeten Unternehmen nur bei 1,2 MitarbeiterInnen. Der Großteil dieses Wachstums bezieht sich dabei auf die erste Phase der Unternehmensgründung (in etwa die ersten 3 Jahre).

\subsubsection{Gründungen von Frauen in Forschung und Technologie}

In den vergangenen Jahren lässt sich für Österreich eine Zunahme der Zahl von Gründungen in forschungs- und technologieintensiven Branchen generell wie der Spinoff-Gründungen im Speziellen beobachten. Über spezifische Gründungen von Frauen in diesem Feld liegen bislang für Österreich keine systematischen Daten vor. Entsprechende Anhaltspunkte bzw. Richtwerte können daher nur aus exemplarischen Betrachtungen oder der Übertragung internationaler Erfahrungen generiert werden.

Im Rahmen einer Studie des ZEW zu HighTech-Gründungen in Deutschland (Metzger et al., 2008) wurden Frauengründungen im HighTech-Sektor beleuchtet. Datengrundlage der Untersuchung ist die ZEW-High-Tech-Befragung 2007. Dabei zeigte sich, dass der Anteil der von Frauen gegründeten Unternehmen im High-Tech-Sektor knapp $10 \%$ beträgt und damit weniger als halb so hoch wie der Anteil von Frauen an Gründungen insgesamt ist. Das Branchenauswahlmuster der High-Tech-Gründerinnen ist dem außerhalb dieses Sektors sehr ähnlich: So finden Frauengründungen vor allem im Bereich der technologieorientierten Dienstleistungen $(66 \%)$ und seltener in der hochwertigen Technik und Spitzentechnik
$(12 \%)$ statt. Frauen gründen - gemessen am Umsatz - auch im High-Tech-Sektor kleinere und langsamer wachsende Unternehmen als Männer. Geschlechtsspezifische Unterschiede zeigen sich auch in der Finanzierung: Der Anteil der Unternehmen, die sich aus dem Cash Flow, also den laufenden Einnahmen des Unternehmens, finanziert haben, ist unter den Frauengründungen geringer als unter den Männergründungen. Eigenmittel werden von Frauen seltener, Mittel von Verwandten und Freunden dagegen häufiger in Anspruch genommen. Beim formalen Bildungsniveau gibt es wenig Unterschiede zwischen weiblichen und männlichen Gründungspersonen, sehr wohl jedoch im Hinblick auf die Kenntnisse, die das Gründungsteam vorwiegend besitzt Bei einem Großteil der Männergründungen (59 \%) ist technisches oder ingenieurwissenschaftliches Fachwissen im Gründungsteam vorherrschend. Frauengründungen weisen deutlich häufiger einen kaufmännischen Kompetenzschwerpunkt $(36 \%)$ als einen technischen oder ingenieurwissenschaftlichen (32\%) auf.

$\mathrm{Zu}$ vermuten ist, dass die Frauenanteile an High-Tech-Gründungen auch in Österreich ähnlich niedrig sein werden wie in Deutschland. Die Gründe dafür sind vielfältig und sowohl auf persönliche wie strukturelle und gesellschaftliche Einflussfaktoren zurückzuführen. Eine wesentliche Ursache für die geringe Präsenz von Frauen im High-Tech-Sektor und speziell in der forschungsintensiven Industrie liegt dabei in der geschlechterspezifisch segregierten Studien- und Berufswahl: Frauen sind in den technisch-ingenieurwissenschaftlichen Ausbildungen und Studiengängen unterrepräsentiert. Dies bedingt in weiterer Folge, dass sie weniger häufig in forschungs- und technologieintensiven Branchen beschäftigt sind. So beträgt der Frauenanteil unter den WissenschafterInnen und IngenieurInnen im F\&E-Bereich im Jahr 2006 in Österreich knapp 24 \%. Auswertungen der Volkszählung 2001 errechnen 
Frauenanteile in technikorientierten Berufen zwischen $14 \%$ bei InformatikerInnen, Datenverarbeitungsfachkräften oder IngenieurInnen und $38 \%$ bei MathematikerInnen, StatistikerInnen und BiowissenschafterInen. Für Metzger et al. (2008) schlagen sich diese geschlechterspezifischen Bildungs- und Beschäftigungsunterschiede direkt in der Qualifikation der GründerInnen von High-Tech-Gründungen nieder. Über HochschulabsolventInnen mit Diplom oder vergleichbarem Abschluss im Gründungsteam verfügen Männer- wie Frauengründungen gleich häufig. Allerdings haben Männergründungen doppelt so häufig ein promoviertes Gründungsmitglied und können häufiger auf Erfahrungen einer Gründerperson aus der Tätigkeit an einer wissenschaftlichen Einrichtung zurückgreifen. Aufgrund ihrer geringeren Verankerung in diesen Bereichen verfügen Frauen damit seltener über Erfahrungen aus einer Tätigkeit in der wissenschaftlichen Forschung, die häufig den Impuls und die Voraussetzungen für eine High-Tech-Gründung schafft.

Die Gründe für die schwach ausgeprägte Gründungsaktivität von Frauen im HighTech-Sektor sind jedoch nicht allein in der Berufs- und Studienwahl von Frauen zu finden. Bei Überlegungen von Frauen, eine Gründung in forschungs- und technologieintensiven Branchen anzustreben, werden häufig mehrdimensionale Barrieren deutlich. Geschlechterspezifische Zuschreibungen im Technikfeld zeigen dabei ebenso ihre Auswirkungen wie Gründungsstereotype. Frauen, die bspw. im High-Tech-Sektor gründen, befinden sich nicht selten in einem Spannungsverhältnis zwischen UnternehmerInnentum und Technik, in welchem sich die unterschiedlichen Geschlechterkonstruktionen je nach Feld mehrfach und gegenseitig bedingen. So sind sowohl Gründungen wie Tätigkeiten in technologieintensiven Branchen traditionell männlich konnotiert. Frauen, die ein Unternehmen in technologieintensiven Branchen gründen, durchbrechen nach Hanappi-Egger et al. (2004) in zweifacher Weise die ihnen traditionell zugedachten Rollen: Zum einem reklamieren sie für sich die männlich kodierten Eigenschaftszuschreibungen von Technik und zum anderen verfügen sie als Unternehmerinnen über mehr Sichtbarkeit und möglicherweise auch Einfluss. Eine schwierige Ausgangssituation, die zum Teil noch dadurch erschwert wird, dass Frauen in einem technischen Beruf unter Umständen stärker durch ihre Frauen- als ihre Berufsrolle wahrgenommen werden.

Dieses Zusammenwirken struktureller, im Ausbildungs- und Berufsverlauf begründeter, sozialer, in Rollenbildern und Stereotypen konstituierter sowie individueller, in persönlichen Wünschen und Vorstellungen verankerter Barrieren erschwert nach wie vor berufliche Selbständigkeit und Unternehmensgründungen von Frauen, insbesondere in jenen Branchen und Bereichen, in denen sie unterrepräsentiert sind, auch wenn sich die Entwicklung der letzten Jahre sehr dynamisch gezeigt hat. So ist der Frauenanteil an den Neugründungen in den letzten Jahren um knapp 14 \%-Punkte angestiegen und im Jahr 2007 wurde bereits jedes dritte Unternehmen von einer Frau gegründet. Die bestehenden geschlechterspezifischen Unterschiede in der Branchenwahl wirken jedoch nicht nur auf die Gründung selbst, sondern haben auch Auswirkungen auf das Unternehmenswachstum. So verfügen Unternehmen in forschungs- und technologieintensiven Branchen tendenziell über höhere Wachstumspotenziale als bspw. der Bereich der persönlichen Dienstleistungen, in denen verstärkt Frauen ihre Unternehmen begründen. Insofern gilt es gerade in diesem Bereich, die Gründungsintensität von Frauen durch eine zielgruppenspezifische Ansprache oder gezielte Angebote $\mathrm{zu}$ erhöhen, um neue Potenziale zu erschließen und dadurch die unternehmerische Dynamik insgesamt zu fördern. Eine wichtige 
Voraussetzung ist die Verbesserung der empirischen Datengrundlagen und die Schaffung einer einheitlichen Gründungsstatistik bzw. eines Monitorings, um ein differenziertes Bild von GründerInnen in Österreich (persönliche
Charakteristika, strukturelle Merkmale etc.) und eine Aufschlüsselung in unterschiedliche Sektoren (z.B. low-/medium-/high-tech, wissensintensiv etc.) zu ermöglichen. 


\section{Evaluierung von Forschungs- und Technologiepolitik in Österreich}

Die Forschungs-, Technologie- und Innovationspolitik (FTI-Politik) hat in den letzten Jahren in Österreich und auch europaweit stark an Bedeutung gewonnen. Besonders der Lissabon-Prozess und die Barcelona-Ziele waren Ansporn dafür, dass FTI auf der politischen Agenda stark nach oben gerückt sind. Auch die mediale Berichterstattung ist erheblich gestiegen. Neben den nachweisbaren positiven Effekten für Wachstum und Beschäftigung ist es vor allem der politische Gestaltungsspielraum von FTI-spezifischen Maßnahmen, der zu einer Steigerung des politischen Interesses geführt hat. Damit und mit der Steigerung der Ausgaben der öffentlichen Hand wächst natürlich auch der Bedarf an Information, Legitimation, Lernen und Kontrolle. Evaluationen werden dafür eingesetzt, um über Erfolg und Misserfolg von Maßnahmen Auskunft zu geben, über die Zweckmäßigkeit der Mittelaufwendung für bestimmte Maßnahmen zu urteilen und Politik-Lernen im Sinne eines Verbesserns von Maßnahmen zu gewährleisten. Und dieser Einsatz ist gerechtfertigt, wenn man bedenkt, dass die öffentliche Hand 2008 über 2,7 Mrd. € für Forschung und technologische Entwicklung ausgegeben hat. Das folgende Kapitel hat daher zum Ziel, einen ausführlichen und umfassenden Überblick über die Entwicklungen in Österreich in den letzten Jahren zu geben und die kategoriale Vielfalt von Evaluierung in diesem Bereich zu beschreiben. Die Ausführungen basieren auf Zinöcker und Dinges (2009), Pichler (2009) und Polt (2009). In einem zweiten Teil - und dies soll zu einem fixen Bestandteil zukünftiger Forschungs- und Technologieberichte werden - werden rezente und relevant erscheinende Evaluierung vorgestellt und deren Hauptergebnisse kurz zusammengefasst.

\subsection{Kontext und Ratio}

Pichler (2009, S. 46) bringt den Einsatz und die Bedeutung von Evaluierung im Rahmen forschungs- und technologiepolitischer Entscheidungen auf den Punkt: Forschungs- und Technologiefinanzierung durch die öffentliche Hand „ist kein Beschaffungsvorgang“. Entscheidet im privaten Sektor der Marktmechanismus über den Erfolg von Investitionen, so bedarf es im öffentlichen Bereich sachgerechter Entscheidungsgrundlagen und spezifischen Wissens, um den Einsatz öffentlicher Mittel zu legitimieren. Daher haben die Politik als Geldgeber, Förderagenturen als Geldverteiler und Forschungseinrichtungen als Geldempfänger gleichermaßen Interesse an funktionierenden Evaluierungsstrukturen. Diese Interessen treffen zunächst bei Projektentscheidungen zusammen, wo Evaluierungen als Selektionsmechanismen bei der Projektauswahl eingesetzt werden.

Evaluierungen erfüllen aber auch eine bestimmte Funktion auf einem projektübergreifenden, programm- oder institutionenbezogenen Niveau, wenn Entscheidungen über neue Maßnahmen oder Einschätzungen über neue Entwicklungen getroffen und diese umgesetzt werden sollen.

Institutionell hat sich die in den letzten Jahren zu beobachtende "Evaluierungskultur" in der Schaffung der Plattform Forschungs- und Technologieevaluierung (fteval) niedergeschla- 
gen $^{87}$. Damit wurde in Österreich der Grundstein für den Anstieg von Evaluierungsaktivitäten gelegt, was sich daran messen lässt, dass im Zeitraum von 2003 bis 2007 über sechzig Evaluationen im Bereich der FTI-Politik durchgeführt wurden. Das ist für ein kleines Land wie Österreich nicht wenig und im Vergleich zu den Jahren zuvor ein bedeutender Anstieg.

Die Plattform ist ein 2006 aus einer 1996 gegründeten Arbeitsgemeinschaft hervorgegangener Verein, dessen Mitglieder vorwiegend die zuständigen Bundesministerien, Förderorganisationen und Forschungsinstitute sind. Die Mitglieder haben sich zur Einhaltung der von der Plattform beschlossenen „Standards der Evaluierung in der Forschungs- und Technologiepolitik" verpflichtet. In ganz allgemeiner Weise sollen damit die Aktivitäten der Plattform zur Steigerung der „Evaluationskultur" in Österreich unterstützt werden. Speziell dienen Standards dazu, sowohl den EvaluatorInnen als auch beauftragenden Einrichtungen und Evaluierten einen Rahmen und Verhaltensmaßgaben bereitzustellen. Damit wird ein höherer Grad an Verbindlichkeit und Sicherheit für alle Beteiligten erreicht.

Die Evaluierungsplattform ermöglicht somit einen Austausch zwischen allen betroffenen Akteuren im forschungs- und technologiepolitischen Prozess und stellt somit im Dreieck Politik - Förderagenturen - Forschung einen wichtigen Balancefaktor dar. Gleichzeitig führt diese Konstellation aber dazu, dass sich keine dieser Akteursebenen die Evaluierungsfunktion als Governance-Instrument überwiegend aneignen kann, womit aber auch die Akzeptanz von Evaluierung sichergestellt bzw. erhöht werden kann.

\subsection{Rechtlicher Rahmen für Evaluation}

Neben der Tätigkeit des Rechnungshofes, der Elemente einer Evaluierungsfunktion ausübt, gab es über lange Zeit keinen gesetzlichen Rahmen für Evaluation in Österreichs FTI-Politik. Die im Bereich der Förderung gestiegene Bedeutung von Evaluierung sowie die Entwicklungen auf Institutionenebene hatten auch legistische Auswirkungen auf die Evaluierungspraxis in den letzten Jahren. Maßgebliche Rechtsgrundlagen sind das Forschungs- und Technologieförderungsgesetz (FTF-G), das Gesetz zur Errichtung der Österreichischen Forschungsförderungsgesellschaft 2004 (FFG-G), das Forschungsorganisationsgesetz (FOG; Berichtswesen: $§ \S ~ 6-9)$ sowie die auf diesen Gesetzen basierenden Richtlinien zur Forschungsförderung ${ }^{88}$ und zur Förderung der wirtschaftlich-technischen Forschung und Technologieentwicklung, die sogenannten FTE-Richtlinien. ${ }^{89}$ Das FTF-G (§ 15 Abs. 2) normiert erstmals auf gesetzlicher Ebene die Evaluierungsgrundsätze als Mindesterfordernisse für die Richtlinien. Die Richtlinien sehen vor, dass „für alle auf den FTE-Richtlinien basierenden Förderungsprogrammen und -maßnahmen ein schriftliches Evaluierungskonzept $\mathrm{zu}$ erstellen ist, das den Zweck, die Ziele und die Verfahren sowie die Termine zur Überprüfung der Erreichung der Förderungsziele enthält und geeignete Indikatoren definiert. Zum Zweck der Erfassung der erforderlichen Informationen ist ein entsprechendes Monitoring aufzubauen" (Abschnitt 2.2., Seite 4). Evaluierungsfunktionen sind darüber hinaus in den durch die genannten Gesetze eingerichteten Förderungsinstitutionen FFG und FWF verankert, die dabei weitgehend unabhängig vorgehen können.

87 http://www.fteval.at

88 Richtlinien der Bundesregierung über die Gewährung und Durchführung von Förderungen gemäß §§ 10-12 FOG, BGBl. Nr. 341/1981

89 Richtlinien zur Förderung der wirtschaftlich-technischen Forschung und Technologieentwicklung (FTE-Richtlinien) gemäß § 11 Z 1 bis 5 des Forschungs- und Technologieförderungsgesetzes (FTFG) des Bundesministers für Verkehr, Innovation und Technologie vom 27.9.2006 (GZ 609.986/0013-III/I2/2006) und des Bundesministers für Wirtschaft und Arbeit vom 28.9.2006 (GZ 97.005/0012$\mathrm{C} 1 / 9 / 2006)$ 
Für Universitäten gab es 1997 eine erste detaillierte Evaluierungsverordnung im Rahmen des Universitätsorganisationsgesetz 1993. Heute wird im Universitätsgesetz 2002 den Universitäten vorgegeben, ein eigenes Qualitätsmanagementsystem aufzubauen, im Rahmen dessen Evaluationen des gesamten Leistungsspektrums der Universität durchzuführen sind (§ 14 Universitätsgesetz 2002).

Darüber hinaus hat der Rat für Forschung und Technologieentwicklung mittels einer Empfehlung die österreichische Bundesregierung dazu angehalten, bewusst mit dem Instrument Evaluation zu arbeiten und es aktiv in die Politikgestaltung einzubeziehen (Ratsempfehlung vom 12. April 2005).

\subsection{Projekte, Programme, Institutionen und das System}

Evaluierungen sind in der Erfüllung ihrer Funktion einem ständigen Entwicklungsprozess unterworfen. Die Berücksichtigung neuer theoretischer Ansätze sowie die Anwendung (bzw. Entwicklung) adäquater Methoden sind unabdingbar, will man die Wirkungsweise staatlicher Fördermaßnahmen entsprechend erfassen. Auch hier konnten in den letzten Jahren neue Akzente in inhaltlich/methodischer Hinsicht gesetzt werden, indem Methoden wie Logic Charts (Zinöcker et al. 2004), Logit-Probit-Analysen (Streicher et al. 2004), Matched Pairs (Pointner und Polt 2005) und Focus Groups erstmalig eingesetzt wurden. Die (möglichen positiven) Wirkungen von Evaluierungen werden auch dadurch unterstrichen, dass sich (einige) EntscheidungsträgerInnen in den Agenturen und Ministerien ernsthaft mit den Ergebnissen von Evaluation auseinander- setzen und Empfehlungen entweder aufgreifen oder begründet verwerfen. Beispiele hierfür:

- Der Umgang der Politik mit den Evaluationsergebnissen (Arnold et al. 2004) zu den Förderorganisationen Fonds zur Förderung der wissenschaftlichen Forschung (FWF) und des Forschungsförderungsfonds für die gewerbliche Wirtschaft (FFF). Diese Evaluierung wurde ergänzt durch eine Prüfung durch den Rechnungshof. Zwischen beiden Untersuchungen bestand ein gewisses Konkurrenzverhältnis, das sich auch in unterschiedlichen Ergebnissen und Empfehlungen niederschlug. So empfahl der Rechnungshof die Zusammenlegung von FWF und FFF, während die internationale Evaluierung davon ausdrücklich abriet; die Politik orientierte sich dabei an der Evaluierung.

- Der Umgang der Nachfolgeorganisation des FFF, der Forschungsförderungsgesellschaft (FFG), und des Wissenschaftsfonds FWF mit den Ergebnissen der jeweiligen Institutionenevaluierung 2004 (vgl. Binder 2005; Novak 2005).

- Das Interesse in Ministerien und Agenturen an Evaluation: So haben sich ab 2004 über 140 Personen (klassische "AnwenderInnen" von Evaluation) im Rahmen von Workshops, die von der Plattform Forschungs- und Technologieevaluierung angeboten wurden, beteiligt (Zinöcker 2004).

- Die Veranstaltung zweier bedeutender internationaler Konferenzen zu Evaluation im Bereich Forschungs- und Technologiepolitik in den Jahren 2003 und 2006: Eine gut genutzte Möglichkeit für österreichische Akteure, sich über den internationalen Stand der Diskussion in Kenntnis zu setzen..$^{90}$

90 Evaluation of Government Funded Activities in R\&D, May 2003. New Frontiers in Evaluation, April 2006. Dokumentationen dieser Konferenzen sind unter www.fteval.at/03conference03 und www.fteval.at/conference06 zugänglich. 


\section{Evaluation auf Projektebene}

Evaluationen auf Projektebene im Sinne einer Auswahl von Projektanträgen sind über weite Strecken avanciert und ausgereift. Der österreichische Wissenschaftsfonds FWF bedient sich seit Jahren eines erprobten Systems von internationalem Peer Review. Hier ist anzumerken, dass gerade diese Evaluierungsmethode in Bezug auf mögliche Befangen- und Voreingenommenheiten einer harschen Kritik ausgesetzt, aber unter ForscherInnen (Arnold et al. 2004), nicht nur in Österreich, weitgehend unumstritten ist (vgl. hierzu Dinges 2006). Die Evaluation des Fonds (Streicher et al. 2004) hat den professionellen Einsatz der Methode zur Projektselektion nachgezeichnet und auch gezeigt, dass keine Unstimmigkeiten (etwa: „Ältere Einreicher werden jüngeren gegenüber bevorzugt") nachweisbar sind.

Für den Bereich der unternehmensnahen Projektforschung gilt Ähnliches: Hier hat die Evaluation (Jörg und Falk 2004) die Professionalität der abwickelnden Stelle FFF $^{91}$ klar nachgezeichnet, jedoch auch auf Probleme der Risikobewertung in der Projektselektion aufmerksam gemacht.

Daneben gibt es eine Reihe von Programmen, die immer mehr danach trachten, die Fördervergabe von Projekten fair und transparent $\mathrm{zu}$ gestalten. Sehr oft werden hierzu Formen von Peer Review (etwa expert panels, extended Peer Review $)^{92}$ herangezogen, Wettbewerbe organisiert, Jurys gebildet. Wichtig ist hierbei die Zusammenarbeit mit der Evaluation auf Programmebene, die eben diese Form der Vergabe von Projekten innerhalb eines solchen Programms klar nachvollziehen und nachzeichnen sollte. Verschiedene Programme (die Sonderforschungsbereiche des FWF, die Kompetenzzentren der FFG, u.ä.) sehen Zwischen- und Endevaluationen ihrer ,Projekte' explizit vor, des Weiteren evaluiert die FFG die Projekte ihres Basisprogramms regelmäßig ex post (Sheikh 2005).

Davon gesondert sollte die Auftragsforschung in den Ministerien gesehen werden: Mit der steigenden Bedeutung von FTI-Programmen seit Anfang der 1990er und den schwierigen budgetären Rahmenbedingungen der Ministerien ist die Auftragsforschung in den Hintergrund getreten. Dennoch gab es noch 2002 über 750 Projekte mit einer Dotierung von etwa 35 Mio. $€$, die auf diesem Wege entstanden sind (Zinöcker und Dinges 2004).

\section{Evaluation auf Programmebene}

In fast allen Programmen wird heute mit Evaluationen gearbeitet. Dies ist maßgeblich auf die Rolle des Rates für Forschung und Technologieentwicklung zurückzuführen, der diese Evaluationen mit Nachdruck eingefordert hat. Als besonders breit diskutierte Beispiele für Programmevaluationen können das Kompetenzzentren-Assessment (Edler et al. 2003) oder die Evaluation des TechnologietransferProgramms (Jörg et al. 2000) gelten, deren Empfehlungen klare Auswirkungen auf die Weiterentwicklung dieser Programme hatten. Im Bereich der Programmevaluationen hatten zwei Auftraggeber, die sehr früh von der Notwendigkeit von Evaluation überzeugt waren, Vorreiterrollen: Erstens das Ministerium für Verkehr, Innovation und Technologie ${ }^{93}$, das Evaluationen jener Programme durchführen ließ, die im Rahmen des (heute nicht mehr existenten) Innovations- und Technologiefonds in-

\footnotetext{
91 Der FFF ist der Fonds zur Förderung der gewerblichen Wirtschaft (FFF), der 2004 Teil der Forschungsförderungsagentur FFG geworden ist.

$92 \mathrm{Zu}$ den methodischen Unterschieden siehe Rigby 2002 und 2004.

93 in einer Vielzahl von Ausformungen und Akronymen in den letzten sieben Legislaturperioden
} 
itiiert wurden (neben dem bmvit trat hier auch das BMWA in Erscheinung). Der zweite zentrale Auftraggeber war der ehemalige Fonds zur Förderung der gewerblichen Wirtschaft $(\mathrm{FFF})^{94}$, der systematisch alle in seinem institutionellen Rahmen etablierten Initiativen (Holzforschung, Mikrotechnik, Lebensmittelinitiative etc.) extern evaluieren ließ.

\section{Evaluation auf Ebene der Institutionen}

Im Bereich der Evaluation von Institutionen hat es mit Sicherheit in den letzten Jahren die größte Bewegung gegeben. Die zentralen Institutionen zur Förderung der grundlagennahen und der angewandten Forschung, FWF und FFF, wurden erstmalig in den vierzig Jahren ihres Bestehens 2004 evaluiert (Arnold et al. 2004). Andere Institutionen der Forschungsförderung, etwa die Christian Doppler Forschungsgesellschaft (Schibany et al. 2005) oder die Betreuungsstrukturen für das 6. EU Rahmenprogramm (Sheikh et al. 2004), zogen nach.

Grund für diese zunehmende Bedeutung der Evaluation von Institutionen war auch eine neue, professionellere Arbeitsaufteilung zwischen Ministerien und Agenturen: Die Programmabwicklung weg aus den Ministerien hin zu Förderagenturen auszulagern (Agenturifizierung) war eine zentrale Forderung in einer Vielzahl von Evaluationen und Studien, der schließlich auch von der Politik entsprochen wurde.

Das Verhältnis Ministerien zu Agenturen, das sich auch als Prinzipal-Agent-Problem darstellen lässt, wird wesentlichen Einfluss auf die Steuerung der österreichischen FTI-Politik haben. All die hier zum Einsatz kommenden Steuerungsmechanismen werden Themen zukünftiger Evaluationen sein.
Im Bereich der Evaluation von Forschungsinstitutionen gibt es auch eine klare Verbindung zu den Universitäten, die mit dem Universitätsgesetz 2002 in die Autonomie entlassen wurden und nunmehr durch Leistungsvereinbarungen an das Wissenschaftsministerium gebunden sind. Das UG 2002 verpflichtet die Universitäten, zur Qualitätsund Leistungssicherung in allen Bereichen ein eigenes Qualitätsmanagementsystem aufzubauen. Dies umfasst universitätsinterne Evaluierungen, externe Evaluierungen auf Veranlassung der Rektorate, der Universitätsräte bzw. des BMWF sowie personenbezogene Leistungsevaluierungen. Die Universitäten verpflichten sich in den Leistungsvereinbarungen mit dem BMWF zu Vorhaben der Qualitätssicherung und Evaluierung und diese sind somit wesentlicher Bestandteil der Leistungsvereinbarungen. Wie auch im Universitätsbericht $2008^{95}$ dargestellt, schreitet der Aufbau von Qualitätsmanagementsystemen an den Universitäten kontinuierlich voran, die Aktivitäten und Instrumente der Qualitätssicherung und Evaluation werden zunehmend systematisiert und sind gut verankert.

Relevante Informationen zu den österreichischen Universitäten etwa auch im Bereich der Qualitätssicherung und Evaluation werden in Form von Wissensbilanzen (Leitner 2007) und Leistungsberichten systematisch erfasst.

Mit der Österreichischen Qualitätssicherungsagentur (AQA) ist eine unabhängige Einrichtung für Qualitätssicherung, Evaluierung und Zertifizierung geschaffen worden, die dem gesamten Hochschulbereich zur Verfügung steht und von den wesentlichen Akteuren (Universitätenkonferenz, Fachhochschulkonferenz, HochschülerInnenschaft, BMWF) getragen wird. Die AQA hat verschiedene Ver-

94 Der FFF ist in die österreichische Forschungsförderungsgesellschaft FFG, Bereich Basisprogramme, übergegangen. 95 Vgl. Universitätsbericht 2008, Kap. 1.4 „Evaluierung und Qualitätssicherung“ 
fahren zur Qualitätssicherung entwickelt und bietet mit dem Quality Audit eine unabhängige Begutachtung und Zertifizierung des Qualitätsmanagementsystems von Universitäten, Fachhochschulen, Privatuniversitäten und Pädagogischen Hochschulen. Im Rahmen des AQA-Pilotprojektes zur Begleitung und Entwicklung von Qualitätsmanagementsystemen lassen sich zwei Universitäten im Leistungsbereich Forschung beim Aufbau ihres QM-Systems begleiten. ${ }^{96}$

Im Auftrag des BMWF wird derzeit eine Studie zur Evaluierung von Forschung an Universitäten erstellt, in der den Fragen nachgegangen wird, wie die Evaluierung von Forschung an österreichischen Universitäten funktioniert und welche Rolle sie im universitären Qualitätsmanagement spielt. Die Universitäten verstehen durchgängig die Evaluierung von Forschung als Teil ihres Qualitätsmanagements und nutzen sie vor allem als Instrument der Reflexion sowie zur Unterstützung ihrer Entwicklung, indem sie die Evaluierungsergebnisse als eine Grundlage für Planung und Entscheidungen verwenden. Viele Universitäten haben in den letzten Jahren bereits Strukturen und Prozesse für die Forschungsevaluierung eingerichtet, andere sind gerade dabei - in jedem Fall wird das damit verbundene institutional learning noch einige Jahre in Anspruch nehmen.

Die wichtigsten institutionellen VertreterInnen der außeruniversitären Forschung in Österreich (Austrian Research Centers, Joanneum Research), die keine unwesentliche Rolle in Österreichs FTI-Politik spielen, sind als Institutionen noch nie extern evaluiert worden. Dies ist bis zu einem gewissen Grad gerechtfertigt, da diese Forschungseinrichtungen nur zum Teil vom Staat basissubventioniert werden und sich ohnehin im Wettbewerb um ihre Finanzierung bemühen müssen.
Andererseits wurde bis dato etwa noch nie an EvaluatorInnen die Frage gestellt, ob etwa der (geringe) Anteil an Basissubvention richtig eingesetzt wird.

\section{Evaluation auf Politik- und Systemebene}

Dieser Bereich der Evaluation ist wohl auch international gesehen noch sehr unterentwickelt, wenngleich hier in Österreich im letzten Jahr wichtige Schritte gesetzt wurden. Herausragend ist wohl die vom BMVIT und BMWA (nunmehr BMWFJ) beauftragte Systemevaluierung der Forschungsförderung und -finanzierung (siehe Kapitel 2.1), deren Ziel es ist, die Forschungsförderung und -finanzierung in Hinblick auf die Leistungsfähigkeit des österreichischen Innovationssystems zu analysieren und allfälligen Handlungsbedarf zu seiner Verbesserung zu identifizieren. Gleichzeitig gab es auch andere diesbezügliche Aktivitäten, welche auf der Systemebene angesiedelt waren. Im Rahmen des von der Bundesregierung getragenen Forschungsdialogs (BMWF 2008; siehe Kapitel 2.2) wurde das Forum für einen breit angelegten Konsultations- und Diskussionsprozess gelegt. Schließlich beschäftigte sich auch ein Policy-Mix-Peer-Review-Team im Rahmen von CREST /Comité de la Recherche Scientifique et Technique ; Ausschuss für wissenschaftliche und technische Forschung der EU) mit dem österreichischen FTI-System und entwickelte spezifische Politikempfehlungen (CREST 2008; siehe Kapitel 2.2.1).

Gleichzeitig ist auf der Politik- und Systemebene auch der Übergang $z u$ anderen Berichten und Studien fließend: Eine ganze Anzahl von Arbeiten, die unter Umständen diesem Bereich zuzuordnen wären, trägt nicht die Bezeichnung „Evaluation" und hat auch keine explizite Evaluationsfunktion vorgesehen, 
enthält aber dennoch Elemente formativer oder summativer Evaluierungen. Hierzu gehören etwa die Forschungs- und Technologieberichte, die jährlich den Status von FTI(-Politik) abbilden, oder auch das Weißbuch des Wirtschaftsforschungsinstitutes WIFO, das klare Empfehlungen für die Weiterentwicklung der FTI-Politik in Österreich beinhaltet. Die Studie „Instrumente der Technologiepolitik und ihr Mix" von Schibany et al. (2005) hat wiederum zu einer nachhaltigen Diskussion darüber beigetragen, wie die einzelnen Instrumente der FTI-Politik zueinander stehen und wie ihr Einsatz optimiert werden könnte.

\section{Resümee}

Auch die Sicht von außen bestätigt, dass „Österreich nun eine der am besten entwickelten und eingebetteten Innovationsevaluierungskulturen in Europa" (CREST 2008, S. 19) besitzt. Eine entwickelte Evaluationskultur stellt einen zentralen Bestandteil einer lernenden und strategisch orientierten FTI-Politik dar. Eine gute Evaluationskultur ist gleichsam Voraussetzung und Folge einer guten, also effizienten, transparenten und fairen Politik. Gleichzeitig - und darauf weist das CREST Peer Review Team ausdrücklich hin - kann die Programmvielfalt und die daraus resultierende Menge an Evaluierungen auch zu einer „Paralyse durch Analyse" führen. Evaluierungen erfüllen nur dann ihren Zweck, wenn ihre Ergebnisse in künftige Politikformulierung und -praxis einfließen und entsprechende Mechanismen dies sicherstellen.

Der Überblick über die FTI-Politik und ihre Evaluation in Österreich zeugt von einem lebhaften Feld, in dem es trotz der bestehenden Aktivitäten an Herausforderungen für die $\mathrm{Zu}$ kunft nicht mangelt. Einige dieser Herausforderung sind beispielsweise:

Daten und Datenzugang: Neidvoll lässt sich aus Österreich beobachten, wie selbstverständ- lich Unternehmen in anderen Ländern (wie z.B. UK) Bilanzdaten und die Politik Förderdaten öffentlich zugänglich machen. In Österreich herrscht hier noch ein gewisses Tabu vor, wenngleich die amtliche Statistik mit gutem Beispiel vorangehen und den Zugang zu FTI-relevanten Datenbeständen erleichtern könnte, setzt der $\S 1$ des Bundesstatistikgesetzes 2000 (BStatG) doch das Ziel, „den Bundesorganen zur Planung, Entscheidungsvorbereitung und Kontrolle von Maßnahmen sowie der Wissenschaft, der Wirtschaft und der Öffentlichkeit" Daten bereitzustellen.

Generierung von Daten: Die Qualität von Evaluationen steht in unmittelbarem Zusammenhang mit der Qualität der Informationen (Daten), die im Programmverlauf gesammelt werden. Hier heißt es jedoch mit großer Sorgfalt vorzugehen: "Collect all data that is needed and need all data that is collected" ist ein schöner Leitsatz, der auch angesichts einer möglichen Überforderung der Evaluierten (siehe nächster Punkt) zu berücksichtigen sein wird.

Evaluationsmüdigkeit versus „Unter-Evaluation": Zu leicht kann man mit zu vielen Interviewwünschen, $\mathrm{zu}$ vielen (und $\mathrm{zu}$ langen) Fragebögen etc. eine „Evaluationsmüdigkeit" bewirken, die nur kontraproduktiv sein kann. Die Anzahl der Evaluationen auf Institutionsund Programmebene hat in den letzten Jahren massiv zugenommen. Gleichzeitig ist es unzureichend, dass zentrale Fördereinrichtungen so wie in Österreich mit FFF und FWF geschehen - nur einmal in vierzig Jahren evaluiert werden. Hier muss ein sinnvoller Rhythmus gefunden werden, in welchen Abständen langfristige Initiativen $\mathrm{zu}$ evaluieren sind.

Realistische Erwartungen: $\mathrm{Zu}$ oft werden in FTI-Programmen nachgerade homöopathische Budgetmittel eingesetzt, um Ziele wie ,Wirtschaftswachstum', ,Hebung des Lebensstandards' oder, Änderung des österreichischen Mindsets' zu erreichen. Hier ist es an der Zeit, 
realistischere Erwartungen an den Tag zu legen und Zielüberfrachtungen in Programmen $z u$ vermeiden. Ex post Evaluationen vergangener Programme und der gezielte Einsatz von ex ante Evaluationen im Rahmen der Programmgestaltung könnten hier einen wichtigen Beitrag leisten.

Offene Diskussion: Evaluierungen dienen auch dazu, die Sichtbarkeit von Programmen, Maßnahmen und Politikbereichen zu erhöhen, was leicht zu einer gefälligen Instrumentalisierung von Evaluierung führen kann. Daher trägt nicht nur die schiere Anzahl von bereits durchgeführten Evaluierung zu einer „Evaluierungskultur" bei, sondern vielmehr die umfassende Bereitschaft und das Interesse der Adressaten von Evaluierung, sich mit kritischen Empfehlungen und Einschätzungen auseinanderzusetzen und diese bereitwillig zu diskutieren.

\subsection{Die Ergebnisse ausgewählter Evaluierungen}

Die im Folgenden vorgestellten Evaluierungen wurden im Laufe des letzten Jahres durchgeführt und haben primär bundespolitische Relevanz. Ein weiteres Auswahlkriterium besteht darin, dass die Evaluierungen bereits abgeschlossen, auf der Homepage der Plattform Forschungsund Technologieevaluierung (http://www.fteval.at) veröffentlicht und zugänglich sind. Die Darstellung erfolgt nach keinem einheitlichen Schema, da Evaluierungen sehr spezifisch sind und sich in ihren Ausrichtungen, Inhalten und letztlich in ihrer jeweiligen Bedeutung unterscheiden.

\subsubsection{Zwischenevaluierung - AplusB Academia Business Spin-off Gründungen}

Zielsetzung der vorliegenden Zwischenevaluierung ${ }^{97}$ war es, den bisherigen Programmver- lauf von AplusB zu reflektieren, um auf dieser Basis Schlussfolgerungen und Empfehlungen für die Weiterentwicklung des Programms im Gesamtkontext des österreichischen Fördersystems zu entwickeln. Unter den mittlerweile zahlreichen Programmen im Bereich industry - science linkages verfolgt das 2001 durch das bmvit initiierte AplusB-Programm das Ziel, sowohl die Anzahl als auch den Erfolg von Gründungen aus dem Forschungsbereich nachhaltig zu steigern. Durch das AplusB Programm soll versucht werden, "Zentren zu schaffen, die WissenschafterInnen aus Universitäten, Fachhochschulen und Forschungseinrichtungen die Möglichkeit geben, sich durch Stimulierung, Qualifizierung und Unterstützung auf dem oft schwierigen Weg von einer guten Idee zu einem Unternehmen helfen und begleiten zu lassen. Dabei geht es nicht nur um konkrete Unterstützung im Gründungsprozess, sondern auch darum, Unternehmertum als Option im akademischen Denken und Handeln stärker zu verankern." (Sonderrichtlinie Juni 2001, S. 4)

Träger von AplusB-Zentren können nur Kapitalgesellschaften in der Form einer $\mathrm{GmbH}$ sein und es muss sichergestellt sein, dass mindestens eine akademische Einrichtung und mindestens eine Einrichtung mit ausgewiesenem Know-how Gesellschafter sind. Gleichzeitig ist sicherzustellen, dass mindestens $50 \%$ der Gesellschaftsanteile von Institutionen gehalten werden, die mit der Wahrnehmung öffentlicher Interessen betraut sind. Die Zentren haben somit große Freiheitsgrade bei der Gesellschafterwahl und können dementsprechend die regionalen Gegebenheiten vor Ort berücksichtigen.

Insgesamt steht hinter AplusB derzeit ein Gesamtbudget von rund 77 Mio. €. Hierbei ist zu berücksichtigen, dass die Laufzeit der

97 Heydebreck, P., K. Petersen (2008): Zwischenevaluierung - AplusB Academia Business Spin-off Gründerprogramm; inno, im Auftrag des bmvit, Karlsruhe. 
Förderung maximal 10 Jahre beträgt, wobei in dem jeweiligen Call zunächst ein Förderansuchen für die ersten 5 Jahre abgegeben wurde. Mit der Zwischenevaluierung der Zentren im
5. Jahr wird über die weitere Zukunft der Zentren entschieden. Bislang fiel diese „Stop-orGo"-Entscheidung im Falle der ersten 5 geförderten Zentren jeweils positiv aus.

\section{Tabelle 45: Gesamtbudget des AplusB Programms}

\begin{tabular}{|c|c|c|c|c|c|c|}
\hline & \multicolumn{2}{|c|}{ 1. Förderperiode } & \multicolumn{2}{|c|}{ 2. Förderperiode } & \multicolumn{2}{|c|}{ Gesamtsumme } \\
\hline genehmigtes Budget & in $€$ & $\%$ & in $€$ & $\%$ & in $€$ & $\%$ \\
\hline Gesamtbudget & 44.506 .333 & 100 & 32.535 .981 & 100 & 77.042 .314 & 100 \\
\hline Bundesförderung & 18.506 .010 & 42 & 10.384 .806 & 32 & 28.890 .816 & 37 \\
\hline Landesförderung & 15.391.020 & 35 & 9.789 .713 & 30 & 25.180 .733 & 33 \\
\hline Eigenmittel & 10.609.304 & 24 & 12.361 .462 & 38 & 22.970 .766 & 30 \\
\hline geplante Gründungsvorhaben & 316 & & 195 & & 511 & \\
\hline
\end{tabular}

Quelle: Heydebreck und Petersen (2008)

Wie Tabelle 45 zeigt, wird das Gesamtbudget aus verschiedenen Quellen gespeist. So verlangt die Sonderrichtlinie AplusB neben der Förderung des Bundes eine finanzielle Unterstützung des Landes sowie einen Eigenmittelanteil:

Der Bundesanteil kann in der ersten Förderperiode der Zentren maximal $45 \%$ der förderbaren Gesamtkosten bei einer Landesförderung von mindestens $35 \%$ sowie einem Eigenmittelanteil von mindestens $20 \%$ betragen.

Während der Jahre 6 bis 10 sinkt der Bundes- anteil auf durchschnittlich maximal $35 \%$ ab, wobei ein Eigenanteil von in der Regel $30 \%$ (mindestens aber $25 \%$ ) gefordert wird.

Insgesamt planen die Zentren mit diesen Mitteln 511 Gründungsvorhaben zu betreuen. Bis Mitte April 2008 haben die neun AplusB Zentren 236 Technologieprojekte initiiert, daraus sind bereits 171 Gründungen von Technologieunternehmen hervorgegangen und 144 Gründungsprojekte sind nach der Betreuung in den AplusB-Zentren mittlerweile am freien Markt tätig.

\section{Tabelle 46: Gründungsprojekte nach Zentren (Stand: 15.4.2008)}

\begin{tabular}{llcccc} 
Zentren & Bundesland & Gründerprojekte & ausgeschieden & im Zentrum & gegründet \\
\hline BUILD! & Kärnten & 29 & 21 & 8 & 26 \\
CAST & Tirol & 28 & 19 & 9 & 22 \\
INiTS & Wien & 64 & 44 & 20 & 50 \\
SPG & Steiermark & 34 & 24 & 10 & 21 \\
tech2b & Oberösterreich & 40 & 24 & 16 & 26 \\
ZAT & Steiermark & 17 & 10 & 7 & 8 \\
accent & Niederösterr. & 8 & 0 & 8 & 5 \\
BCCS & Salzburg & 10 & 2 & 6 & 3 \\
v-start & Vorarlberg & 6 & 0 & 13 & 5 \\
Gesamt & & 236 & & 92 & 171
\end{tabular}

Quelle: FFG, Heydebreck und Petersen (2008) 


\section{Empfehlungen der Evaluierung}

$\mathrm{Zu}$ den Good Practices zählt die Konzeption des AplusB-Programms: Aufgesetzt wurde ein insgesamt zielführendes Programm hinsichtlich Struktur, Inhalt, Maßnahmen, Zielsetzungen, Ressourcen und Zielruppen. Auch das Programmmanagement der Österreichischen Forschungsgesellschaft (FFG) zählt dazu; diese führt und füllt die Projektträgerfunktion adäquat und professionell aus. Dieses gilt gleichermaßen für das den Zentren vorgegebene Berichtswesen - Status- und Jahresberichte, Gründungsmonitoring - sowie die Zwischenevaluierungen der Zentren.

Verbesserungspotentiale und damit Handlungsempfehlungen werden in den folgende Aspekten gesehen: Die Zielgruppe WissenschafterInnen aus Hochschulen und Forschungseinrichtungen sollte geöffnet werden und die Gruppe derer mit akademischem Hintergrund und mehrjähriger Berufserfahrung. Die Aufenthaltsdauer von GründerInnen im Zentrum sollte eine Flexibilisierung erfahren. Die AplusB-Plattform sollte unbedingt intensiviert werden; neben der verbesserten Zusammenarbeit auf nationaler Ebene sollte der Erfahrungsaustausch auf internationaler Ebene ausgebaut werden. Die Vermarktung von AplusB sollte forciert werden im Sinne von Aufbau und Etablierung einer Dachmarke.

Im Hinblick auf AplusB-10+ kann und muss die Empfehlung lauten: Die AplusB-Zentren sollten ihre Arbeit ungehindert fortsetzen können, um die Anfangserfolge im Zuge der Zielsetzung Steigerung der österreichischen Gründungsdynamik weiter ausbauen zu können. Federführend sollte dieses Programm auch zukünftig auf Bundesebene - beim bmvit - angesiedelt sein, damit die erforderliche Kontinuität gewährleistet ist.

\subsubsection{Geistige Eigentumsrechte an den Hochschulen: Evaluierung des Programms uni:invent (2004 - 2006)}

Die im Auftrag des BMWF und BMWA beauftragte Evaluierung ${ }^{98}$ verfolgte das Ziel, einen Überblick über den Output sowie eine Einschätzung des bisherigen Programmverlaufs zu geben. Auf der rechtlichen Grundlage des UG 2002, welches den Universitäten neue Möglichkeiten der Nutzung und Verwertung universitärer Forschungsleistung eröffnete (§ 106), ermöglicht uni:invent durch gezielte Maßnahmen einen kulturellen Wandel an den Universitäten. Durch die Schaffung adäquater Strukturen (,IPR-Management ") sollen die kommerzielle Nutzung von Forschungsergebnissen erleichtert und wirtschaftliche Impulse gesetzt werden. Neben der Etablierung von ErfinderberaterInnen an den Hochschulen spielt auch die aws eine wesentliche und tragende Rolle in diesem Programm.

Die Evaluierung betont, dass Gesetze zwar notwendige, aber keine hinreichenden Grundlagen sind, um Forschungsergebnisse verstärkt einer wirtschaftlichen Umsetzung zuzuführen. Es bedarf flankierender Maßnahmen und einer entsprechenden Anschubfinanzierung, um das Thema IPRs (Intellectual Property Rights) an den Hochschulen nachhaltig $\mathrm{zu}$ etablieren. Das uni:invent Programm ist daher ein wichtiges und notwendiges Programm, das zum richtigen Zeitpunkt und mit einer entsprechenden Dotierung den Intentionen des Gesetzgebers Rechnung trägt.

Auf den untersuchten Zeitraum bezogen lassen sich die messbaren Outputs wie folgt zusammenfassen:

- Für den Zeitraum 2004 bis 2006 wurden 652 Erfindungsmeldungen von 405 ErfinderInnen eingereicht, wobei mit über $60 \%$ der

98 Schibany, A., G. Streicher, B. Nones (2008), Geistige Eigentumsrechte an Hochschulen: Evaluierung des Programms uni:invent; InTeReg Research Report Nr. 74-2008, Joanneum Research, Wien. 
Erfindungsmeldungen die medizinischen und technischen Universitäten den höchsten Anteil haben.

\section{Abbildung 62: Anzahl der Erfindungsmeldungen}

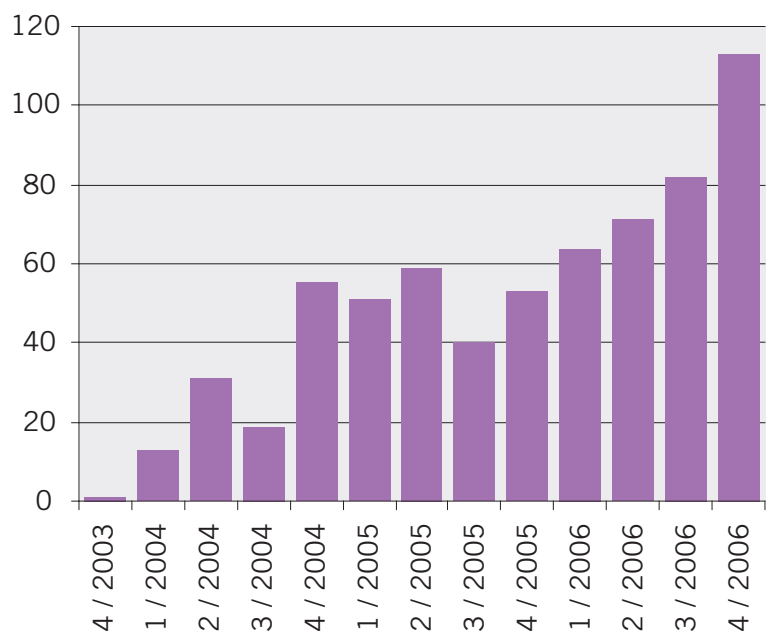

Quelle: Schibany et al. (2008)

- Mit fast einem Drittel aller Meldungen ist die Biotechnologie das deutlich wichtigste Technologiefeld, mit einigem Abstand gefolgt von chemischer Verfahrenstechnik und Physik (16 bzw. 13 \%).

- Aus der Gesamtheit aller Diensterfindungen wurden 281 Diensterfindungen von den Universitäten aufgegriffen.

- 195 dieser Diensterfindungen wurden als Patente angemeldet.
- Die bereits daraus erzielten Erlöse sind unterschiedlich bewertbar: von reinen Lizenzeinnahmen, welche ökonomisch als „Rentenabschöpfung" interpretiert werden können, bis hin zu Forschungskooperationen, welche durch bestehendes Eigentumsrecht induziert werden. Dementsprechend bewegen sich die bisherigen Erlöse zwischen 300 Tsd. $€$ bis zu einer Million $€$.

Den Erfolg des Programms einzig an den bislang erzielten monetären Erlösen dingfest zu machen, wäre zu kurz gegriffen und würde die bisherigen Maßnahmen in der Etablierung einer "Verwertungskultur" an den österreichischen Hochschulen unterschätzen. Denn neben den umfassenden Awareness- und Beratungsmaßnahmen - welche zum "Grundgeschäft" von uni:invent zählen und in unterschiedlicher Intensität und Schwerpunktsetzung beizubehalten sind - sind es vor allem sektorspezifische Charakteristika, welche den Verlauf einer Erlöskurve bestimmt. Im Life-Science-Bereich spielt beispielsweise der Zeitfaktor eine wesentliche Rolle, indem die Entwicklungsphase bis hin zum fertigen Produkt im Schnitt 10 Jahre oder länger dauern kann. Und bevor ein Produkt nicht vertrieben werden kann, fließen auch keine Erlöse. Die folgende Abbildung versucht diesen Sachverhalt zu exemplifizieren. 


\section{Abbildung 63: Eckpunkte des Programms und Erlöskurve}

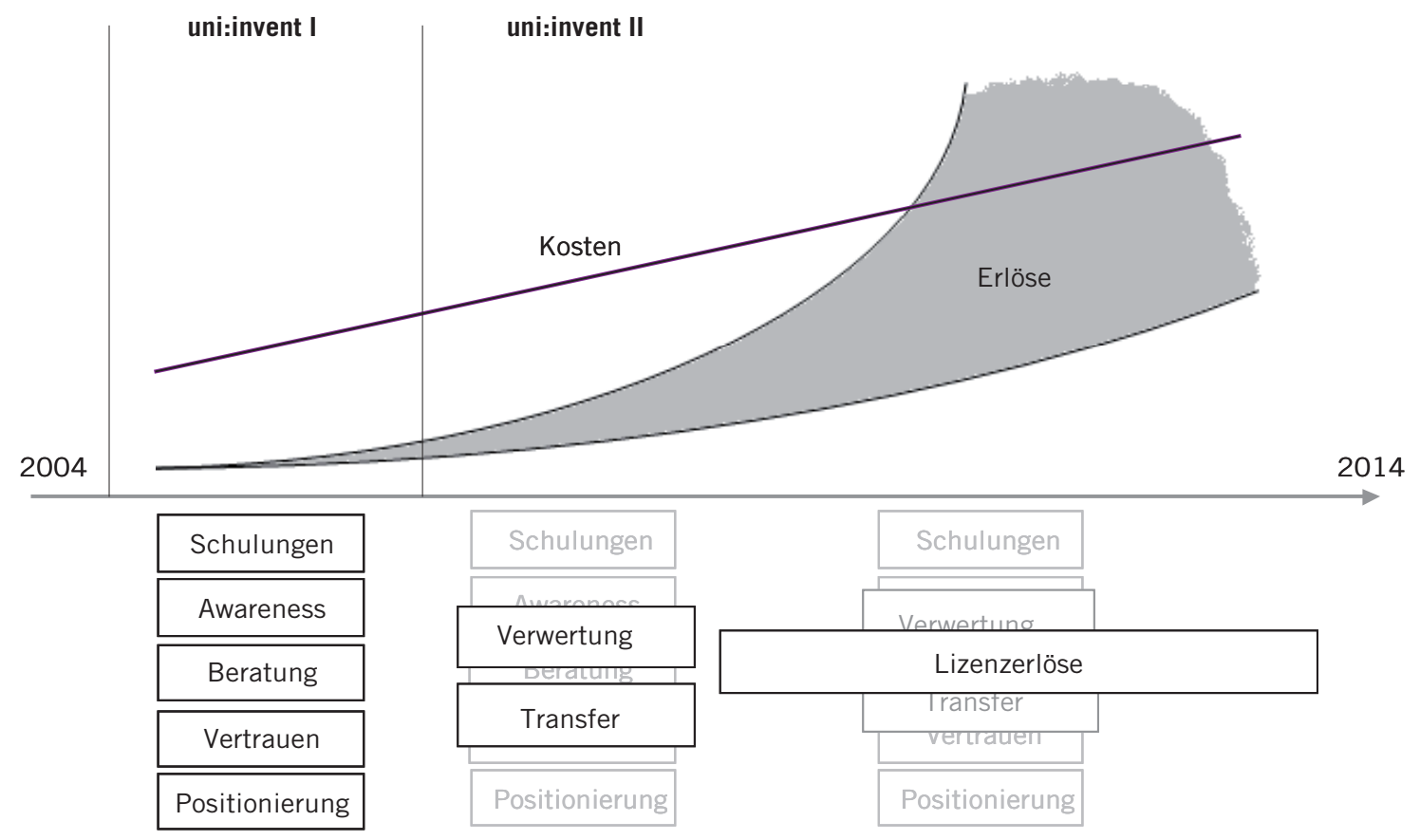

Quelle: Schibany et al. (2008)

Die Evaluierung betont in ihrem Resümee, dass eine Reihe von Förderprogrammen das Thema Wissens- und Technologietransfer an den Universitäten bereits etabliert hat. Die Programme zielen dabei in ihren jeweiligen Ausprägungen auf ein vorhandenes Potential an den Hochschulen und verfolgen unter- schiedliche Verwertungsoptionen. Dabei gilt es jedoch, funktionierende Schnittstellen zu schaffen, die den spezifischen Anforderungen der Universität angepasst sein müssen. Hier befinden sich die Universitäten noch in unterschiedlichen Entwicklungsstadien. 


\section{Referenzen}

Aghion, P., M. Dewatripont, C. Hoxby, A. Mas-Collel, A. Sapir (2007): "Why reform Europe's universities?", Bruegel Policy Brief 04/2007.

Aiginger, K. (Hrsg.) (1987): Die internationale Wettbewerbsfähigkeit Österreichs. Österreichische Strukturberichterstattung, WIFO, Wien.

Aiginger, K. et al. (2009), Endbericht der Systemevaluierung, Österreichisches Institut für Wirtschaftsforschung, Wien.

Aiginger, K., G. Tichy, E. Walterskirchen (2006): Weißbuch: Mehr Beschäftigung durch Wachstum auf Basis von Innovation und Qualifikation, WIFO, Wien.

Apfelthaler G., T. Schmalzer, U. Schneider, R. Wenzel (2008): Global Entrepreneurship Monitor: Bericht 2007 zur Lage des Unternehmertums in Österreich, FH Joanneum, Graz, http://gem.fhjoanneum.at/downloads/GEM_Austria_2007.pdf

arge creativ wirtschaft austria (2008), Dritter österreichischer Kreativwirtschaftsbericht, Wien.

Arnold, E., A. Schibany et al. (ed.) (2004): Evaluation of the Austrian Industrial Research Promotion Fund (FFF) and the Austrian Science Fund (FWF). Synthesis Report of Technopolis Ltd. in partnership with ETH, Joanneum Research, University of Twente and WIFO, Brighton -Wien: Eigenverlag.

Arrow, K. J. (1962): The Economic Implications of Learning by Doing, The Review of Economic Studies 3, pp.155-173.

Arundel, A., C. Bordoy, P. Mohnen, K. Smith (2008): Innovation Surveys and Policy: Lessons from the CIS, in: Nauwelaers, C., R. Wintjes, Innovation Policy In Europe - Measurement and Strategy, Cheltenham. pp. 3-28.

Astor, M., S. Heinrich und G. Klose(2009), Systemische Analyse des Innovations- und Interventionssystems, Prognos AG, Berlin.

Azoulay, P., W. Ding, T. Stuart (2006): "The Impact of Academic Patenting on the Rate, Quality and Direction of (Public) Research", Cambridge, NBER working paper 11917.

Bauwens, L., G. Mion, J.-F. Thisse (2008): "The resistible decline of European science", CEPR Discussion Paper No. 6625.

Belot, M., T. Hatton (2008): Immigrant Selection in the OECD, CEPR Discussion Paper 6675, London.
Berger, M. (2008): Micro data from Innovation studies, Bericht über die Teilnahme am gleichnamigen OECD Projekt im Auftrag des Bundesministeriums für Wirtschaft und Arbeit, Joanneum Research, Wien.

Biffl, G. et al. (2008): Migrantinnen und Migranten auf dem Wiener Arbeitsmarkt, Studie des WIFO und Lechner, Reiter \& Riesenfelder Sozialforschung OEG im Auftrag des Wiener ArbeitnehmerInnen Förderungsfonds (WAFF), Wien.

Biffl, G.( 2006): Small-Scale Study III: Conditions of entry and residence of third country highly-skilled workers in Austria, Study for the International Organisation for Migration Vienna in its function as the National Contact Point Austria within EMN, Wien.

Binder, M. (2005): The Austrian Research Promotion Agency (FFG). Plattform fteval Newsletter 25: 39-40

BMF und BMWA (2008), Österreichs steuerliche F\&E Förderung im Überblick, BMWA, Wien, 2008; download: http://www.bmwfj.gv.at/NR/ rdonlyres/15EC322B-9F71-46B1-A484-428BCC2D74FE/0/IndirekteForschungsf\% C3\%Bórderu ng_Homepage.pdf

BMVIT und BMWA (2007), Systemevaluierung der Forschungsförderung und -Finanzierung - Leitungsbild; download: http://www.bmvit.gv.at/en/ innovation/download/teilBleistungsbild.pdf

BMWF und BMVIT (2007), Österreichischer Forschungs- und Technologiebericht 2007, Wien.

BMWF (2008): Österreichischer Forschungsdialog: Ergebnisdokumentation, Bundesminister für Wissenschaft und Forschung, Wien; http://www. bmwf.gv.at/fileadmin/user_upload/forschung/ forschungsdialog/OESTERR_FORSCHUNGSDIALOG_Ergebnisdokumentation_0808bmwf.pdf und http://www.bmwf.gv.at/fileadmin/user_upload/ forschung/forschungsdialog/ZUKUNFTSbotschaften_des_FORSCHUNGSministers_0808bmwf.pdf

Breschi, S., F. Lissoni, F. Montobbio (2007): „The scientific productivity of academic inventors: New evidence from Italian data", Economics of Innovation and New Technology, 16, (101). 
Caloghirou, Y., S. Ioannides, N. Vonortas (2003): “Research Joint Ventures", Journal of Economic Surveys, 17(4): 541-70.

Carpenter, R.E., B. Petersen (2003): Capital Market Imperfections, High-Tech Investment, and the New Equity Financing; in: The Economic Journal, 2002, 112, p. 54-72

Chesbrough, H. (2003): Open Innovation. "The New Imperative for Creating and Profiting From Technology", Harvard University Press, Boston.

Chiswick, B. R., P.W. Miller (2007): The International Transferability of Human Capital Skills, IZA Discussion Paper 2670, IZA, Bonn.

Cohen, W. M., R. Nelson, J. Walsh (2000): "Protecting Their Intellectual Assets: Appropriability Conditions and Why U.S. Manufacturing Firms Patent (or Not)", NBER Working Paper 7552. Cambridge, Mass.

Crépon, B., E. Duguet, J. Mairesse (1998): Research and Development, Innovation and Productivity: An Econometric Analysis at the Firm Level, Economics of Innovation and New Technology 2, 115-158.

Crespo-Cuaresma, J., J. Wörz (2005): On Export Structure and Growth, Review of World Economics, 141 (1): 33-49.

CREST Expert Group Report (2008): "Policy Mix Peer Reviews: Country Report Austria", A Report of the CREST Policy Mix Expert Group, European Union Scientific and Technical Research Committee; http://www.bmwf.gv.at/fileadmin/user_ upload/forschung/forschungsdialog/CREST_Austrian_Policy_Mix_Report_-_September_2008.pdf

Criscuolo, C., J. Haskel (2002): Innovations and productivity growth in the UK, CeRiBa discussion paper.

David, P.A., Hall, B. H., Toole, A.A. (2000). Is Public R\&D a complement or substitute for private R\&D? A Review of Econometric Evidence, Research Policy 29, 497-529.

De Jong, J., Lokshin, B., Mohnen, P., Verhoeven, W. , 2007, Evaluatie WBSO: Effecten, doelgroep bereik en uitvoering, Onderzoek rapporten van Ministerie van Economische Zaken. Den Haag: Ministerie van Economische Zaken

Dinges, M. (2006): Pressure, Deception and Peer Review; Plattform fteval Newsletter 28: 11-22.

Dornmayer, H., B. Lenger (2006): Evaluierung der Maßnahmen der GründerInnenförderung des AMS. Wien: Institut für Bildungsforschung der Wirtschaft/AMS Österreich

Dosi, G., P. Llerena, M. Sylos Labini (2005): "The relationships between science, technologies and their industrial exploitation: An illustration through the myths and realities of the so-called 'European Paradox'", Research Policy, 35, (1450).

Edler, J., S. Bührer, V. Lo, S. Sheikh (2003): Assessment "Zukunft der Kompetenzzentrenprogramme und Zukunft der Kompetenzzentren" (Assessment „Future of the Competence Centres Programmes and Future of the Competence Centres"). Evaluation Report. Karlsruhe/Vienna: Fraunhofer ISI/ Austrian Institute for SME Research.

Europäische Kommission (1994): "European Report on S\&T Indicators", Luxemburg.

Europäische Kommission (2003a): "Third European Report on S\&T Indicators", Luxemburg.

Europäische Kommission (2003b): Die Rolle der Universitäten im Europa des Wissens", $\mathrm{KOM}(2003) 58$ endgültig, vom 5. Februar 2003.

Europäische Kommission (2004): Aktionsplan: Europäische Agenda für unternehmerische Initiative, $\operatorname{KOM}(2004) 70$

Europäische Kommission (2005): "Das intellektuelle Potential Europas wecken: So können die Universitäten ihren vollen Beitrag zur Lissabonner Strategie leisten", Kommunikation $\operatorname{KOM}(2005) 152$ endgültig, Brüssel

Europäische Kommission (2006): "Ein innovationsfreundliches, modernes Europa", Kommunikation $\operatorname{KOM}(2006) 589$ endgültig, Brüssel.

Falk, M. (2007): R\&D spending in the high-tech sector and economic growth, Research in Economics, 61 (3): 140-147.

Falk, M., F. Unterlass (2006): Determinanten des Wirtschaftswachstums im OECD-Raum, WIFO Weißbuch, Teilstudie 1, Wien.

Falk, M., H. Leo (2004): „Die Innovationsaktivitäten der österreichischen Unternehmen. Empirische Analysen auf Basis der Europäischen Innovationserhebung 1996 und 2000", WIFO, Wien.

Falk, R. (2009), Kohärenz des Instrumentenmix, Österreichisches Institut für Wirtschaftsforschung, Wien.

Falk, R. (2009), Tax Incentives for R\&D, Österreichisches Institut für Wirtschaftsforschung, Wien.

Fritz, O., U. Huemer, K. Kratena, H. Mahringer, N. Prean, G. Streicher (2008): „Mittelfristige Beschäftigungsprognose für Österreich und die Bundesländer", Studie des WIFO in Zusammenarbeit mit Joanneum Research, beauftragt durch das österreichische Arbeitsmarktservice, Wien.

Gächter, A. (2006): Qualifizierte Einwanderinnen und Einwanderer in Österreich und ihre berufliche Stellung, ZSI, Wien. 
Georgieff, P., S. Kimpeler, K. Müller, C. Rammer (2008), Beitrag der Creative Industries zum Innovaitonssystem am Beispiel Österreichs; Endbericht zur Studie im Auftrag der Wirtschaftskammer Österreich arge creativ wirtschaft austria; ZEW, ISI-Frauenhofer, Karlsruhe.

Gerhardter, G., M. Gruber, S. Pohn-Weidinger und G. Wagner (2009), Strategische Governance, convelop, Graz.

Geroski, P. A. (2000): Models of Technology Diffusion. Research Policy, 29, 603-625.

Geroski, P., C.F. Walters (1995): Innovative Activity over the Business Cycle; Economic Journal 105, 916-928.

Geroski, P., P.S. Machin (1993): Innovation, Profitability and Growth over the Business Cycle; Empirica 20, 33-50.

Grossman, G.M., E. Helpman (1991): Innovation and Growth in the Global Economy, Cambridge.

Guellec, D. (2001): Basic Research: Statistical Issues. DSTI/EAS/STP/NESTI(2001)38, OECD, Paris.

Guellec, D., B. van Pottlesberghe de la Potterie (2001): R\&D and Producitivity Growth; Panel Data Analysis of 16 OECD Countries, OECD, Paris.

Guellec, D., E. Ioannidis (1999): Causes of Fluctuations in R\&D Expenditures: A Quantitative Analysis, OECD Economic Studies 29, 123-138.

Guger A. (1998): Economic Policy and Social Democracy: The Austrian Experience, in: Oxford Review of Economic Policy 14, 40-59.

Hagedoorn, J. (2002): "Inter-Firm R\&D Partnership: An Overview of Patterns and Trends since 1960", Research Policy, 371-385.

Hagen, H.-O., C. Ahlstrand, M. Daniels, E. Nilsson, A. Adermon (2007): Innovations matters - An empirical analysis of innovation 2002-2004 and its impact on productivity, in Statistics Sweden, Yearbook of Productivity 2007, Stockholm. Pp. $1-28$.

Hall, B., J. Mairesse (2006): Empirical studies of innovation in the knowledge-driven economy, Economics of Innovation and New Technology 4-5, 289-299.

Hanappi-Egger, E., B. Weiss (2004): Informations- und Kommunikationstechnologien und e-business: Frauenspezifische Wissensbasis unter besonderer Berücksichtigung von Gründerinnen. BMWA, Wien.

Heckl, E., S. Sheikh, A. Dorr, C. Dörflinger (2005): Unternehmerinnen in Österreich. Aktuelle Situation und Entwicklungsmöglichkeiten. BMWA, Wien
Henkel, J., E. von Hippel (2005): "Welfare Implications of User Innovation", Journal of Technology Transfer, 30, 1/2, 73-87.

Herstad, S. J., C. Bloch, B. Ebersberger, E. van de Velde (2008): "Open innovation and globalisation: Theory, evidence and implications", Projektbericht des ERA-Nets Vision, Helsinki.

Hollanders, H., A. van Cruysen (2008), Rethinking the European Innovation Scoreboard: A New Methodology for 2008-2010; Pro Inno Metrics; http:// www.proinno-europe.eu/EIS2008/website/docs/ EIS 2008 Final report.pdf

Hollenstein, H., M. Woerter (2008): Inter- and IntraFirm Diffusion of Technology: The Example of E-Commerce. An Analysis Based on Swiss FirmLevel Data. Research Policy, 37, 545-564.

Huber, P. et al. (2008): Die ökonomischen Auswirkungen der Immigration in Österreich 1989-2007, Österreichisches Institut für Wirtschaftsforschung, Wien.

Hutschenreiter, G. (2004): "Public-Private Partnerships for Research and Innovation: An Evaluation of the Austrian Experience", Organisation for Economic Co-operation and Development, Paris.

Hutschenreiter, G., M. Peneder (1997): Österreichs 'Technologielücke' im Außenhandel, WIFO-Monatsberichte 70 (2): 103-114.

Janger, J. (2007A): Wettbewerbsfähigkeit der Österreichischen Wirtschaft, in: Kompetenzzentrum Forschungsschwerpunkt Internationale Wirtschaft FIW (Hg.): Österreichs Außenwirtschaft 2007, Wien, S. 91-96..

Janger, J. (2007B): Außenhandelsspezialisierung Österreichs im Vergleich mit den anderen Ländern der EU 15 und den neuen EU-Ländern, in: Kompetenzzentrum Forschungsschwerpunkt Internationale Wirtschaft FIW (Hg.): Österreichs Außenwirtschaft 2007, Wien, S. 85-91.

Janger, J. und M. Böheim (2009), Rahmenbedingungen, Österreichische Nationalbank und Österreichisches Institut für Wirtschaftsforschung, Wien.

Janz, N., H. Lööf, B. Peters (2004): Firm level Innovation and Productivity: Is there a Common Story?, Problems and perspectives in management 2, 184204.

Jörg, L. R. Falk (2004): Evaluation of the Austrian Industrial Research Promotion Fund (FFF) and the Austrian Science Fund (FWF). Background report 3.1.2., FFF: Internal functioning and customer satisfaction, Wien: Technopolis/WIFO.

Jörg, L., A. Schibany (2006): Zwischenevaluierung der aws-Technologieprogramme; Technopolis und Joanneum Research, Wien. 
Jörg, L., T. Jud, F. Ohler, W. Pointner, W. Polt, K. Zinöcker (2000): Evaluierung des ITF-Schwerpunktprogrammes Technologietransfers, Wien: Technopolis.

Kattinger, M. (2008): Reha-Klinik für Industriestrukturen, Neue Züricher Zeitung, 22. April 2008, Nr. 93, S. 13.

Klomp, L., G. van Leeuwen (2001): Linking innovation and firm performance: A new approach, International Journal of the Economics of Business 3, 343-364.

Klose, G., U. Glöckner, M. Astor, S. Heinrich und D. Riesenberg (2009), Grundlagenfinanzierte Forschungs-und Wissenschaftseinrichtungen, Prognos AG, Berlin.

Lassnig, L., S. Vogtenhuber (2007): Klassifikation von Ausbildung und Beruf, IHS, Wien.

Laursen, K., A. Salter (2006): "Open for Innovation: The Role of Openness in Explaining Innovation Performance among U.K. Manufacturing Firms", Strategic Management Journal, 27, 131-150.

Lay, G., J. Wengel (1998): Techniktrends in der Produktionsmodernisierung. ISI Fraunhofer Working Paper, Mitteilungen aus der Produktionsinnovationserhebung (Dezember 1998).

Le Bas, C. (2001): How Variations of Economic Activity Can Push or Pull Innovative Activity: A Survey; Lyon, Centre Walras, Université Lyon 2.

Leitner, K.-H. (2003): „Von der Idee zum Markt: Die 50 besten Innovationen Österreichs", Böhlau Verlag, Wien.

Leitner, K.-H. (2007): Intellectual Capital Reporting and Evaluation in Austrian Universities: Relationships and Complementarities, in: Platform Research and Technology Policy Evaluation, Austrian Council for Research and Technology Development (Eds.): Evaluation of Austrian Research and Technology Policies. A Summary of Austrian Evaluation Studies from 2003 to 2007, Vienna, pp. $97-105$

Leitner, K-H: (2009): „Open Innovation und User Innovation: Grundlagen und Perspektiven für Österreich", in: Leitner, K-H., Weber, M., Fröhlich, J. (Hrsg.): Innovationsforschung und Technologiepolitik in Österreich: Neue Perspektiven und Gestaltungsmöglichkeiten, Studienverlag, Innsbruck, 2009, 21-44.

Leo, H., R. Falk, K. Friesenbichler, W. Hölzl (2006): Forschung und Innovation als Motor des Wachstums, WIFO Weißbuch, Teilstudie 8, Wien.

Lööf, H., A. Heshmati (2002): Knowledge Capital and Performance Heterogeneity: A Firm-Level Inno- vation Study, International Journal of Production Economics 1, 61-85.

Lööf, H., A. Heshmati, R. Apslund, S.O. Nås (2003): Innovation and performance in manufacturing firms: A comparison of the Nordic countries, International Journal of Management Research 3, 5-36.

Lueger, M., H. Frank, A. Kessler, C. Korunka (2007): Gründungsrealisierung und Erfolg von jungen Unternehmen in Österreich: Eine Analyse der Entwicklung im Verlauf von acht Jahren (1998-2005): WU Wien, http://www.bmwa. gv.at/NR/rdonlyres/5E08E2A5-FB3F-45A4-B7C48F187606F7CB/0/GrndungsrealisierungundUnternehmenserfolg.pdf

Lundvall, B. (1988): "Innovation as an Interactive Process: from User-producer Interaction to the National System of Innovation", in: Dosi, G., Freeman, C., Nelson, R.R., Silverberg, G., Soete, L. (Hg.): Technical Change and Economic Theory, Pinter, London et al., 348-369.

Mansfield, E. (1995): "Academic research underlying industrial innovation: Sources, characteristics and financing", Review of Economics and Statistics, $77,(55)$.

Marterbauer, M. (2001): Der Verlust des Wachstumsvorsprungs. Österreichs Makroökonomische Entwicklung von 1970 bis 2000, in: Wirtschaft und Gesellschaft 28, 443-465.

Mathieu, A., M. Meyer, B. van Pottelsberghe de la Potterie (2008): "Turining science into business: A case study of a major European research university", Solvy Business School Working Paper WPCEP 07-035.

Mayer, S., I. Fischl, S. Ruhland und S. Sheikh (2009), Das Angebot der direkten FTI-Förderung in Österreich, KMU Forschung Austria, Wien.

Meri, T. (2008), Spitzentechnologie nutzende wissensintensive Dienstleistungen, in Statistik kurz gefaßt - Wissenschaft und Technologie, Vol. 18/2008, Eurostat.

Metzger G., M. Niefert, G. Licht (2008): High-TechGründungen in Deutschland: Trends, Strukturen, Potenziale, Studie in Zusammenarbeit mit der High-Tech-Gründerinitiative „unternimm was." von Microsoft Deutschland, ZEW Mannheim.

Miles, I. (2005): "Innovation in Services", in Fagerberg, J., Movery, D. C. and Nelson, R. R. (Eds.): The Oxford Handbook of Innovation. Oxford University Press, Oxford, 433-458.

Mohnen, P., A. Garcia (2004): Impact of government funding on R\&D and innovation, Study financed by the European Commission in preparation for 
the Competitiveness Report, http://www.eco. uc3m.es/IEEF/Mohnen.pdf

Mohnen, P., J. Mairesse, M. Dagenais (2006): Innovativity: A comparison across seven European countries, Economics of Innovation and New Technology 4-5, 391-413.

Mohnen, P., P. Therrien (2003): Comparing the innovation performance of manufacturing firms in Canada and in selected European countries: An econometric analysis, in: Gault, F., Understanding Innovation in Canadian Industry, Montreal. S. 313-339.

Narin, F., K.S. Hamilton, D. Olivastro (1997): “The increasing linkage between U.S. technology and public science", Research Policy, 26, (317).

Novak, R. (2005): The Austrian Science Fund FWF. Plattform fteval Newsletter 25: 37-38.

OECD (2004): Unternehmerische Initiative und innovative KMU in einer globalen Wirtschaft fördern: Auf dem Weg zu einer verantwortungsbewussteren, integrativeren Globalisierung, Zusammenfassung der Hintergrunddokumente, Zweite OECDKonferenz der für Klein- und Mittelunternehmen (KMU): Istanbul, Türkei, 3.-5. Juni 2004

OECD (2005): "International Policy Conference 'Intellectual Assets and Innovation. Value Creation in the Knowledge Economy'", 20-22. Oktober 2005, Ferrara, Italien, http://www.ferraraonintangibles.net.

OECD (2007): "OECD Economic Surveys AUSTRIA"; Volume 2007/15

OECD (2008a): A Profile of the Immigrant Populations in the 21st Century: Data from the OECD Countries, OECD, Paris.

OECD (2008b): "Education at a Glance 2008", Paris.

OECD (2008c): "OECD Science, Technology and Industry Outlook 2008", Organisation for Economic Co-operation and Development, Paris.

OECD (2008d): Innovation in firms: the OECD innovation microdata project, OECD: Paris (Veröffentlichung Anfang 2009 vorgesehen).

Pavitt, K. (1984): "Sectoral Patterns of Technological Change: Towards a Taxonomy and a Theory." Research Policy 13(6): 343-373.

Pavitt, K. (2001): "Public policies to support basic research: What can the rest of the world learn from US theory and practice (and what they should not learn)", Industrial and Corporate Change 10, (761).

Pavitt, K. (2005): "Innovation Processes", in: Fagerberg, J., Movery, D. C. und Nelson, R. R. (Hrsg.) The Oxford Handbook of Innovation Oxford, Oxford University Press, S. 86-114.
Peneder, M. (1999): The Austrian Paradox: 'Old' Structures but High Performance?, Austrian Economic Quarterly, 1999 (4): 239-247.

Peneder, M. (2003A): Industrial Structure and Aggregate Growth, Structural Change and Economic Dynamics, 14 (4): 427-448.

Peneder, M. (2003B): Wirtschaftliche Entwicklung und Strukturwandel in Österreich aus heutiger Sicht, in: Pichler, R. (Hg): Innovationsmuster in der österreichischen Wirtschaftsgeschichte, StudienVerlag, Innsbruck, 2003, 26-40.

Peneder, M. (2007): A Sectoral Taxonomy of Educational Intensity, Empirica, 34(3): 189-212.

Peneder, M. (2008), Entrepreneurship, Technological Regimes, and Productivity Growth. Integrated Classifications of Firms and Sectors, EU KLEMS Working Paper No. 28.

Peneder, M. (2008a): Entrepreneurship, Technological Regimes and Productivity Growth. Integrated Classifications of Firms and Sectors, EU KLEMS Working Paper.

Peneder, M. (2008b): "Was bleibt vom ÖsterreichParadoxon? Wachstum und Strukturwandel in der Wissensökonomie", Studie im Rahmen des Österreichischen Forschungsdialogs, beauftragt durch das Bundesministerium für Wissenschaft und Forschung (BMWF): Wien.

Peneder, M., M. Falk, M. Hölzl, W., Kaniovski, S., K. Kratena (2006): WIFO-Weißbuch: Mehr Beschäftigung durch Wachstum auf Basis von Innovation und Qualifikation. Teilstudie 3: Wachstum, Strukturwandel und Produktivität. Disaggregierte Wachstumsbeiträge für Österreich von 1990 bis 2004, Wien: Österreichisches Institut für Wirtschaftsforschung.

Pichler, R. (2009): „Institutionelle Dimensionen von Evaluierung in Österreich", in: Widmer, Th., Beywl, W., Fabian C. (Hrsg.) (2009) „Evaluation . Ein systematisches Handbuch, VS Verlag für Sozialwissenschaften, Wiesbaden.

Pointner, W., W. Polt (2005): Diffusionsorientierte Technologiepolitik. Eine vergleichende Wirkungsanalyse für Österreich, die Schweiz, Deutschland und die USA. Joanneum Research InTeReg Bd 5,Wien: Leykam Verlag.

Polt, W. (2009) „Evaluation in den Bereichen Forschung, Technologie und Innovation in Deutschland, Österreich und der Schweiz", in: Widmer, Th., Beywl, W., Fabian C. (Hrsg.) (2009) „Evaluation . Ein systematisches Handbuch, VS Verlag für Sozialwissenschaften, Wiesbaden 
Rammer, C., H. Penzkofer, A. Stephan, C. Grenzmann (2004): FuE- und Innovationsverhalten von KMU und Großunternehmen unter dem Einfluss der Konjunktur; Studien zum deutschen Innovationssystem Nr. 22-2004, Mannheim, München, Berlin, Essen.

Raymond, W., P. Mohnen, F. Palm, S. Schim van der Loeff (2006): Persistence of innovation in Dutch manufacturing: Is it spurious?, UNU-MERIT Working Papers 2006-011, http://www.merit.unu. edu/publications/wppdf/2006/wp2006-011.pdf (19.6.2008)

Reckling, F. (2007): „Der Wettbewerb der Nationen - oder wie weit die österreichische Forschung von der Weltspitze entfernt ist. Eine Analyse der internationalen Wettbewerbsfähigkeit wissenschaftlicher Forschung Österreichs in den Natur- und Sozialwissenschaften", FWF Positionspapier, Wien.

Reidl, S., F. Steyer, H. Schiffbänker(2006): Zwischen Unabhängigkeit und Zukunftsangst. Quantitative Ergebnisse zur Arbeit in den Wiener Creative Industries. Bericht 3, Wien

Reinstaller, A., F. Unterlass, N. Prean (2008): "Gibt es ein „europäisches Paradoxon" in Österreich? Die Beziehung zwischen Wissenschaft und ihrer industriellen Nutzung", Studie im Rahmen des Österreichischen Forschungsdialogs, beauftragt durch das Bundesministerium für Wissenschaft und Forschung (BMWF): Wien.

Riggs, W., E. von Hippel (1996): "The Impact of Scientific and Commercial Value of the Sources of Scientific Instruments Innovation", Research Policy, 23, 459-469.

Romer, P.M. (1990): Endogenous Technological Change, Journal of Political Economy 5, 71-102.

Rosenberg, N. (1982): Inside the Black Box. Technology and Economics. Cambridge, Cambridge University Press.

Rothwell, R. (1994): "Towards the Fifth-Generation Innovation Process", International Marketing Review, 11, 1, 7-31.

Ruhland S., I. Fischl, S. Mayer und S. Sheikh (2009), Governance in der FTI-Politik im Wechselspiel zwischen Ministerien und Agenturen, KMU Forschung Austria, Wien.

Schartinger, D., C. Rammer, M. Firscher, J. Fröhlich (2002), Knowledge interaction between universities and industry in Austria: patterns and determinants; in: Research Policy 31, 303-328.

Schibany, A., B. Nones, J. Schmidmayer, L. Jörg, K. Warta, S. Sheikh, J. Edler (2005): Evaluierung der Christian Doppler Gesellschaft CDG. Joanneum
Research, KMU Forschung Austria, Fraunhofer ISI, Wien: Eigenverlag.

Schibany, A., G. Streicher (2008), The European Scoreboard: drowning by numbers?; in Science and Public Policy, 35(10), 717-732.

Schibany, A., G. Streicher, B. Nones (2008), Geistige Eigentumsrechte an Hochschulen: Evaluierung des Programms uni:invetn; InTeReg Research Report Nr. 74-2008, Joanneum Research, Wien.

Schibany, A., G. Streicher, H. Gassler (2006): Österreich im Kontext des Lissabon- und Barcelonaprozesses; im Auftrag des Rates für Forschung und Technologieentwicklung, InTeReg Research Report Nr. 52-2006, Joanneum Research.

Schibany, A., G. Streicher, H. Gassler (2007): Der European Innovation Scoreboard: Vom Nutzen und Nachteil indikatorgeleiteter Länderrankings; InTeReg Research Report Nr. 65-2007, Joanneum Research, Wien.

Schibany, A., H. Gassler, G. Streicher (2007a): High Tech or Not Tech. Vom fehlenden Strukturwandel und anderen Sorgen, InTeReg Working Paper Nr. 35-2007.

Schibany, A., L. Jörg, B. Nones (2005): Instrumente der Technologieförderung und ihr Mix. Joanneum Research, InTeReg Research Report No. 37, Vienna.

Schiefer, A. (2008), Forschung und experimentelle Entwicklung (F\&E) im Unternehmenssektor 2006, Statistische Nachrichten, Vol. 11, S. 1012 - 1044.

Schiefer, A. (2008): Forschung und experimentelle Entwicklung (F\&E) im Unternehmenssektor 2006; Statistische Nachrichten 11/2008, Statistik Austria, Wien.

Schiffbänker H., B. Woitech, H. Gassler, E. Heckl, A. Dörflinger, C. Dörflinger (2007): Gründerinnen in Österreich - Vergleich internationaler Förderungsmodelle für forschungs- und technologieintensive Unternehmensgründungen, Studie im Auftrag von w-fFORTE in Kooperation mit aws und AplusB, Hrsg. Österreichische Forschungsförderungsgesellschaft (FFG).

Schwarz C. (2006): Gründungsalltag, Gender und Gründungsfinanzierung, ibw-Schriftenreihe Nr. 133, Juni 2006, Wien

Schwarz E., T. Ehrmann, R. Breitenecker R. (2005): Erfolgsdeterminanten junger Unternehmen in Österreich: eine empirische Untersuchung zum Beschäftigungswachstum, ZfB - Zeitschrift für Betriebswirtschaft, Band 75 (11): S. 1077-1098. 
Schwarz, E., E. Grieshuber (2003): Vom Gründungszum Jungunternehmen - Eine explorative Analyse, Institut für Wirtschaftswissenschaften, Universität Klagenfurt, Klagenfurt.

Sheikh, S., I. Mandl, A. Radauer, J. Edler, V. Lo, S. Hafner (2004): Evaluierung der österreichischen Betreuungsstrukturen für das 6. EU-Rahmenprogramm für Forschung, technologische Entwicklung und Demonstration. Im Auftrag des Bundesministeriums für Wirtschaft und Arbeit, Wien/ Karlsruhe: KMU Forschung Austria/Fraunhofer ISI.

Sheikh, S., W. Bornett (2005): FFG - Bereich Basisprogramme - Projektevaluierung 2005. Im Auftrag der Österreichischen Forschungsförderungsgesellschaft (FFG): Bereich Basisprogramme, Wien: KMU Forschung Austria.

Silverberg, G., G. Dosi, L. Orsenigo, L. (1988): Innovation, Diversity and Diffusion: A Self-Organisation Model. The Economic Journal, 98, 1032-1054.

Slaughter, S. (1993): "Innovation and Learning During Implementation: A Comparison of Use and Manufacturer Innovations", Research Policy, 22, 81-95.

Solow, R.M. (1957): Technical change and the aggregate production function, Review of Economics and Statistics 3, 312-320.

Statistik Austria (2002): Innovationen in österreichischen Unternehmen 1998-2000. Ergebnisse der dritten europäischen Innovationserhebung (CIS3), Wien.

Statistik Austria (2007a): Arbeitskräfteerhebung 2006 - Ergebnisse des Mikrozensus, Wien, Eigenverlag

Statistik Austria (2007b): Erfolgsfaktoren österreichischer Jungunternehmen,

Streicher, G. (2007): Additionality of FFG funding, InTeReg Working Paper No. 49-2007, Joanneum Research, http://www.joanneum.at/uploads/tx_ publicationlibrary/WP_49_additionality_ffg.pdf

Streicher, G., A. Schibany, M. Dinges (2004): Evaluation FWF - Impact Analysis. Background Report 4.2. Joanneum Research InTeReg Research Report No. 23. Vienna

Streicher, J., S. Mayer und S. Sheikh (2009), Public RTDI Funding in Austria - the Target Groups' Perspectives, KMU Forschung Austria, Wien.

Tichy, G. (1984): Strategy and Implementation of Employment Policy in Austria, in: Kyklos 37, 363386.

Tichy, G. (2000): The Innovation Potential and Thematic Leadership of Austrian Industries: An Inter- pretation of the Technology Delphi with Regard to the Old Structures/High-performance Paradox, Empirica 27, 411-436.

van Leeuwen, G., L. Klomp (2006): On the contribution of innovation to multi- factor productivity growth, Economics of Innovation and New Technology 4-5, 367-390.

Van Looy, B., J. Callaert, K. Debackere (2007): „Publication and patent behaviour of academic researchers: Conflicting, reinforcing or merely coexisting?" Verfügbar unter http://ssrn.com/abstract $=874905$

Van Pottelsberghe de la Potterie, B. (2007): "Hot 'patent' issues: Quantitative evidence", in: Guellec, D., van Pottelsberghe de la Potterie, B., "The Economics of the European Patent System", Oxford University Press, Oxford.

Verspagen, B. (2005): Innovation and Economic Growth, in: Fagerberg, J., Mowery, D., Nelson, R. (2005): The Oxford Handbook of Innovation, Oxford. S. 487-513.

von Hippel, E. (1986): "Lead Users: a Source of Novel Product Concepts", Management Science, 32, 791-805.

von Hippel, E. (2005): "Democratizing Innovation", MIT Press, Cambridge und London.

Yoo, S.-H. (2008): High-technology exports and economic output: an empirical investigation, Applied Economic Letters 15, 523-525.

Zinöcker, K., M. Dinges (2004): Evaluation of the Austrian Industrial Research Promotion Fund (FFF) and the Austrian Science Fund (FWF). Positionierung des FFF und FWF vis-a-vis anderen Finanzierungsinstrumenten. Modul 2. Joanneum Research InTeReg Research Report No. 23. Vienna.

Zinöcker, K., M. Dinges (2009): „Evaluation von Forschungs- und Technologiepolitik in Österreich", in: Widmer, Th., Beywl, W., Fabian C. (Hrsg.) (2009) „Evaluation . Ein systematisches Handbuch, VS Verlag für Sozialwissenschaften, Wiesbaden.

Zinöcker, K., S. Sheikh, L. Jörg, W. Polt, M. Stampfer (2004): Ausbildungsworkshops Evaluierung - eine Dokumentation, Wien: Plattform fteval.

Zucker, L., M. Darby M.R. Brewer (1998): "Intellectual Capital and the Birth of the U.S. Biotechnology Enterprise", American Economic Review, 88, 290-306. 


\section{Anhang}

\section{Definition der Wirtschaftssektoren}

\begin{tabular}{|c|c|c|c|}
\hline NACE & Branche & Abteilung & Technologiedefinition \\
\hline 01 & Landwirtschaft;Jagd & Land- u.Forstwirtschaft, Fischerei & - \\
\hline 02 & Forstwirtschaft & Land- u.Forstwirtschaft, Fischerei & - \\
\hline 05 & Fischerei und Fischzucht & Land- u.Forstwirtschaft, Fischerei & - \\
\hline 10 & Kohlenbergbau, Torfgewinnung & Bergbau & - \\
\hline $11+13$ & Erdöl, und Erdgasbergbau; Erzbergbau & Bergbau & - \\
\hline 14 & Gewinnung von Steinen und Erden & Bergbau & - \\
\hline 15 & Herstellung von Nahrungs- u. Genussmitteln und Getränken & Sachgütererzeugung & Sonstige Sachgüter \\
\hline 16 & Tabakverarbeitung & Sachgütererzeugung & Sonstige Sachgüter \\
\hline 17 & Herstellung von Textilien und Textilwaren & Sachgütererzeugung & Sonstige Sachgüter \\
\hline 18 & Herstellung von Bekleidung & Sachgütererzeugung & Sonstige Sachgüter \\
\hline 19 & Ledererzeugung und -verarbeitung & Sachgütererzeugung & Sonstige Sachgüter \\
\hline 20 & Be- und Verarbeitung von Holz & Sachgütererzeugung & Sonstige Sachgüter \\
\hline 21 & Herstellung und Verarbeitung von Papier und Pappe & Sachgütererzeugung & Sonstige Sachgüter \\
\hline 22 & Verlagswesen, Druckerei & Sachgütererzeugung & Sonstige Sachgüter \\
\hline 23 & Kokerei, Mineralölverarbeitung & Sachgütererzeugung & Sonstige Sachgüter \\
\hline 24 & Herstellung von Chemikalien und chemischen Erzeugnissen & Sachgütererzeugung & High-Tech \\
\hline 25 & Herstellung von Gummi- und Kunststoffwaren & Sachgütererzeugung & Medium Tech \\
\hline 26 & Herst. u. Bearbeitung von Glas, Waren aus Steinen u. Erden & Sachgütererzeugung & Medium Tech \\
\hline 27 & Metallerzeugung und -bearbeitung & Sachgütererzeugung & Medium Tech \\
\hline 28 & Herstellung von Metallerzeugnissen & Sachgütererzeugung & Medium Tech \\
\hline 29 & Maschinenbau & Sachgütererzeugung & Medium Tech \\
\hline 30 & Herstellung von Büromaschinen & Sachgütererzeugung & High-Tech \\
\hline 31 & Herstellung von Geräten der Elektrizitätserzeugung,-verteilung & Sachgütererzeugung & Medium Tech \\
\hline 32 & Rundfunk-, Fernseh- und Nachrichtentechnik & Sachgütererzeugung & High-Tech \\
\hline 33 & Medizin-,Mess-, Steuer- und Regelungstechnik, Optik & Sachgütererzeugung & High-Tech \\
\hline 34 & Herstellung von Kraftwagen und Kraftwagenteilen & Sachgütererzeugung & Medium Tech \\
\hline 35 & Sonstiger Fahrzeugbau & Sachgütererzeugung & Medium Tech \\
\hline 36 & Herstellung von Möbeln, Schmuck, Musikinstrumenten, Sportgeräten & Sachgütererzeugung & Sonstige Sachgüter \\
\hline 37 & Rückgewinnung (Recycling) & Sachgütererzeugung & Sonstige Sachgüter \\
\hline 40 & Energieversorgung & Energie- und Wasserversorgung & - \\
\hline 41 & Wasserversorgung & Energie- und Wasserversorgung & - \\
\hline 45 & Bauwesen & Bauwesen & - \\
\hline 50 & KFZ-Handel; Instandhaltung u. Reparatur v. KFZ; Tankstellen & Dienstleistungen & Sonstige Dienstleistungen \\
\hline 51 & Handelsvermittlung und Großhandel (o. Handel mit KFZ) & Dienstleistungen & Sonstige Dienstleistungen \\
\hline 52 & Einzelhandel (o. Handel mit KFZ); Rep. v. Gebrauchsg. & Dienstleistungen & Sonstige Dienstleistungen \\
\hline 55 & Beherbergungs- und Gaststättenwesen & Dienstleistungen & Sonstige Dienstleistungen \\
\hline 60 & Landverkehr; Transport in Rohrfernleitungen & Dienstleistungen & Sonstige Dienstleistungen \\
\hline 61 & Schiffahrt & Dienstleistungen & Sonstige Dienstleistungen \\
\hline 62 & Flugverkehr & Dienstleistungen & Sonstige Dienstleistungen \\
\hline 63 & Hilfs- und Nebentätigkeiten für den Verkehr; Reisebüros & Dienstleistungen & Sonstige Dienstleistungen \\
\hline
\end{tabular}




\begin{tabular}{llll}
\hline 64 & Nachrichtenübermittlung & Dienstleistungen & Sonstige Dienstleistungen \\
\hline 65 & Kreditwesen & Dienstleistungen & Sonstige Dienstleistungen \\
\hline 66 & Versicherungswesen & Dienstleistungen & Sonstige Dienstleistungen \\
\hline 67 & Mit dem Kredit- und Versicherungswesen verbundene Tätigkeiten & Dienstleistungen & Sonstige Dienstleistungen \\
\hline 70 & Realitätenwesen & Dienstleistungen & Sonstige Dienstleistungen \\
\hline 71 & Vermietung beweglicher Sachen ohne Bedienungspersonal & Dienstleistungen & Sonstige Dienstleistungen \\
\hline 72 & Datenverarbeitung und Datenbanken & Dienstleistungen & Hi-Tech Knowledge Intensive \\
\hline 73 & Forschung und Entwicklung & Dienstleistungen & Hi-Tech Knowledge Intensive \\
\hline 74 & Erbringung von unternehmensbezogenen Dienstleistungen & Dienstleistungen & Sonstige Dienstleistungen \\
\hline 75 & Öffentliche Verwaltung, Landesverteidigung, Sozialversicherung & Dienstleistungen & Sonstige Dienstleistungen \\
\hline 80 & Unterrichtswesen & Dienstleistungen & Sonstige Dienstleistungen \\
\hline 85 & Gesundheits-, Veterinär- und Sozialwesen & Dienstleistungen & Sonstige Dienstleistungen \\
\hline 90 & Abwasser- und Abfallbeseitigung und sonstige Entsorgung & Dienstleistungen & Sonstige Dienstleistungen \\
\hline 91 & Interessenvertr., kirchl. und sonst. religiöse Vereinig., sonst. Vereine & Dienstleistungen & Sonstige Dienstleistungen \\
\hline 92 & Kultur, Sport und Unterhaltung & Dienstleistungen & Sonstige Dienstleistungen \\
\hline 93 & Erbringung von sonstigen Dienstleistungen & Dienstleistungen & Sonstige Dienstleistungen \\
\hline 95 & Private Haushalte & Dienstleistungen & Sonstige Dienstleistungen \\
\hline
\end{tabular}

Quelle: Statistik Austria; eigene Technologie-Zuordnung (basierend auf OECD) 


\section{Systemevaluierung der Forschungsförderung und -finanzierung: Leistungsbild}

The evaluators are requested to elaborate on the following issues:

- Analysis of system health: Is the Austrian RDTI funding system overall healthy?

- Does the system fit to current and future requirements?

The evaluation should touch upon the framework of governmental intervention (reflecting also the interplay with European and regional levels), the individual instruments and RTDI institutions and their systemic interaction as discussed below. The associated questions are not intended to be comprehensive nor compulsory set of questions. Rather are they intended to illustrate areas of interest of the clients and hence represent a guideline for the evaluator.

\section{Framework}

\section{Framework Conditions}

The field of RTDI is characterized by its diversity and by its linkages to other policy areas and systems like education or the economy.

Question 1: Assessment of the role of the frameworks conditions, human resources, tax and fiscal regimes in the innovation system.

\section{Governance}

In the last few years, the Austrian system experienced a set of administrative and organizational reforms which led to a professional system of division of labour between several ministries and agencies ('Agencyfication').

Question 2: Is there a strategic approach towards governmental intervention in the Austrian system?

- Federal Government ('Bund'), Regional Governments ('Länder') and the European level: How co- herent are federal, and regional policies? How do they correspond to European policies?

Question 3: Are the mechanisms used to manage the relation between the ministries and the agencies effective and efficient?

- Is there a need for more coordination?

- Are the institutional structures and the division of labour in devising and implementing RTDI interventions effective?

\section{Instruments \& Interventions}

The Austrian system has developed a rich system of public funding. The instruments can be classified as:

- Indirect measures, such as the public sector forsaking tax income from the private sector in exchange for approved RTDI investment behaviour. Indirect measures also comprise catalytic Financial RTDI measures.

- Direct measures, such as the direct transfer of financial support for RTDI via grants, conditional loans, warranties and block grants to RTDI institutions.

\section{Indirect Measures}

Instruments of indirect fiscal research advancement (Tax Credits and Tax Allowances ) are crucial and (financially) very important in Austria;

Question 4: What evidence can be given about the structure, the usage, the acceptance and the effects of these instruments?

\section{Direct Measures}

Direct measures are the most elaborate ones of these measures: 'Elaborate' in terms of quantity of instruments used. The clients are interested in the assessment of direct measures which can be classified bottom up, top down, ( including mission oriented, functional approaches) etc. 
Question 5: Are the rationale, strategies, approaches and the thematic focus of these measures still relevant and realistic? Is the choice of bottom-up and top-down programmes appropriate?

Question 6: Are the resource endowment and the size the instruments sufficient to reach the goals of the intervention?

RTDI institutions: As mentioned above, the goal of this evaluation is NOT an assessment of the quality of RTDI institutions such as universities, Austrian Research Centers, competence centers, or the Austrian Academy of Sciences. However, the funding of RTDI institutions (e.g. via the General University Funds, GUF) (in terms of the size of the outlays) feeds back to functionality of the remaining set of direct measures in the Austrian RTDI system: a good reason to pose questions to the evaluator.

Question 7: What are the effects of block grants on the behaviour of beneficiaries?

Question 8: What are the effects of block grants such as GUF on the remaining set of direct measures used?

\section{Systems Perspective}

The assessment of the Austrian RTDI intervention landscape from a systems perspective is the core of this evaluation endeavour. The evaluators are encouraged to use any available information to get a concise picture of the systemic interaction of individual instruments and institutions. Some of the available information sources are collected in the appendix to this document.

\section{Systemic Fit of the Intervention Logic}

Governments do not fund RTDI for its own sake. The most prominent rationale for government intervention is market failure (both in allocation and distribution) and system failures.
Question 9: Are these failures addressed in a sufficient and comprehensive way on the system level?

- Do needs / problem areas and the number of instruments correspond?

- Is there an overlap, or are there white spots concerning problem areas / target groups?

- Is there over / under representation of problem areas / target groups?

Question 10: Is governmental intervention in Austria (taking into account also intervention at the European and regional levels) capable of reducing these failures?

\section{Systemic Interplay of Interventions}

The underlying hypothesis of this evaluation is that the overall systemic effects of governmental intervention cannot be assessed by the simple aggregation of the effects of individual intervention. The effects on the system level accrue through the interplay particularly between tax incentives and bottom-up funding - of individual interventions, their complementarities, their contradictions. The evaluator supplies an assessment of the joint effects of the interventions on the system level.

Question 11: Can contradictions, substitution effects and complementarities be identified?

Question 12: Does the system of governmental interventions structure and support the connectivity of actors and institutions within the innovation system?

Question 13: How coherent is the set of the instruments used? Are the incentive mechanisms compatible if several interventions target the same actors or groups of actors?

\section{Target}

The following aspects are of particular interest to the clients and therefore, should be adressed in the 
evaluation accordingly: In the business sector: large companies (also MNEs), SMEs, start-ups and RTDI novices; in the science and research sector; university sector and the non-university sector ("Ausseruniversitäre Forschung"|), researchers, young researchers, and gender aspects are of particular interest to the clients. These should be addressed in the evaluation accordingly.

Question 14: How does the system of governmental intervention cover the target groups (users)?

- Is there evidence for double founding?

- Is there competition between instruments concerning the attraction of users?

Question 15: What is the users' perspective and their assessment of the system of governmental intervention in Austria?

Question 16: What is the users' behaviour in the system of governmental intervention?

- Is there evidence for strategic / opportunistic behaviour?

\section{Recommendations \& Scenarios}

The recommendations made in this evaluation should lead to continuous improvement in the Austrian innovation system within the European context: through the analysis the clients want to learn a hierarchy of improvement steps ranging from incremental improvement, which can be implemented within a short time horizon to (if necessary) radical changes, which can be considered in a medium to long term perspective. The recommendations should reflect likely or potential changes in the international and national context. The evaluator should link up to national and international strategic discussions about research priorities. The evaluator will structure these developments and develop scenarios in an appropriate way. The recommendation will be linked to these scenarios.

Quelle: BMVIT und BMWA (2007) 


\section{Die neuen Branchentaxonomien im Überblick}

\begin{tabular}{|c|c|c|c|}
\hline Nace & Branche & Ausbildungsintensität & Innovationsintensitätt \\
\hline & & $\mathrm{Al}$ & II \\
\hline 10 & Kohlenbergbau, Torfgewinnung & Mittel & Mittel-gering \\
\hline 11 & Erdöl-, Erdgasbergbau & Mittel & Mittel-gering \\
\hline 14 & Steine, Erden, sonstiger Bergbau & Mittel & Gering \\
\hline 15 & Nahrungs-, Genussmittel, Getränke & Gering & Mittel-gering \\
\hline 16 & Tabakverarbeitung & Gering & Mittel-gering \\
\hline 17 & Textilien, Textilwaren & Gering & Mittel-hoch \\
\hline 18 & Bekleidung & Gering & Gering \\
\hline 19 & Ledererzeugung, -verarbeitung & Gering & Gering \\
\hline 20 & Be-, Verarbeitung von Holz & Gering & Mittel \\
\hline 21 & Papier, Pappe & Mittel & Mittel \\
\hline 22 & Verlagswesen, Druckerei & Mittel & Mittel-gering \\
\hline 23 & Kokerei, Mineralölverarbeitung & Mittel & Mittel-hoch \\
\hline 24 & Chemikalien & Mittel & Mittel-hoch \\
\hline 25 & Gummi-,Kunststoffwaren & Mittel & Mittel-hoch \\
\hline 26 & Glas & Gering & Mittel-hoch \\
\hline 27 & Metallerzeugung, -bearbeitung & Gering & Mittel-hoch \\
\hline 28 & Metallerzeugnisse & Gering & Mittel \\
\hline 29 & Maschinenbau & Mittel & Hoch \\
\hline 30 & Büromaschinen, Datenverarbeitungsgeräte & Hoch & Hoch \\
\hline 31 & Elektrogeräte & Mittel & Hoch \\
\hline 32 & Rundfunk-, Fernseh-, Nachrichtentechnik & Mittel & Hoch \\
\hline 33 & Medizin-, Mess-, Regelungstechnik, Optik & Mittel & Hoch \\
\hline 34 & Kraftwagen, Kraftwagenteile & Mittel & Mittel-hoch \\
\hline 35 & Sonstiger Fahrzeugbau & Mittel & Mittel-hoch \\
\hline 36 & Möbel, Schmuck, Musikinstrumente, Sportgeräte etc. & Mittel & Mittel \\
\hline 37 & Rückgewinnung (Recycling) & Mittel & Gering \\
\hline 40 & Energieversorgung & Mittel & Mittel-gering \\
\hline 41 & Wasserversorgung & Mittel & Mittel-gering \\
\hline 45 & Bauwesen & Gering & - \\
\hline 50 & Kraftfahrzeughandel (inkl. Instandhaltung, Reparatur) & Gering & - \\
\hline 51 & Großhandel & Mittel & Gering \\
\hline 52 & Einzelhandel & Mittel & - \\
\hline 55 & Beherbergungs-, Gaststättenwesen & Gering & - \\
\hline 60 & Landverkehr; Transport in Rohrfernleitungen & Mittel & Gering \\
\hline 61 & Schifffahrt & Mittel & Gering \\
\hline 62 & Flugverkehr & Mittel & Mittel \\
\hline 63 & Verkehr; Reisebüros & Mittel & Gering \\
\hline 64 & Nachrichtenübermittlung & Mittel & Mittel-Hoch \\
\hline 65 & Kreditwesen & Hoch & Mittel \\
\hline 66 & Versicherungswesen & Mittel & Mittel-gering \\
\hline 67 & Sonstiges Kredit-, Versicherungswesen & Mittel & Gering \\
\hline 70 & Realitätenwesen & Mittel & - \\
\hline 72 & Datenverarbeitung & Hoch & Hoch \\
\hline 73 & Forschung und Entwicklung & Hoch & Hoch \\
\hline 74 & Unternehmensbezogene Dienstleistungen & Hoch & Mittel \\
\hline
\end{tabular}

NB: Die Bestimmung der Ausbildungs- bzw. Innovationsintensität als Hoch, Mittel oder Gering beruht auf mehrstufigen und multivariaten statistischen Clusterverfahren. Als Variable wurden z.B. die Bildungsabschlüsse der Beschäftigen, die F\&E-Ausgaben der Unternehmen, die Frage, ob Innovationen für den Markt oder lediglich für das Unternehmen neu sind, die Anmeldung von Patenten und anderen Schutz- rechten, oder die relative Bedeutung externer und interner Wissensquellen berücksichtigt. Die Informationen beruhen auf Individual- und Unternehmensdaten. Theoretische Begründung, Daten, Methode und Validierung der Ergebnisse sind ausführlich in Peneder $(2007,2008)$ dokumentiert.

Quelle: Peneder (2007, 2008). 


\section{Statistischer Anhang}

\section{$1 \quad$ Finanzierung der Bruttoinlandsausgaben für F\&E und Forschungsquote 2009 (Tabellen 1 und 1a) ${ }^{1}$}

Die österreichischen Bruttoinlandsausgaben für Forschung und experimentelle Entwicklung (F\&E) - die Gesamtsumme der Ausgaben für in Österreich durchgeführte F\&E - werden 2009 2,73\% des Bruttoinlandsproduktes (BIP) erreichen und gegenüber 2008 um $1,8 \%$ steigen. Somit werden im heurigen Jahr gemäß der neuesten Schätzung der Statistik Austria für in Österreich durchgeführte F\&E voraussichtlich insgesamt 7,652 Mrd. Euro ausgegeben werden. Die im Vergleich zu den Vorjahren deutlich abgeschwächte Entwicklung der Forschungsausgaben - im Zeitraum 2004 bis 2008 war ein durchschnittlicher jährlicher Anstieg von 9,4\% zu verzeichnen - ist auf den für Österreich aktuell prognostizierten Konjunkturrückgang zurückzuführen.

Von den gesamten Forschungsausgaben 2009 wird mit 45,0\% (rund 3,44 Mrd. Euro) der größte Anteil von der Wirtschaft finanziert werden. 39,9\% (rund 3,1 Mrd. Euro) wird der öffentliche Sektor beitragen (Bund rund 2,55 Mrd. Euro, Bundesländer rund 402 Mio. Euro, sonstige öffentliche Einrichtungen wie Gemeinden, Kammern, Sozialversicherungsträger rund 104 Mio. Euro). 14,8\% werden vom Ausland und 0,4\% (rund 29 Mio. Euro) vom privaten gemein-

1 Auf der Grundlage der Ergebnisse der F\&E-statistischen Vollerhebungen sowie sonstiger aktuell verfügbarer Unterlagen und Informationen, insbesondere der F\&E-relevanten Voranschlags- und Rechnungsabschlussdaten des Bundes und der Bundesländer, wird von der Statistik Austria jährlich die "Globalschätzung der österreichischen Bruttoinlandsausgaben für F\&E" erstellt. Im Rahmen der jährlichen Erstellung der Globalschätzung erfolgen, auf der Basis von neuesten Daten, jeweils auch rückwirkende Revisionen bzw. Aktualisierungen. Den Definitionen des weltweit (OECD, EU) gültigen und damit die internationale Vergleichbarkeit gewährleistenden Frascati-Handbuchs entsprechend wird die Finanzierung der Ausgaben der in Österreich durchgeführten Forschung und experimentellen Entwicklung dargestellt. Gemäß diesen Definitionen und Richtlinien ist die ausländische Finanzierung von in Österreich durchgeführter F\&E sehr wohl einbezogen, hingegen österreichische Zahlungen für im Ausland durchgeführte F\&E sind ausgeschlossen (Inlandskonzept). nützigen Sektor finanziert werden. Die Finanzierung durch das Ausland (rund 1,13 Mrd. Euro) stammt zum überwiegenden Teil von mit heimischen Unternehmen verbundenen europäischen Unternehmen, die Österreich zum Forschungsstandort gewählt haben und schließt die Rückflüsse aus den EU-Rahmenprogrammen für Forschung, technologische Entwicklung und Demonstration ein.

Für Vergleichszwecke werden die Bruttoinlandsausgaben für F\&E als Prozentsatz des Bruttoinlandsprodukts ausgedrückt („Forschungsquote“). Dieser Indikator ist für Österreich seit 1981 von $1,10 \%$ auf $2,73 \%$ im Jahre 2009 angestiegen und hat in den letzten Jahren den EU-Durchschnitt deutlich übertroffen. Die aktuellsten gesamteuropäischen Vergleichsdaten liegen für 2006 vor: Demnach beträgt diese Kennzahl für die Europäische Union (EU-27) im Durchschnitt $1,84 \%$ und für Österreich 2,46\%.

In die aktuelle Globalschätzung der österreichischen Bruttoinlandsausgaben für $\mathrm{F} \& \mathrm{E}$ wurden die endgültigen Ergebnisse der Erhebung über Forschung und experimentelle Entwicklung der Statistik Austria über das Berichtsjahr 2006 einbezogen sowie erste Teilergebnisse der F\&E-Erhebung über das Jahr 2007 im firmeneigenen Bereich. Diese zeigen ein im Vergleich zu den Vorjahren geringeres Wachstum der F\&E-Finanzierung durch den Unternehmenssektor, welches sich auch 2008 fortsetzt. 2009 wird der für Österreich derzeit prognostizierte Konjunkturrückgang auch einen Rückgang des F\&E-Eigenfinanzierungsanteils der Unternehmen nach sich ziehen und mit 3,44 Mrd. Euro wird die Finanzierung durch die Wirtschaft um 1,3\% unter dem Wert von 2008 liegen.

Da die F\&E-Finanzierung aus dem Ausland zum überwiegenden Teil von mit heimischen Unternehmen verbundenen europäischen Unternehmen stammt, ist hier auf Grund der Wirtschaftsprognosen mit einem weitaus stärkeren Rückgang zu rechnen: Nach derzeitigem Informationsstand ist ein Absinken der F\&E-Mittel um 6,2\% zu erwarten. Mit 1,13 Mrd. Euro werden 2009 die Forschungsgel- 
der aus dem Ausland nach leichten Anstiegen 2007 und 2008 unter dem Erhebungsergebnis von 2006 (1,16 Mrd. Euro) liegen.

Die Forschungsfinanzierung durch den Bund wird 2009 nach den der Statistik Austria vorliegenden Informationen über die Entwicklung der F\&E-relevanten Budgetanteile und weiterer F\&E-Fördermaßnahmen - insbesondere die Erstattungen des Bundes an Unternehmen im Rahmen der Forschungsprämie - mit 2,55 Mrd. Euro um 8,9\% über dem Wert von 2008 liegen und maßgeblich zur Stabilisierung der österreichischen Forschungsausgaben beitragen.

\section{F\&E-Ausgaben des Bundes 2009}

2.1. Die in Tabelle 1 ausgewiesenen Ausgaben des Bundes für in Österreich durchgeführte F\&E 2009 setzen sich wie folgt zusammen: Gemäß der der F\&E-Globalschätzung zugrunde liegenden Methodik ist das Kernstück die Gesamtsumme des Teils b der Beilage T (2009 in der Fassung des Bundesvoranschlag-Entwurfes). Zusätzlich wurden die für 2009 zur Verfügung stehenden Mittel der Nationalstiftung für Forschung, Technologie und Entwicklung sowie auf dem Informationsstand Mitte April 2009 beruhende Schätzungen der voraussichtlich 2009 zur Auszahlung gelangenden Forschungsprämien einbezogen (Quelle: BMF).

2.2. Zusätzlich zu den Ausgaben des Bundes für in Österreich durchgeführte F\&E wird der Bund im Jahre 2009 Beitragszahlungen an internationale Organisationen, die Forschung und Forschungsförderung als Ziel haben, in Höhe von 71,1 Mio. Euro leisten, die in der Beilage $\mathrm{T} /$ Teil a dargestellt sind, jedoch gemäß dem Inlandskonzept nicht in die österreichischen Bruttoinlandsausgaben für $\mathrm{F} \& \mathrm{E}$ eingerechnet werden.

2.3. Die in der Beilage $\mathbf{T}^{2}$ (Teil a und Teil b) zusammengefassten forschungswirksamen Ausgaben des Bundes, welche die forschungswirksamen Anteile an den Beitragszahlungen an internationale Organisationen (s.o. Pkt. 2.2) einschließen, werden traditioneller Weise unter der Bezeichnung „Ausgaben des Bundes für Forschung und Forschungsförderung“ zusammengefasst und entsprechen dem auf Basis

2 2009: Beilage T entsprechend der Fassung des Bundesvoranschlag-Entwurfes. des Frascati-Handbuches von OECD und EU angewendeten "GBAORD“-Konzept ${ }^{3}$, welches sich primär auf die Budgets des Zentral- bzw. Bundesstaates bezieht, im Gegensatz zum Inlandskonzept die forschungsrelevanten Beitragszahlungen an internationale Organisationen einschließt und die Grundlage der Klassifizierung von F\&E-Budgetdaten nach sozio-ökonomischen Zielsetzungen für die Berichterstattung an EU und OECD bildet.

2009 kommen folgenden sozio-ökonomischen Zielsetzungen die stärksten Anteile an den Ausgaben des Bundes für Forschung und Forschungsförderung zu:

- Förderung der allgemeinen Erweiterung des Wissens: $29,7 \%$

- Förderung von Handel, Gewerbe und Industrie: $25,7 \%$

- Förderung des Gesundheitswesens: 20,6\%

- Förderung der sozialen und sozio-ökonomischen Entwicklung: 5,3\%

- Förderung der Erforschung der Erde, der Meere, der Atmosphäre und des Weltraumes: 4,5\%

- Förderung des Umweltschutzes: 4,0\%

- Förderung der Land- und Forstwirtschaft: 3,1\%

\section{F\&E-Ausgaben der Bundesländer}

Die als Teilsumme in Tabelle 1 ausgewiesene Forschungsfinanzierung durch die Bundesländer beruht auf den von den Ämtern der Landesregierungen gemeldeten F\&E-Ausgaben-Schätzungen auf Basis der jeweiligen Landesvoranschläge. Die F\&E-Ausgaben der Landeskrankenanstalten werden gemäß einer mit den Landesregierungen vereinbarten Methodik von Statistik Austria jährlich geschätzt.

\section{F\&E-Ausgaben 2006 im internationalen Vergleich (Tabelle 10)}

Die Übersichtstabelle zeigt anhand der wichtigsten F\&E-relevanten Kennzahlen die Position Österreichs im Vergleich zu den anderen Mitgliedstaaten der Europäischen Union bzw. der OECD (Quelle: OECD, MSTI 2008-2).

3 GBAORD: Government Budget Appropriations or Outlays for $\mathrm{R} \& \mathrm{D}=$ „Staatliche Mittelzuweisungen oder Ausgaben für Forschung und Entwicklung" (EU-Übersetzung). 


\section{Tabellenübersicht}

1 Globalschätzung 2009: Bruttoinlandsausgaben für F\&E Finanzierung der in Österreich durchgeführten Forschung und experimentellen Entwicklung 1989-2009

2 Globalschätzung 2009: Bruttoinlandsausgaben für F\&E Finanzierung der in Österreich durchgeführten Forschung und experimentellen Entwicklung 1989-2009 in Prozent des BIP

3 Ausgaben des Bundes für Forschung und Forschungsförderung 2006 bis 2009

4 Ausgaben des Bundes 1993 bis 2009 für Forschung und Forschungsförderung nach sozio-ökonomischen Zielsetzungen

5 Ausgaben des Bundes 2007 für Forschung und Forschungsförderung nach sozio-ökonomischen Zielsetzungen und Ressorts

6 Ausgaben des Bundes 2008 für Forschung und Forschungsförderung nach sozio-ökonomischen Zielsetzungen und Ressorts

7 Ausgaben des Bundes 2009 für Forschung und Forschungsförderung nach sozio-ökonomischen Zielsetzungen und Ressorts

8 Ausgaben für Forschung und experimentelle Entwicklung 1993 bis 2006 nach Durchführungs- und Finanzierungssektoren

9 Allgemeine forschungswirksame Hochschulausgaben des Bundes 1999 - 2009

10 Forschung und experimentelle Entwicklung (F\&E) 2006 im internationalen Vergleich

11 Beschäftigte in F\&E 2006 nach Durchführungssektoren und Beschäftigtenkategorien

12 Beschäftigte in F\&E 2006 nach Durchführungssektoren, Beschäftigtenkategorien und Geschlecht

13 Beschäftigte in F\&E 2006 nach Bundesländern und Beschäftigtenkategorien

14 Ausgaben für F\&E 2006 nach Durchführungssektoren und Ausgabenarten

15 Ausgaben für F\&E 2006 nach Bundesländern und Ausgabenarten

16 Ausgaben für F\&E 2006 nach Durchführungssektoren und Forschungsarten

17 Ausgaben für F\&E 2006 nach Bundesländern und Forschungsarten

18 Ausgaben für F\&E 2006 nach Bundesländern (nach dem Hauptstandort/ nach dem F\&E-Standort)

19 Finanzierung der Ausgaben für Forschung und experimentelle Entwicklung (F\&E) 2006 nach Durchführungssektoren/ Erhebungsbereichen und Finanzierungsbereichen

20 Finanzierung der Ausgaben für F\&E 2006 nach Bundesländern und Finanzierungsbereichen

21 Bruttoregionalprodukt (BRP), Bruttoinlandsausgaben für F\&E und regionale Forschungsquoten 2006

22 Hochschulsektor: Beschäftigte in F\&E 2006 nach Wissenschaftszweigen und Beschäftigtenkategorien

23 Hochschulsektor: Ausgaben für F\&E 2006 nach Wissenschaftszweigen und Ausgabenarten

24 Hochschulsektor: Ausgaben für F\&E 2006 nach Wissenschaftszweigen und Forschungsarten

25 Hochschulsektor: Finanzierung der Ausgaben für F\&E 2006 nach Wissenschaftszweigen und Finanzierungsbereichen

26 Universitäten: Beschäftigte in F\&E 2006 nach Wissenschaftszweigen und Beschäftigtenkategorien

27 Universitäten: Beschäftigte in F\&E 2006 - Arbeitszeitverteilung in Prozent nach Wissenschaftszweigen

28 Universitäten: Wissenschaftliches Personal 2006 nach Wissenschaftszweigen, Geschlecht und Altersgruppen

29 Universitäten: Ausgaben für F\&E 2006 nach Wissenschaftszweigen und Ausgabenarten

30 Universitäten: Ausgaben für F\&E 2006 nach Wissenschaftszweigen und Forschungsarten

31 Universitäten: Finanzierung der Ausgaben für F\&E 2006 nach Wissenschaftszweigen und Finanzierungsbereichen

32 Sektor Staat: Beschäftigte in F\&E 2006 nach Wissenschaftszweigen und Beschäftigtenkategorien

33 Sektor Staat: Beschäftigte in F\&E 2006 nach Rechtsträgern und Beschäftigtenkategorien

34 Sektor Staat: Ausgaben für F\&E 2006 nach Wissenschaftszweigen und Ausgabenarten

35 Sektor Staat: Ausgaben für F\&E 2006 nach Rechtsträgern und Ausgabenarten

36 Sektor Staat: Ausgaben für F\&E 2006 nach Wissenschaftszweigen und Forschungsarten

37 Sektor Staat: Ausgaben für F\&E 2006 nach Rechtsträgern und Forschungsarten

38 Sektor Staat: Finanzierung der Ausgaben für F\&E 2006 nach Wissenschaftszweigen und Finanzierungsbereichen 
39 Sektor Staat: Finanzierung der Ausgaben für F\&E 2006 nach Rechtsträgern und Finanzierungsbereichen

40 Privater gemeinnütziger Sektor: Beschäftigte in F\&E 2006 nach Wissenschaftszweigen und Beschäftigtenkategorien

41 Privater gemeinnütziger Sektor: Ausgaben für F\&E 2006 nach Wissenschaftszweigen und Ausgabenarten

42 Privater gemeinnütziger Sektor: Ausgaben für F\&E 2006 nach Wissenschaftszweigen und Forschungsarten

43 Privater gemeinnütziger Sektor: Finanzierung der Ausgaben für F\&E 2006 nach Wissenschaftszweigen und Finanzierungsbereichen

44 Unternehmenssektor: Beschäftigte in F\&E 2006 nach Wirtschaftszweigen und Beschäftigtengrößenklassen

45 Unternehmenssektor: Wissenschaftler und Ingenieure in F\&E 2006 nach Wirtschaftszweigen, Ausbildung und Geschlecht

46 Unternehmenssektor: Beschäftigte in F\&E 2006 und Ausgaben für F\&E 2006 nach Bundesländern

47 Unternehmenssektor: Ausgaben für F\&E 2006 nach Wirtschaftszweigen, Beschäftigtengrößenklassen und Ausgabenarten

48 Unternehmenssektor: Ausgaben für F\&E 2006 nach Wirtschaftszweigen und Forschungsarten

49 Unternehmenssektor: Finanzierung der Ausgaben für F\&E 2006 nach Wirtschaftszweigen und Finanzierungssektoren

50 Pfad vom 4. zum 7. EU-Forschungsrahmenprogramm

51 Ergebnisse Österreich im 6. RP

52 Ergebnisse Österreich im 7. RP

53 FFG: Förderstatistik 2008 - Gesamtübersicht

54 FFG: Geförderte Projekte 2008 gemäß der Systematik der Wirtschaftstätigkeit (NACE)

55 FFG: Förderstatistik 2008 nach Bundesländern

56 FWF: Bewiligungen nach Forschungsstätten (Mio. €) 2008 - autonomer Bereich

57 FWF: Bewilligungen nach Wissenschaftsdisziplinen (Mio. €) 2006 - 2008 - autonomer Bereich

58 FWF: ERA-NET Beteiligungen

59 aws: Leistungsüberblick Beratung und Service, 2008

60 aws: Hochtechnologie: Beratung, Betreuung, Vermittlung (2008) 


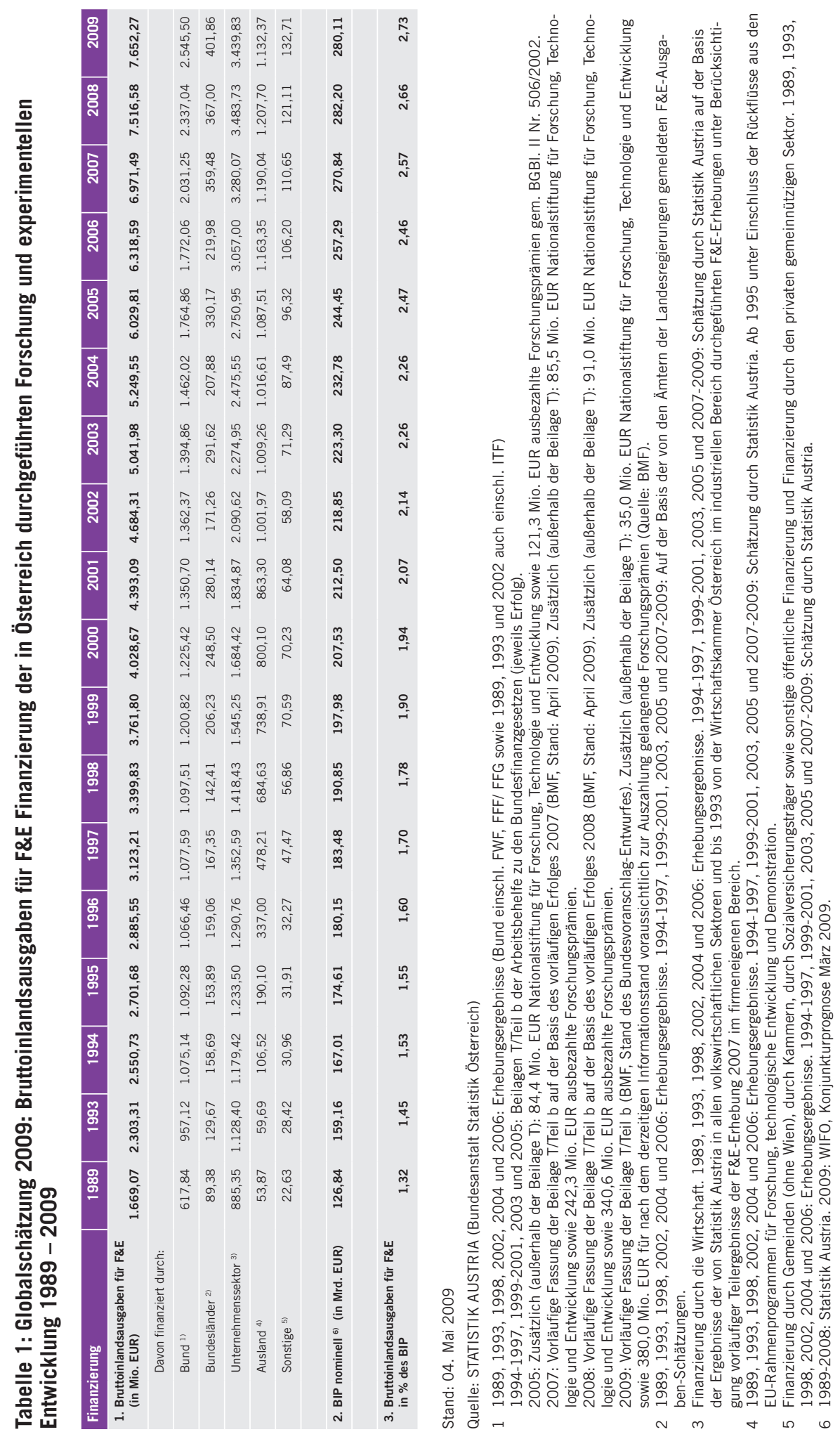




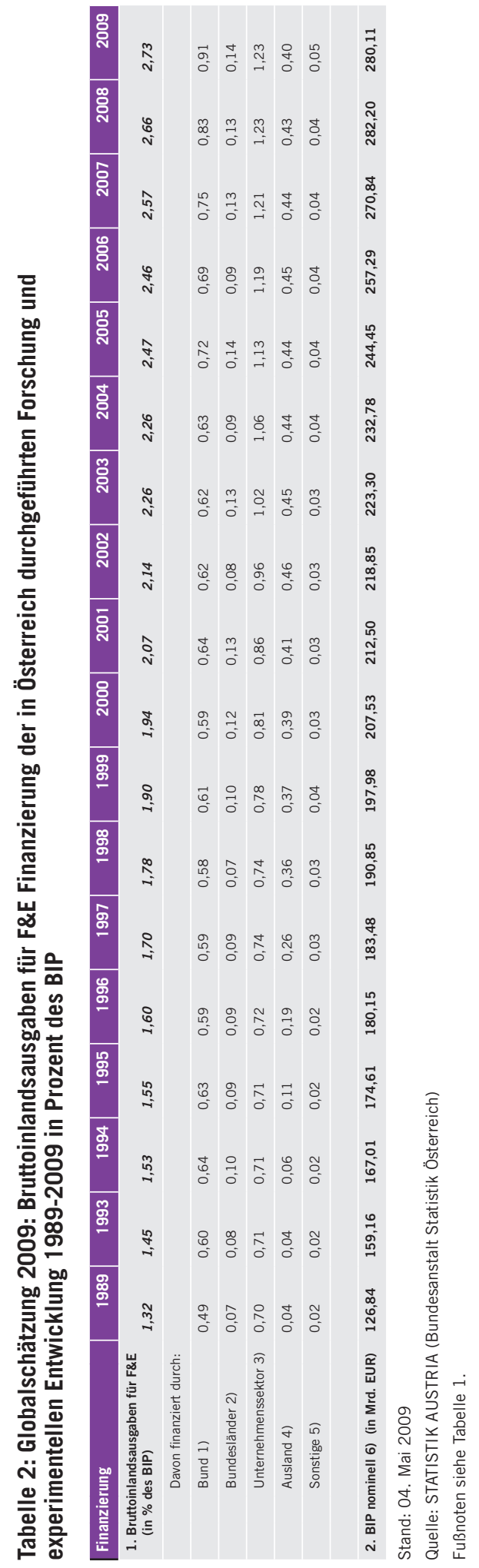




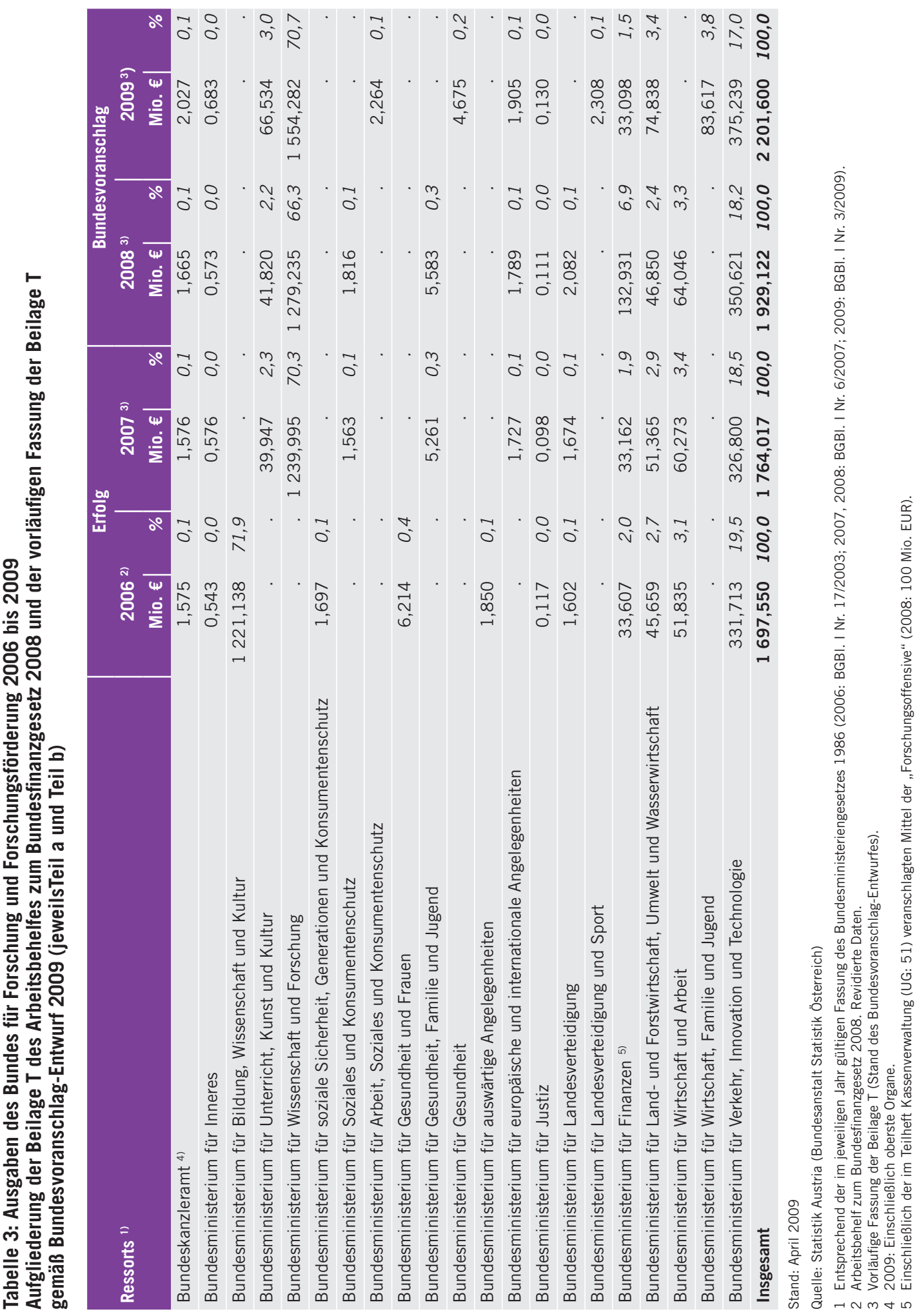




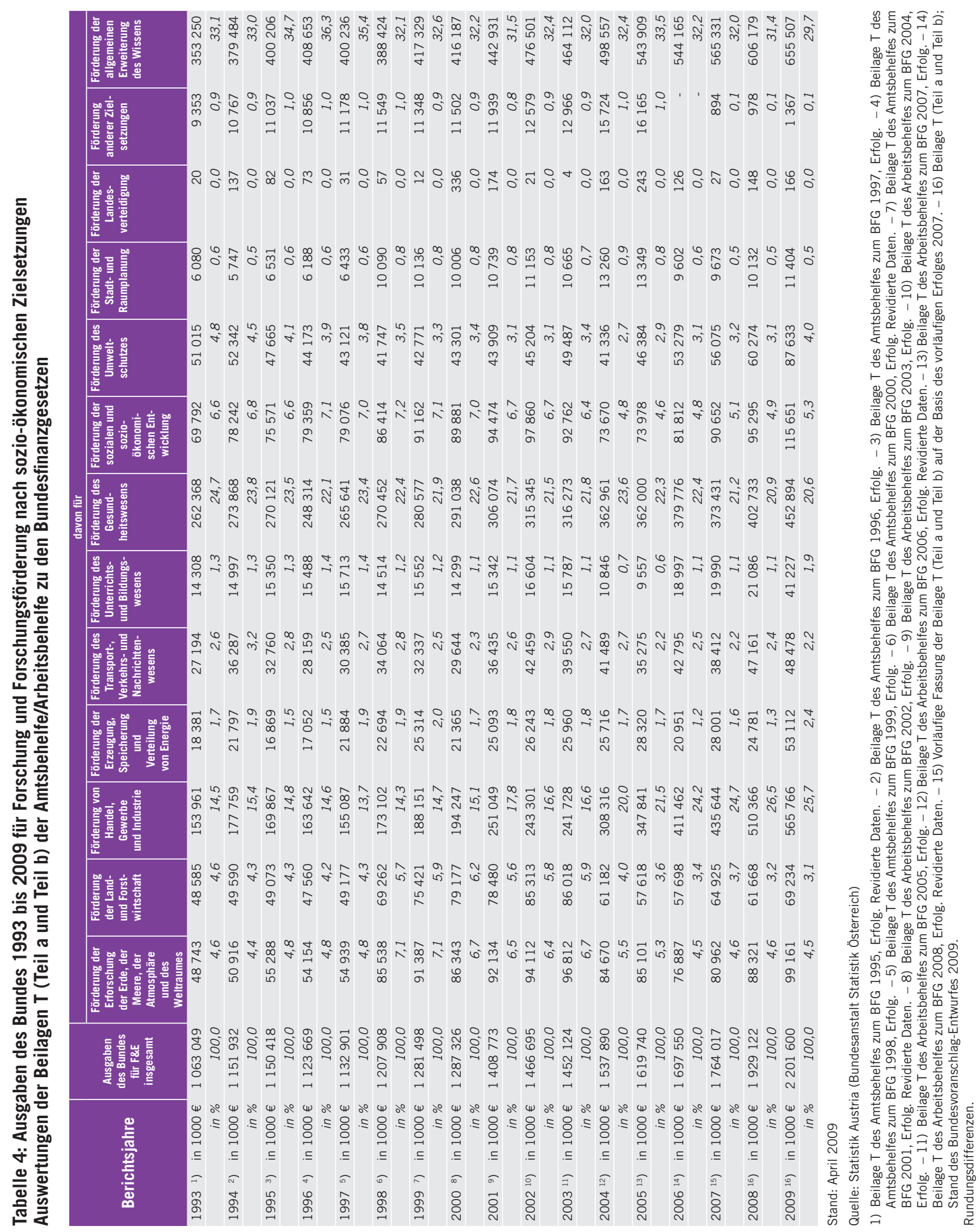




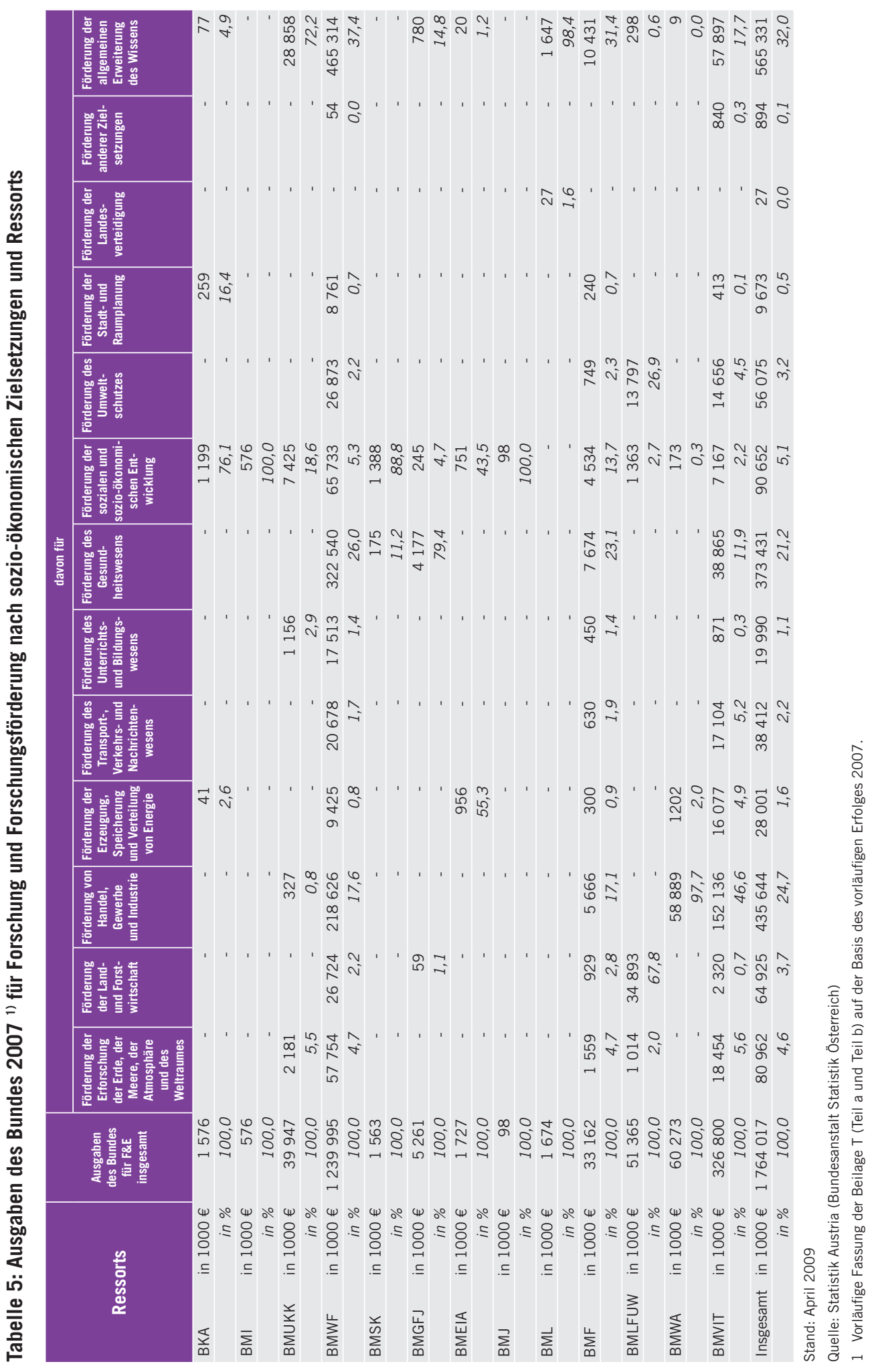




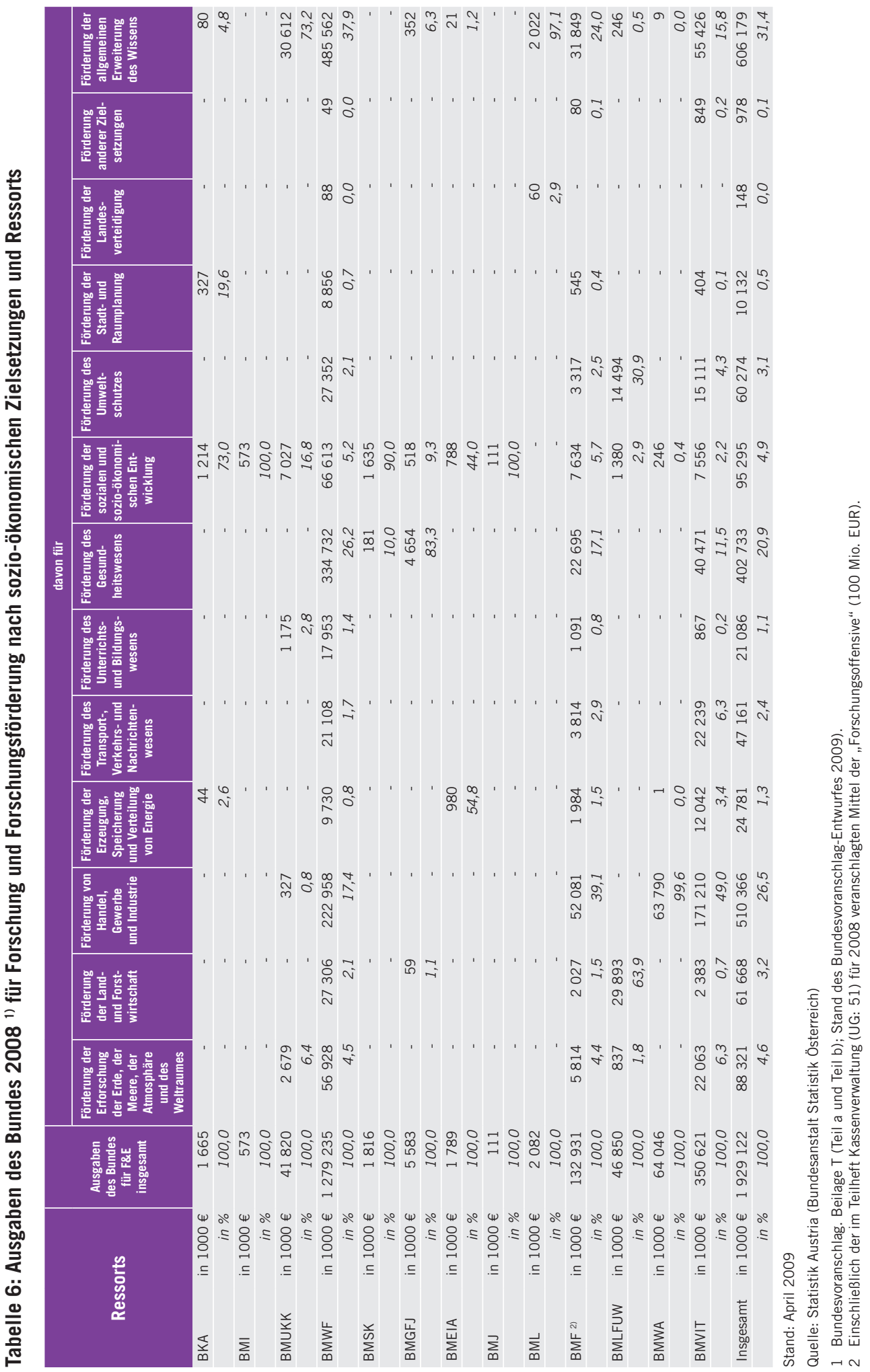




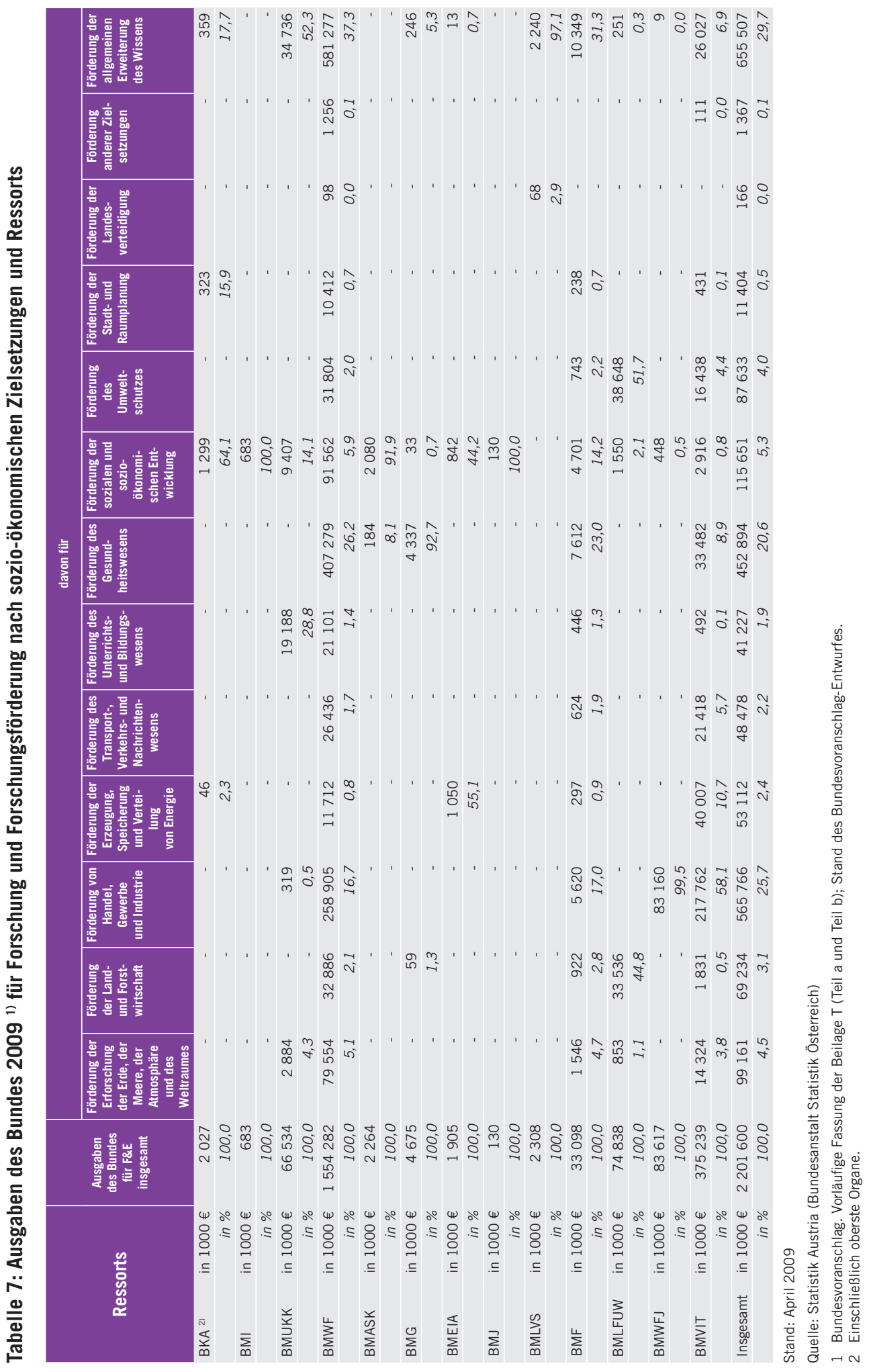




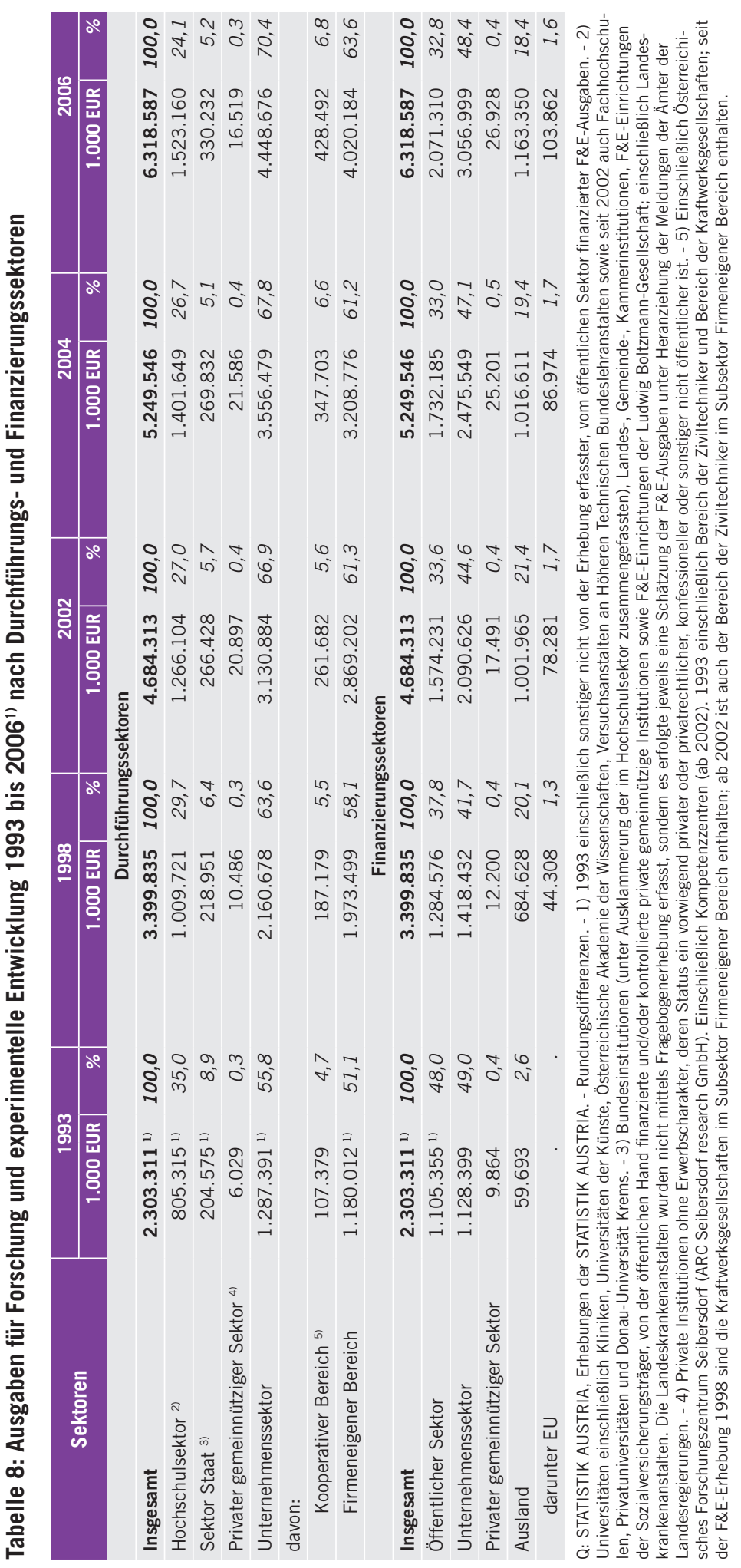


Tabelle 9: Allgemeine forschungswirksame Hochschulausgaben des Bundes 1999 - 2009 1) "General University Funds“

\begin{tabular}{|c|c|c|}
\hline \multirow{3}{*}{ Jahre } & \multicolumn{2}{|c|}{ Allgemeine Hochschulausgaben } \\
\hline & insgesamt & F\&E \\
\hline & \multicolumn{2}{|c|}{ Mio $€$} \\
\hline 1999 & 1960,216 & 834,529 \\
\hline 2000 & 1956,167 & 842,494 \\
\hline 2001 & 2008,803 & 866,361 \\
\hline 2002 & 2104,550 & 918,817 \\
\hline 2003 & 2063,685 & 899,326 \\
\hline 2004 & 2091,159 & 980,984 \\
\hline 2005 & 2136,412 & 1014,543 \\
\hline 2006 & 2157,147 & 1027,270 \\
\hline 2007 & 2314,658 & 1083,258 \\
\hline 2008 & 2352,334 & 1113,728 \\
\hline 2009 & 2637,851 & 1255,162 \\
\hline
\end{tabular}

Quelle: Statistik Austria (Bundesanstalt Statistik Österreich); Stand: April 2009

1 Auf Basis der Beilagen T der Arbeitsbehelfe zu den Bundesfinanzgesetzen.

2007: Vorläufige Fassung der Beilage T auf der Basis des vorl. Erfolges 2007.

2008, 2009: Jeweils Voranschlag. Stand: Bundesvoranschlag-Entwurf 2009. 
Tabelle 10: Forschung und experimentelle Entwicklung (F\&E) 2006 im internationalen Vergleich

\begin{tabular}{|c|c|c|c|c|c|c|c|c|}
\hline \multirow{4}{*}{ Land } & \multirow{4}{*}{$\begin{array}{c}\text { Brutto- } \\
\text { inlands- } \\
\text { ausgaben } \\
\text { für F\&E } \\
\text { in \% des BIP }\end{array}$} & \multirow{2}{*}{\multicolumn{2}{|c|}{$\begin{array}{l}\text { Finanzierung der } \\
\text { Bruttoinlandsausgaben } \\
\text { für F\&E durch }\end{array}$}} & \multirow{4}{*}{$\begin{array}{c}\text { Beschäftigte } \\
\text { in F\&E } \\
\text { in Vollzeit- } \\
\text { äquivalent }\end{array}$} & \multicolumn{4}{|c|}{ Bruttoausgaben für F\&E des } \\
\hline & & & & & \multirow{2}{*}{$\begin{array}{c}\text { Unter- } \\
\text { nehmens- } \\
\text { sektors }\end{array}$} & \multirow{2}{*}{$\begin{array}{l}\text { Hochschul- } \\
\text { sektors }\end{array}$} & \multirow{2}{*}{$\begin{array}{l}\text { Sektors } \\
\text { Staat }\end{array}$} & \multirow{2}{*}{$\begin{array}{c}\text { privaten } \\
\text { gemeinnützigen } \\
\text { Sektors }\end{array}$} \\
\hline & & Staat & Wirtschaft & & & & & \\
\hline & & \multicolumn{2}{|c|}{$\%$} & & \multicolumn{4}{|c|}{ in \% der Bruttoinlandsausgaben für F\&E } \\
\hline Belgien & $1,88^{\mathrm{p})}$ & $24,7^{3)}$ & $59,7^{3)}$ & $55.204^{p)}$ & $69,2^{p)}$ & $22,2^{p)}$ & $7,9^{p)}$ & $0,7^{p)}$ \\
\hline Dänemark & 2,46 & $27,6^{3)}$ & $59,5^{3)}$ & 44.878 & 66,9 & 25,9 & 6,6 & 0,6 \\
\hline Deutschland & 2,54 & 27,8 & 68,1 & 487.260 & 69,9 & 16,3 & $13,9^{\circ}$ &.$^{n)}$ \\
\hline Finnland & 3,45 & 25,1 & 66,6 & 58.257 & 71,3 & 18,7 & 9,4 & 0,6 \\
\hline Frankreich & $2,10^{p)}$ & $38,4^{p)}$ & $52,4^{\mathrm{p})}$ & 363.867 & $63,1^{\mathrm{p})}$ & $19,2^{\mathrm{p})}$ & $16,5^{\mathrm{p})}$ & $1,2^{\mathrm{p})}$ \\
\hline Griechenland & $0,57^{c)}$ & $46,8^{3)}$ & $31,1^{3)}$ & $35.140^{c)}$ & $30,0^{c)}$ & $47,8^{c)}$ & $20,8^{c)}$ & $1,3^{\circ)}$ \\
\hline Irland p) & 1,32 & 30,1 & 59,3 & 17.660 & 67,5 & 26,0 & 6,5 & . \\
\hline Italien & 1,14 & 48,3 & 40,4 & 192.002 & 48,8 & 30,3 & 17,2 & 3,7 \\
\hline Luxemburg & 1,66 & $16,6^{3)}$ & $79,7^{3)}$ & 4.377 & 86,1 & 2,1 & 11,8 & . \\
\hline Niederlande & $1,73^{p)}$ & $36,2^{1)}$ & $51,1^{1)}$ & $96.861^{p)}$ & $59,2^{\mathrm{p})}$ & $27,2^{\mathrm{clp})}$ & 13,6 op) &.$^{\text {n) }}$ \\
\hline Österreich & $2,46^{4)}$ & $32,8^{4)}$ & $48,4^{4)}$ & $49.377^{4)}$ & $70,4^{4)}$ & $24,1^{4)}$ & $5,2^{4)}$ & $0,3^{4)}$ \\
\hline Portugal & $1,00^{c)}$ & $55,2^{3)}$ & $36,3^{3)}$ & 30.160 c) & $46,5^{c)}$ & $32,0^{c)}$ & $11,3^{c)}$ & $10,3^{c)}$ \\
\hline Schweden & 3,74 & 23,2 a)3) & $65,7^{\text {а)3) }}$ & 78.715 & 74,7 & $20,6^{\text {p) }}$ & 4,5 & 0,2 \\
\hline Spanien & 1,20 & 42,5 & 47,1 & 188.978 & 55,5 & 27,6 & 16,7 & 0,2 \\
\hline Vereinigtes Königreich & 1,78 & 31,9 & 45,2 & $334.686^{b)}$ & 61,7 & 26,1 & 10,0 & 2,2 \\
\hline EU $15^{b)}$ & 1,89 & 33,4 & 55,6 & 2.037 .422 & 63,9 & 22,3 & 12,7 & 1,2 \\
\hline Polen & 0,56 & 57,5 & 33,1 & 73.554 & 31,5 & 31,0 & 37,0 & 0,4 \\
\hline Slowakische Republik & 0,49 & 55,6 & 35,0 & 15.028 & 43,1 & 24,1 & $32,8^{d)}$ & 0,1 \\
\hline Slowenien & 1,59 & 34,4 & 59,3 & 9.793 & 60,2 & 15,1 & 24,5 & 0,2 \\
\hline Tschechische Republik & 1,55 & 39,0 & 56,9 & 47.729 & 66,2 & 15,9 & 17,6 & 0,4 \\
\hline Ungarn & 1,00 & 44,8 & 43,3 & 25.971 & $48,3^{v}$ & $24,4^{v)}$ & $25,4^{v)}$ & . \\
\hline EU 25 b) & 1,80 & 34,0 & 55,1 & 2.234 .173 & 63,2 & 22,4 & 13,2 & 1,2 \\
\hline Australien & 2,01 & 38,4 & 57,2 & 125.770 & 57,3 & 25,7 & 14,1 & 2,9 \\
\hline Island ${ }^{3)}$ & 2,77 & 40,5 & 48,0 & 3.226 & 51,5 & 22,0 & 23,5 & 3,0 \\
\hline Japan & 3,39 & $16,2^{\text {e) }}$ & 77,1 & 935.182 & 77,2 & 12,7 & 8,3 & 1,9 \\
\hline Kanada p) & 1,94 & $32,7^{c)}$ & 48,0 & 213.930 c)3) & 54,7 & 35,5 & 9,3 & 0,4 \\
\hline Korea g) & 3,22 & 23,1 & 75,5 & 237.599 & 77,3 & 10,0 & 11,6 & 1,2 \\
\hline Mexiko ${ }^{3)}$ & 0,46 & 45,3 & 46,5 & 89.398 & 49,5 & 27,4 & 22,1 & 1,0 \\
\hline Neuseeland ${ }^{3)}$ & 1,16 & 43,0 & 41,3 & 23.178 & 41,8 & 32,5 & 25,7 & . \\
\hline Norwegen & 1,52 & $44,0^{3)}$ & $46,4^{3)}$ & 31.745 & 54,2 & 30,2 & 15,7 & . \\
\hline Schweiz ${ }^{2)}$ & 2,90 & 22,7 & 69,7 & 52.250 & 73,7 & 22,9 & $1,1^{\text {h) }}$ & 2,3 \\
\hline Türkei & 0,58 & 48,6 & 46,1 & 54.444 & 37,0 & 51,3 & 11,7 & . \\
\hline Vereinigte Staaten j) & 2,66 & 29,1 & $65,2^{\circ}$ & . & 71,0 & 13,5 & $11,3^{\mathrm{h})}$ & 4,2 \\
\hline OECD insgesamt ${ }^{b)}$ & 2,26 & 28,5 & 63,9 & . & 69,1 & 17,2 & 11,4 & 2,6 \\
\hline
\end{tabular}

Quelle: OECD (MSTI 2008-2), Statistik Austria (Bundesanstalt Statistik Österreich)

a) Bruch in der Zeitreihe. - b) Schätzung des OECD-Sekretariates (basierend auf nationalen Quellen). - c) Nationale Schätzung, wenn erforderlich vom OECD-Sekretariat den OECD-Normen angepasst. - d) F\&E-Ausgaben für Landesverteidigung nicht enthalten. - e) Nationale Erhebungsergebnisse; vom OECD-Sekretariat den OECD-Normen angepasst. - g) Nur naturwissenschaftlich-technische Forschung. - h) Nur Bundesmittel oder Mittel der Zentralregierung. - j) Ohne Investitionsausgaben. - n) Anderswo enthalten. - o) Enthält auch andere Kategorien. - p) Vorläufige Werte. - v) Die Summe der Gliederungselemente ergibt nicht die Gesamtsumme.

1) 2003. - 2) 2004. - 3) 2005. - 4) Statistik Austria; Ergebnisse der Erhebung über Forschung und experimentelle Entwicklung 2006.

Vollzeitäquivalent $=$ Personenjahr 
Tabelle 11: Beschäftigte in Forschung und experimenteller Entwicklung (F\&E) in Kopfzahlen und in Vollzeitäquivalenten 2006 nach Durchführungssektoren/ Erhebungsbereichen und Beschäftigtenkategorien

\begin{tabular}{|c|c|c|c|c|c|}
\hline \multirow[b]{2}{*}{ Sektoren, Bereiche } & \multirow{2}{*}{$\begin{array}{c}\text { F\&E } \\
\text { durch- } \\
\text { führende } \\
\text { Erhebungs- } \\
\text { einheiten }\end{array}$} & \multirow[b]{2}{*}{ Insgesamt } & \multicolumn{3}{|c|}{ davon } \\
\hline & & & $\begin{array}{l}\text { Wissenschaft- } \\
\text { liches Personal }\end{array}$ & $\begin{array}{l}\text { Höherqualifiziertes } \\
\text { nichtwissenschaft- } \\
\text { liches Personal }\end{array}$ & $\begin{array}{l}\text { Sonstiges } \\
\text { Hilfspersonal }\end{array}$ \\
\hline & \multicolumn{5}{|c|}{ Kopfzahlen } \\
\hline Insgesamt & 3.863 & 83.966 & 49.597 & 23.884 & 10.485 \\
\hline 1. Hochschulsektor & 1.162 & 32.715 & 23.609 & 4.954 & 4.152 \\
\hline \multicolumn{6}{|l|}{ davon: } \\
\hline 1.1 Universitäten (ohne Kliniken) & 928 & 23.799 & 17.174 & 3.477 & 3.148 \\
\hline 1.2 Universitätskliniken & 89 & 5.454 & 3.706 & 905 & 843 \\
\hline 1.3 Universitäten der Künste & 44 & 827 & 721 & 61 & 45 \\
\hline 1.4 Akademie der Wissenschaften & 61 & 1.167 & 908 & 238 & 21 \\
\hline 1.5 Fachhochschulen & 17 & 961 & 726 & 193 & 42 \\
\hline 1.6 Sonstiger Hochschulsektor ${ }^{1)}$ & 23 & 507 & 374 & 80 & 53 \\
\hline 2. Sektor Staat ${ }^{2)}$ & 254 & 5.511 & 2.789 & 1.129 & 1.593 \\
\hline \multicolumn{6}{|l|}{ davon: } \\
\hline 2.1 Ohne Landeskrankenanstalten & 254 & 5.511 & 2.789 & 1.129 & 1.593 \\
\hline \multicolumn{6}{|l|}{ 2.2 Landeskrankenanstalten } \\
\hline 3. Privater gemeinnütziger Sektor ${ }^{3)}$ & 40 & 404 & 284 & 72 & 48 \\
\hline 4. Unternehmenssektor & 2.407 & 45.336 & 22.915 & 17.729 & 4.692 \\
\hline \multicolumn{6}{|l|}{ davon: } \\
\hline 4.1 Kooperativer Bereich ${ }^{4)}$ & 52 & 4.928 & 2.820 & 1.084 & 1.024 \\
\hline \multirow[t]{2}{*}{ 4.2 Firmeneigener Bereich } & 2.355 & 40.408 & 20.095 & 16.645 & 3.668 \\
\hline & \multicolumn{5}{|c|}{ Vollzeitäquivalente } \\
\hline Insgesamt & 3.863 & $49.377,1$ & $29.198,7$ & $14.821,7$ & $5.356,8$ \\
\hline 1. Hochschulsektor & 1.162 & $12.668,2$ & $9.261,3$ & $1.855,6$ & $1.551,3$ \\
\hline \multicolumn{6}{|l|}{ davon: } \\
\hline 1.1 Universitäten (ohne Kliniken) & 928 & $9.986,2$ & $7.339,6$ & $1.321,8$ & $1.324,9$ \\
\hline 1.2 Universitätskliniken & 89 & $1.333,0$ & 815,8 & 342,3 & 174,9 \\
\hline 1.3 Universitäten der Künste & 44 & 179,5 & 158,4 & 12,5 & 8,6 \\
\hline 1.4 Akademie der Wissenschaften & 61 & 627,9 & 542,7 & 74,9 & 10,3 \\
\hline 1.5 Fachhochschulen & 17 & 384,2 & 288,8 & 76,8 & 18,6 \\
\hline 1.6 Sonstiger Hochschulsektor ${ }^{1)}$ & 23 & 157,4 & 116,0 & 27,3 & 14,1 \\
\hline 2. Sektor Staat ${ }^{2)}$ & 254 & $2.422,6$ & $1.348,5$ & 356,5 & 717,6 \\
\hline \multicolumn{6}{|l|}{ davon: } \\
\hline 2.1 Ohne Landeskrankenanstalten & 254 & $2.422,6$ & $1.348,5$ & 356,5 & 717,6 \\
\hline 2.2 Landeskrankenanstalten & . & . & . & . & \\
\hline 3. Privater gemeinnütziger Sektor ${ }^{3)}$ & 40 & 160,5 & 118,4 & 26,3 & 15,9 \\
\hline 4. Unternehmenssektor & 2.407 & $34.125,8$ & $18.470,5$ & $12.583,3$ & $3.072,0$ \\
\hline \multicolumn{6}{|l|}{ davon: } \\
\hline 4.1 Kooperativer Bereich ${ }^{4)}$ & 52 & $3.342,3$ & $2.095,1$ & 530,6 & 716,6 \\
\hline 4.2 Firmeneigener Bereich & 2.355 & $30.783,5$ & $16.375,4$ & $12.052,7$ & $2.355,4$ \\
\hline
\end{tabular}

Q: STATISTIK AUSTRIA, Erhebung über Forschung und experimentelle Entwicklung 2006. Erstellt am: 08.08.2008. -

1) Privatuniversitäten und Donau-Universität Krems (aus Geheimhaltungsgründen zusammengefasst). - 2) Bundesinstitutionen (unter Ausklammerung der im Hochschulsektor zusammengefassten), Landes-, Gemeinde- und Kammerinstitutionen, F\&E-Einrichtungen der Sozialversicherungsträger, von der öffentlichen Hand finanzierte und/ oder kontrollierte private gemeinnützige Institutionen sowie F\&E-Einrichtungen der Ludwig Boltzmann-Gesellschaft; ohne Landeskrankenanstalten. Die Landeskrankenanstalten wurden nicht mittels Fragebogenerhebung erfasst, sondern es erfolgte eine Schätzung der F\&E-Ausgaben durch Statistik Austria unter Heranziehung der Meldungen der Ämter der Landesregierungen. Daher liegen keine Daten über Beschäftigte in F\&E vor. - 3) Private gemeinnützige Institutionen, deren Status ein vorwiegend privater oder privatrechtlicher, konfessioneller oder sonstiger nicht öffentlicher ist. - 4) Einschließlich ARC Seibersdorf research GmbH sowie Kompetenzzentren. - Rundungsdifferenzen. 
Tabelle 12: Beschäftigte in Forschung und experimenteller Entwicklung (F\&E) in Kopfzahlen und in Vollzeitäquivalenten 2006 nach Durchführungssektoren/ Erhebungsbereichen, Beschäftigtenkategorien und Geschlecht

\begin{tabular}{|c|c|c|c|c|c|c|c|c|c|}
\hline \multirow{3}{*}{ Sektoren, Bereiche } & \multirow{3}{*}{$\begin{array}{c}\text { F\&E } \\
\text { durch- } \\
\text { führende } \\
\text { Erhebungs- } \\
\text { einheiten }\end{array}$} & \multirow{2}{*}{\multicolumn{2}{|c|}{ Insgesamt }} & \multicolumn{6}{|c|}{ davon } \\
\hline & & & & \multicolumn{2}{|c|}{$\begin{array}{l}\text { Wissenschaft- } \\
\text { liches Personal }\end{array}$} & \multicolumn{2}{|c|}{$\begin{array}{l}\text { Höherqualifiziertes } \\
\text { nichtwissenschaft- } \\
\text { liches Personal }\end{array}$} & \multicolumn{2}{|c|}{$\begin{array}{l}\text { Sonstiges } \\
\text { Hilfspersonal }\end{array}$} \\
\hline & & männl. & weibl. & männl. & weibl. & männl. & weibl. & männl. & weibl. \\
\hline & \multicolumn{9}{|c|}{ Kopfzahlen } \\
\hline Insgesamt & 3.863 & 59.236 & 24.730 & 37.056 & 12.541 & 17.105 & 6.779 & 5.075 & 5.410 \\
\hline 1. Hochschulsektor & 1.162 & 18.367 & 14.348 & 15.419 & 8.190 & 1.731 & 3.223 & 1.217 & 2.935 \\
\hline \multicolumn{10}{|l|}{ davon: } \\
\hline 1.1 Universitäten (ohne Kliniken) & 928 & 13.733 & 10.066 & 11.403 & 5.771 & 1.279 & 2.198 & 1.051 & 2.097 \\
\hline 1.2 Universitätskliniken & 89 & 2.633 & 2.821 & 2.306 & 1.400 & 197 & 708 & 130 & 713 \\
\hline 1.3 Universitäten der Künste & 44 & 447 & 380 & 417 & 304 & 15 & 46 & 15 & 30 \\
\hline 1.4 Akademie der Wissenschaften & 61 & 636 & 531 & 532 & 376 & 102 & 136 & 2 & 19 \\
\hline 1.5 Fachhochschulen & 17 & 636 & 325 & 515 & 211 & 110 & 83 & 11 & 31 \\
\hline 1.6 Sonstiger Hochschulsektor ${ }^{1)}$ & 23 & 282 & 225 & 246 & 128 & 28 & 52 & 8 & 45 \\
\hline 2. Sektor Staat ${ }^{2)}$ & 254 & 3.037 & 2.474 & 1.694 & 1.095 & 592 & 537 & 751 & 842 \\
\hline \multicolumn{10}{|l|}{ davon: } \\
\hline 2.1 Ohne Landeskrankenanstalten & 254 & 3.037 & 2.474 & 1.694 & 1.095 & 592 & 537 & 751 & 842 \\
\hline \multicolumn{10}{|l|}{ 2.2 Landeskrankenanstalten } \\
\hline 3. Privater gemeinnütziger Sektor ${ }^{3)}$ & 40 & 172 & 232 & 137 & 147 & 25 & 47 & 10 & 38 \\
\hline 4. Unternehmenssektor & 2.407 & 37.660 & 7.676 & 19.806 & 3.109 & 14.757 & 2.972 & 3.097 & 1.595 \\
\hline \multicolumn{10}{|l|}{ davon: } \\
\hline 4.1 Kooperativer Bereich ${ }^{4)}$ & 52 & 3.584 & 1.344 & 2.270 & 550 & 767 & 317 & 547 & 477 \\
\hline \multirow[t]{2}{*}{ 4.2 Firmeneigener Bereich } & 2.355 & 34.076 & 6.332 & 17.536 & 2.559 & 13.990 & 2.655 & 2.550 & 1.118 \\
\hline & \multicolumn{9}{|c|}{ Vollzeitäquivalente } \\
\hline Insgesamt & 3.863 & $37.771,0$ & $11.606,1$ & $23.529,9$ & $5.668,8$ & $11.335,7$ & $3.486,0$ & $2.905,4$ & $2.451,5$ \\
\hline 1. Hochschulsektor & 1.162 & $7.523,9$ & $5.144,3$ & $6.383,5$ & $2.877,8$ & 642,9 & $1.212,7$ & 497,6 & $1.053,8$ \\
\hline \multicolumn{10}{|l|}{ davon: } \\
\hline 1.1 Universitäten (ohne Kliniken) & 928 & $6.085,0$ & $3.901,2$ & $5.141,8$ & $2.197,8$ & 485,0 & 836,7 & 458,2 & 866,7 \\
\hline 1.2 Universitätskliniken & 89 & 606,9 & 726,1 & 503,5 & 312,3 & 75,1 & 267,3 & 28,3 & 146,6 \\
\hline 1.3 Universitäten der Künste & 44 & 100,6 & 78,9 & 95,6 & 62,9 & 2,8 & 9,7 & 2,2 & 6,4 \\
\hline 1.4 Akademie der Wissenschaften & 61 & 378,0 & 249,8 & 350,9 & 191,8 & 25,3 & 49,6 & 1,8 & 8,4 \\
\hline 1.5 Fachhochschulen & 17 & 264,5 & 119,8 & 214,9 & 73,9 & 44,3 & 32,6 & 5,3 & 13,3 \\
\hline 1.6 Sonstiger Hochschulsektor ${ }^{1)}$ & 23 & 88,9 & 68,5 & 76,8 & 39,3 & 10,4 & 16,8 & 1,7 & 12,4 \\
\hline 2. Sektor Staat ${ }^{2)}$ & 254 & $1.430,2$ & 992,4 & 883,9 & 464,6 & 183,7 & 172,8 & 362,5 & 355,0 \\
\hline \multicolumn{10}{|l|}{ davon: } \\
\hline 2.1 Ohne Landeskrankenanstalten & 254 & $1.430,2$ & 992,4 & 883,9 & 464,6 & 183,7 & 172,8 & 362,5 & 355,0 \\
\hline 2.2 Landeskrankenanstalten & & & & & & & & . & \\
\hline 3. Privater gemeinnütziger Sektor ${ }^{3)}$ & 40 & 65,4 & 95,1 & 55,6 & 62,8 & 6,9 & 19,4 & 2,9 & 13,0 \\
\hline 4. Unternehmenssektor & 2.407 & $28.751,5$ & $5.374,3$ & $16.206,9$ & $2.263,6$ & $10.502,2$ & $2.081,1$ & $2.042,4$ & $1.029,7$ \\
\hline \multicolumn{10}{|l|}{ davon: } \\
\hline 4.1 Kooperativer Bereich ${ }^{4)}$ & 52 & $2.547,4$ & 794,9 & $1.751,5$ & 343,6 & 382,4 & 148,2 & 413,5 & 303,2 \\
\hline 4.2 Firmeneigener Bereich & 2.355 & $26.204,1$ & $4.579,4$ & $14.455,4$ & $1.920,0$ & $10.119,8$ & $1.932,9$ & $1.628,9$ & 726,5 \\
\hline
\end{tabular}

Q: STATISTIK AUSTRIA, Erhebung über Forschung und experimentelle Entwicklung 2006. Erstellt am: 08.08.2008. - 1) Privatuniversitäten und Donau-Universität Krems (aus Geheimhaltungsgründen zusammengefasst). - 2) Bundesinstitutionen (unter Ausklammerung der im Hochschulsektor zusammengefassten), Landes-, Gemeinde- und Kammerinstitutionen, F\&E-Einrichtungen der Sozialversicherungsträger, von der öffentlichen Hand finanzierte und/oder kontrollierte private gemeinnützige Institutionen sowie F\&E-Einrichtungen der Ludwig Boltzmann-Gesellschaft; ohne Landeskrankenanstalten. Die Landeskrankenanstalten wurden nicht mittels Fragebogenerhebung erfasst, sondern es erfolgte eine Schätzung der F\&E-Ausgaben durch Statistik Austria unter Heranziehung der Meldungen der Ämter der Landesregierungen. Daher liegen keine Daten über Beschäftigte in F\&E vor. - 3) Private gemeinnützige Institutionen, deren Status ein vorwiegend privater oder privatrechtlicher, konfessioneller oder sonstiger nicht öffentlicher ist. - 4) Einschließlich ARC Seibersdorf research GmbH sowie Kompetenzzentren. - Rundungsdifferenzen. 
Tabelle 13: Beschäftigte in Forschung und experimenteller Entwicklung (in Vollzeitäquivalenten) in sämtlichen Erhebungsbereichen ${ }^{1)} 2006$ nach Bundesländern"2) und Beschäftigtenkategorien

\begin{tabular}{|c|c|c|c|c|c|}
\hline \multirow{3}{*}{ Bundesländer } & \multirow{3}{*}{$\begin{array}{l}\text { F\&E } \\
\text { durch- } \\
\text { führende } \\
\text { Erhebungs- } \\
\text { einheiten }\end{array}$} & \multicolumn{4}{|c|}{ Vollzeitäquivalente für F\&E } \\
\hline & & \multirow[b]{2}{*}{ Insgesamt } & \multicolumn{3}{|c|}{ davon } \\
\hline & & & $\begin{array}{l}\text { Wissenschaft- } \\
\text { liches Personal }\end{array}$ & $\begin{array}{l}\text { Höherqualifiziertes } \\
\text { nichtwissenschaft- } \\
\text { liches Personal }\end{array}$ & $\begin{array}{c}\text { Sonstiges } \\
\text { Hilfspersonal }\end{array}$ \\
\hline Österreich & 3.863 & $49.377,0$ & $29.198,7$ & $14.821,6$ & $5.356,8$ \\
\hline Burgenland & 53 & 335,7 & 166,4 & 108,4 & 60,9 \\
\hline Kärnten & 185 & $2.326,6$ & $1.728,5$ & 493,1 & 105,0 \\
\hline Niederösterreich & 414 & $3.998,5$ & $1.948,9$ & $1.531,9$ & 517,6 \\
\hline Oberösterreich & 682 & $7.203,0$ & $3.715,5$ & $2.754,4$ & 733,0 \\
\hline Salzburg & 224 & $1.834,6$ & $1.101,3$ & 600,6 & 132,7 \\
\hline Steiermark & 683 & $9.291,7$ & $5.447,0$ & $2.330,1$ & $1.514,6$ \\
\hline Tirol & 339 & $3.751,3$ & $2.332,6$ & $1.042,3$ & 376,5 \\
\hline Vorarlberg & 143 & $1.429,1$ & 668,1 & 699,7 & 61,4 \\
\hline Wien & 1.140 & $19.206,6$ & $12.090,3$ & $5.261,2$ & $1.855,1$ \\
\hline
\end{tabular}

Q: STATISTIK AUSTRIA, Erhebung über Forschung und experimentelle Entwicklung 2006. Erstellt am: 08.08.2008 - 1) Die Landeskrankenanstalten wurden nicht mittels Fragebogenerhebung erfasst, sondern es erfolgte eine Schätzung der F\&E-Ausgaben durch Statistik Austria unter Heranziehung der Meldungen der Ämter der Landesregierungen. Daher liegen keine Daten über Beschäftigte in F\&E vor. - 2) Standardauswertung nach dem Hauptstandort des Unternehmens. - Rundungsdifferenzen. 
Tabelle 14: Ausgaben für Forschung und experimentelle Entwicklung (F\&E) 2006 nach Durchführungssektoren/ Erhebungsbereichen und Ausgabenarten

\begin{tabular}{|c|c|c|c|c|c|c|}
\hline \multirow{3}{*}{ Sektoren, Bereiche } & \multirow{3}{*}{$\begin{array}{c}\text { F\&E } \\
\text { durch- } \\
\text { führende } \\
\text { Erhebungs- } \\
\text { einheiten }\end{array}$} & \multirow[b]{2}{*}{ Insgesamt } & \multicolumn{4}{|c|}{ davon } \\
\hline & & & $\begin{array}{l}\text { Personal- } \\
\text { ausgaben }\end{array}$ & $\begin{array}{c}\text { Laufende } \\
\text { Sachausgaben }\end{array}$ & $\begin{array}{l}\text { Ausgaben für } \\
\text { Ausrüstungs- } \\
\text { investitionen }\end{array}$ & $\begin{array}{c}\text { Bauausgaben } \\
\text { und Ausgaben } \\
\text { für Liegen- } \\
\text { schaftsankäufe }\end{array}$ \\
\hline & & \multicolumn{5}{|c|}{ in 1.000 EUR } \\
\hline Insgesamt & $3.863^{3)}$ & 6.318 .587 & 3.198 .343 & 2.544 .643 & 454.461 & 121.140 \\
\hline 1. Hochschulsektor & 1.162 & 1.523 .160 & 710.514 & 655.102 & 117.422 & 40.122 \\
\hline \multicolumn{7}{|l|}{ davon: } \\
\hline 1.1 Universitäten (ohne Kliniken) & 928 & 1.165 .630 & 555.670 & 514.266 & 93.922 & 1.772 \\
\hline 1.2 Universitätskliniken & 89 & 193.936 & 80.584 & 71.895 & 5.931 & 35.526 \\
\hline 1.3 Universitäten der Künste & 44 & 21.616 & 12.071 & 8.441 & 1.104 & - \\
\hline 1.4 Akademie der Wissenschaften & 61 & 79.629 & 32.358 & 34.649 & 11.573 & 1.049 \\
\hline 1.5 Fachhochschulen & 17 & 43.493 & 20.429 & 18.409 & 4.368 & 287 \\
\hline 1.6 Sonstiger Hochschulsektor ${ }^{1)}$ & 23 & 18.856 & 9.402 & 7.442 & 524 & 1.488 \\
\hline 2. Sektor Staat ${ }^{2)}$ & $254^{3)}$ & 330.232 & 182.296 & 123.416 & 16.234 & 8.286 \\
\hline \multicolumn{7}{|l|}{ davon: } \\
\hline 2.1 Ohne Landeskrankenanstalten & 254 & 215.800 & 121.261 & 78.254 & 12.157 & 4.128 \\
\hline 2.2 Landeskrankenanstalten & . & 114.432 & 61.035 & 45.162 & 4.077 & 4.158 \\
\hline 3. Privater gemeinnütziger Sektor ${ }^{4)}$ & 40 & 16.519 & 7.630 & 8.538 & 348 & 3 \\
\hline 4. Unternehmenssektor & 2.407 & 4.448 .676 & 2.297 .903 & 1.757 .587 & 320.457 & 72.729 \\
\hline \multicolumn{7}{|l|}{ davon: } \\
\hline 4.1 Kooperativer Bereich ${ }^{5)}$ & 52 & 428.492 & 229.416 & 172.220 & 24.067 & 2.789 \\
\hline 4.2 Firmeneigener Bereich & 2.355 & 4.020 .184 & 2.068 .487 & 1.585 .367 & 296.390 & 69.940 \\
\hline
\end{tabular}

Q: STATISTIK AUSTRIA, Erhebung über Forschung und experimentelle Entwicklung 2006. Erstellt am: 19.08.2008. - 1) Privatuniversitäten und Donau-Universität Krems (aus Geheimhaltungsgründen zusammengefasst). - 2) Bundesinstitutionen (unter Ausklammerung der im Hochschulsektor zusammengefassten), Landes-, Gemeinde- und Kammerinstitutionen, F\&E-Einrichtungen der Sozialversicherungsträger, von der öffentlichen Hand finanzierte und/oder kontrollierte private gemeinnützige Institutionen sowie F\&E-Einrichtungen der Ludwig Boltzmann-Gesellschaft; einschließlich Landeskrankenanstalten. Die Landeskrankenanstalten wurden nicht mittels Fragebogenerhebung erfasst, sondern es erfolgte eine Schätzung der F\&E-Ausgaben durch Statistik Austria unter Heranziehung der Meldungen der Ämter der Landesregierungen. - 3) Anzahl der Erhebungseinheiten ohne Landeskrankenanstalten. - 4) Private gemeinnützige Institutionen, deren Status ein vorwiegend privater oder privatrechtlicher, konfessioneller oder sonstiger nicht öffentlicher ist. - 5) Einschließlich ARC Seibersdorf research GmbH sowie Kompetenzzentren. 
Tabelle 15: Ausgaben für Forschung und experimentelle Entwicklung (F\&E) in sämtlichen Erhebungsbereichen 1) 2006 nach Bundesländern'2) und Ausgabenarten

\begin{tabular}{|c|c|c|c|c|c|c|}
\hline \multirow{3}{*}{ Bundesländer } & \multirow{3}{*}{$\begin{array}{c}\text { F\&E } \\
\text { durch- } \\
\text { führende } \\
\text { Erhebungs- } \\
\text { einheiten }{ }^{3)}\end{array}$} & \multirow[b]{2}{*}{ Insgesamt } & \multicolumn{4}{|c|}{ davon } \\
\hline & & & $\begin{array}{l}\text { Personal- } \\
\text { ausgaben }\end{array}$ & $\begin{array}{c}\text { Laufende } \\
\text { Sachausgaben }\end{array}$ & $\begin{array}{l}\text { Ausgaben für } \\
\text { Ausrïstungs- } \\
\text { investitionen }\end{array}$ & $\begin{array}{l}\text { Bauausgaben } \\
\text { und Ausgaben } \\
\text { für Liegen- } \\
\text { schaftsankäufe }\end{array}$ \\
\hline & & \multicolumn{5}{|c|}{ in 1.000 EUR } \\
\hline Österreich & 3.863 & 6.318 .587 & 3.198 .343 & 2.544 .643 & 454.461 & 121.140 \\
\hline Burgenland & 53 & 34.383 & 18.360 & 10.997 & 4.804 & 222 \\
\hline Kärnten & 185 & 393.752 & 170.180 & 168.824 & 51.455 & 3.293 \\
\hline Niederösterreich & 414 & 461.530 & 253.813 & 162.870 & 36.118 & 8.729 \\
\hline Oberösterreich & 682 & 964.932 & 434.488 & 446.951 & 64.583 & 18.910 \\
\hline Salzburg & 224 & 200.919 & 111.175 & 62.562 & 23.056 & 4.126 \\
\hline Steiermark & 683 & 1.121 .674 & 575.445 & 458.407 & 72.433 & 15.389 \\
\hline Tirol & 339 & 556.675 & 226.203 & 248.455 & 51.502 & 30.515 \\
\hline Vorarlberg & 143 & 154.619 & 93.962 & 47.476 & 8.368 & 4.813 \\
\hline Wien & 1.140 & 2.430 .103 & 1.314 .717 & 938.101 & 142.142 & 35.143 \\
\hline
\end{tabular}

Q: STATISTIK AUSTRIA, Erhebung über Forschung und experimenteller Entwicklung 2006. erstellt am: 25.08.2008. - 1) Einschließlich F\&E-Ausgaben-Schätzung für Landeskrankenanstalten. - 2) Standardauswertung nach dem Hauptstandort des Unternehmens. - 3) Anzahl der Erhebungseinheiten ohne Landeskrankenanstalten. 
Tabelle 16: Ausgaben für Forschung und experimentelle Entwicklung (F\&E) 2006 nach Durchführungssektoren/ Erhebungsbereichen und Forschungsarten

\begin{tabular}{|c|c|c|c|c|c|c|c|c|}
\hline \multirow{3}{*}{ Sektoren, Bereiche } & \multirow{3}{*}{$\begin{array}{l}\text { F\&E } \\
\text { durch- } \\
\text { führende } \\
\text { Erhebungs- } \\
\text { einheiten }\end{array}$} & \multirow{3}{*}{$\begin{array}{c}\begin{array}{c}\text { Ausgaben für } \\
\text { F\&E insge- } \\
\text { samt }\end{array} \\
\text { in } 1.000 \text { EUR }\end{array}$} & \multicolumn{6}{|c|}{ davon } \\
\hline & & & \multicolumn{2}{|c|}{ Grundlagenforschung } & \multicolumn{2}{|c|}{$\begin{array}{l}\text { Angewandte For- } \\
\text { schung }\end{array}$} & \multicolumn{2}{|c|}{$\begin{array}{l}\text { Experimentelle Ent- } \\
\text { wicklung }\end{array}$} \\
\hline & & & in 1.000 EUR & in $\%$ & in 1.000 EUR & in $\%$ & in 1.000 EUR & in $\%$ \\
\hline Insgesamt & 3.863 & 6.204 .155 & 1.064 .476 & 17,2 & 2.193 .550 & 35,4 & 2.946 .129 & 47,4 \\
\hline 1. Hochschulsektor & 1.162 & 1.523 .160 & 746.112 & 49,0 & 638.642 & 41,9 & 138.406 & 9,1 \\
\hline \multicolumn{9}{|l|}{ davon: } \\
\hline 1.1 Universitäten (ohne Kliniken) & 928 & 1.165 .630 & 613.773 & 52,6 & 468.344 & 40,2 & 83.513 & 7,2 \\
\hline 1.2 Universitätskliniken & 89 & 193.936 & 52.216 & 26,9 & 110.220 & 56,9 & 31.500 & 16,2 \\
\hline 1.3 Universitäten der Künste & 44 & 21.616 & 6.628 & 30,7 & 9.757 & 45,1 & 5.231 & 24,2 \\
\hline 1.4 Akademie der Wissenschaften & 61 & 79.629 & 64.178 & 80,6 & 9.922 & 12,5 & 5.529 & 6,9 \\
\hline 1.5 Fachhochschulen & 17 & 43.493 & 1.938 & 4,5 & 29.970 & 68,9 & 11.585 & 26,6 \\
\hline 1.6 Sonstiger Hochschulsektor ${ }^{1)}$ & 23 & 18.856 & 7.379 & 39,1 & 10.429 & 55,3 & 1.048 & 5,6 \\
\hline 2. Sektor Staat ${ }^{2)}$ & 254 & 215.800 & 69.532 & 32,2 & 127.711 & 59,2 & 18.557 & 8,6 \\
\hline \multicolumn{9}{|l|}{ davon: } \\
\hline 2.1 Ohne Landeskrankenanstalten & 254 & 215.800 & 69.532 & 32,2 & 127.711 & 59,2 & 18.557 & 8,6 \\
\hline 2.2 Landeskrankenanstalten & & & . & & & & . & \\
\hline 3. Privater gemeinnütziger Sektor ${ }^{3)}$ & 40 & 16.519 & 3.682 & 22,3 & 12.076 & 73,1 & 761 & 4,6 \\
\hline 4. Unternehmenssektor & 2.407 & 4.448 .676 & 245.150 & 5,5 & 1.415 .121 & 31,8 & 2.788 .405 & 62,7 \\
\hline \multicolumn{9}{|l|}{ davon: } \\
\hline 4.1 Kooperativer Bereich ${ }^{4)}$ & 52 & 428.492 & 107.534 & 25,1 & 230.739 & 53,8 & 90.219 & 21,1 \\
\hline 4.2 Firmeneigener Bereich & 2.355 & 4.020 .184 & 137.616 & 3,4 & 1.184 .382 & 29,5 & 2.698 .186 & 67,1 \\
\hline
\end{tabular}

Q: STATISTIK AUSTRIA, Erhebung über Forschung und experimentelle Entwicklung 2006. Erstellt am: 25.08.2008. - 1) Privatuniversitäten und Donau-Universität Krems (aus Geheimhaltungsgründen zusammengefasst). - 2) Bundesinstitutionen (unter Ausklammerung der im Hochschulsektor zusammengefassten), Landes-, Gemeinde- und Kammerinstitutionen, F\&E-Einrichtungen der Sozialversicherungsträger, von der öffentlichen Hand finanzierte und/oder kontrollierte private gemeinnützige Institutionen sowie F\&E-Einrichtungen der Ludwig Boltzmann-Gesellschaft; ohne Landeskrankenanstalten. Die Landeskrankenanstalten wurden nicht mittels Fragebogenerhebung erfasst, sondern es erfolgte eine Schätzung der F\&E-Ausgaben durch Statistik Austria unter Heranziehung der Meldungen der Ämter der Landesregierungen. Eine Aufgliederung der F\&E-Ausgaben nach Forschungsarten liegt nicht vor. - 3) Private gemeinnützige Institutionen, deren Status ein vorwiegend privater oder privatrechtlicher, konfessioneller oder sonstiger nicht öffentlicher ist. - 4) Einschließlich ARC Seibersdorf research GmbH sowie Kompetenzzentren. 
Tabelle 17: Ausgaben für Forschung und experimentelle Entwicklung (F\&E) in sämtlichen Erhebungsbereichen ${ }^{1)} 2006$ nach Bundesländern ${ }^{2)}$ und Forschungsarten

\begin{tabular}{|c|c|c|c|c|c|c|c|c|}
\hline \multirow{3}{*}{ Bundesländer } & \multirow{3}{*}{$\begin{array}{l}\text { F\&E } \\
\text { durch- } \\
\text { führende } \\
\text { Erhebungs- } \\
\text { einheiten }\end{array}$} & \multirow{3}{*}{$\begin{array}{c}\begin{array}{c}\text { Ausgaben für } \\
\text { F\&E insgesamt }{ }^{1)}\end{array} \\
\text { in } 1.000 \text { EUR }\end{array}$} & \multicolumn{6}{|c|}{ davon } \\
\hline & & & \multicolumn{2}{|c|}{ Grundlagenforschung } & \multicolumn{2}{|c|}{$\begin{array}{c}\text { Angewandte } \\
\text { Forschung }\end{array}$} & \multicolumn{2}{|c|}{$\begin{array}{c}\text { Experimentelle } \\
\text { Entwicklung }\end{array}$} \\
\hline & & & in 1.000 EUR & in $\%$ & in 1.000 EUR & in $\%$ & in 1.000 EUR & in $\%$ \\
\hline Österreich & 3.863 & 6.204 .155 & 1.064 .476 & 17,2 & 2.193 .550 & 35,4 & 2.946 .129 & 47,4 \\
\hline Burgenland & 53 & 33.211 & 2.006 & 6,0 & 12.646 & 38,1 & 18.559 & 55,9 \\
\hline Kärnten & 185 & 386.440 & 19.976 & 5,2 & 68.472 & 17,7 & 297.992 & 77,1 \\
\hline Niederösterreich & 414 & 445.444 & 40.473 & 9,1 & 178.005 & 40,0 & 226.966 & 50,9 \\
\hline Oberösterreich & 682 & 957.176 & 82.135 & 8,6 & 358.948 & 37,5 & 516.093 & 53,9 \\
\hline Salzburg & 224 & 197.003 & 46.159 & 23,4 & 60.816 & 30,9 & 90.028 & 45,7 \\
\hline Steiermark & 683 & 1.100 .380 & 245.662 & 22,3 & 420.659 & 38,2 & 434.059 & 39,5 \\
\hline Tirol & 339 & 543.806 & 144.242 & 26,5 & 219.191 & 40,3 & 180.373 & 33,2 \\
\hline Vorarlberg & 143 & 151.797 & 6.623 & 4,4 & 59.800 & 39,4 & 85.374 & 56,2 \\
\hline Wien & 1.140 & 2.388 .898 & 477.200 & 20,0 & 815.013 & 34,1 & 1.096 .685 & 45,9 \\
\hline
\end{tabular}

Q: STATISTIK AUSTRIA, Erhebung über Forschung und experimentelle Entwicklung 2006. Erstellt am: 25.08.2008. - 1) Ohne F\&E-Ausgaben-Schätzung für Landeskrankenanstalten. Die Landeskrankenanstalten wurden nicht mittels Fragebogenerhebung erfasst, sondern es erfolgte eine Schätzung der F\&E-Ausgaben durch Statistik Austria unter Heranziehung der Meldungen der Ämter der Landesregierungen. Eine Aufgliederung der F\&E-Ausgaben nach Forschungsarten liegt nicht vor. - 2) Standardauswertung nach dem Hauptstandort des Unternehmens. 
Tabelle 18: Ausgaben für Forschung und experimentelle Entwicklung (F\&E) in sämtlichen Erhebungsbereichen 2006 nach Bundesländern (nach dem Hauptstandort/ nach dem F\&E-Standort)

\begin{tabular}{|c|c|c|c|c|}
\hline \multirow[t]{2}{*}{ Bundesländer } & \multicolumn{2}{|c|}{$\begin{array}{l}\text { Nach dem Hauptstandort } \\
\text { der Erhebungseinheit/ des Unternehmens }{ }^{1)}\end{array}$} & \multicolumn{2}{|c|}{$\begin{array}{c}\text { Nach dem F\&E-Standort/ } \\
\text { den F\&E-Standorten des Unternehmens }\end{array}$} \\
\hline & in 1.000 EUR & in $\%$ & in 1.000 EUR & in $\%$ \\
\hline Österreich & 6.318 .587 & 100,0 & 6.318 .587 & 100,0 \\
\hline Burgenland & 34.383 & 0,5 & 30.520 & 0,5 \\
\hline Kärnten & 393.752 & 6,2 & 377.668 & 6,0 \\
\hline Niederösterreich & 461.530 & 7,3 & 514.874 & 8,1 \\
\hline Oberösterreich & 964.932 & 15,3 & 996.913 & 15,8 \\
\hline Salzburg & 200.919 & 3,2 & 230.737 & 3,7 \\
\hline Steiermark & 1.121 .674 & 17,8 & 1.265 .426 & 20,0 \\
\hline Tirol & 556.675 & 8,8 & 554.722 & 8,8 \\
\hline Vorarlberg & 154.619 & 2,4 & 153.947 & 2,4 \\
\hline Wien & 2.430 .103 & 38,5 & 2.193 .780 & 34,7 \\
\hline
\end{tabular}

Q: STATISTIK AUSTRIA, Erhebung über Forschung und experimentelle Entwicklung 2006. Erstellt am: 04.09.2008 - 1) Die regionale Zuordnung der Erhebungseinheiten, auch der Unternehmen des firmeneigenen Bereichs, erfolgte ausschließlich nach dem Bundesland, in dem sich der Hauptstandort befindet (Standardauswertung).

- 2) Im Rahmen dieser verfeinerten Regionalauswertung erfolgte für die Unternehmen des firmeneigenen Bereichs, welche in mehr als einem Bundesland F\&E durchführten, die Aufteilung der F\&E-Ausgaben zu den Bundesländern, in denen sich die F\&E-Standorte befinden. Für die Erhebungseinheiten in den anderen Bereichen war die Frage „F\&E-Standorte auch in anderen Bundesländern“ nicht relevant. 


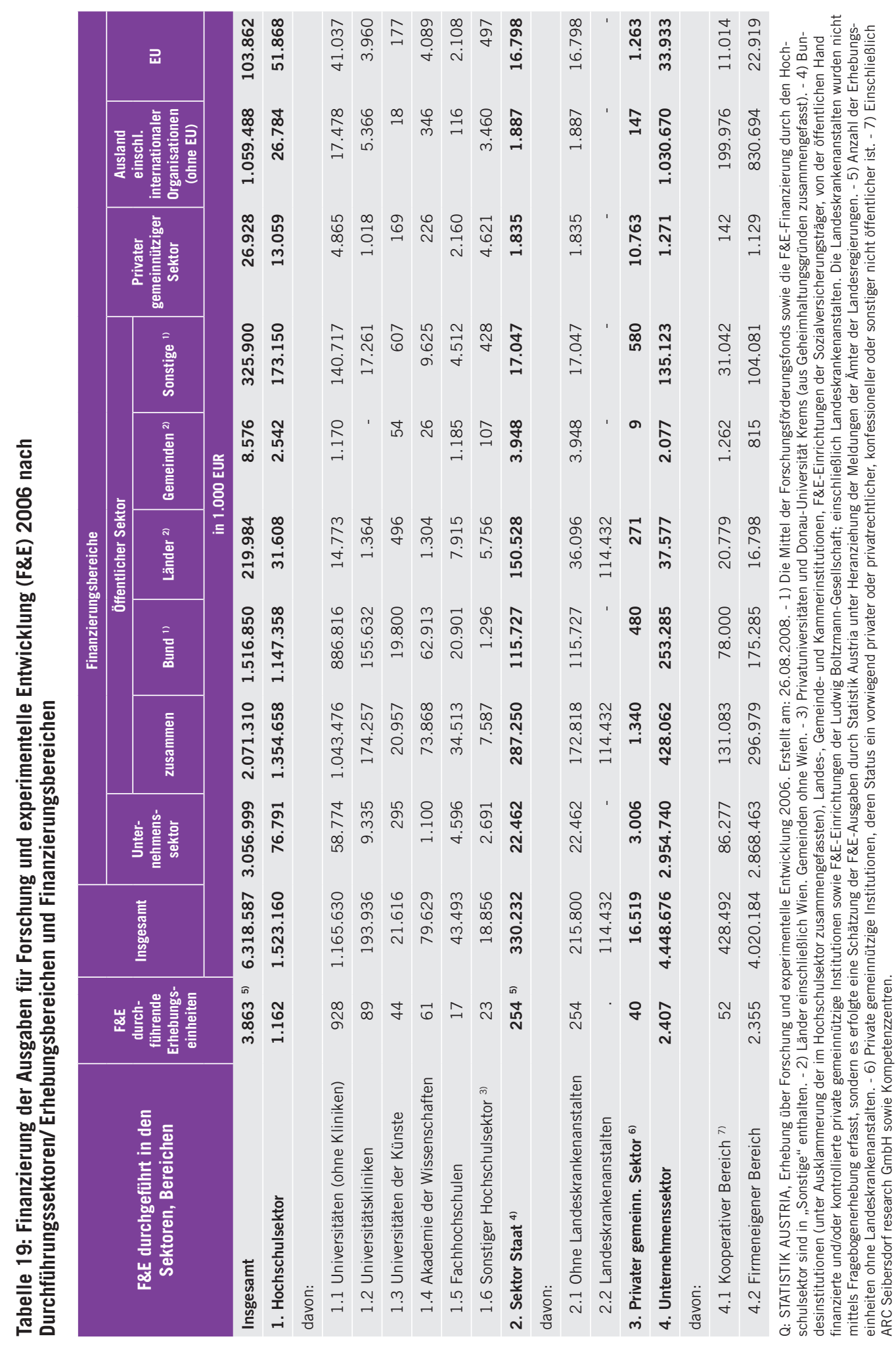




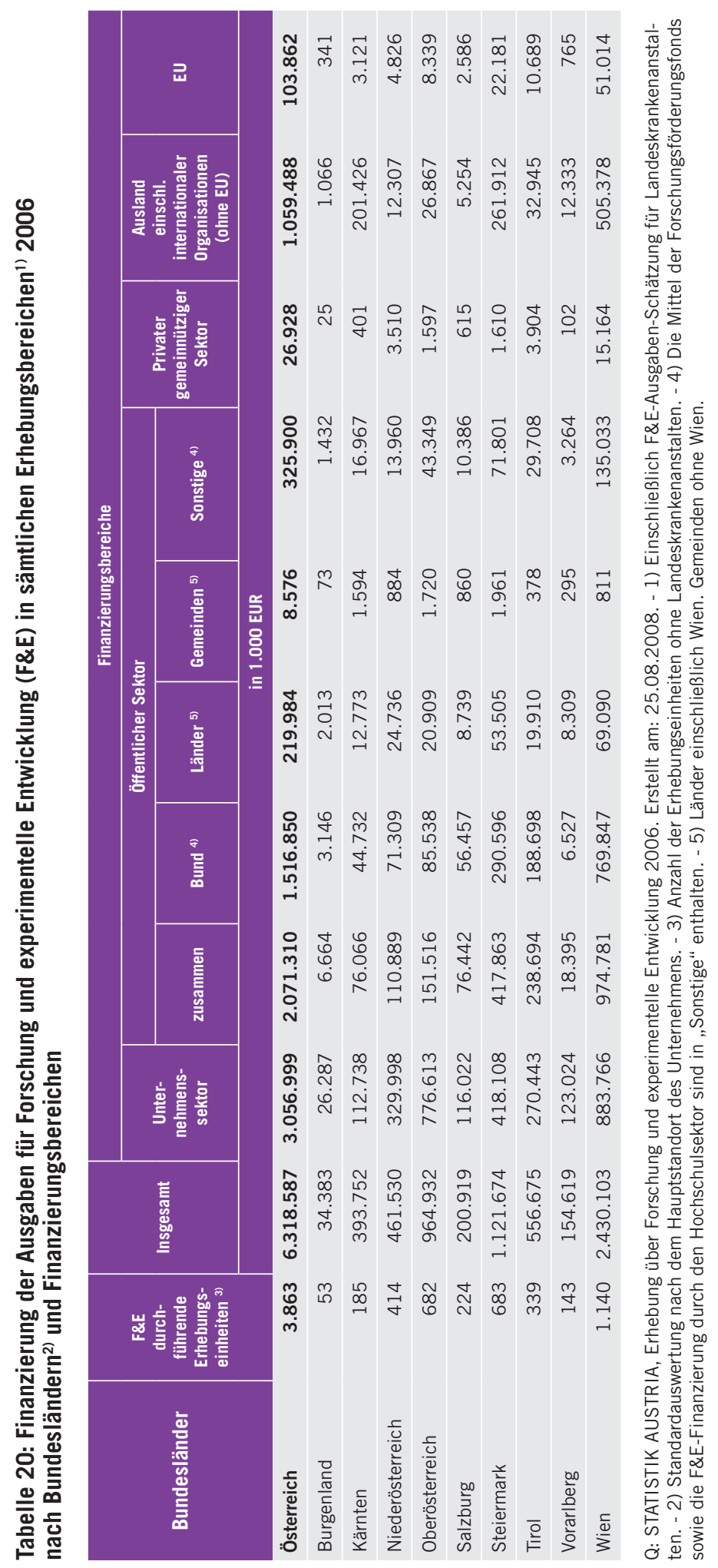


Tabelle 21: Bruttoregionalprodukt (BRP), Bruttoinlandsausgaben für F\&E und regionale Forschungsquoten 2006

\begin{tabular}{|c|c|c|c|}
\hline \multirow{2}{*}{$\begin{array}{l}\text { Regionen, Bundesländer } \\
\text { (NUTS 1, NUTS 2) }\end{array}$} & \multirow{2}{*}{$\begin{array}{c}\text { Bruttoregionalprodukt } \\
\left(\text {,regionales BIP“4 }{ }^{1)}\right. \\
\text { in Mio. EUR }\end{array}$} & \multicolumn{2}{|c|}{ Bruttoinlandsausgaben für F\&E ${ }^{2)}$} \\
\hline & & in Mio. EUR & in $\%$ des BRP \\
\hline Österreich & 257294 & 6318,59 & 2,46 \\
\hline Ostösterreich & 114640 & 2739,17 & 2,39 \\
\hline Burgenland & 5739 & 30,52 & 0,53 \\
\hline Niederösterreich & 40158 & 514,87 & 1,28 \\
\hline Wien & 68743 & 2193,78 & 3,19 \\
\hline Südösterreich & 47193 & 1643,09 & 3,48 \\
\hline Kärnten & 14838 & 377,67 & 2,55 \\
\hline Steiermark & 32355 & 1265,43 & 3,91 \\
\hline Westösterreich & 95460 & 1936,32 & 2,03 \\
\hline Oberösterreich & 42560 & 996,91 & 2,34 \\
\hline Salzburg & 18427 & 230,74 & 1,25 \\
\hline Tirol & 22683 & 554,72 & 2,45 \\
\hline Vorarlberg & 11790 & 153,95 & 1,31 \\
\hline
\end{tabular}

Q: STATISTIK AUSTRIA. Erstellt am: 12.01.2009. - 1) Stand 22.12.2008. - 2) Firmeneigener Bereich: Regionale Zuordnung nach dem F\&E-Standort/ den F\&E-Standorten des Unternehmens.

- Rundungsdifferenzen. 
Tabelle 22: Hochschulsektor ${ }^{1)}$ : Beschäftigte in Forschung und experimenteller Entwicklung (F\&E) 2006 nach Wissenschaftszweigen und Beschäftigtenkategorien

\begin{tabular}{|c|c|c|c|c|c|}
\hline \multirow[b]{2}{*}{ Wissenschaftszweige } & \multirow[b]{2}{*}{$\begin{array}{l}\text { F\&E } \\
\text { durch- } \\
\text { führende } \\
\text { Erhebungs- } \\
\text { einheiten }\end{array}$} & \multirow[b]{2}{*}{ Insgesamt } & \multicolumn{3}{|c|}{ davon } \\
\hline & & & $\begin{array}{l}\text { Wissenschaft- } \\
\text { liches Personal }\end{array}$ & $\begin{array}{c}\text { Höherqualifiziertes } \\
\text { nichtwissenschaft- } \\
\text { liches Personal }\end{array}$ & $\begin{array}{l}\text { Sonstiges } \\
\text { Hilfspersonal }\end{array}$ \\
\hline & \multicolumn{5}{|c|}{ Kopfzahlen } \\
\hline 1.0 bis 6.0 Insgesamt & 1.162 & 32.715 & 23.609 & 4.954 & 4.152 \\
\hline 1.0 bis 4.0 zusammen & 673 & 23.429 & 16.148 & 4.062 & 3.219 \\
\hline 1.0 Naturwissenschaften & 252 & 8.551 & 6.371 & 1.416 & 764 \\
\hline 2.0 Technische Wissenschaften & 192 & 4.578 & 3.369 & 547 & 662 \\
\hline 3.0 Humanmedizin & 172 & 9.128 & 5.731 & 1.894 & 1.503 \\
\hline 4.0 Land- und Forstwirtschaft, Veterinärmedizin & 57 & 1.172 & 677 & 205 & 290 \\
\hline 5.0 und 6.0 zusammen & 489 & 9.286 & 7.461 & 892 & 933 \\
\hline 5.0 Sozialwissenschaften & 274 & 5.429 & 4.363 & 532 & 534 \\
\hline \multirow[t]{2}{*}{ 6.0 Geisteswissenschaften } & 215 & 3.857 & 3.098 & 360 & 399 \\
\hline & \multicolumn{5}{|c|}{ Vollzeitäquivalente } \\
\hline 1.0 bis 6.0 Insgesamt & 1.162 & $12.668,2$ & $9.261,3$ & $1.855,6$ & $1.551,3$ \\
\hline 1.0 bis 4.0 zusammen & 673 & $9.570,5$ & $6.747,2$ & $1.583,6$ & $1.239,7$ \\
\hline 1.0 Naturwissenschaften & 252 & $4.092,9$ & $3.178,2$ & 547,3 & 367,4 \\
\hline 2.0 Technische Wissenschaften & 192 & $2.012,6$ & $1.502,7$ & 215,1 & 294,8 \\
\hline 3.0 Humanmedizin & 172 & $2.934,6$ & $1.744,7$ & 733,2 & 456,7 \\
\hline 4.0 Land- und Forstwirtschaft, Veterinärmedizin & 57 & 530,4 & 321,5 & 88,0 & 120,8 \\
\hline 5.0 und 6.0 zusammen & 489 & $3.097,7$ & $2.514,2$ & 272,0 & 311,6 \\
\hline 5.0 Sozialwissenschaften & 274 & $1.847,1$ & $1.476,2$ & 176,1 & 194,7 \\
\hline 6.0 Geisteswissenschaften & 215 & $1.250,7$ & $1.037,9$ & 95,9 & 116,9 \\
\hline
\end{tabular}

Q: STATISTIK AUSTRIA, Erhebung über Forschung und experimentelle Entwicklung 2006. Erstellt am: 08.08.2008. - 1) Universitäten einschließlich Kliniken, Universitäten der Künste, Akademie der Wissenschaften, Fachhochschulen, Privatuniversitäten und Donau-Universität Krems. - Rundungsdifferenzen. 
Tabelle 23: Hochschulsektor ${ }^{1)}$ : Ausgaben für Forschung und experimentelle Entwicklung (F\&E) 2006 nach Wissenschaftszweigen und Ausgabenarten

\begin{tabular}{|c|c|c|c|c|c|c|}
\hline \multirow{3}{*}{ Wissenschaftszweige } & \multirow{3}{*}{$\begin{array}{c}\text { F\&E } \\
\text { durch- } \\
\text { führende } \\
\text { Erhebungs- } \\
\text { einheiten }\end{array}$} & \multirow[b]{2}{*}{ Insgesamt } & \multicolumn{4}{|c|}{ davon } \\
\hline & & & $\begin{array}{l}\text { Personal- } \\
\text { ausgaben }\end{array}$ & $\begin{array}{c}\text { Laufende } \\
\text { Sachausgaben }\end{array}$ & $\begin{array}{l}\text { Ausgaben für } \\
\text { Ausrüstungs- } \\
\text { investitionen }\end{array}$ & $\begin{array}{c}\text { Bauausgaben } \\
\text { und Ausgaben } \\
\text { für Liegen- } \\
\text { schaftsankäufe }\end{array}$ \\
\hline & & \multicolumn{5}{|c|}{ in 1.000 EUR } \\
\hline 1.0 bis 6.0 Insgesamt & 1.162 & 1.523 .160 & 710.514 & 655.102 & 117.422 & 40.122 \\
\hline 1.0 bis 4.0 zusammen & 673 & 1.162 .243 & 523.163 & 501.304 & 98.214 & 39.562 \\
\hline 1.0 Naturwissenschaften & 252 & 477.341 & 221.736 & 200.462 & 53.571 & 1.572 \\
\hline 2.0 Technische Wissenschaften & 192 & 218.368 & 107.122 & 86.642 & 24.110 & 494 \\
\hline 3.0 Humanmedizin & 172 & 396.862 & 167.455 & 174.971 & 17.107 & 37.329 \\
\hline 4.0 Land- und Forstwirtschaft, Veterinärmedizin & 57 & 69.672 & 26.850 & 39.229 & 3.426 & 167 \\
\hline 5.0 und 6.0 zusammen & 489 & 360.917 & 187.351 & 153.798 & 19.208 & 560 \\
\hline 5.0 Sozialwissenschaften & 274 & 209.340 & 108.633 & 87.399 & 12.976 & 332 \\
\hline 6.0 Geisteswissenschaften & 215 & 151.577 & 78.718 & 66.399 & 6.232 & 228 \\
\hline
\end{tabular}

Q. STATITISK AUSTRIA, Erhebung über Forschung und experimentelle Entwicklung 2006. Erstellt am: 29.08.08. - 1) Universitäten einschließlich Kliniken, Universitäten der Künste, Akademie der Wissenschaften, Fachhochschulen, Privatuniversitäten und Donau-Universität Krems. 
Tabelle 24: Hochschulsektor ${ }^{1)}$ : Ausgaben für Forschung und experimentelle Entwicklung (F\&E) 2006 nach Wissenschaftszweigen und Forschungsarten

\begin{tabular}{|c|c|c|c|c|c|c|c|c|}
\hline \multirow{3}{*}{ Wissenschaftszweige } & \multirow{3}{*}{$\begin{array}{c}\text { F\&E } \\
\text { durch- } \\
\text { futhrende } \\
\text { Erhebungs- } \\
\text { einheiten }\end{array}$} & \multirow{3}{*}{$\begin{array}{c}\begin{array}{c}\text { Ausgaben fuir } \\
\text { F\&E insgesamt }\end{array} \\
\text { in } 1.000 \text { EUR }\end{array}$} & \multicolumn{6}{|c|}{ davon } \\
\hline & & & \multicolumn{2}{|c|}{ Grundlagenforschung } & \multicolumn{2}{|c|}{ Angewandte Forschung } & \multicolumn{2}{|c|}{$\begin{array}{l}\text { Experimentelle Ent- } \\
\text { wicklung }\end{array}$} \\
\hline & & & in 1.000 EUR & in \% & in 1.000 EUR & in $\%$ & in 1.000 EUR & in $\%$ \\
\hline 1.0 bis 6.0 Insgesamt & 1.162 & 1.523 .160 & 746.112 & 49,0 & 638.642 & 41,9 & 138.406 & 9,1 \\
\hline 1.0 bis 4.0 zusammen & 673 & 1.162 .243 & 542.563 & 46,7 & 496.761 & 42,7 & 122.919 & 10,6 \\
\hline 1.0 Naturwissenschaften & 252 & 477.341 & 295.271 & 61,9 & 143.332 & 30,0 & 38.738 & 8,1 \\
\hline 2.0 Technische Wissenschaften & 192 & 218.368 & 58.806 & 26,9 & 129.597 & 59,4 & 29.965 & 13,7 \\
\hline 3.0 Humanmedizin & 172 & 396.862 & 160.783 & 40,5 & 186.774 & 47,1 & 49.305 & 12,4 \\
\hline 4.0 Land- und Forstwirtschaft, Veterinärmedizin & 57 & 69.672 & 27.703 & 39,8 & 37.058 & 53,2 & 4.911 & 7,0 \\
\hline 5.0 und 6.0 zusammen & 489 & 360.917 & 203.549 & 56,4 & 141.881 & 39,3 & 15.487 & 4,3 \\
\hline 5.0 Sozialwissenschaften & 274 & 209.340 & 97.227 & 46,4 & 102.673 & 49,1 & 9.440 & 4,5 \\
\hline 6.0 Geisteswissenschaften & 215 & 151.577 & 106.322 & 70,1 & 39.208 & 25,9 & 6.047 & 4,0 \\
\hline
\end{tabular}

Q: STATISTIK AUSTRIA, Erhebung über Forschung und experimentelle Entwicklung 2006. Erstellt am: 04.09.2008 - 1) Universitäten einschließlich Kliniken, Universitäten der Künste, Akademie der Wissenschaften, Fachhochschulen, Privatuniversitäten und Donau-Universität Krems. 


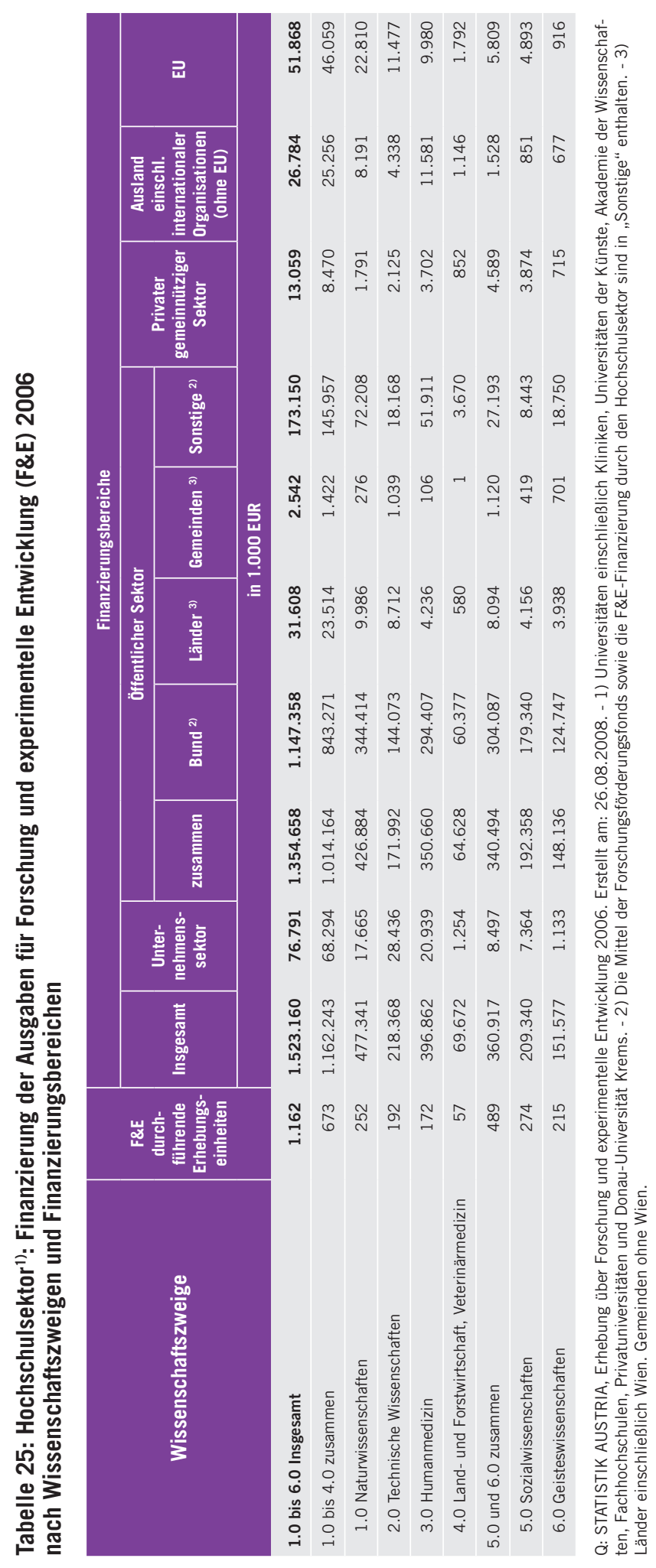




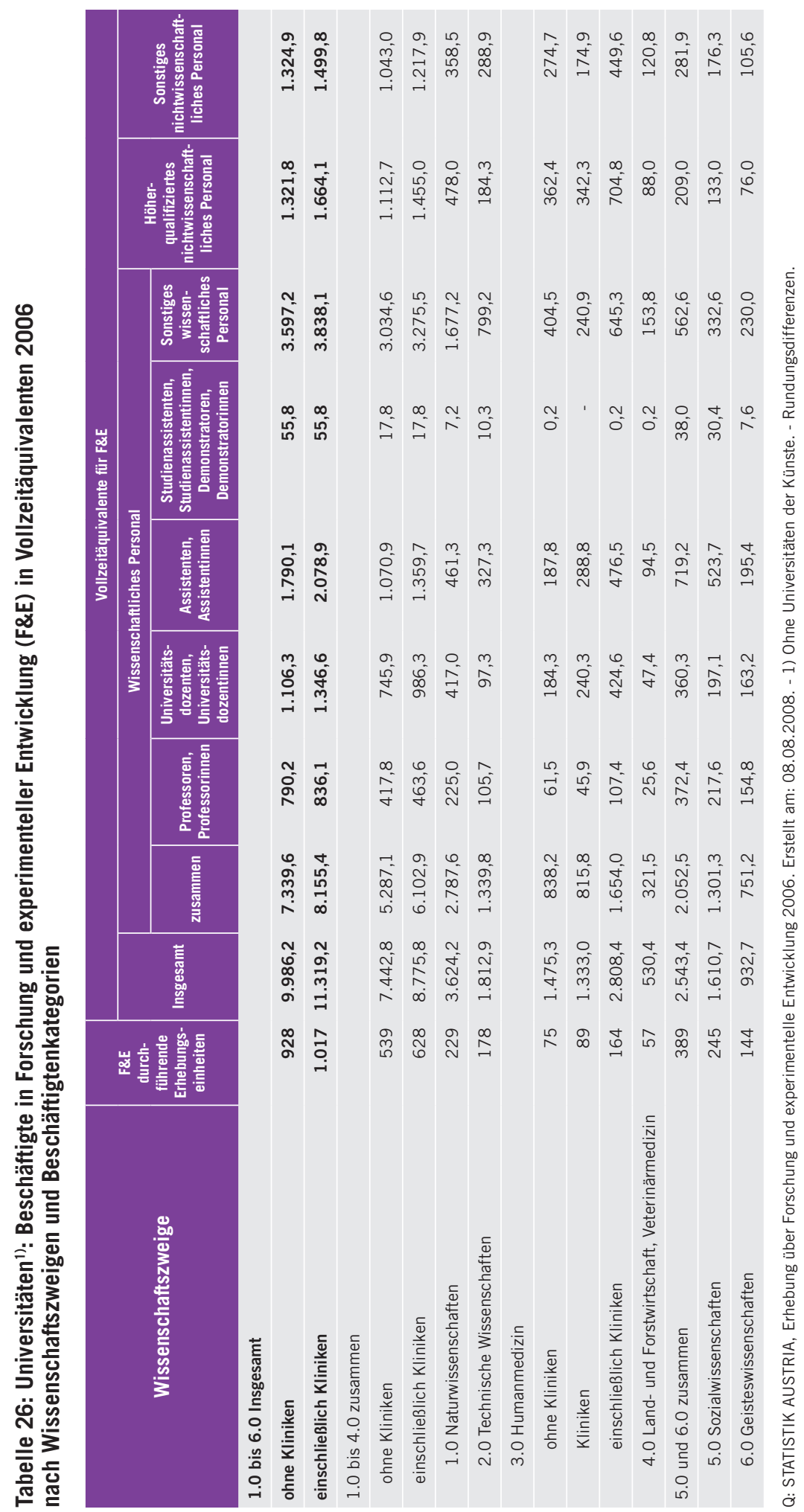




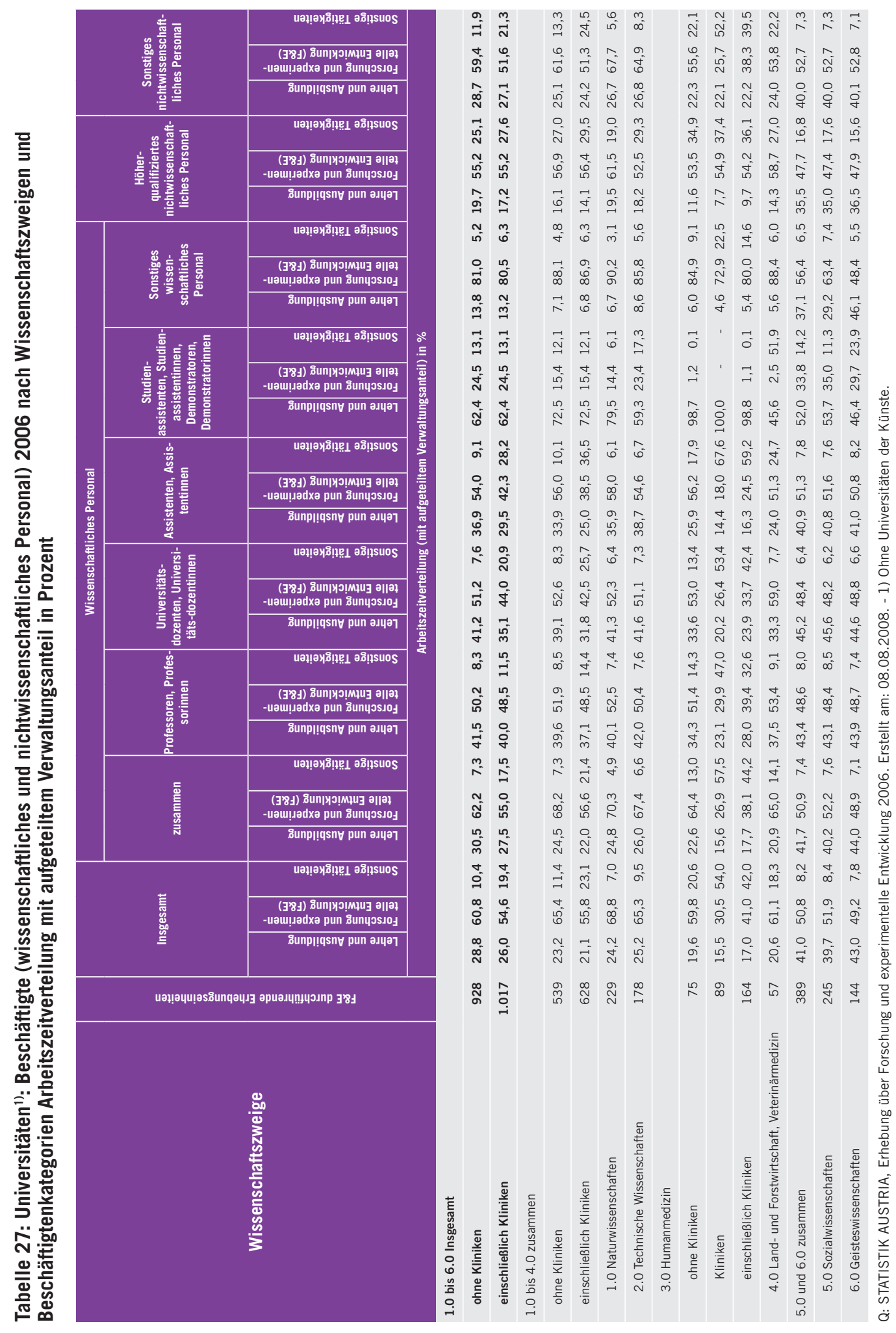




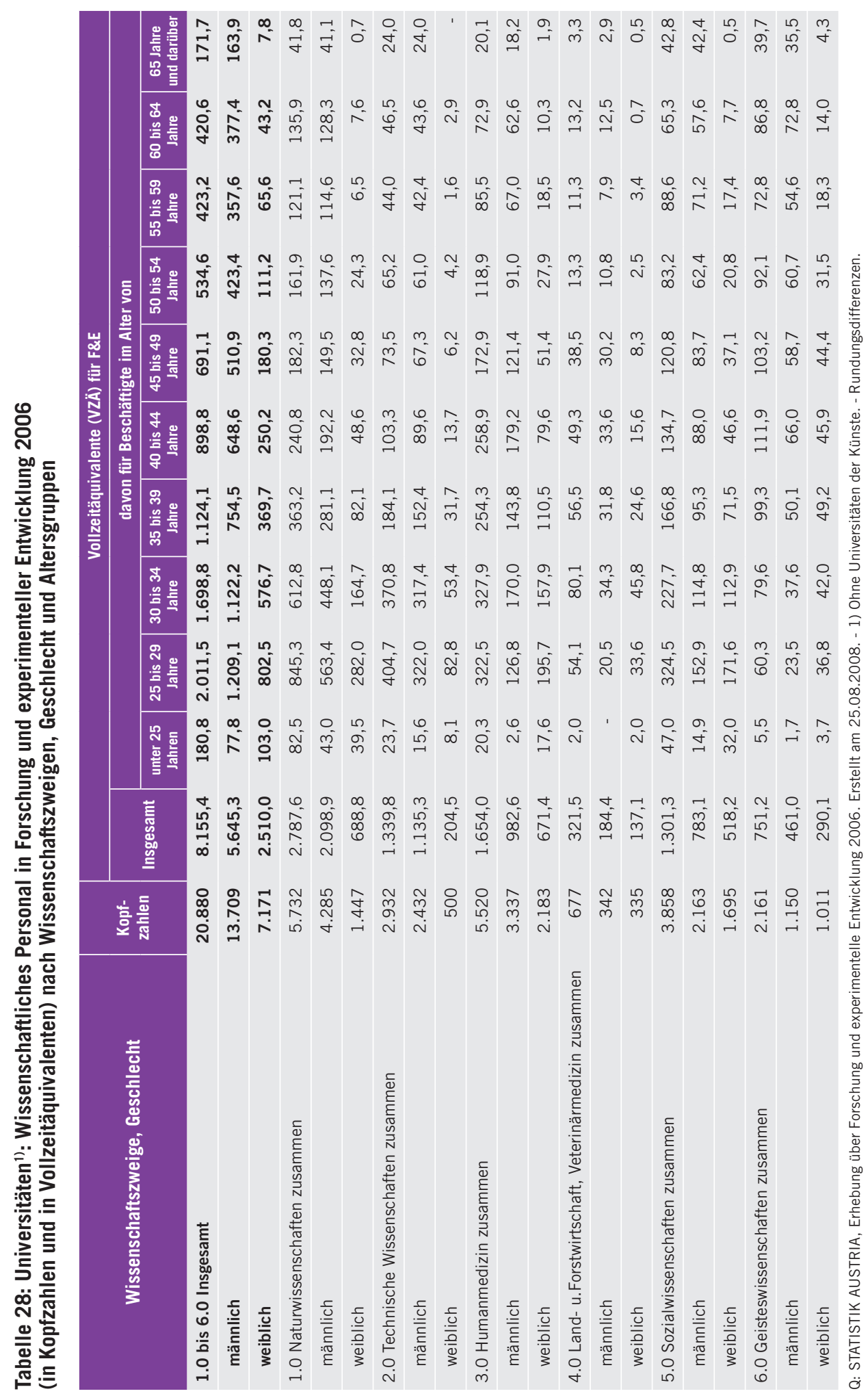


Tabelle 29: Universitäten 1): Ausgaben für Forschung und experimentelle Entwicklung im Jahr 2006 gegliedert nach Wissenschaftszweigen und Ausgabenarten

\begin{tabular}{|c|c|c|c|c|c|c|}
\hline \multirow{3}{*}{ Wissenschaftszweige } & \multirow{3}{*}{$\begin{array}{c}\text { F\&E } \\
\text { durch- } \\
\text { führende } \\
\text { Erhebungs- } \\
\text { einheiten }\end{array}$} & \multirow[b]{2}{*}{ Insgesamt } & \multicolumn{4}{|c|}{ davon } \\
\hline & & & $\begin{array}{l}\text { Personal- } \\
\text { ausgaben }\end{array}$ & $\begin{array}{c}\text { Laufende } \\
\text { Sachausgaben }\end{array}$ & $\begin{array}{l}\text { Ausgaben für } \\
\text { Ausrüstungs- } \\
\text { investitionen }\end{array}$ & $\begin{array}{l}\text { Bauausgaben } \\
\text { und Ausgaben } \\
\text { für Liegen- } \\
\text { schaftsankäufe }\end{array}$ \\
\hline & & \multicolumn{5}{|c|}{ in 1.000 EUR } \\
\hline \multicolumn{7}{|l|}{1.0 bis 6.0 Insgesamt } \\
\hline ohne Kliniken & 928 & 1.165 .630 & 555.670 & 514.266 & 93.922 & 1.772 \\
\hline einschließlich Kliniken & 1.017 & 1.359 .566 & 636.254 & 586.161 & 99.853 & 37.298 \\
\hline \multicolumn{7}{|l|}{1.0 bis 4.0 zusammen } \\
\hline ohne Kliniken & 539 & 864.096 & 400.346 & 385.042 & 77.212 & 1.496 \\
\hline einschließlich Kliniken & 628 & 1.058 .032 & 480.930 & 456.937 & 83.143 & 37.022 \\
\hline 1.0 Naturwissenschaften & 229 & 415.499 & 198.129 & 174.559 & 42.310 & 501 \\
\hline 2.0 Technische Wissenschaften & 178 & 191.340 & 95.247 & 74.536 & 21.171 & 386 \\
\hline \multicolumn{7}{|l|}{ 3.0 Humanmedizin } \\
\hline ohne Kliniken & 75 & 187.585 & 80.120 & 96.718 & 10.305 & 442 \\
\hline Kliniken & 89 & 193.936 & 80.584 & 71.895 & 5.931 & 35.526 \\
\hline einschließlich Kliniken & 164 & 381.521 & 160.704 & 168.613 & 16.236 & 35.968 \\
\hline 4.0 Land- und Forstwirtschaft, Veterinärmedizin & 57 & 69.672 & 26.850 & 39.229 & 3.426 & 167 \\
\hline 5.0 und 6.0 zusammen & 389 & 301.534 & 155.324 & 129.224 & 16.710 & 276 \\
\hline 5.0 Sozialwissenschaften & 245 & 182.189 & 95.670 & 74.897 & 11.461 & 161 \\
\hline 6.0 Geisteswissenschaften & 144 & 119.345 & 59.654 & 54.327 & 5.249 & 115 \\
\hline
\end{tabular}

Q: STATISTIK AUSTRIA, Erhebung über Forschung und experimentelle Entwicklung 2006. Erstellt am 28.08.2008. - 1) Ohne Universitäten der Künste.

Tabelle 30: Universitäten ${ }^{1)}$ : Ausgaben für Forschung und experimentelle Entwicklung 2006 nach Wissenschaftszweigen und Forschungsarten

\begin{tabular}{|c|c|c|c|c|c|c|c|c|}
\hline \multirow{3}{*}{ Wissenschaftszweige } & \multirow{3}{*}{$\begin{array}{c}\text { F\&E } \\
\text { durch- } \\
\text { führende } \\
\text { Erhebungs- } \\
\text { einheiten }\end{array}$} & \multirow{3}{*}{$\begin{array}{c}\text { Ausgaben für } \\
\text { F\&E insgesamt } \\
\text { in } 1.000 \text { EUR }\end{array}$} & \multicolumn{6}{|c|}{ davon } \\
\hline & & & \multicolumn{2}{|c|}{ Grundlagenforschung } & \multicolumn{2}{|c|}{ Angewandte Forschung } & \multicolumn{2}{|c|}{$\begin{array}{c}\text { Experimentelle } \\
\text { Entwicklung }\end{array}$} \\
\hline & & & in 1.000 EUR & in $\%$ & in 1.000 EUR & in $\%$ & in 1.000 EUR & in $\%$ \\
\hline \multicolumn{9}{|l|}{1.0 bis 6.0 Insgesamt } \\
\hline ohne Kliniken & 928 & 1.165 .630 & 613.773 & 52,6 & 468.344 & 40,2 & 83.513 & 7,2 \\
\hline einschließlich Kliniken & 1.017 & 1.359 .566 & 665.989 & 48,9 & 578.564 & 42,6 & 115.013 & 8,5 \\
\hline \multicolumn{9}{|l|}{1.0 bis 4.0 zusammen } \\
\hline ohne Kliniken & 539 & 864.096 & 433.277 & 50,2 & 355.550 & 41,1 & 75.269 & 8,7 \\
\hline einschließlich Kliniken & 628 & 1.058 .032 & 485.493 & 45,9 & 465.770 & 44,0 & 106.769 & 10,1 \\
\hline 1.0 Naturwissenschaften & 229 & 415.499 & 249.109 & 60,0 & 135.572 & 32,6 & 30.818 & 7,4 \\
\hline 2.0 Technische Wissenschaften & 178 & 191.340 & 56.643 & 29,6 & 112.611 & 58,9 & 22.086 & 11,5 \\
\hline \multicolumn{9}{|l|}{ 3.0 Humanmedizin } \\
\hline ohne Kliniken & 75 & 187.585 & 99.822 & 53,2 & 70.309 & 37,5 & 17.454 & 9,3 \\
\hline Kliniken & 89 & 193.936 & 52.216 & 26,9 & 110.220 & 56,9 & 31.500 & 16,2 \\
\hline einschließlich Kliniken & 164 & 381.521 & 152.038 & 39,9 & 180.529 & 47,3 & 48.954 & 12,8 \\
\hline 4.0 Land- und Forstwirtschaft, Veterinärmedizin & 57 & 69.672 & 27.703 & 39,8 & 37.058 & 53,2 & 4.911 & 7,0 \\
\hline 5.0 und 6.0 zusammen & 389 & 301.534 & 180.496 & 59,9 & 112.794 & 37,4 & 8.244 & 2,7 \\
\hline 5.0 Sozialwissenschaften & 245 & 182.189 & 90.492 & 49,6 & 84.496 & 46,4 & 7.201 & 4,0 \\
\hline 6.0 Geisteswissenschaften & 144 & 119.345 & 90.004 & 75,4 & 28.298 & 23,7 & 1.043 & 0,9 \\
\hline
\end{tabular}

Q: STATISTIK AUSTRIA, Erhebung über Forschung und experimentelle Entwicklung 2006. Erstellt am: 29.08.08. - 1) Ohne Universitäten der Künste. 


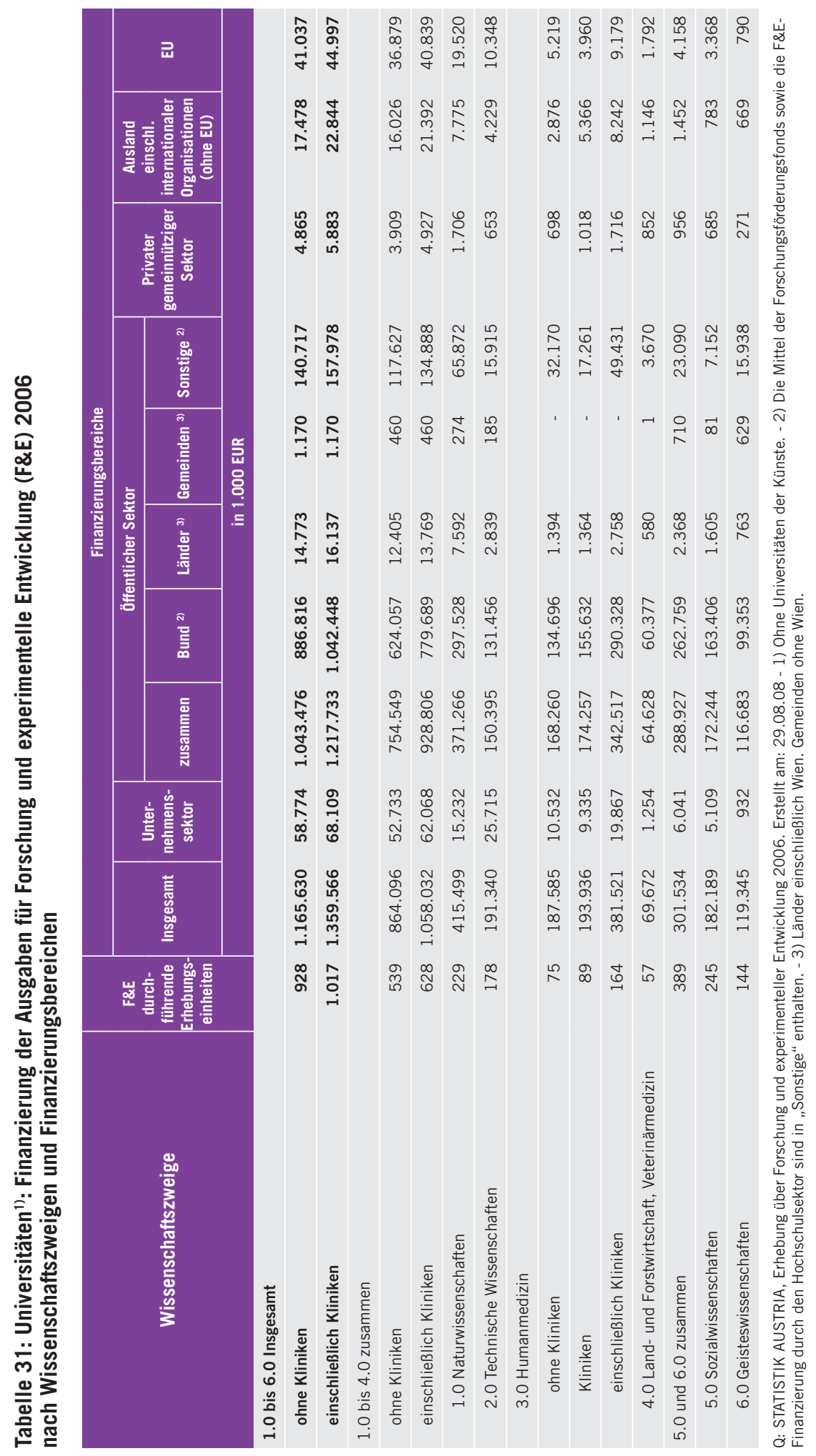


Tabelle 32: Sektor Staat ${ }^{1)}$ : Beschäftigte in Forschung und experimenteller Entwicklung (F\&E) 2006 nach Wissenschaftszweigen und Beschäftigtenkategorien

\begin{tabular}{|c|c|c|c|c|c|}
\hline \multirow[b]{2}{*}{ Wissenschaftszweige } & \multirow{2}{*}{$\begin{array}{c}\text { F\&E } \\
\text { durch- } \\
\text { führende } \\
\text { Erhebungs- } \\
\text { einheiten }\end{array}$} & \multirow[b]{2}{*}{ Insgesamt } & \multicolumn{3}{|c|}{ davon } \\
\hline & & & $\begin{array}{l}\text { Wissenschaft- } \\
\text { liches Personal }\end{array}$ & $\begin{array}{l}\text { Höherqualifiziertes } \\
\text { nichtwissenschaft- } \\
\text { liches Personal }\end{array}$ & $\begin{array}{c}\text { Sonstiges } \\
\text { Hilfspersonal }\end{array}$ \\
\hline & \multicolumn{5}{|c|}{ Kopfzahlen } \\
\hline 1.0 bis 6.0 Insgesamt & 254 & 5.511 & 2.789 & 1.129 & 1.593 \\
\hline 1.0 bis 4.0 zusammen & 97 & 2.831 & 1.325 & 633 & 873 \\
\hline 1.0 Naturwissenschaften & 34 & 928 & 481 & 215 & 232 \\
\hline 2.0 Technische Wissenschaften & 12 & 579 & 328 & 129 & 122 \\
\hline 3.0 Humanmedizin & 32 & 266 & 166 & 71 & 29 \\
\hline 4.0 Land- und Forstwirtschaft, Veterinärmedizin & 19 & 1.058 & 350 & 218 & 490 \\
\hline 5.0 und 6.0 zusammen & 157 & 2.680 & 1.464 & 496 & 720 \\
\hline 5.0 Sozialwissenschaften & 85 & 1.093 & 752 & 205 & 136 \\
\hline \multirow[t]{2}{*}{ 6.0 Geisteswissenschaften } & 72 & 1.587 & 712 & 291 & 584 \\
\hline & \multicolumn{5}{|c|}{ Vollzeitäquivalente } \\
\hline 1.0 bis 6.0 Insgesamt & 254 & $2.422,6$ & $1.348,5$ & 356,5 & 717,6 \\
\hline 1.0 bis 4.0 zusammen & 97 & $1.329,1$ & 679,4 & 205,8 & 443,9 \\
\hline 1.0 Naturwissenschaften & 34 & 363,9 & 245,6 & 39,9 & 78,4 \\
\hline 2.0 Technische Wissenschaften & 12 & 266,3 & 183,8 & 40,8 & 41,6 \\
\hline 3.0 Humanmedizin & 32 & 100,1 & 69,3 & 19,8 & 11,0 \\
\hline 4.0 Land- und Forstwirtschaft, Veterinärmedizin & 19 & 598,8 & 180,7 & 105,3 & 312,8 \\
\hline 5.0 und 6.0 zusammen & 157 & $1.093,5$ & 669,1 & 150,7 & 273,7 \\
\hline 5.0 Sozialwissenschaften & 85 & 479,6 & 357,8 & 76,2 & 45,6 \\
\hline 6.0 Geisteswissenschaften & 72 & 613,9 & 311,3 & 74,6 & 228,1 \\
\hline
\end{tabular}

Q: STATISTIK AUSTRIA, Erhebung über Forschung und experimentelle Entwicklung 2006. Erstellt am: 08.08.2008. - 1) Bundesinstitutionen (unter Ausklammerung der im Hochschulsektor zusammengefassten), Landes-, Gemeinde- und Kammerinstitutionen, F\&E-Einrichtungen der Sozialversicherungsträger, von der öffentlichen Hand finanzierte und/oder kontrollierte private gemeinnützige Institutionen sowie F\&E-Einrichtungen der Ludwig Boltzmann-Gesellschaft; ohne Landeskrankenanstalten. Die Landeskrankenanstalten wurden nicht mittels Fragebogenerhebung erfasst, sondern es erfolgte eine Schätzung der F\&E-Ausgaben durch Statistik Austria unter Heranziehung der Meldungen der Ämter der Landesregierungen. Daher liegen keine Daten über Beschäftigte in F\&E vor. - Rundungsdifferenzen. 
Tabelle 33: Sektor Staat ${ }^{1)}$ : Beschäftigte in Forschung und experimenteller Entwicklung (F\&E) 2006 nach Rechtsträgern und Beschäftigtenkategorien

\begin{tabular}{|c|c|c|c|c|c|}
\hline \multirow[b]{2}{*}{ Rechtsträger } & \multirow{2}{*}{$\begin{array}{l}\text { F\&E } \\
\text { durch- } \\
\text { führende } \\
\text { Erhebungs- } \\
\text { einheiten }\end{array}$} & \multirow[b]{2}{*}{ Insgesamt } & \multicolumn{3}{|c|}{ davon } \\
\hline & & & $\begin{array}{l}\text { Wissenschaft- } \\
\text { liches Personal }\end{array}$ & $\begin{array}{c}\text { Höherqualifiziertes } \\
\text { nichtwissenschaft- } \\
\text { liches Personal }\end{array}$ & $\begin{array}{l}\text { Sonstiges } \\
\text { Hilfspersonal }\end{array}$ \\
\hline & \multicolumn{5}{|c|}{ Kopfzahlen } \\
\hline Insgesamt & 254 & 5.511 & 2.789 & 1.129 & 1.593 \\
\hline Bund & 55 & 2.649 & 1.129 & 557 & 963 \\
\hline Länder (einschließlich Wien) & 39 & 740 & 269 & 120 & 351 \\
\hline Gemeinden (ohne Wien) & 8 & 155 & 76 & 30 & 49 \\
\hline Kammern & 3 & 29 & 17 & - & 12 \\
\hline Sozialversicherungsträger & - & - & - & - & - \\
\hline PloE öffentlich ${ }^{2)}$ & 108 & 1.563 & 1.026 & 338 & 199 \\
\hline \multirow[t]{2}{*}{ Ludwig Boltzmann-Gesellschaft } & 41 & 375 & 272 & 84 & 19 \\
\hline & \multicolumn{5}{|c|}{ Vollzeitäquivalente } \\
\hline Insgesamt & 254 & $2.422,6$ & $1.348,5$ & 356,5 & 717,6 \\
\hline Bund & 55 & $1.190,2$ & 507,8 & 181,0 & 501,4 \\
\hline Länder (einschließlich Wien) & 39 & 204,6 & 94,1 & 16,4 & 94,2 \\
\hline Gemeinden (ohne Wien) & 8 & 61,5 & 36,7 & 8,3 & 16,5 \\
\hline Kammern & 3 & 9,6 & 7,1 & - & 2,6 \\
\hline Sozialversicherungsträger & - & - & - & - & - \\
\hline PloE öffentlich ${ }^{2)}$ & 108 & 789,3 & 574,0 & 122,4 & 92,9 \\
\hline Ludwig Boltzmann-Gesellschaft & 41 & 167,3 & 128,8 & 28,4 & 10,0 \\
\hline
\end{tabular}

Q: STATISTIK AUSTRIA, Erhebung über Forschung und experimentelle Entwicklung 2006. Erstellt am: 08.08.2008. - 1) Bundesinstitutionen (unter Ausklammerung der im Hochschulsektor zusammengefassten), Landes-, Gemeinde- und Kammerinstitutionen, F\&E-Einrichtungen der Sozialversicherungsträger, von der öffentlichen Hand finanzierte und/oder kontrollierte private gemeinnützige Institutionen sowie F\&E-Einrichtungen der Ludwig Boltzmann-Gesellschaft; ohne Landeskrankenanstalten. Die Landeskrankenanstalten wurden nicht mittels Fragebogenerhebung erfasst, sondern es erfolgte eine Schätzung der F\&E-Ausgaben durch Statistik Austria unter Heranziehung der Meldungen der Ämter der Landesregierungen. Daher liegen keine Daten über Beschäftigte in F\&E vor. - 2) Private gemeinnützige Institutionen, die überwiegend von der öffentlichen Hand finanziert und/oder kontrolliert werden. - Rundungsdifferenzen. 
Tabelle 34: Sektor Staat'): Ausgaben für Forschung und experimentelle Entwicklung 2006 nach Wissenschaftszweigen und Ausgabenarten

\begin{tabular}{|c|c|c|c|c|c|c|}
\hline \multirow{3}{*}{ Wissenschaftszweige } & \multirow{3}{*}{$\begin{array}{c}\text { F\&E } \\
\text { durch- } \\
\text { führende } \\
\text { Erhebungs- } \\
\text { einheiten }\end{array}$} & \multirow[b]{2}{*}{ Insgesamt } & \multicolumn{4}{|c|}{ davon } \\
\hline & & & $\begin{array}{l}\text { Personal- } \\
\text { ausgaben }\end{array}$ & $\begin{array}{l}\text { Laufende } \\
\text { Sachausgaben }\end{array}$ & $\begin{array}{l}\text { Ausgaben für } \\
\text { Ausrüstungs- } \\
\text { investitionen }\end{array}$ & $\begin{array}{l}\text { Bauausgaben } \\
\text { und Ausgaben } \\
\text { für Liegen- } \\
\text { schaftsankäufe }\end{array}$ \\
\hline & & \multicolumn{5}{|c|}{ in $1.000 \mathrm{EUR}$} \\
\hline 1.0 bis 6.0 Insgesamt & $254^{2)}$ & 330.232 & 182.296 & 123.416 & 16.234 & 8.286 \\
\hline 1.0 bis 4.0 zusammen & $97^{2)}$ & 218.420 & 122.972 & 77.649 & 11.394 & 6.405 \\
\hline 1.0 Naturwissenschaften & 34 & 38.390 & 17.244 & 16.273 & 3.910 & 963 \\
\hline 2.0 Technische Wissenschaften & 12 & 22.029 & 14.545 & 6.155 & 1.296 & 33 \\
\hline 3.0 Humanmedizin & $32^{2)}$ & 121.429 & 65.453 & 47.189 & 4.629 & 4.158 \\
\hline 4.0 Land- und Forstwirtschaft, Veterinärmedizin & 19 & 36.572 & 25.730 & 8.032 & 1.559 & 1.251 \\
\hline 5.0 und 6.0 zusammen & 157 & 111.812 & 59.324 & 45.767 & 4.840 & 1.881 \\
\hline 5.0 Sozialwissenschaften & 85 & 44.692 & 29.319 & 14.601 & 675 & 97 \\
\hline 6.0 Geisteswissenschaften & 72 & 67.120 & 30.005 & 31.166 & 4.165 & 1.784 \\
\hline
\end{tabular}

Q: STATISTIK AUSTRIA, Erhebung über Forschung und experimentelle Entwicklung 2006. Erstellt am: 29.08.08. - 1) Bundesinstitutionen (unter Ausklammerung der im Hochschulsektor zusammengefassten), Landes-, Gemeinde- und Kammerinstitutionen, F\&E-Einrichtungen der Sozialversicherungsträger, von der öffentlichen Hand finanzierte und/oder kontrollierte private gemeinnützige Institutionen sowie F\&E-Einrichtungen der Ludwig Boltzmann-Gesellschaft; einschließlich Landeskrankenanstalten. Die Landeskrankenanstalten wurden nicht mittels Fragebogenerhebung erfasst, sondern es erfolgte eine Schätzung der F\&E-Ausgaben durch Statistik Austria unter Heranziehung der Meldungen der Ämter der Landesregierungen. - 2) Anzahl der Erhebungseinheiten ohne Landeskrankenanstalten.

\section{Tabelle 35: Sektor Staat ${ }^{1)}$ : Ausgaben für Forschung und experimentelle Entwicklung (F\&E) 2006 nach Rechtsträgern und Ausgabenarten}

\begin{tabular}{|c|c|c|c|c|c|c|}
\hline \multirow{3}{*}{ Rechtsträger } & \multirow{3}{*}{$\begin{array}{l}\text { F\&E } \\
\text { durch- } \\
\text { führende } \\
\text { Erhebungs- } \\
\text { einheiten }\end{array}$} & \multirow[b]{2}{*}{ Insgesamt } & \multicolumn{4}{|c|}{ davon } \\
\hline & & & $\begin{array}{l}\text { Personal- } \\
\text { ausgaben }\end{array}$ & $\begin{array}{l}\text { Laufende } \\
\text { Sachausgaben }\end{array}$ & $\begin{array}{l}\text { Ausgaben für } \\
\text { Ausrüstungs- } \\
\text { investitionen }\end{array}$ & $\begin{array}{l}\text { Bauausgaben } \\
\text { und Ausgaben } \\
\text { für Liegen- } \\
\text { schaftsankäufe }\end{array}$ \\
\hline & & \multicolumn{5}{|c|}{ in 1.000 EUR } \\
\hline Insgesamt & $254^{2)}$ & 330.232 & 182.296 & 123.416 & 16.234 & 8.286 \\
\hline Bund & 55 & 100.070 & 56.469 & 32.650 & 8.586 & 2.365 \\
\hline Länder (einschließlich Wien) & $39^{2)}$ & 145.165 & 70.922 & 63.498 & 4.934 & 5.811 \\
\hline Gemeinden (ohne Wien) & 8 & 4.995 & 2.444 & 2.303 & 248 & - \\
\hline Kammern & 3 & 4.402 & 654 & 3.748 & - & - \\
\hline Sozialversicherungsträger & - & - & - & - & - & - \\
\hline PloE öffentlich ${ }^{3)}$ & 108 & 64.328 & 44.707 & 17.818 & 1.693 & 110 \\
\hline Ludwig Boltzmann-Gesellschaft & 41 & 11.272 & 7.100 & 3.399 & 773 & - \\
\hline
\end{tabular}

Q: STATISTIK AUSTRIA, Erhebung über Forschung und experimentelle Entwicklung 2006. Erstellt am: 04.09.2008 - 1) Bundesinstitutionen (unter Ausklammerung der im Hochschulsektor zusammengefassten), Landes-, Gemeinde- und Kammerinstitutionen, F\&E-Einrichtungen der Sozialversicherungsträger, von der öffentlichen Hand finanzierte und/oder kontrollierte private gemeinnützige Institutionen sowie F\&E-Einrichtungen der Ludwig Boltzmann-Gesellschaft; einschließlich Landeskrankenanstalten. Die Landeskrankenanstalten wurden nicht mittels Fragebogenerhebung erfasst, sondern es erfolgte eine Schätzung der F\&E-Ausgaben durch Statistik Austria unter Heranziehung der Meldungen der Ämter der Landesregierungen. - 2) Anzahl der Erhebungseinheiten ohne Landeskrankenanstalten. - 3) Private gemeinnützige Institutionen, die überwiegend von der öffentlichen Hand finanziert und/oder kontrolliert werden. 
Tabelle 36: Sektor Staat ${ }^{1)}$ : Ausgaben für Forschung und experimentelle Entwicklung (F\&E) 2006 nach Wissenschaftszweigen und Forschungsarten

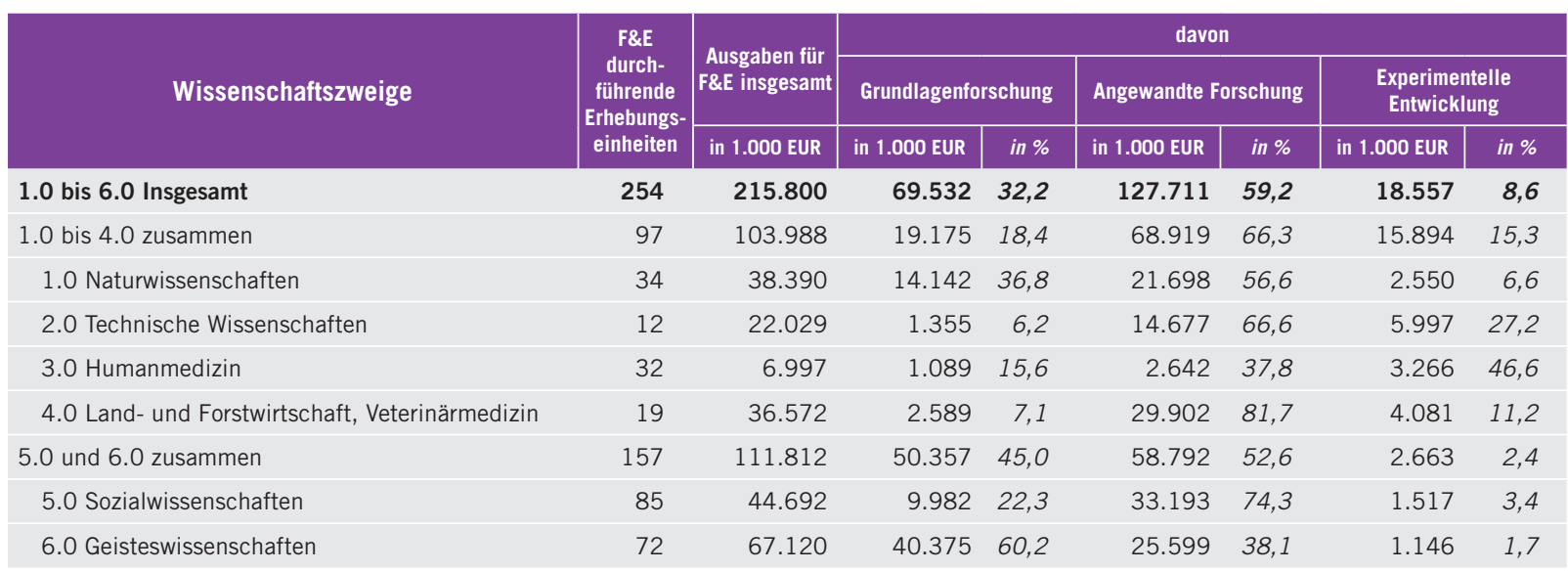

Q: STATISTIK AUSTRIA, Erhebung über Forschung und experimentelle Entwicklung 2006. Erstellt am: 04.09.2008 - 1) Bundesinstitutionen (unter Ausklammerung der im Hochschulsektor zusammengefassten), Landes-, Gemeinde- und Kammerinstitutionen, F\&E-Einrichtungen der Sozialversicherungsträger, von der öffentlichen Hand finanzierte und/oder kontrollierte private gemeinnützige Institutionen sowie F\&E-Einrichtungen der Ludwig Boltzmann-Gesellschaft; ohne Landeskrankenanstalten. Die Landeskrankenanstalten wurden nicht mittels Fragebogenerhebung erfasst, sondern es erfolgte eine Schätzung der F\&E-Ausgaben durch Statistik Austria unter Heranziehung der Meldungen der Ämter der Landesregierungen. Eine Aufgliederung der F\&E-Ausgaben nach Forschungsarten liegt nicht vor.

Tabelle 37: Sektor Staat ${ }^{1}$ : Ausgaben für Forschung und experimentelle Entwicklung (F\&E) 2006 nach Rechtsträgern und Forschungsarten

\begin{tabular}{|c|c|c|c|c|c|c|c|c|}
\hline \multirow{3}{*}{ Rechtsträger } & \multirow{3}{*}{$\begin{array}{l}\text { F\&E } \\
\text { durch- } \\
\text { führende } \\
\text { Erhebungs- } \\
\text { einheiten }\end{array}$} & \multirow{3}{*}{$\begin{array}{c}\text { Ausgaben für } \\
\text { F\&E insgesamt } \\
\text { in } 1.000 \text { EUR }\end{array}$} & \multicolumn{6}{|c|}{ davon } \\
\hline & & & \multicolumn{2}{|c|}{ Grundlagenforschung } & \multicolumn{2}{|c|}{ Angewandte Forschung } & \multicolumn{2}{|c|}{$\begin{array}{l}\text { Experimentelle } \\
\text { Entwicklung }\end{array}$} \\
\hline & & & in 1.000 EUR & in $\%$ & in 1.000 EUR & in $\%$ & in 1.000 EUR & in $\%$ \\
\hline Insgesamt & 254 & 215.800 & 69.532 & 32,2 & 127.711 & 59,2 & 18.557 & 8,6 \\
\hline Bund & 55 & 100.070 & 33.983 & 34,0 & 59.708 & 59,6 & 6.379 & 6,4 \\
\hline Länder (einschließlich Wien) & 39 & 30.733 & 14.627 & 47,6 & 15.499 & 50,4 & 607 & 2,0 \\
\hline Gemeinden (ohne Wien) & 8 & 4.995 & 1.916 & 38,4 & 2.127 & 42,5 & 952 & 19,1 \\
\hline Kammern & 3 & 4.402 & 1.006 & 22,9 & 3.384 & 76,8 & 12 & 0,3 \\
\hline Sozialversicherungsträger & - & - & - & - & - & - & - & - \\
\hline PloE öffentlich 2) & 108 & 64.328 & 13.942 & 21,7 & 42.843 & 66,6 & 7.543 & 11,7 \\
\hline Ludwig Boltzmann-Gesellschaft & 41 & 11.272 & 4.058 & 36,0 & 4.150 & 36,8 & 3.064 & 27,2 \\
\hline
\end{tabular}

Q: STATISTIK AUSTRIA, Erhebung über Forschung und experimentelle Entwicklung 2006. Erstellt am: 04.09.2008 - 1) Bundesinstitutionen (unter Ausklammerung der im Hochschulsektor zusammengefassten), Landes-, Gemeinde- und Kammerinstitutionen, F\&E-Einrichtungen der Sozialversicherungsträger, von der öffentlichen Hand finanzierte und/oder kontrollierte private gemeinnützige Institutionen sowie F\&E-Einrichtungen der Ludwig Boltzmann-Gesellschaft; ohne Landeskrankenanstalten. Die Landeskrankenanstalten wurden nicht mittels Fragebogenerhebung erfasst, sondern es erfolgte eine Schätzung der F\&E-Ausgaben durch Statistik Austria unter Heranziehung der Meldungen der Ämter der Landesregierungen. Eine Aufgliederung der F\&E-Ausgaben nach Forschungsarten liegt nicht vor. - 2) Private gemeinnützige Institutionen, die überwiegend von der öffentlichen Hand finanziert und/oder kontrolliert werden. 


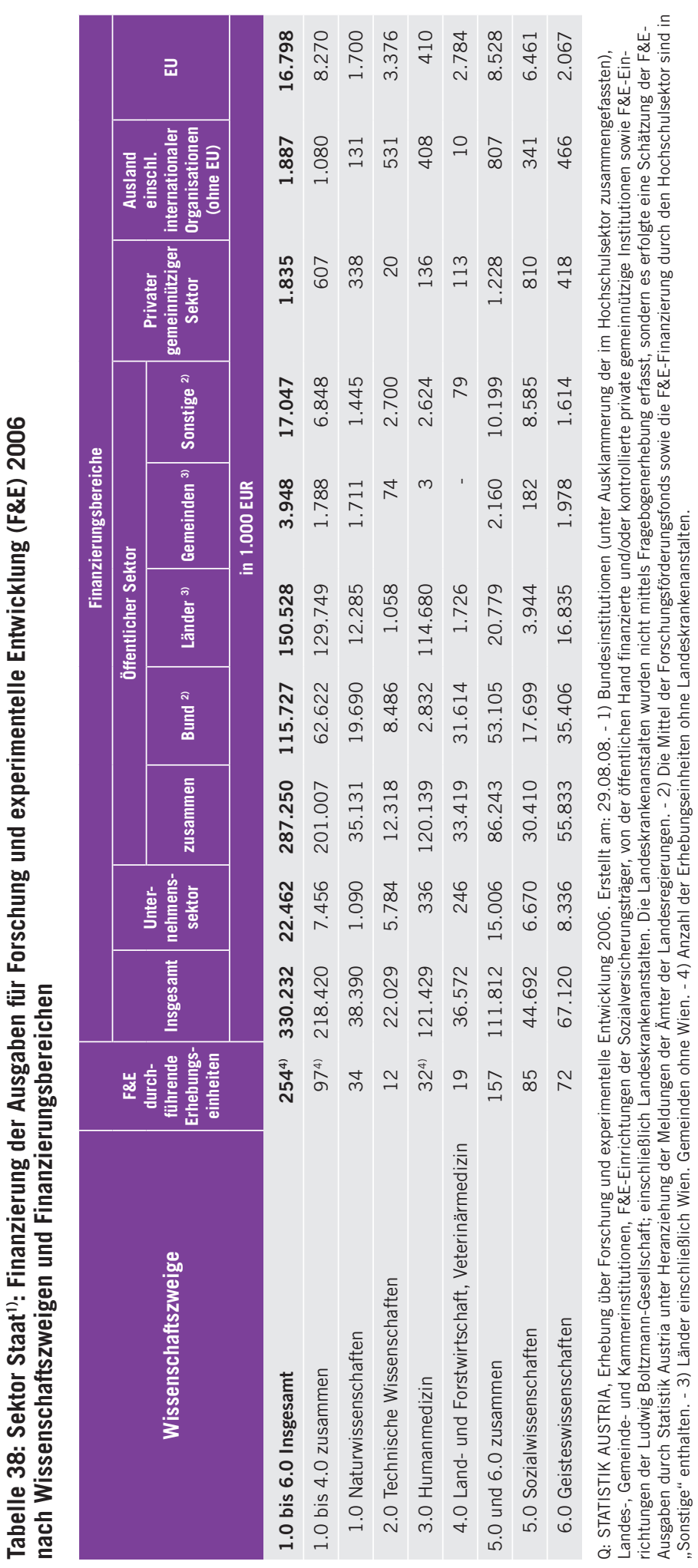




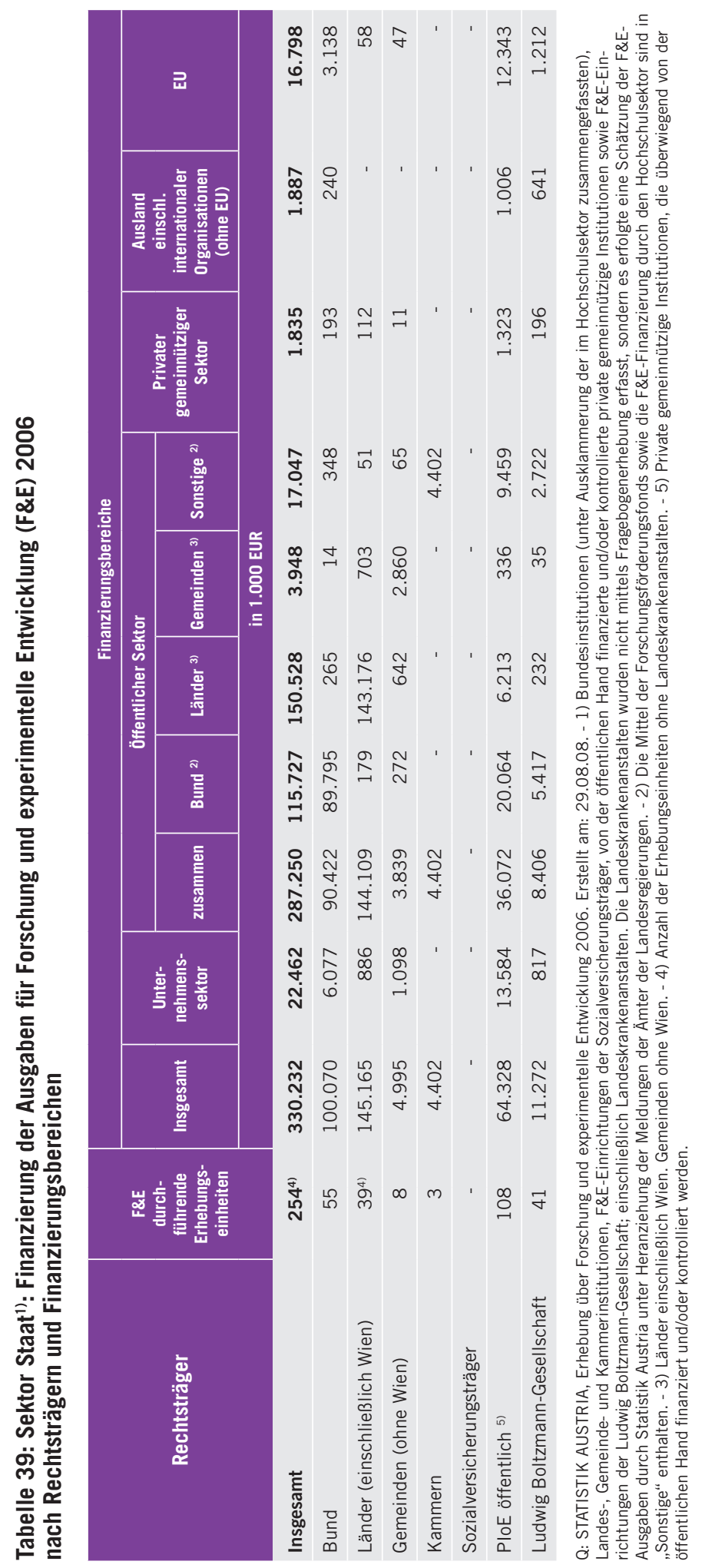


Tabelle 40: Privater gemeinnütziger Sektor ${ }^{1)}$ : Beschäftigte in Forschung und experimenteller Entwicklung (F\&E) 2006 nach Wissenschaftszweigen und Beschäftigtenkategorien

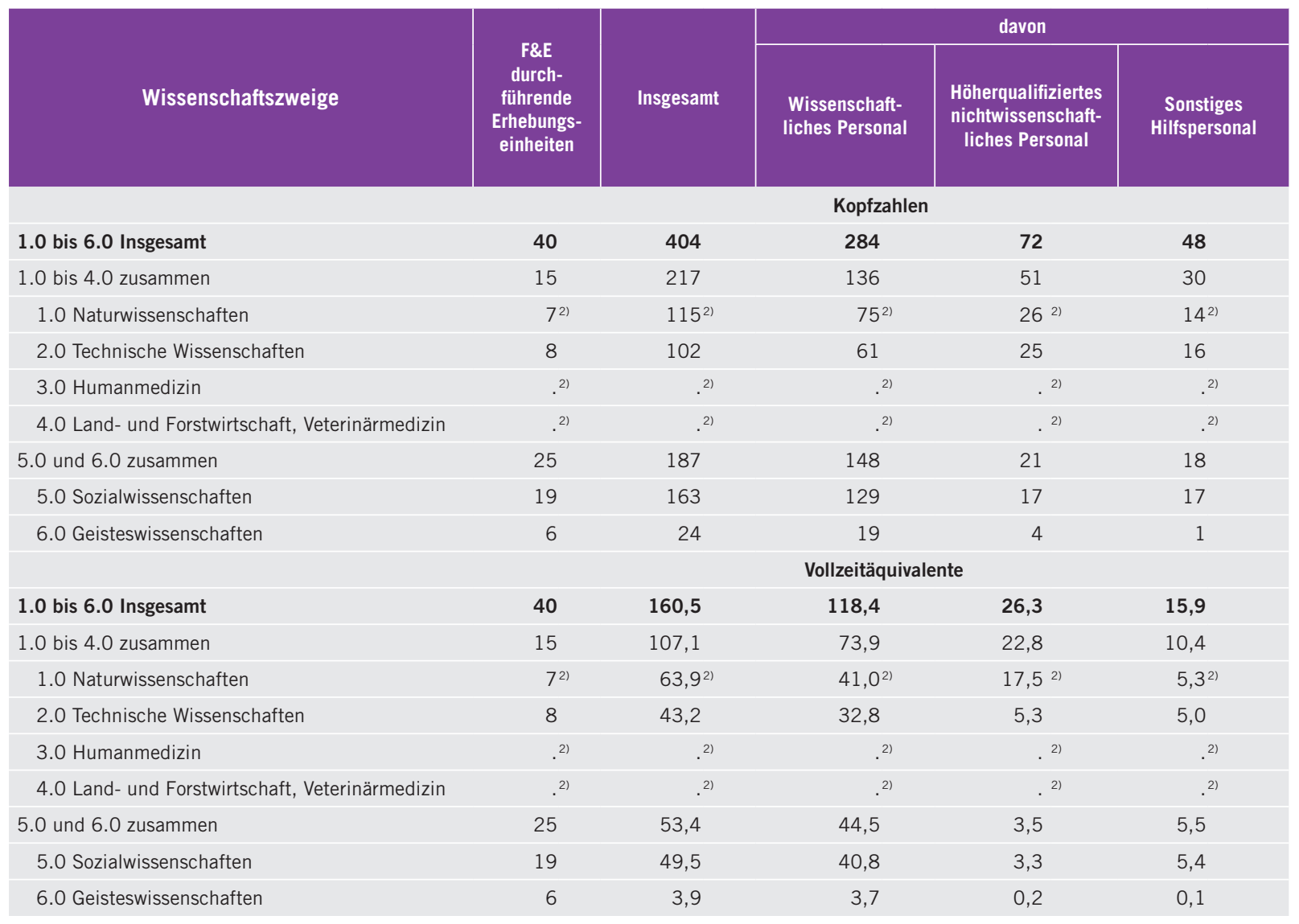

Q: STATISTIK AUSTRIA, Erhebung über Forschung und experimentelle Entwicklung 2006. Erstellt am: 08.08.2008. - 1) Private gemeinnützige Institutionen, deren Status ein vorwiegend privater oder privatrechtlicher, konfessioneller oder sonstiger nicht öffentlicher ist. - 2) Aus Geheimhaltungsgründen können die Daten nur gemeinsam ausgewiesen werden. - Rundungsdifferenzen. 
Tabelle 41: Privater gemeinnütziger Sektor ${ }^{1)}$ : Ausgaben für Forschung und experimentelle Entwicklung (F\&E) 2006 nach Wissenschaftszweigen und Ausgabenarten

\begin{tabular}{|c|c|c|c|c|c|c|}
\hline \multirow{3}{*}{ Wissenschaftszweige } & \multirow{3}{*}{$\begin{array}{l}\text { F\&E } \\
\text { durch- } \\
\text { führende } \\
\text { Erhebungs- } \\
\text { einheiten }\end{array}$} & \multirow[b]{2}{*}{ Insgesamt } & \multicolumn{4}{|c|}{ davon } \\
\hline & & & $\begin{array}{l}\text { Personal- } \\
\text { ausgaben }\end{array}$ & $\begin{array}{c}\text { Laufende } \\
\text { Sachausgaben }\end{array}$ & $\begin{array}{l}\text { Ausgaben für } \\
\text { Ausruistungs- } \\
\text { investitionen }\end{array}$ & $\begin{array}{l}\text { Bauausgaben } \\
\text { und Ausgaben } \\
\text { für Liegen- } \\
\text { schaftsankäufe }\end{array}$ \\
\hline & & \multicolumn{5}{|c|}{ in 1.000 EUR } \\
\hline 1.0 bis 6.0 Insgesamt & 40 & 16.519 & 7.630 & 8.538 & 348 & 3 \\
\hline 1.0 bis 4.0 zusammen & 15 & 12.472 & 5.123 & 7.068 & 278 & 3 \\
\hline 1.0 Naturwissenschaften & 4 & 804 & 427 & 335 & 39 & 3 \\
\hline 2.0 Technische Wissenschaften & 8 & 6.921 & 2.058 & 4.660 & 203 & - \\
\hline 3.0 Humanmedizin & $.2)$ & .2) & $.2)$ &.$^{2)}$ &.$^{2)}$ & $.2)$ \\
\hline 4.0 Land- und Forstwirtschaft, Veterinärmedizin & $.2)$ & $.2)$ & $.2)$ &.$^{2)}$ &.$^{2)}$ & $.2)$ \\
\hline 5.0 und 6.0 zusammen & 25 & 4.047 & 2.507 & 1.470 & 70 & - \\
\hline 5.0 Sozialwissenschaften & 19 & 3.859 & 2.444 & 1.353 & 62 & - \\
\hline 6.0 Geisteswissenschaften & 6 & 188 & 63 & 117 & 8 & - \\
\hline
\end{tabular}

Q: STATISTIK AUSTRIA, Erhebung über Forschung und experimentelle Entwicklung 2006. Erstellt am: 29.08.08. - 1) Private gemeinnützige Institutionen, deren Status ein vorwiegend privater oder privatrechtlicher, konfessioneller oder sonstiger nicht öffentlicher ist. - 2) Daten können aus Geheimhaltungsgründen nicht gesondert ausgewiesen werden, sind jedoch in den Zwischen- und Endsummen enthalten. 
Tabelle 42: Privater gemeinnütziger Sektor ${ }^{1}$ : Ausgaben für Forschung und experimentelle Entwicklung (F\&E) 2006 nach Wissenschaftszweigen und Forschungsarten

\begin{tabular}{|c|c|c|c|c|c|c|c|c|}
\hline \multirow{3}{*}{ Wissenschaftszweige } & \multirow{3}{*}{$\begin{array}{l}\text { F\&E } \\
\text { durch- } \\
\text { führende } \\
\text { Erhebungs- } \\
\text { einheiten }\end{array}$} & \multirow{3}{*}{$\begin{array}{c}\begin{array}{c}\text { Ausgaben für } \\
\text { F\&E insgesamt }\end{array} \\
\text { in } 1.000 \text { EUR }\end{array}$} & \multicolumn{6}{|c|}{ davon } \\
\hline & & & \multicolumn{2}{|c|}{ Grundlagenforschung } & \multicolumn{2}{|c|}{ Angewandte Forschung } & \multicolumn{2}{|c|}{$\begin{array}{l}\text { Experimentelle } \\
\text { Entwicklung }\end{array}$} \\
\hline & & & in 1.000 EUR & in $\%$ & in 1.000 EUR & in \% & in 1.000 EUR & in \% \\
\hline 1.0 bis 6.0 Insgesamt & 40 & 16.519 & 3.682 & 22,3 & 12.076 & 73,1 & 761 & 4,6 \\
\hline 1.0 bis 4.0 zusammen & 15 & 12.472 & 2.790 & 22,4 & 9.019 & 72,3 & 663 & 5,3 \\
\hline 1.0 Naturwissenschaften & 4 & 804 & 742 & 92,3 & 62 & 7,7 & - & - \\
\hline 2.0 Technische Wissenschaften & 8 & 6.921 & 2.036 & 29,4 & 4.326 & 62,5 & 559 & 8,1 \\
\hline 3.0 Humanmedizin & $.2)$ &.$^{2)}$ & $.2)$ &.${ }^{2)}$ & $.2)$ &.$^{2)}$ &.$^{2)}$ &.$^{2)}$ \\
\hline 4.0 Land- und Forstwirtschaft, Veterinärmedizin &.$^{2)}$ & $.2)$ &.$^{2)}$ &..$^{2)}$ & $.2)$ &.$^{2)}$ & $.2)$ &..$^{2)}$ \\
\hline 5.0 und 6.0 zusammen & 25 & 4.047 & 892 & 22,0 & 3.057 & 75,6 & 98 & 2,4 \\
\hline 5.0 Sozialwissenschaften & 19 & 3.859 & 833 & 21,6 & 2.970 & 76,9 & 56 & 1,5 \\
\hline 6.0 Geisteswissenschaften & 6 & 188 & 59 & 31,4 & 87 & 46,3 & 42 & 22,3 \\
\hline
\end{tabular}

Q: STATISTIK AUSTRIA, Erhebung über Forschung und experimentelle Entwicklung 2006. Erstellt am: 04.09.2008 - 1) Private gemeinnützige Institutionen, deren Status ein vorwiegend privater oder privatrechtlicher, konfessioneller oder sonstiger nicht öffentlicher ist. - 2) Daten können aus Geheimhaltungsgründen nicht gesondert ausgewiesen werden, sind jedoch in den Zwischen- und Endsummen enthalten. 


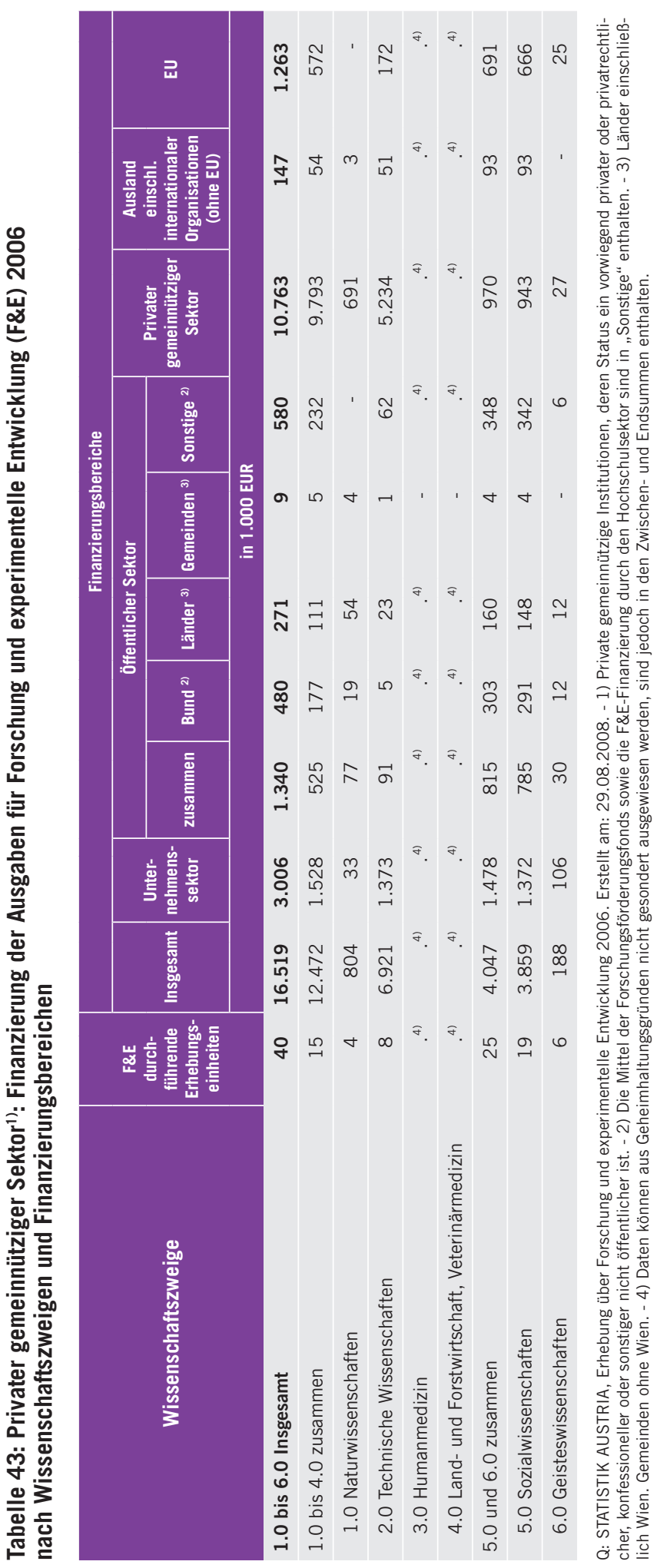


Tabelle 44: Unternehmenssektor ${ }^{1)}$ : Beschäftigte in Forschung und experimenteller Entwicklung (F\&E) 2006 nach Wirtschaftszweigen, Beschäftigtengrößenklassen und Beschäftigtenkategorien

\begin{tabular}{|c|c|c|c|c|c|c|c|}
\hline \multirow{3}{*}{ 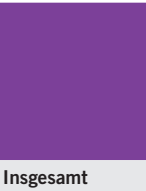 } & \multirow{2}{*}{$\begin{array}{l}\text { Wirtschaftszweige, } \\
\text { Beschäftigtengrößenklassen }\end{array}$} & \multirow[b]{2}{*}{$\begin{array}{l}\text { F\&E durch- } \\
\text { führende } \\
\text { Erhebungs- } \\
\text { einheiten }\end{array}$} & \multirow[b]{2}{*}{$\begin{array}{c}\text { Kopfzahlen } \\
\text { für F\&E } \\
\text { insgesamt }\end{array}$} & \multicolumn{4}{|c|}{ Vollzeitäquivalente für F\&E } \\
\hline & & & & \begin{tabular}{|l|} 
Insgesamt \\
\end{tabular} & \begin{tabular}{|c|} 
Wissenschaftler, \\
Wissenschaft- \\
lerinnen und \\
Ingenieure, \\
Ingenieurinnen'2)
\end{tabular} & \begin{tabular}{|c|} 
Höher \\
qualifiziertes \\
nichtwissen- \\
schaftliches $^{\text {Personal }}$ (3) $^{3)}$ \\
\end{tabular} & \begin{tabular}{|c|}
$\begin{array}{c}\text { Sonstiges } \\
\text { Hilfs- } \\
\text { personal }\end{array}$ \\
\end{tabular} \\
\hline & & 2.407 & 45.336 & $34.125,8$ & $18.470,5$ & $12.583,3$ & $3.072,0$ \\
\hline \multicolumn{8}{|l|}{ Wirtschaftszweige } \\
\hline $01+02+05$ & Land- und Forstwirtschaft, Fischerei & 3 & 46 & 13,5 & 4,9 & 8,6 & - \\
\hline $10-14$ & Bergbau und Gewinnung von Steinen und Erden & 10 & 117 & 50,5 & 7,7 & 41,9 & 0,9 \\
\hline $15-37$ & Sachgütererzeugung & 1.324 & 29.360 & $23.779,8$ & $12.480,1$ & $9.368,9$ & $1.930,8$ \\
\hline 15 & Nahrungs- und Genussmittel, Getränke & 87 & 573 & 281,6 & 151,6 & 107,6 & 22,4 \\
\hline 16 & Tabakverarbeitung & .4) & .4) & .4) & $.4)$ & $.4)$ & .4) \\
\hline 17 & Textilien und Textilwaren (ohne Bekleidung) & 28 & 307 & 229,7 & 70,0 & 140,4 & 19,3 \\
\hline $18+19$ & Bekleidung, Leder, Schuhe & 14 & 96 & 57,9 & 19,5 & 27,9 & 10,5 \\
\hline 20 & Holz (ohne Herstellung von Möbeln) & 52 & 278 & 136,9 & 60,4 & 50,9 & 25,6 \\
\hline 21 & Papier und Pappe & 27 & 189 & 136,9 & 55,8 & 79,8 & 1,3 \\
\hline 22 & Verlagswesen, Druckerei, Vervielfältigung von bespielten Ton-, Bild- und Datenträgern & 13 & 144 & 114,0 & 39,2 & 64,8 & 10,0 \\
\hline 23 & Kokerei, Mineralölverarbeitung, Spalt- und Brutstoffe & $.4)$ & $.4)$ & .4) & $.4)$ & .4) & . ${ }^{4)}$ \\
\hline 24 ohne 24.4 & Chemikalien und chemische Erzeugnisse (ohne pharmazeutische Erzeugnisse) & 73 & 1.383 & $1.143,5$ & 469,4 & 587,6 & 86,5 \\
\hline 24.4 & Pharmazeutische Erzeugnisse & 25 & 1.514 & $1.323,9$ & 751,1 & 451,2 & 121,6 \\
\hline 25 & Gummi- und Kunststoffwaren & 76 & 1.081 & 836,3 & 301,0 & 353,1 & 182,2 \\
\hline 26 & Glas, Waren aus Steinen und Erden & 64 & 826 & 666,1 & 483,1 & 144,5 & 38,5 \\
\hline $\begin{array}{l}27.1-27.3 \text { und } \\
27.51 / 52\end{array}$ & $\begin{array}{l}\text { Roheisen, Stahl, Ferrolegierungen, Rohre, } \\
\text { Eisen-, Stahlgießerei }\end{array}$ & 29 & 774 & 416,9 & 222,3 & 134,0 & 60,6 \\
\hline $27.4+27.53 / 54$ & NE-Metalle, Leicht-, Schwermetallgießerei & 26 & 414 & 275,1 & 130,0 & 105,5 & 39,6 \\
\hline 28 & Metallerzeugnisse & 138 & 1.522 & 905,2 & 413,2 & 424,3 & 67,7 \\
\hline 29 & Maschinenbau & 298 & 5.308 & $4.159,4$ & $1.751,7$ & $2.064,8$ & 342,9 \\
\hline 30 & Büromaschinen, Datenverarbeitungsgeräte und -einrichtungen & 12 & 229 & 183,1 & 80,4 & 97,5 & 5,2 \\
\hline 31 & Geräte der Elektrizitätserzeugung, -verteilung u.Ä. & 82 & 2.194 & $1.827,1$ & 957,2 & 739,7 & 130,2 \\
\hline 32 ohne 32.1 & Rundfunk-, Fernseh- und Nachrichtentechnik (ohne elektronische Bauelemente) & 28 & 4.070 & $3.928,1$ & $2.461,3$ & $1.400,8$ & 66,0 \\
\hline 32.1 & Elektronische Bauelemente & 31 & 1.818 & $1.701,8$ & $1.481,5$ & 111,4 & 108,9 \\
\hline 33 ohne 33.1 & Mess-, Steuer- und Regelungstechnik, Optik & 75 & 1.169 & 827,1 & 430,1 & 382,9 & 14,1 \\
\hline 33.1 & Medizintechnik & 31 & 606 & 534,0 & 376,5 & 112,0 & 45,5 \\
\hline 34 & Kraftwagen und Kraftwagenteile & 38 & 2.678 & $2.522,4$ & $1.209,2$ & 924,5 & 388,7 \\
\hline 35 & Sonstiger Fahrzeugbau & 16 & 1.138 & 877,4 & 271,1 & 512,1 & 94,2 \\
\hline 36 ohne 36.1 & Schmuck, Musikinstrumente, Sportgeräte, Spielwaren, sonstige Erzeugnisse & 30 & 694 & 455,3 & 201,1 & 236,2 & 18,0 \\
\hline 36.1 & Möbel & 26 & 238 & 148,2 & 42,5 & 78,3 & 27,4 \\
\hline 37 & Rückgewinnung (Recycling) & $.4)$ & $.4)$ & .4) & $.4)$ & $.4)$ & .4) \\
\hline $40+41$ & Energie- und Wasserversorgung & 25 & 220 & 62,8 & 37,8 & 23,8 & 1,2 \\
\hline 45 & Bauwesen & 82 & 407 & 188,1 & 86,6 & 76,2 & 25,3 \\
\hline $50-93$ & Dienstleistungen & 963 & 15.186 & $10.031,1$ & $5.853,4$ & $3.063,9$ & $1.113,8$ \\
\hline $50-52$ & Handel; Instandhaltung und Reparatur von Kraftfahrzeugen und Gebrauchsgütern & 205 & 1.866 & $1.373,1$ & 668,3 & 611,7 & 93,1 \\
\hline 55 & Beherbergungs- und Gaststättenwesen & - & - & - & - & - & - \\
\hline $60-64$ & Verkehr und Nachrichtenübermittlung & 25 & 508 & 397,6 & 336,7 & 43,8 & 17,1 \\
\hline $65-67$ & Kredit- und Versicherungswesen & 7 & 451 & 289,8 & 181,3 & 87,2 & 21,3 \\
\hline $70+71+74$ & $\begin{array}{l}\text { Realitätenwesen, Vermietung beweglicher Sachen, unternehmensbezogene } \\
\text { Dienstleistungen }\end{array}$ & 243 & 3.433 & $2.338,7$ & $1.369,0$ & 528,7 & 441,1 \\
\hline 72 ohne 72.2 & Datenverarbeitung und Datenbanken (ohne Softwarehäuser) & 56 & 491 & 265,6 & 133,2 & 107,6 & 24,8 \\
\hline 72.2 & Softwarehäuser & 210 & 3.326 & $1.904,7$ & 964,1 & 884,2 & 56,4 \\
\hline 73 & Forschung und Entwicklung & 193 & 4.898 & $3.378,7$ & $2.158,4$ & 779,9 & 440,4 \\
\hline $75-93$ & $\begin{array}{l}\text { Öffentliche Verwaltung, Unterrichtswesen, Gesundheitswesen, sonstige öffentliche und } \\
\text { persönliche Dienstleistungen }\end{array}$ & 24 & 213 & 82,8 & 42,4 & 20,8 & 19,6 \\
\hline \multicolumn{8}{|c|}{ Beschäftigtengrößenklassen } \\
\hline \multicolumn{2}{|c|}{1 - 49 Beschäftigte } & 1.278 & 7.773 & $4.406,9$ & $2.746,3$ & $1.363,7$ & 296,9 \\
\hline \multicolumn{2}{|c|}{50 - 249 Beschäftigte } & 721 & 10.770 & $7.098,9$ & $3.505,7$ & $3.057,5$ & 535,7 \\
\hline \multicolumn{2}{|c|}{250 und mehr Beschäftigte } & 408 & 26.793 & $22.620,0$ & $12.218,5$ & $8.162,1$ & $2.239,5$ \\
\hline
\end{tabular}

Q: STATISTIK AUSTRIA, Erhebung über Forschung und experimentelle Entwicklung 2006. Erstellt am: 08.08.2008. - 1) Umfasst den kooperativen Bereich und den firmeneigenen Bereich. - 2) Akademiker, Akademikerinnen und gleichwertige Kräfte. - 3) Maturanten und Maturantinnen, Techniker und Technikerinnen, Laboranten und Laborantinnen. - 4) Daten können aus Geheimhaltungsgründen nicht gesondert ausgewiesen werden, sind jedoch in den Zwischen- und Endsummen enthalten. - Rundungsdifferenzen. 
Tabelle 45: Unternehmenssektor ${ }^{1}$ : Wissenschaftler, Wissenschaftlerinnen, Ingenieure und Ingenieurinnen in Forschung und experimenteller Entwicklung (F\&E) 2006 nach Wirtschaftszweigen, Ausbildung und Geschlecht

\begin{tabular}{|c|c|c|c|c|c|c|c|c|c|c|c|c|c|c|c|c|}
\hline \multirow{4}{*}{ 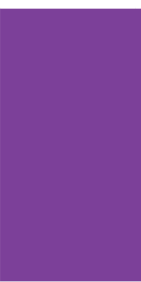 } & \multirow{4}{*}{ Wirtschaftszweige } & \multirow{4}{*}{ 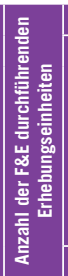 } & \multicolumn{14}{|c|}{ Vollzeitäquivalente für F\&E } \\
\hline & & & \multirow{2}{*}{\multicolumn{2}{|c|}{ 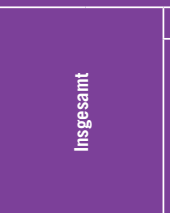 }} & \multicolumn{12}{|c|}{ davon } \\
\hline & & & & & \multicolumn{2}{|c|}{ 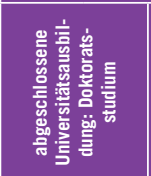 } & \multicolumn{2}{|c|}{ 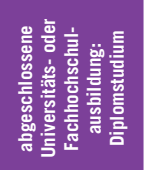 } & \multicolumn{2}{|c|}{ 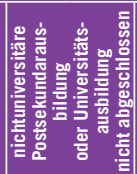 } & \multicolumn{2}{|c|}{ 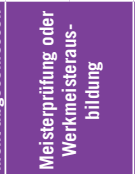 } & \multicolumn{2}{|c|}{ 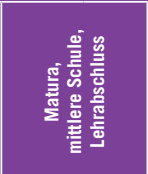 } & \multicolumn{2}{|c|}{ 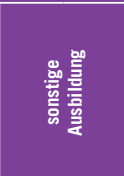 } \\
\hline & & & männl. & weibl. & männl. & weibl. & männl. & weibl. & männl. & weibl. & männl. & weibl. & männl. & weibl. & männl. & weibl. \\
\hline Insgesamt & & 2.407 & $16.206,8$ & $2.263,6$ & $2.608,2$ & 532,7 & $6.976,7$ & 841,2 & 616,1 & 111,9 & 431,0 & 24,1 & $5.012,1$ & 511,1 & 562,7 & 242,7 \\
\hline $01+02+05$ & Land- und Forstwirtschaft, Fischerei & 3 & 3,4 & 1,5 & 0,1 & 0,5 & 3,3 & 1,0 & - & - $\quad-$ & - & - & - & - & - & - \\
\hline $10-14$ & Bergbau und Gewinnung von Steinen und Erden & 10 & 7,5 & 0,2 & 0,2 & - & 5,7 & - & - & - & 0,4 & - & 0,4 & 0,2 & 0,8 & - \\
\hline $15-37$ & Sachgütererzeugung & 1.324 & $11.230,7$ & $1.249,3$ & $1.435,6$ & 250,3 & $4.484,5$ & 399,3 & 349,9 & 69,9 & 374,6 & 21,6 & $4.159,7$ & 379,8 & 426,4 & 128,4 \\
\hline 15 & Nahrungs- und Genussmittel, Getränke & 87 & 108,2 & 43,4 & 15,0 & 4,0 & 34,7 & 23,1 & 8,6 & 2,9 & 24,6 & 3,0 & 19,1 & 5,1 & 6,2 & 5,3 \\
\hline 16 & Tabakverarbeitung & .2) & $.2)$ & .2) & .2) & .2) & .2) & $.2)$ & .2) & $.2)$ & .2) & $.2)$ & .2) & .2) & .2) & $.2)$ \\
\hline 17 & Textilien und Textilwaren (ohne Bekleidung) & 28 & 56,4 & 13,6 & 4,5 & 0,7 & 11,9 & 5,0 & - & 3,9 & 6,8 & 0,3 & 28,0 & 3,0 & 5,2 & 0,7 \\
\hline $18+19$ & Bekleidung, Leder, Schuhe & 14 & 16,5 & 3,0 & 2,0 & - & 1,3 & 1,5 & - & - & 6,2 & - & 2,0 & 1,5 & 5,0 & - \\
\hline 22 & $\begin{array}{l}\text { Verlagswesen, Druckerei, Vervielfältigung von } \\
\text { bespielten Ton-, Bild- und Datenträgern }\end{array}$ & 13 & 34,2 & 5,0 & 5,0 & 2,0 & 19,2 & 3,0 & 3,1 & - & - & - & 5,9 & - & 1,0 & - \\
\hline 23 & $\begin{array}{l}\text { Kokerei, Mineralölverarbeitung, } \\
\text { Spalt- und Brutstoffe }\end{array}$ & .) & .2) & .2) & .2) &.$^{2)}$ & .2) &.$^{2)}$ & .2) & .2) &.$^{2)}$ & $.2)$ & .2) & .) & $.2)$ & $.2)$ \\
\hline $\begin{array}{l}24 \\
\text { ohne } 24.4\end{array}$ & $\begin{array}{l}\text { Chemikalien und chemische Erzeugnisse } \\
\text { (ohne pharmazeutische Erzeugnisse) }\end{array}$ & 73 & 363,8 & 105,6 & 170,6 & 43,1 & 72,4 & 33,7 & 17,8 & 5,0 & 9,7 & 1,0 & 86,1 & 19,3 & 7,2 & 3,5 \\
\hline 24.4 & Pharmazeutische Erzeugnisse & 25 & 395,3 & 355,8 & 217,2 & 115,2 & 21,0 & 37,1 & 18,2 & 27,3 & 14,0 & 14,0 & 55,8 & 122,4 & 69,1 & 39,8 \\
\hline 25 & Gummi- und Kunststoffwaren & 76 & 272,6 & 28,4 & 24,0 & 1,1 & 111,1 & 9,2 & 9,1 & 4,5 & 21,6 & - & 102,6 & 11,9 & 4,2 & 1,7 \\
\hline 26 & Glas, Waren aus Steinen und Erden & 64 & 400,0 & 83,1 & 28,5 & 6,2 & 77,6 & 11,8 & 4,1 & - & 4,5 & - & 109,5 & 8,8 & 175,8 & 56,3 \\
\hline $\begin{array}{l}27.1-27.3 \text { und } \\
27.51 / 52\end{array}$ & $\begin{array}{l}\text { Roheisen, Stahl, Ferrolegierungen, Rohre, } \\
\text { Eisen-, Stahlgießerei }\end{array}$ & 29 & 204,7 & 17,6 & 51,8 & 3,0 & 83,6 & 8,8 & 1,7 & 2,0 & 3,0 & - & 62,9 & 2,8 & 1,7 & 1,0 \\
\hline $27.4+27.53 / 54$ & NE-Metalle, Leicht-, Schwermetallgießerei & 26 & 120,6 & 9,4 & 23,3 & 1,3 & 39,3 & 2,0 & 2,2 & - & 5,7 & - & 48,6 & 6,1 & 1,5 & - \\
\hline 28 & Metallerzeugnisse & 138 & 391,6 & 21,6 & 28,7 & 4,6 & 99,0 & 5,3 & 9,2 & 0,2 & 23,2 & - & 228,6 & 10,5 & 2,9 & 1,0 \\
\hline 29 & Maschinenbau & 298 & $1.697,4$ & 54,3 & 135,4 & 4,8 & 749,5 & 25,0 & 52,9 & 5,0 & 93,4 & - & 629,3 & 18,0 & 36,9 & 1,5 \\
\hline 30 & $\begin{array}{l}\text { Büromaschinen, Datenverarbeitungs- geräte } \\
\text { und -einrichtungen }\end{array}$ & 12 & 75,0 & 5,4 & 2,4 & 2,7 & 34,3 & 2,7 & 13,0 & - & - & - & 22,3 & - & 3,0 & - \\
\hline 31 & Geräte der Elektrizitätserzeugung, -verteilung u.Ä. & 82 & 926,1 & 31,1 & 38,8 & 2,8 & 357,3 & 13,8 & 26,8 & 3,0 & 12,2 & - & 471,0 & 9,1 & 20,0 & 2,4 \\
\hline 33 ohne 33.1 & Mess-, Steuer- und Regelungstechnik, Optik & 75 & 404,9 & 25,2 & 61,5 & 6,0 & 181,1 & 14,9 & 22,0 & 1,8 & 9,6 & 1,0 & 123,7 & 1,5 & 7,0 & - \\
\hline 33.1 & Medizintechnik & 31 & 338,1 & 38,4 & 45,5 & 5,9 & 143,0 & 13,1 & 13,1 & - & 19,2 & - & 76,9 & 11,6 & 40,4 & 7,8 \\
\hline 34 & Kraftwagen und Kraftwagenteile & 38 & $1.170,6$ & 38,6 & 119,5 & 4,0 & 520,4 & 22,5 & 60,5 & - & 48,5 & - & 420,7 & 12,1 & 1,0 & - \\
\hline 35 & Sonstiger Fahrzeugbau & 16 & 259,1 & 12,0 & 32,7 & 1,0 & 83,3 & 3,0 & 11,0 & - & 34,1 & 2,0 & 93,0 & 5,0 & 5,0 & 1,0 \\
\hline 36 ohne 36.1 & $\begin{array}{l}\text { Schmuck, Musikinstrumente, Sportgeräte, } \\
\text { Spielwaren, sonstige Erzeugnisse }\end{array}$ & 30 & 179,0 & 22,1 & 7,0 & 1,0 & 57,6 & 11,2 & 19,7 & 2,1 & 6,7 & 0,3 & 84,4 & 7,5 & 3,6 & - \\
\hline 36.1 & Möbel & 26 & 39,1 & 3,4 & 1,1 & - & 9,6 & 1,1 & 2,0 & - & 8,4 & - & 12,7 & 0,9 & 5,3 & 1,4 \\
\hline 37 & Rückgewinnung (Recycling) & .2) & .2) & $.2)$ & .2) & .2) & $.2)$ & $.2)$ & .2) & $.2)$ & .2) & .2) & .2) & .2) & .2) & .2) \\
\hline $40+41$ & Energie- und Wasserversorgung & 25 & 33,7 & 4,1 & 7,1 & 0,5 & 16,7 & 2,3 & 3,7 & 1,0 & 3,0 & - & 3,2 & 0,3 & - & - \\
\hline 45 & Bauwesen & 82 & 85,3 & 1,3 & 10,0 & 0,1 & 37,0 & 0,2 & 6,9 & - & 3,1 & - & 27,9 & 0,9 & 0,4 & 0,1 \\
\hline $50-93$ & Dienstleistungen & 963 & $4.846,2$ & $1.007,2$ & $1.155,1$ & 281,3 & $2.429,5$ & 438,4 & 255,6 & 41,0 & 49,9 & 2,5 & 821,0 & 129,9 & 135,1 & 114,2 \\
\hline $50-52$ & $\begin{array}{l}\text { Handel; Instandhaltung und Reparatur von } \\
\text { Kraftfahrzeugen und Gebrauchsgütern }\end{array}$ & 205 & 523,5 & 144,8 & 136,3 & 44,5 & 159,2 & 51,5 & 52,6 & 1,6 & 29,4 & 1,0 & 112,5 & 30,6 & 33,5 & 15,6 \\
\hline 55 & Beherbergungs- und Gaststättenwesen & - & - & - & - & - & - & - & - & - & - & - & - & - & - & - \\
\hline $60-64$ & Verkehr und Nachrichtenübermittlung & 25 & 293,5 & 43,2 & 17,1 & 1,0 & 84,7 & 8,8 & 7,3 & - & 1,3 & - & 170,8 & 25,4 & 12,3 & 8,0 \\
\hline $65-67$ & Kredit- und Versicherungswesen & 7 & 86,7 & 94,6 & 15,9 & 5,5 & 20,4 & 3,6 & 0,6 & - & - & - & 1,8 & - & 48,0 & 85,5 \\
\hline $70+71+74$ & $\begin{array}{l}\text { Realitätenwesen, Vermietung beweglicher } \\
\text { Sachen, unternehmensbezogene Dienstleistungen }\end{array}$ & 243 & $1.248,1$ & 120,8 & 228,3 & 14,9 & 789,7 & 83,1 & 61,4 & 10,8 & 11,1 & - & 148,7 & 10,4 & 8,9 & 1,6 \\
\hline 72 ohne 72.2 & $\begin{array}{l}\text { Datenverarbeitung und Datenbanken } \\
\text { (ohne Softwarehäuser) }\end{array}$ & 56 & 122,1 & 11,1 & 15,8 & 3,5 & 45,6 & 4,3 & 1,8 & - & 2,5 & 0,5 & 45,8 & 2,4 & 10,6 & 0,4 \\
\hline 72.2 & Softwarehäuser & 210 & 870,3 & 93,8 & 103,7 & 11,1 & 408,0 & 43,6 & 72,1 & 4,7 & 3,3 & 1,0 & 270,1 & 31,4 & 13,1 & 2,0 \\
\hline 73 & Forschung und Entwicklung & 193 & $1.666,7$ & 491,7 & 630,9 & 200,3 & 908,0 & 239,5 & 58,1 & 22,8 & 2,3 & - & 63,3 & 28,1 & 4,0 & 1,0 \\
\hline 75-93 & $\begin{array}{l}\text { Öffentliche Verwaltung, Unterrichtswesen, } \\
\text { Gesundheitswesen, sonstige öffentliche und } \\
\text { persönliche Dienstleistungen }\end{array}$ & 24 & 35,3 & 7,1 & 7,2 & 0,5 & 13,8 & 4,0 & 1,7 & 1,0 & - & - & 7,9 & 1,5 & 4,7 & 0,1 \\
\hline
\end{tabular}

Q: STATISTIK AUSTRIA, Erhebung über Forschung und experimentelle Entwicklung (F\&E) 2006. Erstellt am 01.09.2008. - 1) Umfasst den firmeneigenen Bereich und den kooperativen Bereich. - 2) Daten können aus Geheimhaltungsgründen nicht gesondert ausgewiesen werden, sind jedoch in den Zwischen- und Endsummen enthalten. - Rundungsdifferenzen. 
Tabelle 46: Unternehmenssektor ${ }^{11}$ : Beschäftigte in Forschung und experimenteller Entwicklung (F\&E) und Ausgaben für F\&E 2006 nach Bundesländern²)

\begin{tabular}{|c|c|c|c|c|c|c|c|c|}
\hline \multirow{3}{*}{ Bundesländer } & \multicolumn{4}{|c|}{ Beschäftigte in F\&E } & \multicolumn{4}{|c|}{ F\&E-Ausgaben } \\
\hline & \multicolumn{2}{|c|}{$\begin{array}{l}\text { nach dem Haupt- } \\
\text { standort des } \\
\text { Unternehmens }\end{array}$} & \multicolumn{2}{|c|}{$\begin{array}{l}\text { nach dem F\&E-Standort/ } \\
\text { den F\&E-Standorten } \\
\text { des Unternehmens }\end{array}$} & \multicolumn{2}{|c|}{$\begin{array}{l}\text { nach dem Haupt- } \\
\text { standort des } \\
\text { Unternehmens }\end{array}$} & \multicolumn{2}{|c|}{$\begin{array}{l}\text { nach dem F\&E-Standort/ } \\
\text { den F\&E-Standorten } \\
\text { des Unternehmens }{ }^{3)}\end{array}$} \\
\hline & Kopfzahl & in $\%$ & Kopfzahl & in $\%$ & in 1.000 EUR & in $\%$ & in 1.000 EUR & in $\%$ \\
\hline Österreich & 45.336 & 100,0 & 45.336 & 100,0 & 4.448 .676 & 100,0 & 4.448 .676 & 100,0 \\
\hline Burgenland & 396 & 0,9 & 358 & 0,8 & 30.094 & 0,7 & 26.231 & 0,6 \\
\hline Kärnten & 2.519 & 5,6 & 2.510 & 5,5 & 352.765 & 7,9 & 336.681 & 7,6 \\
\hline Niederösterreich & 4.957 & 10,9 & 5.310 & 11,7 & 419.846 & 9,4 & 473.190 & 10,6 \\
\hline Oberösterreich & 8.872 & 19,6 & 9.182 & 20,3 & 873.162 & 19,6 & 905.143 & 20,3 \\
\hline Salzburg & 1.641 & 3,6 & 1.922 & 4,2 & 123.870 & 2,8 & 153.688 & 3,5 \\
\hline Steiermark & 8.475 & 18,7 & 9.607 & 21,2 & 759.020 & 17,1 & 902.772 & 20,3 \\
\hline Tirol & 2.664 & 5,9 & 2.624 & 5,8 & 311.086 & 7,0 & 309.133 & 6,9 \\
\hline Vorarlberg & 1.897 & 4,2 & 1.881 & 4,1 & 143.916 & 3,2 & 143.244 & 3,2 \\
\hline Wien & 13.915 & 30,6 & 11.942 & 26,4 & 1.434 .917 & 32,3 & 1.198 .594 & 27,0 \\
\hline
\end{tabular}

Q: STATISTIK AUSTRIA, Erhebung über Forschung und experimentelle Entwicklung (F\&E) 2006. Erstellt am: 29.08.08. - 1) Umfasst den firmeneigenen Bereich und den kooperativen Bereich. - 2) Die regionale Zuordnung der Einheiten des kooperativen Bereichs erfolgt ausschließlich nach dem Bundesland, in dem das Unternehmen seinen Hauptstandort hat. Für die Unternehmen des firmeneigenen Bereichs ist sowohl die Gliederung nach dem Bundesland des Hauptstandorts als auch eine alternative Gliederung nach dem Bundesland des F\&E-Standorts/den Bundesländern der F\&E-Standorte verfügbar. - 3) Die Ausgaben für F\&E nach dem(n) F\&E-Standort(en) wurden auf der Basis der Verteilung der Beschäftigten in F\&E auf die F\&E-Standorte berechnet. 
Tabelle 47: Unternehmenssektor ${ }^{1)}$ : Ausgaben für Forschung und experimentelle Entwicklung (F\&E) 2006 nach Wirtschaftszweigen, Beschäftigtengrößenklassen und Ausgabenarten

\begin{tabular}{|c|c|c|c|c|c|c|c|}
\hline \multirow{2}{*}{\multicolumn{2}{|c|}{$\begin{array}{c}\text { Wirtschaftszweige/ } \\
\text { Beschäftigtengrößenklassen }\end{array}$}} & \multirow{2}{*}{$\begin{array}{l}\text { Anzahl der } \\
\text { F\&E durch- } \\
\text { führenden } \\
\text { Erhebungs- } \\
\text { einheiten }\end{array}$} & Insgesamt & $\begin{array}{l}\text { Personal- } \\
\text { ausgaben }\end{array}$ & $\begin{array}{l}\text { Laufende } \\
\text { Sach- } \\
\text { ausgaben }\end{array}$ & $\begin{array}{l}\begin{array}{l}\text { Ausgaben } \\
\text { fuir } \\
\text { Anlagen } \\
\text { und } \\
\text { Ausstattung }\end{array} \\
\end{array}$ & $\begin{array}{l}\text { Ausgaben } \\
\text { für } \\
\text { Gebäude } \\
\text { und } \\
\text { Grundstücke }\end{array}$ \\
\hline & & & \multicolumn{5}{|c|}{ in 1.000 EUR } \\
\hline \multicolumn{2}{|l|}{ Insgesamt } & 2.407 & 4.448 .676 & 2.297 .903 & 1.757 .587 & 320.457 & 72.729 \\
\hline \multicolumn{8}{|l|}{ Wirtschaftszweige } \\
\hline $10-14$ & Bergbau und Gewinnung von Steinen und Erden & 10 & 7.146 & 3.417 & 2.693 & 1.036 & - \\
\hline $15-37$ & Sachgütererzeugung & 1.324 & 3.159 .392 & 1.646 .093 & 1.234 .121 & 225.916 & 53.262 \\
\hline 15 & Nahrungs- und Genussmittel, Getränke & 87 & 23.540 & 15.493 & 5.112 & 2.251 & 684 \\
\hline 16 & Tabakverarbeitung & $.2)$ & $.2)$ & $.2)$ & $.2)$ & $.2)$ & $.2)$ \\
\hline 17 & Textilien und Textilwaren (ohne Bekleidung) & 28 & 26.465 & 13.541 & 9.217 & 3.679 & 28 \\
\hline $18+19$ & Bekleidung, Leder, Schuhe & 14 & 5.345 & 2.637 & 1.976 & 322 & 410 \\
\hline 20 & Holz (ohne Herstellung von Möbeln) & 52 & 20.162 & 7.154 & 3.750 & 7.562 & 1.696 \\
\hline 22 & Verlagswesen, Druckerei, Vervielfältigung von bespielten Ton-, Bild- und Datenträgern & 13 & 21.057 & 9.838 & 9.801 & 1.418 & - \\
\hline 23 & Kokerei, Mineralölverarbeitung, Spalt- und Brutstoffe & $.2)$ & $.2)$ & $.2)$ & $.2)$ & $.2)$ &.$^{2)}$ \\
\hline 24 ohne 24.4 & Chemikalien und chemische Erzeugnisse (ohne pharmazeutische Erzeugnisse) & 73 & 124.462 & 67.575 & 43.057 & 11.147 & 2.683 \\
\hline 24.4 & Pharmazeutische Erzeugnisse & 25 & 277.195 & 89.251 & 154.698 & 25.098 & 8.148 \\
\hline 25 & Gummi- und Kunststoffwaren & 76 & 88.010 & 45.646 & 25.158 & 15.827 & 1.379 \\
\hline 26 & Glas, Waren aus Steinen und Erden & 64 & 61.635 & 36.338 & 16.525 & 7.629 & 1.143 \\
\hline $\begin{array}{l}27.1-27.3 \text { und } \\
27.51 / 52\end{array}$ & Roheisen, Stahl, Ferrolegierungen, Rohre, Eisen-, Stahlgießerei & 29 & 91.820 & 28.988 & 49.770 & 8.411 & 4.651 \\
\hline $27.4+27.53 / 54$ & NE-Metalle, Leicht-, Schwermetallgießerei & 26 & 32.775 & 16.652 & 11.734 & 4.365 & 24 \\
\hline 28 & Metallerzeugnisse & 138 & 99.691 & 57.660 & 35.267 & 6.163 & 601 \\
\hline 29 & Maschinenbau & 298 & 483.072 & 269.722 & 163.523 & 45.865 & 3.962 \\
\hline 30 & Büromaschinen, Datenverarbeitungsgeräte und -einrichtungen & 12 & 17.536 & 11.844 & 4.625 & 817 & 250 \\
\hline 31 & Geräte der Elektrizitätserzeugung, -verteilung u.Ä. & 82 & 195.106 & 113.322 & 67.511 & 10.781 & 3.492 \\
\hline 32 ohne 32.1 & Rundfunk-, Fernseh- und Nachrichtentechnik (ohne elektronische Bauelemente) & 28 & 542.261 & 383.137 & 140.951 & 10.413 & 7.760 \\
\hline 32.1 & Elektronische Bauelemente & 31 & 338.014 & 128.945 & 181.091 & 27.917 & 61 \\
\hline 34 & Kraftwagen und Kraftwagenteile & 38 & 366.364 & 168.830 & 172.985 & 12.474 & 12.075 \\
\hline 35 & Sonstiger Fahrzeugbau & 16 & 115.775 & 50.231 & 62.569 & 2.560 & 415 \\
\hline 36 ohne 36.1 & Schmuck, Musikinstrumente, Sportgeräte, Spielwaren, sonstige Erzeugnisse & 30 & 44.950 & 26.680 & 13.148 & 5.102 & 20 \\
\hline 36.1 & Möbel & 26 & 12.084 & 7.435 & 3.497 & 1.152 & - \\
\hline 37 & Rückgewinnung (Recycling) & $.2)$ & $.2)$ & $.2)$ &.$^{2)}$ & $.2)$ &.$^{2)}$ \\
\hline $40+41$ & Energie- und Wasserversorgung & 25 & 9.223 & 3.899 & 2.328 & 2.090 & 906 \\
\hline 45 & Bauwesen & 82 & 25.700 & 8.960 & 15.595 & 1.068 & 77 \\
\hline $50-93$ & Dienstleistungen & 963 & 1.246 .395 & 635.039 & 502.535 & 90.337 & 18.484 \\
\hline $50-52$ & Handel; Instandhaltung und Reparatur von Kraftfahrzeugen und Gebrauchsgütern & 205 & 206.227 & 86.126 & 95.246 & 14.597 & 10.258 \\
\hline 55 & Beherbergungs- und Gaststättenwesen & - & - & - & - & - & - \\
\hline $60-64$ & Verkehr und Nachrichtenübermittlung & 25 & 46.468 & 25.873 & 7.808 & 12.787 & - \\
\hline $65-67$ & Kredit- und Versicherungswesen & 7 & 30.211 & 22.418 & 6.358 & 1.435 & - \\
\hline $70+71+74$ & $\begin{array}{l}\text { Realitätenwesen, Vermietung beweglicher Sachen, } \\
\text { unternehmensbezogene Dienstleistungen }\end{array}$ & 243 & 350.902 & 165.677 & 161.826 & 19.809 & 3.590 \\
\hline 72 ohne 72.2 & Datenverarbeitung und Datenbanken (ohne Softwarehäuser) & 56 & 25.280 & 14.757 & 7.816 & 2.697 & 10 \\
\hline 72.2 & Softwarehäuser & 210 & 173.035 & 115.123 & 51.134 & 6.032 & 746 \\
\hline 73 & Forschung und Entwicklung & 193 & 407.056 & 201.244 & 170.353 & 31.579 & 3.880 \\
\hline 75-93 & $\begin{array}{l}\text { Öffentliche Verwaltung, Unterrichtswesen, Gesundheitswesen, sonstige öffentliche } \\
\text { und persönliche Dienstleistungen }\end{array}$ & 24 & 7.216 & 3.821 & 1.994 & 1.401 & - \\
\hline \multicolumn{8}{|c|}{ Beschäftigtengrößenklassen } \\
\hline \multicolumn{2}{|c|}{1 - 49 Beschäftigte } & 1.278 & 413.807 & 225.890 & 135.466 & 41.474 & 10.977 \\
\hline \multicolumn{2}{|c|}{50 - 249 Beschäftigte } & 721 & 840.900 & 433.716 & 326.347 & 67.482 & 13.355 \\
\hline \multicolumn{2}{|c|}{250 und mehr Beschäftigte } & 408 & 3.193 .969 & 1.638 .297 & 1.295 .774 & 211.501 & 48.397 \\
\hline
\end{tabular}

Q: STATISTIK AUSTRIA, Erhebung über Forschung und experimentelle Entwicklung (F\&E) 2006. Erstellt am: 29.08.08. - 1) Umfasst den firmeneigenen Bereich und den kooperativen Bereich. - 2) Daten können aus Geheimhaltungsgründen nicht gesondert ausgewiesen werden, sind jedoch in den Zwischen- und Endsummen enthalten. 
Tabelle 48: Unternehmenssektor ${ }^{1}$ : Ausgaben für Forschung und experimentelle Entwicklung (F\&E) 2006 nach Wirtschaftszweigen und Forschungsarten

\begin{tabular}{|c|c|c|c|c|c|c|c|c|c|}
\hline & \multirow{3}{*}{ Wirtschaftszweige } & \multirow{3}{*}{$\begin{array}{l}\text { Anzahl der } \\
\text { F\&E durch- } \\
\text { führenden } \\
\text { Erhebungs- } \\
\text { einheiten }\end{array}$} & \multirow{3}{*}{$\begin{array}{c}\text { Interne F\&E- } \\
\begin{array}{c}\text { Ausgaben } \\
\text { insgesamt }\end{array} \\
\text { in } 1.000 \text { EUR }\end{array}$} & \multicolumn{6}{|c|}{ Davon für } \\
\hline & & & & \multicolumn{2}{|c|}{$\begin{array}{l}\text { Grundlagen- } \\
\text { forschung }\end{array}$} & \multicolumn{2}{|c|}{$\begin{array}{l}\text { angewandte } \\
\text { Forschung }\end{array}$} & \multicolumn{2}{|c|}{$\begin{array}{l}\text { experimentelle } \\
\text { Entwicklung }\end{array}$} \\
\hline & & & & in 1.000 EUR & in $\%$ & in 1.000 EUR & in $\%$ & in 1.000 EUR & in $\%$ \\
\hline Insgesamt & & 2.407 & 4.448 .676 & 245.150 & 5,5 & 1.415 .121 & 31,8 & 2.788 .405 & 62,7 \\
\hline $01+02+05$ & Land- und Forstwirtschaft, Fischerei & 3 & 820 & - & - & 719 & 87,7 & 101 & 12,3 \\
\hline $10-14$ & Bergbau und Gewinnung von Steinen und Erden & 10 & 7.146 & 657 & 9,2 & 3.312 & 46,3 & 3.177 & 44,5 \\
\hline 15 & Nahrungs- und Genussmittel, Getränke & 87 & 23.540 & 300 & 1,3 & 6.229 & 26,5 & 17.011 & 72,3 \\
\hline 16 & Tabakverarbeitung & $.2)$ &.$^{2)}$ &.$^{2)}$ & $.2)$ & $.2)$ &.$^{2)}$ &.$^{2)}$ &.$^{2)}$ \\
\hline 17 & Textilien und Textilwaren (ohne Bekleidung) & 28 & 26.465 & 1.493 & 5,6 & 6.279 & 23,7 & 18.693 & 70,6 \\
\hline $18+19$ & Bekleidung, Leder, Schuhe & 14 & 5.345 & 303 & 5,7 & 1.304 & 24,4 & 3.738 & 69,9 \\
\hline 20 & Holz (ohne Herstellung von Möbeln) & 52 & 20.162 & 787 & 3,9 & 4.191 & 20,8 & 15.184 & 75,3 \\
\hline 21 & Papier und Pappe & 27 & 17.075 & 301 & 1,8 & 4.627 & 27,1 & 12.147 & 71,1 \\
\hline 22 & $\begin{array}{l}\text { Verlagswesen, Druckerei, Vervielfälti-gung von bespielten Ton-, } \\
\text { Bild- und Datenträgern }\end{array}$ & 13 & 21.057 & 294 & 1,4 & 2.457 & 11,7 & 18.306 & 86,9 \\
\hline 23 & Kokerei, Mineralölverarbeitung, Spalt- und Brutstoffe &.$^{2)}$ &.$^{2)}$ &.$^{2)}$ &.$^{2)}$ &.$^{2)}$ &.$^{2)}$ & .2) & .2) \\
\hline 24 ohne 24.4 & $\begin{array}{l}\text { Chemikalien und chemische Erzeugnisse } \\
\text { (ohne pharmazeutische Erzeugnisse) }\end{array}$ & 73 & 124.462 & 4.304 & 3,5 & 51.618 & 41,5 & 68.540 & 55,1 \\
\hline 24.4 & Pharmazeutische Erzeugnisse & 25 & 277.195 & 269 & 0,1 & 101.299 & 36,5 & 175.627 & 63,4 \\
\hline 25 & Gummi- und Kunststoffwaren & 76 & 88.010 & 3.758 & 4,3 & 29.761 & 33,8 & 54.491 & 61,9 \\
\hline 26 & Glas, Waren aus Steinen und Erden & 64 & 61.635 & 5.620 & 9,1 & 13.443 & 21,8 & 42.572 & 69,1 \\
\hline $\begin{array}{l}27.1-27.3 \text { und } \\
27.51 / 52\end{array}$ & Roheisen, Stahl, Ferrolegierungen, Rohre, Eisen-, Stahlgießerei & 29 & 91.820 & 6.548 & 7,1 & 24.130 & 26,3 & 61.142 & 66,6 \\
\hline $27.4+27.53 / 54$ & NE-Metalle, Leicht-, Schwermetallgießerei & 26 & 32.775 & 1.267 & 3,9 & 10.823 & 33,0 & 20.685 & 63,1 \\
\hline 28 & Metallerzeugnisse & 138 & 99.691 & 3.420 & 3,4 & 27.742 & 27,8 & 68.529 & 68,7 \\
\hline 29 & Maschinenbau & 298 & 483.072 & 20.734 & 4,3 & 174.536 & 36,1 & 287.802 & 59,6 \\
\hline 30 & Büromaschinen, Datenverarbeitungsgeräte und -einrichtungen & 12 & 17.536 & 760 & 4,3 & 4.929 & 28,1 & 11.847 & 67,6 \\
\hline 31 & Geräte der Elektrizitätserzeugung, -verteilung u.Ä. & 82 & 195.106 & 4.266 & 2,2 & 100.406 & 51,5 & 90.434 & 46,4 \\
\hline 32 ohne 32.1 & $\begin{array}{l}\text { Rundfunk-, Fernseh- und Nachrichten-technik } \\
\text { (ohne elektronische Bauelemente) }\end{array}$ & 28 & 542.261 & 1.633 & 0,3 & 68.348 & 12,6 & 472.280 & 87,1 \\
\hline 33.1 & Medizintechnik & 31 & 56.987 & 2.394 & 4,2 & 10.559 & 18,5 & 44.034 & 77,3 \\
\hline 34 & Kraftwagen und Kraftwagenteile & 38 & 366.364 & 14.722 & 4,0 & 84.144 & 23,0 & 267.498 & 73,0 \\
\hline 35 & Sonstiger Fahrzeugbau & 16 & 115.775 & 13.149 & 11,4 & 22.348 & 19,3 & 80.278 & 69,3 \\
\hline 36 ohne 36.1 & $\begin{array}{l}\text { Schmuck, Musikinstrumente, Sportgeräte, Spielwaren, } \\
\text { sonstige Erzeugnisse }\end{array}$ & 30 & 44.950 & 2.647 & 5,9 & 14.313 & 31,8 & 27.990 & 62,3 \\
\hline 36.1 & Möbel & 26 & 12.084 & 569 & 4,7 & 5.060 & 41,9 & 6.455 & 53,4 \\
\hline 37 & Rückgewinnung (Recycling) & $.2)$ &..$^{2)}$ &.$^{2)}$ & $.2)$ & .2) & $.2)$ &.$^{2)}$ &.$^{2)}$ \\
\hline $40+41$ & Energie- und Wasserversorgung & 25 & 9.223 & 39 & 0,4 & 7.489 & 81,2 & 1.695 & 18,4 \\
\hline 45 & Bauwesen & 82 & 25.700 & 1.841 & 7,2 & 6.913 & 26,9 & 16.946 & 65,9 \\
\hline $50-93$ & Dienstleistungen & 963 & 1.246 .395 & 143.443 & 11,5 & 538.313 & 43,2 & 564.639 & 45,3 \\
\hline $50-52$ & $\begin{array}{l}\text { Handel; Instandhaltung und Reparatur von Kraftfahrzeugen } \\
\text { und Gebrauchsgütern }\end{array}$ & 205 & 206.227 & 5.498 & 2,7 & 76.924 & 37,3 & 123.805 & 60,0 \\
\hline 55 & Beherbergungs- und Gaststättenwesen & - & - & - & - & - & - & - & - \\
\hline $60-64$ & Verkehr und Nachrichtenübermittlung & 25 & 46.468 & 392 & 0,8 & 9.216 & 19,8 & 36.860 & 79,3 \\
\hline $65-67$ & Kredit- und Versicherungswesen & 7 & 30.211 & - & - & 8.830 & 29,2 & 21.381 & 70,8 \\
\hline $70+71+74$ & $\begin{array}{l}\text { Realitätenwesen, Vermietung beweglicher Sachen, } \\
\text { unternehmensbezogene Dienstleistungen }\end{array}$ & 243 & 350.902 & 48.978 & 14,0 & 163.445 & 46,6 & 138.479 & 39,5 \\
\hline 72 ohne 72.2 & Datenverarbeitung und Datenbanken (ohne Softwarehäuser) & 56 & 25.280 & 462 & 1,8 & 7.733 & 30,6 & 17.085 & 67,6 \\
\hline 72.2 & Softwarehäuser & 210 & 173.035 & 6.478 & 3,7 & 64.063 & 37,0 & 102.494 & 59,2 \\
\hline 73 & Forschung und Entwicklung & 193 & 407.056 & 81.146 & 19,9 & 205.151 & 50,4 & 120.759 & 29,7 \\
\hline $75-93$ & $\begin{array}{l}\text { Öffentliche Verwaltung, Unterrichtswesen, Gesundheitswesen, } \\
\text { sonstige öffentliche und persönliche Dienstleistungen }\end{array}$ & 24 & 7.216 & 489 & 6,8 & 2.951 & 40,9 & 3.776 & 52,3 \\
\hline
\end{tabular}

Q: STATISTIK AUSTRIA, Erhebung über Forschung und experimentelle Entwicklung (F\&E) 2006. Erstellt am: 29.08.08. - 1) Umfasst den firmeneigenen Bereich und den kooperativen Bereich. - 2) Daten können aus Geheimhaltungsgründen nicht gesondert ausgewiesen werden, sind jedoch in den Zwischen- und Endsummen enthalten. 


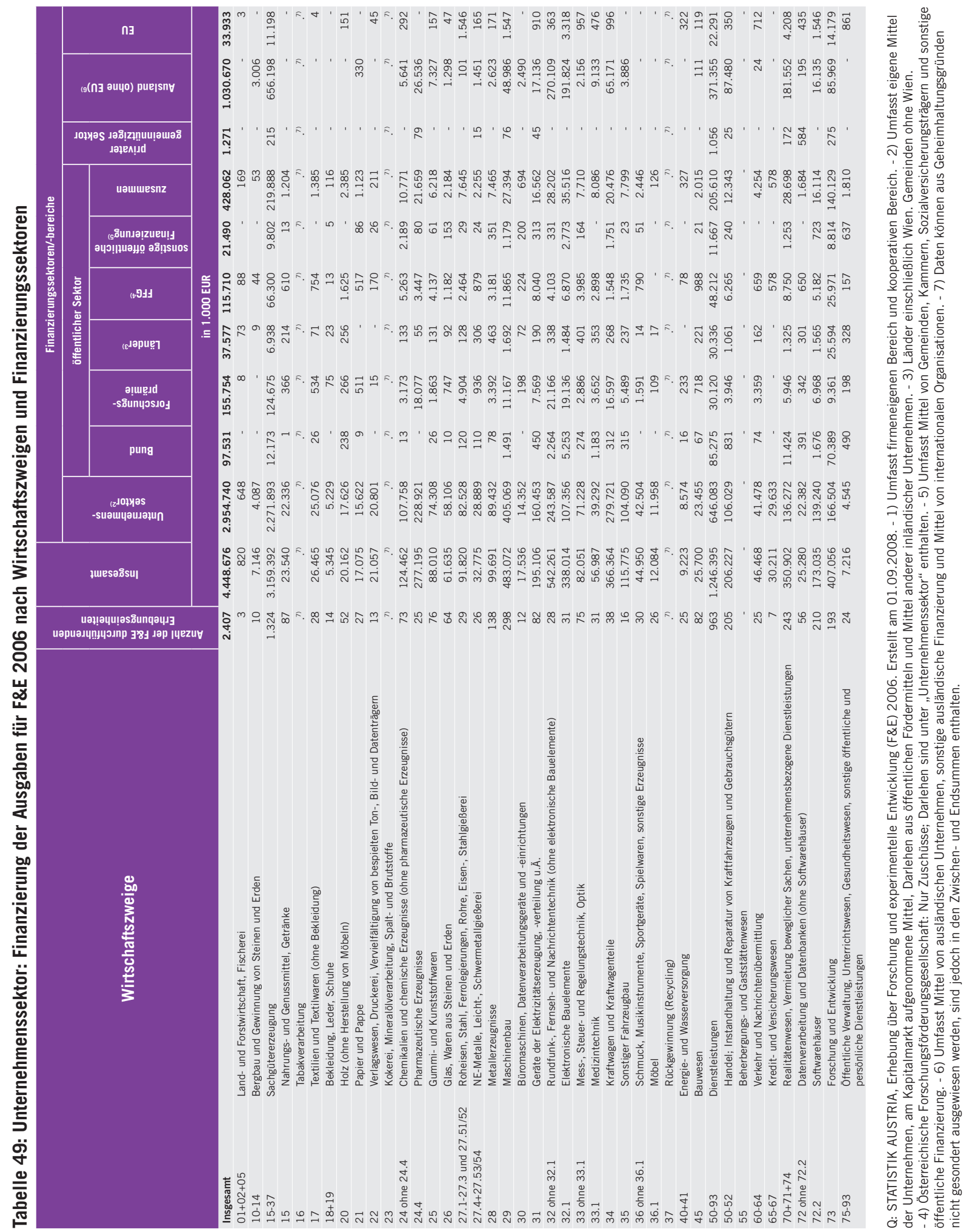




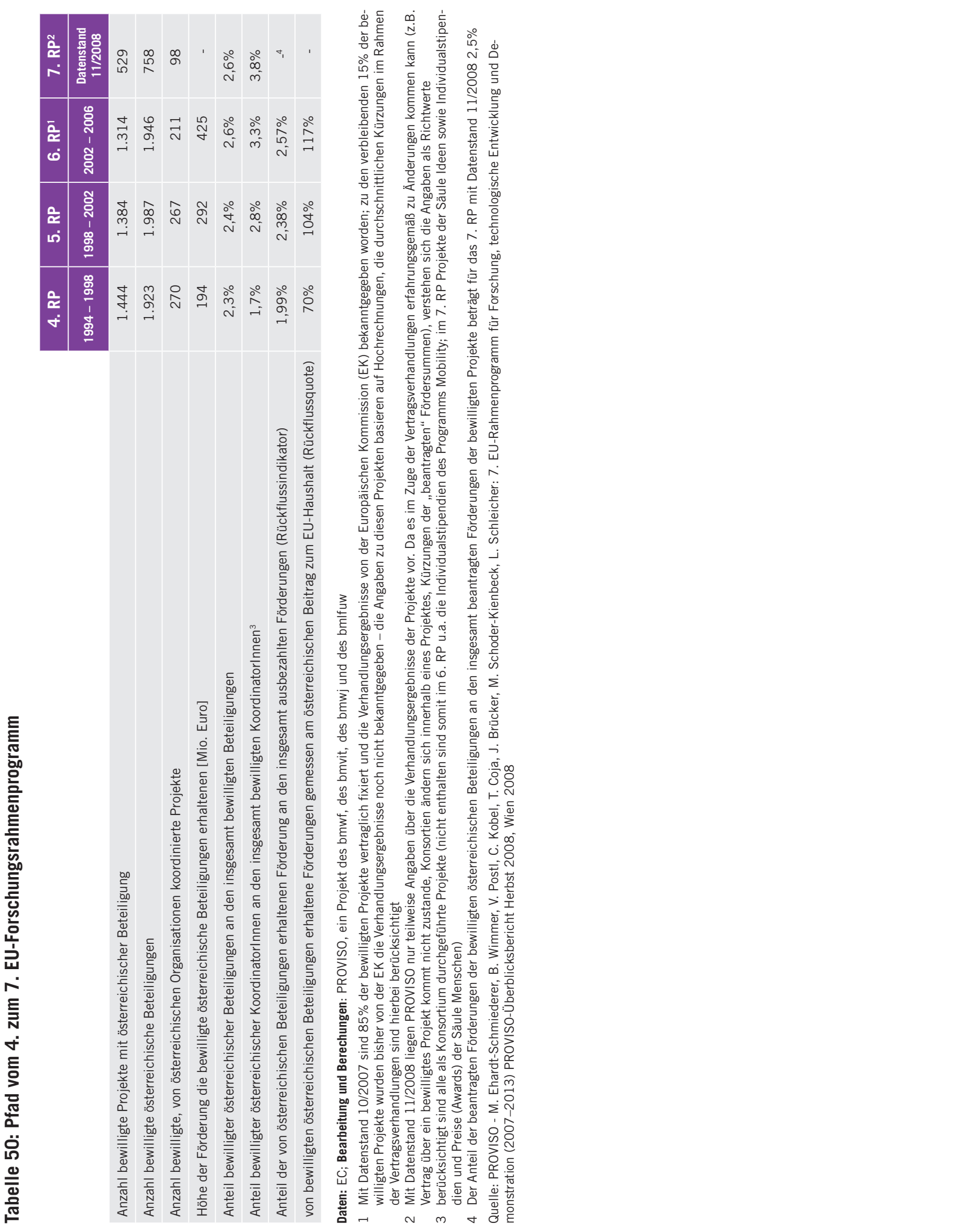



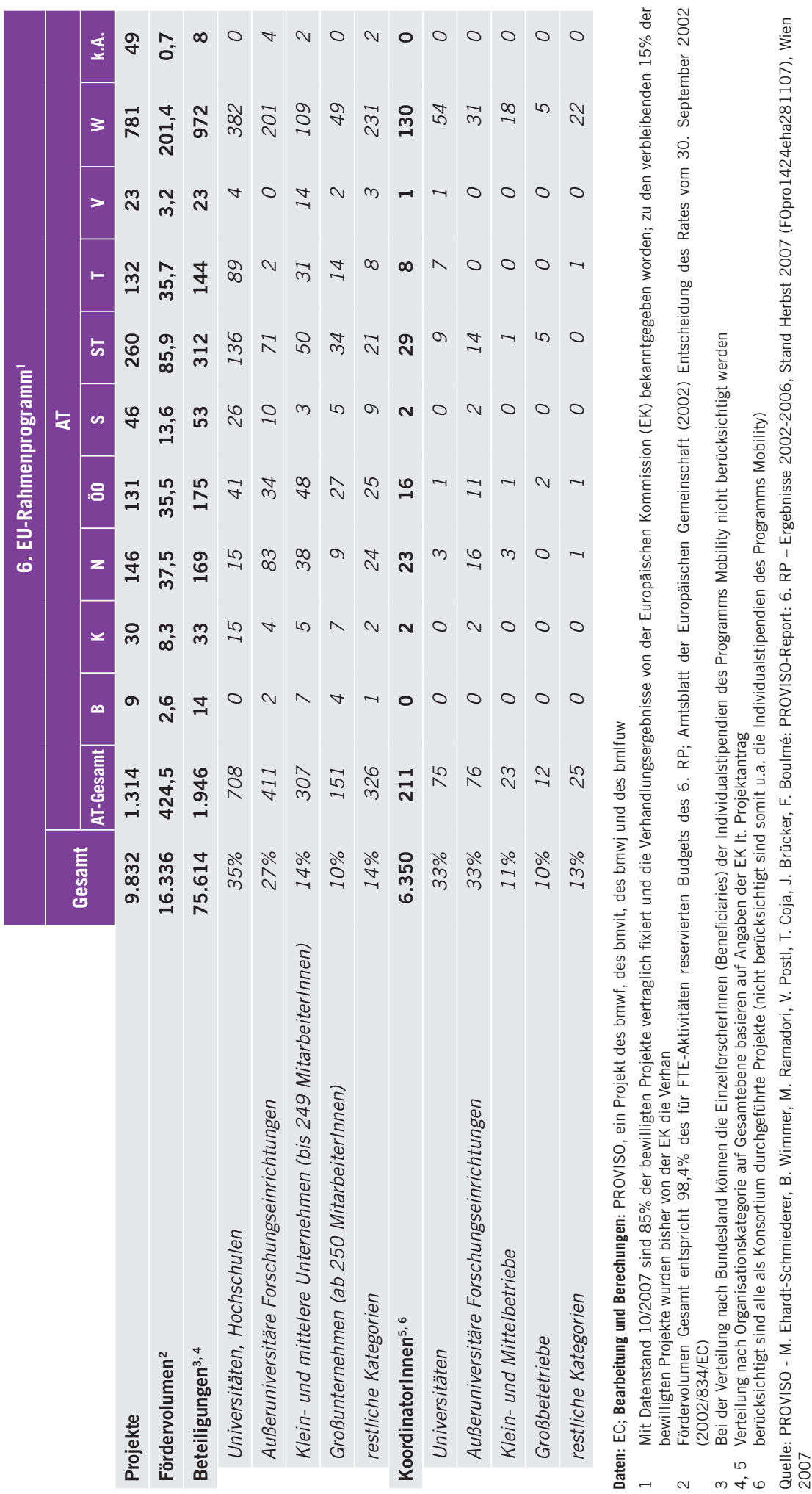

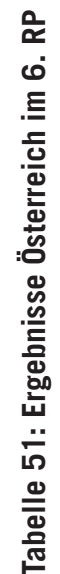

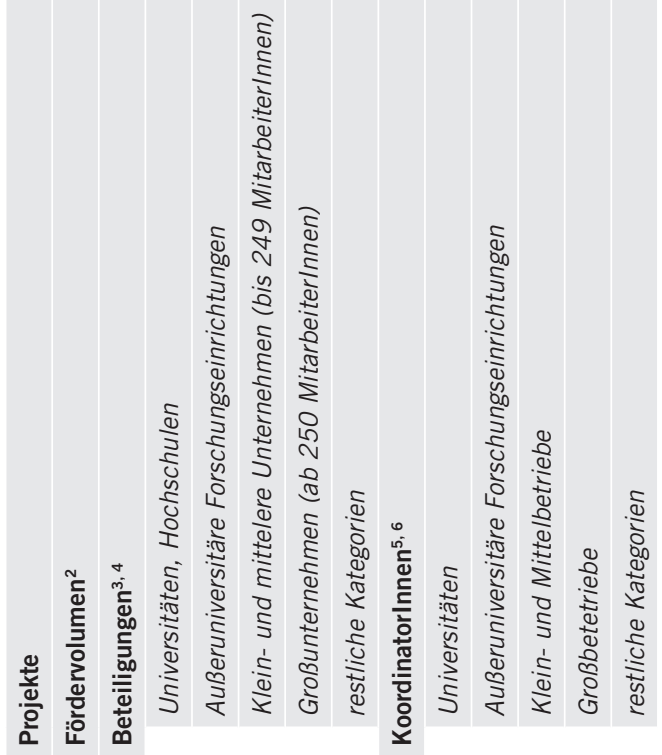




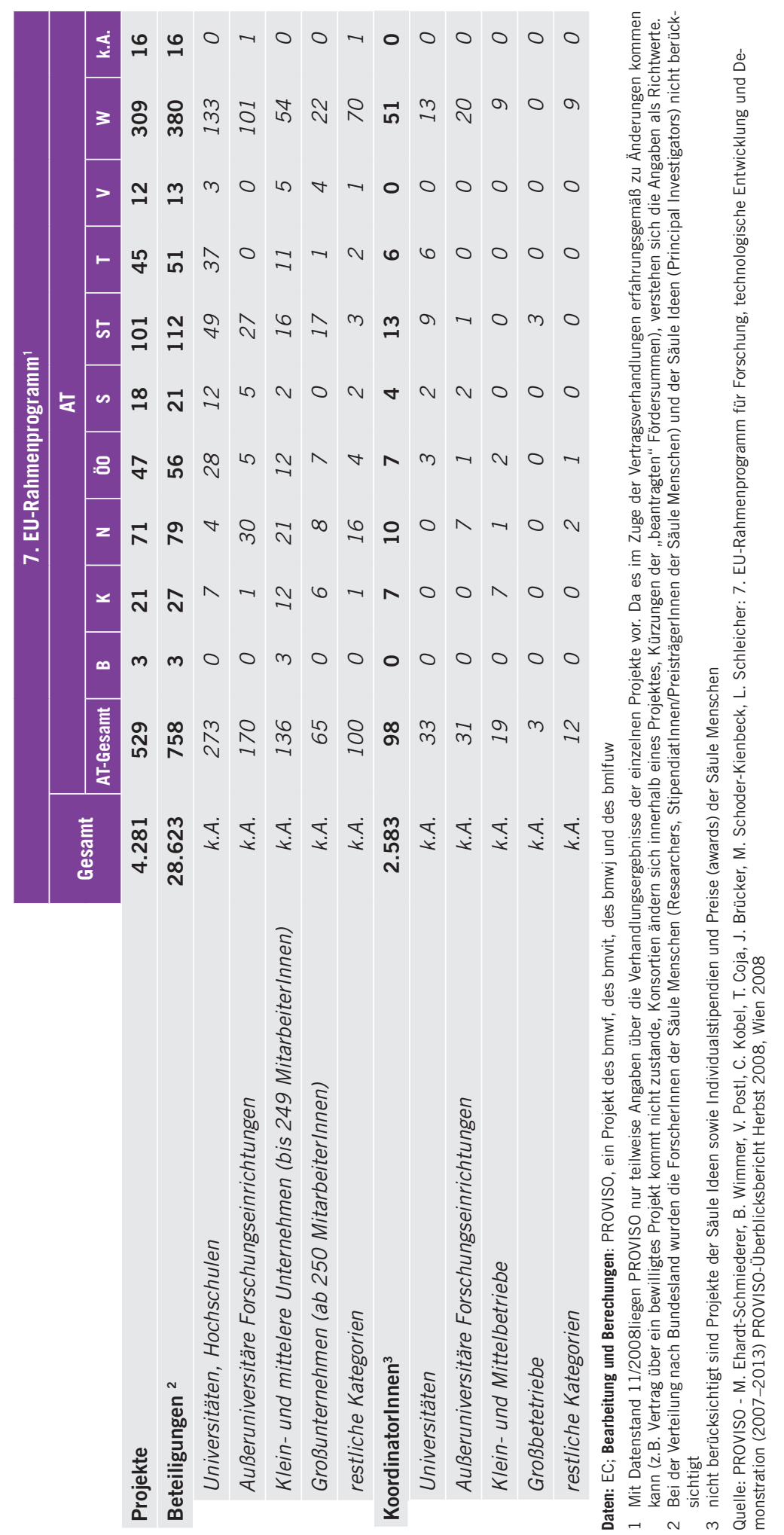




\section{Tabelle 53: FFG: Förderstatistik 2008 - Gesamtübersicht}

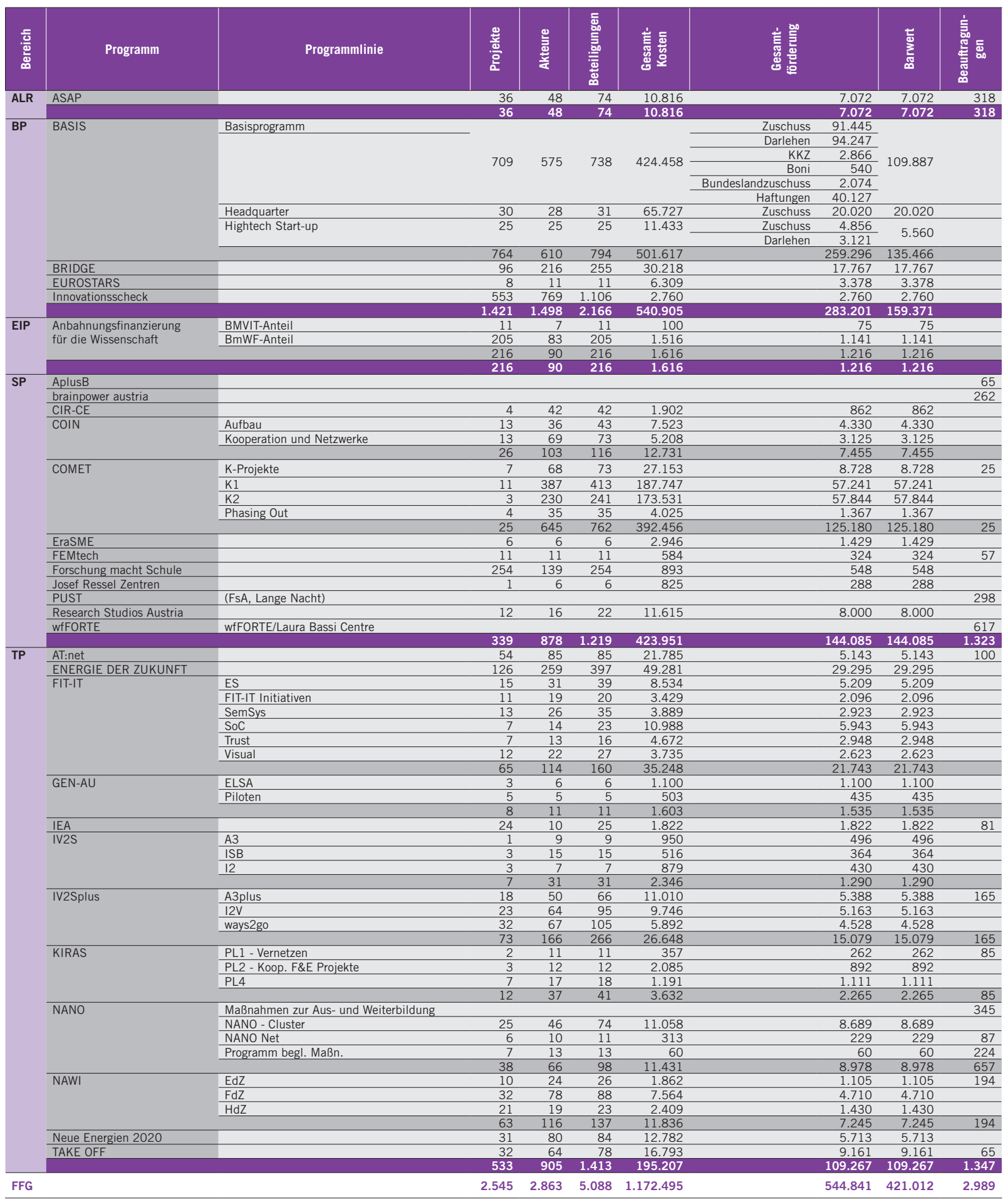


Tabelle 54: FFG: Geförderte Projekte 2008 gemäß der Systematik der WIRTSCHAFTSTÄTIGKEIT (NACE)

\begin{tabular}{|c|c|c|c|c|c|c|c|c|c|c|c|}
\hline Bezeichnung & 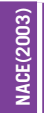 & $\begin{array}{l}\text { 崖 } \\
\frac{\mathrm{u}}{2} \\
\frac{8}{\alpha}\end{array}$ & $\begin{array}{l}\frac{9}{2} \\
\frac{\pi}{2} \\
\frac{0}{2} \\
\therefore \circ\end{array}$ & $\begin{array}{l}\text { 害 } \\
\text { 兽 } \\
\text { 总 }\end{array}$ & 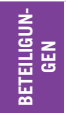 & 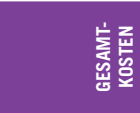 & 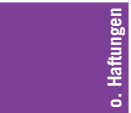 & 差总 & 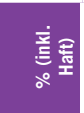 & 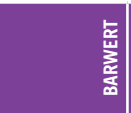 & \\
\hline ohne Zuordnung & & 621 & $24,4 \%$ & 916 & 1417 & 164.015 .244 & 89.174 .380 & 89.174 .380 & $16,4 \%$ & 89.174 .380 & $21,2 \%$ \\
\hline Landwirtschaft, Jagd & 01 & 24 & $0,9 \%$ & 26 & 31 & 4.203 .405 & 2.438 .950 & 2.438 .950 & $0,4 \%$ & 2.371 .368 & $0,6 \%$ \\
\hline Forstwirtschaft & 02 & 12 & $0,5 \%$ & 14 & 20 & 679.240 & 469.600 & 469.600 & $0,1 \%$ & 469.600 & $0,1 \%$ \\
\hline Fischerei und Fischzucht & 05 & 1 & $0,0 \%$ & 2 & 2 & 5.000 & 5.000 & 5.000 & $0,0 \%$ & 5.000 & $0,0 \%$ \\
\hline Kohlebergbau, Torfgewinnung & 10 & 2 & $0,1 \%$ & 4 & 4 & 10.000 & 10.000 & 10.000 & $0,0 \%$ & 10.000 & $0,0 \%$ \\
\hline $\begin{array}{l}\text { Erdöl- und Erdgasbergbau, sowie damit verbundene } \\
\text { Dienstleistungen }\end{array}$ & 11 & 1 & $0,0 \%$ & 2 & 2 & 5.000 & 5.000 & 5.000 & $0,0 \%$ & 5.000 & $0,0 \%$ \\
\hline Erzbergbau & 13 & 1 & $0,0 \%$ & 2 & 2 & 5.000 & 5.000 & 5.000 & $0,0 \%$ & 5.000 & $0,0 \%$ \\
\hline Gewinnung von Steinen und Erden, sonstiger Bergbau & 14 & 2 & $0,1 \%$ & 4 & 4 & 461.200 & 291.600 & 291.600 & $0,1 \%$ & 291.600 & $0,1 \%$ \\
\hline Herstellung von Nahrungs- und Genussmitteln und Getränken & 15 & 37 & $1,5 \%$ & 51 & 66 & 8.592 .590 & 4.374 .519 & 4.374 .519 & $0,8 \%$ & 2.769 .086 & $0,7 \%$ \\
\hline Tabakverarbeitung & 16 & 1 & $0,0 \%$ & 2 & 2 & 5.000 & 5.000 & 5.000 & $0,0 \%$ & 5.000 & $0,0 \%$ \\
\hline Herstellung von Textilien und Textilwaren (ohne Bekleidung) & 17 & 7 & $0,3 \%$ & 7 & 7 & 3.564 .300 & 1.875 .800 & 1.875 .800 & $0,3 \%$ & 889.549 & $0,2 \%$ \\
\hline Herstellung von Bekleidung & 18 & 2 & $0,1 \%$ & 1 & 2 & 1.998 .000 & 998.000 & 998.000 & $0,2 \%$ & 433.919 & $0,1 \%$ \\
\hline Ledererzeugung und -verarbeitung & 19 & 1 & $0,0 \%$ & 1 & 1 & 424.600 & 297.100 & 297.100 & $0,1 \%$ & 156.906 & $0,0 \%$ \\
\hline $\begin{array}{l}\text { Be- und Verarbeitung von Holz (ohne Herstellung von } \\
\text { Möbeln) }\end{array}$ & 20 & 27 & $1,1 \%$ & 34 & 45 & 5.933 .000 & 2.987 .500 & 2.987 .500 & $0,5 \%$ & 2.256 .374 & $0,5 \%$ \\
\hline $\begin{array}{l}\text { Verlagswesen, Druckerei, Vervielfältigung von bespielten Ton-, } \\
\text { Bild- und Datenträgern }\end{array}$ & 22 & 4 & $0,2 \%$ & 7 & 7 & 212.000 & 113.000 & 113.000 & $0,0 \%$ & 67.841 & $0,0 \%$ \\
\hline $\begin{array}{l}\text { Kokerei, Mineralölverarbeitung, Herstellung und Verarbeitung } \\
\text { von Spalt- und Brutstoffen }\end{array}$ & 23 & 4 & $0,2 \%$ & 6 & 7 & 1.710 .600 & 856.500 & 856.500 & $0,2 \%$ & 522.290 & $0,1 \%$ \\
\hline Herstellung von Chemikalien und chemischen Erzeugnissen & 24 & 116 & $4,6 \%$ & 146 & 183 & 103.092.937 & 44.504 .826 & 51.365 .826 & $9,4 \%$ & 31.341 .324 & $7,4 \%$ \\
\hline Herstellung von Gummi- und Kunststoffwaren & 25 & 50 & $2,0 \%$ & 91 & 99 & 12.788 .264 & 6.591 .207 & 6.924 .207 & $1,3 \%$ & 4.523 .752 & $1,1 \%$ \\
\hline $\begin{array}{l}\text { Herstellung und Bearbeitung von Glas, Herstellung von Waren } \\
\text { aus Steinen und Erden }\end{array}$ & 26 & 35 & $1,4 \%$ & 31 & 47 & 20.105 .100 & 8.392 .400 & 9.691 .400 & $1,8 \%$ & 6.009 .320 & $1,4 \%$ \\
\hline Metallerzeugung und -bearbeitung & 27 & 40 & $1,6 \%$ & 52 & 64 & 19.983 .293 & 8.963 .391 & 12.302 .391 & $2,3 \%$ & 7.526 .562 & $1,8 \%$ \\
\hline Herstellung von Metallerzeugnissen & 28 & 39 & $1,5 \%$ & 47 & 52 & 11.057 .800 & 5.794 .400 & 5.794 .400 & $1,1 \%$ & 3.429 .791 & $0,8 \%$ \\
\hline Maschinenbau & 29 & 141 & $5,5 \%$ & 174 & 202 & 81.415 .787 & 37.957 .654 & 40.735 .554 & $7,5 \%$ & 24.047 .226 & $5,7 \%$ \\
\hline $\begin{array}{l}\text { Herstellung von Büromaschinen, Datenverarbeitungsgeräten } \\
\text { und -einrichtungen }\end{array}$ & 30 & 8 & $0,3 \%$ & 11 & 11 & 5.150 .345 & 2.247 .614 & 3.077 .614 & $0,6 \%$ & 1.756 .009 & $0,4 \%$ \\
\hline $\begin{array}{l}\text { Herstellung von Geräten der Elektrizitätserzeugung, } \\
\text {-verteilung u.ä. }\end{array}$ & 31 & 33 & $1,3 \%$ & 40 & 46 & 21.495 .478 & 8.915 .903 & 11.195 .903 & $2,1 \%$ & 5.774 .000 & $1,4 \%$ \\
\hline Rundfunk-, Fernseh- und Nachrichtentechnik & 32 & 99 & $3,9 \%$ & 66 & 129 & 114.278 .509 & 43.651 .716 & 49.812 .716 & $9,1 \%$ & 31.227 .848 & $7,4 \%$ \\
\hline Medizin-, Mess-, Steuer- und Regelungstechnik, Optik & 33 & 133 & $5,2 \%$ & 126 & 163 & 63.087 .444 & 26.439 .352 & 33.777 .352 & $6,2 \%$ & 19.159 .468 & $4,6 \%$ \\
\hline Herstellung von Kraftwagen und Kraftwagenteilen & 34 & 48 & $1,9 \%$ & 32 & 56 & 32.070 .400 & 14.108 .000 & 17.269 .000 & $3,2 \%$ & 7.883 .672 & $1,9 \%$ \\
\hline Sonstiger Fahrzeugbau & 35 & 64 & $2,5 \%$ & 60 & 102 & 34.662 .077 & 13.120 .730 & 18.867 .730 & $3,5 \%$ & 12.979 .532 & $3,1 \%$ \\
\hline Energieversorgung & 40 & 17 & $0,7 \%$ & 87 & 90 & 17.607 .021 & 6.804 .436 & 6.804 .436 & $1,2 \%$ & 6.294 .300 & $1,5 \%$ \\
\hline Wasserversorgung & 41 & 5 & $0,2 \%$ & 9 & 9 & 1.074 .930 & 705.700 & 705.700 & $0,1 \%$ & 386.619 & $0,1 \%$ \\
\hline Bauwesen & 45 & 58 & $2,3 \%$ & 107 & 122 & 19.869 .755 & 8.146 .279 & 8.146 .279 & $1,5 \%$ & 7.151 .074 & $1,7 \%$ \\
\hline $\begin{array}{l}\text { Kraftfahrzeughandel; Instandhaltung und Reparatur von } \\
\text { Kraftfahrzeugen; Tankstellen }\end{array}$ & 50 & 2 & $0,1 \%$ & 4 & 4 & 10.000 & 10.000 & 10.000 & $0,0 \%$ & 10.000 & $0,0 \%$ \\
\hline $\begin{array}{l}\text { Handelsvermittlung und Großhandel (ohne Handel mit } \\
\text { Kraftfahrzeugen) }\end{array}$ & 51 & 51 & $2,0 \%$ & 84 & 102 & 255.000 & 255.000 & 255.000 & $0,0 \%$ & 255.000 & $0,1 \%$ \\
\hline $\begin{array}{l}\text { Einzelhandel (ohne Handel mit Kraftfahrzeugen und ohne } \\
\text { Tankstellen); Reparatur von Gebrauchsgütern }\end{array}$ & 52 & 29 & $1,1 \%$ & 51 & 57 & 142.000 & 141.000 & 141.000 & $0,0 \%$ & 141.000 & $0,0 \%$ \\
\hline Beherbergungs- und Gaststättenwesen & 55 & 4 & $0,2 \%$ & 7 & 8 & 20.000 & 20.000 & 20.000 & $0,0 \%$ & 20.000 & $0,0 \%$ \\
\hline Landverkehr; Transport in Rohrfernleitungen & 60 & 35 & $1,4 \%$ & 82 & 119 & 10.968 .060 & 5.592 .093 & 5.592 .093 & $1,0 \%$ & 5.592 .093 & $1,3 \%$ \\
\hline Flugverkehr & 62 & 4 & $0,2 \%$ & 7 & 7 & 130.700 & 82.200 & 82.200 & $0,0 \%$ & 82.200 & $0,0 \%$ \\
\hline Hilfs- und Nebentätigkeiten für den Verkehr; Reisebüros & 63 & 7 & $0,3 \%$ & 12 & 13 & 32.000 & 31.000 & 31.000 & $0,0 \%$ & 31.000 & $0,0 \%$ \\
\hline Nachrichtenübermittlung & 64 & 2 & $0,1 \%$ & 7 & 7 & 2.478 .000 & 661.733 & 661.733 & $0,1 \%$ & 661.733 & $0,2 \%$ \\
\hline Kreditwesen & 65 & 1 & $0,0 \%$ & 2 & 2 & 5.000 & 5.000 & 5.000 & $0,0 \%$ & 5.000 & $0,0 \%$ \\
\hline Versicherungswesen & 66 & 1 & $0,0 \%$ & 2 & 2 & 5.000 & 5.000 & 5.000 & $0,0 \%$ & 5.000 & $0,0 \%$ \\
\hline Realitätenwesen & 70 & 10 & $0,4 \%$ & 20 & 20 & 50.000 & 50.000 & 50.000 & $0,0 \%$ & 50.000 & $0,0 \%$ \\
\hline Vermietung beweglicher Sachen ohne Bedienungspersonal & 71 & 9 & $0,4 \%$ & 17 & 18 & 45.000 & 45.000 & 45.000 & $0,0 \%$ & 45.000 & $0,0 \%$ \\
\hline Datenverarbeitung und Datenbanken & 72 & 209 & $8,2 \%$ & 281 & 339 & 52.604 .082 & 31.461 .038 & 31.461 .038 & $5,8 \%$ & 22.768 .884 & $5,4 \%$ \\
\hline Forschung und Entwicklung & 73 & 290 & $11,4 \%$ & 631 & 863 & 311.109 .846 & 107.715 .372 & 107.715 .372 & $19,8 \%$ & 107.679.996 & $25,6 \%$ \\
\hline Erbringung von unternehmensbezogenen Dienstleistungen & 74 & 173 & $6,8 \%$ & 272 & 338 & 3.162 .245 & 2.120 .347 & 2.120 .347 & $0,4 \%$ & 1.770 .325 & $0,4 \%$ \\
\hline Unterrichtswesen & 80 & 5 & $0,2 \%$ & 9 & 9 & 41.196 & 25.299 & 25.299 & $0,0 \%$ & 25.299 & $0,0 \%$ \\
\hline Gesundheits-, Veterinär- und Sozialwesen & 85 & 16 & $0,6 \%$ & 83 & 84 & 26.046 .688 & 7.367 .956 & 7.367 .956 & $1,4 \%$ & 7.367 .956 & $1,8 \%$ \\
\hline Abwasser- und Abfallbeseitigung und sonstige Entsorgung & 90 & 22 & $0,9 \%$ & 21 & 26 & 10.759 .350 & 6.361 .100 & 6.361 .100 & $1,2 \%$ & 3.457 .393 & $0,8 \%$ \\
\hline $\begin{array}{l}\text { Interessenvertretungen, kirchliche und sonstige religiöse } \\
\text { Vereinigungen, sonstige Vereine (ohne Sozialwesen, Kultur } \\
\text { und Sport) }\end{array}$ & 91 & 1 & $0,0 \%$ & 2 & 2 & 5.000 & 5.000 & 5.000 & $0,0 \%$ & 5.000 & $0,0 \%$ \\
\hline Kultur, Sport und Unterhaltung & 92 & 8 & $0,3 \%$ & 24 & 24 & 1.870 .340 & 585.602 & 585.602 & $0,1 \%$ & 585.602 & $0,1 \%$ \\
\hline Erbringung von sonstigen Dienstleistungen & 93 & 10 & $0,4 \%$ & 13 & 14 & 573.873 & 382.000 & 382.000 & $0,1 \%$ & 382.000 & $0,1 \%$ \\
\hline Private Haushalte & 95 & 1 & $0,0 \%$ & 3 & 3 & 723.683 & 434.210 & 434.210 & $0,1 \%$ & 434.210 & $0,1 \%$ \\
\hline Exterritoriale Organisationen und Körperschaften & 99 & 1 & $0,0 \%$ & 1 & 1 & 30.000 & 15.000 & 15.000 & $0,0 \%$ & 15.000 & $0,0 \%$ \\
\hline Gesamt & & 2.545 & $100,0 \%$ & 3.822 & 5.088 & 1.172 .494 .512 & 504.714 .589 & 544.841 .489 & $100,0 \%$ & 421.011 .700 & $100,0 \%$ \\
\hline
\end{tabular}


Tabelle 55: FFG: Förderstatistik 2008 nach Bundesländern

\begin{tabular}{|lrrrr|}
\hline & $\begin{array}{r}\text { Projekt- } \\
\text { beteiligungen }\end{array}$ & $\begin{array}{r}\text { Gesamtförderung } \\
\text { (Zuschïsse, Darlehen und Haftungen) }\end{array}$ & Förderbarwert & Anteil in \% \\
\hline Burgenland & 95 & 6.599 .309 & 5.616 .012 & 1,3 \\
\hline Kärten & 229 & 32.007 .899 & 26.267 .839 & 6,2 \\
\hline Niederösterreich & 426 & 32.948 .663 & 26.762 .807 & 6,4 \\
\hline Oberösterreich & 903 & 136.619 .229 & 94.942 .232 & 22,6 \\
\hline Salzburg & 190 & 10.600 .774 & 7.466 .630 & 1,8 \\
\hline Steiermark & 1.069 & 165.526 .605 & 133.432 .918 & 31,7 \\
\hline Tirol & 249 & 25.864 .476 & 20.236 .642 & 4,8 \\
\hline Vorarlberg & 110 & 14.476 .537 & 8.955 .067 & 2,1 \\
\hline Wien & 1.548 & 118.792 .522 & 95.926 .078 & 1.405 .475 \\
\hline Ausland & 269 & 1.405 .475 & 421.011 .700 & \\
\hline FFG - Gesamt & 5.088 & 544.841 .489 & & 100 \\
\hline
\end{tabular}




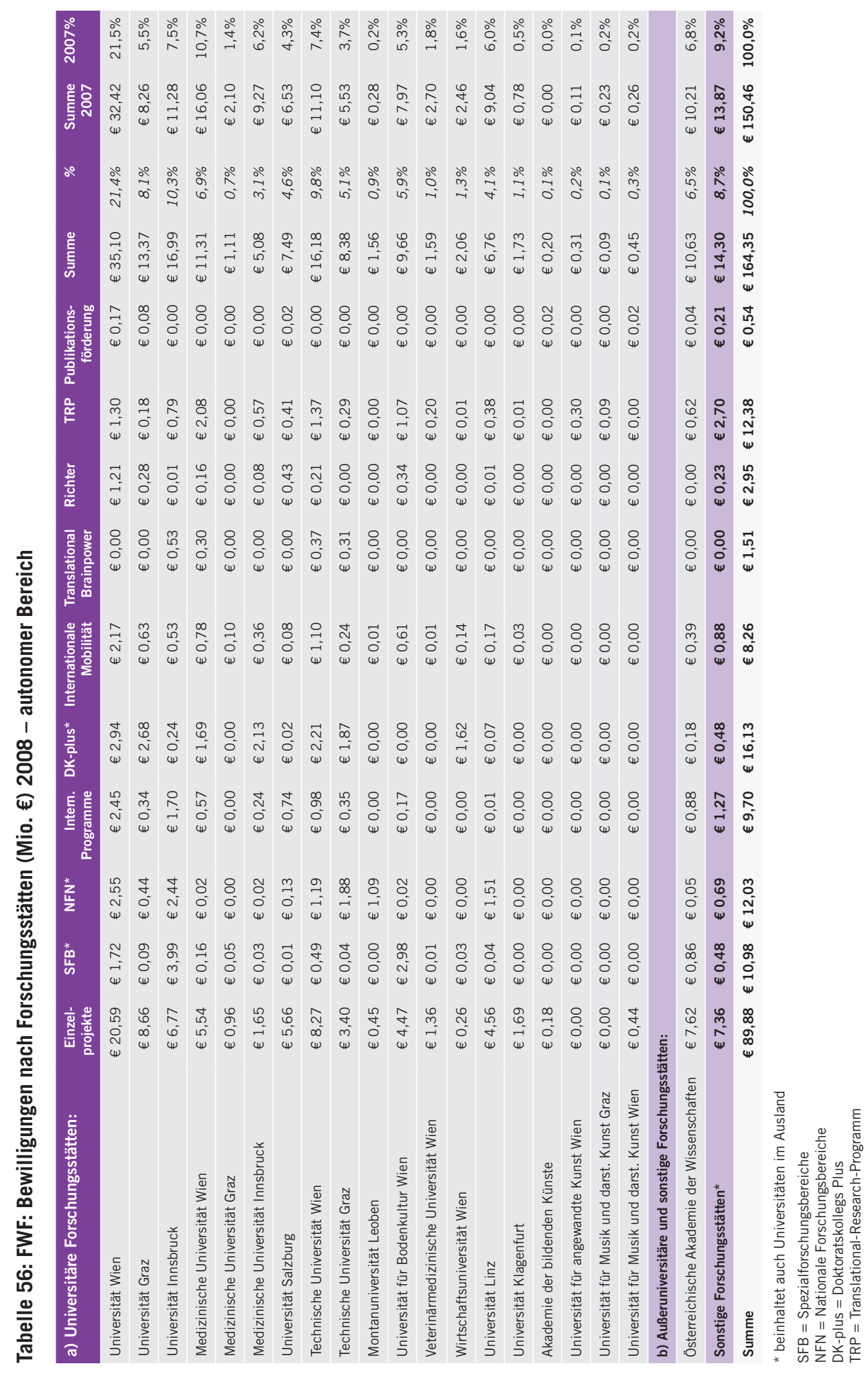




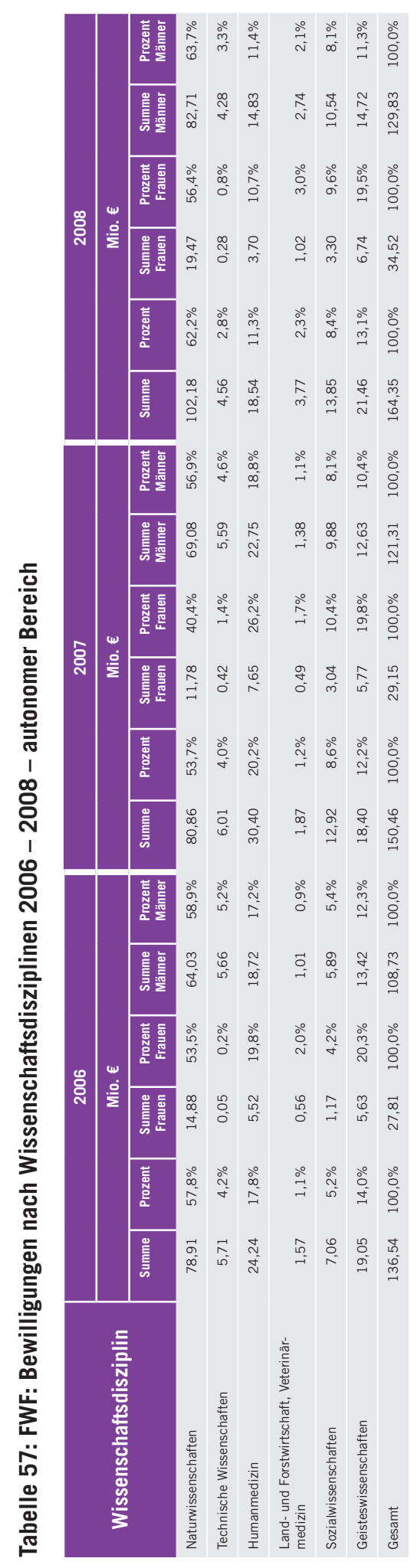


Tabelle 58: FWF: ERA-NET-Beteiligungen

\begin{tabular}{lllll}
\hline ERA-NET & Themenbereich & Beginn & Laufzeit & FWF-Beteiligung \\
\hline ERA-Chemistry & Chemie & 2004 & 5 Jahre & Work Package Leader \\
\hline PathoGenoMics & Pathogenomik & 2004 & 5 Jahre & Partner \\
\hline NanoSci-ERA & Nanowissenschaften & 2005 & 3 Jahre & Work Package Leader \\
\hline EUROPOLAR & Polarforschung & 2005 & 4 Jahre & Task Leader \\
\hline HERA & Geisteswissenschaften & 2005 & 4 Jahre & Partner \\
\hline BioDivERsA & Biodiversität & 2005 & 4 Jahre & Partner \\
NEURON & Neurowissenschaften & 2007 & 4 Jahre & Work Package Leader \\
\hline ASTRONET & Astronomie & 2005 & 4 Jahre & Associate Partner (seit 2007) \\
NORFACE & Sozialwissenschaften & 2004 & 5 Jahre & Associate Partner (seit 2007) \\
\hline Plant Genomics & Pflanzengenomik & 2006 & 4 Jahre & Call Teilnahme (2008) \\
\hline E-Rare & Seltene Krankheiten & 2006 & 4 Jahre & Call Teilnahme (2008)
\end{tabular}




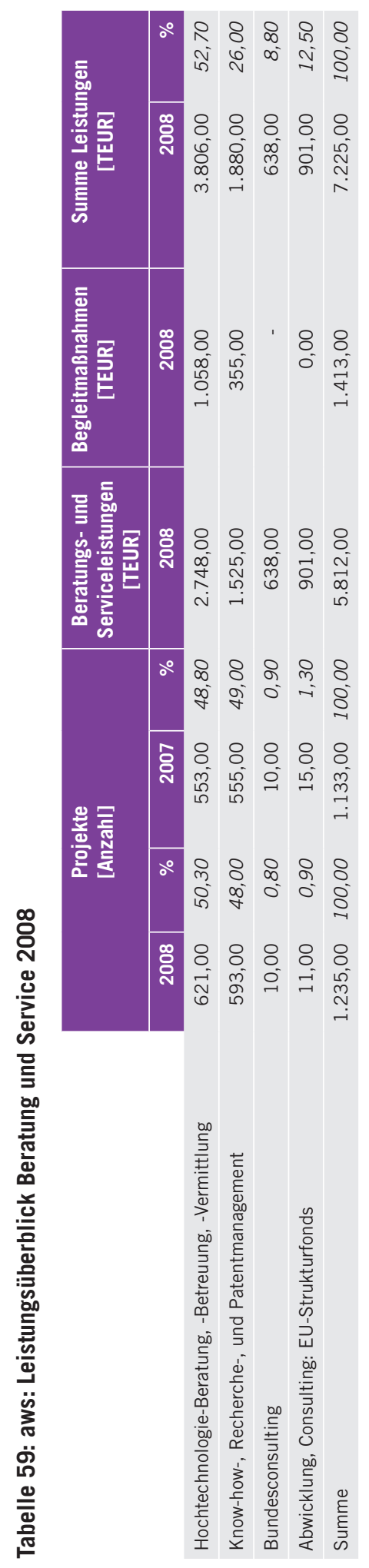


Tabelle 60: aws: Hochtechnologie: Beratung, Betreuung, Vermittlung (2008)

\begin{tabular}{|c|c|c|c|c|c|c|c|c|}
\hline & \multicolumn{4}{|c|}{$\begin{array}{l}\text { Projekte } \\
\text { [Anzahl] }\end{array}$} & \multirow{2}{*}{\begin{tabular}{|c}
$\begin{array}{c}\text { Beratungs- und } \\
\text { Serviceleistungen } \\
\text { [TEUR] }\end{array}$ \\
2008
\end{tabular}} & \multirow{2}{*}{$\begin{array}{c}\begin{array}{c}\text { Begleit- } \\
\text { maßnahmen } \\
\text { [TEUR] }\end{array} \\
2008\end{array}$} & \multicolumn{2}{|c|}{$\begin{array}{l}\text { Summe } \\
\text { Leistungen } \\
\text { [TEUR] }\end{array}$} \\
\hline & 2.008 & $\%$ & 2007 & $\%$ & & & 2008 & $\%$ \\
\hline Jugend Innovativ & 296 & 47,7 & 288 & 52,1 & 537 & - & 537 & 14,1 \\
\hline Staatspreis Innovation & 30 & 4,8 & & & 263 & - & 263 & 6,9 \\
\hline Life Science Austria & 114 & 18,4 & 122 & 22,1 & 1.021 & 300 & 1.321 & 34,7 \\
\hline Nano Netzwerke & - & - & 3 & 0,5 & - & - & - & - \\
\hline Beteiligungsvermittlung Business Angels & 54 & 8,6 & 55 & 9,9 & 286 & - & 286 & 7,5 \\
\hline Seedfinanzierung für High-Tech-Gründungen ${ }^{1}$ & 126 & 20,3 & 85 & 15,4 & 641 & 141 & 782 & 20,5 \\
\hline ImpulsProgramm creativwirtschaft & 1 & 0,2 & n.a. & n.a. & - & 617 & 617 & 16,3 \\
\hline Summe & 621 & 100,0 & 553 & 100,0 & 2.748 & 1.058 & 3.806 & 100,0 \\
\hline
\end{tabular}

1 Seedfinancing für High-Tech-Gründungen: 36 Neuanfragen, die in die laufende Betreuung übernommen werden, 90 aktuelle Portfolioprojekte in laufender Betreuung 